\title{
Phylogeny of Paleozoic limbed vertebrates reassessed through revision and expansion of the largest published relevant data matrix
}

\author{
David Marjanović Corresp., 1 , Michel Laurin ${ }^{2}$ \\ ${ }^{1}$ Science Programme "Evolution and Geoprocesses", Museum für Naturkunde - Leibniz Institute for Evolutionary and Biodiversity Research, Berlin, \\ Germany \\ 2 Centre de Recherches sur la Paléobiologie et les Paléoenvironnements (CR2P), Centre national de la Recherche scientifique (CNRS)/Muséum national \\ d'Histoire naturelle (MNHN)/Sorbonne Université, Paris, France \\ Corresponding Author: David Marjanović \\ Email address: david.marjanovic@gmx.at
}

The largest published phylogenetic analysis of early limbed vertebrates (Ruta M, Coates MI. 2007. Journal of Systematic Palaeontology 5:69-122) recovered e.g. Seymouriamorpha, Diadectomorpha and (in some trees) Caudata as paraphyletic and found the "temnospondyl hypothesis" on the origin of Lissamphibia (TH) to be more parsimonious than the "lepospondyl hypothesis" (LH) - though only, as we show, by one step.

We report 4200 misscored cells, over half of them due to typographic and similar accidental errors. Further, some characters were duplicated; some had only one described state; for one, most taxa were scored after presumed relatives. Even potentially continuous characters were unordered, the effects of ontogeny were not sufficiently taken into account, and data published after 2001 were mostly excluded.

After these issues are improved - we document and justify all changes to the matrix -, but no characters are added, we find (Analysis R1) much longer trees with e.g. monophyletic Caudata, Diadectomorpha and (in some trees) Seymouriamorpha; Ichthyostega either crownward or rootward of Acanthostega; and Anthracosauria either crownward or rootward of Temnospondyli. The LH is 9 steps shorter than the TH (R2; constrained) and 12 steps shorter than the "polyphyly hypothesis" (PH - R3; constrained).

Brachydectes (Lysorophia) is not found next to Lissamphibia; instead, a large clade that includes the adelogyrinids, urocordylid "nectrideans" and aïstopods occupies that position. As expected from the taxon/character ratio, most bootstrap values are low.

Adding 56 OTUs to the original 102 increases the resolution (and decreases most bootstrap values). The added taxa range in completeness from complete articulated skeletons to an incomplete lower jaw. Even though the lissamphibian-like temnospondyls Gerobatrachus, Micropholis and Tungussogyrinus and the extremely peramorphic salamander Chelotriton are added, the difference between LH (R4;

unconstrained) and $\mathrm{TH}$ (R5) rises to 10 steps, that between $\mathrm{LH}$ and $\mathrm{PH}$ (R6) to 15; the TH also requires several more regains of lost bones than the LH. Casineria, in which we tentatively identify a postbranchial lamina, emerges rather far from amniote origins in a gephyrostegid-chroniosuchian grade.

Bayesian inference (Analysis EB, settings as in R4) mostly agrees with R4. High posterior probabilities are found for Lissamphibia (1.00) and the LH (0.92); however, many branches remain weakly supported, and most are short, as expected from the small character sample. 
We discuss phylogeny, approaches to coding, methods of phylogenetics (Bayesian inference vs. equally weighted vs. reweighted parsimony), some character complexes (e.g. preaxial/postaxial polarity in limb development), and prospects for further improvement of this matrix. Even in its revised state, the matrix cannot provide a robust assessment of the phylogeny of early limbed vertebrates. Sufficient improvement will be laborious - but not difficult. 


\section{Phylogeny of Paleozoic limbed vertebrates reassessed 2 through revision and expansion of the largest 3 published relevant data matrix}

David Marjanović1 ${ }^{1}$ Michel Laurin ${ }^{2}$

${ }^{1}$ Science Programme "Evolution and Geoprocesses", Museum für Naturkunde - Leibniz

8 Institute for Evolutionary and Biodiversity Research, Berlin, Germany; ORCID: 0000-0001-

$9 \quad 9720-7726$

$10{ }^{2}$ Centre de Recherches sur la Paléobiologie et les Paléoenvironnements (CR2P), Centre national

11 de la Recherche scientifique (CNRS)/Muséum national d'Histoire naturelle (MNHN)/Sorbonne

12 Université, Paris, France; ORCID: 0000-0003-2974-9835

13

14 Corresponding author:

15 David Marjanović

16 david.marjanovic@gmx.at 


\section{Abstract}

The largest published phylogenetic analysis of early limbed vertebrates (Ruta M, Coates MI. 2007. Journal of Systematic Palaeontology 5:69-122) recovered e.g. Seymouriamorpha, Diadectomorpha and (in some trees) Caudata as paraphyletic and found the "temnospondyl hypothesis" on the origin of Lissamphibia (TH) to be more parsimonious than the "lepospondyl hypothesis" (LH) - though only, as we show, by one step.

We report 4200 misscored cells, over half of them due to typographic and similar accidental errors. Further, some characters were duplicated; some had only one described state; for one, most taxa were scored after presumed relatives. Even potentially continuous characters were unordered, the effects of ontogeny were not sufficiently taken into account, and data published after 2001 were mostly excluded.

After these issues are improved - we document and justify all changes to the matrix -, but no characters are added, we find (Analysis R1) much longer trees with e.g. monophyletic Caudata, Diadectomorpha and (in some trees) Seymouriamorpha; Ichthyostega either crownward or rootward of Acanthostega; and Anthracosauria either crownward or rootward of Temnospondyli. The LH is 9 steps shorter than the TH (R2; constrained) and 12 steps shorter than the "polyphyly hypothesis" (PH - R3; constrained). Brachydectes (Lysorophia) is not found next to Lissamphibia; instead, a large clade that includes the adelogyrinids, urocordylid "nectrideans" and aïstopods occupies that position. As expected from the taxon/character ratio, most bootstrap values are low.

Adding 56 OTUs to the original 102 increases the resolution (and decreases most bootstrap values). The added taxa range in completeness from complete articulated skeletons to an incomplete lower jaw. Even though the lissamphibian-like temnospondyls Gerobatrachus, Micropholis and Tungussogyrinus and the extremely peramorphic salamander Chelotriton are added, the difference between LH (R4; unconstrained) and TH (R5) rises to 10 steps, that between $\mathrm{LH}$ and $\mathrm{PH}$ (R6) to 15; the TH also requires several more regains of lost bones than the LH. Casineria, in which we tentatively identify a postbranchial lamina, emerges rather far from amniote origins in a gephyrostegid-chroniosuchian grade.

Bayesian inference (Analysis EB, settings as in R4) mostly agrees with R4. High posterior probabilities are found for Lissamphibia (1.00) and the LH (0.92); however, many branches remain weakly supported, and most are short, as expected from the small character sample.

We discuss phylogeny, approaches to coding, methods of phylogenetics (Bayesian inference vs. equally weighted vs. reweighted parsimony), some character complexes (e.g. preaxial/postaxial polarity in limb development), and prospects for further improvement of this matrix. Even in its revised state, the matrix cannot provide a robust assessment of the phylogeny of early limbed vertebrates. Sufficient improvement will be laborious - but not difficult.

\section{Introduction}

\footnotetext{
This ancient inhabitant of the coal swamps of Nova Scotia, was, in short, as we often find to be the case with the earliest forms of life, the possessor of powers and structures not usually, in the modern world, combined in a single species. It was certainly not a fish, yet its bony scales, and the form of its vertebræ, and of its teeth, might, in the absence of other evidence, cause it to be mistaken for one. We call it a batrachian, yet its dentition, the sculpturing of the bones of its skull, which were certainly no more external plates than the similar bones of a crocodile, its ribs, and the structure of
} 
64

65

66

67

68

69

70

71

72

73

74

75

76

77

78

79

80

81

82

83

84

85

86

87

88

89

90

91

92

93

94

95

96

97

98

99

100

101

102

103

104

105

106

107

108

109

110

111

112

113

114

115

its limbs, remind us of the higher reptiles; and we do not know that it ever possessed gills, or passed through a larval or fish-like condition. Still, in a great many important characters, its structures are undoubtedly batrachian. It stands, in short, in the same position with the Lepidodendra and Sigillarice under whose shade it crept, which though placed by palæo-botanists in alliance with certain modern groups of plants, manifestly differed from these in many of their characters, and occupied a different position in nature. In the coal period, the distinctions of physical and vital conditions were not well defined - dry land and water, terrestrial and aquatic plants and animals, and lower and higher forms of animal and vegetable life, are consequently not easily separated from each other.

- Dawson (1863: 23-24) about Dendrerpeton acadianum

Homoplasy Is even More Common than I, or perhaps Anyone, Has ever Imagined - section headline in Wake (2009: 343)

What is required is a more complete discussion of the character coding of previous data matrices, and a thorough reanalysis based on those matrices. This would enable a well-founded discussion of lissamphibian origins in light of a supermatrix based on all the current and pertinent data. - Sigurdsen \& Green (2011: 459)

Giant morphological data matrices are increasingly common in cladistic analyses of vertebrate phylogeny, reporting numbers of characters never seen or expected before. However, the concern for size is usually not followed by an equivalent, if any, concern for character construction/selection criteria. Therefore, the question of whether quantity parallels quality for such influential works remains open.

- Simões et al. (2016a: abstract)

Not too surprisingly, as it is yet a youthful paradigm shift, modern phylogenetic systematics is still evolving to improve on the lack of precision, rigour and objectivity it inherited from the pre-cladistic period. Furthermore, transforming a descriptive science (morphological description) bounded by language as a means of outlining empirical observations into hopefully objectively delimited characters and character-states is a difficult task; every effort to do so is to be commended, while at the same time rigorously scrutinized and improved upon.

- Simões et al. (2016a: 215)

Phylogenetic trees are hypotheses that attempt to explain a data matrix. Much work has gone, to great success, into the methods for generating and testing phylogenetic hypotheses from a given data matrix; and a matrix of molecular data can, apart from the problem of alignment, be largely taken for granted as a set of observed facts. But molecular data are not always available. In many cases phylogeneticists have to rely on morphological data - and a matrix of morphological data is a matrix of hypotheses. The characters, their states, and the relationships between the states are hypotheses that rely on hypotheses about homology, about independent evolution from other characters, about ontogeny and even preservation (especially in the case of fossils); the terminal taxa (OTUs) are hypotheses that rely on hypotheses of monophyly, ontogeny and again preservation; and even given all these, each cell in a data matrix is still a hypothesis that relies on hypotheses of homology, ontogeny and preservation - some are close enough to observed facts, others less so. In addition, morphological data matrices can only be compiled by hand - there is no equivalent to sequencer machines or alignment programs. This makes human error inevitable. Consequently, morphological data matrices must not be taken for granted as sets of objective data; the hypotheses of which they consist must be identified and carefully tested.

The analysis by Ruta \& Coates (2007 - hereinafter RC07) has played a large role in shaping current ideas on the phylogeny and early evolution (Late Devonian to Cisuralian, with a few younger taxa) of the limbed vertebrates, including the origins of amniotes and 
116 lissamphibians. Being based on the largest matrix so far applied to this problem, its results have

117 been widely treated as a consensus and even used as the basis for further work in evolutionary

118 biology (Bernardi et al., 2016; MacIver et al., 2017). However, several conflicting phylogenetic

119 results have persisted in other analyses based on different matrices (Vallin \& Laurin, 2004;

120 Marjanović \& Laurin, 2008, 2009; Sigurdsen \& Green, 2011; Pardo et al., 2017; Pardo, Small \&

121 Huttenlocker, 2017: fig. 2, S6, S7). Although the large differences in character sampling between

122 any two of these analyses may be the greatest contribution to their discrepancies (Pardo et al.,

123 2017; compare also Cau, 2018a), it is also possible that some of the differences between these

124 trees may be a function of taxon sampling, analytical parameters like ordering, choice of

125 optimization criterion (parsimony or Bayesian inference), correlation between characters (due to

126 different treatments of ontogeny and heterochrony or other sources of large-scale convergence,

127 or to outright duplication of characters in the same matrix), or accidental misscores (Vallin \&

128 Laurin, 2004; Wiens, Bonett \& Chippindale, 2005; Tykoski, 2005; Pawley, 2006; Marjanović \&

129 Laurin, 2008, 2009, 2013a; Sigurdsen \& Green, 2011; Langer et al., 2017; Spindler et al., 2018:

130 online resource 3 ). We have aimed to test this complex of hypotheses rigorously by reevaluating

132

133

134

135

136

137

138

139

140

Exhaustive treatment of characters and taxa is the most appropriate way to disentangle contrasting phylogenetic signals in large matrices.

$-\mathrm{RC} 07$ (abstract)

Ruta, Coates \& Quicke (2003) and RC07 presented two successive versions of a new matrix, discussed taxa and characters, analyzed their matrices with various methods and constraints, and used the resulting trees as a starting point for a review of the phylogeny of limbed vertebrates in general and the origin of lissamphibians in particular. Similarly, we have investigated the following questions.

\section{Accuracy of analysis procedure}

\section{- Did RC07 find all of the most parsimonious trees (MPTs) that fit their matrix?}

This may seem trivial, but Matsumoto et al. (2013) reported that the software PAUP* 4.0b10 found three times as many MPTs as TNT 1.0 did when used on their matrix; conversely, Schoch (2013: 682) reported that PAUP 3.1 did not find any trees as short as those recovered by TNT; Baron, Norman \& Barrett (2017) found only 93 of 16,632 MPTs, having neglected to run a second round of tree bisection and reconnection on their TNT trees (Watanabe, 2017a, b; Langer et al., 2017: supplementary information: 26); likewise using TNT, Cau (2018a) found only 3,072 of 10,872 MPTs (Mortimer, 2018; Cau, 2018b); and Skutschas \& Gubin (2012) found that the "parsimony ratchet", a procedure for reducing calculation time (Ruta, Coates \& Quicke, 2003), did not find any trees less than 35 steps longer than the MPTs. RC07 used the parsimony ratchet. We therefore repeated their analysis without using the ratchet (Analysis O1 - see Table 1 and Fig. 1). 
172

173

174

- What is the difference in tree length between the MPTs of RC07, which find Lissamphibia nested among the temnospondyls (Fig. 1), and the shortest trees compatible with their matrix that are constrained to place Lissamphibia among the "lepospondyls"?

RC07 reported that this difference is both nine steps (p. 85) and 15 steps (p. 86). The first resulted from an unpublished constraint compatible with the tree of Laurin (1998a), which is not the only possibility for where and how Lissamphibia could be placed among the "lepospondyls". RC07 did not publish or describe the constraint they used for the second. We therefore created a new constraint, which we describe explicitly below, and used it in a second analysis of their matrix (Analysis O2). A third (Analysis O3) is constrained against lissamphibian monophyly and is useful for certain comparisons.

\section{Accuracy of the matrix of $R C 07$}

Analogously to the problem of alignment in molecular analyses, morphological analyses begin with the construction of a dataset, where characters need to be defined in ways that prevent them from being redundant. (When the state of a character is predictable from the state of another character, the characters are redundant for the purposes of phylogenetic analysis; to use redundant characters amounts to counting the same character at least twice, doubling or multiplying its influence on the results.) Observations need to be interpreted in terms of these characters, and then these interpretations need to be inserted in the data matrix by hand. In our own practice, we have every once in a while caught ourselves making typographic errors, being momentarily confused about which state is 0 and which is 1 (because of faulty memory as well as conflicting conventions on how to assign such numbers -0 can for instance mean "absent" or "presumably plesiomorphic"), inserting the right value in the wrong column or line, and committing similar blunders; additionally, we have on occasion misinterpreted the descriptive literature and its illustrations (line drawings, but even photographs, can give misleading threedimensional impressions), overlooked poorly known publications, had language barriers or conflicting terminologies prevent us from being sure if a published sentence said one thing or the opposite, or simply relied on the then current state of research that was later overturned when the next publication came out. It stands to reason that these things also happen to other people.

Following the reevaluations by Marjanović \& Laurin (2008, 2009: supplementary information) of the matrices by McGowan (2002) and Anderson et al. (2008a), those by Sigurdsen \& Green (2011) of the matrices by Vallin \& Laurin (2004), RC07 and Anderson et al. (2008a) and the one by Langer et al. (2017) of the matrix by Baron, Norman \& Upchurch (2017), we scrutinized the matrix of $\mathrm{RC} 07$ in the light of the following questions:

- Are there redundant characters or accidental misscores in the matrix of RC07?

- If there are any, and if we revise them, does that change the resulting trees?

These questions account for the bulk of the work we present here. All changes to the matrix, most of which are of these kinds, are documented and justified in Appendix S1, the commented character list, which comprises more than half of the total text of the present publication. The revised matrix is presented in human-readable form as Appendix S2, where the changes are highlighted in color, and in NEXUS format as Data S3. 
206

207

208

209

210

211

212

213

214

215

216

217

218

219

220

221

222

223

224

225

226

227

228

229

230

231

232

233

234

235

236

237

238

239

240

241

242

243

244

245

246

247

248

249

250

We should stress that we did not make changes to the matrix in order to test whether they are sufficient for obtaining different MPTs from the ones found by RC07 (as a reviewer put it: what it takes to "break" their matrix). Neither did we restrict our changes to information Ruta \& Coates could have known in 2007 (or 2006, when they submitted their manuscript); the context in which the matrix was made is not a subject of our study. Rather, we have tried to identify all redundant characters and all misscores (regardless of whatever their sources may be), deal with all of these potential problems to the best of our current abilities, perform new phylogenetic analyses on the revised matrix (Analyses R1-R3; see Table 1), and report how the resulting MPTs differ from the ones found by RC07 in lengths, topologies, indices and bootstrap support (Analysis B1). The question of how many additional steps are required to obtain different hypotheses of lissamphibian origins is tested by constrained analyses (R2, R3) as in RC07; these numbers are far lower than the total of our changes to the matrix.

In all likelihood, accidental misscores should be a good approximation to random noise. Such noise is expected to produce many weak false signals which cancel each other out instead of accumulating into a challenge to the true signal. However, when the true signal is weak to begin with (perhaps due to a character sample which is small enough to cause accidental sampling bias) and one or a few strong false signals are already present (due to large-scale evolutionary convergence or redundant characters), random noise added to the true and false signals may change the balance from slightly in favor of the true signal to slightly in favor of a false signal - or indeed from one false signal to another, so that efforts to reduce the strength of the first false signal will not make the true signal stand out.

Our methods for identifying and attempting to deal with redundant characters - in some cases a hard problem on which we expect future advances - are explained below (Material and methods: Treatment of characters). This includes ontogeny-related characters: taxa known only from immature or paedomorphic individuals will predictably have "immature" states of many characters, with dramatic consequences for the resulting trees such as clustering of these taxa into spurious clades (Wiens, Bonett \& Chippindale, 2005). Our approach to this difficult problem, modified from the recommendation of Wiens, Bonett \& Chippindale (2005: 96), was independently proposed by Tykoski (2005), Pawley (2006: 206) and Marjanović \& Laurin (2008); it was explained in more detail by Marjanović \& Laurin (2013a) and is presented again below (Material and methods: Treatment of characters: "Ontogeny discombobulates phylogeny"). The changes made to the matrix for this reason are likewise documented and justified in Appendix S1; they are also marked in blue in Appendix S2 and counted in Data S4.

As mentioned, Sigurdsen \& Green (2011) performed their own reevaluation of the matrix of RC07. That work, however, had a much more limited scope than ours (see Marjanović \& Laurin, 2013a, for discussion). We have incorporated most, though not all, of the changes to individual cells suggested in it (as discussed under the respective characters in Appendix S1). Unlike Sigurdsen \& Green (2011), we have not deleted characters of unclear value.

\section{Phylogeny of early limbed vertebrates}

By total number of scores, the matrix of RC07 is the largest published one that concerns the phylogeny of early (roughly Paleozoic) limbed vertebrates other than amniotes. If we have come satisfactorily close to solving the problems presented above, our modified matrix should therefore be better suited to investigating the following questions, among others, than any other 
251 matrix published so far, even though it cannot treat all of them in sufficient depth. Compare Fig.

252

253

254

255

256

257

258

259

260

261

262

263

264

265

266

267

268

269

270

271

272

273

274

275

276

277

278

279

280

281

282

283

284

285

286

287

288

289

290

291

292

293

294

295

1:

- Are lissamphibians temnospondyls, "lepospondyls" or (diphyletically) both?

- How strong is the support for each of these hypotheses?

- Are the albanerpetids lissamphibians? What are their closest relatives?

- Do the traditional diadectomorphs form a clade?

- Do the traditional "microsaurs" form a clade (including or excluding any lissamphibians)?

- Do the traditional "lepospondyls", or some of them, form a clade (including or excluding any lissamphibians)?

- Do the traditional seymouriamorphs form a clade?

- Are the traditional seymouriamorphs or any traditional "lepospondyls", especially "microsaurs", closer to Amniota?

- What can be said about temnospondyl phylogeny?

- Is Anthracosauria or Temnospondyli closer to Amniota?

- What are the phylogenetic positions of Solenodonsaurus, Gephyrostegus, Bruktererpeton, Caerorhachis, Silvanerpeton and Tulerpeton (all have been connected to anthracosaur origins at one point or another)?

- Are Adelogyrinidae and Acherontiscus "lepospondyls" or close to the colosteids?

- Is Colosteidae or Whatcheeriidae closer to the tetrapod crown-group?

- Is Ichthyostega or Acanthostega closer to the tetrapod crown-group?

- What happens to the above questions when taxa are added (Analyses R4-R6 and B2; see Table 1 and the "Phylogenetic background" section below)?

- The addition of taxa allows us to test further questions such as:

- What is the phylogenetic position of Chroniosuchia and several other taxa?

- Are the traditional diadectomorphs amniotes, or are they the closest relatives of Amniota?

- Is Casineria close to amniote origins?

These questions are presented in more detail in the "Phylogenetic background" section below and reviewed in the Discussion (section "Phylogenetic relationships").

The addition of taxa required doubling the number of analyses of the revised matrix. To avoid another duplication, we did not add any characters; adding characters will be part of future work. However, we discuss a few characters - both inside and outside the present matrix - that have recently been connected to lissamphibian origins (Discussion: Characters: subsections other than the first and part of the second).

\section{The effects of different methods of analysis}

- Does a Bayesian analysis of our revised matrix support a different tree than parsimony?

All of the analyses mentioned above used the nonparametric method somewhat misleadingly called "parsimony" or "maximum parsimony". For comparison, we also applied Bayesian inference to our revised matrix. The behavior of Bayesian inference under the conditions of this 
296

297

298

299

300

301

302

303

304

305

306

307

308

309

310

311

312

313

314

315

316

317

318

319

320

321

322

323

324

325

326

327

328

329

330

331

332

333

334

335

336

337

338

339

340

matrix are not well understood (Discussion: Bayesian inference and parsimony in comparison), and Bayesian analyses are time-consuming; we therefore ran only one analysis (under the same conditions as R4 and B2: enlarged taxon sample, no constraints) which we consider exploratory (Analysis EB; see Table 1). According to recent simulations, Bayesian inference has advantages over parsimony (Wright \& Hillis, 2014; O'Reilly et al., 2016, 2017; Puttick et al., 2017; but see Simmons, 2011a, b; Brown et al., 2017; Goloboff, Torres \& Arias, 2017); in particular, it is much less sensitive to long-branch attraction, which may be a concern with some of the "weirder" taxa in our sample like adelospondyls, aïstopods or indeed lissamphibians.

\section{Phylogenetic background}

The early phylogeny of the limbed vertebrates contains a number of open questions on which there is either no consensus, or the existing consensus is weakly supported. Most famously, the origin of the modern amphibians (Lissamphibia and its possible member or sister-group Albanerpetidae - see below on that name) remains a vexing problem (Fig. 2). From the late $19^{\text {th }}$ century to now, the modern amphibians have been considered temnospondyls by some (Fig. $2 \mathrm{C}-$ "temnospondyl hypothesis", abbreviated as TH below; most recently found by: RC07; Sigurdsen \& Green, 2011; Pardo, Small \& Huttenlocker, 2017: fig. S6; Pardo et al., 2017), lepospondyls by others (Fig. 2D - "lepospondyl hypothesis", abbreviated as LH below; Vallin \& Laurin, 2004; Pawley, 2006: appendix 16; Marjanović \& Laurin, 2008, 2009, 2013a), and polyphyletic by yet others (Fig. 2E - "polyphyly hypothesis", abbreviated as PH below; Carroll, 2007; Huttenlocker et al., 2013), with Salientia being nested among the temnospondyls, Gymnophionomorpha among the lepospondyls, and Caudata either in the lepospondyls (all early works, e.g. Carroll \& Holmes, 1980) or in the temnospondyls (works published in the $21^{\text {st }}$ century).

This particular question has far-reaching implications for the interpretation of most of our taxon sample. For example, the seymouriamorphs - considered close to amniote origins for most of the $20^{\text {th }}$ century, though known to have gilled juveniles and possibly neotenes -indeed lie on the amniote stem under the TH (Fig. 1); from phylogenetic bracketing, it follows that the unfossilized parts of their anatomy and behavior were more amniote-like than found in lissamphibians and lay within the range of other crown-group tetrapods in the absence of fossil evidence to the contrary. Under the $\mathrm{PH}$, the seymouriamorphs lie on the common stem of amniotes and caecilians to the exclusion of batrachians (salientians + caudates); the last common ancestor of the extant amphibians was thus the last common ancestor of all crown-group tetrapods, and those of its features retained by both batrachians and caecilians should be expected to have persisted in the seymouriamorphs unless there is evidence to the contrary. Under the LH, in contrast, the seymouriamorphs are (most likely, see below) stem-tetrapods, bracketed by extant tetrapods on only one side, and may have been less similar to extant tetrapods than the $\mathrm{TH}$ or the $\mathrm{PH}$ predict. Likewise, the diverse temnospondyls are stemamphibians under the TH (Fig. 1, 2C), bracketed by lissamphibians and amniotes among extant taxa; under the PH (Fig. 2E), they belong to the batrachian stem, bracketed by extant amphibians on both sides, so that a great many features found among extant amphibians should be expected to have been shared by temnospondyls (validating a large amount of existing literature and artwork); under the LH, however (Fig. 2D), they are not crown-group tetrapods at all, but lie fairly far rootward on their stem and should be expected to be unlike anything alive today in an unknown but large number of aspects. (The new version of the TH by Pardo, Small \& 
341 Huttenlocker, 2017: fig. 2, S7 but not S6, makes much the same predictions as the PH in this

342

343

344

345

346

347

348

349

350

351

352

353

354

355

356

357

358

359

360

361

362

363

364

365

366

367

368

369

370

371

372

373

374

375

376

377

378

379

380

381

382

383

384

385

386 regard because it turns most temnospondyls into lissamphibians.)

Among other more or less open questions are the phylogenies of Temnospondyli (e.g. Pawley, 2006; Ruta, 2009; Dilkes, 2015a; Pardo, Small \& Huttenlocker, 2017), "Lepospondyli" (RC07; Huttenlocker et al., 2013; Marjanović \& Laurin, 2013a; Pardo, Small \& Huttenlocker, 2017: fig. S6) and Devonian limbed vertebrates (e.g., Ahlberg \& Clack, 1998; Clack et al., 2012a; Pardo et al., 2017), as well as the relative positions of Anthracosauria and Temnospondyli (Laurin \& Reisz, 1999; RC07; Pardo et al., 2017) and the position of Diadectomorpha inside or next to Amniota (Berman, Sumida \& Lombard, 1992; Berman, 2013), not to mention the positions of confusing (Andrews \& Carroll, 1991; Smithson et al., 1994; Clack, 2001; Ruta, Milner \& Clack, 2002; Vallin \& Laurin, 2004; RC07) or fragmentary Carboniferous taxa (Smithson, 1980; Paton, Smithson \& Clack, 1999; Bolt \& Lombard, 2006; Clack et al., 2012b, 2016; Sookias, Böhmer \& Clack, 2014).

Molecular data are of limited use for tackling these questions: of all tetrapodomorphs (tetrapods and everything closer to them than to lungfish), only frogs, salamanders, caecilians and amniotes still have living members (Fig. 2). Thus, molecular data cannot test, say, whether Amniota is closer to Anthracosauria or to Temnospondyli, or if the many disparate taxa classified as "Lepospondyli" constitute a clade, a grade, or a wastebasket. When it comes to the origins of the extant amphibians, molecular data can distinguish the PH (Fig. 2E) from the other hypotheses, because the $\mathrm{PH}$ predicts that the extant amphibians are paraphyletic with respect to Amniota, while the other two hypotheses of course predict monophyly under recent conceptions of the affinities of temnospondyls, lepospondyls and amniotes (Fig. 2B; Laurin, 2002; see also Marjanović \& Laurin, 2007, 2013a); but molecular data cannot distinguish the two monophyly hypotheses, because too many relevant taxa have been extinct for too long. Finding the extant amphibians monophyletic with respect to Amniota (Fig. 2A), analyses of molecular data support both monophyly hypotheses equally (Fig. 2A, C, D); only paleontological data can distinguish them. Existing analyses of paleontological data, however, disagree greatly on this question (see above; Fig. 2C-E) as well as on others. To some extent, no doubt, this is due to the many differences in their taxon and character samples. Another possible reason, however, are problems in datasets of the kinds presented above (Aims: Accuracy of the matrix of RC07). When such misscores and miscodings are removed from matrices - without, as far as possible, changing the taxon or character sample -, do the results change?

The largest published morphological data matrix that has been applied to the problems of the phylogeny of limbed vertebrates in general and the origin of the modern amphibians in particular is that by RC07; it supported the $\mathrm{TH}$ and is often cited for this result. Here we reevaluate this matrix in order to test, and explain within the limitations of the dataset, to what degree this result - and others that together constitute the consensus tree of RC07 (fig. 5, 6; our Fig. 1) - continues to follow from their dataset after a thorough effort to improve the accuracy of the scoring and reduce character redundancy has been carried out to the best of our current knowledge.

Naturally, this effort will not suffice to solve the question of lissamphibian origins or any other of the many controversies in the phylogeny of early limbed vertebrates. A quick look at matrices such as those of Ruta (2009), Sigurdsen \& Green (2011), Maddin, Jenkins \& Anderson (2012), Sookias, Böhmer \& Clack (2014), Dilkes (2015a), Clack et al. (2016), Pardo, Small \& Huttenlocker (2017) or Pardo et al. (2017), or at reinvestigations of anatomy such as those of Witzmann (2007, 2010, 2013), Bolt \& Lombard (2010), Mondéjar-Fernández, Clément \& 
387 Sanchez (2014), Dilkes (2015a) or Pardo et al. (2017) and references therein will demonstrate 388 that many characters which are known to carry phylogenetic signal for the present taxon sample remain absent from this matrix; this even includes some of the very few (41) characters used by McGowan (2002). Adding them (as well as yet more taxa) will be part of future work, and may well lead to trees with a different topology. However, we think the present work forms a necessary step towards solving any of those problems. Further progress may come from larger matrices - if and only if the increase in the number of cells is not accompanied by a proportional decrease in the care that goes into scoring them (Simões et al., 2016a).

Originally we did not intend to add any taxa to the matrix, just as we have not added any characters. However, soon after the work of RC07 was published, the intriguing amphibamid temnospondyl Gerobatrachus was described and was argued to add strong support to the $\mathrm{PH}$ (Anderson et al., 2008a). Phylogenetic analyses that included Gerobatrachus in different versions of the same matrix have supported the PH (Anderson et al., 2008a), the LH (Marjanović \& Laurin, 2009), or more recently the TH (Maddin \& Anderson, 2012; Maddin, Jenkins \& Anderson, 2012); the latter work even found Gerobatrachus to be nested within Lissamphibia (partially replicated by Pardo, Small \& Huttenlocker, 2017: fig. S6B, S7B; trivially replicated by Pardo, Small \& Huttenlocker, 2017: fig. 2, S7A, where most temnospondyls are lissamphibians; not replicated by Pardo, Small \& Huttenlocker, 2017: fig. S6A or by Pardo et al., 2017, where Gerobatrachus and Lissamphibia are sister-groups as in Maddin \& Anderson, 2012). Clearly, Gerobatrachus is too important to be left out. Following the example of Marjanović \& Laurin (2008) and Langer et al. (2017), we have therefore performed a separate series of analyses (R4R6, B2) for which we added Gerobatrachus to the matrix; at that opportunity, for the same series of additional analyses, we added a further 55 OTUs as detailed and justified in Material and methods: OTUs added for a separate set of analyses, bringing the total from 102 to 158 .

In the Discussion section, we explore the relationships of the sampled taxa and the distributions of certain character states in the light of our findings and other recent publications. By presenting the current areas of uncertainty (some expected, some unexpected), we hope to highlight opportunities for future research.

\section{Abbreviations}

AMNH: properly AMNH DVP or AMNH FARB - Department of Vertebrate Paleontology or Collection of Fossil Amphibians, Reptiles and Birds at the American Museum of Natural History, New York. App./app., Appendix (our S1 or S2)/appendix (of cited works); all App.-Tables are part of App. S1.

BEG: Vertebrate Paleontology Laboratory, The University of Texas at Austin (formerly Bureau of Economic Geology).

BPE: bootstrap percentage given the expanded taxon sample (bootstrap analysis B2). BPO: bootstrap percentage given the original taxon sample (bootstrap analysis B1). CG78: Carroll \& Gaskill (1978). ch.: character. ci: consistency index (in lowercase to make the numbers stand out). CM: Carnegie Museum of Natural History, Pittsburgh. LH: "lepospondyl hypothesis" on the origin of lissamphibians. hi: homoplasy index. 
433 ITRI: inter-tree retention index (Grand et al., 2013). This is an asymmetric measure of how many 434 of the three-item statements that constitute a reference tree are shared by the tree in question, 435 taking into account the number of degrees of freedom.

436 MB.Am.: "amphibian" in the vertebrate paleontology collection of the Museum für Naturkunde, 437 Berlin.

438 MCZ: properly MCZ VPRA - "reptile" or "amphibian" in the vertebrate paleontology collection 439 of the Museum of Comparative Zoology, Harvard University, Cambridge (Massachusetts).

440 MGUH: Geologisk Museum, Statens Naturhistoriske Museum, Københavns Universitet, 441 Copenhagen ("Museum Geologicum Universitatis Hafniensis").

442 MNHN: Muséum national d'Histoire naturelle, Paris.

443 MNN MOR: Moradi collection of the Musée national du Niger, currently kept at the Burke 444 Museum, University of Washington, Seattle.

445 MPT: most parsimonious tree.

446 NHMW: Naturhistorisches Museum Wien, Vienna.

447 NMS: National Museum of Scotland, Edinburgh.

448 NSM: Nova Scotia Museum, Halifax.

449 OTU: operational taxonomic unit (a line in a data matrix).

450 PH: "polyphyly hypothesis" on the origin of "lissamphibians".

451 PIN: Paleontological Institute of the Academy of Sciences of the Russian Federation, Moscow.

452 PP: posterior probability in percent given the expanded taxon sample (exploratory Bayesian

453 analysis EB).

454 PU-ANF: "amphibian" specimens from Puertollano (Spain) in the vertebrate collection of the

455 Department of Paleontology, Geology division, Universidad Complutense, Madrid; formerly

456 kept at the Museum für Naturkunde, Berlin.

457 rc: rescaled consistency index.

458 RC07: Ruta \& Coates (2007).

459 ri: retention index.

460 TH: "temnospondyl hypothesis" on the origin of lissamphibians.

461 TMM: Vertebrate Paleontology Laboratory, The University of Texas at Austin (formerly Texas

462 Memorial Museum).

463 USNM: National Museum of Natural History, Smithsonian Institution, Washington (DC).

464

465

466

467

468

469

470

471

472

473

474

475

476

477

478 YPM: Yale Peabody Museum, New Haven (Connecticut).

Abbreviations not listed here that consist of at least three letters, at least one space and a number designate characters, following the practice of RC07; see App. S1. Note that merged characters keep the abbreviations of all their constituents: e.g., PREMAX 1-2-3 (ch. 1) is built from the three characters PREMAX 1, PREMAX 2 and PREMAX 3 of RC07, and MAX 5/PAL 5 (ch. 22) consists of MAX 5 and PAL 5 of RC07.

\section{Nomenclature}

A few remarks are necessary to explain our use of certain terms.

\section{Taxonomic nomenclature}

Without mentioning the fact that they were doing so, Averianov \& Sues (2012: 466) corrected the spelling of Albanerpetontidae Fox \& Naylor, 1982, to Albanerpetidae. We follow this in 
479 analogy to several corrections by Martín, Alonzo-Zarazaga \& Sanchiz (2012) as discussed by 480 Marjanović \& Laurin (2013b: 543), who did not know of Averianov \& Sues (2012) and therefore 481 incorrectly claimed that "no other spelling [than Albanerpetontidae] has ever been used", as well as in analogy to several further corrections by Schoch \& Milner (2014). Assuming that this correction is a "justified emendation", the name Albanerpetidae must continue to be attributed to Fox \& Naylor, 1982 (International Commission on Zoological Nomenclature, 1999: articles 19.2, 29).

Likewise, the spelling Hapsidopareiontidae must be corrected to Hapsidopareiidae Daly, 1973: there is no basis for -ont- in Homeric Greek $\pi \alpha \rho$ ïiov ("cheek") (Perseus Digital Library, accessed 5 November 2017). This name has been used so rarely that the question of common usage does not arise.

The name Diploradus Clack \& T. R. Smithson in Clack et al., 2016, was explained as follows (Clack et al., 2016: 3): "Genus from diplo (Greek) 'double' and radus (Greek) 'row' referring to the double coronoid tooth row." We have not been able to find a word similar to "radus" in the Greek or for that matter Latin dictionaries in the Perseus Digital Library ("Dictionary Entry Lookup" and "English-to-[Language] lookup" in the "General Search Tools" http://www.perseus.tufts.edu/hopper/search accessed 5 November 2017). The closest in form and meaning appears to be Latin radius, originally meaning "staff, rod". (The one language we have found where rad means "row" is Slovak.) However, this does not affect the validity of the name.

\section{Formal and informal phylogenetic nomenclature}

Because of the length of this paper and because the International Code of Phylogenetic Nomenclature ("PhyloCode") has not yet taken effect, we refrain from proposing new clade names or definitions. Having, however, noticed that the names Adelogyrinidae and Adelospondyli currently refer to indistinguishable taxa, we follow Ruta, Coates \& Quicke (2003, especially p. 284: "Acherontiscus is an adelospondyl"), RC07: 81 (but not fig. 5) and Coates, Ruta \& Friedman (2008: fig. 2: "Adelospondyli") in informally referring to a clade composed of Acherontiscus and Adelogyrinidae as "adelospondyls" for brevity, always excluding the historically included lysorophians.

As in a previous paper (Marjanović \& Laurin, 2013a), where we failed to make this explicit, we use "modern amphibians" for Lissamphibia and its possible member or sister-group Albanerpetidae.

Several names for temnospondyl clades, including Temnospondyli itself, have been given very different definitions by Yates \& Warren (2000) and Schoch (2013). We have generally applied the definitions by Yates \& Warren (2000). The important exceptions are Dissorophoidea and Stereospondylomorpha, which we use in the text for clades containing all those OTUs that have traditionally been regarded as members of clades with these names and exclude most or all OTUs that have traditionally not been regarded as members. For Dissorophoidea, we indicate both definitions and our usage in the tree figures (our usage usually, but not always, coincides with the definition by Yates \& Warren, 2000).

\section{Anatomical nomenclature}

Consistently or nearly so, RC07 (as also Pardo, Szostakiwskyj \& Anderson, 2015) exchanged the terms "skull roof" and "skull table". The former refers, in most other works, to the 
525 dermatocranium except its ventral side (the palate); the latter refers to the dorsal side of the skull

526

527

528

529

530

531

532

533

534

535

536

537

538

539

540

541

542

543

544

545

546

547

548

549

550

551

552

553

554

555

556

557

558

559

560

561

562

563

564

565

566

567

568

569

570

roof, often demarcated from the lateral sides by distinct edges.

RC07 (and Ruta, Coates \& Quicke, 2003) also almost consistently used "mesial" when they were aiming at "medial". This appears to be a common British practice; internationally, however, it is "medial" that means "toward the sagittal plane at a right angle to it", while "mesial" belongs to toothrow nomenclature and means "toward the symphysis along the curvature of the jaw", the opposite of the poorly chosen term "distal". Many instances of "mesial" apply to the lower jaw and actually mean "lingual", "proximal at a $90^{\circ}$ angle to the curvature of the jaw", the opposite of "labial"; only at the symphysis is mesial medial.

We have tried to rectify these issues in the annotated character list (App. S1). However, we use the pairs "anterior - posterior" and "cranial/rostral - caudal" interchangeably (despite a preference for the latter) because there is, in our taxon sample, no danger of confusion.

Our ambiguous usage of “orbit” (Marjanović \& Laurin, 2008, 2013a) confused Pardo \& Anderson (2016), who claimed that we had first thought that Brachydectes had very large eyes which filled its orbitotemporal fenestrae (ascribed to our 2008 paper) and that we had later amended this view (ascribed to 2013a); in reality, we explicitly stated (2008: 194-195) that we considered the "orbits (or 'orbitotemporal fenestrae' as they are sometimes called in salientians and caudates)" to have "presumably" contained jaw muscles in Brachydectes, not just the eyeballs, and we implied no changes in the later paper (Marjanović \& Laurin, 2013a: 239, 241). - In the present work we consistently speak of "orbitotemporal fenestrae" to mean skull openings that appear to have contained the eyes as well as jaw muscles, regardless of the inferred homologies of these openings.

\section{Material and methods}

The data matrices were edited in successive versions of Mesquite up to 3.31 (Maddison \& Maddison, 2017); this program was also used to display and visually compare trees and to optimize characters on them.

\section{Treatment of characters}

\section{Character interdependence; redundant characters}

Characters in a data matrix for phylogenetic analysis are interdependent when a state of a character (other than "unknown") is predictable - without prior knowledge of phylogeny - from the state of another character. Because phylogenetic analysis operates on the assumption that all characters are independent of each other, the presence of interdependent characters in a matrix amounts to counting the same apomorphy at least twice, which can distort the resulting tree topology and will almost inevitably distort at least some of its support values. While this fact seems to be universally acknowledged in principle, we find (Marjanović \& Laurin, 2008, 2009, 2013a; and below) that it is underappreciated in practice.

Different kinds of character interdependence require different amounts of effort to detect. O'Keefe \& Wagner (2001: 657; and references therein) distinguished "logical correlations among characters" from "[b]iological correlations"; Pardo (2014: 52-60) distinguished four kinds of interdependence. We call Pardo's first three kinds, which include logical interdependence, "redundancy" and biological interdependence "correlation" hereinafter. 
571

572

573

574

575

576

577

578

579

580

581

582

583

584

585

586

587

588

589

590

591

592

593

594

595

596

597

598

599

600

601

602

603

604

605

606

607

608

609

610

611

612

613

614

615

616

It can be very difficult to determine whether characters are correlated; studies of development genetics are sometimes, perhaps often, required (Kangas et al., 2004; Harjunmaa et al., 2014). We expect, therefore, that all of our best efforts will be unable to completely eliminate character interdependence from any morphological matrix. However, many cases of redundant characters are much more obvious; and although RC07 noticed and removed several cases from the preceding version (Ruta, Coates \& Quicke, 2003), we present a considerable number of additional cases in App. S1. We have merged each pair (or multiple) of redundant characters that we identified into a single character.

To make our mergers more transparent, we have created abbreviations for merged characters from those of all their constituents: e.g., PREMAX 1-2-3 (ch. 1) is built from the three characters PREMAX 1, PREMAX 2 and PREMAX 3 of RC07, and MAX5/PAL5 (ch. 22) consists of MAX 5 and PAL 5 of RC07. The extreme cases are VOM 5-10/PTE 10-12-18/INT VAC 1 (ch. 105), assembled from the six characters VOM 5, VOM 10, PTE 10, PTE 12, PTE 18 and INT VAC 1 of RC07, and EXOCC 2-3-4-5/BASOCC 1-5 (ch. 134).

\section{Ordering of multistate characters}

RC07 (p. 78), like Ruta, Coates \& Quicke (2003: 271), followed the widespread practice (e.g. Sigurdsen \& Green, 2011; Schoch, 2013; stated but unexplained preference of Simões et al., 2016a: 211; Pardo et al., 2017; Pardo, Small \& Huttenlocker, 2017) of treating all multistate characters as unordered. Following another widespread practice, they did not spell out any reasons for this decision. Presumably they adhered to the common assumption (already identified and discussed by Slowinski, 1993) that ordering a character is to make an assumption, while "leaving" it unordered means not to make an assumption - which is incorrect (Slowinski, 1993). In particular, potentially continuous (clinal) characters should be ordered, because the basic assumption behind ordering - that it is easier to change from any state to a similar state than to a less similar one - has already been used to partition the observed spread of data into discrete states in the first place; it would be incoherent to reject this assumption in one place but not the other (Slowinski, 1993; Wiens, 2001). This also holds for certain meristic characters (Wiens, 2001). As advocated by Slowinski (1993), Wiens (2001) and Baron, Norman \& Barrett (2017: supplementary information: 4-9), we have ordered many, but not all, multistate characters; see App. S1 for discussion of each case. Two characters (ch. 32, 134: PAR 2/POSFRO 3/INTEMP 1/SUTEMP 1, EXOCC 2-3-4-5/BASOCC 1-5) have part of their state range ordered (e. g. Slowinski, 1993: fig. 1a, d); this is accomplished by creating stepmatrices (App. 1: App.-Tables $2,4)$. We have marked these decisions in the name of each multistate character by adding "(ordered)", "(unordered)" or "(stepmatrix)" to its end.

The consequences of ordering potentially continuous characters are largely unpredictable. Empirically, ordering such characters can reveal additional signal and thus increase the resolution of the consensus tree (Slowinski, 1993; Fröbisch \& Schoch, 2009a; Grand et al., 2013; Simões et al., 2016a: fig. 2b, 3b, 4; Baron, Norman \& Barrett, 2017: fig. 1, extended data fig. 4); on the other hand, and even at the same time, it can also reveal previously hidden character conflict and thus decrease the resolution, showing that the original resolution was not supported by the data (Slowinski, 1993; Marjanović \& Laurin, 2008; Baron, Norman \& Barrett, 2017: fig. 1, extended data fig. 4). Both of these results are congruent with the finding of the simulation studies by Grand et al. (2013) and Rineau et al. (2015) that ordering clinal characters decreases the rate of artefactual resolution and increases the power to detect real clades. 
617

618

619

620

621

622

623

624

625

626

627

628

629

630

631

632

633

634

635

636

637

638

639

640

641

642

643

644

645

646

647

648

649

650

651

652

653

654

655

656

657

658

659

660

661

662

663

664

\title{
Continuous characters
}

Due to the limits of available computation power, we have not been able to analyze continuous characters "as such" (as recommended by Simões et al., 2016a; Brocklehurst, Romano \& Fröbisch, 2016; and references therein), but have broken them up into a small number of discrete states. Such characters were ordered if multistate (see above). Where RC07 had defined reproducible state limits that did not render the characters parsimony-uninformative (see below), we have not changed them; in the cases of PREMAX 7 and SKU TAB 1 (our ch. 3 and 95), which we have wholly recoded (see below: "Deleted, recoded and split characters"), we divided the observed range into several equal parts (not many - Bardin et al., 2014) at round numbers because there are no gaps in the distribution. This is an obvious opportunity for future improvement. See Brocklehurst, Romano \& Fröbisch (2016) for further discussion.

\section{Inapplicable characters and mergers}

Perhaps the eventual solution will be to write new algorithms for computer programs that will allow the characters to be coded independently but that will consider interactions between characters and count steps in some characters only on those portions of the tree on which they are applicable. - Maddison (1993: 580)

\begin{abstract}
Character-taxon matrices and their accompanying character lists should be viewed as formatted data, and not just a table of observations. That is, they should be constructed with an understanding of how that information will be interpreted by the algorithm that is receiving them. For many multistate characters, authors should consider how character state information is (or is not) distributed to other transformation series. The problem with ' 0 ' being used as a catch-all for anything that simply 'isn't 1 ' should be borne in mind when using binary characters (see discussion below).
\end{abstract}

$$
\text { - Brazeau (2011: 494) }
$$

Maddison (1993) concluded that addressing this problem would require modification of phylogenetic software; 25 years later, there are still few signs of progress on this problem. - Brazeau, Guillerme \& Smith (2017: 5)

It is a common situation that a character is inapplicable to part of a taxon sample: when, say, the ectopterygoid bone in the palate is absent, it does not have a shape, and it is not toothed or toothless. Strong \& Lipscomb (1999) reviewed the methods for dealing with such cases:

- Reductive coding: inapplicable scores are treated as missing data, using the symbols "?" or "-". (The latter designates a gap in a molecular sequence, which is treated either as missing data - the default setting in available software - or as a fifth nucleotide $/ 21^{\text {st }}$ amino acid - which is absence coding, see below.)

- Composite coding: characters that are inapplicable to any OTU are merged with the characters they depend on, producing multistate characters. For instance, "ectopterygoid toothed (0); toothless (1)" might be merged with "ectopterygoid present (0); absent (1)" as the unordered multistate character "ectopterygoid toothed (0); toothless (1); absent (2)".

- Nonadditive binary coding: the presence or absence of each character state is treated as a binary character of its own that can be scored for all OTUs. For example, "toothed ectopterygoid: present (0); absent (1)" and "toothless ectopterygoid: present (0); absent 
665

666

667

668

669

670

671

672

673

674

675

676

677

678

679

680

681

682

683

684

685

686

687

688

689

690

691

692

693

694

695

696

697

698

699

700

701

702

703

704

705

706

707

708

709

710
(1)" can be scored for all OTUs - taxa that lack an ectopterygoid have state 1 of both of these characters.

- Absence coding: inapplicability is coded as an additional state of each concerned character.

As Strong \& Lipscomb (1999) pointed out, absence coding creates redundant characters by counting each condition that makes characters inapplicable several times, once for each character that it makes inapplicable. Absence coding must therefore be avoided.

Nonadditive binary coding runs the same risk. In the example given above, an OTU cannot have state 0 of both characters: having state 0 of one unfailingly predicts state 1 of the other. The characters are thus redundant. It also treats nonhomologous conditions as the same state, which runs the risk of creating spurious apomorphies which may increase artefactual resolution (Brazeau, 2011, and references therein); Strong \& Lipscomb (1999: 368-369) went so far as to state that "non-additive binary coding denies homology and the hierarchical relationships between states. The result are cladograms and character interpretations that are absurd and inaccurate representations of our observations." Brazeau (2011: 495) further performed a reductio ad absurdum by asking what would happen if molecular data were coded as, e.g., "adenine at site 121: absent (0); present (1)", where state 0 would treat guanine, cytosine, thymine, and the absence of site 121 (a deletion) as identical and primarily homologous for no defensible reason.

Composite coding eliminates the redundancy that is sometimes created by nonadditive binary coding. Furthermore, it has the advantage over reductive coding that it prevents contradictory optimization of ancestors. With reductive coding, an ancestor may be optimized as having a toothed ectopterygoid and at the same time as having no ectopterygoid at all (Brazeau, Guillerme \& Smith, 2017, and references therein); composite coding makes this impossible and may thus make arguably nonsensical trees less parsimonious. However, composite coding is not always feasible to its full extent. In our example, if the character that represents the presence of the ectopterygoid is merged with the character that describes its dentition, what is to become of size and shape? Merging all of the affected characters would often yield a huge multistate character that would, in many cases, require a complex stepmatrix and be difficult to interpret in evolutionary terms (as already noted by Maddison, 1993), not to mention the adverse effects of stepmatrices on calculation times (pers. obs.).

RC07 used reductive, composite and nonadditive binary coding in different cases. The numerous occurrences of nonadditive binary coding may be a side effect of taking characters from diagnoses and synapomorphy lists (Bardin, Rouget \& Cecca, 2014), where they are usually presented in the form of a single (presumedly derived) state, while the other states are not mentioned. We have greatly reduced the amount of nonadditive binary coding. There are a few cases we have left for the time being; these are cases where the effort needed to rectify the situation would probably be out of proportion to the gain in phylogenetic signal. Examples are PREMAX 4 (ch. 2 in App. S1), "Premaxilla with flat, expanded anteromedial dorsal surface and elongated along its lateral margin but not along its medial margin, when observed in dorsal aspect: absent (0); present (1)", PASPHE 11 (ch. 143), "Basipterygoid processes of the basisphenoid shaped like anterolaterally directed stalks, subtriangular to rectangular in ventral view and projecting anterior to the insertion of the cultriform process: absent (0); present (1)", TIB 6 (ch. 235), "Outline of tibia medial margin shaped like a distinct, subsemicircular embayment contributing to interepipodial space and the diameter of which is less than one-third of bone length: absent (0); present (1)", and TRU VER 30 (ch. 275), “Transverse processes stout 
711 and abbreviated, the length of which is less than $30 \%$ of neural arch height: absent (0); present 712 (1)". We would need to survey the range of morphologies and in some cases their considerable 713 ontogenetic transformations - see below - in detail in order to determine how many characters,

714 let alone how many states, should be distinguished within state 0 of each of these. All these

715

We have slightly redefined some characters to avoid predictable scores. An example is PAR 1, our ch. 31. In RC07 it was called "Parietal/tabular suture: absent (0); present (1)" (their ch. 38). When the parietal and the tabular are present but the supratemporal is absent, the parietal and the tabular inevitably touch, so the score of 1 is wholly predictable from the scores of the characters that code the presence/absence of the supratemporal (our ch. 32; ch. 63 of RC07) and the tabular (our ch. 53; ch. 67 of RC07); yet, RC07 scored 1 in almost all these cases. Conversely, of the nine OTUs which lack not only the supratemporal but also the tabular, eight were scored as unknown, but one (Albanerpetidae) was scored 0: lacking a tabular, it cannot have a parietal-tabular suture. If this is not a typographic or similar error, perhaps state 0 was in this case understood as every condition that is not state 1 (nonadditive binary coding). We have made state 0 explicit in redefining the character as: "Supratemporal/postparietal suture (0); parietal/tabular suture (1)." This is reductive coding: if any of these four bones is absent, the character is inapplicable (which we spell out in App. S1 for this and all comparable characters). The scores we changed to unknown because our redefinition makes them unambiguously inapplicable - Albanerpetidae, all "microsaurs" except Odonterpeton (which lacks tabulars and was already scored as unknown), Brachydectes, Scincosaurus, all diplocaulids - are marked in green in App. S2 (and counted as such in Data S4), as are all scores that involve the redefinition, or possible redefinition, of a state of any character (see below: Modifications to individual cells).

Others we have simply interpreted as reductive instead of as nonadditive binary. An example is POSORB 5, our ch. 64 (ch. 81 of RC07). Its name remains unchanged: "Postorbital/tabular suture: absent (0); present (1)." All OTUs scored in RC07 as having state 0 indeed lack a contact between the postorbital and the tabular; almost all of them, however, have a supratemporal, and whenever the supratemporal is present, it lies between the other two bones and prevents them from reaching each other. No cases are known in our taxon sample, and no cases are known to us outside our taxon sample, where the postorbital or the tabular would reach around the supratemporal and separate it from the parietal or the squamosal. We consider state 0 predictable from the presence of the supratemporal (our ch. 32; ch. 63 of RC07), have changed it to unknown in all OTUs that have a supratemporal, mentioned this in App. S1, marked these changes in green in App. S2 and counted them accordingly in Data S4.

On a few occasions we have changed massively non-binary to composite coding. RC07 used, for example, the following characters (p. 110, italics omitted):

333. DIG 1. Digits: absent (0); present (1).

334. DIG 2. Manus with no more than four digits: absent (0); present (1).

335. DIG 3. Manus with no more than five digits: absent (0); present (1).

336. DIG 4. Manus with no more than three digits: absent (0); present (1). Despite having "no more than" in their names, DIG 2-DIG 4 were scored in RC07 as if they meant "exactly four fingers per hand: no (0); yes (1)", "exactly five" and so on: each OTU scored as 1 for any of these three characters was scored as 0 for both of the others. In other words, state 1 of any of these three unfailingly predicted state 0 of the other two - and also state 1 of DIG 1. We have therefore merged DIG 2-DIG 4 and most of DIG 1 as follows (App. S1): 
756

757

758

759

760

761

762

763

764

765

766

767

768

769

770

771

772

773

774

775

776

777

778

779

780

781

782

783

784

785

786

787

788

789

790

791

792

793

794

795

796

797

798

799

800

801

276. DIG 1-2-3-4: "Independent radials" (0); polydactyly (1); pentadactyly (2);

tetradactyl forelimb (3); tridactyl forelimb (4) (ordered).

The term "independent radials" refers to Johanson et al. (2007). The complete absence of extremities, previously part of DIG $1(0)$, is now part of the limb-reduction/body-elongation character:

219. HUM 18/DIG 1: Forelimb absent (0); humerus present, length smaller (1) or greater (2) than combined length of two and a half mid-trunk vertebrae (ordered).

In a few other cases we have changed fully reductive to partially composite coding. This includes the following characters of RC07:

140. ECT 1. Separately ossified ectopterygoid: present (0); absent (1).

141. ECT 2. Ectopterygoid with (0) or without (1) fangs [...].

142. ECT 3. Ectopterygoid without (0) or with (1) small teeth (denticles) [...].

143. ECT 4. Ectopterygoid longer than/as long as (0) or shorter than (1) palatine.

144. ECT 5. Ectopterygoid with (0) or without (1) row of teeth $(3+)[\ldots]$.

145. ECT 6. Ectopterygoid/maxilla contact: present (0); absent (1).

146. ECT 7. Ectopterygoid narrowly wedged between palatine and pterygoid: no (0); yes

(1).

The presence (ECT 1) and size of the ectopterygoid (ECT 4) are now a single character, to which we have added an additional state (ultimately from McGowan, 2002):

115. ECT 1-4: Ectopterygoid at least as long as palatine (0); at least about a third as long as but shorter than palatine (1); at most about a third as long as palatine (2); absent (3) (ordered).

The interpretation of ECT 1 and ECT 4 as parts of a potentially continuous character fits the observation that taxa with very small ectopterygoids tend to be the closest relatives of those with no ectopterygoids and nested among taxa with middle-sized ones: absence, in this particular case, seems to be in effect a length of zero (as forelimb absence is a humerus length of zero in HUM 18/DIG 1). ECT 2, ECT 3 and ECT 5, however, are unaffected; they continue to be scored as unknown for OTUs that lack ectopterygoids. Restudy of the literature and a specimen has shown that states ECT 6(1) and ECT 7(1) can only be scored for at most one OTU each (App. $\mathrm{S} 1)$; this makes the characters ECT 6 and ECT 7 parsimony-uninformative, so we have deleted them (see below).

Too recently for us to use, Brazeau, Guillerme \& Smith (2017) published a new approach to dealing with reductively coded inapplicability in phylogenetics software. We are looking forward to further developments of its implementation. However, we strongly disagree that socalled "neomorphic characters" should be scored as having the presumedly plesiomorphic state when they are inapplicable. This requires identifying the plesiomorphic state in advance, which increases the danger that the phylogenetic analysis will conform to one's preconceptions just as much as an all-zero outgroup would. It is also much less easy than Brazeau, Guillerme \& Smith (2017: 23) implied when they stated that in their analysis "every inapplicable token in each neomorphic character was replaced with the token corresponding to the presumed non-derived condition (typically 'absent')" - for example, our matrix contains many characters for the presence or absence of bones that are, in our taxon sample, plesiomorphically present and are apomorphically lost several times, while different taxon samples (e.g. vertebrates generally, or actinopterygians) would support the opposite polarization or none at all. Further, this method creates redundancy just like absence coding does: in the example by Brazeau, Guillerme \& Smith (2017: table 1), absence of the tail unfailingly predicts absence of eyespots on the tail. 
802

803

804

805

806

807

808

809

810

811

812

813

814

815

816

817

818

819

820

821

822

823

824

825

826

827

828

829

830

831

832

833

834

835

836

837

838

839

840

841

842

843

844

845

846

847
“Ontogeny discombobulates phylogeny” (Wiens, Bonett \& Chippindale, 2005)

Heterochrony can result in misleading scores if morphologically immature (juvenile or paedomorphic) individuals are scored at face value, which can result in large-scale character correlation that can strongly distort phylogenetic trees (Wiens, Bonett \& Chippindale, 2005). We have tried to deal with this problem as described by Marjanović \& Laurin (2008) and in more detail by Marjanović \& Laurin (2013a), modified from the recommendation of Wiens, Bonett \& Chippindale (2005: 96) and independently proposed by Tykoski (2005: 276): observed states are treated as unknown if they are restricted to immature stages in non-paedomorphic close relatives of the OTU in question. Thus, OTUs known from growth series including skeletally mature individuals are therefore scored as only having the most mature state of ontogeny-affected characters instead of as polymorphic; OTUs known only from apparently morphologically immature individuals are scored as having the most mature observed state or any more mature one, as partial uncertainty (or complete uncertainty if the only observed state is the least mature one the character has to offer), instead of just the most mature observed state. Pawley (2006: 206) appears to have used the same approach, explaining for binary characters: "If it was possible that a derived [...] characteristic may be absent in a particular specimen due to the morphogenetic immaturity of the specimen, then the character state was coded '?', to avoid confusing morphogenetic immaturity with the plesiomorphic state."

The ontogeny of most of our OTUs and their closest relatives is insufficiently well known; despite this, and in spite of the additional complications discussed by Marjanović \& Laurin (2013a), we think that this approach offers the greatest chance to escape character correlation caused by heterochrony. Each of the rather few cases (79 in the taxon sample of RC07, 18 in the taxa we added) is discussed in App. S1 and marked in blue in App. S2; Data S4 additionally lists our scores of deleted characters that would be marked in blue (three in the taxon sample of RC07, one in an OTU we added).

\section{Deleted, recoded and split characters}

Our matrix has only 277 characters, a strong decrease from the 339 of RC07. For the most part, this is due to our mergers of redundant characters and does not entail a loss of information (see above; detailed in App. S1). The characters IFN 1, ILI 10, ISC 1, DOR FIN 1 and BAS SCU 1, however, were parsimony-uninformative in the matrix of RC07 and remain so after our corrections (all of them documented and justified in App. S1); we have deleted them rather than carrying these currently irrelevant characters along and inflating the character count (see Marjanović \& Laurin, 2013a, for discussion). The characters PREFRO 6, PREFRO 9, LAC 6, NOS 1, VOM 11, PAL 6, ECT 6, ECT 7, BASOCC 6 and DEN 1 were parsimony-informative as scored by RC07, but this is no longer the case after our corrections (likewise documented and justified in App. S1), so we have deleted them as well. (For LAC 6 and VOM 11, one of the two states turns out not to occur in the original taxon sample at all, and only once or never in the expanded taxon sample.) Conversely, the character ANG 3 was parsimony-uninformative in RC07, but is parsimony-informative in our matrix even for the original taxon sample (see App. S1); we have kept it (though merged it with ANG 2, now ch. 161).

Characters INT FEN 1 and TEETH 3 were composites of two independent characters each; we have split INT FEN 1 into the redefined INT FEN 1 (ch. 84) and the new MED ROS 1 
848 (ch. 85), and TEETH 3 into the redefined TEETH 3 (ch. 183) and the new TEETH 10 (ch. 190). 849 Similarly, we have reversed the merger of PIN FOR 1 and PIN FOR 2 of Ruta, Coates \& Quicke 850 (2003), our ch. 91 and 92, into PIN FOR 2 of RC07. We have also partitioned several characters 851 into more states than before.

852 Character PREMAX 7, a ratio of two measurements, was parsimony-uninformative as defined (one of the two original states is limited to an OTU that was scored as unknown by RC07), but not as described or scored; we have measured all OTUs, defined new state limits (as discussed in App. S1: ch. 3), and rescored the entire taxon sample. SKU TAB 1, another ratio, was defined and described in contradictory ways, neither of which matched the scores; we have treated it the same way (see App. S1: ch. 95). The measurements, sources, calculations and state changes are presented and compared to the original and revised scores in Data S5; the ratios,

859 sources and state changes are also shown in App.-Tables 1 (for PREMAX 7) and 3 (for SKU

860

861

862 TAB 1), which are placed in App. S1 under the characters they refer to.

PTE 15 is not reproducible; we have not figured out, either from the description or from the scores, what exactly the difference between the two states was meant to be (see App. S1 for details). The most likely meaning would be a duplicate of PTE 14 (ch. 123); we have deleted PTE 15.

Similarly, TAB 4 was described in a confusing way (see App. S1). Most likely, it is either

865

866

867

868

869

870

871

872

873

874

875

876

877

878

879

880

881

882

883

884

885

886

887

888

889

890

891

892

893

\section{Blockwise scoring of taxa}

In RC07, the binary character PREMAX 7 (our ch. 3, mentioned in the preceding subsection), was (except for a few cases of missing data) scored 1 in all amniotes, diadectomorphs, "microsaurs", seymouriamorphs, anthracosaurs, Silvanerpeton, Gephyrostegus, Diplocaulus, Diploceraspis and Eocaecilia, and state 0 in all other OTUs. We have measured every OTU anew (Data S5; App.-Table 1). As detailed in App. S1, we have not been able to find an interpretation of the description of this character by RC07, or the different description by Ruta, Coates \& Quicke (2003), that would generate such a neat pattern or anything close. No matter how we interpret the character, most of the original scores have no particular relationship with our measurements (App.-Table 1; Data S5). Evidently, most OTUs were scored after presumed close relatives instead of on their own terms.

We have not found evidence of blockwise scoring in any other character. In this matrix, fortunately, blockwise scoring does not seem to be a recurrent problem.

\section{Modifications to individual cells}

All modifications (including those made by Damien Germain [2008a: chapter V], except where we found them unjustified) are documented and justified in App. S1, with citations of the literature and/or specimens we used to rescore each cell. For the sake of brevity, the original scores (RC07) are usually not mentioned there, only our modified ones are; scores we did not change are not mentioned in App. S1 except where they could be controversial.

All modifications to individual cells are further presented in App. S2 (deleted characters excepted) and Data S4, separated by type of change: Green font in App. S2 (second sheet of Data 
894 S4) marks new scores when a state is new, redefined or possibly redefined (in cases where we 895 are not sure of the meaning intended by RC07) and is also used to mark all but the most trivial 896 newly recognized cases of inapplicability (usually changes away from nonadditive binary 897 coding) as well as, in App. S2 but not Data S4, the scores changed to unknown following the 898 removal of all postcranial material from Rhynchonkos (see below under Treatment of OTUs:

899 Rhynchonkos). Blue font (third sheet of Data S4) indicates scores changed in order to account for 900 ontogeny (see above under "Ontogeny discombobulates phylogeny"); red font (first sheet of Data S4) shows scores changed at face value (unaffected by any redefinition or ontogenetic considerations). The distinction between green and red should not be mistaken for different degrees of certainty; those are instead discussed in App. S1. Only green is shown when red and green apply, only blue is shown when red or green and blue apply; blue font is also used for the added OTUs where appropriate (their other scores are all black).

POSPAR 9, our ch. 43, is an example of a character to which we have made changes of

906

907

908

909

910

911

912

913

914

915

916

917

918

919

920

921

922

923

924

925

926

927

928

929

930

931

932

933

934

935

936

937

938 all three types. (See App. S1 for details, discussions and references.) RC07 (p. 96: ch. 54) called it "Postparietals without (0) or with (1) broad, concave posterior emargination." As the presence of such an emargination does not depend on the presence of postparietals or their participation in the dorsal surface of the skull, we have redefined the character (see App. S1) as: "Edge between the dorsal and the caudal surfaces of the skull without (0) or with (1) broad, concave posterior emargination in the central bones." This has allowed us to score Triadobatrachus (which lacks identifiable postparietals and has our state 0), Captorhinus (whose postparietals lie entirely on the occipital surface, i.e. ventral of the emargination that gives it our state 1) and Batropetes (which lacks identifiable postparietals and has our state 1); the resulting changes (from original scores as unknown) are marked in green.

Even though RC07 scored state 1 exclusively for diplocaulids other than Keraterpeton, it is in fact present both under their definition and under ours in a large number of other OTUs. The resulting 20 changes are marked in red font; this includes three from state 0 to polymorphism, and four (to state 1) that we comment as "arguably borderline", "weakly", "borderline" or "marginally". Also marked in red is the change to unknown in Tseajaia, where, as we discuss in App. S1, the position of the suture between postparietal and tabular is unclear so that we cannot determine if the central bones (the postparietals in this case) have a concave or a straight caudal edge, i.e. if they are wide enough to visibly participate in the curvature. Likewise red is the change from unknown to 0 in Ventastega, which was required under both definitions by material published only in 2008.

Finally, Microphon changed from state 0 (scored by RC07) to state 1 in ontogeny, so we have scored state 1 and marked this in blue. Only state 0 (scored by RC07) has been observed in its fellow seymouriamorph Leptoropha, but all known individuals are ontogenetically comparable to those of Microphon that show state 0 ; as we do not know when the skeletal ontogeny of Leptoropha would have ended, or if Leptoropha reached morphological maturity at all instead of remaining paedomorphic in the adult stage, we have scored it as unknown (i.e. uncertainty between the two available states) and marked this in blue as well.

While we have not systematically compared every cell in the matrix to the literature or specimens, our attention was not limited to particular taxa or characters; we ended up making changes to the scoring of all OTUs, and of all characters except 96 (FONT 1), 150 (PSYM 4), 187 (TEETH 7) and the deleted parsimony-uninformative ISC 1, DOR FIN 1 and BAS SCU 1. In the end we have probably compared almost all cells in the matrix to the literature and/or to

939 specimens. Shying away from the inordinate amount of time this work ended up taking, we 
940 initially concentrated our efforts on characters and OTUs relevant to lissamphibian origins, as 941 determined, mostly, by optimizations on trees in Mesquite (Maddison \& Maddison, 2017). Soon, 942 though, we branched out to characters with subjectively suspicious state distributions (it goes 943 without saying that many turned out to be entirely or partially correct), characters we did not 944 immediately understand, characters we recoded while merging them with others and taxa that

945

946

947

948

949

950

951

952

953

954

955

956

957

958

959

960

961

962

963

964

965

966

967

968

969

970

971

972

973

974

975

976

977

978

979

980

981

982

983

984

985 had been redescribed (recently or sometimes not so recently). Furthermore, we investigated characters that supported conflicting or unusual hypotheses of relationships apart from lissamphibian origins, and the taxa implicated in these; examples are Seymouriamorpha and Diadectomorpha, both surprisingly found to be paraphyletic by RC07, and Adelogyrinidae and Acherontiscus, found for the first time to clade with colosteids rather than "lepospondyls" by RC07. Finally, we checked the scores of OTUs that temporarily did strange things in preliminary analyses of ours, like Tulerpeton, Edops, Trimerorhachis or Ossinodus (unsurprisingly, some of this strangeness has remained). Whenever we investigated a character, we usually verified its scores for all OTUs, and whenever we investigated an OTU, we verified its scores for all characters except as restricted in the two subsections below.

As expected, we found cells where the correct score may be a matter of interpretation rather than being straightforwardly testable. (These are discussed in App. S1, or at least marked by such terms as "borderline", "weakly", "arguably", "probably", "most likely" or "almost certainly"; see above for examples.) The homology of a few bones and processes is a matter of interpretation, and sometimes we had to deal with such factors as taphonomic distortion; we argue for our interpretations in App. S1, but we recognize that some of our arguments may not be significantly stronger than potential counterarguments.

However, we must stress that a very large proportion of our changes to the matrix - most of the 2404 (see Data S4) that are marked in red in App. S2 and the 67 changes to deleted characters that would be marked red (Data S4), as well as many of the green or blue ones where those colors also apply - concern cases where scores in RC07 contradict their definitions and descriptions of the characters, according to all sources we had access to (literature and/or pers. obs. of specimens), without RC07 having provided an explanation (e.g. a new interpretation of homology or pers. obs. of specimens). We expect that many readers will share our surprise at this fact, and invite them to double-check our claims in App. S1.

\section{Literature}

For the following OTUs - and quite possibly others - we have compared most or all cells to the literature (see below for specimens). Only the most important sources are cited here, a few more scores were changed based on sources cited in App. S1:

- Panderichthys (Vorobyeva \& Schultze, 1991; Boisvert, 2005, 2009; Brazeau \& Ahlberg, 2006; Boisvert, Mark-Kurik \& Ahlberg, 2008; Ahlberg, 2011 - mostly occiput, extremities and girdles)

- Ventastega (Ahlberg, Lukševičs \& Lebedev, 1994; Lukševičs, Ahlberg \& Clack, 2003; Ahlberg et al., 2008)

- Acanthostega (Coates, 1996; Porro, Rayfield \& Clack, 2015)

- Ichthyostega (skull roof: Clack \& Milner, 2015; lower jaw: Ahlberg \& Clack, 1998; Clack et al., 2012a; humerus: Jarvik, 1996; Callier, Clack \& Ahlberg, 2009; Ahlberg, 2011; Pierce, Clack \& Hutchinson, 2012; vertebrae: Pierce et al., 2013)

- Tulerpeton (Lebedev \& Coates, 1995) 
986 - Colosteus (Langston, 1953; Hook, 1983; Lombard \& Bolt, 2010)

987 - Greererpeton (Smithson, 1982; Godfrey, 1989; Lombard \& Bolt, 2001, 2010)

988 - Crassigyrinus (Panchen, 1985; Panchen \& Smithson, 1990; Clack, 1998)

989 - Whatcheeria (Lombard \& Bolt, 1995, 2006)

990 - Baphetes (Beaumont, 1977; Milner \& Lindsay, 1998; Milner, Milner \& Walsh, 2009)

991 - Megalocephalus (Beaumont, 1977)

992 - Eucritta (Clack, 2001)

993 - Edops (Romer \& Witter, 1942)

994

995

996

997

998

999

1000

1001

1002

1003

1004

1005

1006

1007

1008

1009

1010

1011

1012

1013

1014

1015

1016

1017

1018

1019

1020

1021

1022

1023

1024

1025

1026

1027

1028

1029

- Chenoprosopus (Hook, 1993; Reisz, Berman \& Henrici, 2005 - previously missing data, including all postcranial material, and data mentioned as having been hitherto misinterpreted)

- Cochleosaurus (Sequeira, 2004, 2009)

- Isodectes (Sequeira, 1998)

- Neldasaurus (Chase, 1965)

- Trimerorhachis (Pawley, 2007; Milner \& Schoch, 2013)

- Balanerpeton (Milner \& Sequeira, 1994)

- Dendrerpetidae (Carroll, 1967; A. R. Milner, 1980, 1996; Godfrey, Fiorillo \& Carroll, 1987; Holmes, Carroll \& Reisz, 1998; Robinson, Ahlberg \& Koentges, 2005 - especially to make sure no polymorphisms were overlooked; see below and Schoch \& Milner, 2014)

- Eryops (Sawin, 1941; Pawley \& Warren, 2006)

- Acheloma (Olson, 1941; Maddin, Reisz \& Anderson, 2010; Polley \& Reisz, 2011 mostly filled in previously missing data)

- Phonerpeton (Dilkes, 1990)

- Broiliellus (Carroll, 1964; Schoch, 2012)

- Amphibamus (Daly, 1994)

- Doleserpeton (Sigurdsen, 2008; Sigurdsen \& Bolt, 2009, 2010; Sigurdsen \& Green, 2011: app. 2)

- Eoscopus (Daly, 1994)

- Platyrhinops (Clack \& Milner, 2010; remaining missing data filled in from Hook \& Baird, 1984, and from Werneburg, 2012a, where not likely ontogeny-dependent)

- Micromelerpeton (Boy, 1972, 1995; Schoch, 2009)

- Apateon, Leptorophus, Schoenfelderpeton (Boy, 1972, 1986, 1987; Werneburg, 1991, 2007a; Schoch \& Fröbisch, 2006; Schoch \& Milner, 2008; Fröbisch \& Schoch, 2009b)

- Albanerpetidae (Estes \& Hoffstetter, 1976; Fox \& Naylor, 1982; Gardner, 2001; McGowan, 2002; Gardner, Evans \& Sigogneau-Russell, 2003; Venczel \& Gardner, 2005; Maddin et al., 2013a; note that we follow this latter reference in interpreting the "cultriform process of the parasphenoid" [McGowan, 2002: fig. 13] as a hyobranchial element, contra Marjanović \& Laurin, 2008)

- Eocaecilia (Jenkins, Walsh \& Carroll, 2007)

- Triadobatrachus (Rage \& Roček, 1989; Roček \& Rage, 2000; Sigurdsen, Green \& Bishop, 2012; Ascarrunz et al., 2016)

- Valdotriton (Evans \& Milner, 1996)

- Karaurus (Ivachnenko, 1978)

- Caerorhachis (Ruta, Milner \& Coates, 2002) 
1030

1031

1032

1033

1034

1035

1036

1037

1038

1039

1040

1041

1042

1043

1044

1045

1046

1047

1048

1049

1050

1051

1052

1053

1054

1055

1056

1057

1058

1059

1060

1061

1062

1063

1064

1065

1066

1067

1068

1069

1070

1071

1072

1073

- Bruktererpeton (Boy \& Bandel, 1973)

- Gephyrostegus (Carroll, 1970; Godfrey \& Reisz, 1991; Klembara et al., 2014)

- Eoherpeton (Panchen, 1975; Smithson, 1985)

- Proterogyrinus (Holmes, 1980, 1984)

- Archeria (Romer, 1957; Holmes, 1989)

- Pholiderpeton attheyi (Panchen, 1972)

- Anthracosaurus (Panchen, 1977; Clack, 1987a)

- Pholiderpeton scutigerum (Clack, 1987b)

- Solenodonsaurus (Laurin \& Reisz, 1999; Danto, Witzmann \& Müller, 2012 - mostly filled in previously missing data)

- Kotlassia (Bystrow, 1944; Bulanov, 2003)

- Discosauriscus (Klembara, 1997, 2009; Klembara \& Bartík, 2000)

- Seymouria (White, 1939; Laurin, 1996a, 2000; Klembara et al., 2005, 2006, 2007)

- Diadectes (Case, 1910, 1911; Case \& Williston, 1912; Olson, 1947; Moss, 1972; Berman, Sumida \& Lombard, 1992; Berman, Sumida \& Martens, 1998; Berman et al., 2004)

- Limnoscelis (Fracasso, 1983; Berman \& Sumida, 1990; Reisz, 2007; Berman, Reisz \& Scott, 2010; Kennedy, 2010)

- Westlothiana (Smithson et al., 1994)

- Batropetes (Glienke, 2013, 2015)

- Stegotretus (Berman, Eberth \& Brinkman, 1988)

- Rhynchonkos (CG78; Szostakiwskyj, Pardo \& Anderson, 2015)

- Microbrachis (CG78; Vallin \& Laurin, 2004; Olori, 2015)

- Hyloplesion (CG78; Olori, 2015)

- Brachydectes (Wellstead, 1991; Pardo \& Anderson, 2016)

- Acherontiscus (Carroll, 1969a)

- Adelospondylus, Adelogyrinus, Dolichopareias (Andrews \& Carroll, 1991)

- Scincosaurus (Milner \& Ruta, 2009)

- Diplocaulus (postcranium and lower jaw: Williston, 1909; Douthitt, 1917)

- Diploceraspis (mostly postcranium and lower jaw: Beerbower, 1963)

- Lethiscus (Wellstead, 1982; Anderson, Carroll \& Rowe, 2003; Pardo et al., 2017)

- Oestocephalus (Carroll, 1998a; Anderson, Carroll \& Rowe, 2003; Anderson, 2003a)

- Phlegethontia (Anderson, 2002, 2007a)

- Notobatrachus (Estes \& Reig, 1973; Báez \& Basso, 1996; Báez \& Nicoli, 2004, 2008)

- $\quad$ Vieraella (Estes \& Reig, 1973; Báez \& Basso, 1996)

- Ossinodus (Warren \& Turner, 2004; Warren, 2007; Bishop, 2014)

- Pederpes (Clack \& Finney, 2005)

- Orobates (Berman et al., 2004; Nyakatura et al., 2015: digital model)

- Ariekanerpeton (Laurin, 1996b; Klembara \& Ruta, 2005a, b)

- Leptoropha (Bulanov, 2003)

- Microphon (Bulanov, 2003, 2014)

- Capetus (Sequeira \& Milner, 1993)

- Tseajaia (Moss, 1972; Berman, Sumida \& Lombard, 1992)

- Utegenia (Laurin, 1996c; Klembara \& Ruta, 2004a, b) 
1074 Parts of the above list surprise us. For instance, Ruta, Coates \& Quicke (2003) cited Ahlberg,

1075 Lukševičs \& Lebedev (1994) as their source for scoring Ventastega, but only used that

1076 publication to code not much more than half of the bones that are described and illustrated in that

1077 work; large parts of the skull, e.g. almost the entire palate, were scored as entirely unknown by

1078 Ruta, Coates \& Quicke (2003) and RC07 for reasons that we could not determine. This goes so

1079 far that in some instances some of the bones belonging to a skull fragment were scored while

1080 others sutured to them in the same fragment were not.

1081 RC07 cited the paper by Sequeira (2004), but only for its phylogenetic analysis, not for

1082 its redescription of the skull of Cochleosaurus based on "several large, presumably adult, skulls"

1083 (Sequeira, 2004: abstract) - all previous descriptions "were based almost entirely on small,

1084 subadult [...] specimens" (ibid.). Instead, RC07 kept the outdated scores of Cochleosaurus by

1085 Ruta, Coates \& Quicke (2003). In total, to the best of our knowledge, RC07 made only two

1086 changes to individual cells of the preceding version, the two mentioned on their p. 93 for

1087 PREMAX 1 (ch. 1).

$1088 \quad$ Ruta, Coates \& Quicke (2003) cited Boy \& Bandel (1973) as their source for the scoring

1089 of Bruktererpeton. That publication is written in German, which may explain some of the

1090 differences between it and the scoring by Ruta, Coates \& Quicke (2003) and RC07. (Fortunately,

1091 German is D. M.'s native language.) Some of the figures, however, contradict the matrix by

1092 RC07 as well.

1093 Despite the overlap in authors, many discrepancies exist between the matrix by RC07 and

1094 the detailed redescriptions of Caerorhachis (Ruta, Milner \& Coates, 2002 - not cited by RC07,

1095 but cited as "2001", the year of publication originally intended by the journal, by Ruta, Coates \&

1096 Quicke, 2003), Silvanerpeton (Ruta \& Clack, 2006 - not cited, not even as "in press"),

1097 Ariekanerpeton (Klembara \& Ruta, 2005a, b - not cited, although personal observations are

1098 cited) and Utegenia (Klembara \& Ruta, 2004a, b - cited).

1099 Ruta, Coates \& Quicke (2003) scored Kotlassia after the description by Bystrow (1944);

1100 RC07 did not change this. Bystrow explicitly synonymized Kotlassia and Karpinskiosaurus and

1101 did not document for all parts of his description on which specimen(s) they were based. Bulanov

1102 (2003) disagreed with Bystrow and separated the two taxa again, considering them rather distant

1103 relatives, but he merely mentioned the existence of postcrania of Kotlassia (the entire monograph

1104 describes only the cranial anatomy of a wide range of seymouriamorphs). Similarly, Klembara

1105 (2011) redescribed only the skull of Karpinskiosaurus. A useful description of the postcrania of

1106 either taxon does not exist as far as we know. We have accepted the scores by RC07 at face

1107 value for the time being and scored all postcranial characters of Karpinskiosaurus, a taxon we

1108 have added to the matrix (see below), as unknown, except for those few that Bystrow (1944)

1109 explicitly commented on.

1110

1111

1112

1113

1114

1115

1116

1117

1118

1119

\section{Specimens}

"This has not been a literature-based exercise", wrote Ruta, Coates \& Quicke (2003: 292) in their conclusions; and while they and RC07 did have to rely on the literature to score some taxa (if only because the taxon sample is simply too large for anyone to visit all relevant specimens within realistic time and budget constraints), both of their publications contain lists of specimens they had studied firsthand. Strangely, however, the results of almost none of these observations are mentioned in the papers; there is no way of extracting information on which characters are described or illustrated incorrectly in the literature or which ones are visible in the fossils but not 
1120 presented in a publication (so that scoring from the literature would produce spurious missing

1121

1122

1123

1124

1125

1126

1127

1128

1129

1130

1131

1132

1133

1134

1135

1136

1137

1138

1139

1140

1141

1142

1143

1144

1145

1146

1147

1148

1149

1150

1151

1152

1153

1154

1155

1156

1157

1158

1159

1160

1161

1162

1163

1164

1165 data). We must insist that a data matrix is not an appropriate place to publish new observations: in a matrix there is no way to tell if a surprising score is a new observation or just a typographic error, a momentary confusion of contradictory conventions about which state is called 0 and which is called 1, or one of a number of other very common problems (see above and Marjanović \& Laurin, 2013a, for discussion). For each of the scores we have changed based on our observations of specimens, we have therefore documented the change in App. S1 along with the inventory numbers of specimens that show the states we have scored.

For the following taxa in the original sample we have compared most or all cells to specimens (fossils or casts) which are, where necessary, mentioned under the respective characters in App. S1:

- Ichthyostega (palate only)

- Edops (as Romer \& Witter [1942] noted, the sutures on the only known adult skull roof that of MCZ 1378 - are for the most part extremely difficult to trace, so we have accepted their interpretations wherever D. M. was unable to confirm them; D. M. did not detect any contradictions)

- Isodectes (postcranium only)

- Ecolsonia (previously missing data only)

- Triadobatrachus

- Karaurus (everything except the palate, which is not reproduced by the cast we had access to)

Diceratosaurus is about to undergo redescription by Jason Anderson's lab, D. M. and Florian Witzmann; we have not preempted that work, but only changed the scores of characters of particular interest based on D. M.'s observations of specimens, mostly filling in missing data and often confirming the redescription by Jaekel (1903; not cited by RC07 or Ruta, Coates \& Quicke, 2003).

The complete list of specimens we have used to change scores in the matrix forms Table 2. We cannot provide a list of all specimens we have seen; specimens that agree with the scores of RC07 are therefore only mentioned in App. S1 when a score could be controversial, or when other specimens of the same OTU show another state and we have consequently scored polymorphism. However, whenever D. M. visited a collection, he saw all or almost all specimens that are broadly relevant to this study. One exception to this is the NMS, where the collection is kept in a building on the other side of the city and specimens that are not on exhibit are brought to the museum building only on request; the only NMS specimen D. M. has seen is Casineria (which we added to the matrix as described below). The other exception is the Anderson lab at the University of Calgary, which currently houses many important specimens of which D. M. has only taken the time to study a referred specimen of Asaphestera in any detail.

\section{The albanerpetid neck}

Though this concerns very few characters, our coding of the albanerpetids (a single OTU as in RC07) assumes our reinterpretation of their unique atlas-axis complex. As in mammals, this complex accommodated dorsoventral and lateral movements of the head at separate joints. Traditionally (e.g. Estes \& Hoffstetter, 1976; Fox \& Naylor, 1982), this complex is considered to consist of the atlas (a complete vertebra consisting of a centrum and fused, fully formed, full-size neural arch), the axis (a centrum that lacks any trace of a neural arch), and the third vertebra 
1166 (again a complete vertebra consisting of a centrum and fused, fully formed, full-size neural arch).

1167 The "axis" is commonly sutured to the "third vertebra". Dorsoventral movements of the head

1168 occurred between the skull and the atlas, lateral ones between the atlas and the "axis". By

1169 comparison to amniotes and "microsaurs", we think it is more parsimonious to interpret the

1170

1171 "axis" as only the intercentrum of the axis, even though intercentra are otherwise unknown in albanerpetids, while the "third vertebra" would be the pleurocentrum and neural arch of the axis.

1172

1173 Comparison to temnospondyls is more difficult; in both Doleserpeton (Sigurdsen \& Bolt, 2010)

1174

1175

1176

1177

1178

1179

1180

1181

1182

1183

1184

1185

1186

1187 and Gerobatrachus (Anderson et al., 2008a), the only dissorophoids with pleurocentrumdominant vertebrae, the axis is very incompletely preserved, but the relative sizes of pleuro- and intercentra elsewhere in the column are at least not incompatible with our interpretation.

\section{The skull roof of Brachydectes}

I have not hesitated to criticise former interpretations of structures and sutures, and I need not add that my own are likewise open to criticism.

$$
\text { - Romer (1930: 80) }
$$

Wellstead (1991) reconstructed several unusual features in the skull roof of Brachydectes. We previously tried (Marjanović \& Laurin, 2008, 2013a) to resolve these problems by reinterpreting the homologies of the bones that Wellstead (1991) had identified. For example, the supposed postparietals do not meet in Wellstead's reconstruction, but are separated by a contact between the suproccipital and the parietals; we suggested that they were tabulars instead, while the supposed tabulars would be postorbitals (thought absent by Wellstead), and postparietals would be absent. As a byproduct, this would restore normal spatial relationships to the posttemporal foramen identified by Wellstead (1991).

Based on new material and $\mu \mathrm{CT}$ scans, Pardo \& Anderson (2016) have shown that Wellstead (1991) had overlooked a few sutures and misjudged certain 3D relationships; our reinterpretation of Wellstead's reconstruction is therefore moot. In particular, Wellstead correctly identified the postparietals, which do, however, meet in the midline in most or all specimens for a variable part of their length; there is no posttemporal foramen at all; and postorbitals are, like in lissamphibians, absent. We have updated the scores of Brachydectes following Pardo \& Anderson (2016).

\section{Treatment of OTUs}

OTUs are hypotheses; the delimitations of some require comments.

\section{Paleothyris/Protorothyris}

One OTU of RC07 is (explicitly) a composite of Paleothyris acadiana and Protorothyris archeri. Protorothyris is, according to the only phylogenetic analysis that has included both so far (Müller \& Reisz, 2006), more closely related to Petrolacosaurus - another OTU in this matrix - than to Paleothyris; Paleothyris could even be more closely related to Captorhinus also included in this matrix - than to either Protorothyris or Petrolacosaurus.

Still, these two species are morphologically similar, and most of their differences could

1212 Protorothyris. Our changes to this OTU, however, are exclusively based on the description of 
1213 Paleothyris by Carroll (1969b) and D. M.'s observations of Paleothyris specimens, especially

1214 TMM 45955-2 (a cast of MCZ 3482, which is on display and was not accessible at the time),

1215 cursorily confirmed against MCZ 3473, MCZ 3475, MCZ 3477, MCZ 3481 (the holotype), MCZ

1216 3483, MCZ 3486, MCZ 3487, MCZ 3488, MCZ 3490, MCZ 3492 and the apparently

1217 uncatalogued unlabeled specimen B-17 (also kept at the MCZ). Some of these changes are from

1218 known to unknown, so they likely concern characters whose state is known in the more

1219 completely preserved Protorothyris but not in Paleothyris.

1220

1221

1222

1223

1224

1225

1226

1227

1228

1229

1230

1231

1232

1233

1234

1235

1236

1237

1238

1239

1240

1241

1242

1243

1244

1245

1246

1247

1248

1249

1250

1251

1252

1253

1254

1255

1256

1257

\section{Dendrerpetidae}

Milner (1996) revised the specimens from Joggins (Nova Scotia, Canada) that were at that time included in Dendrerpeton acadianum (following Carroll, 1967), but had originally been described as several genera with many species. "The majority of specimens are still attributable to Dendrerpeton acadianum but three other forms are represented by one or two specimens each. These are Dendrerpeton confusum sp. nov., based on a single large skull, Dendrerpeton helogenes (Steen) comb. nov., based on two specimens, and an indeterminate cochleosaurid, based on a set of cranial fragments" (Milner, 1996: abstract). One of the specimens newly referred to $D$. helogenes was the well preserved skull that had been described by Godfrey, Fiorillo \& Carroll (1987) as D. acadianum. "Subsequently Holmes et al. [ = Holmes, Carroll \& Reisz] (1998) described an exquisitely preserved skeleton of a temnospondyl from Joggins and referred it to Dendrerpeton acadianum. By doing so, they lumped all the Joggins material together again. In this work, it is argued that the Joggins material includes two distinct genera, Dendrerpeton sensu MILNER 1996 and Dendrysekos (Dendrerpeton helogenes of MILNER 1996), which differ in a number of characters. The specimens of GODFREY et al. [ = Godfrey, Fiorillo \& Carroll] (1987) and HolmEs et al. (1998) are referable to Dendrysekos. This effectively means that Dendrysekos rather than Dendrerpeton has formed the outgroup in many recent cladistic analyses of temnospondyls." (Schoch \& Milner, 2014: 25)

Ruta, Coates \& Quicke (2003) did not cite Milner (1996). Their "Dendrerpeton acadianum" OTU, kept unchanged by RC07 (who did not cite Milner [1996] either), is in part based on their personal observations of specimens, of which at least some - notably the lectotype - really do belong to D. acadianum according to Milner (1996) and Schoch \& Milner (2014). However, Ruta, Coates \& Quicke (2003) also used and cited the descriptions by Godfrey, Fiorillo \& Carroll (1987) and Holmes, Carroll \& Reisz (1998). Assuming that the personal observations were in fact used to score the taxon and not merely to cursorily confirm the literature, this OTU is thus a chimera of Dendrerpeton acadianum and Dendrysekos helogenes.

Schoch \& Milner (2014) continued to maintain that Dendrerpeton and Dendrysekos are sister-groups; for this reason, we have not split the OTU for the time being, but merely renamed it Dendrerpetidae, the name recommended by Schoch \& Milner (2014), and compared it to the literature to make sure no polymorphisms had been overlooked (see above and Watanabe, 2015). In future work, however, it may be a good idea to treat all dendrerpetid species separately (including Dendrerpeton rugosum from Jarrow in Ireland: A. R. Milner, 1980; Schoch \& Milner, 2014): Ruta (2009) added Dendrerpeton confusum to a temnospondyl matrix derived from the matrix of RC07 and found that it was not the sister-group of the "D. acadianum" OTU. We have not done this yet because the present matrix most likely does not contain enough characters to place them accurately with respect to each other. 
1258

1259

1260

1261

1262

1263

1264

1265

1266

1267

1268

1269

1270

1271

1272

1273

1274

1275

1276

1277

1278

1279

1280

1281

1282

1283

1284

1285

1286

1287

1288

1289

1290

1291

1292

1293

1294

1295

1296

1297

1298

1299

1300

1301

1302

1303

(Whether the "D. acadianum" OTU of Ruta [2009] included any other dendrerpetids is unclear to us. The matrix of Ruta [2009] is a slight modification of that of Ruta \& Bolt [2006]. The latter's app. 1 lists all OTUs, except $D$. confusum, and the literature sources used to score them. For "D. acadianum", these are Carroll, 1967; Milner, 1980, 1996; Godfrey, Fiorillo \& Carroll, 1987; and Holmes, Carroll \& Reisz, 1998. In addition, this OTU is marked with an asterisk for having "been examined directly by one or both authors (using either casts or original specimens)"; which specimens those are is not mentioned, but presumably they are the same as those consulted by Ruta, Coates \& Quicke [2003]. However, given the absence of D. confusum from this appendix, we wonder if the appendix was written before Ruta \& Bolt decided to separate the two Dendrerpeton OTUs; if so, it is possible that the " $D$. acadianum" OTU really is just $D$. acadianum and not a chimera.)

Balanerpeton has sometimes (Pawley, 2006; Clack et al., 2012b; Dilkes, 2015a), but not always (Milner \& Sequeira, 1994; Ruta, 2009), been found as the sister-group of

"Dendrerpeton"; we therefore follow Schoch \& Milner (2014) in not including it in the Dendrerpetidae OTU.

Rhynchonkos

Szostakiwskyj, Pardo \& Anderson (2015) have shown that previous conceptions of Rhynchonkos (e.g. CG78; considered to contain a single species) were chimeric. They restricted Rhynchonkos to its holotype, a skull with lower jaw. The other skulls were referred to the new taxa Aletrimyti and Dvellecanus; a further lower jaw turned out to belong to none of the three. Szostakiwskyj, Pardo \& Anderson (2015) did not mention the postcranial material that had been referred to Rhynchonkos (e.g. CG78). A poorly preserved but largely articulated presacral skeleton (CG78: fig. 67) belongs to the same specimen as the referred skull of Aletrimyti (Szostakiwskyj, Pardo \& Anderson, 2015: 5). We therefore wondered whether to restrict our Rhynchonkos OTU to Rhynchonkos or instead to Aletrimyti (the type material of which is also slightly better preserved than that of Rhynchonkos). J. Pardo (pers. comm. 2015) has, however, pointed out that the postcranial material needs to be revisited. We have therefore restricted the Rhynchonkos OTU to the type material of Rhynchonkos and scored all postcranial characters as unknown (changes marked in green in App. S2 but not counted in Data S4).

We considered adding Aletrimyti and Dvellecanus as separate OTUs to our analyses with added taxa. Most likely, however, this would require also adding several at least superficially similar "microsaurs" (Nannaroter, Tambaroter, Huskerpeton, Proxilodon) and several characters; we prefer to leave this to future work.

RC07 had scored a few characters that remain unknown for all of the specimens referred to Rhynchonkos by CG78. An example is CAU FIN 1 (our ch. 277; see App. S1), for which they scored state 1, the absence of lepidotrichia in the tail - to the best of our knowledge, only the most proximal tail vertebrae are represented in the previously attributed material (CG78: 103104, 109-110, fig. 67-72). This is, incidentally, a common issue that affects several other OTUs and several other tail characters (App. S1).

Taxa added as parts of existing OTUs

We have interpreted almost all OTUs as genera (or larger taxa in the cases of Albanerpetidae and Dendrerpetidae) rather than species. This has allowed us to fill in some missing data and to 
1304 approach more plesiomorphic morphotypes. We do not think the polymorphisms this has 1305 occasionally introduced are a problem; quite the opposite - they are a better representation of the 1306 scope of the matrix. With the exceptions of Megalocephalus (Milner, Milner \& Walsh, 2009), 1307 Broiliellus (Schoch, 2012; Holmes, Berman \& Anderson, 2013; Dilkes, 2015b), Oestocephalus 1308 (Anderson, 2003a) and of course Pholiderpeton, the monophyly of the few genera in this matrix 1309 that are not monospecific is fairly obvious, at least with respect to the other OTUs in this matrix, 1310 and has not been disputed in the literature. Pholiderpeton was already coded as two separate 1311 OTUs (Ph. scutigerum, Ph. attheyi) by RC07 and indeed Ruta, Coates \& Quicke (2003).

1312 Megalocephalus is, if at all, only paraphyletic with respect to Kyrinion (Milner, Milner \& Walsh, 1313 2009) which is not included in this matrix (we have refrained from adding it - see below -

1314 because it would, given the present character sample, provide almost no new information on

1315 baphetid morphodiversity and thus baphetid relationships); also, we have not used any

1316 information from ?M. lineolatus, but only from the type species M. pachycephalus. We have not

1317 used information from any species referred to Broiliellus other than the one used by RC07, $B$.

1318 brevis - which is, incidentally, not the type species of Broiliellus and might therefore end up

1319 outside that taxon. In the figures, App. S2 and Data S4, we explicitly call this OTU "Broiliellus

1320 brevis". We have also ignored the incompletely preserved ?Oestocephalus nanus (Boyd, 1982),

1321 which is likely closer to Phlegethontia than to Oestocephalus (Anderson, 2003a), and the well

1322 preserved and stunningly well prepared but inadequately described ?O. guettleri (Krätschmer,

1323

1324

1325

1326

1327

1328

1329

1330

1331

1332

1333

1334 2006) and have - no doubt like RC07 - restricted ourselves to the type species, O. amphiuminus. (Naturally, the intriguing large skull referred to $O$. by Anderson, Pardo \& Holmes [2018] was unknown to us; we are looking forward to its complete description.) Because information about the specimen called Scincosaurus spinosus was unavailable to us, we only used the type species, S. crassus (used by RC07). Further, while the monophyly of Micromelerpeton has not been doubted, we have ended up using only M. credneri (the type species, used by RC07) because the other species do not add any information, being known from fewer individuals which are all skeletally immature.

Milner \& Schoch (2013) found that all recognized species of Trimerorhachis except $T$. sandovalensis form a clade which may or may not be the sister-group of $T$. sandovalensis to the exclusion of Neldasaurus. Because Neldasaurus is an OTU in this matrix, we have not used information from ?T. sandovalensis to score the Trimerorhachis OTU. We considered adding ?T. sandovalensis as an OTU to our analyses with added taxa, but its scores would hardly differ from the Trimerorhachis OTU at all. - Because Milner \& Schoch (2013) presented a phylogeny of the four species in the undoubted Trimerorhachis clade, we have avoided scoring Trimerorhachis as polymorphic and instead tried to identify the plesiomorphic condition (as we have done with Albanerpetidae and Batropetes); this concerns very few characters, however.

\section{OTUs added for a separate set of analyses}

Several theoretical considerations suggest that taxon exemplars should be as diverse as possible [...] [five references]. Importantly, a recent study based on simulations of true phylogenies (Salisbury \& Kim, 2001) indicates that dense and random taxon sampling increases the probability of retrieving correctly the plesiomorphic condition of characters as well as the ancestral state near the tree root. Furthermore, Salisbury \& Kim's (2001) simulations show that in the analysis of small clades, estimates of ancestral states are strongly affected by cladogram topology and by the number of descendent [sic] branches in progressively more distal internal nodes.

- Ruta, Coates \& Quicke (2003: 255) 
1352

1353

1354

1355

1356

1357

1358

1359

1360

1361

1362

1363

1364

1365

1366

1367

1368

1369

1370

1371

1372

1373

1374

1375

1376

1377

1378

1379

1380

1381

1382

1383

1384

1385

1386

1387

1388

1389

1390

1391

1392

1393

1394

1395

1396

1397

1398

1399

1400

Also, taxon removal because of incomplete preservation and missing character scores may be undesirable, because such taxa may have a positive effect on cladogram resolution [...] [five references].

$$
\text { - Ruta, Coates \& Quicke (2003: 257) }
$$

We recommend the inclusion of as many fossils as possible in any phylogenetic analysis. - Conrad \& Norell (2015)

\begin{abstract}
Numerous phylogenetic analyses demonstrate that the systematic position of Paranthodon is highly labile and subject to change depending on which exemplifier for the clade Stegosauria is used. The results indicate that the use of a basal exemplifier may not result in the correct phylogenetic position of a taxon being recovered if the taxon displays character states more derived than those of the basal exemplifier, and we recommend the use, minimally, of one basal and one derived exemplifier per clade.

- Raven \& Maidment (2018: abstract)
\end{abstract}

Analogously to that of Marjanović \& Laurin (2008b, 2009), the most important of the aims of the present work is to find out which hypothesis on the origin of the modern amphibians the matrix by RC07 supports after revision if we keep, as far as possible, its taxon and character sample intact. However, the Early Permian (Cisuralian) Gerobatrachus was described too late (Anderson et al., 2008a) to be included in the matrix of RC07, yet it is highly relevant because the phylogenetic analysis included in its description supported the PH. Being a temnospondyl with many similarities to lissamphibians, Gerobatrachus may also be considered to support the TH over the LH; indeed, the analysis by Maddin, Jenkins \& Anderson (2012) found it to be the oldest known lissamphibian while supporting the TH. However, this is based on a further development of the matrix by Anderson et al. (2008a) that did not take the reevaluations by Marjanović \& Laurin (2009) and Sigurdsen \& Green (2011) into account; and Marjanović \& Laurin (2009) found the LH despite including Gerobatrachus. Following the example of Marjanović \& Laurin (2008), we have therefore added Gerobatrachus to the present matrix in order to test if it changes the results.

At this opportunity we also added the following taxa:

\title{
Further temnospondyls other than stereospondylomorphs
}

Micropholis is an amphibamid, which means that the TH and the PH consider it close to the ancestry of some or all extant amphibians. It is the only dissorophoid known to have two widely spaced occipital condyles (like the modern amphibians), and it shares the Early Triassic age of the oldest known lissamphibians (the stem-salientians Triadobatrachus and Czatkobatrachus), unlike the Cisuralian Doleserpeton and Gerobatrachus or the Pennsylvanian Amphibamus. Growth series spanning most of the skeleton are known (Schoch \& Rubidge, 2005). - It is particularly surprising that RC07 did not add Micropholis to their matrix; they speculated that it might "turn out to occupy a more derived position than Doleserpeton on the amphibian stem group" (RC07: 86) because of its stratigraphic position.

Nigerpeton was described (Steyer et al., 2006; Sidor, 2013) as a cochleosaurid edopoid temnospondyl. The edopoids are thought to be close to the base of Temnospondyli - indeed, some analyses of temnospondyl phylogeny have used one or more edopoids as the outgroup or part thereof (e.g. Schoch \& Witzmann, 2009a, b) - so we expected an influence on the position of Temnospondyli and on the interrelationships of its largest constituent groups. The skull and lower jaw have been described, though their surface is mostly badly preserved. D. M. has seen 
1401 all known specimens - including postcranial material - and compared all scores to them; in App.

1402

1403

1404

1405

1406

1407

1408

1409

1410

1411

1412

1413

1414

1415

1416

1417

1418

1419

1420

1421

1422

1423

1424

1425

1426

1427

1428

1429

1430

1431

1432

1433

1434

1435

1436

1437

1438

1439

1440

1441

1442

1443

1444

1445

S1 we report a number of previously unpublished observations.

Saharastega, of which likewise the entire skull is known, though the preservation of the surface is again bad (Damiani et al., 2006), has had an unstable phylogenetic position close to the root of Temnospondyli. Based on a few character states that are rather odd for a temnospondyl, it has even been suggested to be a seymouriamorph, though not in the peer-reviewed literature (Yates, 2007). It almost certainly is a temnospondyl, but, as for Nigerpeton, we expected an influence on the relationships and the large-scale phylogeny of the temnospondyls. In addition, this is of course an opportunity to clarify the position of Saharastega itself. - D. M. has seen the only known specimen and compared all scores to it; in App. S1 we report a number of previously unpublished observations.

Iberospondylus is a rather early temnospondyl known from most of the skull and various postcranial remains, preserving an unusual combination of plesiomorphies and apomorphies such as the dissorophoid-like dorsal process on the quadrate. The eight phylogenetic analyses that have included it so far (Laurin \& Soler-Gijón, 2001, 2006; Pawley, 2006: fig. 44; Schoch \& Witzmann, 2009a, b; Dilkes, 2015a; Pardo, Small \& Huttenlocker, 2017: fig. 2, S7) have given seven different results, and two of them were based on very small matrices. - Both of us have studied all three specimens.

Tungussogyrinus has occasionally been considered a caudate, which would be highly interesting considering its Early Triassic age. Werneburg (2009) redescribed it as the sister-group to all other branchiosaurids, but noted similarities to lissamphibians, especially one apomorphy shared with Salientia that is coded in this matrix. - RC07 (p. 86) mentioned Tungussogyrinus together with Micropholis.

Acanthostomatops (Witzmann \& Schoch, 2006a) is a Cisuralian zatracheid temnospondyl thought to lie close to Dissorophoidea and/or Eryops. As pointed out but not tested by Witzmann \& Schoch (2006a: 365), it may thus influence temnospondyl phylogeny as well as the position of Lissamphibia.

Palatinerpeton is a temnospondyl known from an incompletely preserved, immature skeleton (Boy, 1996). Endowed with an unusual combination of characters and suggested to be a stereospondylomorph (Schoch \& Milner, 2000) or in a trichotomy with Stereospondylomorpha and an Eryopidae-Zatracheidae-Parioxys clade (Boy 1996; see "Taxa that were not added", below, for Parioxys), it has the potential to influence temnospondyl phylogeny.

Erpetosaurus, redescribed by Milner \& Sequeira (2011) on the basis of many flattened specimens that range from skull fragments to incomplete articulated skeletons, is a dvinosaurian temnospondyl, thought to be most closely related to Isodectes in the taxon sample of RC07. We have added it in order to confuse things - in other words, to test the robusticity of this matrix against homoplasy: Erpetosaurus shows a confusing mix of unexpected plesiomorphies and unexpected similarities to colosteids (among others).

Mordex was neglected for a long time because it is mostly known from larvae that were confused with Branchiosaurus, Platyrhinops and other dissorophoids from the same site. Recently, however, it has been understood as the oldest known trematopid (Milner \& Sequeira, 2003; Milner, 2007; Werneburg, 2012a); Milner (2007) called it "most similar to Ecolsonia" (tentatively confirmed by Schoch, 2012, and Schoch \& Milner, 2014), which implies that adding Mordex to the present analysis will influence the phylogenetic position of Ecolsonia. Although it is not very well known - the largest specimen is an incomplete skull roof, the others are all much 
1446 smaller and incompletely ossified (Werneburg, 2012a) -, it differs from all other taxa in this 1447 matrix.

Branchiosaurus used to be the name given to almost all skeletally immature

\section{Stereospondylomorphs} temnospondyls from the Czech Pennsylvanian, as well as occasionally the Pennsylvanian and Cisuralian of other places. Building on and greatly expanding the work of Milner \& Sequeira (2003), Werneburg (2012a) tried to sort out this confusion and provided a preliminary redescription of the type species, B. salamandroides from the Czech Republic. This redescription confirms that Branchiosaurus differs in several features from all other branchiosaurids (see Schoch \& Milner, 2008); some of these features are coded in the present matrix. Branchiosaurus is further necessary to test the phylogenetic position of Tungussogyrinus (see above). - As Werneburg (2012a) noted, B. fayoli from Commentry and " $B$. aff. fayoli" from Montceau-lesMines (both France) need to be redescribed before it can be assessed whether they form a clade with $B$. salamandroides; we have therefore not used any information from them.

Temnospondyl phylogeny is a vexing question; most mathematically possible permutations of Edopoidea (which may not be a clade; Pawley, 2006: chapter 6), Capetus, Dendrerpetidae + Balanerpeton (together mono- or paraphyletic), Dvinosauria, Eryopidae, Stereospondylomorpha and Dissorophoidea have been supported by recent phylogenetic analyses, and a few taxa have been found close to Eryopidae or as early stereospondylomorphs. This was, in part, discussed by Ruta, Coates \& Quicke (2003) (as well as by Pawley, 2006: chapter 5; Ruta, 2009; Schoch, 2013; Dilkes, 2015a; and references in all five). It is strange, then, that the matrices of Ruta, Coates \& Quicke (2003) and RC07 do not contain a single possible stereospondylomorph despite the presence of representatives of all other clades listed above. We have therefore added:

Sclerocephalus, variously considered an eryopid relative and/or a stereospondylomorph, is known from complete articulated skeletons and was redescribed by Meckert (1993; shoulder girdle and forelimb only) and Schoch \& Witzmann (2009a); D. M. compared some scores to the specimen MB.Am.1346 (a skull roof in dorsal view, a left lower jaw in medial and a right lower jaw in lateral view). Because Sclerocephalus is a weakly supported clade of four species (Schoch \& Witzmann, 2009a) - ignoring the poorly known ?S. stambergi, whose phylogenetic position is even less clear (Klembara \& Steyer, 2012) -, we have only considered the type species, $S$. haeuseri, which also happens to be the best-known one..

Cheliderpeton vranyi, the type species of Cheliderpeton, was redescribed by Werneburg $\&$ Steyer (2002). Like Sclerocephalus, it has variously been considered an eryopid relative or a stereospondylomorph. The present matrix may be able to decide this question. Further, Werneburg considered Cheliderpeton an intasuchid, Steyer preferred to consider it an archegosaurid (Werneburg \& Steyer, 2002); this question is testable in this matrix insofar as Cheliderpeton may form an exclusive clade with Archegosaurus (see below) or not. Growth series of the skull roof and a few other bones are known. We have not been able to use information from the putative second species, Ch. lellbachae, which "[i]n most respects [...] resembles Sclerocephalus haeuseri" and needs to be (re)described (Schoch \& Witzmann, 2009b: 122).

Glanochthon was variously referred to Archegosaurus, Actinodon, Sclerocephalus or Cheliderpeton till Werneburg \& Steyer (2002) recognized it as distinct from all four and Schoch \& Witzmann (2009b) redescribed it. (For example, it appears as "Cheliderpeton latirostre" in the 
1492 analysis by Ruta, 2009.) Found to be closer to Archegosaurus and Stereospondyli than 1493 Cheliderpeton and Sclerocephalus by Schoch \& Witzmann (2009b), the two species (G.

1494 latirostris, G. angusta; Schoch \& Witzmann, 2009b) are known from growth series of many 1495 skulls (including lower jaws: Boy, 1993) and most of the postcranium except, apparently, the 1496 tail.

Archegosaurus is known from complete articulated skeletons and was redescribed by Witzmann (2006: head skeleton) and Witzmann \& Schoch (2006b: postcranium). Together with the other potential archegosaurids, all of which are much less completely known, it is considered a close relative of Stereospondyli. We have not used information from Memonomenos, which was often referred to Archegosaurus in earlier times but belongs elsewhere in the tree (Schoch \& Milner, 2000: fig. 52; Schoch \& Witzmann, 2009b).

Platyoposaurus, known from rich cranial and postcranial material (Efremov, 1932; Konzhukova, 1955; Gubin, 1991), is superficially very similar to Archegosaurus and was found as its sister-group by Pardo, Small \& Huttenlocker (2017: fig. 2, S7), but may actually be closer to Stereospondyli (Schoch \& Witzmann, 2009b; Pereira Pacheco et al., 2016).

Konzhukovia has been allied to Archegosaurus and to Stereospondyli in various sources. Gubin (1991) illustrated (drawings 6,15) and described the well preserved type skull of the type species, K. vetusta; we have not used other information except for two comments on the same species by Pereira Pacheco et al. (2016: app. 2). First, further information on K. vetusta is not available (Gubin [1991: fig. 2 of plate II] only showed a small photo of an additional skull fragment of $K$. vetusta and merely mentioned the existence of a lower-jaw fragment and postcranial fragments); second, the referral of $K$. tarda and a fortiori $K$. sangabrielensis to Konzhukovia is doubtful (Pereira Pacheco et al., 2016, found K. tarda outside a clade consisting of $K$. vetusta and Tryphosuchus paucidens, and $K$. sangabrielensis outside a clade containing all three); third, K. sangabrielensis is only known from a partial skull that hardly seems to differ from K. vetusta in characters included in this matrix, while Gubin (1991: fig. 1 of plate II) only showed small photos of a skull of $K$. tarda and merely mentioned the existence of another skull. The diagnoses of the three species do not differ in characters that the present matrix contains, and the illustrations apparently do not either (except for the lateral-line grooves of $K$. sangabrielensis, which K. vetusta actually shares: Pereira Pacheco et al., 2016: app. 2).

Lydekkerina is within a few internodes of the origin of Stereospondyli according to all recent analyses. It is known from complete skeletons that are unusually well ossified for a stereospondyl. The postcranium was recently described by Pawley \& Warren (2005) and Hewison (2008), and the skull and lower jaw by Jeannot, Damiani \& Rubidge (2006) and Hewison (2007). Shishkin, Rubidge \& Kitching (1996) showed that various proportions of the skull roof of Lydekkerina are paedomorphic, a hypothesis further supported for other characters by Hewison (2007); this probably does not affect any characters in this matrix. Miniaturization by progenesis may explain the well ossified endochondral bones.

Australerpeton was recently identified as a rhinesuchid stereospondyl (Eltink et al., 2016) after being thought to lie close to Archegosaurus by Schoch \& Milner (2000). Almost the entire skeleton is known (Barberena, 1998; Dias \& Schultz, 2003; Eltink \& Langer, 2014; Eltink et al., 2016).

\section{Chroniosuchians}


1537 Chroniosuchia is an enigmatic clade that has long been known from fragmentary remains. Only

1538 recently has more complete material been published, without, however, clarifying the

1539 phylogenetic position of the group. These animals have occasionally (e.g. Laurin, 2000;

1540 Klembara, Clack \& Čerňanský, 2010) been considered embolomeres (thus anthracosaurs) mainly

1541 because of their embolomerous centra, but their confusing mosaic of character states is

1542 compatible with a number of other phylogenetic positions as well; indeed, Klembara et al. (2014)

1543 recovered them as the sister-group to a gephyrostegid-seymouriamorph clade, Witzmann \&

1544 Schoch (2017) found them in three positions (next to Anthracosauria, Silvanerpeton or a clade

1545 containing Gephyrostegus, seymouriamorphs, amniotes and "microsaurs" among others), and

1546 Schoch, Voigt \& Buchwitz (2010) and Buchwitz et al. (2012) even found them to be

1547 lepospondyls. Moreover, the two chroniosuchians we added could influence the positions of the

1548 embolomeres, Gephyrostegus, Bruktererpeton, Silvanerpeton, perhaps Caerorhachis, and

1549 possibly Solenodonsaurus in our tree.

$1550 \quad$ Chroniosaurus is by far the most thoroughly described representative (Clack \&

1551 Klembara, 2009; Klembara, Clack \& Čerňanský, 2010) of the clade, with most of the skeleton

1552

1553

1554

1555

1556

1557

1558

1559

1560

1561

1562

1563

1564

1565

1566

1567 being preserved. - The specimens described and figured by Clack \& Klembara (2009) did not preserve much of the lower jaws; Clack \& Klembara (2009: 21) therefore stated that they had scored the reconstruction by Ivachnenko \& Tverdochlebova (1980) in their phylogenetic analysis. This reconstruction (Ivachnenko \& Tverdochlebova, 1980: drawing 16) would allow unambiguous scoring of our characters 147 (PSYM 1) and 155 (SPL 2), which Clack \& Klembara (2009) scored as unknown, and 156 (SPL 3-4), which they scored as having our state 1 or 2. However, we have kept only the scores of Clack \& Klembara (2009) because all of the drawings in Ivachnenko \& Tverdochlebova (1980) are reconstructions which often do not indicate which parts are actually known; the characters in question concern those parts of the lower jaw that are most likely to be incompletely preserved or crisscrossed by fractures (as chroniosuchian dermal bones usually are). We should note that the reconstruction of the skull proper in all three views (dorsal, ventral, lateral: drawings 1a, б, в) differs appreciably from those of Clack \& Klembara (2009) and Klembara, Clack \& Čerňanský (2010) in the shape and proportions of the skull, the course of some sutures, and the size, shape and relative positions of all openings, in two cases even their presence. The text (Ivachnenko \& Tverdochlebova, 1980: 22) only briefly describes the chroniosuchian lower jaw in general, without mentioning which

1568 information is based on Chroniosaurus or Chroniosuchus, and does not refer to the characters in question. Specimen drawings or photos of the lower jaw of any chroniosuchian have never been published to our knowledge, except for part of the labial side of that of Chroniosaurus in Clack \& Klembara (2009: fig. 2) which does not provide further information.

Bystrowiella, originally described from rather limited postcranial material (Witzmann, Schoch \& Maisch, 2008), has recently become another well-understood chroniosuchian following the discovery of cranial as well as further postcranial material (Witzmann \& Schoch, 2017). As a bystrowianid, it shortens the potentially long branch of the chroniosuchid

\section{Chroniosaurus.}

\section{"Microsaurs"}

Utaherpeton was a surprising omission by $\mathrm{RC} 07$, given the facts that it is among the oldest known "microsaurs" and has been considered a basal "microsaur" (Carroll, Bybee \& Tidwell, 1991; Carroll \& Chorn, 1995; Anderson, 2001; Anderson et al., 2008a) or the sister-group of 
1583 Microbrachis and thus a basal member of the "microsaur"-lysorophian-lissamphibian clade 1584 (Vallin \& Laurin, 2004); in more recent analyses it fell out close to a "nectridean"-aïstopod1585 lysorophian-lissamphibian clade (Marjanović \& Laurin, 2009: Electronic Supplementary 1586 Material 2) or as a "lepospondyl" outside the major groups (Pardo, Small \& Huttenlocker, 2017: 1587 fig. S6, and references therein). Similarities to the "nectrideans" were already noted in the 1588 original description (Carroll, Bybee \& Tidwell, 1991). A variety of interesting effects on

1589 lepospondyl intra- and perhaps even interrelationships could be expected from its addition to the 1590 present matrix based on the two descriptions (Carroll, Bybee \& Tidwell, 1991; Carroll \& Chorn, 1995). - We have tentatively accepted the referral by Carroll \& Chorn (1995) of the specimen they described to Utaherpeton. Carroll \& Chorn (1995) did not offer any evidence for this referral other than the fact that their specimen is a "microsaur" from the same locality as Utaherpeton, and did not discuss or even mention the question. However, the unusual proportions of the hindlimb mentioned in the diagnosis by Carroll, Bybee \& Tidwell (1991) do appear to fit, the difference in the shape of the interclavicle may well be ontogenetic, and while the figures do not make clear if either specimen has precisely 26 presacral vertebrae as described, they do not rule out that both at least have the same or almost the same count.

The "Goreville microsaur", too, is among the oldest known "microsaurs". Although it was deliberately not named by Lombard \& Bolt (1999), it differs from all other OTUs in this matrix; its somewhat unusual combination of plesio- and apomorphic character states (noted in its description) could change the topology of the tree. Eight badly preserved specimens, amounting to most of the skeleton, are known.

Sparodus is another "microsaur" that is not obviously deeply nested in one of the universally recognized clades, showing affinities to both Pantylidae and Gymnarthridae but having fewer apomorphies than the undisputed members of both (and being older than most of them). - Personal observation by D. M. of NHMW 1899/0003/0006 (Fig. 3, 4), the referred specimen described by Carroll (1988), shows that Carroll's (1988: fig. 1) drawing and reconstruction of the skull are probably optimistic, unless the sutures are better visible on the latex cast Carroll used (which we have not seen) than on the fossil itself. However, this concerns very few characters.

Carrolla is a brachystelechid "microsaur" known only from a skull with lower jaw. Few differences between it and Batropetes (the brachystelechid that was already included in the matrix) are known, and few of those concern characters in the present matrix, but the redescription by Maddin, Olori \& Anderson (2011) based on high-resolution computed tomography has made a large amount of information available. This is especially important because it allows us to better test the results by Vallin \& Laurin (2004), who found Brachystelechidae to be the sister-group of Lysorophia + Lissamphibia, as well as the results by Maddin, Jenkins \& Anderson (2012), who found the brachystelechids to be nested in a clade (Recumbirostra) that contains all the other "microsaurs" with a snout tip that protrudes beyond the premaxillary toothrow (state PREMAX 8(1) in the present matrix). - D. M. has seen the only known specimen and was consequently able to score some characters that concern externally visible anatomy and were insufficiently addressed by Maddin, Olori \& Anderson (2011); these scores are described in App. S1.

Crinodon is a "microsaur" that is rich in plesiomorphies. Known from a well-preserved dermatocranium and partial lower jaws (CG78) - parts of the body that the present craniocentric matrix is well equipped to deal with -, it may help shed light on "microsaurian" intra- and interrelationships. CG78 classified it as a tuditanid, but the two "tuditanids" in the present matrix 
1629 (Tuditanus and Asaphestera) were not found as sister-groups by RC07, leaving us wondering

1630 whether the diagnostic characters of "Tuditanidae" might all be symplesiomorphies or

1631 homoplasies.

1632

1633

Trihecaton is a large "microsaur" known from an articulated skeleton that lacks the skull

1634

1635

1636

1637

1638

1639

1640

1641

1642

1643

1644

1645

1646

1647

1648

1649

1650

1651

1652

1653

1654

1655

1656

1657

1658

1659

1660

1661

1662

1663

1664

1665

1666

1667

1668

1669

1670

1671

1672

1673

1674 (apart from the maxilla, whose dorsal edge is not prepared), part of the lower jaw, most of the distal limbs and much of the tail - although further preparation would probably reveal additional bones. Presumably because most of the skull is unknown, Trihecaton has never featured in a phylogenetic hypothesis since CG78 gave it its own family in Tuditanomorpha; yet, it differs from all other OTUs in this matrix, so we see no reason to continue its exclusion from phylogenetic analyses. D. M. scored Trihecaton directly from the two known specimens (which most likely belong to the same individual); it is possible that additional preparation has been done since the description by CG78.

Quasicaecilia, known from an isolated skull without lower jaws (except an articular fragment), was redescribed by Pardo, Szostakiwskyj \& Anderson (2015). It is a very small brachystelechid that may contribute to resolving the relationships of brachystelechids, other burrowing "microsaurs", lysorophians and lissamphibians; additionally or alternatively, it could highlight the effects of miniaturized taxa on phylogenetic analyses.

Llistrofus is very similar to Hapsidopareion in the original taxon sample, but while Bolt $\&$ Rieppel (2009) found very few differences in their redescription of the skull, they also found very few synapomorphies. The postcranium is scored after CG78.

\section{Synapsids}

The diadectomorphs (in the original and the expanded sample: Diadectes, Orobates, Tseajaia, Limnoscelis) are usually thought to lie on the amniote stem. It has, however, been proposed (Berman, Sumida \& Lombard, 1992; Berman, 2013) that they are actual amniotes - in particular, the closest known relatives of Synapsida, thus part of Theropsida, the sister-group of Sauropsida (Goodrich, 1916). Surprisingly, all unquestioned amniotes in the matrix by RC07-

Petrolacosaurus, Paleothyris, Captorhinus - are sauropsids; the lack of unambiguous theropsids means that the original taxon sample is unable to test the mentioned hypothesis, even though its large amount of non-amniote OTUs would have made it very well suited for such a purpose. The two synapsid OTUs we have added help to solve this problem. Furthermore, they might further influence diadectomorph monophyly - which was not found by Ruta, Coates \& Quicke (2003), Pawley (2006), RC07, or even Germain (2008a).

We have included Eothyris, Oedaleops and Eocasea as a single OTU called Caseasauria. Eothyris is known from an isolated, almost complete skull and lower jaw (Reisz, Godfrey \& Scott, 2009); Oedaleops is known from a less complete skull and lower jaw, additional skull fragments, vertebrae, ribs, and girdle and limb elements (Reisz, Godfrey \& Scott, 2009; Sumida, Pelletier \& Berman, 2014; we follow the latter in not including the ilium tentatively referred by Langston, 1965); Eocasea is known from a skeleton lacking the shoulder girdle, the forelimbs, the distal part of the tail and most of the skull (Reisz \& Fröbisch, 2014). The overlapping parts of these three taxa score almost identically in our matrix; where they disagree, we have simply scored polymorphism (and noted this in App. S1), because the relationships of these taxa to each other and to the herbivorous members of Caseidae, which are not considered here, remain unclear (Reisz, Godfrey \& Scott, 2009; Sumida, Pelletier \& Berman, 2014; Reisz \& Fröbisch, 2014; Brocklehurst, Romano \& Fröbisch, 2016; though see Brocklehurst et al., 2016). - Some 
1675 data of Eothyris not presented in the cited publications were filled in by D. M. by observation of

1676 the holotype; they agree with the conditions in Oedaleops (Reisz, Godfrey \& Scott, 2009) and

1677 Eocasea (Reisz \& Fröbisch, 2014). We did not consider Callibrachion or Datheosaurus, which

1678 are poorly preserved and incompletely ossified (Spindler, Falconnet \& Fröbisch, 2015), or the

1679 recently (re)described Vaughnictis (Brocklehurst et al. 2016); they should be taken into account

1680 in future analyses, however, because all the analyses by Brocklehurst et al. (2016: fig. 10, 11)

1681 and Spindler et al. (2018: fig. 30) fully resolved the phylogenetic positions of all taxa mentioned

1682 in this paragraph.

1683 Supplementing the Caseasauria OTU is Archaeovenator, the oldest known varanopid and 1684 sister-group to all other varanopids, known from a largely complete, mostly articulated skeleton

1685 described by Reisz \& Dilkes (2003) and recently found nearly as close to the origin of Synapsida 1686 as Caseasauria (Reisz \& Fröbisch, 2014: app. S5; Brocklehurst et al., 2016; Spindler et al., 1687 2018).

1688

1689

1690

1691

1692

1693

1694

1695

1696

1697

1698

1699

1700

1701

1702

1703

1704

1705

1706

1707

1708

1709

1710

1711

1712

1713

1714

1715

1716

1717

1718

1719

\section{Lissamphibians}

Liaobatrachus is an Early Cretaceous salientian known from plentiful, very well preserved articulated skeletons (Dong et al., 2013). Although it was found to form a trichotomy with the two component clades of the crown-group of frogs, which means it is not very close to the root of Salientia, it may still influence the position of Salientia in particular and Lissamphibia in general, because few of the more rootward salientians are well preserved. - Dong et al. (2013) lumped what had been described as five monospecific genera (Liaobatrachus, Dalianbatrachus, Callobatrachus, Mesophryne, Yizhoubatrachus) as three species of Liaobatrachus and added a fourth. Chen et al. (2016) and Gao \& Chen (2017) preferred to consider Liaobatrachus and Dalianbatrachus nomina dubia and include the other three nominal genera as separate OTUs in their phylogenetic analyses; rather than finding them as a clade, they found them as a grade on the bombinanuran stem just within the anuran crown-group. However, there are no other crowngroup frogs in our matrix; if the mentioned analyses are correct, our Liaobatrachus OTU merely bears the wrong name and contains too much missing data. Further, Gao \& Chen (2017) found little if any support in this part of the tree, while Chen et al. (2016) did not perform any robustness analyses; both analyses contain less than twice as many characters as taxa and were focused on more highly nested crown-group frogs; the arrangement of Mesophryne, Yizhoubatrachus and Callobatrachus as an uninterrupted grade differs starkly from the positions found in earlier literature (summarized in Marjanović \& Laurin, 2013b: fig. 3), which were widely dispersed through the salientian stem and the base of the crown; and both analyses peculiarly lack L. zhaoi Dong et al., 2013, which is preserved in three dimensions and therefore is the source of most of the scores of our Liaobatrachus OTU.

Beiyanerpeton is a neotenic salamandroid of apparently Late Jurassic age (Gao \& Shubin, 2012), in any case older than its Early Cretaceous relative Valdotriton which was already included in the matrix. It is the only known lissamphibian, extant or extinct, to possess grooves for the lateral-line organ on the skull. Among the lissamphibians in the matrix, it is the only one known to possess opisthotics that are not fused to the prootics or exoccipitals (or otherwise absent). These factors make it important enough to add it to this matrix (which contains both of these characters), even though its paedomorphosis makes some characters difficult to interpret for purposes of phylogenetics (see Gao \& Shubin, 2012: supplementary information). 
1720

1721

1722

1723

1724

1725

1726

1727

1728

1729

1730

1731

1732

1733

1734

1735

1736

1737

1738

1739

1740

1741

1742

1743

1744

1745

1746

1747

1748

1749

1750

1751

1752

1753

1754

1755

1756

1757

1758

1759

1760

1761

1762

1763

1764

1765

The even older (Late or Middle Jurassic) Pangerpeton was found by Jia \& Gao (2016a) to be a stem-hynobiid salamander, similarly close to the root of Urodela as Beiyanerpeton and Valdotriton. Several features of the only known skeleton (which lacks most of the tail, but is accompanied by a body outline) suggest that it had undergone metamorphosis, although it remained aquatic (Wang \& Evans, 2006). Indeed, in having the jaw joints and the occiput in the same vertical transverse plane, it is more peramorphic than Beiyanerpeton, Valdotriton and even Karaurus. The skeleton is preserved in ventral view, so that most of the skull roof remains unknown, and it is unfortunate that the photos (even in the PDF version) have such low resolution that, for example, the ribs are entirely invisible and had to be scored from sparse remarks in the text that were not meant for comparison with our taxon sample. Still, Pangerpeton does not score redundantly with any other OTU in this matrix.

Even more peramorphic than Pangerpeton is Chelotriton. Scoring of this late Oligocene pleurodeline salamandrid (newt) is based mostly on MB.Am.45 (Marjanović \& Witzmann, 2015). This specimen exhibits peramorphic traits that are otherwise rare or even entirely unknown in salamanders (in a few cases even in lissamphibians as a whole), such as very long ribs, some of which are ventrally curved, and jaw joints that lie far caudal of the occiput. In 1981, it was misidentified as a "Dissorophid temnospondyl" "?Amphibamus" on a label. MB.Am.45 is indeed strikingly amphibamid-like in appearance, with some similarity also to certain diplocaulid "nectrideans". We have added Chelotriton to the matrix to see the effects, if any, of its unexpected peramorphic features on caudate and lissamphibian intra- and interrelationships. For this purpose, we took MB.Am.45 (scored mostly from the silicone cast MB.Am.45.3) at face value wherever possible rather than scoring polymorphism; missing data were filled in from the less extremely peramorphic Chelotriton individuals described and figured by Roček \& Wuttke (2010) and Schoch, Poschmann \& Kupfer (2015).

\section{Seymouriamorph}

Karpinskiosaurus may help clarify seymouriamorph phylogeny and relationships. The skulls of the type species, $K$. secundus, including an adult, non-paedomorphic one, were redescribed by Klembara (2011); according to the same work, the type specimen of $K$. ultimus requires revision, so this species is not considered here. Much of the axial skeleton was illustrated and commented on by Bystrow (1944).

\section{Undoubted colosteids}

Deltaherpeton is one of the oldest known colosteids, represented by a skull roof and lower jaw (Bolt \& Lombard, 2001, 2010). It shows a few plesiomorphies as well as several unique features that are absent or not preserved in Colosteus and Greererpeton. Bolt \& Lombard (2010) drew attention to this fact, but then undertook only a comparison instead of a phylogenetic analysis to justify their conclusion that "[t]he morphology of Deltaherpeton and the revised data presented for colosteids do not clarify the relationship of colosteids to other early tetrapods" (Bolt \& Lombard, 2010: abstract). Indeed, there are characters that indicate a very rootward position of Colosteidae (as found by RC07), but others support a position more crownward than those of Whatcheeriidae and Crassigyrinus (as found by Ruta, 2009, if we assume that the temnospondyls would be close to or within the crown), and a few have led to the traditional concept of colosteids as temnospondyls (as the sister-group to all other members of that clade). 
1766

1767

1768

1769

1770

1771

1772

1773

1774

1775

1776

1777

1778

1779

1780

1781

1782

1783

1784

1785

1786

1787

1788

1789

1790

1791

1792

1793

1794

1795

1796

1797

1798

1799

1800

1801

1802

1803

1804

1805

1806

1807

1808

1809

1810

1811

Pholidogaster has been known for over 150 years; it was redescribed by Romer (1964) and, after additional preparation of the skull and shoulder girdle, Panchen (1975). Its identity as a colosteid has not been questioned since the latter publication; and yet, to the best of our knowledge, it has never been included in a phylogenetic analysis. Although it is generally less well known than Colosteus, Greererpeton and Deltaherpeton, it scores differently from all three in our matrix; unlike in any known specimen of the other three colosteids (Bolt \& Lombard, 2010), the septomaxilla and parts of its surroundings are preserved. Its age, close to that of Deltaherpeton, makes it a particularly interesting potential source of information on the phylogeny and affinities of Colosteidae.

\section{Anthracosaurs}

Holmes \& Carroll (2010) have described an unusually small but adult (or nearly so) incomplete skeleton of an anthracosaur (embolomere) from Joggins (Late Carboniferous). Unfortunately, NSM 994 GF 1.1 cannot be assigned to a named genus, because it does not overlap with diagnostic parts of the only known anthracosaur from the same site, Calligenethlon; however, because it is considerably more complete than any specimen which can be assigned to Calligenethlon (all of which are slightly smaller and possibly less mature), we have added it to this matrix.

Palaeoherpeton is less well known than the originally included anthracosaurs, but the material was very thoroughly described by Panchen (1964, with a correction 1972) and scores differently from any other OTU in our matrix.

Neopteroplax, an anthracosaur described by Romer (1963) from a largely complete skull with lower jaw, has been mostly ignored ever since. In spite of having a skull shape quite similar to that of Pholiderpeton attheyi, however, Neopteroplax shows a number of features that are unusual for embolomeres; it makes an interesting addition to the question of anthracosaur phylogeny and affinities. We consider the referral of isolated jaw fragments and centra (not clear if pleuro- or intercentra) from a younger, very distant site to Neopteroplax (Romer, 1963: 451) highly doubtful; for the centra in particular it requires a rather long chain of inference from a single potentially diagnostic feature (the distance between the teeth). The jaw fragments would not add any information to our scoring of Neopteroplax; we have ignored the centra to avoid creating a chimera.

\section{Aistopods}

The skull of Coloraderpeton was redescribed by Anderson (2003a) and reinterpreted from $\mu \mathrm{CT}$ data by Pardo et al. (2017). Like Lethiscus (Pardo et al., 2017), it preserves unexpected plesiomorphies; among those not known in Lethiscus are an at least mostly enclosed mandibular lateral-line canal and a bone that is most parsimoniously interpreted as a preopercular. Unfortunately, information on the postcranium is very scarce in publications (Carroll, 1998b; Anderson, 2003a; Anderson, Carroll \& Rowe, 2003), and D. M. only briefly saw the specimens (at the $\mathrm{CM}$ ) years before we decided to add Coloraderpeton to the matrix.

Pseudophlegethontia (Anderson, 2003b) may fill the morphological gap between Phlegethontia and the other aïstopods; we have reinterpreted a few characters (as detailed in App. S1) in the light of Lethiscus and Coloraderpeton (Pardo et al., 2017). 


\section{Devonian enigmas}

1813

1814

1815

1816

1817

1818

1819

1820

1821

1822

1823

1824

1825

1826

1827

1828

1829

1830

1831

1832

1833

1834

1835

1836

1837

1838

1839

1840

1841

1842

1843

1844

1845

1846

1847

1848

1849

1850

1851

1852

1853

1854

1855

1856

1857

Ruta, Coates \& Quicke (2003: 262) listed Metaxygnathus among Devonian taxa that are "known mainly from lower jaw rami and/or incomplete postcranial remains" and "are omitted" from their matrix, presumably because they are so incompletely known. As with Sigournea and

Doragnathus, however, the isolated lower jaw ramus called Metaxygnathus differs from all other lower jaws (isolated or not) in this matrix. Indeed, one character, ANG 3, seems to have been deliberately included to potentially hold Acanthostega and Metaxygnathus together in "future, expanded versions of our matrix" (RC07: 103). Our source was the redescription by Ahlberg \& Clack (1998).

Ymeria is an "Ichthyostega-grade" animal known from premaxillae, a palate in dorsal view and lower jaws (plus shoulder girdle remains that are too fragmentary to be scored in this matrix). It was found one node rootward of Ichthyostega in those analyses by Clack et al. (2012a) and Sookias, Böhmer \& Clack (2014) that had enough resolution to tell.

Densignathus, much like Metaxygnathus, is known only from lower-jaw material (Daeschler, 2000; Ahlberg, Friedman \& Blom, 2005) and was listed among the excluded taxa by Ruta, Coates \& Quicke (2003: 262), but differs from all other lower jaws in the matrix.

Elginerpeton has been described, from successively greater amounts of isolated material and jaw fragments, as a Devonian animal close to the origin of limbs (Ahlberg, 1995, 1998; Ahlberg \& Clack, 1998; Ahlberg, Friedman \& Blom, 2005). It could serve to establish character polarities for the other Devonian OTUs. Following Ahlberg, Friedman \& Blom (2005) and Ahlberg (2011), we have included the postorbital bone and the postcranial material described by Ahlberg (1998), except for the supposed humerus.

\section{Mississippian enigmas}

Sigournea is known only from an isolated lower jaw (Bolt \& Lombard, 2006). Although similarities to the baphetoids, especially Spathicephalus (see below), and to the equally mysterious jaw material called Doragnathus (see below) have been noted (Bolt \& Lombard, 2006; Milner, Milner \& Walsh, 2009), these conjectures have only been tested twice in a phylogenetic analysis. Sookias, Böhmer \& Clack (2014) found it in the whatcheeriid region of the tree (more rootward than Baphetes, the only included baphetoid). That analysis did not include Doragnathus and had a generally insufficient taxon and, likely, character sample to address this question. Clack et al. (2016), who included Doragnathus, found Sigournea as a colosteid (unweighted parsimony) or in several places in the grade between the more rootward whatcheeriids and the more crownward colosteids (Bayesian inference, as well as parsimony with various degrees of reweighting). Our matrix contains enough lower-jaw and tooth characters to show that Sigournea is distinct from any other taxon for which more than a few such characters can be scored.

Doragnathus is another mysterious animal known only from lower jaws and parts of upper jaws. It has only once before (Clack et al., 2016) been included in a phylogenetic analysis, even though it was described quite some time ago (Smithson, 1980), and even though it differs from Sigournea, Spathicephalus (see below) and all other potential relatives in characters that are included in the present matrix. As recommended by Smithson \& Clack (2013), we have not scored the isolated postcranial material that was found at the same site and may or may not belong to the same taxon (described and illustrated by Smithson \& Clack, 2013). 
1858

1859

1860

1861

1862

1863

1864

1865

1866

1867

1868

1869

1870

1871

1872

1873

1874

1875

1876

1877

1878

1879

1880

1881

1882

1883

1884

1885

1886

1887

1888

1889

1890

1891

1892

1893

1894

1895

1896

1897

1898

1899

1900

1901

1902
Spathicephalus is a baphetoid known from skull and lower-jaw material with several strange autapomorphies (Baird, 1962; Beaumont \& Smithson, 1998; Smithson et al., 2017). Still, in a few characters it may be more plesiomorphic than at least Baphetes and Megalocephalus, which may help test baphetoid relationships (see Milner, Milner \& Walsh, 2009); it may also influence the positions of Sigournea and Doragnathus.

MB.Am.1441, the "St. Louis tetrapod", is a natural mold of a skull with lower jaws in ventral view that was described by Clack et al. (2012b). The description noted several similarities to colosteids, but also to temnospondyls, and ultimately did not commit to either hypothesis of relationships; the phylogenetic analysis in that paper, which used a very small taxon sample and a rather repetitive character sample, found it to be the sister-group of a novel (Ptyonius, (Adelogyrinus, Greererpeton)) clade, which together with the "St. Louis tetrapod" formed the sister-group to the only two included temnospondyls - an unusual arrangement as well. The matrix presented here may be better suited to resolving the relationships of MB.Am.1441, which D. M. was able to study firsthand (both the natural mold, MB.Am.1441.1, and the silicone cast, MB.Am.1441.2). Despite the small number of characters that can be scored, the "St. Louis tetrapod" differs from all other OTUs in this matrix.

Perittodus, Diploradus and Aytonerpeton are three of the many new taxa that were briefly presented by Clack et al. (2016). Their middle Tournaisian age lies in Romer's Gap, which is a temporal as well as morphological gap between the Devonian and the post-Tournaisian limbed vertebrates. Perittodus is mostly known from a very plesiomorphic, at most "Ichthyostegagrade" lower jaw; Diploradus has a lower jaw reminiscent of Sigournea and Doragnathus, but also preserves fragments of the rest of the skeleton; Aytonerpeton is known from scattered postcrania as well as a snout which resembles the "St. Louis tetrapod" and preserves, as we discuss in App. S1 (ch. 6, 7), the youngest anterior tectal identified in any limbed vertebrate.

Casineria is generally thought to be close to the origin of amniotes or at least seymouriamorphs (Paton, Smithson \& Clack, 1999; Clack et al., 2012b, 2016; Witzmann \& Schoch, 2017: fig. 16). In stark contrast, Pawley (2006: 207) reported that it scored identically to Caerorhachis in her matrix, apart from the (quite different) distribution of missing data, and considered them "indistinguishable based on the available evidence" (Pawley, 2006: 195, 239), pointing out further that the supposedly amniote-like features of Casineria have a wider distribution. In her phylogenetic analysis, it came out as a temnospondyl (in a trichotomy with Caerorhachis and a clade formed by all other temnospondyls). D. M. has seen the only known specimen, a headless and largely tailless, mostly articulated skeleton. We present new photos (Fig. 5-7), interpretations and comparisons to the description and redescription of Caerorhachis (Holmes \& Carroll, 1977; Ruta, Milner \& Coates, 2002) in the Discussion (The interrelationships of Anthracosauria, Silvanerpeton, Caerorhachis, Gephyrostegidae, Casineria and

Temnospondyli); notably, we identify what seems to be a postbranchial lamina on the cleithrum (Fig. 7).

\section{Pennsylvanian enigma}

The "Parrsboro jaw" is an incomplete impression of a lower jaw of Pennsylvanian age, consistently called NSM 987GF65 in the original description (Godfrey \& Holmes, 1989) but NSM 987GH65 in the partial redescription (Sookias, Böhmer \& Clack, 2014). It has a unique combination of characters and differs from all other OTUs in this matrix, which may therefore 
1903 have the potential to resolve the relationships of this mysterious fragment that has warranted

1904

1905

1906

1907

1908

1909

1910

1911

1912

1913

1914

1915

1916

1917

1918

1919

1920

1921

1922

1923

1924

1925

1926

1927

1928

1929

1930

1931

1932

1933

1934

1935

1936

1937

1938

1939

1940

1941

1942

1943

1944

1945

1946

1947 redescription but defied the phylogenetic analysis of Sookias, Böhmer \& Clack (2014: fig. 5A).

\section{Taxa that were not added}

We have not added taxa that likely could not be handled by the character sample of the present matrix (e.g. too deeply nested taxa, such as any further baphetoids, cochleosaurids, eryopids, dvinosaurs, dissorophids, stereospondylomorphs, "microsaurs", amniotes or lissamphibians beyond those we did add, or taxa too far outside the clade of limbed vertebrates). Still, a few taxa would have been intuitive candidates for addition, but we have left them out for the following reasons:

Because of the state of preparation of the specimens, the original description of the enigmatic and interesting "anthracosaur" Eldeceeon (Smithson, 1994) is very preliminary and contains little information; following the discovery of additional specimens, Eldeceeon is being redescribed according to Ruta \& Clack (2006) and Clack \& Milner (2015).

Although Madygenerpeton is only the second chroniosuchian for which a thorough description exists, we have not included it because it seems to lie at the tip of a long branch. According to the oldest phylogenetic analysis of Chroniosuchia (Schoch, Voigt \& Buchwitz, 2010), it is highly nested within that clade as the sister-group of Chroniosaurus, so that its seemingly plesiomorphic aquatic adaptations are probably reversals and would likely, perhaps together with the many other autapomorphies that Madygenerpeton has, distort the tree in general and the position of Chroniosaurus in particular. Indeed, Madygenerpeton lacks traces of the lateral-line organ (Schoch, Voigt \& Buchwitz, 2010; D. M., pers. obs.) which would be expected to be present in a primarily aquatic vertebrate; the dorsal rims of the eye sockets are raised high above the skull table, implying a crocodile-like predator that looked for terrestrial prey rather than staying underwater, an interpretation further corroborated by the nostrils which are apparently raised above the roof of the snout. In temnospondyls, for example, the nostrils are always well below eye level, and, with the borderline exception of Glaukerpeton (Werneburg \& Berman, 2012), the eye sockets are never noticeably raised (D. M., pers. obs., and see below). The second analysis that included Madygenerpeton (Buchwitz et al., 2012) instead found it as the sister-group to all other chroniosuchians, but - much like its predecessor - had a very small sample of outgroups. Conversely, the present matrix undersamples characters relevant to chroniosuchian phylogeny: the osteoderms and the antorbital fenestra, for example, are not coded. Indeed, a test run failed to find Madygenerpeton as a chroniosuchian. Only osteoderms and the mentioned skull roof (with distorted parts of the palate) are known of Madygenerpeton.

The temnospondyl Parioxys ferricolus, variously connected to Eryops and/or Dissorophoidea and more specifically found as the sister-group of Iberospondylus by Pawley (2006: chapter 5), is most likely a chimeric taxon (J. Pardo, pers. comm. 2012). The lectotype (AMNH 4309) is covered by a crust that would be difficult to prepare away and completely obscures the sutures as well as most other details (D. M., pers. obs. 2013; Schoch \& Milner, 2014: 77), and referred specimens (AMNH 2445 and almost all of the MCZ material) are being restudied by M. Ruta (D. M., pers. obs. of loan slips in 2013). "Preparation of USNM material has revealed many similarities to Cacops" (a dissorophid like Broiliellus; Schoch \& Milner, 2014: 77); it remains to be seen if the same will hold true of the lectotype. - AMNH 7118, the type and only known specimen of P. bolli, consists only of encrusted (D. M., pers. obs. 2013) 
1948 assorted postcrania, and whether it (or any other material currently assigned to Parioxys) can be 1949 referred to Parioxys awaits investigation.

Onchiodon is well known, but so similar to the even better known Eryops (Werneburg, 2007b) that it would most likely not add any new information to this matrix. Pretty much the same holds for the less well known Glaukerpeton (Werneburg \& Berman, 2012). The rest of Eryopidae - and also the diversity within Eryops - is very poorly understood and currently undergoing revision (Werneburg, 2007b, 2012b; Werneburg \& Berman, 2012; Schoch \& Milner, 2014; Rasmussen, Huttenlocker \& Irmis, 2016).

Comparison to character lists in Fröbisch \& Reisz (2012), Schoch (2012), Maddin et al. (2013b) and Holmes, Berman \& Anderson (2013) shows that many characters relevant to dissorophoid phylogeny are lacking from this matrix. Adding these characters and a better and more even sample of dissorophoid temnospondyls - micromelerpetids (in our matrix represented only by Micromelerpeton), dissorophids (Broiliellus brevis), trematopids (original sample: Ecolsonia, Acheloma, Phonerpeton; added: Mordex) and amphibamids (Amphibamus, Eoscopus, Doleserpeton, Platyrhinops, three "branchiosaurids"; added: Gerobatrachus, Micropholis, two more "branchiosaurids") - will be part of future work. As Schoch (2012), Werneburg (2012a), Maddin et al. (2013b) and Holmes, Berman \& Anderson (2013) pointed out, some of these taxa need to be (or are being) redescribed, and some material probably even needs additional preparation, which is in many cases as difficult to do as for Parioxys (see above). In particular, as mentioned above, we have not used information from species currently referred to Broiliellus other than B. brevis.

Kirktonecta (Clack, 2011a), "the oldest known microsaur", is so poorly preserved (and split through the bone like Eldeceeon) that it would not differ from several other OTUs in this matrix if we added it.

The characters in which Czatkobatrachus differs from Triadobatrachus (Evans \& Borsuk-Białynicka, 2009) are not represented in this matrix. It would be possible to score Czatkobatrachus for a few characters that are unknown in Triadobatrachus, but in all of these, Czatkobatrachus has the state expected (under all hypotheses) for the ancestral lissamphibian and the ancestral salientian, so it is not relevant here.

"Several other taxa [of Mesozoic salamanders] (e.g., Laccotriton, Sinerpeton, and Jeholotriton) are excluded from this analysis because they are anatomically uncertain and are currently under taxonomic revision" (Gao \& Shubin, 2012: supplementary information: 3 ). This revision is ongoing (Jia \& Gao, 2016b).

Adding Chinlestegophis (Pardo, Small \& Huttenlocker, 2017) to our matrix would currently be pointless in the absence of stereospondyls (other than Lydekkerina and Australerpeton in the expanded taxon sample) and characters pertinent to stereospondyl phylogeny.

\section{Phylogenetic analyses}

\section{Maximum-parsimony analyses}

These analyses were conducted in PAUP* 4.0b10 (Swofford, 2003) on a desktop computer with an Intel ${ }^{\circledR}$ Core $^{\mathrm{TM}} 2$ Duo processor $(2.67 \mathrm{GHz})$ and $3.87 \mathrm{~GB}$ of usable RAM. Test runs in PAUP* 4.0a158 (Swofford, 2017) were sometimes slower and sometimes found fewer MPTs. Alpha 
1993 versions of PAUP* 4.0 up to a158 were, however, used to generate initial tree figures for further 1994 processing from the results of all phylogenetic and robustness analyses.

We did not use any additional weighting procedures (such as reweighting, see

1997 Discussion: Reweighting and equal weighting in comparison); each character contains at least one state transition that costs one step, and there are no state transitions that cost less.

Six analyses (Table 1) of our modified matrix were conducted with (Analyses R4-R6) and without the added taxa (Analyses R1-R3), and with and without backbone constraints. The first constraint (Fig. 8A), used in Analyses R2 and R5, forced the dissorophoid temnospondyl Doleserpeton to be closer to the three salientians (Triadobatrachus, Notobatrachus, Vieraella; their monophyly with respect to Doleserpeton was specified, but not their relationships to each other) than the lysorophian lepospondyl Brachydectes, making the LH impossible but allowing both the TH and the PH. The second constraint (Fig. 8B), used in Analyses R3 and R6, only allowed the PH by additionally forcing the gymnophionomorph Eocaecilia to be closer to Brachydectes than to Doleserpeton. Both constraints contain Eusthenopteron in the outgroup position. In order to find all optimal islands and all optimal trees within each island, each heuristic search used 10,000 addition-sequence replicates (with random addition sequences), each of which was restricted to 50 million rearrangements by tree bisection and reconnection (25 million turned out to be too few to find all MPTs in some cases). This limit was hit in almost all replicates of the analyses with added taxa and close to two thirds of the replicates in the analyses without added taxa. Analysis R1 took 25:58:25 of calculation time, Analysis R6 lasted for 47:25:19.

We also reanalyzed (Analysis O1; Table 1) the original, unmodified matrix of RC07 without any constraints to test whether their procedure for reducing calculation time (the "parsimony ratchet"; see Ruta, Coates \& Quicke, 2003) had overlooked any MPTs. This was deemed important because, when Skutschas \& Gubin (2012) applied the same procedure to their own matrix which was small enough for a branch-and-bound analysis, it found a single tree that was no less than 35 steps longer than the MPTs found by branch-and-bound - a method which is guaranteed to find all MPTs. (To our surprise, Skutschas \& Gubin [2012] reported the length difference without commenting on it and without discarding the crassly suboptimal tree or offering a reason why it should be retained in consideration.) Calculation time with 5,000 addition-sequence replicates and up to a hundred million rearrangements per replicate was 21:48:07.

On p. 85, RC07 reported that constraining their analysis to find a topology congruent with that of Laurin (1998a) - a version of the LH - required "the addition of a mere nine steps" and yielded 60 suboptimal trees. On the next page, however, they wrote: "Using our own data, the 720 suboptimal trees placing lissamphibians with lepospondyls are 15 steps longer than the most parsimonious trees from the original analysis." (RC07 did not publish or describe the constraint they used to obtain this result.) To resolve this contradiction, we reanalyzed (Analysis O2; Table 1) the original, unmodified matrix of RC07 under a backbone constraint that required the salientians to be closer to Brachydectes than to Doleserpeton. Eusthenopteron was again included in the constraint in the outgroup position. The salientians are Triadobatrachus, Notobatrachus and Vieraella; their monophyly with respect to Doleserpeton was specified, but not their relationships to each other (Fig. 8A with the positions of Brachydectes and Doleserpeton exchanged). Calculation time was 23:48:00.

For completeness and to be able optimize characters on a tree supporting the $\mathrm{PH}$, we reanalyzed (Analysis O3; Table 1) the same matrix under a backbone constraint that required 
2039 Eocaecilia to be closer to Brachydectes than to Doleserpeton and the salientians (Fig. 8B).

2040 Calculation time was 23:22:13.

2041

2042

2043

2044

2045

2046

2047

2048

2049

Robustness analyses

José Grau (Museum für Naturkunde, Berlin) kindly performed two parsimony bootstrap analyses on our modified matrix (Table 1), one each for the original (Analysis B1) and for the expanded taxon sample (Analysis B2). Both were carried out on an Intel ${ }^{\circledR}$ Xeon ${ }^{\circledR}$ CPU E5-4667 v4 (2.20 $\mathrm{GHz}, 512 \mathrm{~GB}$ of available RAM) using PAUP* 4.0a158 for Unix/Linux (Swofford, 2017), and used 1,000 bootstrap replicates consisting of 500 addition-sequence replicates which were limited to 50 million rearrangements each. To keep this within a reasonable timeframe, J. Grau

2050 divided each analysis into 100 runs of 10 bootstrap replicates, which were run on a total of 50

2051

2052 processors by means of GNU parallel v20130522, followed by concatenation and calculating the majority-rule consensus to obtain the final bootstrap tree. To maximize the amount of visible

2053

2054 information, we included not only clades with bootstrap values of 0.5 and above in the bootstrap trees, but also clades with lower bootstrap values that do not contradict any of those with higher

2055

2056

2057

2058 ones (PAUP* setting: contree all /majrule=yes le 50=yes; equivalent to bootstrap keepall=yes for a non-parallel analysis).

The total running time for each bootstrap run was between 27 and 38 hours; the

2059 consensus took around 40 minutes to compute. Each bootstrap run produced between 30,111 and 114,768 trees; the consensus was calculated from a total of 6,870,699 trees.

In order to test whether the differences between MPTs from different analyses with the

2060

2061

2062

2063

2064

2065

2066

2067

2068

2069

2070

2071

2072

2073

2074

2075

2076

2077

2078

2079

2080

2081

2082

2083

2084 same taxon sample and the same reversibility coding are statistically significant, we ran the tests implemented in PAUP*: a Kishino-Hasegawa test, a Templeton test and a winning-sites test. The $\mathrm{p}$ value we report for each is the probability (Kishino-Hasegawa) or approximate probability (Templeton, winning sites) of finding a more extreme test statistic; the null hypothesis is that the differences between the two tested trees are indistinguishable from random. The MPTs used for these tests are part of the data file (Data S3) and were chosen to differ as little as possible from each other topologically.

Eduardo Ascarrunz (pers. comm.) and the reviewer Michael Buchwitz kindly notified us of problems with our first (2015) applications of these tests. Goldman, Anderson \& Rodrigo (2000) and Planet (2006) have shown that all three tests, as implemented in PAUP* and elsewhere, are inappropriate to use for our questions and indeed for the vast majority of the questions they have been applied to in the phylogenetics literature. To the best of our knowledge, better tests are not available for practical purposes. Thus, we cannot reject the abovementioned null hypothesis in any case. However, the error caused by using these tests is one-sided: the $p$ values found by better tests are always greater than the $p$ values found by the inappropriately used one-tailed tests - greater by an amount that cannot be estimated in advance (Goldman, Anderson \& Rodrigo, 2000). This allows us to look at the problem from the other side and distinguish the cases where the null hypothesis can certainly not be rejected from those where it remains rejectable: when the inappropriate tests do not reject the null hypothesis, we can be certain that a better test would not reject it either, but when the inappropriate tests do reject the null hypothesis, the null hypothesis may or may not be rejected by a better test.

PAUP* performs two-tailed versions of all three tests; we report $p$ values from the onetailed versions, derived by halving the p values put out by PAUP* (Goldman, Anderson \& Rodrigo, 2000; Planet, 2006). 
2085

2086

2087

2088

2089

2090

2091

2092

2093

2094

2095

2096

2097

2098

2099

2100

2101

2102

2103

2104

2105

2106

2107

2108

2109

2110

2111

2112

2113

2114

2115

2116

2117

2118

2119

2120

2121

2122

2123

2124

2125

2126

2127

2128

2129
Measure of similarity

Valentin Rineau (CR2P, Paris) kindly calculated ITRIs for selected pairwise comparisons of trees in the software LisBeth 01/2013 (see Zaragüeta-Bagils et al., 2012). The ITRI (Grand et al., 2013) is a measure of the similarity of a tree in question to a reference tree (not the other way around, which could give a different value). If the tree in question contains all of the three-item statements (3is) that constitute the reference tree, meaning that it is identical to or a superset of the reference tree, its ITRI compared to that reference tree is $100 \%$. However, the ITRI is not simply the fraction of shared 3is. Combinations of 3is imply further 3 is in the same tree; this limitation of the degrees of freedom is used to weight the ratios of shared 3is before calculating the ITRI (Grand et al., 2013).

Previously (e.g. Grand et al., 2013; Rineau et al., 2015), the ITRI has been used for cases where the true tree is known (e.g. simulations). This is of course not the case here. In each of our comparisons we have used the "older" tree (higher up in Table 1) as the reference tree: O trees as the references for $\mathrm{R}$ trees, $\mathrm{O} 1$ as the reference for $\mathrm{O} 2, \mathrm{O} 1$ and $\mathrm{O} 2$ as the references for $\mathrm{O} 3$ (in two separate comparisons) and so on; $\mathrm{O} 1$ is the reference tree in each comparison that contains it, EB never is. Thus, we quantify how similar "newer" trees are to "older" ones.

If the reference tree is larger than the tree in question, the latter's ITRI can never reach $100 \%$ because it cannot contain all 3is found in the reference tree. We have always used reference trees equal in size to or smaller than the trees in question (see Table 1), so such cases do not arise here.

From each analysis we used only one tree. Analysis EB (see the following section) of course yielded a single tree; from the parsimony analyses we used the same MPTs as for the statistical tests described in the preceding section. Because those trees (included in Data S3) were chosen to be as similar as possible, this ensures that the ITRIs we find are upper bounds on a range. Lower bounds could only be found by an exhaustive search. We did not compare strict consensus trees because they are poorly resolved (which would exaggerate the similarities) in some of the very ways the ITRI is designed to avoid. The majority-rule consensus is not a good representation of the result of a parsimony analysis (see Marjanović \& Laurin, 2018); the Adams consensus must not be mistaken for an actual tree.

\section{Exploratory Bayesian analysis}

As a preliminary test of whether the method of analysis has an impact on our results, J. Grau kindly ran an unconstrained Bayesian-inference analysis on our revised matrix including the added taxa (Analysis EB; Table 1). He used MrBayes 3.2.6 (Huelsenbeck et al., 2015) in parallel on 16 AMD Opteron ${ }^{\mathrm{TM}} 6172$ processors $(2.1 \mathrm{GHz})$, using $16 \mathrm{~GB}$ of RAM. Instead of the usual two, we chose to use four simultaneous runs; all other settings remained at the factory defaults, notably the specification of the first quarter of the generations as burn-in. Calculation time was 20 hours and 6 minutes.

MrBayes cannot handle stepmatrices. We therefore had to split the two characters with stepmatrices into two to three characters each, which were ordered or unordered. The results of this nontrivial task are documented in App. S1 under characters 32 and 134; the scores of the new characters are wholly predictable from those of the stepmatrix characters used for all other 
2130 analyses, and are therefore not presented separately except in the matrix file (Data S6). The total

2131

2132

2133

2134

2135

2136

2137

2138

2139

2140

2141

2142

2143

2144

2145

2146

2147

2148

2149

2150

2151

2152

2153

2154

2155

2156

2157

2158

2159

2160

2161

2162

2163

2164

2165

2166

2167

2168

2169

2170

2171

2172

2173

2174

number of characters for this analysis was 280 .

The average standard deviation of the split frequencies decreased to 0.05 between six and seven million generations into the analysis. Because it had not reached stationarity, we extended the analysis in the hope of reaching the recommended value of 0.01 ; after some twenty million generations of fluctuation around 0.013 , we ended the analysis after a total of 40 million generations (including ten million of burn-in). Convergence was achieved according to two other metrics: the plot of the logarithm of probability against generation number (excluding the burnin) did not show a trend, and the potential scale reduction factor (PSRF) of the tree length was 1.000 as recommended.

\section{Results}

A very brief overview and comparison of our results is presented as Table 3 . The results of statistical tests for lack of distinguishability of topologies are reported below and shown in Table 4; the similarities of topologies are reported below as ITRI and shown in Table 5.

\section{Reanalyses of the matrix of Ruta \& Coates (2007)}

Our unconstrained reanalysis of the original, unmodified matrix (Analysis O1) found 324 MPTs, as RC07 did; their strict consensus (Fig. 1) is identical to that reported by RC07 (fig. 5, 6). Their length is 1621 steps when we distinguish polymorphism from partial uncertainty (both occur in the matrix; PAUP* setting: pset mstaxa=variable), but 1584 steps - as reported by RC07 - when we treat both as partial uncertainty (pset mstaxa=uncertain). The indices we find are slightly different from those reported by $\mathrm{RC} 07$, and differ again depending on the treatment of polymorphism: when polymorphism is treated as uncertainty, the ci excluding the parsimonyuninformative characters is 0.2281 rather than 0.22 , the ri is 0.6768 rather than 0.67 and the rc is 0.1564 rather than 0.15 ; when polymorphism and uncertainty are distinguished, the ri is unaffected, but the ci excluding the parsimony-uninformative characters rises to 0.2458 and the rc becomes 0.1683 .

Our analysis constrained for the $\mathrm{LH}$ (Analysis O2) found 60 most parsimonious trees. Their strict consensus (Fig. 9) is quite unlike what RC07 reported on p. 86, and also unlike that of Laurin (1998a), part or all of which RC07 used as a constraint on p. 85: we find Lissamphibia as the sister-group of Albanerpetidae, both of which together form the sister-group to Holospondyli ("nectrideans" including aïstopods). All of these together are found as the sistergroup of Brachydectes; in other words, lepospondyl topology is identical to that in the shortest trees, except for the addition of Lissamphibia + Albanerpetidae next to Holospondyli, and except for the loss of resolution in Urocordylidae. The resolution of Temnospondyli is greatly improved. The length of these suboptimal trees is 1622 steps ( 1585 when polymorphism is treated as uncertainty); ci excluding the parsimony-uninformative characters $=0.2280$ (polymorphism treated as uncertainty) or 0.2457 (polymorphism and uncertainty distinguished), $\mathrm{ri}=0.6766, \mathrm{rc}=0.1562$ or 0.1681 .

In short, the matrix of RC07 supports the temnospondyl hypothesis over the lepospondyl hypothesis by one single step, rather than either nine or fifteen steps as originally claimed (RC07: 85, 86). 
2175

2176

2177

2178

2179

2180

2181

2182

2183

2184

2185

2186

2187

2188

2189

2190

2191

2192

2193

2194

2195

2196

2197

2198

2199

2200

2201

2202

2203

2204

2205

2206

2207

2208

2209

2210

2211

2212

2213

2214

2215

2216

2217

2218

2219

2220

To test this result, we manually drew a most parsimonious rooted tree and a rooted tree that fulfills the constraint in Mesquite (Maddison \& Maddison, 2017). Mesquite reported the same lengths as PAUP* (distinguishing polymorphism from partial uncertainty, as Mesquite always does).

This result is perhaps not as surprising as it may seem. Although they only published the values for seven nodes, RC07 (app. 4 - p. 122) did conduct a bootstrap and a decay analysis. While Lissamphibia (bootstrap percentage $=67$, Bremer index $=8$ ) and most of the nodes within it are well supported, the sister-group relationship of Lissamphibia and Doleserpeton has a Bremer index of 1 and "no boots[t]rap support compatible with a 50\% majority-rule consensus", and the sister-group relationship of both of these to Amphibamus has a Bremer index of 2 and "no bootstrap support in a 50\% majority-rule consensus". Values for more rootward nodes were not reported, but are not likely to be any higher given the limited resolution of this part of the tree (Fig. 1).

For completeness and for the purposes of the next section, we also ran an analysis constrained for the PH (Analysis O3). It yielded 4627 MPTs of 1633 steps (1596 when polymorphism is treated as uncertainty), twelve steps more than the MPTs from O1; ci excluding the parsimony-uninformative characters $=0.2264$ (polymorphism treated as uncertainty) or 0.2468 (polymorphism and uncertainty distinguished), $\mathrm{ri}=0.6737, \mathrm{rc}=0.1545$ or 0.1662 . Although less well resolved among seymouriamorphs, diadectomorphs and "lepospondyls", the strict consensus is very similar to those of $\mathrm{O} 1$ and $\mathrm{O} 2$; Salientia and the two caudates form a trichotomy nested next to Doleserpeton as in O1, Albanerpetidae and Eocaecilia form a clade nested in a trichotomy with Holospondyli and Brachydectes (all three resolutions occur in different MPTs). Indeed, some MPTs are identical to those from O2 except for the position of Batrachia. In some MPTs, the "lepospondyl" clade (including Albanerpetidae and Eocaecilia but not the adelospondyls) and Solenodonsaurus lie outside the "seymouriamorph""diadectomorph"-amniote clade.

Unsurprisingly, trees from $\mathrm{O} 1$ and $\mathrm{O} 2$ are statistically indistinguishable: $\mathrm{p}=0.4500$ (Kishino-Hasegawa and Templeton tests), $\mathrm{p}=0.5000$ (winning-sites test). Trees from $\mathrm{O} 2$ and O3, however, may be distinct: $p=0.0468$ (Kishino-Hasegawa), 0.0467 (Templeton), 0.0637 (winning sites). Interestingly, despite being one step farther apart than $\mathrm{O} 2$ and $\mathrm{O} 3$, trees from $\mathrm{O} 1$ and $\mathrm{O} 3$ are not distinguishable at the 0.05 level: $\mathrm{p}=0.0545$ (Kishino-Hasegawa), 0.0547 (Templeton), 0.0599 (winning sites). The tree from O2 used for these tests (included in Data S3) has an ITRI of $86.7 \%$ compared to the tree from O1; the tree from $\mathrm{O} 3$ has an ITRI of $95.5 \%$ compared to $\mathrm{O} 1$ and an ITRI of only $92.8 \%$ compared to O2, fitting the tests for lack of distinguishability.

Data S7 contains the unmodified matrix, the constraints, our other analysis settings, a most parsimonious tree (O1, 1621 steps), a tree that fulfills the constraint for the LH $(\mathrm{O} 2,1622$ steps) and two that fulfill the constraint for the $\mathrm{PH}(\mathrm{O} 3,1633$ steps), the first chosen to be as similar as possible to those from $\mathrm{O} 1$ and $\mathrm{O} 2$ and used in the statistical tests and in the section following below, the other very different in topology (together, the differences between these two trees account for almost all the polytomies in the strict consensus). Executing this file in PAUP* repeats all three analyses and performs the statistical tests on the trees that are already stored in the file.

\section{Amount, distribution and impact of revised scores}


2221 Excepting the deleted postcranium of Rhynchonkos (Material and methods: Treatment of OTUs:

2222 Rhynchonkos), App. S2 contains 4125 colored scores (2404 red, 1642 green, 79 blue) for the

2223 original taxon sample. (See Material and methods: Modifications to individual cells or below for

2224 the meanings of the colors; the scores were counted in Data S4.) Additionally, 67 changes to

2225 deleted characters are counted as red in Data S4, five as green and three as blue; the total number

2226 of individual changed scores in the matrix is thus 4200 .

2227

2228

2229

2230

2231

2232

2233

2234

2235

2236

2237

2238

2239

2240

2241

2242

2243

2244

2245

2246

2247

2248

2249

2250

2251

2252

2253

2254

2255

2256

2257

2258

2259

2260

2261

2262

2263

2264

2265

Table 6 shows the OTUs with the highest numbers of red changes (in App. S2, i.e. not counting deleted characters). These are due to contradictions between the scores of RC07 and their character or state definitions, without any redefinitions by us. At the top lies Ventastega, due to the description of new material by Ahlberg et al. (2008), but also due to the fact that Ruta, Coates \& Quicke (2003) only scored about half as much as they could have based on the original description by Ahlberg, Lukševičs \& Lebedev (1994) as mentioned above (Material and methods: Modifications to individual cells: Literature). The close second is Lethiscus, whose skull was redescribed from $\mu \mathrm{CT}$ data by Anderson et al. (2017), though some of the changes we had earlier made based on Wellstead (1982; also describing the postcranium) and Anderson, Carroll \& Rowe (2003) were confirmed by the new paper. Data published after 2001 - not always after 2006 - account for most of the rest of the table as well, although we should highlight the fact that most of the 43 red changes we made to Diplocaulus come from the detailed descriptions of the lower jaw and postcranium by Williston (1909) and Douthitt (1917), neither of which was cited by RC07 or Ruta, Coates \& Quicke (2003).

Table 7 does the same for characters. The highest rank by a large margin belongs to ch. 257 (TRU VER 4), "Haemal spines not fused (0) or fused (1) to caudal centra". RC07 considered state 1 to be "almost exclusively observed in nectrideans", but this is not defensible unless the character is extensively reinterpreted. It is possible that RC07 only meant "pleurocentra" by "centra", and identified the single-piece centra of "nectrideans" as pleurocentra (but did not score them accordingly, except in Scincosaurus; see ch. 259, TRU VER 7, in App. S1). Indeed, it has sometimes been assumed that hemal arches are homologous to intercentra or parts thereof (most recently by Olori, 2015: 57). However, that is not the case, nor do hemal arches always fuse to intercentra when intercentra are present (e.g. Pawley \& Warren, 2005; Schoch, 2006: fig. 6H; Witzmann \& Schoch, 2006a; Holmes \& Carroll, 2010; apparently Clack, 2011b). Therefore, we have taken the definition literally and scored state 1 whenever the hemal spines are fused to intercentra or to single-piece centra, keeping it for all "nectrideans" but expanding it to most of the rest of the sampled OTUs with known tails - and scoring it as unknown in 13 OTUs that had been given state 0 despite lacking sufficiently preserved tails, as well as in the one OTU (Triadobatrachus) that had a tail without any hemal arches and to which this character, here originally scored 0 as well, therefore cannot apply. At the third rank in Table 7, the problem of the homology of the "dorsal process" of the ilium has a somewhat similar impact on ch. 225 (ILI 3); the other character at the third rank, the mandibular lateral-line canal (ch. 101: SC 2), was misscored for almost all "microsaurs", apparently because Ruta, Coates \& Quicke (2003) had misinterpreted statements by CG78 about pits and grooves clearly referring, from context, to ornament or nerves and blood vessels. All characters mentioned in this paragraph are discussed at some length in App. S1.

For the characters in the rest of Table 7, we cannot find such a single main cause or any common pattern for the incorrect scores. Literature from 2006 or later is the main source for changes in only one, literature from 2003 or later only in a total of three of these characters. 
2266

2267

2268

2269

2270

2271

2272

2273

2274

2275

2276

2277

2278

2279

2280

2281

2282

2283

2284

2285

2286

2287

2288

2289

2290

2291

2292

2293

2294

2295

2296

2297

2298

2299

2300

2301

2302

2303

2304

2305

2306

2307

2308

2309

2310

2311

Green changes are at least in part due to redefinitions or possible redefinitions of states (or entire characters). As we made such changes to characters rather than to taxa, many of the OTUs with the highest numbers of such scores (Table 8) are among those we rescored wholesale. Indeed, many are familiar from Table 6 - less than half of the 20 OTUs in the latter (Edops, Cochleosaurus, Isodectes, Doleserpeton, Eocaecilia, Cardiocephalus, Diplocaulus and Ossinodus) are missing from the former. Also well represented in Table 8, however, are well known OTUs to which many characters are inapplicable; this is because we have changed many scores from known to unknown in attempts to move away from nonadditive binary coding or avoid redundancy. Thus, five of the seven modern amphibian OTUs and all three aisstopods have made it into the 20 highest ranks, and at the very top lies Brachydectes, which combines unusual anatomy, lost skull bones and a recent redescription (Pardo \& Anderson, 2016).

Naturally, the two characters with the most green changes (Table 9) are the two we split off from others (Material and methods: Treatment of characters: Deleted, recoded and split characters), one of them having fresh scores for all 102 OTUs. We redefined and completely rescored the next six as well; similar but less drastic changes, such as reinterpretation as reductive instead of nonadditive binary, account for the rest of the table.

Blue changes represent our attempts to take ontogeny into account. These affect mostly "branchiosaurs" and "discosauriscids" (Table 10), and characters that depend on the extent of ossification (Table 11). Notable are also the two characters describing the lateral-line canals at ranks 3 and 5 in Table 11, which are affected by the disappearance of grooves from the skull (ch. $100, \mathrm{SC} 1$ ) and the lower jaw (ch. 101, SC 2) in the ontogeny of "branchiosaurs" and "discosauriscids"; also at rank 5 lies ch. 146 (JAW ART 1/SQU 2/DEN 8), which describes the gradual caudal shift of the jaw joint as the suspensorium grows with positive allometry in the ontogeny of many vertebrates.

\section{Analyses of our revised matrix}

Note that the tree lengths we report throughout are those given by PAUP* (distinguishing polymorphism from partial uncertainty). Mesquite consistently reports one fewer step for trees from Analyses R1-R3 (original taxon sample; see Table 1) and one or two fewer steps for trees from Analyses R4-R6 (expanded taxon sample); apparently, the programs handle the stepmatrices differently. (As mentioned, PAUP* and Mesquite report the same lengths for the MPTs of Analyses O1-O3, where stepmatrices do not occur.)

Comparison of the results to literature other than $\mathrm{RC} 07$ is part of the Discussion (section Phylogenetic relationships).

\section{Analysis R1}

The unconstrained analysis of our presumably improved matrix without added taxa yielded 1120 MPTs with a length of 2182 steps (a drastic increase over the 1621 steps required by the unmodified matrix of RC07), a ci of 0.2181 (likewise revealing increased character conflict), a hi of 0.8327 , a ri of 0.62118 , and a rc of 0.1355 .

Figures 10 and 11 presents the similarities and differences between the MPTs, based on inspection of the strict consensus and the first, the $25^{\text {th }}$, the $75^{\text {th }}$, the $125^{\text {th }}$, the last and every $50^{\text {th }}$ MPT $(1,25,50,75,100,125,150,200 \ldots 1000,1050,1100,1120)$ in Mesquite. They show that, in all MPTs, Panderichthys is the most rootward member of the ingroup as in RC07. More 
2312 crownwards, however, the positions of Ichthyostega, Ventastega and Acanthostega are not fully

2313 resolved: Ventastega can be the sister-group of Acanthostega or one node more rootward;

2314 Ichthyostega can be more crownward than both, more rootward than both, or (Fig. 11) between

2315 the two when they are not sister-groups. Interestingly, a Ventastega-Acanthostega clade is only

2316 recovered when Ichthyostega lies crownward of it.

2317

2318

2319

2320

2321

2322

2323

2324

2325

2326

2327

2328

2329

2330

2331

2332

2333

2334

2335

2336

2337

The next more crownward branch is the Mississippian Whatcheeriidae (resolved as in RC07), followed crownward by the Devonian Tulerpeton. Next more crownward is Crassigyrinus, followed by Colosteidae (which was instead rootward of Whatcheeriidae in $\mathrm{RC} 07)$.

Crownward of these, the MPTs share a consistent backbone, which is a Hennigian comb of - from rootward to crownward - Baphetidae, Anthracosauria, Silvanerpeton, Gephyrostegus, Solenodonsaurus, Seymouriamorpha, and then the crown-group consisting of Amphibia, Diadectomorpha and Sauropsida (Fig. 10, 11). Parts of this arrangement contradict the findings of RC07 (Fig. 1); the position of Solenodonsaurus is altogether novel.

In different MPTs, Temnospondyli is either rootward of Anthracosauria (one node crownward of Baphetidae) as in RC07, or crownward of Anthracosauria (one node rootward of Solenodonsaurus). These two positions are part of two classes of topologies that have limited overlap other than the mentioned backbone and the almost complete resolution of the tetrapod crown-group.

When Temnospondyli is rootward of Anthracosauria (Fig. 11), Eucritta is one node rootward of Baphetidae; Edops is the sister-group of all other temnospondyls, which form a Hennigian comb of Cochleosauridae, Eryops, Capetus, Dvinosauria, Dendrerpetidae and Dissorophoidea, where Balanerpeton can be the sister-group of either of the last two. Caerorhachis is one node rootward of the anthracosaurs (as in RC07); within the latter, Pholiderpeton scutigerum is the sister-group of either (Proterogyrinus + Archeria) or $(P h$. attheyi + Anthracosaurus), both unlike in RC07. Bruktererpeton is either the sister-group of

2338

2339

2340

2341

2342

2343

2344

2345

2346

2347 Gephyrostegus or one node more rootward (never crownward as in RC07), both lying rootward of Solenodonsaurus and crownward of Silvanerpeton; the traditional seymouriamorphs show a remarkable diversity of mono- and paraphyletic arrangements, though never the one found by RC07.

When Temnospondyli is crownward of Anthracosauria (Fig. 10), Eucritta is a nonbaphetid baphetoid (as in RC07). Caerorhachis is one node rootward of the anthracosaurs in some MPTs (Fig. 10: main tree and upper inset), in which case Dissorophoidea forms the sistergroup to all other temnospondyls (among which Dvinosauria is highly nested), or to all except Dvinosauria, or to all except (Isodectes + Neldasaurus), in which latter case Trimerorhachis is the sister-group of a highly nested (Balanerpeton + Dendrerpetidae) clade. In the other MPTs

2348 (Fig. 10: lower inset), Caerorhachis is the sister-group of all other temnospondyls, among which

2349

2350

2351

2352

2353

2354

2355

2356 Dvinosauria is sister to the remainder, followed by Dissorophoidea. Edops is far from the temnospondyl root in both cases. Anthracosauria is resolved as in RC07; Bruktererpeton and Gephyrostegus are sister-groups and lie one node rootward of Temnospondyli; the traditional seymouriamorphs form a monophyletic Hennigian comb, except that Kotlassia is either one node rootward of Seymouriamorpha or a member of it (the sister-group to the rest) - a subset of the resolutions in the other class of topologies.

In both cases (Fig. 10, 11), the dissorophoid Ecolsonia can emerge as a trematopid or (as in $\mathrm{RC} 07$ ) one node closer to all other dissorophoids. 
2357

2358

2359

2360

2361

2362

2363

2364

2365

2366

2367

2368

2369

2370

2371

2372

2373

2374

2375

2376

2377

2378

2379

2380

2381

2382

2383

2384

2385

2386

2387

2388

2389

2390

2391

2392

2393

2394

2395

2396

2397

2398

2399

2400

2401

2402

All MPTs support the LH. Lissamphibia is highly nested among the "lepospondyls"; within Temnospondyli, Amphibamidae is monophyletic and highly nested.

The tetrapod crown-group consists of Amphibia (the lissamphibian total group, here including all "lepospondyls") and the sister-groups Diadectomorpha and Sauropsida. Sauropsida is resolved as in RC07; Diadectomorpha is resolved as (Limnoscelis (Tseajaia (Orobates, Diadectes))).

Unchanged from RC07, Westlothiana is the most rootward amphibian and is followed crownward by two "microsaur" clades, which comprise all "microsaurs" in the taxon sample except (unlike in RC07) Batropetes; the composition and especially the topologies of these clades are quite different from those found by RC07, however. The larger and more rootward clade has a basal split into (Microbrachis + Hyloplesion) on one side and (Odonterpeton (Saxonerpeton (Hapsidopareion (Gymnarthridae, Ostodolepididae)))) on the other, where Rhynchonkos is the sister-group of Gymnarthridae or (Gymnarthridae + Ostodolepididae); the smaller and more crownward one is composed of Pantylidae (as in RC07) and Tuditanidae (Tuditanus + Asaphestera).

Next more crownward lie the sister-groups Scincosaurus and Diplocaulidae. Diplocaulidae is resolved as in RC07, except that Keraterpeton and Diceratosaurus are sistergroups.

The mentioned brachystelechid "microsaur" Batropetes and the lysorophian Brachydectes lie successively closer to the modern amphibians. However, the sister-group of Lissamphibia is a clade that contains, one could say, all the strangest "lepospondyls": the adelospondyls (which, in $\mathrm{RC} 07$, were the sister-group of the colosteids) form the sister-group to Urocordylidae + Aïstopoda. Unlike in RC07, Adelospondylus is resolved as the sister-group to the other adelogyrinids and Lethiscus as the sister-group of the other aïstopods.

Lissamphibia is fully resolved as (Eocaecilia (Albanerpetidae (Caudata, Salientia))).

The tree from R1 included in Data S3 has an ITRI of 72.0\% compared to the tree from $\mathrm{O} 1$ (supporting the TH) and an ITRI of $92.8 \%$ compared to the tree from O2 (LH).

\section{Analysis R2}

Constraining the analysis of the original taxon sample against the LH yielded 64 MPTs with a length of 2191 steps - nine more than in the unconstrained Analysis R 1 - and a ci of 0.2173 , a hi of 0.8334 , a ri of 0.6191 , and a rc of 0.1345 . Figure 12 presents the similarities and differences between the MPTs, based on inspection of all of them in Mesquite. All MPTs have a bundle of highly unusual features.

Specifically, when the salientians move closer to Doleserpeton than to Brachydectes, they drag not merely the other lissamphibians along, but also the aïstopods, urocordylids and adelospondyls; and this assemblage nests not within the amphibamids or the dissorophoids more generally, but within the dvinosaurs. The modern amphibians form the sister-group to the aïstopod Phlegethontia; all together are joined on the outside by Oestocephalus, then Lethiscus, then Urocordylidae (where Ptyonius is resolved as the sister-group to the rest), then the adelospondyls, and then the dvinosaurian temnospondyl Trimerorhachis, followed by Isodectes and then Neldasaurus. The clade that contains all non-dvinosaurian temnospondyls is still recovered as in Analysis R1, but is almost fully resolvedas in a subset of the trees from Analysis R1, with highly nested edopoids. Temnospondyli lies crownward of Gephyrostegidae, Silvanerpeton, Anthracosauria and (most rootward) Caerorhachis. Eucritta is the sister-group of 
2403 Baphetidae. In some trees, the (Microbrachis + Hyloplesion) clade falls out as the sister-group to

2404

2405

2406

2407

2408

2409

2410

2411

2412

2413

2414

2415

2416

2417

2418

2419

2420

2421

2422

2423

2424

2425

2426

2427

2428

2429

2430

2431

2432

2433

2434

2435

2436

2437

2438

2439

2440

2441

2442

2443

2444

2445

2446

2447

all lepospondyls except Westlothiana.

Other than this, the topology is consistent with trees from Analysis R1 (Fig. 10). The clade formed by Diplocaulidae and Scincosaurus does not follow the other holospondyls into Temnospondyli, but stays behind as the sister-group of (Batropetes + Brachydectes). Ichthyostega and Ecolsonia have the same positions as in Analysis R1; Kotlassia is always rootward of Seymouriamorpha; Lissamphibia and Albanerpetidae are sister-groups in all MPTs.

Statistical tests find the difference between a tree from Analysis R2 and a tree from Analysis R1 insignificant at a p level of 0.05: $p$ is 0.2248 under the Kishino-Hasegawa test, 0.1854 under the Templeton test and 0.1332 under the winning-sites test (Table 4). The same tree from R2 has an ITRI of $76.7 \%$ compared to a tree from O1, 82.0\% compared to O2 and $86.5 \%$ compared to R1.

Analysis R3

An analysis of the original taxon sample that was constrained to be compatible with the $\mathrm{PH}$ yielded 736 MPTs with a length of 2194 steps - only three steps more than those from Analysis R2, 12 more than those from Analysis R1 - and a ci of 0.2170 , a hi of 0.8336 , a ri of 0.6184 and a rc of 0.1342 .

Judging from the first, the last and every $25^{\text {th }}$ MPT, the different MPTs (Fig. 13) are very similar to those of R1. Salientia forms the sister-group to Doleserpeton and is nested within the amphibamid dissorophoid temnospondyls, not within the dvinosaurian temnospondyls as it is in R2. All other modern amphibians have the same positions as in R1.

The difference between a tree from Analysis R3 and a tree from Analysis R1 is potentially significant at the level of $p=0.05$ according to the statistical tests: $p=0.0352$ (Kishino-Hasegawa), 0.0362 (Templeton), 0.0175 (winning sites). The difference between Analyses R3 and R2 is far from significance: $\mathrm{p}=0.4074$ (Kishino-Hasegawa), 0.4255 (Templeton), 0.5000 (winning sites). The same tree from R3 has an ITRI of $78.9 \%$ compared to O3, $95.7 \%$ compared to $\mathrm{R} 1$ and $85.3 \%$ compared to $\mathrm{R} 2$.

\section{Analysis R4}

From here on, the OTUs we have added are marked with an asterisk; taxa absent even from the expanded taxon sample are marked with two asterisks.

An unconstrained analysis of the increased taxon sample found 401 MPTs with a length of 3011 steps, a ci of 0.1860 , a hi of 0.8788 , a ri of 0.6186 , and a rc of 0.1150 .

Figure 14 presents the similarities and differences between the MPTs, based on the strict and Adams consensus trees and on the first, the last and every $10^{\text {th }}$ tree.

Despite containing several OTUs known only from isolated lower jaws, the Devonian area of the tree is remarkably well resolved (Fig. 14): *Elginerpeton, *Metaxygnathus, (Acanthostega + Ventastega), *Ymeria, *Perittodus, Ichthyostega, Whatcheeriidae, *Densignathus, Tulerpeton and Crassigyrinus are successively more crownward. This pattern is partially obscured by Ossinodus, which has four positions: as a whatcheeriid (the sister-group to the rest as in R1), as the sister-group of *Densignathus, or one node more crownward or more rootward than the latter. 
2448

2449

2450

2451

2452

2453

2454

2455

2456

2457

2458

2459

2460

2461

2462

2463

2464

2465

2466

2467

2468

2469

2470

2471

2472

2473

2474

2475

2476

2477

2478

2479

2480

2481

2482

2483

2484

2485

2486

2487

2488

2489

2490

2491

Crownward of Crassigyrinus follows a dichotomy between an unusually enlarged Temnospondyli and its sister-group, which contains the tetrapod crown-group (all MPTs support the LH).

Within Temnospondyli, the sister-group of all others is Eucritta, followed by a novel and fully resolved colosteid-baphetid clade. The *"St. Louis tetrapod" (MB.Am.1441; Clack et al., 2012 b) is the sister-group to all other colosteids, among which *Pholidogaster is the sister-group to (*Aytonerpeton (*Deltaherpeton (Colosteus, Greererpeton))). Within Baphetidae, Baphetes, Megalocephalus, *Diploradus and *Sigournea are successively closer to *Doragnathus and *Spathicephalus.

The traditional temnospondyls form a clade which is resolved as an unusual Hennigian comb where Edops, Cochleosauridae, Eryopiformes, Capetus, Dvinosauria, (Balanerpeton + Dendrerpetidae) and *Iberospondylus are successively closer to Dissorophoidea.

* Palatinerpeton has two positions as the sister-group of Dendrerpetidae or of (*Iberospondylus + Dissorophoidea). *Nigerpeton and *Saharastega form a novel clade with three novel positions: as the sister-group of Eryops, of Stereospondylomorpha or of all traditional temnospondyls except Edops and Cochleosauridae.

Dissorophoidea is fully resolved. Trematopidae, including *Mordex and Ecolsonia, is the sister-group of the remainder, in which *Micropholis clusters with the "branchiosaurs" of the original taxon sample, while (*Branchiosaurus $+*$ Tungussogyrinus) is the sister-group of *Acanthostomatops. The latter three together form the sister-group of (Broiliellus + Amphibamidae).

The *Parrsboro jaw (Godfrey \& Holmes, 1989; Sookias, Böhmer \& Clack, 2014) is found in two very distinct positions: as the sister-group to the clade of all traditional temnospondyls, or next to Caerorhachis.

Caerorhachis (and optionally the *Parrsboro jaw) is one node crownward of Temnospondyli. It is followed crownward by Anthracosauria, Silvanerpeton, Bruktererpeton, Gephyrostegus, Chroniosuchia (*Chroniosaurus, *Bystrowiella), Solenodonsaurus and Seymouriamorpha. Anthracosauria is fully resolved as in RC07, with the added taxa forming a clade (*NSM 994 GF 1.1 (*Palaeoherpeton, *Neopteroplax)) that nests with Anthracosaurus. * Casineria has four positions: one node rootward of Bruktererpeton, one node crownward of Gephyrostegus, between the two, or as the sister-group of Bruktererpeton.

Just outside the tetrapod crown-group lies Seymouriamorpha, in which (Leptoropha + Microphon) and a (Seymouria (*Karpinskiosaurus, Kotlassia)) clade lie successively closer to Discosauriscidae. Within the crown, the amniote + diadectomorph clade is fully resolved; curiously, however, the added OTUs *Caseasauria and *Archaeovenator emerge as the sistergroup to Limnoscelis, followed by the clade of the other diadectomorphs and then by Sauropsida - in other words, the diadectomorphs are found as amniotes, specifically as synapsids that lack the synapsid condition (Berman, 2013), but they do not form a clade (unlike in Berman, 2013).

On the amphibian side of the crown, Westlothiana remains the sister-group to the remainder, which has a topology somewhat reminiscent of Vallin \& Laurin (2004). Instead of having a "microsaur" grade at the base as in Analyses R1-R3, this clade shows a basal dichotomy: (Holospondyli (Microbrachis, Hyloplesion)) lies on one side, while the other branch groups the remaining "microsaurs" and Brachydectes (which is the only lysorophian even in the expanded taxon sample) with the fully resolved Lissamphibia. 
2492

2493

2494

2495

2496

2497

2498

2499

2500

2501

2502

2503

2504

2505

2506

2507

2508

2509

2510

2511

2512

2513

2514

2515

2516

2517

2518

2519

2520

2521

2522

2523

2524

2525

2526

2527

2528

2529

2530

2531

2532

2533

2534

2535

2536

2537

The basal dichotomy of Holospondyli is into a (Scincosaurus + Diplocaulidae) clade, which is fully resolved as in R1-R3, and a clade of (*Utaherpeton (adelospondyls (Urocordylidae, Aïstopoda))). Aïstopoda is poorly resolved.

On the other side of the amphibian branch, Pantylidae and Tuditanidae are sister-groups like in R1-R3; Tuditanidae is augmented by *Crinodon and the *Goreville microsaur. A (Gymnarthridae + Ostodolepididae) clade lies next to the remainder, in which Saxonerpeton, an unexpected (Odonterpeton $+{ }^{*}$ Sparodus) clade, an expected (Hapsidopareion $+{ }^{*}$ Llistrofus) clade, Rhynchonkos and finally a clade composed of Brachydectes and Brachystelechidae (composed of Batropetes $+(*$ Carrolla $+*$ Quasicaecilia); named after a synonym of Batropetes) are successively closer to Lissamphibia, unlike in R1. Within Lissamphibia, Albanerpetidae emerges as the sister-group of Batrachia. The closest relative of Salientia is the caudate *Chelotriton; together they are the sister-group to a clade formed by all other caudates, in which Karaurus is as highly nested as possible.

*Trihecaton has two positions as the sister-group of (Holospondyli (Microbrachis, Hyloplesion)) or one node rootward of Saxonerpeton.

A tree from R4 has an ITRI of 75.6\% compared to O1, 88.2\% compared to $\mathrm{O} 2$ and $92.7 \%$ compared to R1.

Analysis R5

An analysis of the increased taxon sample with a constraint against the LH found 6778 MPTs which have 3021 steps - 10 more than the MPTs of Analysis R4 - as well as a ci of 0.1854 , a hi of 0.8792 , a ri of 0.6170 , and a rc of 0.1144 .

As shown by comparison of the strict and Adams consensus trees and the first, the last and every $250^{\text {th }}$ MPT (Fig. 15, 16), all of which support the TH, the constraint has not had a strong effect on the tree; most of the differences to the results of R4 consist of loss of resolution among the temnospondyls. This is in stark contrast to earlier versions of this matrix (Marjanović \& Laurin, 2015, 2016) or to the differences between R1 and R2.

The clade of *Nigerpeton and *Saharastega gains two new positions within Stereospondylomorpha (Fig. 15: lower inset; Fig. 16: middle inset); in one of them (Fig. 16), Dvinosauria joins it. These two clades can also form a clade or grade just crownward of Cochleosauridae, followed crownward by Eryops and then Stereospondylomorpha (Fig. 16: main tree, upper inset); or they can form a clade next to Stereospondylomorpha, which then, too, lies crownward of Eryops (Fig. 16: upper inset). The trematopids sometimes (Fig. 16) form a grade (with Ecolsonia at its crownward end as in RC07 and some MPTs of R1), *Tungussogyrinus, *Branchiosaurus and *Micropholis gain positions within the non-trematopid non-lissamphibian dissorophoid grade, *Acanthostomatops sometimes leaves it. Unlike in Analysis R2, which was performed under the same constraint, Lissamphibia is not nested among the dvinosaurs, and no "lepospondyls" are found among the temnospondyls; instead, either Doleserpeton or * Gerobatrachus, but never both together, is the sister-group of Lissamphibia. Lissamphibia is resolved with a Procera topology, where an (Albanerpetidae + Eocaecilia) clade is nested within a caudate grade or next to a caudate clade, which always excludes * Chelotriton, the sister-group to all other procerans.

* Trihecaton has a single position as the sister-group of (Holospondyli (Microbrachis, Hyloplesion)). The other large lepospondyl clade is slightly rearranged from R4: the sister-group of the Brachydectes-brachystelechid clade is Hapsidopareiidae, followed by (Odonterpeton 
2538 (*Goreville microsaur, *Sparodus)), then (Rhynchonkos (Gymnarthridae, Ostodolepididae)), and

2539

2540

2541

2542

2543

2544

2545

2546

2547

2548

2549

2550

2551

2552

2553

2554

2555

2556

2557

2558

2559

2560

2561

2562

2563

2564

2565

2566

2567

2568

2569

2570

2571

2572

2573

2574

2575

2576

2577

2578

2579

2580

2581

2582

2583

then Saxonerpeton followed by (Tuditanidae + Pantylidae) as in R4.

The statistical tests find the difference between a tree from R5 and a tree from R4 insignificant: $p=0.1831$ (Kishino-Hasegawa), 0.2278 (Templeton), 0.0975 (winning sites). The same tree from R5 has an ITRI of $90.2 \%$ when compared to a tree from O1, 80.1\% compared to O2, $79.8 \%$ compared to $\mathrm{R} 2$ and $87.8 \%$ compared to $\mathrm{R} 4$.

\section{Analysis R6}

With a constraint for the $\mathrm{PH}$, an analysis of the increased taxon sample found 1816 MPTs with 3026 steps - 5 more than those from Analysis R5, 15 more than those from Analysis R4. The MPTs have a ci of 0.1851 , a hi of 0.8794 , a ri of 0.6162 and a rc of 0.1140 .

Inspection of the first, the last and every $50^{\text {th }}$ MPT (we later examined every $10^{\text {th }}$ MPT up to the $640^{\text {th }}$ for the position of * Cheliderpeton) shows topologies mostly identical to those of R4, allowing us to greatly simplify Fig. 17. In addition to its positions in R4, the *Nigerpeton* Saharastega clade can lie inside a rearranged Stereospondylomorpha (as in some MPTs of R5). Batrachia is nested as the sister-group to Doleserpeton, followed by *Gerobatrachus. Within Batrachia, *Chelotriton is the sister-group of Salientia. Albanerpetidae is highly nested in the clade of all remaining caudates, as the sister-group of Valdotriton.

Lepospondyli is resolved much like in Analysis R1, with Eocaecilia as the sister-group of Aïstopoda; Brachydectes sometimes entersBrachystelechidae as the sister-group of Batropetes. Aïstopoda is better resolved than in R4 and R5 in that there is always a dichotomy between (Oestocephalus + Phlegethontia) and the remainder. *Trihecaton lies next to Gymnarthridae or next to Micraroter.

Trees from R6 and R4 are indistinguishable at the level of $p=0.05: p=0.1328$ (KishinoHasegawa), 0.0565 (Templeton), 0.0966 (winning sites). Unsurprisingly, trees from R6 and R5 are hardly distinguishable: $\mathrm{p}=0.3515$ (Kishino-Hasegawa), 0.3174 (Templeton), 0.4538 (winning sites). The same tree from R6 has an ITRI of $88.0 \%$ compared to O3, 88.6\% compared to $\mathrm{R} 3,87.5 \%$ compared to $\mathrm{R} 4$ and $97.5 \%$ compared to R5.

\section{Bootstrap analyses $B 1$ and $B 2$}

Figures 18 and 19 present the bootstrap trees for the original and the expanded taxon samples, respectively (Analyses B1 and B2). They are fully resolved because clades with bootstrap values below $0.5(50 \%)$ are included if they do not contradict the majority-rule consensus (see above). In Fig. 18 (Analysis B1), support is skewed towards peripheral nodes, while the "trunk" of the tree has bootstrap percentages well below 50. Still, together with many uncontroversial results, the position of Whatcheeriidae rootward of Colosteidae, Crassigyrinus and even Tulerpeton is supported $(67 \%, 54 \%$ and $57 \%$, respectively) as well as the membership of Ossinodus (52\%), the position of Eucritta as a baphetoid (55\%), Dissorophoidea sensu lato excluding Lissamphibia (84\%), Ecolsonia as a trematopid (56\%), Trematopidae as the sister-group to Dissorophoidea sensu stricto (55\%), Amphibamidae excluding Branchiosauridae (60\%), Micromelerpeton as the sister-group of Branchiosauridae (61\%), Bruktererpeton and Gephyrostegus as sister-groups (53\%), the monophyly of Diadectomorpha (59\%) and Sauropsida (80\%) as well as their sistergroup relationship (68\%), Microbrachis and Hyloplesion as sister-groups (67\%), Lissamphibia including Albanerpetidae (65\%), Batrachia + Albanerpetidae (53\%), Batrachia excluding 
2584 Albanerpetidae (77\%), Caudata (74\%) and the sister-group relationship of Scincosaurus and

2585 Diplocaulidae (56\%). Further, Acanthostega as crownward of Ventastega (45\%), Ichthyostega as

2586 crownward of both (47\%), Balanerpeton + Dendrerpetidae (49\%), Isodectes + Neldasaurus

2587 (47\%), the adelospondyl clade (47\%) and the sister-group relationship of Urocordylidae and

2588 Aïstopoda (43\%) are each almost certainly better supported than any single alternative. In

2589

2590

2591

2592

2593

2594

2595

2596

2597

2598

2599

2600

2601

2602

2603

2604

2605

2606

2607

2608

2609

2610

2611

2612

2613 contrast, Kotlassia is found within Seymouriamorpha only in $28 \%$ of the replicates.

Adding taxa (Analysis B2) slightly increases the bootstrap support of four nodes, but unsurprisingly - generally depresses the support of almost the entire tree (Fig. 19). Of its potentially controversial branches, only the sister-group relationship of *Nigerpeton and *Saharastega (50\%), that of Microbrachis and Hyloplesion (66\%), that of Scincosaurus and Diplocaulidae (63\%), the clade of modern amphibians (Albanerpetidae + Lissamphibia: 67\%) and Batrachia (54\%) are found by $50 \%$ or more of the bootstrap replicates; Lissamphibia is found in $44 \%$ of the replicates, Karaurus $+*$ Beiyanerpeton in $46 \%$, Chroniosuchia and Gephyrostegidae in $42 \%$ each, and the adelospondyl clade (40\%) and Doleserpeton + ${ }^{*}$ Gerobatrachus in $40 \%$ each. The addition of two synapsids (Synapsida: $56 \%$ ) has made Limnoscelis a diadectomorph (24\%) outside of Amniota (30\%) and reduced the support for the monophyly of Sauropsida to $26 \%$, although support for Amniota + Diadectomorpha is considerably higher at $44 \%$.

Analysis EB

The results of our exploratory Bayesian analysis (Fig. 20,21) are remarkably similar to those of Analysis B2, which was conducted under the same settings. The most conspicuous difference may be how strong the support for the LH is: a node with a posterior probabilities of $92 \%$ (Fig. 20) would need to be broken to move the modern amphibians into Temnospondyli, not to mention one each with posterior probabilities of $76 \%, 74 \%, 73 \%$ and finally $64 \%$ (Dissorophoidea incl. *Iberospondylus).

Posterior probabilities of $75 \%$ or higher support *Elginerpeton crownward of Panderichthys (100\%) and outside the rest of the ingroup (77\%), Acanthostega crownward of Ventastega (75\%), Ichthyostega crownward of Acanthostega (76\%), Tulerpeton crownward of 2614 all whatcheeriids and *Densignathus (77\%), Colosteidae including the *St. Louis tetrapod (97\%)

2615 forming an exclusive clade with Baphetidae and Eucritta (74\%) which lies crownward of

2616

2617

2618

2619

2620

2621

2622

2623

2624

2625

2626

2627

2628

2629 Whatcheeriidae and Crassigyrinus (86\%) and rootward of Caerorhachis (again 86\%), Eucritta in Baphetoidea (94\%), *Sigournea + *Doragnathus $+*$ Spathicephalus $+*$ Diploradus $(87 \%)$ lying next to Megalocephalus (82\%) in Baphetidae (again 82\%), Anthracosauria (97\%), Embolomeri (99\%), Proterogyrinus outside a clade of all other embolomeres (94\%), Balanerpeton + Dendrerpetidae (83\%), Dvinosauria (77\%), *Nigerpeton + *Saharastega $(99 \%),{ }^{*}$ Konzhukovia + *Platyoposaurus (98\%) next to *Australerpeton (81\%), Ecolsonia as a trematopid (94\%), Phonerpeton + Acheloma (100\%), Kotlassia inside Seymouriamorpha (99\%), Discosauriscidae excluding Kotlassia, Seymouria and * Karpinskiosaurus (91\%), Leptoropha + Microphon $(100 \%),{ }^{*}$ Caseasauria $+*$ Archaeovenator $(75 \%)$, Diadectomorpha $(96 \%)$, a clade composed of Limnoscelis and the two diadectids (76\%), Tuditanidae (89\%) containing the *Goreville microsaur $+{ }^{*}$ Crinodon $(88 \%)$, Gymnarthridae (96\%), Ostodolepididae (87\%), Hapsidopareion $+{ }^{*}$ Llistrofus (100\%), Microbrachis + Hyloplesion (97\%), *Sparodus as a pantylid (88\%), Pantylus + Stegotretus (93\%), a "Holospondyli" clade including all "nectrideans", "aïstopods", adelospondyls, *Utaherpeton, Brachydectes, "brachystelechids" and modern amphibians (76\%), 
2630 the adelospondyl clade (100\%), Adelogyrinidae (95\%), Urocordylidae incl. Aïstopoda (96\%), 2631 Aïstopoda (100\%), Scincosaurus + Diplocaulidae (86\%), Diplocaulidae (100\%), Diplocaulus + 2632 Diploceraspis (100\%) as the sister-group of Batrachiderpeton (94\%), a clade of Brachydectes, 2633 the "brachystelechids" and the modern amphibians (92\%), a novel clade formed by the modern 2634 amphibians, *Quasicaecilia and *Carrolla to the exclusion of Batropetes and Brachydectes $(88 \%)$, Lissamphibia (100\%), Batrachia (81\%), Salientia excluding *Chelotriton $(99 \%)$ and also excluding Triadobatrachus (100\%). Amphibia including Westlothiana has $74 \%$ posterior

2636

2637

2638

2639 probability, the clade of all remaining amphibians has $73 \%$, the colosteid clade of *Aytonerpeton, *Deltaherpeton, Colosteus and Greererpeton has $71 \%$, as does Isodectes + Neldasaurus; Amniota incl. Diadectomorpha has $70 \%$, as do Colosteidae + Baphetidae and Ossinodus $+*$ Densignathus. Gephyrostegidae reaches $69 \%$, Chroniosuchia to $67 \%$.

Posterior probabilities below 50\% are most common in Temnospondyli and its

2641

2642

2643

2644

2645

2646

2647

2648

2649

2650

2651

2652

2653

2654

2655

2656

2657

2658

2659

2660

2661

2662

2663

2664

2665

2666

2667

2668

2669

2670

2671

2672

2673

2674

2675 surroundings as well as in the "microsaur backbone". The strange finding of urocordylid paraphyly is weakly supported $(65 \%)$. The Ossinodus $+*$ Densignathus clade is poorly supported as crownward of Whatcheeriidae (57\%), *Perittodus as rootward of it (again 57\%), and

*Perittodus as crownward of Ichthyostega (58\%); Chroniosuchia has weak support (55\%) for its position crownward of Temnospondyli and *Casineria. Solenodonsaurus is kept together with Seymouriamorpha by a posterior probability of $51 \%$.

The longest branch is the terminal branch of Phlegethontia ( 0.172 expected changes per character; Fig. 21), followed by those of Eocaecilia (0.169), Brachydectes (0.146), Ichthyostega and ${ }^{*}$ Chelotriton $\left(0.132\right.$ each), *Neopteroplax $(0.126),{ }^{*}$ Tungussogyrinus $(0.125)$ and *Erpetosaurus (0.124). Internal branches with at least 0.1 expected changes per character are limited to Aïstopoda (0.155), Diplocaulus + Diploceraspis (0.145), Hapsidopareion + *Llistrofus (0.133), the ingroup except Panderichthys (0.129), Lissamphibia (0.114), the adelospondyls (0.111), the clade of non-traditional baphetids (0.107) and Urocordylidae incl. Aïstopoda (0.100).

The tree has an ITRI of $71.2 \%$ compared to O1, $81.3 \%$ compared to O2, 96.7\% compared to $\mathrm{R} 1$ and $84.2 \%$ compared to R4.

\section{Discussion}

Reevaluating the matrix of RC07 has revealed additional character conflict and polymorphism (on the latter's impact see Watanabe, 2015) and greatly increased the length of the most parsimonious trees. Evidently, the MPTs found by RC07 painted an oversimplified picture of the evolution of the limbed vertebrates.

The addition of taxa in Analyses R3-R6, B2 and EB has had unexpected effects that may have improved the reliability of the tree. As previously demonstrated (Mortimer, 2006; Butler \& Upchurch, 2007; Raven \& Maidment, 2018), every OTU in a data matrix can influence the position of every other OTU in the resulting trees.

The ITRIs (Table 5) show that our revisions of the scores had a noticeable but moderate impact on tree topology. Comparisons among trees from the original matrix (O1-O3) always reveal ITRIs above $85 \%$, and similarly, nine of the 11 comparisons performed among trees obtained from the revised matrix (R1-R6, EB) yield ITRIs above 85\%; in contrast, of the 12 comparisons of trees from the revised matrix to trees from the original matrix, only four show ITRIs above $85 \%$, and two have the lowest values of the entire Table $(71.2 \%$ and $72 \%)$. The method of analysis apparently played a lesser role: the two comparisons of the Bayesian tree 
2676 (EB) to parsimony trees based on the same matrix (with or without added taxa) yield ITRIs

2677

2678

2679

2680

2681

2682

2683

2684

2685

2686

2687

2688

2689

2690

2691

2692

2693

2694

2695

2696

2697

2698

2699

2700

2701

2702

2703

2704

2705

2706

2707

2708

2709

2710

2711

2712

2713

2714

2715

2716

2717

2718

2719

2720

2721

between $84 \%$ and $97 \%$. The topological constraints also apparently played only a moderate role in determining tree similarity. This is shown by the fact that comparisons between trees supporting the same hypothesis (TH, $\mathrm{LH}$ or $\mathrm{PH}$ ) have similarly large and widely overlapping ranges: $76.7 \%$ to $90.2 \%$ (TH), $81.3 \%$ to $96.7 \%(\mathrm{LH})$ and $78.9 \%$ to $88.6 \%(\mathrm{PH})$. Further, some trees supporting different hypotheses have high ITRIs (e.g. R6, supporting the PH, has an ITRI of $97.5 \%$ with respect to R5, which supports the $\mathrm{TH}$ ), while other such comparisons yield very low ITRIs (e.g. 72\% for R1 [LH] compared to O1 [TH]). Strikingly, among the trees that support the LH, EB is much more similar to R1 (96.7\%), which contains fewer taxa, than to R4 (84.2\%), while R4 has an intermediate ITRI of $92.7 \%$ with respect to R1. Thus, the ITRIs suggest that the observed similarities between trees result from a complex interplay between all the factors mentioned above, and possibly others not considered here.

Unstable areas of the tree and other phenomena highlight promising areas for future research. These include redescription of Westlothiana (currently being undertaken following the discovery of additional specimens: M. Ruta, pers. comm. 2015; Clack \& Milner, 2015), **Eldeceeon (currently being undertaken, see Material and methods: Treatment of OTUs: Taxa that were not added above), the "microsaurs" Asaphestera, Tuditanus, Odonterpeton and *Trihecaton (see below), **Sauravus (the presumed sister-group of Scincosaurus), *Casineria (especially in order to determine whether it is distinguishable from Caerorhachis), *Utaherpeton (see below), ${ }^{* *}$ Macrerpeton (see below), and quite possibly others.

\section{Bias in the matrices?}

Given the fact that the present work bears on one of the most controversial questions that remain in vertebrate phylogeny, the origin of the modern amphibians, it is not surprising that several colleagues have wondered during the long genesis of this work whether various kinds of bias are present in the original matrix or in our revision of it. To some extent, we can test this: if our changes have mostly gone in one direction (say, increasing support for the LH or decreasing support for the $\mathrm{TH}$ ), then either the matrix of $\mathrm{RC} 07$ was in some way biased against that direction, or our changes have been biased in that direction, or both. While we could perhaps not objectively distinguish between these three possibilities, we can address whether our revisions show such a preferential direction in the first place.

Our answer is firmly negative (see next section, "Bias in the scores?"). Indeed, we consider one of the most important results of our present work, together with the work of Sigurdsen \& Green (2011), Langer et al. (2017) and Spindler et al. (2018: online resource 3) as well as our previous work (Marjanović \& Laurin, 2008, 2009), to be the observation that accidental, unsystematic misscores are very common in published matrices - common enough to change the most parsimonious topologies.

Naturally, in many cases, RC07 simply could not have avoided incorrect scores because the correct ones were only published after 2006. We have not differentiated these in App. S2 or Data S4, because our goal was to reevaluate the matrix and the trees that result from it, not the context in which it was made. Needless to say, however, this information could be extracted from the dates of the sources we cite in App. S1; some of it is summarized in Tables 6-8 and 10.

Bias in the scores? 
2722 Tables 7, 9 and 11 list the characters at the top 20 ranks of changes marked red in App. S2, the

2723

2724

2725

2726

2727

2728

2729

2730

2731

2732

2733

2734

2735

2736

2737

2738

2739

2740

2741

2742

2743

2744

2745

2746

2747

2748

2749

2750

2751

2752

2753

2754

2755

2756

2757

2758

2759

2760

2761

2762

2763

2764

2765

2766 characters at the top 20 ranks of changes marked green and all characters with changes marked blue, respectively (as explained in Results: Amount, distribution and impact of revised scores). In columns $\mathrm{O}$ and $\mathrm{R}$ of each table, we present by how many steps each of these characters supports the $\mathrm{LH}$, the $\mathrm{TH}$ and/or the PH over its alternatives. Strikingly often, e.g. for the top four characters in Table 7, that number is zero both before $(\mathrm{O})$ and after all our revisions put together (R) even if those revisions have caused dozens of changes. In all three tables, the only numbers larger than two in columns $\mathrm{O}$ and $\mathrm{R}$ are found in characters HUM 18/DIG 1 (ch. 219), which favored the TH over both of its alternatives by one step in RC07 (HUM 18 by one step, DIG 1 by none), but now disfavors it, again compared to both alternatives, by three steps after 24 red changes (Table 7), and JAW ART 1/SQU 2/DEN 8 (ch. 146), which favored the LH over both alternatives by one step in RC07, but now favors the PH by one step over the $\mathrm{LH}$ and the TH by four steps over the LH after 25 red and five blue changes (Tables 7, 11), not to mention a green one (App. S2, Data S4). It is evident from Tables 7, 9 and 11 that support for all three hypotheses was present in the matrix of RC07, and that our revision has both added and removed support for all three, all in similar amounts. Table 9 shows in particular that many of our redefinitions of characters, including the most drastic ones, have had negligible effects, or none, on support for the hypotheses about the origins of the modern amphibians; Table 11 shows the same for our approach to ontogeny and heterochrony, and Table 7 for the many, many scattered changes that do not depend on interpretations of characters.

To quantify this impression, we performed binomial tests on the data from Tables 7, 9 and 11, both separately and on all these data simultaneously (Table 12), in QuickCalcs (Motulsky, 2018). As reported in Table 12, none of the separate tests are significant ( $p>0.10$ in all cases), and neither is the test on the pooled data $(p=0.6835)$. The method of our test consists in counting how many parsimony steps were gained by the LH and TH by our rescoring. For instance, if the original score of a character in RC07 favored the TH over the LH by one step and if, after our rescoring, it favored the LH over the TH by one step, we scored a two-step difference in favor of the LH. We did this over all the characters listed in Tables 7, 9 and 11, and tested, in each case, if the observed distribution could be explained by changes that randomly favored either of the hypotheses $\left(\mathrm{H}_{0}\right)$. In these tests, we ignored characters whose rescoring did not alter the relative support of $\mathrm{LH}$ and $\mathrm{TH}$. We did not test if our scores altered the relative support of the PH because there is no reason to expect bias in favor of the PH either in $\mathrm{RC} 07$ or in our revision (Tables 1, 4). We performed all tests as two-tailed because there are a priori reasons to think that we could have favored either hypothesis: the LH because this is the hypothesis that we have supported in the past, and the TH because we tried to avoid biasing the results (e.g. by bold interpretations of morphological data) in favor of the $\mathrm{LH}$, which may have resulted in a bias against the LH. Regardless, performing the tests as one-tailed yields $p>0.05$ in all cases.

There is very little evidence, too, of disagreements between Ruta \& Coates and us about how to interpret the homology of the morphological features coded here. Almost all of them belong to TRU VER 4, our ch. 257, which is discussed above (Results: Amount, distribution and impact of revised scores) and at greater length in App. S1; Table 7 shows that the 50 changes we have made to its scores (all red, no green or blue ones: App. S2, Data S4) have not had any impact on its irrelevance to the origins of the modern amphibians. Even the character for which the OTUs were evidently scored blockwise (PREMAX 7, our ch. 3; see Material and methods: 
2767 Treatment of characters: Blockwise scoring of taxa and App. S1) has no net bearing on this

2768 question either before or after our extensive revision of this character (Table 9).

2769

2770

Further evidence of accidental, unsystematic misscores is constituted by the numerous discrepancies (App. S1) between the matrix of RC07 and the careful, detailed, splendidly

2771

2772 illustrated works by the same authors (Lebedev \& Coates, 1995; Coates, 1996; Ruta, Milner \& Coates, 2002; Klembara \& Ruta, 2004a, b, 2005a, b; Ruta \& Clack, 2006; Milner \& Ruta, 2009) that we have used as sources for our revision.

Therefore, we are certain that the vast majority of, at least, the straightforwardly

2774

2775

2776

2777

2778

2779

2780

2781

2782

2783

2784

2785

2786

2787

2788

2789

2790

2791

2792

2793

2794

2795

2796

2797

2798

2799

2800

2801

2802

2803

2804

2805

2806

2807

2808

2809

2810

2811 indefensible scores in the matrix of RC07 are typographic or similar errors as described in the Introduction (Aims: Accuracy of the matrix of RC07). Their large number simply underscores that morphological phylogenetics, as great as its rewards are, is extremely work-intensive - not so much in terms of difficulty as in terms of sheer amount of time required.

\section{Bias in character selection?}

While the character sample is much smaller than it could be (discussed below: Characters: Persisting problems with the character sample), we see no evidence to suggest that it is biased for or against a hypothesis on lissamphibian origins. Characters supporting all three of these hypotheses are represented in similar numbers (Tables $7,9,11$ ), and we have not noticed any glaring omissions of relevant characters. It is not the case either that characters supporting one of these hypotheses are systematically overcounted by being duplicated or multiplied as redundant characters. Instead, even though we have found and redefined or merged several redundant characters all across the matrix, there is evidence for systematic avoidance of redundancy: the matrices of Ruta, Coates \& Quicke (2003) and RC07 lack many characters that had been used in previous matrices for analysis of the phylogeny of limbed vertebrates and that would have been obvious choices to include except for the fact that they would be redundant with others. For example, the length ratio of the antorbital and postorbital parts of the skull has been popular (to pick some of the most recent examples: Clack et al., 2012b: ch. 328; Clack et al., 2016: ch. 164 and 165; Pardo et al., 2017: ch. 32; Pardo, Small \& Huttenlocker, 2017: ch. 25; Spindler et al., 2018: ch. 3), but would have made a correlated mess of FRO 2, TAB 7, NOS 4, ORB 5, SKU TAB 1, VOM 1, VOM 13 (our ch. 27, 57, 83, 90, 95, 102, again 102) and possibly others. We think it was deliberately kept out of the matrix for this reason. Of the redundant characters that did make it into the matrix of Ruta, Coates \& Quicke (2003), several were eliminated by RC07; an example is PAL 3 (ch. 129 of Ruta, Coates \& Quicke, 2003: "Palatine excluded from (0) or contributing to (1) interpterygoid vacuities"), which was almost identical to VOM 10 (ch. 124 of Ruta, Coates \& Quicke, 2003: "Vomer in contact with anterior ramus of pterygoid (0) or not (1)"; ch. 129 of RC07: "Vomer contact with pterygoid palatal ramus: present (0); absent (1)").

\section{Methods of phylogenetic analysis}

\section{Bayesian inference and parsimony in comparison}

We consider our Bayesian analysis exploratory because the behavior and performance of Bayesian inference on datasets like ours have not been studied. Firstly, the amount and distribution of missing data may be a matter of concern. We are aware of three studies of its

2812 effects on Bayesian inference: 
2813

2814

2815

2816

2817

2818

2819

2820

2821

2822

2823

2824

2825

2826

2827

2828

2829

2830

2831

2832

2833

2834

2835

2836

2837

2838

2839

2840

2841

2842

2843

2844

2845

2846

2847

2848

2849

2850

2851

2852

2853

2854

2855

2856

2857

2858
Wright \& Hillis (2014) explicitly intended to study the performance of Bayesian inference with morphological data. They simulated matrices of exclusively binary characters and scored all characters that evolved at a given rate as unknown for selected taxa; this may be somewhat realistic for molecular data, where different genes (each with its own rate) may have been sequenced for different taxa in a supermatrix, but makes limited sense for morphological data, where missing data are clustered by body parts, not by rate of evolution. Wright \& Hillis (2014) found that Bayesian inference outperforms parsimony under the conditions they studied. Using contrived, simulated and empirical DNA datasets, Simmons (2011a, b) studied what happens when different taxa are scored for different characters so that some taxa have no scored characters in common at all, as may (again) happen when different genes have been sequenced for different taxa in a supermatrix. Given a matrix with non-overlapping taxa, parsimony cannot find only a single MPT which has them as sister-groups; instead, it will return consensus trees with polytomies when such taxa are not kept far enough apart by character states they share with other taxa. Parametric methods (Bayesian inference and maximum likelihood) instead try to compensate and can therefore find a single optimal tree which contains such taxa as sister-groups. Sometimes this turns out to be correct; however, Simmons (2011a, b) found that this situation routinely led parametric methods to finding strong support for wholly spurious clades - even under ideal conditions (no homoplasy, a perfectly fitting model of evolution, a perfect alignment, no rate heterogeneity). Moreover, which spurious clades were found was very sensitive to small changes in the scores of a matrix, despite the high support values. For datasets with missing data that are distributed as in his studies, Simmons (2011a, b) recommended that nodes and support values found by parametric analyses in parts of the tree that are not resolved in the strict consensus of a parsimony analysis of the same dataset should only be accepted after special scrutiny of their causes.

In our matrix, missing data are clustered by body parts, so the findings of Wright $\&$ Hillis (2014) may not translate to our situation; all OTUs except * Casineria and the isolated lower jaws we added are scored at least for part of the dermal skull, so Simmons's (2011a, b) almost opposite conclusion may not translate to our situation either.

A second issue was raised by Puttick et al. (2017), who attempted to investigate the impact of tree shape (full symmetry vs. maximum asymmetry) on the performance of different methods. They found that all methods performed badly on the most basal nodes of a Hennigian comb, and that Bayesian inference performed least badly. Goloboff, Torres \& Arias (2017: $14^{\text {th }}$ page) showed that Puttick et al. (2017; later also O'Reilly et al., 2017) had actually tested something completely different: their symmetric tree had unitary branch length, while their asymmetric tree was ultrametric, so that the least nested terminal branches were many times longer than the internal branches (which had unitary length). Their experiment thus amounts to a test of susceptibility to long-branch attraction, not to tree shape. The trees we find are much closer to the asymmetric than to the symmetric end of the spectrum; the branch lengths, however, are much closer to unitary than to ultrametric, with short terminal branches making up the majority of the Devonian part and being scattered over the rest of the "trunk" (Fig. 21). Indeed, apart from the adelospondyls and the aïstopods, our Bayesian tree (Fig. 21) places most OTUs remarkably close to their relative stratigraphic positions.

Of the four most recent studies of the performance of Bayesian inference on matrices of morphological characters, two (O'Reilly et al., 2016; Puttick, et al., 2017) did not mention the problem of missing data at all, and Goloboff, Torres \& Arias (2017) omitted it from most of their comparisons of Bayesian inference to other methods. O'Reilly et al. (2017: 106) stated that "our 
2859 experiments do not attempt to simulate non-contemporary taxa or address the problem of missing 2860 data, qualities of palaeontological data that are of a level of complexity that is beyond the current 2861 debate." For us, of course, this is most of the current debate; we are quite surprised that O'Reilly 2862 et al. (2017) chose to publish their statement in the journal Palaeontology, whose very name 2863 suggests non-contemporary taxa and missing data.

2864

2865

Thirdly, it remains unknown whether Bayesian inference outperforms parsimony on datasets whose amount and distribution of homoplasy is like that of ours. For matrices with

2866

2867

2868

2869

2870

2871

2872

2873

2874

2875

2876

2877

2878

2879

2880

2881

2882

2883

2884

2885

2886

2887

2888

2889 average evolutionary rates approaching three changes per character per tree, the performance of Bayesian inference and parsimony converges when the number of characters increases toward 1000 (O’Reilly et al., 2016, 2017; Puttick et al., 2017; perhaps also Wright \& Hillis, 2014: fig. 6 ). Even with its many multistate characters, our matrix does not contain a number of states equivalent (or close) to 1000 binary characters. However, as listed above, the consistency indices are below 0.22 in all our parsimony analyses and below 0.19 for all those with the expanded taxon sample; this translates to an average of about five changes per character per tree, well outside the range studied by Wright \& Hillis (2014: two to three changes). Similarly, O'Reilly et al. (2016, 2017) and Puttick et al. (2017) studied datasets with consistency indices between 0.26 and 1.0; most of this range occurs only in very small datasets. Individual characters in our matrix show a wide range of rates - some change states only once per tree, some change states 30 to 40 times on every tree, the extreme being 51 (Analyses R1, R3) to 73 changes (R4) for SKU TAB 1 (ch. 95, five states).

To keep their calculations in a feasible timeframe, O'Reilly et al. $(2016,2017)$ and Puttick et al. (2017) reduced all their parsimony results to the majority-rule consensus. This must have overestimated the precision of parsimony and may have underestimated its accuracy.

Goloboff, Torres \& Arias (2017) noted that all preceding studies had assumed a common branch length parameter for all characters to simulate their datasets, so that if one character had an increased probability of changing along a given branch, all others had proportionally increased probabilities of changing along that same branch. As Goloboff, Torres \& Arias (2017) pointed out, this is highly unrealistic for morphological data; indeed, it does not fit our matrix (where different characters are stable or labile in different parts of the same tree). O'Reilly et al. (2017) responded to several criticisms by Goloboff, Torres \& Arias (2017), but left this one unaddressed.

2890

2891

2892

2893

2894

2895

2896

2897

2898

2899

Finally, ordered characters (let alone stepmatrices) were not used in any of the studies cited here, so the impact of ordering remains unknown. In Wright \& Hillis (2014) and O'Reilly et al. (2016), all characters were binary; in Goloboff, Torres \& Arias (2017: $5^{\text {th }}$ page), interestingly, all characters had four states, which is far from realistic as well.

Parametric methods of phylogenetics are generally less sensitive to long-branch attraction than the nonparametric method called "maximum parsimony" is (as inadvertently confirmed by Puttick et al., 2017, and O'Reilly et al., 2017). Long-branch attraction is not unknown in morphological data. Indeed, we suspect that long-branch attraction could be responsible for parts of our MPTs; in particular, our results concerning the placement of adelospondyls, aïstopods (Pardo et al., 2017) and urocordylids (Pardo et al., 2018) should be taken with caution, as discussed below. However, these very results are supported by Analysis EB (posterior probability of $76 \%$ for all these taxa being highly nested amphibians close to Lissamphibia, and $69 \%$ for their forming an exclusive clade with *Utaherpeton; Fig. 20), and the internal branches

2903

2904 in that region of the tree are long but not extremely long (Fig. 21). Although we are looking forward to further developments of parametric methods and further tests of their performance 
2905

2906

2907

2908

2909

2910

2911

2912

2913

2914

2915

2916

2917

2918

2919

2920

2921

2922

2923

2924

2925

2926

2927

2928

2929

2930

2931

2932

2933

2934

2935

2936

2937

2938

2939

2940

2941

2942

2943

2944

2945

2946

2947

2948

2949

2950

2951

2952

with paleontological datasets, the extent to which the trees we present here are wrong will more likely be discovered through improvements to our matrix than improvements to the methods of analysis. Indeed, before we updated the scores of the aïstopod Lethiscus after Pardo et al. (2017) and added two more $\left({ }^{*}\right.$ Coloraderpeton and $*$ Pseudophlegethontia $)$, the abovementioned posterior probabilities were $98 \%$ and $97 \%$, respectively; this may have been a case of "garbage in, garbage out", a law to which no method is immune.

\title{
Reweighting and equal weighting in comparison
}

\begin{abstract}
Our review of their datasets indicates that [...] Goloboff et al. (2017) [...] their simulated datasets are not individually empirically realistic, with many matrices dominated by characters with very high consistency and an unrealistically small proportion of characters exhibiting high levels of homoplasy. The datasets simulated by Goloboff et al. (2017) have qualities that strongly bias in favour of parsimony phylogenetic inference, and implied-weights parsimony in particular, as the presence of large numbers of characters that are congruent with the tree allows implied weights to increase the power of these 'true' congruent characters. This effect will not be possible when increased levels of homoplasy are present $[\ldots]$.
\end{abstract}

- O'Reilly et al., 2017: 106-107, about Goloboff, Torres \& Arias, 2017

Any method of phylogeny inference works when its assumptions are met. Therefore, the fact that the method variously called reweighting, implied weighting or a-posteriori weighting is logically circular (see below) is not an argument against using it when its assumptions are met; when they are, it outperforms all others (Goloboff, Torres \& Arias, 2017). We think they are not met in our dataset and have therefore not used this method.

Specifically, in the empirical matrices Goloboff, Torres \& Arias (2017) cited and the matrices they simulated, there is an exponential or nearly exponential distribution of characters so that a plurality of characters has, on the MPTs found by unweighted parsimony, no homoplasy at all, i.e. as few steps as theoretically possible for a parsimony-informative character with the given number of states, in other words a consistency index of 1 ; next most common are characters with one extra step ( $\mathrm{ci}=0.5$ ), then two and so on (Goloboff, Torres \& Arias, 2017: especially fig. 1). In our matrix, homoplasy-free characters are much less common (Data S8). Given the original taxon sample (Analysis R1; Fig. 22A), the distribution zigzags around an exponential one, but the most common number of extra steps is one, followed by two and only then (by a large margin) by zero; when taxa (and thus homoplasy) are added (Analysis R4; Fig. 22B), the most common number is still one, then three, then two and four (equally), then nine, then eleven, then zero, five, seven and eight (all equally), then the rest vaguely approaches an exponential distribution with a wide variance. In both taxon samples, several binary characters have the maximum possible number of steps given our matrix (i.e., of one state, no two occurrences are unambiguously optimized as homologous, or those that are are canceled out by reversals).

Even if it retrieves an accurate topology, we think that reweighting is prone to underestimating homoplasy and overestimating the support for this topology. Accurate topologies are not the only goal of our research.

(Reweighting attempts to downweight characters depending on how homoplastic they are. The circularity consists in the fact that the amount of homoplasy for each character is determined by optimizing the characters on the MPTs found by unweighted parsimony, which amounts to assuming that these trees are correct or nearly so. By downweighting characters that are incongruent with those trees, reweighting reduces character conflict and thus increases 
2953 support and resolution, but not necessarily accuracy. As Goloboff, Torres \& Arias [2017]

2954

2955

2956

2957

2958

2959

2960

2961

2962

2963

2964

2965

2966

2967

2968

2969

2970

2971

2972

2973

2974

2975

2976

2977

2978

2979

2980

2981

2982

2983

2984

2985

2986

2987

2988

2989

2990

2991

2992

2993

2994

2995

2996

2997

2998

emphasized, unweighted and reweighted parsimony are equally sensitive to long-branch attraction; furthermore, characters can be falsely congruent with each other for reasons other than long-branch attraction - redundancy and correlation in particular.)

\section{Phylogenetic relationships}

In this section we mention the bootstrap percentages (BPO and BPE from Analyses B1 and B2, shown in Fig. 18 and 19, respectively) that support or contradict clades found by our analyses. Most of them are below 50, especially for the expanded taxon sample (Fig. 19); this highlights the suboptimal ratio between number of characters and number of taxa, as well as character conflict and wildcard taxa. As in Fig. 18 and 19 as well as Tables 3 and 4, bootstrap percentages of 50 and above are presented in boldface.

Likewise, we mention the posterior probabilities (in \%: PP from Analysis EB, shown in Fig. 20) that support or contradict clades found by our analyses. As in Fig. 20 and Table 3, PPs of 75 and above are presented in boldface.

\section{Devonian taxa, Whatcheeriidae and Perittodus}

At the base of the ingroup, RC07 found the "textbook" Hennigian comb (Fig. 1, 23A):

(Panderichthys (Ventastega (Acanthostega (Ichthyostega (Tulerpeton, post-Devonian clade))))). However, the position of Ichthyostega soon began to be questioned (Ahlberg et al., 2008: supplementary information; Clack et al., 2012a) as more information about it was discovered and published (Callier, Clack \& Ahlberg, 2009; Ahlberg, 2011; Clack et al., 2012a; Pierce, Clack \& Hutchinson, 2012; Pierce et al., 2013; Clack \& Milner, 2015). In our analyses of the original taxon sample (Fig. 23B), Ichthyostega is equally parsimoniously crownward of Ventastega (BPO $=47 ; \mathrm{BPE}=11 ; \mathrm{PP}=76)$ and Acanthostega $(\mathrm{BPO}=47 ; \mathrm{BPE}=10 ; \mathrm{PP}=76)$, which are - only in this case - sometimes found as sister-groups (contradicted by a BPO of 45, a BPE of 19 and a PP of 75), or rootward of Acanthostega but still crownward of Ventastega, or rootward of both. We will not be surprised if the last of these three options will be upheld by analyses with larger character and outgroup samples, as indeed it has been (in the absence of Ventastega) in the analysis of Pardo et al. (2017). The picture of Ichthyostega as the Godzilla of mudskippers (Pierce, Clack \& Hutchinson, 2012; see also Coates \& Clack, 1995), an animal that acquired an amphibious lifestyle wholly independently from more crownward walking and limbless tetrapodomorphs that lived after Romer's Gap, may well be accurate.

However, in our expanded taxon sample (Fig. 23C), the position of Ichthyostega is stabilized crownward of Ventastega, Acanthostega, *Ymeria and even the Tournaisian *Perittodus (BPE = 10; contradicting a PP of 58, which holds *Perittodus crownward).

The added Devonian taxa are all poorly known, as is *Perittodus. Yet, all of them have fully resolved positions. This appears to be due to a combination of the relatively dense sample of lower-jaw characters and our similarly dense taxon sample.

Within this mostly Devonian grade, the Carboniferous whatcheeriids nest rootward of *Densignathus ( $\mathrm{PP}=57$; against a $\mathrm{BPE}$ of 13$)$. This is particularly intriguing in the light of the "whatcheeriid-grade" skull bones that were found with *Densignathus; we are much less certain than Daeschler, Clack \& Shubin (2009) that they do not actually belong to *Densignathus.

Ossinodus is found as a whatcheeriid in the original taxon sample $(\mathrm{BPO}=\mathbf{5 2} ; \mathrm{BPE}=26)$,

PeerJ reviewing PDF | (2015:12:8254:6:0:NEW 20 Jul 2018) 
2999 specifically as the sister-group to the other two as in $\mathrm{RC} 07(\mathrm{BPO}=\mathbf{7 2} ; \mathrm{BPE}=45)$. This contrasts

3000 with the topology (Pederpes (Ossinodus, Whatcheeria)) found by Pawley (2006) before the

3001 recent descriptions of further Ossinodus material (Warren, 2007; Bishop, 2014). When we add

3002 taxa, Ossinodus gains three additional positions around *Densignathus (PP for the two as sister-

3003 groups $=70$; against a BPE of 13).

3004

3005

In all analyses, the Devonian Tulerpeton emerges crownward of Whatcheeriidae $(\mathrm{BPO}=$

3006

3007

3008

3009

3010

3011

3012

3013

3014

3015

3016

3017

3018

3019

3020

3021

3022

3023

3024

3025

3026

3027

3028

3029

3030

3031

3032

3033

3034

3035

3036

3037

3038

3039

3040

3041

3042

3043

3044 57, $\mathrm{BPE}=18$; PP = 77), neither rootward as in RC07 and Pawley (2006) nor as its sister-group as in Ruta \& Bolt (2006) and the almost identical Ruta (2009) or in Clack \& Klembara (2009). Creating the former topology in Mesquite adds two steps each to Analyses R1 and R4; for the latter, three extra steps need to be added to Analysis R1 and four to R4 (three are required to make Tulerpeton a member of an Ossinodus-*Densignathus clade one node crownward of Whatcheeriidae).

Taken at face value, our results support the idea (Anderson et al., 2015; Clack et al., 2016) that more than one clade of limbed vertebrates survived the Hangenberg event at the Devonian-Carboniferous boundary. We eagerly await the forthcoming redescription of **Elpistostege based on a whole articulated CT-scanned skeleton (Cloutier \& Béchard, 2013; Cloutier et al., 2016); the improved sample of Devonian stem-tetrapods and probably characters in the accompanying phylogenetic analysis will likely contribute to a better understanding of this part of the tree.

\section{More Mississippian mysteries}

RC07 found Colosteidae, Crassigyrinus and Whatcheeriidae to be successively more crownward (Fig. 1, 24A). Like Ruta (2009), we find them successively more rootward in all analyses (Fig. 23B, C, 24B, C; $\mathrm{PP}=\mathbf{8 6} ; \mathrm{BPO}=\mathbf{6 7}$ for Colosteidae + more crownward taxa including Eucritta; $\mathrm{BPE}=22$ for Eucritta + more crownward taxa including Colosteidae). This agrees with other recently published analyses; we attribute this to our corrections. For example, RC07 (and Ruta, Coates \& Quicke, 2003) had scored Whatcheeria as lacking the preopercular bone in the skull (state PREOPE 1(1), which is shared by the colosteids and Crassigyrinus), so that its presence (PREOPE 1(0)) in Pederpes appeared as an isolated reversal. This was apparently due to a misreading of Clack (1998), who explicitly confirmed that the preopercular is present in Whatcheeria as described by Lombard \& Bolt (1995) and Bolt \& Lombard (2000); see ch. 81 (PREOPE 1) in App. S1 for discussion. The third sampled whatcheeriid-grade animal, Ossinodus, was scored as unknown, which was correct at the time; it is now known to have retained the preopercular as well (Warren, 2007), and we have scored it accordingly.

RC07 also found Eucritta as the sister-group of Baphetes + Megalocephalus, as did Ruta (2009), Milner, Milner \& Walsh (2009), Witzmann \& Schoch (2017) and the original full description by Clack (2001). Given the original taxon sample, we find this as one of two options $(\mathrm{BPO}=\mathbf{5 6})$. Unexpectedly, the other option is one node rootward of Baphetidae; a position comparable to this is the only one found when we increase the taxon sample (BPE $=8)$, except in Analysis EB, which finds a (Eucritta (Baphetidae, Colosteidae)) clade with a PP of 74.

Like Eucritta, the extremely broad-headed *Spathicephalus has been considered a nonbaphetid baphetoid (Beaumont \& Smithson, 1998; Milner, Milner \& Walsh, 2009; following the nomenclature of Milner, Milner \& Walsh, 2009). *Spathicephalus is consistently a baphetoid in our analyses $(\mathrm{BPE}=30 ; \mathrm{PP}=\mathbf{8 7})$; however, it is highly nested within Baphetidae $(\mathrm{PP}=\mathbf{8 7}$; against a BPE of 31), closer to the long-snouted Megalocephalus than to the broad-headed 
3045 Baphetes $(\mathrm{PP}=\mathbf{8 2})$. Milner, Milner \& Walsh (2009) sampled baphetids and baphetoid-related

3046

3047

3048

3049

3050

3051

3052

3053

3054

3055

3056

3057

3058

3059

3060

3061

3062

3063

3064

3065

3066

3067

3068

3069

3070

3071

3072

3073

3074

3075

3076

3077

3078

3079

3080

3081

3082

3083

3084

3085

3086

3087

3088

3089

3090 characters more densely than we did; however, it is possible that these advantages are outweighed by the disadvantages of their much smaller outgroup sample (only Acanthostega and Crassigyrinus), the fact that they did not order any characters (of the 24 characters, seven are continuous or meristic multistate characters), redundancy between ch. 3 and 4, and the scoring of juvenile morphology as adult (affecting at least ch. 19).

A further factor that may affect the position of * Spathicephalus may be the enigmatic *Doragnathus and *Sigournea, the latter known from an isolated lower jaw, the former from fragments of lower and upper jaws. Both are found as baphetoids in of our all analyses that include them $(\mathrm{BPE}=30 ; \mathrm{PP}=\mathbf{8 7})$, indeed as baphetids closer to Megalocephalus than Baphetes is $(\mathrm{PP}=\mathbf{8 2}$; against a $\mathrm{BPE}$ of 31$)$, forming a clade with $*$ Spathicephalus $(\mathrm{BPE}=30 ; \mathrm{PP}=44)$ and $*$ Diploradus $(\mathrm{BPE}=19 ; \mathrm{PP}=\mathbf{8 7})$. Similarities between all these taxa have long been noted, but only tested once in a phylogenetic analysis (even apart from the fact that $*$ Diploradus was very recently published): using a rather small character sample, Clack et al. (2016) found the three as a grade not very close to Baphetidae in some of their analyses, but widely scattered in others. Although our results remain to be tested with a larger sample of characters and of baphetoids, we think that *Doragnathus, *Sigournea and *Diploradus will likely be upheld as baphetoids, possibly as baphetids and likely as a clade with *Spathicephalus (BPE =19; PP = 87). It is noteworthy in this respect that Smithson \& Clack (2013) noted similarities to baphetoids in the isolated postcranial material from the site where *Doragnathus was found; perhaps it belongs to *Doragnathus after all.

A very unexpected finding of our analyses with added taxa (Fig. 24C) is a clade composed of Colosteidae and Baphetidae $(\mathrm{BPE}=3 ; \mathrm{PP}=70)$, which is furthermore a member of Temnospondyli (the sister-group to all others combined; contradicting a BPE of 20 and a PP of 86). This ties into the problem of the mutual positions of Temnospondyli and Anthracosauria discussed below.

We are looking forward to the ongoing redescription of Crassigyrinus based on computed tomography. The abstract by Porro, Clack \& Rayfield (2015) promises previously unknown character states, but the only new information it makes explicit is that "all three coronoids bear teeth"; we were unable to use this information, because our matrix contains separate characters for the presence or absence of tusks, denticles and toothrows.

\section{Colosteidae}

Given the original taxon sample, Colosteidae is consistently found in a position one node rootward of Baphetoidea (or Eucritta followed by Baphetidae) and one node crownward of Crassigyrinus (Fig. 24B), as found e.g. by Pawley (2006) and Ruta (2009) and discussed immediately above. Adding taxa (Fig. 24C) pushes them and the baphetids further crownward, where both together form a surprising clade of temnospondyls (discussed below).

Among the added taxa are two uncontroversial colosteids. Analyses R4-R6 and EB, the first phylogenetic analyses to include them, unsurprisingly find $*$ Pholidogaster $(\mathrm{BPE}=30 ; \mathrm{PP}=$ $50)$ and $*$ Deltaherpeton $(\mathrm{BPE}=48 ; \mathrm{PP}=71)$ as successively closer to Colosteus + Greererpeton (a clade with a BPE of $\mathbf{7 6}$ and a PP of 98).

Also included in the enlarged taxon sample are *Erpetosaurus and the unnamed *St. Louis tetrapod (MB.Am.1441). The latter was described (Clack et al., 2012b) as having several similarities to colosteids, but also some to temnospondyls, while lacking several features 
3091 otherwise found in colosteids; the former was most recently redescribed as a dvinosaurian

3092

3093

3094

3095

3096

3097

3098

3099

3100

3101

3102

3103

3104

3105

3106

3107

3108

3109

3110

3111

3112

3113

3114

3115

3116

3117

3118

3119

3120

3121

3122

3123

3124

3125

3126

3127

3128

3129

3130

3131

3132

3133

3134

3135

3136

3137 temnospondyl with convergent similarities to colosteids, including features that the *St. Louis tetrapod lacks (Milner \& Sequeira, 2011). In our analyses, the *St. Louis tetrapod consistently emerges as the sister-group to all other colosteids $(\mathrm{BPE}=25 ; \mathrm{PP}=97)$, while ${ }^{*}$ Erpetosaurus is a dvinosaur $(\mathrm{BPE}=14, \mathrm{PP}=\mathbf{7 7} ; \mathrm{BPE}=20$ and $\mathrm{PP}=\mathbf{8 6}$ for not lying next to Colosteidae, $\mathrm{BPE}=$ 25 and $\mathrm{PP}=\mathbf{9 7}$ for not being nested within it).

One feature that may be relevant to the relationships of the *St. Louis tetrapod is not coded in this matrix: as pointed out by Clack et al. (2012b), denticle-covered platelets of dermal bone that fill the interpterygoid vacuities are only known in that specimen and in temnospondyls. We speculate that such platelets appear more or less automatically when the interpterygoid vacuities are wide enough (the *St. Louis tetrapod has the proportionally widest ones of any colosteid) and denticles are present throughout the roof of the mouth (they are entirely absent in Batropetes, *Carrolla, apparently *Quasicaecilia, Diplocaulus, Diploceraspis and lissamphibians except Eocaecilia). This character deserves further investigation. It may be relevant that Eocaecilia, which fairly clearly lacks such platelets, has denticles on the pterygoids and the parasphenoid but not on the vomers or palatines (Jenkins, Walsh \& Carroll, 2007).

We have also added * Aytonerpeton, which Clack et al. (2016) described as sharing several similarities with the colosteids, but found far from Colosteidae in most of their analyses. One of these features - the premaxillary caniniform region, not coded in our matrix - represents an intermediate state between plesiomorphic homodonty and the premaxillary tusk which is found in traditional colosteids (and *Erpetosaurus), where it is accommodated by a notch in the dentary. This notch is a character in our matrix, and scored as absent for *Aytonerpeton (Clack et al., 2016); yet, *Aytonerpeton nests surprisingly highly within Colosteidae, as the sister-group of (*Deltaherpeton (Colosteus, Greererpeton) ) to the exclusion of *Pholidogaster $(\mathrm{BPE}=35 ; \mathrm{PP}=$ 71), which has the tusk and the notch, and the *St. Louis tetrapod, which has a W-shaped notch (see ch. 153, DEN 4, in App. S1 for discussion). To test this further, it will probably be necessary to fully describe *Aytonerpeton and to reinvestigate the difficult specimens of *Pholidogaster.

Whether the middle Tournaisian *Aytonerpeton, which is older than the Viséan *St. Louis tetrapod, can claim the title of oldest known colosteid depends on two other specimens: the **“Type 3 humerus" from Blue Beach in Nova Scotia (middle Tournaisian) and possibly **"Ribbo" from the early middle Tournaisian site of Willie's Hole in (Old) Scotland (Anderson et al., 2015; Clack et al., 2016).

The interrelationships of Anthracosauria, Silvanerpeton, Caerorhachis, Gephyrostegidae, Casineria and Temnospondyli

Although [anthracosaurs have] long [been] associated with the amniote stem, this link appears to be increasingly tenuous.

$$
\text { - Coates, Ruta \& Friedman, 2008: } 579
$$

Ever since the early $20^{\text {th }}$ century, Anthracosauria (which traditionally encompassed at least Embolomeri and the more recently discovered Eoherpeton) has generally been considered closer to Amniota than Temnospondyli is. The analysis by RC07 supported this "textbook" consensus (Fig. 24A). However, both the anthracosaurs and the temnospondyls share features with Amniota, Diadectomorpha, "Lepospondyli", Seymouriamorpha and/or Solenodonsaurus that the other taxon lacks (Fig. 24). Consequently, Temnospondyli has been found closer to Amniota than Anthracosauria is in a few analyses (Ahlberg \& Clack, 1998; Laurin \& Reisz, 1999: 
3138 bootstrap tree and discussion; Pawley, 2006; Klembara et al., 2014, with poor support; some 3139 MPTs of Pardo et al., 2017).

3140 Our results are best considered inconclusive on this point. Analyses R1 and R3 find

3141 Anthracosauria equally parsimoniously rootward $(\mathrm{BPE}=6 ; \mathrm{PP}=48)$ or crownward of

3142 Temnospondyli $(\mathrm{BPO}=12)$, while R2 fixes the rootward and R4-R6 the crownward position.

3143

3144

Part of the reason may be character conflict: not only Anthracosauria and Temnospondyli

3145

3146

3147

3148

3149

3150

3151

3152

3153

3154

3155

3156

3157

3158

3159

3160

3161

3162

3163

3164

3165

3166

3167

3168

3169

3170

3171

3172

3173

3174

3175

3176

3177

3178

3179

3180

3181

3182

3183 have conflicting combinations of character states, but so do other taxa found in the same area of the tree (Fig. 24).

Silvanerpeton, found as an anthracosaur (the sister-group to all others) by RC07 (BPO = 10 ; $\mathrm{BPE}=6)$, lies one node more crownward $(\mathrm{PP}=48)$ in all of our analyses except $\mathrm{B} 1$ and $\mathrm{B} 2$ (Fig. 24B, C). This is tenuous, however; earlier versions of our matrix (Marjanović \& Laurin, $2015,2016)$ show Silvanerpeton as an anthracosaur, one node more crownward or one node more rootward in different analyses or often in the same ones, indicating that its position depends on changes to a few scores as well as the taxon sample and constraints on other taxa.

Caerorhachis is variously considered to be close to the origin of temnospondyls and/or anthracosaurs (Ruta, Milner \& Coates, 2002, and references therein; Clack et al., 2016). RC07 found Caerorhachis one node closer to Amniota than Temnospondyli and one node farther away than Anthracosauria (Fig. 1, 24A). This is the only position found by Analyses R4-R6 (Fig. 24C). In Analyses R1 and R3, however, Caerorhachis can also be the sister-group to all other temnospondyls (Fig. 24B; BPO of Temnospondyli including Caerorhachis: 14), as found by Pawley (2006) and suggested earlier by Godfrey, Fiorillo \& Carroll (1987). Analyses R2, B2 and EB place Caerorhachis rootward of both Temnospondyli $(\mathrm{BPE}=6$; $\mathrm{PP}=48)$ and Anthracosauria $(\mathrm{BPE}=4 ; \mathrm{PP}=44)$.

RC07, and more recently Witzmann \& Schoch (2017), found Gephyrostegus one node rootward of Bruktererpeton. In Analyses R1-R3, they form either a clade (BPO = 53; $\mathrm{BPE}=42$; $\mathrm{PP}=69$ ) as found by Klembara et al. (2014) in their redescription of the skull of Gephyrostegus, or (in some MPTs from R1 and R3) Bruktererpeton is one node rootward of Gephyrostegus (Fig. 24B). Only the latter arrangement occurs in R4-R6 (Fig. 24C). As found by RC07 and Witzmann \& Schoch (2017), Gephyrostegus and Bruktererpeton are crownward of Anthracosauria in all analyses. A sister-group relationship of Gephyrostegus (or Gephyrostegidae) to Anthracosauria, as found by Pawley (2006), does not occur (though it is only contradicted by $\mathrm{BPO}=16$; $\mathrm{BPE}=6$; $\mathrm{PP}=48$ ); it may be relevant that Pawley's (2006) taxon sample lacked Silvanerpeton and Bruktererpeton.

* Casineria emerges in the gephyrostegid grade (Fig. 24C): variously one node crownward of Gephyrostegus (BPE $=4$ and $\mathrm{PP}=27$ for lying crownward of Gephyrostegidae, $\mathrm{BPE}=7$ and $\mathrm{PP}=55$ for lying rootward of Chroniosuchia), one node rootward of Bruktererpeton, between the two, or as the sister-group of Bruktererpeton. This is novel, but perhaps not too surprising. Originally, *Casineria was thought to lie very close to the origin of Amniota (Paton, Smithson \& Clack, 1999; against a BPE of 9 and a PP of 55). Clack et al. (2012b) and Witzmann \& Schoch (2017, using a very similar matrix) found it slightly more distant, i.e. as a "lepospondyl" (more precisely in a clade with Westlothiana and both of the sampled "microsaurs"; against a BPE of 13 and a PP of 55). Clack et al. (2016) increased the distance slightly farther, finding it as a seymouriamorph (against a BPE of 9 and a PP of 55) or in the same grade (against a BPE of 7 and still the same PP of 55) in all analyses. In the meantime, Pawley (2006: 207) noted that *Casineria scored identically to Caerorhachis in her matrix, apart from the (quite different) distribution of missing data; the matrix contains an unusually large 
3184 amount of postcranial characters, well suited for the headless skeleton called * Casineria. In our 3185 matrix, for which D. M. studied the only known * Casineria specimen (part and counterpart:

3186 NMS G 1993.54.1p, NMS G 1993.54.1cp) for a full day, Caerorhachis and * Casineria are

3187 likewise indistinguishable. Pawley's (2006: fig. 62) phylogenetic analysis featured a trichotomy

3188 of * Casineria, Caerorhachis and all other temnospondyls, with strong bootstrap and Bremer

3189 support for Temnospondyli (fig. 63). Between Caerorhachis on one side and the

3190 seymouriamorph-"lepospondyl"-amniote clade on the other, the positions we find for *Casineria

3191 lie in the middle.

$3192 \quad$ Paton, Smithson \& Clack (1999) emphasized that * Casineria had gastrocentrous

3193

3194

3195

3196

3197

3198

3199 vertebrae and claws, highlighting especially the latter as an amniote-like feature. Pawley (2006: 239; see also 195) remarked: "As in Caerorhachis bairdi, none of the postcranial characteristics claimed to be 'reptiliomorph' in Casineria kiddi are truly apomorphic for the amniote lineage. All are present in temnospondyls [...], or potentially may be present in basal temnospondyls (including the five[-]digit manus), because they are plesiomorphic for early tetrapods." We confirm that the vertebrae of *Casineria with their large intercentra are more reminiscent of Caerorhachis and animals in vaguely the same grade like the anthracosaur Proterogyrinus or, to a lesser degree, the temnospondyl Neldasaurus than of amniotes, seymouriamorphs or even chroniosuchians (Fig. 5); indeed, they differ from those of Caerorhachis at most in a slightly lesser degree of ossification. Although the terminal phalanges are distally pinched and curved plantarly, they are neither pointed as expected of a claw, nor rounded as incorrectly shown in the interpretative drawing by Paton, Smithson \& Clack (1999: fig. 3b). Instead, their ends are squared off (Fig. 6). This shape is intriguingly similar to the outline drawings of the terminal phalanges of Caerorhachis by Holmes \& Carroll (1977: fig. 13), who unfortunately did not provide more detailed illustrations or any description. These two character complexes, thus, are not obstacles to placing * Casineria far from amniote origins. Moreover, if our very tentative identification of distal carpal 1 in Fig. 6 is correct, the first distal carpal began to ossify before the fourth in * Casineria, a plesiomorphic condition not found in amniotes or diadectomorphs (see below, Discussion: Characters: Preaxial polarity in limb development).

Going beyond her matrix, Pawley (2006: 195, 239) called Caerorhachis and * Casineria "indistinguishable based on the available evidence". Now that D. M. has seen the *Casineria specimen (Fig. 5-7), we can only find four differences to the thorough redescription of Caerorhachis by Ruta, Milner \& Coates (2002): Caerorhachis is larger and has more elongate (but otherwise identical) ventral scales, a slightly more prominent dorsal process on the ilium and taller ossified parts of the neural spines. All four can effortlessly be ascribed to ontogeny. Indeed, the neural spines of * Casineria are capped by a black material (Fig. 5; likely an iron sulfide) that is also found on the incompletely ossified ends of long bones (Fig. 6) and may be related to cartilage decay. (For scale ontogeny, see Witzmann [2007, 2010].) Lack of difference is not a synapomorphy; our analyses do not currently find Caerorhachis and *Casineria as sistergroups - if only, perhaps, because the skull of * Casineria is unknown and postcranial characters are underrepresented in our matrix -, and the two specimens come from close but not identical ages and localities. Still, the possibility should be seriously considered that * Casineria, once redescribed, could turn out to be either a synonym or the closest known relative of Caerorhachis. Rearranging a tree from Analysis R4 to form a clade of Caerorhachis, *Casineria and optionally the *Parrsboro jaw (see below) takes two extra steps in Mesquite, moving this clade next to the traditional temnospondyls (Pawley, 2006) takes one more; both actions contradict nodes with a BPE of 6 and a PP of 48. 
3230

3231

3232

3233

3234

3235

3236

3237

3238

3239

3240

3241

3242

3243

3244

3245

3246

3247

3248

3249

3250

3251

3252

3253

3254

3255

3256

3257

3258

3259

3260

3261

3262

3263

3264

3265

3266

3267

3268

3269

3270

3271

3272

3273

3274
Character conflict in this part of the tree concerns, among others, features that are probably relevant to the origin of terrestriality (Fig. 24). At least one anthracosaur $\left({ }^{* *} \mathrm{CM} 34638\right.$ : Clack, 2011b; see also Holmes \& Carroll, 2010) retained a dermal and endochondral tail fin skeleton (state CAU FIN 1(0) in this matrix, see ch. 277 in App. S1); unknown in Silvanerpeton (Ruta \& Clack, 2006), Bruktererpeton and Gephyrostegus, it is absent in all temnospondyls that are well enough known to tell. The anthracosaur Archeria is known (Pawley, 2006: chapter 6) to retain the postbranchial lamina on the cleithrum (CLE 2(0), ch. 192); due to preservation and publication bias (the lamina is practically only visible in cranial or caudal view), this character is unknown in many OTUs in this matrix, but the lamina is absent in Gephyrostegus and all sufficiently well known unquestioned temnospondyls (e.g. Pawley, 2006: chapter 6). Because D. M. (pers. obs.) has identified what seems to be a postbranchial lamina quite similar to that of Archeria (depicted by Pawley, 2006: fig. 70-2.4) on the cleithrum of *Casineria, presented here in Fig. 7, we have scored *Casineria as possessing the lamina; this will require further investigation. If correct, it is a further argument for removing *Casineria from amniote origins. Anthracosauria, Silvanerpeton and Gephyrostegidae retain flat humeri (HUM 10(0), ch. 212), while * Casineria (D. M., pers. obs.) shares a waisted (HUM 10(1)), twisted humerus shape with all skeletally mature temnospondyls (unknown in Caerorhachis); the anthracosaurs Eoherpeton, Proterogyrinus and *NSM 994 GF 1.1 additionally retain an L-shaped humerus (HUM 12-15(0), ch. 214) where the entepicondyle is about as long (measured along its proximal margin) as the part of the humerus proximal to it, which is not seen in Archeria or Pholiderpeton scutigerum (unknown in Ph. attheyi, Anthracosaurus, *Palaeoherpeton and *Neopteroplax) or in the other taxa listed in this paragraph. Finally, pleurocentra that fuse in the ventral midline (TRU VER $8(1)$, ch. 260) are found in all the taxa listed in this paragraph except the unquestioned temnospondyls. (Among the latter, TRU VER 8(1) does occur in Doleserpeton and *Gerobatrachus, as well as in some **brachyopid stereospondyls and some **tupilakosaurid dvinosaurs, but these are too highly nested to be relevant here [Warren, 1999; Werneburg et al., 2007; Warren, Rozefelds \& Bull, 2011].) However, while state TRU VER 8(1) presumably increases the resistance of the vertebral column to compression, this may be dorsoventral compression from bearing weight just as well as mediolateral compression from e.g. anguilliform swimming. Various amphibious and terrestrial temnospondyls, on the other hand, elaborated the neural arches and (in Dissorophidae) neomorphic osteoderms into a weight-bearing apparatus that evidently did not need extensively ossified centra; furthermore, the vertebral columns of Gephyrostegus and Bruktererpeton in particular look rather supple in reconstructions despite showing state TRU VER 8(1).

Outside our matrix, character conflict in this part of the tree is further increased by the sacral vertebra described by Holmes, Godfrey \& Baird (1995: 919, fig. 6) which shows a unique combination of similarities to temnospondyls and anthracosaurs.

A postbranchial lamina on the clavicle, not coded here, has been identified in the dvinosaur **Thabanchuia (Witzmann, 2013) and in a number of *stereospondyls: Schoch \& Witzmann (2010) described it in **Plagiosuchus and **Trematolestes; D. M. has seen it on a clavicle of **Metoposaurus that, as of 2015, was to be catalogued by the University of Opole (Poland); Yates \& Warren (2000: fig. 7) depicted it in **Benthosuchus, ${ }^{* *}$ Paracyclotosaurus, **Lyrocephaliscus and **Koskinonodon - they only called it "anterior flange (prescapular process)", but Florian Witzmann (pers. comm. 2015) confirms that this structure is most likely identical to the postbranchial lamina. The homology of the postbranchial laminae on the 
3275 cleithrum of non-temnospondyls and the clavicle of temnospondyls remains unclear at the 3276 moment.

3277 Interestingly, the parasymphysial bone in the lower jaw (PSYM 1(0), ch. 147) has the

3278 same distribution as the postbranchial lamina on the cleithrum, missing data and aïstopods

3279 excepted (Fig. 24).

3280

3281

Noting the presence of anthracosaurs and Tulerpeton-like animals soon after the

3282 almost all limbed vertebrates are part of the smallest clade that contains Anthracosauria and

3283 Temnospondyli. "If Tulerpeton represents the earliest occurrence of embolomeres (as appears to

3284

3285

3286

3287

3288

3289

3290

3291

3292

3293

3294

3295

3296

3297

3298

3299

3300

3301

3302

3303

3304

3305

3306

3307

3308

3309

3310

3311

3312

3313

3314

3315

3316

3317

3318

3319 be suggested by the close similarity between humeral and femoral morphology)" (Anderson et al., 2015: 24), the last common ancestor of anthracosaurs and temnospondyls would have lived in the Devonian. However, Anderson et al. (2015) provided no support for this complex of ideas, which contradicts the current consensus (including RC07), beyond citing Lebedev \& Coates (1995); they did not mention the reanalysis and refutation of the latter by Laurin (1998b). Unsurprisingly, then, these historical hypotheses are not supported by our analyses; as discussed by Pawley (2006), the similarities between Tulerpeton and Anthracosauria are synapomorphic at that level, but were further modified by Temnospondyli and various other branches - they represent intermediate states. A fortiori, even if some or all extant amphibians are temnospondyls, there is currently no strong reason to think that the origin of the tetrapod crowngroup happened in the Devonian as Anderson et al. (2015) speculated. The highest support values that keep Tulerpeton away from Anthracosauria are $\mathrm{BPO}=\mathbf{6 7}, \mathrm{BPE}=22$ and $\mathrm{PP}=\mathbf{8 6}$ (Fig. 18-20); the lack of support in the parsimony analyses of the extended taxon sample is likely due to the unstable positions of Eucritta, Temnospondyli, Caerorhachis and the *Parrsboro jaw which can all intervene between Tulerpeton and Anthracosauria.

A topology somewhat similar to the above scenario was found by Klembara et al. (2014), where a weakly supported (Crassigyrinus (Whatcheeria, Anthracosauria)) clade and a weakly supported clade composed of Baphetidae and Temnospondyli were found. However, the second clade came out - likewise with weak support - as closer to Amniota than the first clade, which appeared just one node crownward of Ichthyostega. Containing 36 ingroup taxa (a quarter of them seymouriamorphs; Tulerpeton was not sampled), an all-zero outgroup (Klembara \& Ruta, 2004b: 86 - see Marjanović \& Laurin, 2008, for discussion of this practice) and 156 characters (many of course focused on seymouriamorphs), their matrix seems at least a priori less well suited to resolving this problem than ours.

At present, the oldest known anthracosaurs date (as mentioned) from the middle Tournaisian (Anderson et al., 2015), while the oldest known temnospondyls appear only after Romer's Gap (Balanerpeton, possibly Caerorhachis, perhaps *Casineria: Milner \& Sequeira, 1994; Paton, Smithson \& Clack, 1999; Ruta, Milner \& Coates, 2002; no temnospondyls have been identified in the middle Tournaisian material reported by Anderson et al., 2015, or Clack et al., 2016). This is congruent with the hypothesis that Temnospondyli and the tetrapod crowngroup, which is likewise currently unknown before the end of Romer's Gap, are more closely related to each other than to Anthracosauria. However, current understanding of the diversity of limbed vertebrates during Romer's Gap is still very poor; the current absence of evidence may turn out not to be evidence of absence.

3320

\section{The "Parrsboro jaw"}


3321 An incomplete natural mold of a partly crushed lower jaw, the *Parrsboro jaw in our expanded

3322

3323

3324

3325

3326

3327

3328

3329

3330

3331

3332

3333

3334

3335

3336

3337

3338

3339

3340

3341

3342

3343

3344

3345

3346

3347

3348

3349

3350

3351

3352

3353

3354

3355

3356

3357

3358

3359

3360

3361

3362

3363

3364

3365 taxon sample, has only two positions in our parsimony analyses (Fig. 24C): it is either the sistergroup of Caerorhachis $(\mathrm{BPE}=31$; contradicting a node with a $\mathrm{PP}$ of 48 ) or that of all traditional temnospondyls (against a PP of 45). Both positions lie within the large range found by Sookias, Böhmer \& Clack (2014) in their much smaller analysis. Analysis EB recovers what amounts to an intermediate position, rootward of Temnospondyli and Gephyrostegidae but crownward of Silvanerpeton.

Immediately, a character comes to mind that is absent from the present matrix but would influence the position of the *Parrsboro jaw: the presence/absence of denticles on the prearticular. Presence, a plesiomorphy shared with e.g. colosteids (Bolt \& Lombard, 2001), is found in the *Parrsboro jaw and in Caerorhachis but not, to our knowledge, in (other) temnospondyls or for that matter any anthracosaur-grade animals. A denticle field that covers most of the lingual face of the prearticular (extending onto the splenial at least in Caerorhachis), instead of being restricted to the dorsal side, even seems to be entirely limited to Caerorhachis and the *Parrsboro jaw among all sarcopterygians.

\section{Anthracosaurian phylogeny}

In the analyses of the original taxon sample (R1-R3 and B1), Anthracosauria is resolved as in RC07 when it lies rootward of Temnospondyli (the only option in R2), but in two other ways, with Pholiderpeton scutigerum as the sister-group of either (Proterogyrinus + Archeria $)$ or $(P h$. attheyi + Anthracosaurus), when it lies crownward of Temnospondyli. Bootstrap support greater than $50 \%$ or a posterior probability greater than $75 \%$ exists for three nodes: Anthracosauria (not including Silvanerpeton; $\mathrm{BPO}=\mathbf{5 4} ; \mathrm{BPE}=20 ; \mathrm{PP}=\mathbf{9 7})$, Embolomeri $(\mathrm{BPO}=\mathbf{5 2} ; \mathrm{BPE}=21 ; \mathrm{PP}$ = 99), Embolomeri except Proterogyrinus ( $\mathrm{PP}=\mathbf{9 4}$; against a BPO of 33 and a BPE of 31) and Pholiderpeton attheyi + Anthracosaurus excluding Ph. scutigerum $(\mathrm{BPO}=\mathbf{5 7} ; \mathrm{PP}=\mathbf{6 8}$; against a BPE of 18).

Adding taxa narrows these topologies down to that of RC07, even though Anthracosauria is consistently crownward of Temnospondyli in Analyses R4-R6 (against a BPE of 6 and a PP of 48); the three added OTUs form a clade (*NSM 994 GF 1.1 (*Palaeoherpeton, ${ }^{*}$ Neopteroplax); against a BPE of 28 and a PP of 62), which nests with Anthracosaurus (BPE = 28; PP =58).

Like RC07, we consistently find that Pholiderpeton scutigerum and Ph. attheyi are not sister-groups $(\mathrm{BPO}=\mathbf{5 7} ; \mathrm{BPE}=25 ; \mathrm{PP}=68)$. A logical consequence (if para- or polyphyletic genera are to be avoided) would be to reinstate the genus name Eogyrinus Watson, 1926, for $P h$. attheyi. However, we refrain from performing a taxonomic act because Pawley (2006: chapter 6) did find these two species as sister-groups using a different and larger character sample (but the same sample of possible anthracosaurs as RC07, minus Silvanerpeton), not to mention the weak support for our result.

We are more confident that the traditional taxon Eogyrinidae, comprising all embolomeres except Proterogyrinus, Archeria and Anthracosaurus, is paraphyletic (BPO = 57; $\mathrm{BPE}=28 ; \mathrm{PP}=68)$. These taxa were only found to form a clade in the much smaller analysis of Buchwitz et al. (2012), which was focused on chroniosuchians.

Temnospondyl large-scale phylogeny 
3366

3367

3368

3369

3370

3371

3372

3373

3374

3375

3376

3377

3378

3379

3380

3381

3382

3383

3384

3385

3386

3387

3388

3389

3390

3391

3392

3393

3394

3395

3396

3397

3398

3399

3400

3401

3402

3403

3404

3405

3406

3407

3408

3409

3410

3411

3412
I shall refrain here from discussion of the temnospondyls, although work being done at present, by Baird and Carroll, for example, suggests progress toward sorting out true phyletic lines among the Rhachitomi in preference to the somewhat artificial grouping which I used in my 1947 classification. - Romer (1964: 154)

More than fifty years after this hopeful statement, temnospondyl phylogeny remains poorly understood (Fig. 25). RC07, fittingly, found the base of Temnospondyli to be a polytomy of Edopoidea ( = Edops + Cochleosauridae), Dvinosauria, Balanerpeton, Dendrerpetidae and an Eryops-Dissorophoidea clade (Fig. 25A); *stereospondylomorphs were not included in the matrix. Most of the mathematically possible resolutions of this polytomy have been found in more focused but still large analyses: Pawley (2006: chapter 5; Fig. 25B) found Edops alone as the sister-group to all other temnospondyls (except Caerorhachis, see above and below), among which a (Capetus (Dendrerpetidae, Balanerpeton)) clade, Cochleosauridae, Dvinosauria, paraphyletic *stereospondylomorphs and Eryops were successively closer to Dissorophoidea. Ruta (2009; Fig. 25C) found Edopoidea as a whole to be the sister-group to all other temnospondyls, among which Capetus, (Eryops $+{ }^{*}$ Stereospondylomorpha), Dendrerpeton confusum and "D. acadianum" were successively closer to a trichotomous clade formed by Balanerpeton, Dvinosauria and Dissorophoidea; the only two *stereospondylomorphs in the matrix were *Sclerocephalus and *Glanochthon. (The OTU called "Dendrerpeton acadianum" most likely included Dendrysekos.) McHugh (2012; Fig. 25D) found Neldasaurus, otherwise considered a dvinosaur, to be the sister-group of all other temnospondyls; those were divided into, on the one hand, a clade that contained the *stereospondyls and the neotenic dissorophoids inside a clade formed by the remaining dvinosaurs, and on the other hand a clade that contained (Dendrerpetidae + non-aquatic dissorophoids), (Balanerpeton + Capetus $)$, Edopoidea and Eryops as successively closer relatives of the remaining * stereospondylomorphs. After deleting the colosteid Greererpeton from his taxon sample, Schoch (2013) recovered Edopoidea and (Balanerpeton + Dendrerpetidae) successively closer to a trichotomy of Dvinosauria, Dissorophoidea and Eryopiformes (=Eryops + Stereospondylomorpha) - when he used TNT; PAUP 3.1 failed to find any of the shortest trees (Schoch, 2013: 682). Dilkes (2015a) analyzed an expanded and corrected version of Schoch's (2013) dataset. Given the full sample of 73 OTUs (Dilkes, 2015a: fig. 10), he found a polytomy of Capetus, *Iberospondylus, (Dendrerpetidae + Balanerpeton), Edopoidea and a clade which contained all other temnospondyls; that clade was itself a polytomy of *Zatracheidae, Eryopidae, Dissorophoidea, a clade containing the *nonstereospondyl stereospondylomorphs and three separate clades of *stereospondyls. Deleting 25 OTUs, but not Greererpeton, resulted (Dilkes, 2015a: fig. 11A) in a single eryopiform clade that contained Eryops (the remaining eryopid) and the *non-stereospondyl stereospondylomorphs as a grade within which a single *stereospondyl clade was nested, and also revealed a (Dvinosauria (*Zatracheidae, Dissorophoidea)) clade, but continued to resolve the relationships of these clades in two very different ways (Fig. 25E, F). Only the additional deletion of Capetus and *Iberospondylus (Dilkes, 2015a: fig. 11B) made Eryopiformes consistently the sister-group of the (Dvinosauria (*Zatracheidae, Dissorophoidea)) clade, followed on the outside by (Dendrerpetidae + Balanerpeton) and finally Edopoidea as in Schoch (2013) and in Fig. 25E. Using the dendrerpetid Dendrysekos as the outgroup, Eltink \& Langer (2014) found the ingroup to be divided into (Eryops + Dissorophoidea) and (Dvinosauria + *Stereospondylomorpha); theirs was a *stereospondylomorph-focused analysis where Trimerorhachis was the only included dvinosaur. The same holds for Pereira Pacheco et al. (2016; Fig. 25G) and for Eltink et al. (2016), who omitted Dissorophoidea altogether. Pardo, Small \& Huttenlocker (2017: 
3413 supplementary information part D; Fig. 25H) accepted most of the corrections by Dilkes (2015a

3414 - not mentioned by Pardo, Small \& Huttenlocker, 2017) but added taxa, omitted others, and

3415 omitted the characters Dilkes had added. Dvinosauria emerged as the sister-group to the rest of

3416 the ingroup under both parsimony and Bayesian inference (Pardo, Small \& Huttenlocker, 2017:

3417 fig. S7B and $2=$ S7A, respectively); (Dendrerpetidae + Balanerpeton), Capetus, Edopoidea,

3418 *Iberospondylus, **Peltobatrachus, Eryopiformes, *Zatracheidae and **Lapillopsis (previously

3419 considered a stereospondyl) were found successively closer to Dissorophoidea in all MPTs, with

3420

3421

3422

3423

3424

3425

3426

3427

3428

3429

3430

3431

3432

3433

3434

3435

3436

3437

3438

3439

3440

3441

3442

3443

3444

3445

3446

3447

3448

3449

3450

3451

3452

3453

3454

3455

3456

3457 the positions of Capetus and **Peltobatrachus slightly destabilized in the Bayesian analysis.

This diversity of topologies appears to result in large part from the use of different outgroups. Pawley (2006) used Caerorhachis as the outgroup in chapter 5 after finding it to be in a trichotomy with *Casineria and all other temnospondyls in chapter 6 (her main analysis of the phylogeny of the limbed vertebrates). Ruta (2009) included a variety of early limbed vertebrates, but entirely omitted the taxa that form the amniote total group in the trees of RC07 (see Fig. 1), including Gephyrostegidae, Anthracosauria and Caerorhachis - the closest relative of Temnospondyli in his trees is Baphetoidea. McHugh (2012) used Greererpeton as the outgroup. Schoch (2013) chose the anthracosaur Proterogyrinus; in analyses where he included Greererpeton in the ingroup, Greererpeton either fell out as the sister-group to Temnospondyli or, "in some variant analyses of the large dataset (66 taxa, 'no swapping' option in PAUP), Greererpeton nests with the dvinosaurs, and in another variant Greererpeton and Neldasaurus form a 'clade' at the very base of the tree (66 taxa, 'no swapping' option, TNT). Whereas this reflects similarities in the postorbital skull shared by the two taxa, it also shows that aquatic taxa with particular features are sometimes attracted despite the obvious homoplastic status of these characters (indicated by multiple conflicting evidence)." This attraction between Greererpeton and Neldasaurus replicates the result of McHugh (2012), as does, to a lesser extent, the attraction of Greererpeton to the dvinosaurs as a whole. Unfortunately, however, Schoch (2013: 693) did not report the lengths of these trees; the lack of branch-swapping means that the local optima found in each tree-building replicate were not explored any further, which makes it likely that the reported trees were suboptimal. Notably, however, Greererpeton did not have such effects in the analyses of a version of that matrix improved by Dilkes (2015a). In the next version of the same matrix, Pardo, Small \& Huttenlocker (2017: supplementary information part D) kept

Proterogyrinus as the outgroup, and Greererpeton emerged as the sister-group to the rest of the ingroup, followed as mentioned by the monophyletic dvinosaurs.

Our analyses of the original taxon sample (R1-R3, B1; Fig. 26A-C) consistently place the colosteids far away from Temnospondyli. Yet, of those MPTs in which Temnospondyli is crownward of Anthracosauria (which is all in R2), some (all in R2; Fig. 26A) find Dvinosauria (which has a BPO of 35) as the sister-group to all other temnospondyls (except Caerorhachis in some from R1; $\mathrm{BPO}=40)$. This clade of all other temnospondyls $(\mathrm{BPO}=18)$ consists (against a $\mathrm{BPO}$ of 30) of a dichotomy of Dissorophoidea on the one side and an unusual clade on the other side in which (Balanerpeton + Dendrerpetidae), Capetus and Eryops $(\mathrm{BPO}=19)$ are successively closer to a surprisingly highly nested Edopoidea (monophyletic: BPO $=26$ ). Other MPTs from R1 and R3 (Fig. 26B) find Temnospondyli (always excluding Caerorhachis) as containing a backbone of (Dissorophoidea (Eryops (Cochleosauridae (Capetus (Trimerorhachis (Balanerpeton, Dendrerpetidae)))))) (against a BPO of 30); (Isodectes + Neldasaurus) is the sister-group of Trimerorhachis or of all other temnospondyls, among which Edops is one node closer to or one node farther from Dendrerpetidae than Capetus is. Finally, those MPTs (from R1 3458 and R3; Fig. 26C) where Temnospondyli is rootward of Anthracosauria consistently resolve it as 
3459 a Hennigian comb of (Edops (Cochleosauridae (Eryops (Capetus (Dvinosauria (Dendrerpetidae, 3460 Dissorophoidea)))))), with Balanerpeton as the sister-group of either of the last two. This 3461 position of Edops is reminiscent of Pawley (2006: chapter 5; Fig. 25B). Analysis B1, where 3462 Temnospondyli is again rootward of Anthracosauria, finds a sort of compromise topology, with 3463 Dvinosauria on the outside $(\mathrm{BPO}=18)$ except for Caerorhachis, Eryops next to Edopoidea $3464(\mathrm{BPO}=19)$, Dissorophoidea next to $($ Balanerpeton + Dendrerpetidae $)(\mathrm{BPO}=30)$, and very 3465 weak support overall (Fig. 18).

Analyses R4-R6 (Fig. 26C, D) find Temnospondyli rootward of Anthracosauria - so far rootward that Temnospondyli contains Eucritta and (Colosteidae + Baphetidae) successively closer to the clade of traditional temnospondyls (a surprising return to $20^{\text {th }}$-century classifications, contradicting a BPE of 20 and a PP of 86) and optionally the *Parrsboro jaw (see above). In keeping with its rootward position, the clade of traditional temnospondyls is resolved in R4 and R6 as in corresponding trees from R1 and R3, namely as (Edops (Cochleosauridae (Eryopiformes (Capetus (Dvinosauria ((Balanerpeton, Dendrerpetidae) (*Iberospondylus, Dissorophoidea))))))); in R5, Dvinosauria sometimes (Fig. 26D) enters Stereospondylomorpha, and Eryopiformes sometimes becomes a grade (with Stereospondylomorpha closer to Dissorophoidea than to Eryops), in which latter case Dvinosauria can fall out rootward of it or as the sister-group of Stereospondylomorpha, i.e. as part of Limnarchia Yates \& Warren, 2000. The corresponding bootstrap and Bayesian trees (Analyses B2, EB: Fig. 19, 20) find Temnospondyli rootward of Eucritta, Colosteidae, Baphetidae, Caerorhachis and Anthracosauria and consequently find a quite different temnospondyl topology, with an almost basal dichotomy of Dissorophoidea against most of the rest, but the highest BPE throughout the base of the temnospondyl tree is only 14 (for Dvinosauria); similarly, only five very small temnospondyl clades have PPs above $\mathbf{7 5}$ - Dvinosauria has 77, but (Isodectes + Neldasaurus) has 71, (Dissorophoidea (*Iberospondylus, *Mordex)) has 64, Stereospondylomorpha has 62, Dvinosauria except Trimerorhachis has 57, a novel (Edops + Cochleosaurus) clade has 47, Temnospondyli has 39, Dissorophoidea has 38, Temnospondyli excluding *Palatinerpeton has 35, (Chenoprosopus (*Nigerpeton, *Saharastega)) has 33.

Dissorophoidea is never found closer to Eryops than Stereospondylomorpha is; in other words, Euskelia Yates \& Warren, 2000, is always limited to Eryops (BPE of Eryopiformes = 2, $\mathrm{PP}=14)$ or to (Eryops (*Nigerpeton, *Saharastega)) (against a BPE of 15 and a PP of 33).

We conclude that progress in resolving temnospondyl phylogeny may come from analyses that have not only dense sampling of temnospondyls and a long list of maximally independent characters, but also a large, dense sample of other taxa, because the closest relatives of Temnospondyli have not been identified and the position of Temnospondyli rootward or crownward of the anthracosaurs, most evidently, has an enormous impact on temnospondyl phylogeny. In other words, the problem of temnospondyl phylogeny cannot be solved in isolation.

Schoch (2013: 689) defined the name Temnospondyli as applying to "[t]he least inclusive clade containing Edops craigi and Mastodonsaurus giganteus". This definition may be applied to our trees - as done in Fig. 26 - by assuming that **Mastodonsaurus would group with the other *stereospondylomorphs or, in their absence, with Eryops. In Analyses R4-R6 and some MPTs from R1 and R3 (Fig. 26C, D), in other words all those where the anthracosaurs are closer to Amniota than the temnospondyls are, Temnospondyli would then have its usual contents, because Edops is found as the sister-group to all other traditional temnospondyls. Conversely, in Analyses R2, B1, B2, EB and the remaining MPTs from R1 and R3 (Fig. 26A, B), 
3505 Temnospondyli would always exclude Dissorophoidea; in R2, B1, B2, EB and some MPTs from

3506 R1 and R3, Temnospondyli would even contain nothing but Eryops, Stereospondylomorpha and

3507 Edopoidea (Fig. 26A). Furthermore, Capetus, Balanerpeton, Dendrerpetidae and Dvinosauria

3508 fall outside of Temnospondyli under this definition in the MPTs found by Pardo, Small \&

3509 Huttenlocker (2017: fig. S7B; Fig. 25H). Therefore, we strongly recommend against using this

3510 definition, or any other minimum-clade definition with so few internal anchors, for this name.

3511 We have used the definition by Yates \& Warren (2000), which applies the name Temnospondyli

3512 to the clade encompassing all organisms that are more closely related to Eryops than to the

3513 "microsaur" Pantylus.

3514

3515

3516

3517

3518

3519

3520

3521

3522

3523

3524

3525

3526

3527

3528

3529

3530

3531

3532

3533

3534

3535

3536

3537

3538

3539

3540

\section{Stereospondylomorpha}

Throughout the analyses with added taxa (Fig. 14-17, 26C, D), the stereospondylomorph temnospondyls - *Sclerocephalus, *Cheliderpeton, *Glanochthon, *Archegosaurus, *Platyoposaurus, ${ }^{*}$ Konzhukovia, and the stereospondyls *Lydekkerina and *Australerpetonform a clade, either alone $(\mathrm{BPE}=14 ; \mathrm{PP}=62)$ or with $*$ Nigerpeton $+*$ Saharastega (some MPTs from R5 and R6) and Dvinosauria (some MPTs from R5), even though no characters intended to resolve stereospondylomorph phylogeny were included in the matrix.

* Sclerocephalus or, in R4 and some trees from Analyses R5 and R6, a clade formed by $*$ Sclerocephalus and *Cheliderpeton $(\mathrm{BPE}=27$; against $\mathrm{PP}=50)$ is consistently found as the sister-group to the rest. Within this remainder $(\mathrm{BPE}=11$; $\mathrm{PP}=57)$, those trees where *Nigerpeton and *Saharastega do not join, namely all from Analyses EB and R4 as well as some from R5 and R6, group *Konzhukovia with *Platyoposaurus with astonishing support (PP = 98), and both of them with *Australerpeton $(\mathrm{PP}=\mathbf{8 1})$ and *Archegosaurus $(\mathrm{PP}=60)$; Analysis EB also groups * Glanochthon with *Lydekkerina with negligible support $(\mathrm{PP}=31)$; otherwise, * Lydekkerina is the sister-group of all the above. Despite keeping *Nigerpeton and *Saharastega out $(\mathrm{BPE}=14)$, Analysis $\mathrm{B} 2$ groups * Australerpeton and *Archegosaurus $(\mathrm{BPE}=30)$ with the likewise extremely long-snouted *Platyoposaurus $(\mathrm{BPE}=11)$. In the remaining MPTs from R5 and R6, a clade of *Archegosaurus and *Glanochthon (or, perhaps surprisingly, (*Archegosaurus $\left(*\right.$ Cheliderpeton, ${ }^{*}$ Glanochthon $\left.)\right)$ ) as well as a clade of *Konzhukovia and *Lydekkerina $(\mathrm{BPE}=28)$ occur.

* Lydekkerina and *Australerpeton are never found as sister-groups. Most likely we have exhausted the scope of the present character sample, and the stereospondylomorphs are sorted by noise more than by signal. Still, the position of *Sclerocephalus with respect to the others agrees - ignoring the dvinosaurs in Analysis R5 - with Eltink \& Langer (2014), Dilkes (2015a), Pardo, Small \& Huttenlocker (2017) and arguably McHugh (2012).

Cisneros et al. (2015) found Dvinosauria nested within their small sample of

3541

3542

3543

3544

3545

3546

3547

3548

3549 stereospondylomorphs. We do not interpret this result as corroborating our finding of the same in the mentioned trees from R5, because it could easily be due to their small sample of nondvinosaurs in general - or to the fact that Eryops was included in the outgroup so that Eryopiformes was forced to include Dvinosauria a priori. Furthermore, Cisneros et al. (2015) did not order any characters.

\section{Dissorophoidea}


3550 Something of a consensus on dissorophoid phylogeny has been forming. Recent analyses (Ruta,

3551

3552

3553

3554

3555

3556

3557

3558

3559

3560

3561

3562

3563

3564

3565

3566

3567

3568

3569

3570

3571

3572

3573

3574

3575

3576

3577

3578

3579

3580

3581

3582

3583

3584

3585

3586

3587

3588

3589

3590

3591

3592

3593

3594

3595 2009; Maddin et al., 2013b; Holmes, Berman \& Anderson, 2013; Dilkes, 2015a; Pardo, Small \& Huttenlocker, 2017) have generally found Dissorophidae (represented here by Broiliellus brevis alone) and Trematopidae (Acheloma, Phonerpeton, Ecolsonia, *Mordex - the latter here included in a phylogenetic analysis for the first time) as sister-groups (forming Olsoniformes Anderson et al., 2008b), and many have found the traditional "branchiosaur" Micromelerpeton to have no connection to the branchiosaurids (Apateon, Leptorophus, Schoenfelderpeton, *Tungussogyrinus, *Branchiosaurus); sometimes, Micromelerpeton is placed as the sister-group to all other dissorophoids together (Maddin et al., 2013b; Dilkes, 2015a). RC07, however, upheld the more traditional topology where the trematopids lie outside a clade formed by the other dissorophoids while Micromelerpeton forms the sister-group of Branchiosauridae (most recently found by both analyses presented in Pardo, Small \& Huttenlocker, 2017: fig. S6, and by the Bayesian but not the parsimony analysis in their fig. S7). Further, in the dispute over whether Ecolsonia is a trematopid or a dissorophid (e.g. Berman, Reisz \& Eberth, 1985), RC07 found a sort of compromise position in that Ecolsonia came out one node closer to Broiliellus brevis and the remaining dissorophoids than to (Acheloma + Phonerpeton).

This position remains a possibility for Ecolsonia in some trees from Analyses R1-R3 and R5. The other trees, as well as all trees from Analyses R4, R6 and EB, place Ecolsonia as a trematopid ( $\mathrm{BPO}=\mathbf{5 6} ; \mathrm{BPE}=38 ; \mathrm{PP}=\mathbf{9 4})$ like Ruta (2009), Maddin et al. (2013b), Holmes, Berman \& Anderson (2013), Dilkes (2015a) and Pardo, Small \& Huttenlocker (2017), but continue to find a clade of all non-trematopid dissorophoids $(\mathrm{BPO}=\mathbf{5 5} ; \mathrm{BPE}=14 ; \mathrm{PP}=48$ excluding *Branchiosaurus and *Acanthostomatops, see below). Within that clade, Broiliellus can be the sister-group to most or all of the rest (which has the values $\mathrm{BPO}=39, \mathrm{PP}=60$ ) or to Amphibamidae alone (BPE =9); the latter option is the only one in Analyses R4 and R6. Despite our best efforts to eliminate the effects of ontogeny on their scores, Micromelerpeton and Branchiosauridae remain sister-groups throughout the analyses with the original taxon sample $(\mathrm{BPO}=\mathbf{6 1})$. This does not change when taxa are added $(\mathrm{BPE}=27, \mathrm{PP}=55)$, except that *Branchiosaurus, as it turns out, never enters "Branchiosauridae" (see below).

As expected, *Mordex settles at the base of the trematopid clade (R4, R6, some MPTs from R5: (*Mordex (Ecolsonia (Acheloma, Phonerpeton))); BPE = 28) or grade (some MPTs from R5). Analysis EB finds it slightly more rootward, next to *Iberospondylus $(\mathrm{PP}=46)$.

Interestingly, none of our trees find all branchiosaurids to form a clade; such a clade is contradicted by a BPE of 8 and a PP of $60 .{ }^{*}$ Branchiosaurus is found closer to Broiliellus + Amphibamidae (R4, R6, some MPTs from R5) or closer to the root of Dissorophoidea (the other MPTs from R5; BPE $=8 ; \mathrm{PP}=48$ ), yet Micromelerpeton always stays with the bulk of "branchiosaurids" as described above $(\mathrm{BPE}=27 ; \mathrm{PP}=55) . *$ Tungussogyrinus, its somewhat froglike ilium (state ILI 9(1)) and Early Triassic age notwithstanding, nests with *Branchiosaurus in R4, R6 and some MPTs from R5 (BPE = 16), next to the three branchiosaurids from the original sample in other trees from R5, next to them plus Micromelerpeton in yet others from R5 and in EB ( $\mathrm{PP}=54)$, and next to Schoenfelderpeton in the remaining MPTs from R5. We think that the present matrix cannot test whether the branchiosaurids are a clade (Fröbisch \& Schoch, 2009a; Maddin et al., 2013b) or a polyphyletic assemblage of larval and neotenic dissorophoids.

RC07 did not determine if the conventional amphibamids in their sample (Amphibamus, Platyrhinops, Eoscopus, Doleserpeton) formed a clade with respect to Micromelerpeton and the branchiosaurids. The analyses with the original taxon sample (R1-R3) find them as a clade (BPO 
$3596=60$; also containing the salientians in R3 as the sister-group of Doleserpeton). This remains the 3597 case when taxa are added ( $\mathrm{BPE}=25$ and $\mathrm{PP}=43$ for a clade that also contains *Gerobatrachus). 3598 *Micropholis, however, joins this clade only in some MPTs from R5 (as the sister-group of 3599 Eoscopus, or one node rootward of *Gerobatrachus); elsewhere, it is the sister-group of the 3600 "branchiosaur" clade described above. In Analyses R4, Doleserpeton and *Gerobatrachus are 3601 sister-groups (BPE = 40; against a PP of 51); in R5, Doleserpeton and *Gerobatrachus are 3602 equally parsimoniously found as the sister-group of Lissamphibia, with the other one of the two 3603

3604

3605

3606

3607

3608

3609

3610

3611

3612

3613

3614

3615

3616

3617

3618

3619

3620

3621

3622

3623

3624

3625

3626

3627

3628

3629

3630

3631

3632

3633

3634

3635

3636

3637

3638

3639 being the next more distant relative; in R6, Doleserpeton is closer to Batrachia. All this remains to be tested with much larger samples of potential amphibamids and amphibamid-related characters, without reducing the sample of other temnospondyls.

While we are quite skeptical about a close relationship of Micromelerpeton to some or all branchiosaurids (compare with Wiens, Bonett \& Chippindale, 2005), the main alternative in the literature - Micromelerpetidae as the sister-group to all other dissorophoids (e.g. Schoch, 2013; Maddin et al., 2013b; Dilkes, 2015a) - does not seem to be well supported either; it never occurs in our MPTs and contradicts the bootstrap and Bayesian trees as well $(\mathrm{BPO}=\mathbf{8 4} ; \mathrm{BPE}=27$; EB $=60$ ). The name Dissorophoidea should certainly not be defined with Micromelerpeton as one of only two specifiers, as Schoch (2013) has done. That said, the older definition of Dissorophoidea by Yates \& Warren (2000: 86) as all organisms closer to **Dissorophus than to Eryops (**Dissorophus being very closely related to Broiliellus) applies to a clade that contains, in the trees from some of our analyses, not only the traditional dissorophoids but also various other temnospondyls, like Balanerpeton, Dendrerpetidae, Capetus, Dvinosauria and, in some MPTs from R5, even Stereospondylomorpha (Fig. 16).

Schoch (2013) listed dvinosaur-like character states of micromelerpetids and used them to argue for a sister-group relationship of Micromelerpetidae to the rest of Dissorophoidea (accepted without comment by Fröbisch et al., 2015) together with a sister-group relationship (or nearly so) of Dissorophoidea and Dvinosauria. A close relationship of Dvinosauria and Dissorophoidea is never found in our MPTs (contradicted only, however, by BPO $=30, \mathrm{BPE}=6$ and PP =64). We wonder if the similarities listed by Schoch (2013) are instead homoplastic and indicate that the micromelerpetids are a "dissorophoid version of a dvinosaur" - a clade that generally had a dvinosaur-like lifestyle as aquatic predators with more or less anguilliform locomotion but, unlike dvinosaurs, was capable of dispersing over land in the adult stage (at least in the one species, M. credneri, that is known to have had non-neotenic, skeletally mature adults in one population: Lillich \& Schoch, 2007; Schoch, 2009). This might even explain why dvinosaurs and micromelerpetids have not been found in the same sites (see Schoch \& Milner, 2014; Cisneros et al., 2015): maybe micromelerpetids were competitively excluded from bodies of water that dvinosaurs could reach, but were able to diversify in inland water bodies out of the reach of dvinosaurs.

In the matrix of Dilkes (2015a), a revision and extension of that by Schoch (2013), Dvinosauria and Dissorophoidea are held together exclusively or almost exclusively by paedomorphic adaptations to an aquatic lifestyle ("Node D" in Dilkes, 2015a: 81): hypobranchials ossified in adults; lack of ossification of the glenoid facet of the scapula and of the carpals, pubis and tarsals; and weak torsion of the humerus. The remaining character state, jaw joints and occipital condyle(s) being at the same transverse level, may also belong here; it is in fact absent in the more mature known Micromelerpeton specimens (Boy, 1995; Schoch, 2009:

3640 fig. 2a, b; see also our ch. 146 in App. S1). While the sister-group of Dissorophoidea in the tree 3641 of Dilkes (2015a: fig. 13) is the terrestrial Zatracheidae (see below), followed by Dvinosauria on 
3642 the outside, Dissorophoidea would be reconstructed as ancestrally aquatic because

3643 Micromelerpetidae is found as the sister-group to the other dissorophoids, among which

3644 Branchiosauridae is sister to an amphibious/terrestrial clade composed of Amphibamidae,

3645 Dissorophidae and Trematopidae; we consider this position of Branchiosauridae unlikely

3646 because of the evidence for a lack of a clear distinction between amphibamids and

3647 branchiosaurids (Vallin \& Laurin, 2004; Fröbisch \& Schoch, 2009a; Werneburg, 2012a; Maddin

3648 et al., 2013b; and references therein; these results are more or less congruent with the MPTs from

3649

3650

3651

3652

3653

3654

3655

3656

3657

3658

3659

3660

3661

3662

3663

3664

3665

3666

3667

3668

3669

3670

3671 our analyses with added taxa, where such a clear distinction is likewise absent).

\section{Other added temnospondyls}

*Nigerpeton was described as a cochleosaurid (Steyer et al., 2006; Sidor, 2013). This was confirmed by Pawley (2006), Schoch (2013), Dilkes (2015a) and Pardo, Small \& Huttenlocker (2017); McHugh (2012) found it and Edops together to form the sister-group of Cochleosauridae (Fig. 25D). In our analyses (Fig. 26C, D), it is always found next to *Saharastega $(\mathrm{BPE}=\mathbf{5 0}$; PP =99).

* Saharastega was described as a temnospondyl of rather uncertain position (Damiani et al., 2006). In dorsal view, its skull is unusual for a temnospondyl in some ways, which led Yates (2007) to suggest that it was a seymouriamorph. Its palate, however, is unremarkable for a temnospondyl and quite unlike that of any seymouriamorph; we also note that the skull roof is very reminiscent of the temnospondyl **Macrerpeton (Schoch \& Milner, 2014: fig. 38B, E). Pawley (2006; Fig. 25B) found it to be the sister-group of all other cochleosaurids; McHugh (2012; Fig. 25D) recovered it as the sister-group of Zatracheidae, in this matrix represented by *Acanthostomatops (see below); Schoch (2013), followed by Dilkes (2015a), explicitly excluded it as too incomplete.

The *Saharastega $*$ Nigerpeton clade is not found in or close to Cochleosauridae in any MPTs (Fig. 26C, D). R4 places it next to Eryops, next to Stereospondylomorpha or next to all traditional temnospondyls except Edops and Cochleosauridae; R6 adds a position inside Stereospondylomorpha, R5 a further one, where it can be joined by Dvinosauria. Analysis B2, finally, finds it as the sister-group of Edopoidea $(\mathrm{BPE}=4)$, and $\mathrm{EB}$ places it next to the

3672 cochleosaurid Chenoprosopus ( $\mathrm{PP}=33)$, together forming the sister-group $(\mathrm{PP}=25)$ of a novel

3673

3674

3675

3676

3677 Edops-Cochleosaurus clade ( $\mathrm{PP}=47)$. We hope for larger matrices and a restudy of **Macrerpeton more detailed than that by Montanari (2012: chapter II). *Saharastega and *Nigerpeton are both unusually large for Paleozoic temnospondyls, have rather long snouts, and are known almost only from skulls with unusual and not very good preservation; and while

3678

3679

3680

3681

3682 *Nigerpeton shares rare features with the cochleosaurids, a fully stereospondylous intercentrum (state TRU VER 13-14(0)) is catalogued as part of the holotype (D. M., pers. obs.).

*Iberospondylus is better preserved than the above two, but no less enigmatic. In both descriptions (Laurin \& Soler-Gijón, 2001, 2006) it was recovered as the sister-group to a clade that contained Eryops, **Parioxys (on which see Materials and methods: Treatment of OTUs:

3683

3684

3685

3686 Taxa that were not added), **Zatrachys (*Zatracheidae; see below), the included dissorophoids and the stereospondylomorph *Sclerocephalus - a position identical (but for the taxon sample) to that found by Pardo, Small \& Huttenlocker (2017; Fig. 25H). Pawley (2006) found *Iberospondylus as the sister-group of Dissorophoidea (Fig. 25B, together with **Parioxys); McHugh (2012) did not include or mention it; Dilkes (2015a: 69) recovered it as an edopoid or 3687 an eryopid with a reduced taxon sample (Fig. 25E, F). Our analyses all side with Pawley's in 
3688 finding *Iberospondylus (Fig. 26C, D), or in the case of EB an *Iberospondylus-*Mordex clade 3689 (with PP = 46), as the sister-group of Dissorophoidea (BPE = 17; PP =64). *Iberospondylus and 3690 Dissorophoidea share the dorsal process on the caudoventral tip of the quadrate (state QUA

3691

3692

3693 $1(1))$.

3694

3695

3696

3697

3698

3699

3700

3701

3702

3703

3704

3705

3706

3707

3708

3709

3710

3711

3712

3713

3714

3715

3716

3717

3718

3719

3720

3721

3722

3723

3724

3725

3726

3727

3728

3729

3730

3731

3732

3733

* Acanthostomatops represents Zatracheidae, a clade with terrestrial adults long thought to lie close to Eryops and/or Dissorophoidea. Pawley (2006; Fig. 25B) found it next to (*Iberospondylus + Dissorophoidea), McHugh (2012; Fig. 25D) found it nested within Eryopidae together with *Saharastega, Dilkes (2015a: fig. 11; Fig. 25E, F) recovered (Dvinosauria (*Zatracheidae, Dissorophoidea)) in his analyses of reduced taxon samples, Pardo, Small \& Huttenlocker (2017: fig. 2, S7; Fig. 25H) found (Eryopiformes (*Zatracheidae (**Lapillopsis, Dissorophoidea))) based on a closely related matrix. In our Analyses R4 and R6, and some MPTs from R5, unexpectedly, *Acanthostomatops is highly nested within Dissorophoidea, forming part of the sister-group of Broiliellus + Amphibamidae together with a *Branchiosaurus-*Tungussogyrinus clade. In other MPTs from R5, *Acanthostomatops, *Branchiosaurus and optionally *Tungussogyrinus lie one node rootward (instead of crownward) of the "branchiosaur" clade. In yet others, *Acanthostomatops is the sister-group of (Capetus (Balanerpeton, Dendrerpetidae)), together forming the sister-group to (*Palatinerpeton (*Iberospondylus, Dissorophoidea)); yet others have a (Balanerpeton $(*$ Palatinerpeton (Dendrerpetidae, *Acanthostomatops))) clade next to *Iberospondylus + Dissorophoidea. Analysis B2 finds *Acanthostomatops alone as the sister-group of *Iberospondylus + Dissorophoidea (BPE $=11$ ), while EB places it next to all dissorophoids except Trematopidae $(\mathrm{PP}=34)$. While we never find *Acanthostomatops next to Eryops, we occasionally did so in the previous version of our matrix (Marjanović \& Laurin, 2016), even though practically all that has changed in the temnospondyl part of the matrix since then is a series of corrections to *Nigerpeton and *Saharastega. Evidently, the position of *Acanthostomatops is not strongly constrained by our character sample (Fig. 26C, D) despite concerning the least modified of all zatracheids.

* Palatinerpeton is poorly known and has an unexpected combination of features. Unsurprisingly, then, it has never before been included in a large phylogenetic analysis. Boy (1996) presented a detailed phylogenetic hypothesis that put it close to Stereospondylomorpha and to an eryopid-*zatracheid-**Parioxys clade, and less close to Capetus; of all other temnospondyls, however, only Edopoidea was included in the investigation which polarized its characters with reference to non-temnospondyl outgroups. Schoch \& Witzmann (2009a) found it as the sister-group of Eryopiformes, not close to *Acanthostomatops, which instead emerged as the sister-group of Dissorophoidea. Despite the dearth of data, Analyses R4 and R6 as well as some MPTs from R5 place *Palatinerpeton as the sister-group to either Dendrerpetidae (against a BPE of 37 and a PP of 83) or *Iberospondylus + Dissorophoidea (against a BPE of 11 and a PP of 64; Fig. 26C, D); the bootstrap analysis conducted under the same conditions (B2) similarly places * Palatinerpeton as the sister-group to (Capetus (Balanerpeton, Dendrerpetidae)) with a BPE of 5, all together forming the sister-group of (*Acanthostomatops (*Iberospondylus, Dissorophoidea)) with a BPE of 6, an arrangement similar to some MPTs from R5 (Fig. 26C). The remaining MPTs from R5 (also Fig. 26C) find *Palatinerpeton next to Dendrerpetidae + *Acanthostomatops. Analysis EB, in contrast, places it next to all other temnospondyls together ( $\mathrm{PP}=35$; Fig. 20). *Palatinerpeton could become important for the question of temnospondyl phylogeny; a $\mu \mathrm{CT}$ scan could perhaps reveal the unexposed surfaces and fill in much of its missing data. 
3734

3735

3736

3737

3738

3739

3740

3741

3742

3743

3744

3745

3746

3747

3748

3749

3750

3751

3752

3753

3754

3755

3756

3757

3758

3759

3760

3761

3762

3763

3764

3765

3766

3767

3768

3769

3770

3771

3772

3773

3774

3775

3776

3777

3778

3779

*Erpetosaurus was originally thought to be a colosteid, but has long been recognized as an unusual dvinosaur with a few colosteid-like features and was redescribed as such by Milner \& Sequeira (2011). We confirm this as discussed above under Colosteidae $(\mathrm{BPE}=14 ; \mathrm{PP}=77)$ and shown in Fig. 26C, D.

\section{Chroniosuchia}

A clade composed of *Chroniosaurus and $*$ Bystrowiella $(\mathrm{BPE}=42 ; \mathrm{PP}=67)$ has a fully resolved position (Fig. 27G) one node crownward of Gephyrostegus or *Casineria (depending on the latter's position) (BPE $=7 ; \mathrm{PP}=55$ ) and one node rootward of Solenodonsaurus (or, in Analysis EB, of Seymouriamorpha including Solenodonsaurus; $\mathrm{BPE}=6$; $\mathrm{PP}=44$ ). This forms a kind of compromise between the positions found in earlier phylogenetic analyses, while disagreeing with all of them: the closely related analyses of Clack \& Klembara (2009), Clack et al. (2012b) and Witzmann \& Schoch (2017) found the included chroniosuchians in a polytomy with Silvanerpeton, Anthracosauria and a "gephyrostegid"-seymouriamorph-amniote"microsaur" clade (Fig. 27D); Klembara, Clack \& Čerňanský (2010) resolved them as anthracosaurs, agreeing with earlier suggestions (Laurin, 2000, and references therein) that the chroniosuchians were the geologically youngest anthracosaurs; Schoch, Voigt \& Buchwitz (2010) and Buchwitz et al. (2012) found them to be the sister-group of the only included lepospondyl - which Buchwitz et al. (2012) and table 1 of Schoch, Voigt \& Buchwitz (2010) stated to be the poorly known Asaphestera, while the text of Schoch, Voigt \& Buchwitz (2010) mentioned Pantylus instead -; Klembara et al. (2014; Fig. 27E) recovered them as the sistergroup of a gephyrostegid-seymouriamorph clade, though their bootstrap tree has them as the sister-group of Gephyrostegidae alone. All these analyses relied on quite small data matrices; however, unlike us, Schoch, Voigt \& Buchwitz (2010) and Buchwitz et al. (2012) included more than two chroniosuchians in their analyses (the latter's matrix building on the former's).

Our analyses thus place Chroniosuchia as the sister-group to a clade with terrestrial adults, with which they share various adaptations to walking and bearing weight that all more rootward clades, including the "gephyrostegids" and *Casineria, lack (with exceptions nested within the temnospondyls).

Solenodonsaurus

Solenodonsaurus was long thought to lie particularly close to Diadectomorpha + Amniota (Laurin \& Reisz, 1999; Vallin \& Laurin, 2004; Fig. 27A). RC07 found it just outside the smallest clade of diadectomorphs, amniotes and "lepospondyls" (Fig. 27C); we consistently find it just outside the smallest clade formed by the above and all seymouriamorphs (which has BPO $=22$; $\mathrm{BPE}=3$; Fig. 27F, G), except in Analysis EB, where Solenodonsaurus is the sister-group to all other seymouriamorphs $(\mathrm{PP}=51)$.

Danto, Witzmann \& Müller (2012), who redescribed Solenodonsaurus, unexpectedly found it to be a lepospondyl instead; however, their changes to the scores of Solenodonsaurus in the matrix of Ruta, Coates \& Quicke (2003) clearly contradict their own text and/or illustrations in several cases (App. S1).

\section{Seymouriamorpha}


3780 Uniquely, RC07 found Seymouriamorpha to be a grade, with Seymouria and Kotlassia

3781 successively closer to a (Solenodonsaurus (Lepospondyli (Amniota, Diadectomorpha))) clade

3782 than a clade formed by the remaining seymouriamorphs (Fig. 1, 27C). In all trees, Seymouria is

3783 part of a clade with some or all other traditional seymouriamorphs; in Analyses R1 and R3 (Fig.

3784 27F, G), Kotlassia can be the sister-group of that clade or one node rootward of

3785 Seymouriamorpha (this is the only option in R2), and the (Leptoropha + Microphon) clade can

3786 be nested within it (always in R2; Fig. 27F) or lie one node crownward of it. The corresponding

3787 bootstrap tree (B1) shows (Kotlassia + Seymouria), supported by a BPO of 26, as the sister-

3788 group to all other seymouriamorphs (BPO of Seymouriamorpha $=28$ ). The addition of

3789 *Karpinskiosaurus, however, rearranges seymouriamorph phylogeny and prevents Kotlassia,

3790 Leptoropha and Microphon from leaving (Fig. 27G): throughout Analyses R4-R6, the latter two

3791 are the sister-group of all other seymouriamorphs, among which a clade of (Seymouria

3792 (Kotlassia, ${ }^{*}$ Karpinskiosaurus)) is the sister-group to the remainder (Discosauriscidae; BPE $=$

3793 27).

3794

3795

3796

3797

3798

3799

3800

3801

3802

3803

3804

3805

3806

3807

3808

3809

3810

3811

3812

3813

3814

3815

3816

3817

3818

3819

3820

3821

3822

3823

3824

3825

The only seymouriamorph clade with good support is (Leptoropha + Microphon) at a $\mathrm{BPO}$ of 88, a BPE of $\mathbf{8 5}$ and a PP of $\mathbf{1 0 0}$. All other bootstrap percentages, including those for Seymouriamorpha as a whole, are below 40 in both analyses; Analysis EB finds a PP of $\mathbf{9 1}$ for Discosauriscidae and one of $\mathbf{9 9}$ for, as mentioned, Seymouriamorpha excluding Solenodonsaurus, while the other branches stay below a PP of 60 .

Neither the classification of Bulanov (2003), which was not based on a phylogenetic analysis, nor - with the exception of the Seymouria $-{ }^{*}$ Karpinskiosaurus clade found in Analysis EB $(\mathrm{PP}=27)$ - the results of the analysis by Klembara et al. (2014: fig. 8) are congruent with any of our results. From this and the very low support values (though see above for posterior probabilities), we conclude that at least the relationships of Utegenia, Ariekanerpeton and (Leptoropha + Microphon) to each other and to Discosauriscus remain unclear; while the matrix by Klembara et al. (2014) has a denser sampling of seymouriamorphs and of characters that are relevant to their phylogeny, our matrix has a much larger sample of non-seymouriamorphs to root the seymouriamorph tree.

Curiously, Analysis B2 finds Seymouriamorpha inside the amniote-"lepospondyl" clade, as the sister-group to the diadectomorph-amniote clade (Fig. 19); in other words, Seymouriamorpha and the "lepospondyls" switch places compared to all other MPTs and B1. This signal is not, or not only, due to our revision, but was already present in the original matrix, as shown by its occurrence in some MPTs from O3 (Data S7). If corroborated by future studies, this topology would amount to a reversal to the textbook hypothesis of the mid-late $20^{\text {th }}$ century (which was still assumed, for unstated reasons, by Fröbisch \& Schoch, 2009a: fig. 1, and by Berman, 2013). However, the sample of characters where seymouriamorphs and diadectomorphs share the same apomorphic state may already be exhaustive or nearly so in the present matrix, and the bootstrap support for this topology is very low (BPE $=9$; contradicting a BPO of 19 and a PP of 46).

\section{Amniota and Diadectomorpha}

Uniquely, RC07 found the diadectomorphs to form a Hennigian comb with respect to the sauropsids (Fig. 1, 27D): (Tseajaia (Limnoscelis (Orobates (Diadectes (Captorhinus (Paleothyris/Protorothyris, Petrolacosaurus)))))). This was due to numerous misscores, at least some of which were evidently caused by reliance on ancient literature about badly preserved 
3826

3827

3828

3829

3830

3831

3832

3833

3834

3835

3836

3837

3838

3839

3840

3841

3842

3843

3844

3845

3846

3847

3848

3849

3850

3851

3852

3853

3854

3855

3856

3857

3858

3859

3860

3861

3862

3863

3864

3865

3866

3867

3868

3869

3870

3871

specimens. In Analyses R1-R3 we find (Fig. 27F, G) a monophyletic Diadectomorpha (BPO = 59; $\mathrm{BPE}=24 ; \mathrm{PP}=96)$, resolved as (Limnoscelis (Tseajaia (Orobates, Diadectes))) like in the latest published analyses (Reisz, 2007; Kissel, 2010; Berman, 2013; further supported by Berman, Reisz \& Scott, 2010), as the sister-group of Sauropsida; the clade of diadectomorphs except Limnoscelis is found in all of our parsimony analyses and supported in all bootstrap analyses $(\mathrm{BPO}=\mathbf{6 0} ; \mathrm{BPE}=\mathbf{5 2}$; against a $\mathrm{PP}$ of $\mathbf{7 6})$, and the (Diadectes + Orobates) clade enjoys some of the highest values in the entire trees $(\mathrm{BPO}=\mathbf{7 7} ; \mathrm{BPE}=\mathbf{7 3}$; against a $\mathrm{PP}$ of 59).

As mentioned (Material and methods: Treatment of OTUs: Taxa added as new OTUs for a separate set of analyses), the three amniotes included by RC07 are all sauropsids, so RC07 were unable to test whether the diadectomorphs are amniotes. We therefore added the synapsid OTUs *Caseasauria (composite) and *Archaeovenator, which robustly come out as sister-groups $(\mathrm{BPE}=\mathbf{5 6} ; \mathrm{PP}=\mathbf{7 5})$. Surprisingly, however, diadectomorph monophyly breaks down; more precisely, throughout the parsimony analyses with added taxa, Limnoscelis never emerges as a diadectomorph (Fig. 27G). Instead, Analyses R4-R6 find both clades as amniotes, specifically recovering Amniota as (Sauropsida (Diadectomorpha (Limnoscelis (*Caseasauria, *Archaeovenator)))); in terms of the topology found by Analyses R1-R3, *Caseasauria and *Archaeovenator are not placed next to Sauropsida (as they are in Analysis B2: BPE $=30$ ), but next to Limnoscelis.

Under the conditions of Analysis R4, only one additional step is required in Mesquite to restore Amniota and Diadectomorpha as sister-groups - at the cost of rendering Captorhinus a synapsid; moving it back into Sauropsida needs one step more. Analysis EB weakly supports a very different arrangement where Limnoscelis is a diadectid so that Tseajaia is the sister-group to all other diadectomorphs ( $\mathrm{PP}=\mathbf{7 6}$ ), Diadectomorpha is the sister-group of Captorhinus ( $\mathrm{PP}=$ 49), and both together lie on the synapsid side of Amniota ( $\mathrm{PP}=44)$. All this is in stark contrast to the much stronger support for the smallest clade that contains all of the taxa mentioned in this section: $\mathrm{BPO}=\mathbf{6 8}, \mathrm{BPE}=44, \mathrm{PP}=70$. The support for Captorhinus as a sauropsid depends very strongly on the taxon sample $(\mathrm{BPO}=\mathbf{8 0} ; \mathrm{BPE}=26$; contradicted by a $\mathrm{PP}$ of 49$)$, as does that for (Paleothyris + Petrolacosaurus) to a lesser extent: $\mathrm{BPO}=\mathbf{8 4} ; \mathrm{BPE}=\mathbf{5 5} ; \mathrm{PP}=55$.

Many of the characters presented by Berman (2013) as supporting the membership of Diadectomorpha in Amniota are not parsimony-informative given his taxon sample; the others are already included in our matrix. Any satisfactory resolution of amniote origins will require a larger sample of amniotes and possibly hitherto unrecognized characters. Further, several of those features of the description of Tseajaia by Moss (1972) that were not revisited by Berman, Sumida \& Lombard (1992) seem unusual in the light of more recent work on diadectomorphs and may warrant redescription.

\section{Westlothiana}

Despite numerous changes to its scores, Westlothiana stays (Fig. 27) in the position found by RC07 and Pawley (2006): it is the sister-group to all other "lepospondyls". Although bootstrap support is low $(\mathrm{BPO}=26)$ or only exists for a similar topology $(\mathrm{BPE}=10$ for Westlothiana + $*$ Utaherpeton; $\mathrm{BPE}=7$ for both + all other amphibians except the adelospondyls; $\mathrm{BPE}=13$ for Amphibia), there is moderate Bayesian support $(\mathrm{PP}=74)$.

\section{The lepospondyl problem}


3872

3873

3874

3875

3876

3877

3878

3879

3880

3881

3882

3883

3884

3885

3886

3887

3888

3889

3890

3891

3892

3893

3894

3895

3896

3897

3898

3899

3900

3901

3902

3903

3904

3905

3906

3907

3908

3909

3910

3911

3912

3913

3914

3915

3916

3917

3918

3919

3920

[...] the fact that we find throughout the Carboniferous and early [sic] Permian varied series of small, non-labyrinthodont amphibians, which I have classed as lepospondyls in a broad use of that term, presents an evolutionary problem for which we have at present no solution.

- Romer (1964: 158)

Recent research is beginning to shed new light on the anatomy and relationships of rare and problematic forms, such as lepospondyls [...] [four references]. Several issues related to lepospondyl interrelationships are likely to undergo extensive revision in the near future. Published analyses of lepospondyls reveal a disconcerting lack of agreement, to the point that almost any pattern of relationships has been proposed [...] [11 references].

- Ruta, Coates \& Quicke (2003: 290)

And what is a lepo...?

- Gary Johnson, candidate for president of the USA, in a TV interview in 2016

For much of the $20^{\text {th }}$ century, all limbed vertebrates (or nearly so) except Lissamphibia and Amniota were divided into Labyrinthodontia and Lepospondyli. When phylogenetic analysis began to be introduced in the mid-1990s, these two taxa had largely come to be seen as wastebaskets, Labyrinthodontia basically containing the large-bodied taxa and Lepospondyli the small-bodied ones (except small temnospondyls). It was expected that Labyrinthodontia would turn out to be paraphyletic and Lepospondyli to be polyphyletic. The first prediction has held up, if perhaps less radically than expected; the latter has fared much less well, in that analyses like those of Ruta, Coates \& Quicke (2003), Vallin \& Laurin (2004; Fig. 28A) or Maddin, Jenkins \& Anderson (2012; Fig. 28F) turned up a lepospondyl clade lying not close to Temnospondyli or Colosteidae as some had surmised, but right next to Diadectomorpha + Amniota (Fig. 27), reminiscent of the old idea of a close relationship between amniotes and "microsaurs" that Romer had so thoroughly excluded in the mid-20 $0^{\text {th }}$ century. (See, however, Pardo \& Anderson [2016] and Pardo et al. [2017, 2018].)

RC07 found a novel variant supported by previously neglected characters: they upheld the picture described above, except for finding that the adelospondyls (Acherontiscus and Adelogyrinidae) were not lepospondyls or anywhere near, forming instead a clade with the faraway colosteids (Fig. 1, 28D). However, several plesiomorphic scores for the adelospondyls, which kept them away from the remaining "lepospondyls" and were shared with the colosteids, appear indefensible. For example, RC07 scored the adelospondyls as having partially covered lateral-line canals on the skull (our ch. 100: state SC 1(1) for Dolichopareias, SC 1(1 or 2) for Acherontiscus and Adelospondylus; state 1 is "mostly enclosed with short sections in grooves", state 2 is "mostly in grooves with short sections enclosed"), but the grooves (Carroll, 1969a; Andrews \& Carroll, 1991) appear wide and shallow and were most likely genuinely interrupted, with the lateral-line organ perhaps continuing in the skin away from the bone - rather than lying under the bone surface - or perhaps being interrupted itself. This condition, which we have scored as part of the existing state SC 1(3) - lateral-line organ entirely in grooves, not covered by bone - is best seen in *Archegosaurus (Witzmann, 2006) and was evidently also present in Proterogyrinus (Holmes, 1984), which RC07 had also misscored as having state 1 or 2.

Analysis R2 (Fig. 29B) groups the adelospondyls with the aïstopods and urocordylids among the "lepospondyls" that are dragged into Temnospondyli by the constraint on Lissamphibia. All other phylogenetic analyses place the adelospondyls with the urocordylids and aïstopods in highly nested positions within a "lepospondyl"/amphibian clade (PP = 76; Fig. 29A, C). Moving the adelospondyls next to Colosteidae on a tree from Analysis R1 requires only two extra steps; moving them into Colosteidae on a tree from R4 (as the sister-group to 
3921 *Aytonerpeton, together forming the sister-group of Colosteus + Greererpeton) requires no less 3922 than six extra steps. The bootstrap analyses do not strongly support any position; Analysis B1

3923

3924

3925

3926

3927

3928

3929

3930

3931

3932

3933

3934

3935

3936

3937

3938

3939

3940

3941

3942

3943

3944

3945

3946

3947

3948

3949

3950

3951

3952

3953

3954

3955

3956

3957

3958

3959

3960

3961

3962

3963

3964

3965

3966 places the adelospondyls just rootward of Holospondyli (the highest BPO that keeps them away from Holospondyli is 25, the highest that keeps them away from Colosteidae is 35), Analysis B2 finds the adelospondyls next to a clade of all other amphibians (BPE $=13$ rootward, 7 crownward).

In spite of our results, including a node with $\mathrm{PP}=\mathbf{8 6}$ (Caerorhachis and everything more crownward: Fig. 20), we consider it possible that the adelospondyls do not belong together with most or all of the other "lepospondyls", but are rather colosteid-grade animals (as found again in Witzmann \& Schoch, 2017). One reason is the fact that their ceratobranchial bones, not considered in the present matrix, retain longitudinal grooves for gill arteries (Witzmann, 2013). Such grooves indicate internal gills (Schoch \& Witzmann, 2010) and are not found in the only other possibly aquatic "lepospondyl" from which hyobranchial bones are known, the lysorophian Brachydectes (Wellstead, 1991; Witzmann, 2013); there is further no trace of internal gills in extant amphibians - the gills of tadpoles, although soon covered by a lid, are homologous to external gills and thus to the septa between internal gills (Schoch \& Witzmann, 2010, and references therein). In this light, we would like to draw attention to the embolomerous vertebrae of Acherontiscus, where the intercentra are much larger (state TRU VER 13-14(0), our ch. 265) than in any other "lepospondyls" that are known to have intercentra. We furthermore wonder if the single-piece centra of adelogyrinids are pleuro- or intercentra - in the adelogyrinid **Palaeomolgophis, many centra articulate with two successive neural arches (Andrews \& Carroll, 1991), so perhaps the adelogyrinids were stereospondylous. (We have scored them as unknown for this question, as we have done with all other OTUs that have single-piece centra throughout the entire column; for details and discussion, see ch. 259 - TRU VER 7 - in App. S1.)

In any case, the adelospondyls themselves are relatively well supported as a clade $(\mathrm{BPO}=$ 47; $\mathrm{BPE}=40 ; \mathrm{PP}=\mathbf{1 0 0})$, as is Adelogyrinidae to the exclusion of Acherontiscus $(\mathrm{BPO}=\mathbf{7 6}$; $\mathrm{BPE}=\mathbf{7 5}$; PP =95). These results agree with those of RC07 (Fig. 1).

Other than Westlothiana and (exclusively in B2) the adelospondyls and *Utaherpeton, all "lepospondyls" form a clade - with or without some or all modern amphibians, depending on our constraints - in all of our analyses except $\mathrm{R} 2(\mathrm{BPO}=22$; $\mathrm{BPE}=1$; $\mathrm{PP}=73$; Fig. 29B). The relationships within this clade remain unclear. A basic pattern that emerges from our results, however (Fig. 29), is a basal (or, in some MPTs from R2, nearly basal) dichotomy between a clade that contains most "microsaurs" and another that contains Holospondyli. This is shared with RC07 (Fig. 28D) and the Anderson-lab matrices (Marjanović \& Laurin, 2009; Maddin, Jenkins \& Anderson, 2012; Huttenlocker et al., 2013; Pardo, Small \& Huttenlocker, 2017: fig. S6; Fig. 28F); only Analysis EB has Holospondyli deeply nested in a long "microsaur" grade (PP $=40)$. Analyses R1, R3 and sometimes R2 (BPO = 23; BPE = 15) find Microbrachis and Hyloplesion on the "microsaur" side like RC07 (Fig. 28D, 29A, B); the remaining MPTs from R2 find them outside the dichotomy (Fig. 29B); R4-R6 place them on the holospondyl side (Fig. 29C). RC07 found the tuditanids on the "microsaur" side but the pantylids on the holospondyl side; we find both on the holospondyl side (or diplocaulid-scincosaurid side) in R1-R3 (BPO = 2), but, like the Anderson-lab matrices, both on the "microsaur" side in R4-R6 (BPE = 1). RC07 and the Anderson-lab matrices recovered Brachydectes on the holospondyl side but Batropetes on the "microsaur" side; we find both on the holospondyl side (or diplocaulid-scincosaurid side) in $\mathrm{R} 1-\mathrm{R} 3$ and $\mathrm{R} 6(\mathrm{BPO}=25 ; \mathrm{BPE}=6, \mathrm{PP}=\mathbf{7 6})$, but both on the "microsaur" side in R4 and R5. 
3967 This latter topology is reminiscent of the one found by Vallin \& Laurin (2004), where Aïstopoda, 3968 Adelogyrinidae and Nectridea - each a single composite OTU - clustered at the "lepospondyl" 3969 base, from which a long "microsaurian" stem then separated a Lysorophia-Lissamphibia clade 3970 (Fig. 28A).

The alternative to the basal dichotomy, the long "microsaur" grade of Analysis EB, may be considered suspicious: the "microsaurs" are phenetically similar to the amniotes, and remain close to them (except for the brachystelechids), while the other "lepospondyl" groups cluster as many internodes away from Amniota as possible (BPO =19; against a BPE of 7; $\mathrm{PP}=\mathbf{7 6})$.

Even so, the limbless aïstopods and the adelospondyls, for which extremities are unknown and among which Adelogyrinus is well enough preserved that we have scored it as limbless (state HUM 18/DIG 1(0); for discussion see ch. 219 in App. S1 as well as Andrews \& Carroll, 1991: 252), never form an exclusive clade in our analyses (Fig. 29) - even though they did in Germain (2008a: fig. 5.15; simplified in Marjanović \& Laurin, 2013a: fig. 4C).

As in RC07, "nectridean" monophyly is never found; instead (Fig. 29) there is a strong attraction between the urocordylid "nectrideans" and the aïstopods, which form an exclusive clade in all analyses $(\mathrm{BPO}=43 ; \mathrm{BPE}=30 ; \mathrm{PP}=96)$. Except in Analysis R2 (Fig. 29B), however, all "nectrideans", aïstopods, adelospondyls (except in B2) and the "microsaur" *Utaherpeton (except in B2) consistently form either an exclusive clade (Analyses R4 and R5) or a grade towards Brachydectes, brachystelechid "microsaurs" and/or modern amphibians (Analyses R1, R3, R6 and EB; BPO = 25; BPE = 15; PP = 57; Fig. 29A, C). Arguably, R2 is an example of the second pattern, because there, too, Diplocaulidae and Scincosaurus are closer to Brachydectes and the brachystelechid Batropetes than to Urocordylidae (Fig. 29B). In support of the second pattern, five extra steps are needed in Mesquite under the conditions of R1 to create the first pattern, i.e. to make (Scincosaurus + Diplocaulidae) the sister-group of (adelospondyls (Urocordylidae, Aïstopoda)); one more is required to make the latter clade the sister-group of Diplocaulidae alone as in RC07.

Consistently, and unlike in RC07, Diplocaulidae and Scincosaurus are sister-groups $(\mathrm{BPO}=\mathbf{5 6} ; \mathrm{BPE}=\mathbf{6 3} ; \mathrm{PP}=\mathbf{8 6})$. This agrees with other recent analyses (e.g. Ruta, Coates \& Quicke, 2003; Pawley, 2006; Milner \& Ruta, 2009; Pardo, Small \& Huttenlocker, 2017: fig. S6A but not B - Fig. 28B, E). Unlike in all earlier versions of our revised matrix (e.g. Marjanović \& Laurin, 2015, 2016), urocordylid phylogeny is resolved, with the original taxon sample making Ptyonius the sister-group of the other two as in RC07 and Germain (2008a: fig. 5.15), while adding taxa puts Sauropleura in that position; urocordylid monophyly, unsurprisingly, is well supported $(\mathrm{BPO}=\mathbf{5 6} ; \mathrm{BPE}=\mathbf{6 3}$; but contradicting a $\mathrm{PP}$ of 65$)$. Diplocaulidae $(\mathrm{BPO}=\mathbf{5 7} ; \mathrm{BPE}=$ 58; $\mathrm{PP}=\mathbf{1 0 0})$ contains (Diplocaulus + Diploceraspis $)(\mathrm{BPO}=\mathrm{BPE}=\mathrm{PP}=\mathbf{1 0 0})$ and $($ Keraterpeton + Diceratosaurus $)(\mathrm{BPO}=\mathrm{BPE}=43 ; \mathrm{PP}=68)$, the latter clade contradicting RC07 and Germain (2010); Batrachiderpeton is the sister-group of the former clade (BPO = 46; $\mathrm{BPE}=39$; $\mathrm{PP}=94)$. Sampling almost no *stereospondyls, our matrix cannot test the hypothesis by Pardo $(2011,2014)$ and Pardo et al. (2018) that Diplocaulus, Diploceraspis and **Ductilodon - but not the "keraterpetids" - are **brachyopoid temnospondyls; we await full publication of that work (see also Marjanović \& Laurin, 2013a), which will contain a redescription of Diploceraspis and **Ductilodon.

Even though we updated the scores of Lethiscus based on Pardo et al. (2017), who for the first time found the aïstopods as whatcheeriid-grade animals, and even though we added *Coloraderpeton mostly from the same source, we recover Aïstopoda nested as deeply among the "lepospondyls" as in all previous analyses. Attempts to move the aïstopods, the urocordylids, 
4013 *Utaherpeton and the adelospondyls next to the colosteids in Mesquite appear to require ten 4014 additional steps on a tree from Analysis R1 and 22 on one from R4, contradicting a BPO of 35, a 4015 BPE of 20 and a PP of 86; placing Aïstopoda + Urocordylidae next to Ossinodus (crownward of 4016 Whatcheeriidae and optionally of Tulerpeton) while leaving the adelospondyls with the 4017 colosteids takes a total of 25 extra steps in R1 as far as we can determine in Mesquite, against a 4018 BPO of 67 and a PP of 86; moving all these "lepospondyls" next to Ossinodus (one node 4019 crownward of *Densignathus, two of Whatcheeriidae) takes an impressive total of 32 extra steps 4020 in R4, despite contradicting a BPE of only 22. Trees illustrating these topologies are contained in 4021 Data S3.

4022 This could, on the one hand, be due to the fact that our matrix almost completely lacks 4023 4024 4025 4026 4027 4028 4029 4030 4031 4032 4033 4034 4035 4036 4037 braincase characters, which Pardo et al. (2017) sampled more densely than ever before. Most conspicuously, we were unable to score the remarkable persistence of the buccohypophyseal canal in (at least) Lethiscus and * Coloraderpeton, a plesiomorphy not otherwise reported from anywhere crownward of **Tiktaalik. We have, however, scored such features as the exposure of the ventral cranial fissure/suture caudal to the parasphenoid (at least in the midline) in Lethiscus, *Coloraderpeton and apparently *Pseudophlegethontia (Anderson, 2003b: fig. 2A), the lack of a lingual lamina on the angular in all three, the spiracular notches and palatal dentition of the first two, or the mostly or fully enclosed mandibular lateral-line canal and the preopercular bone of *Coloraderpeton (see ch. 142, 161, 69, 103, 104, 107, 108, 110, 118, 101, 81 - PASPHE 9, ANG 2-3, SQU 3, VOM 3, VOM 4, VOM 8, VOM 9, PAL 1, ECT 5, SC 2, PREOPE 1 - in App. S1). Although we suspect that the supposed parasymphysial of Lethiscus and *Coloraderpeton - a very large, massive, toothless bone - is actually a mentomandibular ossification of Meckel's cartilage, we are currently unable to test this idea and have scored the bone as the parasymphysial, which has not otherwise been reported from anywhere crownward of the anthracosaurs (teal in Fig. 24).

Conversely, however, postcranial characters are underrepresented in the matrix by Pardo et al. (2017) even compared to ours. Being deliberately restricted to taxa with good braincase data (Pardo et al., 2017: supplementary information part A), their taxon sample lacks any "lepospondyls" other than Lethiscus, *Coloraderpeton and a few "recumbirostrans" (on which see below).

On the other hand, the quality of our data for Oestocephalus could have misled our analyses. Although Carroll (1998a) expressed strong doubts about "lepospondyl" affinities for aïstopods and a preference for much more rootward placements not unlike the ones eventually found by Pardo et al. (2017), he described - and illustrated in his specimen drawings - several apomorphies of Oestocephalus that are unexpected for such a rootward position, for example a large lingual lamina on the angular. To test these anatomical interpretations, it will be necessary to restudy the specimens. Pardo et al. (2017) did not include Oestocephalus in their matrix; Anderson, Pardo \& Holmes (2018) reported a large, well preserved skull which will add valuable information once fully described.

It will be interesting to see in future analyses what the braincase characters used by Pardo et al. (2017) will do to this area of the tree once they are added to our matrix. In particular, will the urocordylids follow the aïstopods rootward (Pardo et al., 2018), in keeping with such plesiomorphies (not coded in our matrix) as the prearticular denticle row or the huge distal Meckelian fenestra of Sauropleura (Bossy, 1976; Bossy \& Milner, 1998), or will their vertebral 4057 similarities to the other "nectrideans" keep them in a rest-"lepospondyl" clade, or will all 
4058 "nectrideans" come with them in spite of the similarities between the brachystelechid 4059 "microsaurs", the lysorophians, the modern amphibians and Scincosaurus in particular?

$4060 \quad$ Plesiomorphies unexpected for "lepospondyls" are not limited to the head skeleton in 4061 aïstopods. Wellstead (1982: 204, fig. 8A) reported a cololite in Lethiscus, commenting only that 4062 "[i]ts segmented appearance is likely due to a spiral valve in the intestine". The spiral valve is 4063 plesiomorphic for gnathostomes, found today in chondrichthyans and lungfish; in extant 4064 tetrapods, however, it is absent, and cololites suggest its absence in temnospondyls as well (e.g. 4065 Godfrey, 2003: fig. 2). Independently of Pardo et al. (2017), this finding - if correct - implies a 4066 4067 4068 4069 4070 4071

4072

4073

4074

4075

4076

4077

4078

4079

4080

4081

4082

4083

4084

4085

4086

4087

4088

4089

4090

4091

4092

4093

4094

4095

4096

4097

4098

4099

4100

4101

4102 phylogenetic position for Aïstopoda rootward of Temnospondyli.

Aïstopoda is perhaps of particular interest because some of its members were terrestrial beyond reasonable doubt, even though *Coloraderpeton must have been obligatorily aquatic on account of its mandibular lateral-line canal (Pardo et al., 2017): Rößler et al. (2012) reported two indeterminate aïstopods from Chemnitz (eastern Germany), where a forest was covered by volcanic ash around the Artinskian-Sakmarian boundary in the Cisuralian (see also Spindler et al., 2018: 319). The forest floor is preserved in situ; no water was involved in the deposition. This confirms the taphonomic and morphological arguments reviewed by Anderson (2002) and Germain (2008a: chapters II, III; 2008b), to which we add the absence of lateral and ventral keels on the centra, which are found (state TRU VER 15(1)) in all other potential anguilliform swimmers in this matrix. Considering the age of Lethiscus, which lies at the very end of Romer's gap, it is conceivable that the "first step on land" was done "without limbs" (Germain, 2008a: chapter III) - and that Aïstopoda contains a separate origin of a terrestrial lifestyle.

Following our corrections to Lethiscus after Pardo et al. (2017), aïstopod monophyly is well supported $(\mathrm{BPO}=\mathbf{7 0} ; \mathrm{BPE}=\mathbf{6 3} ; \mathrm{PP}=\mathbf{1 0 0})$, yet not found in any trees from Analysis $\mathrm{R} 2$, where the aïstopods form a grade towards the modern amphibians. The phylogeny of Aïstopoda is unchanged from $\mathrm{RC} 07$ given the original taxon sample, but the resolution largely breaks down when taxa are added (Fig. 29).

In addition to finding the aïstopods to be whatcheeriid-grade animals, Pardo et al. (2017) found all other "lepospondyls" they sampled - Brachydectes and a selection of "microsaurs" - to form a clade within Sauropsida. Although this was supported (Pardo et al., 2017: extended data figure $7 \mathrm{~b}$ ) by bootstrap percentages of 71 for Sauropsida including the animals in question ("Recumbirostra"; see below), 95 for Amniota and 96 for Amniota + Limnoscelis (the only sampled diadectomorph), we wonder if this result is an artefact of the taxon and character samples. For instance, the hyobranchial apparatus of Brachydectes or Pantylus would imply several reversals from the ancestral amniote condition or massive homoplasy among all other amniotes; similarly, many of the sampled "microsaurs" retain well-developed dorsal scales (e.g. CG78), but not one traditional amniote does. A much larger matrix will be required to test this question. It is puzzling, too, that Pardo et al. (2017) derived a large part of their matrix from that of Huttenlocker et al. (2013), which is in turn derived from that of Anderson et al. (2008a), but did not implement or mention any of the changes to the latter that were proposed (or repeated from Marjanović \& Laurin, 2009) by Sigurdsen \& Green (2011), even though they cited Sigurdsen \& Green (2011) as the source of a new character.

\section{The interrelationships of the "microsaurs"}

The Tuditanomorpha appears to be a natural assemblage [...]. Among the Microbrachomorpha, the four genera are so distinct from one another that each has been placed in a monotypic family. It is 
4104

4105

4106

4107

4108

4109

4110

4111

4112

4113

4114

4115

4116

4117

4118

4119

4120

4121

4122

4123

4124

4125

4126

4127

4128

4129

4130

4131

4132

4133

4134

4135

4136

4137

4138

4139

4140

4141

4142

4143

4144

4145

4146

4147

4148

4149

4150

4151 possible that this is not a natural assemblage, but these forms share more features in common with each other than any do with other groups of Paleozoic tetrapods.

- CG78: 11-12

\begin{abstract}
Although there is considerable question concerning the nature and degree of interrelationship within the Tuditanomorpha, the included families share a great many features. [...] The remaining microsaurs, in contrast, are a more varied group, and may or may not have a significant common heritage. [...] They are here classified as a natural group, but this may not prove to be the case.
\end{abstract}

- CG78: 113

It is not an overstatement to say that "microsaur" phylogeny is a mess. No two analyses, usually even if based on successive versions of the same matrix, have recovered the same topology or nearly so (Fig. 28). Ours are no exception (Fig. 29).

Microsaur monophyly does not occur in any of our trees. A clade with a membership similar to that of Tuditanomorpha Carroll \& Gaskill, 1978, is not found in any of our analyses either, although Analysis R6 comes close by finding a clade composed of all "tuditanomorphs" and the "microbrachomorph" Odonterpeton. Analyses B1, B2 and EB feature an (Odonterpeton (Microbrachis, Hyloplesion)) clade reminiscent of Microbrachomorpha Carroll \& Gaskill, 1978 $(\mathrm{BPO}=23, \mathrm{BPE}=15$ and $\mathrm{PP}=35$ for the whole), and under the conditions of R1 three extra steps suffice in Mesquite to create such a clade; but even so, this clade (which was also found by RC07) is not close to the sampled brachystelechids (Batropetes, ${ }^{*}$ Carrolla and *Quasicaecilia, the first two observed by D. M.), of which Batropetes was cautiously included in Microbrachomorpha by CG78 (the others were not yet known).

That said, the discrepancies between the reconstructions by CG78, the specimen drawings by CG78, and D. M.'s personal observations of the holotype of Odonterpeton are such that Odonterpeton will have to be redescribed. For example, the supposed suture between the left parietal and the supposed postparietal is a series of unconnected cracks; Odonterpeton consequently has no identifiable postparietal at all (state POSPAR 1-2(2); see ch. 39 in App. S1 for details), and symmetry is restored by an additional curve to the left at the caudal end of the suture between the parietals that is omitted from the drawings by CG78 (presumably because it is not visible in strict dorsal view).

Following the redescription of Microbrachis and Hyloplesion by Olori (2015), these two OTUs are found as sister-groups throughout all of our analyses, as they were by RC07; the support for this clade is unusually high $(\mathrm{BPO}=67 ; \mathrm{BPE}=66 ; \mathrm{PP}=97)$.

Anderson (2007b: 205-206) coined the name Recumbirostra and carefully defined it as applying to "the clade descended from the most recent common ancestor of Pantylus, Cardiocephalus sternbergi, Rhynchonkos, and Micraroter, but not including Tuditanus or Microbrachis". This definition, and therefore this name, cannot be applied to the trees from any of our analyses: while Rhynchonkos, Gymnarthridae (incl. Cardiocephalus) and Ostodolepididae (incl. Micraroter) form an exclusive clade of elongate "microsaurs" in all analyses except R4 (variously joined by *Trihecaton in R6 and EB; BPO for clade $=22, \mathrm{BPE}=26, \mathrm{PP}=64$; Fig. 29), the pantylids (Pantylus and Stegotretus; see below for *Sparodus) are never closer to it than Tuditanus and/or Microbrachis are $(\mathrm{BPO}=12 ; \mathrm{BPE}=1 ; \mathrm{PP}=40)$ - indeed, as described below, Pantylidae and Tuditanidae always fall out as sister-groups (Fig. 29) except in EB.

The original taxon sample contained a single brachystelechid, Batropetes. In Analyses R1 and R3, it is the sister-group of a clade consisting of Brachydectes (the only included lysorophian), the adelospondyls, Urocordylidae, Aïstopoda and all available modern amphibians (Fig. 29A). Analysis B1, however, recovers Batropetes and Brachydectes as sister-groups (BPO 
4152 = 37). These two arrangements cannot be distinguished in R2, where the entire clade that forms 4153 the sister-group of Brachydectes in R1 lies within the temnospondyls. When taxa are added, the 4154 three brachystelechids (Batropetes, ${ }^{*}$ Carrolla,${ }^{*}$ Quasicaecilia) form an exclusive clade (BPE $=$ 4155 37) in R4, R5 and some MPTs from R6 (Fig. 29C). In the remaining MPTs from R6, they form a 4156 grade toward Brachydectes; in EB, they form a grade toward the modern amphibians instead, 4157 with Brachydectes on the outside ( $\mathrm{PP}=\mathbf{8 8})$. Only in R2, R4, R5 and perhaps R6 can the 4158 brachystelechids even be considered part of the "microsaur" grade.

4159

4160

4161

Pawley (2006: 239) reported to have found the "microsaurs" Tuditanus and Asaphestera (both classified in Tuditanidae by CG78) to score identically except for missing data. They differ in our matrix. Indeed, the added tuditanid *Crinodon (see below) has more in common with Tuditanus than Asaphestera does (Fig. 29C), despite being easy to distinguish from both.

Although Asaphestera is found as a tuditanid in all parsimony analyses (see below), Analysis EB finds it one node crownward of Tuditanidae ( $\mathrm{PP}=36)$.

As in RC07, though unlike in the Anderson-lab matrices (Fig. 28F), Hapsidopareion (together with *Llistrofus, see below) is never the sister-group of the supposed (CG78) hapsidopareiid Saxonerpeton (kept apart by BPO =26), except in Analysis B2 (BPE = 10) and $\mathrm{EB}(\mathrm{PP}=35)$, although they are only one internode apart in R1 and R2. In all parsimony analyses, Tuditanidae (Tuditanus, Asaphestera; in R4-R6 also *Crinodon; in R4 and B2 further the *Goreville microsaur) and Pantylidae (Pantylus, Stegotretus; in B2 also *Sparodus) consistently form a clade, though it is less well supported $(\mathrm{BPO}=12 ; \mathrm{BPE}=1$; against a $\mathrm{PP}$ of 40) than either Tuditanidae ( $\mathrm{BPO}=24, \mathrm{BPE}=15 ; \mathrm{PP}=\mathbf{8 9}$ excluding Asaphestera) or Pantylidae $(\mathrm{BPO}=\mathbf{9 2} ; \mathrm{BPE}=36, \mathrm{BPE}=\mathbf{7 7}$ without $*$ Sparodus $; \mathrm{PP}=\mathbf{8 8}, \mathrm{PP}=\mathbf{9 3}$ without $*$ Sparodus $)$ on their own. In EB, Tuditanidae forms the sister-group to all other "lepospondyls" (PP = 36 excluding Asaphestera $)$ except Westlothiana $(\mathrm{PP}=73)$.

4176

4177 Added "microsaurs"

4178

*Carrolla, ${ }^{*}$ Quasicaecilia and ${ }^{*}$ Crinodon have been treated above (see also the next section for the first two).

4180

*Utaherpeton was described as one of the oldest "microsaurs"; the original description

4182 (Carroll, Bybee \& Tidwell, 1991) already noted similarities to the "nectrideans". Our results 4183 (Fig. 29C), much like those of Marjanović \& Laurin (2009; Fig. 28F) but unlike those of Vallin 4184 \& Laurin (2004; Fig. 35A) or Pardo, Small \& Huttenlocker (2017: fig. S6), place *Utaherpeton inside the holospondyl clade, next to the clade composed of the adelospondyls, urocordylids, aïstopods and, in R6, Eocaecilia ( $\mathrm{PP}=69$; against a BPE of 10). We deduce that, rather than being just another "microbrachomorph" "microsaur", *Utaherpeton could occupy a crucial position close to the origin of Holospondyli and deserves future attention. The corresponding bootstrap tree, however, interestingly makes it the sister-group of Westlothiana, a position that agrees vaguely with the original description but has negligible support $(\mathrm{BPE}=10$; against a PP of 76).

The *"Goreville microsaur" (Lombard \& Bolt, 1999) either falls out as a tuditanid next to *Crinodon (R4; PP = 88; Fig. 29C) or nests with *Sparodus (R5, R6). The bootstrap trees place it in Tuditanidae, with very low support $(\mathrm{BPE}=15)$, although Bayesian support is considerable $(\mathrm{PP}=\mathbf{8 9})$.

*Sparodus was considered a gymnarthrid by CG78 and Carroll (1988). In our analyses such a relationship is never found. In R4-R6, *Sparodus forms a clade with Odonterpeton and 
4198 (in R5 and R6) the *Goreville microsaur; the same cautionary notes about Odonterpeton as

4199

4200

4201

4202

4203

4204

4205

4206

4207

4208

4209

4210

4211

4212

4213

4214

4215

4216

4217

4218

4219

4220

4221

4222

4223

4224

4225

4226

4227

4228

4229

4230

4231

4232

4233

4234

4235

4236

4237

4238

4239

4240

4241

4242 above apply. Pantylus and Stegotretus nest next to * Sparodus in Analyses B2 and EB (BPE $=$ 36; $\mathrm{PP}=\mathbf{8 8}$ ). A pantylid position would not be surprising considering the enormous palatine and especially coronoid tusks (Carroll, 1988; D. M., pers. obs. of the same specimen - Fig. 3, 4).

* Trihecaton has hitherto been neglected since CG78 because its skull is almost entirely unknown. The rest of the skeleton including the lower jaw, however, is mostly preserved and articulated. Because the postcranium is so close to complete and lower-jaw characters are well represented in this matrix, D. M. has scored *Trihecaton directly from the specimens (which probably belong to the same individual). Analyses R4 and R5 resolve its position next to (Holospondyli (Microbrachis, Hyloplesion)) or, in R4, one node rootward of Saxonerpeton; R6 places it next to Micraroter within Ostodolepididae or next to Gymnarthridae in different MPTs (Fig. 29C). Analyses B2 and EB place it next to the Gymnarthridae-RhynchonkosOstodolepididae clade $(\mathrm{BPE}=10 ; \mathrm{PP}=64)$. Evidently, further taxa and further postcranial characters should be added to future analyses to resolve the potentially interesting position of this animal.

*Llistrofus is always the sister-group of Hapsidopareion $(\mathrm{BPE}=\mathbf{8 7} ; \mathrm{PP}=\mathbf{1 0 0})$, as expected (Bolt \& Rieppel, 2009).

\section{Lissamphibian origins}

As summarized in Tables 1, 3 and 4, our revision of the matrix of RC07 supports the lepospondyl hypothesis over the temnospondyl hypothesis, which in turn is more parsimonious than the polyphyly hypothesis. Adding OTUs, including *Gerobatrachus, to the matrix increases the difference between the LH and the TH by one step and the difference between the TH and the $\mathrm{PH}$ by two steps, still favoring the LH over its alternatives, contrary to the results of RC07 (Fig. 1), Sigurdsen \& Green (2011), Maddin, Jenkins \& Anderson (2012), Pardo, Small \& Huttenlocker (2017: fig. S6) and Pardo et al. (2017); the LH further has a posterior probability of 92 (Fig. 20).

As discussed above (Materials and methods: Robustness analyses), a reliable statistical test for whether the differences between these trees are distinguishable from random is not available. The null hypothesis (that they are not) cannot currently be rejected, except perhaps for the difference between the $\mathrm{LH}$ and the $\mathrm{PH}$, and that only under the original taxon sample (Table 4).

Monophyly of the modern amphibians is found in all analyses (except of course those constrained against it, see Table 1: R3, R6) and is well supported: $\mathrm{BPO}=6 \mathbf{6 5}$; $\mathrm{BP}=67$; $\mathrm{PP}=$ 100. Note that adding taxa does not decrease the support.

In Analysis R1 (Fig. 10, 11, 29A, 30L), the "lepospondyls" closest to Lissamphibia are a clade of adelospondyls, urocordylids and aïstopods, a novel and unexpected result. Next closest is Brachydectes, the only sampled lysorophian. A sister-group relationship between lissamphibians and lysorophians has long been considered an integral part of the lepospondyl hypothesis (though see Marjanović \& Laurin, 2013a, for discussion) and is consistent with the results of Vallin \& Laurin (2004), Pawley (2006: fig. 91) and Marjanović \& Laurin (2008, 2009: supplementary figure), as shown in Fig. 28 and 30A, C, E. On the outside follows Batropetes, the only brachystelechid "microsaur" in the original taxon sample; this is congruent with the results of Vallin \& Laurin (2004). Three extra steps are needed in Mesquite to render Batropetes and 
4243 Brachydectes each other's sister-group, one more is required to move Batropetes closer to

4244

4245

4246

4247

4248

4249

4250

4251

4252

4253

4254

4255

4256

4257

4258

4259

4260

4261

4262

4263

4264

4265

4266

4267

4268

4269

4270

4271

4272

4273

4274

4275

4276

4277

4278

4279

4280

4281

4282

4283

4284

4285

4286

4287

4288

Lissamphibia than Brachydectes.

However, when taxa are added to the parsimony analysis (R4; Fig. 14, 29C, 30M), Brachydectes is the sister-group of Brachystelechidae, which includes Batropetes $(\mathrm{BPO}=37$; $\mathrm{BPE}=23)$, both together forming the sister-group of Lissamphibia $(\mathrm{BPO}=20 ; \mathrm{BPE}=14)$. Analysis EB uniquely finds the modern amphibians nested inside the brachystelechids, closest to *Quasicaecilia $+*$ Carrolla $(\mathrm{PP}=\mathbf{8 8})$, followed by Batropetes $(\mathrm{PP}=45)$ and then by Brachydectes $(\mathrm{PP}=\mathbf{9 2})$.

The sister-group of the smallest clade formed by all modern amphibians, Brachydectes and the brachystelechids is Scincosaurus + Diplocaulidae in Analyses R1 and EB (PP = 57). R4 instead places Rhynchonkos in this position (contradicting a BPE of 26 and a PP of 76); it has historically played a large role in the PH (Holmes \& Carroll, 1980; Carroll, 2007) and was recovered in the same position by Vallin \& Laurin (2004; Fig. 30A). B1 and B2 place Holospondyli as a whole next to (Lissamphibia (Batropetes, Brachydectes)) with very weak support $(\mathrm{BPO}=25 ; \mathrm{BPE}=6)$.

When the TH is enforced on the original taxon sample through a constraint (R2; Fig. 12, 29B, 30N), the lissamphibians do not nest with Doleserpeton as expected, or even within Dissorophoidea at all. Instead, they nest within the dvinosaurs - and take the aïstopods (which become paraphyletic with respect to Lissamphibia), the urocordylids and the adelospondyls with them. While it seems remarkable that such a surprising novel topology is more parsimonious than any conventional version of the $\mathrm{TH}$, the modest magnitude of this difference - to move all lissamphibians next to Doleserpeton (and restore lepospondyl monophyly) requires three extra steps in Mesquite, as many as to enforce the PH (Analysis R3; Fig. 30L) - and the lack of statistical significance for the difference to Analysis R1 suggest that we should ascribe this result to insufficiencies in the matrix. Indeed, adding taxa (R5; Fig. 15, 30O) "rectifies" the constrained topology by placing Lissamphibia among the amphibamid dissorophoids, far from the dvinosaurs and without turning any "lepo-" into temnospondyls; however, both Doleserpeton and *Gerobatrachus are found as sister-groups of Lissamphibia with equal parsimony, failing to resolve the disagreement between Sigurdsen \& Bolt (2010), who favored Doleserpeton in this role, and Anderson et al. (2008a), Maddin \& Anderson (2012), Maddin, Jenkins \& Anderson (2012 - Fig. 30G), Pardo, Small \& Huttenlocker (2017 - Fig. 30I-K) and Pardo et al. (2017), who found *Gerobatrachus to lie closer to (or even within) Lissamphibia.

Pardo, Small \& Huttenlocker (2017: fig. S6) added **Chinlestegophis and **Rileymillerus to a matrix slightly modified from that of Maddin, Jenkins \& Anderson (2012), which contains almost only dissorophoids and "lepospondyls", and found them as temnospondyls - though not as lissamphibians, despite finding the TH. Satisfied that **Chinlestegophis, **Rileymillerus and the lissamphibians were temnospondyls, Pardo, Small \& Huttenlocker (2017) then added them to a matrix that contained temnospondyls and modern amphibians but no "lepospondyls"; that matrix is based on Schoch (2013), accepting (J. Pardo, pers. comm.; D. M., pers. obs.), though not citing, most of the score changes proposed by Dilkes (2015a) but omitting the characters Dilkes had added and greatly changing the taxon sample. Bayesian analysis and some of the MPTs resulting from this second matrix found the caecilians, **Chinlestegophis and **Rileymillerus as stereospondyls while the batrachians remained dissorophoids (Pardo, Small \& Huttenlocker, 2017: fig. 2C, S7). However, lissamphibian monophyly - within Stereospondyli or Dissorophoidea - is equally parsimonious (Marjanović \& Laurin, 2018; Fig. 30I-K). While **Chinlestegophis and **Rileymillerus are clearly

PeerJ reviewing PDF | (2015:12:8254:6:0:NEW 20 Jul 2018) 
4289 stereospondyls, we therefore - and for a number of anatomical reasons - remain uncertain for the

4290

4291

4292

4293

4294

4295

4296

4297

4298

4299

4300

4301

4302

4303

4304

4305

4306

4307

4308

4309

4310

4311

4312

4313

4314

4315

4316

4317

4318

4319

4320

4321

4322

4323

4324

4325

4326

4327

4328

4329

4330

4331

4332

4333

time being whether the caecilians are closely related to them. However, it is clear that a very large matrix - sampling stereospondyls, "lepospondyls" and many characters - will be necessary to adequately test this question.

Such a matrix will also need to be carefully scrutinized for accuracy before analysis because accidental misscores are, as we show here, common and because small changes routinely have disproportionate and unpredictable impacts on the results. In this context, we note that even though the matrices of Maddin \& Anderson (2012), Maddin, Jenkins \& Anderson (2012), Huttenlocker et al. (2013) and Pardo, Small \& Huttenlocker (2017: fig. S6) are largely derived from that of Anderson et al. (2008a), and that of Pardo et al. (2017) is by half, none of these publications addressed or mentioned the changes to that matrix proposed by Marjanović \& Laurin (2009) and Sigurdsen \& Green (2011), despite their impact on the results in the latter publications. Indeed, the matrix and character list of Pardo, Small \& Huttenlocker (2017: fig. S6) remain altogether unpublished.

Other than new braincase characters and one character from Maddin \& Anderson (2012), the matrix of Pardo et al. (2017) was composed from those of Clack et al. (2012a) and Huttenlocker et al. (2013). It is thus not much better equipped to test lissamphibian origins than the latter.

The albanerpetid ** Shirerpeton was published too recently for us to score (Matsumoto \& Evans, 2018). It reinforces our impression that Albanerpetidae should not be omitted from phylogenetic analyses of lissamphibian origins (as done by Pardo, Small \& Huttenlocker, 2017: fig. 2, S7), but instead has potentially crucial new information to offer. For example, a suproccipital bone had never been documented in a modern amphibian, its presence was scored as unknown for Albanerpetidae in RC07, and we have kept this score (despite the suggestive median caudodorsal thickening in the fused braincase of Albanerpeton - Maddin et al., 2013); ** Shirerpeton unambiguously has a suproccipital, which, as Matsumoto \& Evans (2018) noted, strongly resembles that of Brachydectes in its shape and spatial relationships. The suproccipital, or at least its exposure on the skull surface, is absent in all temnospondyls that are well enough known, including Doleserpeton; among "microsaurs", it is absent in Gymnarthridae and not exposed in Stegotretus, but present and exposed elsewhere, including in Batropetes.

\section{Lissamphibian phylogeny}

All salientians form a clade $(\mathrm{BPO}=\mathbf{9 0} ; \mathrm{BPE}=\mathbf{8 7} ; \mathrm{PP}=99)$ in all analyses. The salientian *Liaobatrachus is consistently found as the sister-group of Notobatrachus + Vieraella $(\mathrm{BPE}=$ 82; $\mathrm{PP}=100)$. A clade formed by the latter two $(\mathrm{BPO}=96)$ to the exclusion of more crownward salientians (like *Liaobatrachus: $\mathrm{BPE}=\mathbf{6 8}$; PP = 58) has not been found elsewhere (e.g. Dong et al., 2013); this may be due to the character sample, though perhaps the taxon sample with its many but distant outgroups also plays a role.

Contrary to RC07, or to Pardo, Small \& Huttenlocker (2017: fig. 2C, S6A, S7; Fig. 30J, $\mathrm{K})$, the two caudates Karaurus and Valdotriton form a clade in all MPTs derived from the original taxon sample $(\mathrm{BPO}=\mathbf{7 4})$. Seemingly bizarre things happen when taxa are added, including the further caudates *Beiyanerpeton, *Pangerpeton and *Chelotriton, but this is not surprising: because only two caudates were originally included, the matrix does not contain any characters that are intended to resolve caudate phylogeny. 
4334

4335

4336

4337

4338

4339

4340

4341

4342

4343

4344

4345

4346

4347

4348

4349

4350

4351

4352

4353

4354

4355

4356

4357

4358

4359

4360

4361

4362

4363

4364

4365

4366

4367

4368

4369

4370

4371

4372

4373

4374

4375

4376

4377

4378

4379

The extremely peramorphic salamandrid *Chelotriton (Marjanović \& Witzmann, 2015) is the most salientian- and temnospondyl-like caudate, falling out as the sister-group of Salientia in Analyses R4, R6 and EB (BPE = 38; PP =67; Fig. 30M, P), and as the sister-group of a clade that contains all other non-salientian modern amphibians in Analysis R5. Evidently, its peramorphosis pulls it out of Caudata given the present character sample, and indeed toward the base of Lissamphibia or Batrachia in constrained trees where the closest relatives of Lissamphibia or Batrachia are temnospondyls; but this is not enough to pull the modern amphibians into the temnospondyls, or to support the Procera hypothesis (on which see below) unless the TH is enforced (Fig. 30O). We conclude that ontogeny does not as severely "discombobulate" phylogeny (Wiens, Bonett \& Chippindale, 2005: title) in this case as one could have expected.

The more moderately peramorphic *Pangerpeton is always found within a caudate clade

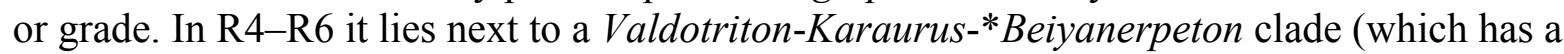
BPE of 21 and, in R5, is either the sister-group of Albanerpetidae + Eocaecilia or contains it as the sister-group of Valdotriton); Analysis EB places it next to *Beiyanerpeton + Karaurus ( $\mathrm{PP}=$ 57).

* Beiyanerpeton, on the other hand, combines paedomorphosis with unexpected plesiomorphies. Possibly due to this balance, it is neither attracted to nor repelled from the caudate root: in R4-R6, it is always found as the sister-group of Karaurus (BPE = 46; $\mathrm{PP}=46$ ), which is normally considered a stem-caudate, but found in various nested positions in the caudate clade or grade in our analyses (a result we ascribe to the character sample).

In trees that support the LH (all unconstrained searches: Analyses R1, R4, B1, B2, EB; Fig. 30L, M), the Batrachia hypothesis is consistently recovered: Salientia and all caudates form a clade to the exclusion of Albanerpetidae and Eocaecilia $(\mathrm{BPO}=\mathbf{7 7} ; \mathrm{BPE}=\mathbf{5 4} ; \mathrm{PP}=\mathbf{8 1})$. This agrees with all recent molecular analyses (e.g. Pyron, 2014: supplementary file amph_shl.tre; Irisarri et al., 2017), with Pardo, Small \& Huttenlocker (2017: fig. 2, S7, but not S6A) and, apart from Albanerpetidae, with Maddin, Jenkins \& Anderson (2012). Given the original taxon sample, Salientia nests as the sister-group of Caudata (R1); otherwise, it forms the sister-group of the unusually peramorphic and therefore frog-like caudate *Chelotriton (see above) followed by a clade containing all other caudates. The sister-group of Batrachia is Albanerpetidae (R1, R4 and $\mathrm{EB} ; \mathrm{BPO}=\mathbf{5 3} ; \mathrm{PP}=55)$ or Eocaecilia $(\mathrm{BPE}=44)$.

In Analysis R5 (Fig. 15, 30O), which is constrained against the LH and supports the TH, Salientia forms the sister-group to all other modern amphibians (Procera); in other words, it lies as close as possible to the amphibamids, while Albanerpetidae and Eocaecilia (both not constrained) nest within the caudate grade. Procera was likewise found by Vallin \& Laurin (2004), Pardo, Small \& Huttenlocker (2017: fig. S6A, but not S7) and Pardo et al. (2017: extended data fig. 7). In R2 (Fig. 30N), in which various holospondyls are the closest relatives of lissamphibians and all of the above are nested among temnospondyls, Eocaecilia always lies outside Batrachia, while Albanerpetidae is the sister-group to Lissamphibia as a whole; moving Albanerpetidae back into Lepospondyli (as the sister-group of Brachydectes) cannot be done in Mesquite without adding at least eight steps.

The constraint for the PH specified only the backbone ((Salientia, Doleserpeton) (Brachydectes, Eocaecilia)); the positions of the caudates and Albanerpetidae were left open. Both groups fall out as lepospondyls (next to Eocaecilia) in the original taxon sample (R3; Fig. 30L). This version of the $\mathrm{PH}$, where Salientia alone belongs to the temnospondyls while Caudata and Gymnophionomorpha are lepospondyls, has not been proposed since Carroll \& Holmes 
4380 (1980). To move Caudata into Temnospondyli (as the sister-group of Salientia) requires two

4381

4382

4383

4384

4385

4386

4387

4388

4389

4390

4391

4392

4393

4394

4395

4396

4397

4398

4399

4400

4401

4402

4403

4404

4405

4406

4407

4408

4409

4410

4411

4412

4413

4414

4415

4416

4417

4418

4419

4420

4421

4422

4423

4424

4425

4426

4427

extra steps in Mesquite; to move Caudata + Albanerpetidae into Temnospondyli costs one more step and comes at the cost of nesting this clade within Salientia (next to Notobatrachus + Vieraella). However, the caudates including albanerpetids become temnospondyls when taxa*Chelotriton? - are added (R6; Fig. 30P).

In summary, Salientia is attracted to Amphibamidae and to Caudata (especially *Chelotriton), and possibly pushed away by "lepospondyls"; Albanerpetidae is drawn towards brachystelechids + lysorophians and Eocaecilia as well as towards Caudata, and repelled by Amphibamidae.

Several of the attractions of Albanerpetidae will likely be reinforced by ** Shirerpeton, which was published too recently for us to score (Matsumoto \& Evans, 2018). For example, ** Shirerpeton is the only modern amphibian known to possess a suproccipital, arguing for a position outside Lissamphibia; the postfrontal is absent as in batrachians but not caecilians; the supratemporal or tabular, retained in Eocaecilia but no other known lissamphibians so far, appears to be present; at the same time, the squamosal and the pterygoid of **Shirerpeton form a tube filled by the rod-like quadrate, a feature shared specifically with Caudata.

\title{
Characters
}

\section{Continued problems with the character sample}

Taxon and character sets are now large enough to be mined for large-scale evolutionary trends [...]. - Coates, Ruta \& Friedman, 2008: 572

\begin{abstract}
As the number of analysed characters increases, the accuracy and resolution of all methods also increase $[\ldots]$. These results suggest that no matter which method is applied to a dataset, it should be a goal for morphological datasets to include as many characters as possible if the most accurate estimates of topology are to be obtained.
\end{abstract}

- O’Reilly et al., 2017: 116

Our mergers of redundant characters are the largest factor in the decrease of the number of parsimony-informative characters from 333 to 277 (re-split to 280 for Analysis EB). This is less than three times the number of taxa (which is 102 in the original sample, 158 in the expanded sample). Many of the characters now have multiple states, so the total number of apomorphic character states has decreased much less than the number of characters suggests; still, the low number of characters (compared with the number of taxa) explains why the present matrix does not provide a fully resolved, robust large-scale phylogeny of the limbed vertebrates. That the number is indeed low for our taxon samples is confirmed by the low bootstrap values and by the short internal branches found in Analysis EB (Fig. 18, 19, 21). It has become common for phylogenetic analyses of a similarly large or smaller sample of extinct amniotes to have far larger numbers of characters (Godefroit et al., 2013: 101 OTUs, 992 characters [all binary]; Lee et al., 2014: 120 OTUs, 1251 characters [all binary]; Tschopp, Mateus \& Benson, 2015: 81 OTUs, 477 characters; Ezcurra, 2016: 96 OTUs, 600 characters; Simões et al., 2016b: 193 OTUs, 610 characters; Shelley, Williamson \& Brusatte, 2016: 169 OTUs, 693 characters; Baron, Norman \& Barrett, 2017: 74 OTUs, 404 parsimony-informative characters [Mortimer, 2017]; Buscalioni, 2017: 97 OTUs, 425 characters; Cau et al., 2017, "first dataset": 199 OTUs, 1314 parsimony-informative characters [all binary; pers. obs.]; Cau, 2018a: 132 OTUs, 1431 parsimony-informative characters [all binary]!). Indeed, the matrices of McGowan (2002), Vallin 
4428 \& Laurin (2004), RC07, and Anderson et al. (2008a) each contain characters that the three others

4429

4430

4431

4432

4433

4434

4435

4436

4437

4438

4439

4440

4441

4442

4443

4444

4445

4446

4447

4448

4449

4450

4451

4452

4453

4454

4455

4456

4457

4458

4459

4460

4461

4462

4463

4464

4465

4466

4467

4468

4469

4470

4471

4472

4473

lack, and a cursory look at those of Pardo, Small \& Huttenlocker (2017 - 76 OTUs, 322

parsimony-informative characters [pers. obs.]) and Pardo et al. (2017 - 58 OTUs, 340

parsimony-informative characters) reveals that even they lack characters present in ours; the supermatrix by Sigurdsen \& Green (2011), compiled from those of Vallin \& Laurin (2004), RC07 and Anderson et al. (2008a), has 335 characters that are parsimony-informative even though the taxon sample is restricted to 25 OTUs. Clearly, then, the present character sample could easily be greatly expanded. Therefore, we strongly doubt the first statement quoted above.

Unfortunately, the character sample is not only small, it is not random. We think that postcranial characters in particular are underused in the present matrix. This is implied by its craniocentricity - 190 of the 277 characters, a bit more than two thirds, describe the skull, lower jaw or teeth. Further, the endochondral braincase (Maddin, Jenkins \& Anderson, 2012;

Szostakiwskyj, Pardo \& Anderson, 2015; Pardo, Szostakiwskyj \& Anderson, 2015; Ascarrunz et al., 2016; Pardo \& Anderson, 2016; Pardo et al., 2017, 2018; Anderson, Pardo \& Holmes, 2018) is represented by only three characters (which all concern the occiput), and the hyobranchial skeleton (Witzmann, 2013; Anderson, Pardo \& Holmes, 2018) - even the much-discussed stapes (e.g. Lombard \& Bolt, 1979, 1988; Bolt \& Lombard, 1985, 1992; Laurin, 1998a; Schoch, 2002; Clack, 2003; Robinson, Ahlberg \& Koentges, 2005; Witzmann, 2006; Sigurdsen, 2008) - has not been considered at all. The analyses of Pawley (2006), who added a net total of 53 postcranial characters to the 95 of the matrix of Ruta, Coates \& Quicke (2003 - the preceding version of RC07) and found different results, support our suspicion that the present matrix and the resulting trees are affected by accidental sampling bias. For an overlapping taxon sample, Ruta (2011) found 157 characters from the appendicular skeleton alone, where the present matrix has 53.

Being the largest one available of its kind and having been scrutinized for problems of coding and scoring, our matrix is currently the best - or least bad - starting point for comparisons and discussions as carried out below. Future analyses of the phylogeny of early limbed vertebrates will, however, certainly need much larger character samples to reach reliable conclusions.

\section{Surprising reversals}

It is not surprising that homoplasy is omnipresent (as shown by the tree indices) in a matrix with 102 or 158 OTUs that span 380 million years. Some reversals, though, are unexpected even within this context. In this section we present bones that are unambiguously optimized (by parsimony in Mesquite on the MPTs from Analyses R1-R6; Mesquite presents uncertainty as such rather than restricting itself to ACCTRAN or DELTRAN) as having been lost and then reappeared. Some of these have been discussed before (Sigurdsen \& Green, 2011, and references therein), mostly in the context of lissamphibian origins. We do not, at present, take any position on whether such reversals should be considered particularly implausible (see Wiens, 2011; Botelho et al., 2014; Diaz \& Trainor, 2015; Ossa-Fuentes, Mpodozis \& Vargas, 2015).

RC07 did not specify any state changes as irreversible (or weighted them in other ways), and their trees (Fig. 1) already require some of the reappearances of lost bones that we find in our parsimony analyses. We have preferred not to make changes to the matrix or the analysis procedures that are not clearly necessary; because the development genetics of most (if not all) of the characters in this matrix are insufficiently well understood, and because supernumerary skull bones - some of which may or may not be atavistic reappearances - are known in several

PeerJ reviewing PDF | (2015:12:8254:6:0:NEW 20 Jul 2018) 
4474 taxa with sufficiently large sample sizes (e.g. Greererpeton: Smithson, 1982; Micromelerpeton 4475 and other "branchiosaurs": Boy, 1972; Discosauriscus: Klembara, 1993; Klembara, Tomášik \& 4476 Kathe, 2002), we have not coded any state changes as impossible.

4477

4478

One of the apomorphies which support the sister-group relationship of Urocordylidae and

4479 Aïstopoda, and thus "nectridean" paraphyly, in Analyses R1 and R3-R6 is the reappearance of the supratemporal bone in the skull roof (ch. 32: transition from state PAR 2/POSFRO 3/INTEMP 1/SUTEMP 1(3) to state (2), plotted in Fig. 29; for details on all characters see App. $\mathrm{S} 1)$; this bone is lost in all other "lepospondyls" except the most rootward one, Westlothiana (and lost again in the aïstopod Phlegethontia, along with several other skull roof bones). Note that this reversal is inherited from RC07 (Fig. 1, 28D). Yet more surprising is the fact that the supratemporal is long and unusually narrow in urocordylids, aïstopods (except Lethiscus), and probably Westlothiana (ch. 51: state SUTEMP 3(1), otherwise found only in Orobates and *Archaeovenator); this character state adds support to the hypothesis that the supratemporal of urocordylids and aïstopods is indeed a supratemporal rather than a neomorph.

In some trees from R5, the intertemporal, too, reappears up to twice (transitions to state PAR 2/POSFRO 3/INTEMP 1/SUTEMP 1(0); Fig. 26D). This happens when Capetus, Balanerpeton and Dendrerpetidae are nested close to Dissorophoidea; in some of those trees, * Nigerpeton and *Saharastega (and optionally Dvinosauria) are highly nested among the stereospondylomorphs, necessitating a second reappearance.

The lost parasymphysial bone in the lower jaw, if correctly identified, reappeared once in the aïstopods Lethiscus and *Coloraderpeton (ch. 147: state PSYM 1(0); compare Fig. 24).

The postsplenial bone of the lower jaw reappears (ch. 157: transitions to state POSPL $1(0)$ ) up to three times in the original taxon sample, and up to twice in the expanded sample. The loss in Diadectomorpha + Amniota is reportedly reversed in Petrolacosaurus (Reisz, 1981; see discussion in App. S1), concerning all MPTs from R1-R6. In Analyses R1-R3 and R6, where the diplocaulids are close enough to Batropetes and Brachydectes which lack postsplenials, the presence of the postsplenial in Diplocaulus (Douthitt, 1917) - contrasting with absence in the diplocaulids Diploceraspis and Batrachiderpeton (as correctly scored by RC07: Beerbower, 1963; Bossy \& Milner, 1998) - is unambiguously optimized as a reversal as well. The third reappearance occurs in the adelogyrinids in R1 and R3.

The anterior tectal is optimized as disappearing early and then reappearing in the colosteid * Aytonerpeton (see ch. 6-TEC 1). This happens not only in the MPTs from Analyses R4-R6, where Colosteidae and Baphetidae are sister-groups within Temnospondyli, but also when we move Colosteidae in these trees to the more traditional place it has in Analyses R1-R3, because the anterior tectal is scored as absent in Pederpes and Crassigyrinus. The homology of this bone is not quite clear, however, and most snout tips in this part of the tree are suboptimally preserved; see ch. 6 and 7 in App. S1 for discussion.

The preopercular bone reappears (ch. 81: state PREOPE 1(0)) in the aïstopod * Coloraderpeton after a very long absence. We suspect that this is an artefact of our character sample and that Aïstopoda belongs far rootward of where we find it (Pardo et al., 2017); further research on other aïstopods, notably **Ophiderpeton, may be helpful. The same holds for the return of the mandibular lateral-line canal and its fully or mostly enclosed condition in *Coloraderpeton; with all other aïstopods currently scored as lacking this canal altogether, the transition from state 4 to state 1 or 0 of the ordered ch. 101 (SC 2) counts as (at least) three 4518 autapomorphies. 
4519

4520

4521

4522

4523

4524

4525

4526

4527

4528

4529

4530

4531

4532

4533

4534

4535

4536

4537

4538

4539

4540

4541

4542

4543

4544

4545

4546

4547

4548

4549

4550

4551

4552

4553

4554

4555

4556

4557

4558

4559

4560

4561

4562

4563

The suture between the basisphenoid and the basioccipital ("ventral cranial suture") is usually covered ventrally by the parasphenoid in limbed vertebrates crownward of Crassigyrinus. The parasphenoid is, at least in the midline, too short to cover it (ch. 142: state PASPHE 9(1)) in the aïstopods *Coloraderpeton and probably *Pseudophlegethontia, and Lethiscus even has an exposed open fissure there if the scan presented by Pardo et al. (2017) has successfully distinguished spongy bone from rock (which may not be the case; J. Pardo, pers. comm.). (We have kept state 2, the covered condition, for Oestocephalus; this has more recently turned out to be wrong [Anderson, Pardo \& Holmes, 2018]. In Phlegethontia, the braincase is fused up to such an extent that the caudal margin of the parasphenoid cannot be recognized, so we have kept its score unknown.) While this is an unexpected reversal in our trees, it also occurs in the diadectomorph Tseajaia and some individuals of Diadectes, including apparently all of $D$. absitus (Moss, 1972; Berman, Sumida \& Martens, 1998); and in the dissorophoid temnospondyl Acheloma the lateral parts of the suture are covered very late in ontogeny (Maddin, Reisz \& Anderson, 2010).

Unlike in the earlier version of this matrix discussed by Marjanović \& Laurin (2013a), losses of the posterior (or rather distal) coronoid (ch. 175: POST COR 1(1)) are no longer necessarily reversed on any of our MPTs except those of R6, where the bone reappears in * Coloraderpeton. The precise homology of the bone that bears the lingual toothrow in the caecilian lower jaw and fuses to the dentary in early ontogeny remains, as far as we know, to be investigated; in the absence of evidence for separate bones, we have scored Eocaecilia as lacking any coronoids despite the presence of the lingual toothrow on the so-called pseudodentary (a bone of compound ontogenetic origin in extant caecilians). We have scored Diplocaulus as unknown; the dentary is unusually broad in dorsal view and bears a short lingual toothrow around the symphysis (Douthitt, 1917: fig. 2.5), and the area is damaged in all specimens that Douthitt (1917: 17) had at his disposal, so we cannot exclude that part of the supposed dentary belongs to a coronoid (or indeed several) and the suture is simply too eroded to be visible.

Sigurdsen \& Green (2011) noted that their reevaluation of the matrix by Vallin \& Laurin (2004) continued to support the LH, but required the return of the lost postparietal (ch. 39 in our matrix: POSPAR 1-2(0)), postfrontal (ch. 45: POSFRO 1(0)) and tabular (ch. 53: TAB 1/SQU 4(0); see also below) in Eocaecilia. Our unconstrained Analyses R1 and R4 do not require any of these reversals, and neither do R3 and R6, which are constrained for the PH (Fig. 30L, M, P). Conversely, the same three reversals are required on all MPTs from R2 and R5 (both constrained against the LH, supporting the TH; Fig. 30N, O). In other words, the losses of these three bones actually favor the $\mathrm{LH}$ and the $\mathrm{PH}$ over the $\mathrm{TH}$.

It remains to be seen, however, if the possible tabular of Eocaecilia - here scored as such (as explained by Marjanović \& Laurin, 2008) - really is a tabular or instead a supratemporal. According to Jenkins, Walsh \& Carroll (2007), it is not known if that bone reaches the caudal margin of the skull table (as expected of a tabular), although it seems to D. M. (pers. obs. of the holotype, MNA V8066) that it does; see ch. 53 (TAB 1/SQU 4) in App. S1 for details. Pardo, Small \& Huttenlocker (2017) misinterpreted the reconstruction in Jenkins, Walsh \& Carroll (2007: fig. 1) as indicating that the shape and size of this bone were known, and argued for considering it a supratemporal based on this erroneous premise. Whether the similarity of the unusually large squamosal of Eocaecilia to the squamosal and the unusually large tabular of **Chinlestegophis combined (Pardo, Small \& Huttenlocker, 2017: fig. 3; not mentioned in the text) is indicative of homology remains to be tested. 
4564

4565

4566

4567

4568

4569

4570

4571

4572

4573

4574

4575

4576

4577

4578

4579

4580

4581

4582

4583

4584

4585

4586

4587

4588

4589

4590

4591

4592

4593

4594

4595

4596

4597

4598

4599

4600

4601

4602

4603

4604

4605

4606

4607

4608

4609
Analysis R5 always nests Eocaecilia and Albanerpetidae within Caudata (Fig. 30O). Albanerpetidae was not included in the matrix of Vallin \& Laurin (2004). The jugal is present (ch. 71: JUG 1(0)) in Eocaecilia and Albanerpetidae, but lost in Batrachia; thus, Analysis R5 requires that an exclusive common ancestor of Eocaecilia and the albanerpetids has regained the lost jugal (which yields a total of three steps for this character over the entire tree). This reversal does not occur in any trees from R1 or R4 (Fig. 30L, M); Brachydectes has lost the jugal, but is never found as the sister-group of the modern amphibians (unlike in Vallin \& Laurin, 2004 - Fig. $30 \mathrm{~A}$ ), so its loss is always optimized as a separate event from the loss at the root of Batrachia (for a total of two steps). Thus, the presence of the jugal in Eocaecilia and Albanerpetidae indirectly but unambiguously supports the $\mathrm{LH}$ over the $\mathrm{TH}$, quite contrary to the conclusions of Sigurdsen \& Green (2011). (Similarly, R6 nests Albanerpetidae within Caudata, requiring the same reversal for the $\mathrm{PH}$.)

Of the other characters mentioned by Sigurdsen \& Green (2011) in this context, the losses of the lacrimal (ch. 18: LAC 1(1); Eocaecilia ${ }^{*}$ Chelotriton + some or all salientians - unknown in Triadobatrachus -, Diploceraspis, Phlegethontia), the prefrontal (ch. 12: PREFRO 1(1); limited to salientians crownward of Triadobatrachus, which retains the prefrontal: Ascarrunz et al., 2016) and the quadratojugal (ch. 77: QUAJUG 1(1); Valdotriton, *Beiyanerpeton, part of Notobatrachus, Batropetes, Brachydectes - unknown in *Carrolla and *Quasicaecilia) do not unambiguously reverse in any of our trees regardless of constraints (Fig. 30L-P).

The palatine is scored as present (ch. 114: PAL 8(0)) in only two batrachians in this matrix: the salientian Triadobatrachus and the caudate *Beiyanerpeton. No reversal is required in any MPTs from Analyses R1-R3. In R4 and R6, these occurrences of state 0 are optimized as two separate reversals; in R5, only that of *Beiyanerpeton remains an unambiguous reversal, to which some MPTs from R5 (supporting the TH) add the occurrence in Eocaecilia as a further one. Judging from comparisons to the literature, all this appearance of reversals seems to be clearly due to our small sample of salientians, caudates and caudate-related characters.

An angular (ch. 160: ANG 1(0)) has been reported in the caudates Karaurus and *Chelotriton (see App. S1), but in no other lissamphibians in our sample (see, however, Jia \& Gao, 2016a, b, for further occurrences in caudates). The former's condition is consistently optimized as a reversal; so is the latter's in Analysis R4 (Fig. 30M). The same cautionary notes about our taxon and character sample apply, particularly because *Chelotriton is a crown-group salamandrid (Marjanović \& Witzmann, 2015, and references therein); moreover, the ventral view of Karaurus has not been adequately described or illustrated and is not visible on the cast we had access to.

Only three "microsaurs" are scored for the presence (ch. 167: ANT COR 1(0)) or absence (state 1) of the anterior (mesial) coronoid: presence has been described in Microbrachis, absence in Pantylus and Batropetes. An unambiguous reversal does not occur among "microsaurs" according to the analyses of the original taxon sample (but does happen in Lethiscus); the condition of Microbrachis is optimized as a reversal in R4-R6, where Microbrachis and Hyloplesion are placed next to Holospondyli rather than among other "microsaurs". As far as the crushed material allows, the lower jaw of Microbrachis should be reinvestigated.

The "nectrideans" Urocordylus (Bossy \& Milner, 1998, and references therein) and Diceratosaurus (Jaekel, 1903; Bossy, 1976; Angela Milner, pers. comm. 2009; D. M., pers. obs. of MB.Am.776, CM 25468, CM 34617, CM 81504 and CM 81508; contra Bossy \& Milner, 1998: 99) have five fingers per hand (state 2 of ch. $276=$ DIG 1-2-3-4), even though many other "lepospondyls", including other "nectrideans" like Sauropleura and Keraterpeton, are known to 
4610 have four or three (states 3 and 4). For Diceratosaurus at least, this is not a case of malformation 4611 during limb regeneration as sometimes found in the temnospondyl Micromelerpeton (Fröbisch, 4612 Bickelmann \& Witzmann, 2014): all five fingers, and indeed metacarpals, have distinct lengths 4613 and widths, with the longest and thickest in the middle (Jaekel, 1903; D. M., pers. obs. of 4614 MB.Am.776, the same specimen). Furthermore, Urocordylus and Diceratosaurus are not close 4615 relatives; Urocordylus is a urocordylid like Sauropleura $(\mathrm{BPO}=\mathbf{8 3}$; $\mathrm{BPE}=\mathbf{8 1}$; PP for Urocordylidae incl. Aïstopoda $=\mathbf{9 6}$ ), while Diceratosaurus is a diplocaulid like Keraterpeton $(\mathrm{BPO}=\mathbf{5 7} ; \mathrm{BPE}=\mathbf{6 3} ; \mathrm{PP}=\mathbf{1 0 0})$.

Thus, according to our unconstrained results, the supratemporal reappeared once after

4620 having been lost, and pentadactyly even did so twice; the parasymphysial and the anterior

4621

4622

4623

4624

4625

4626

4627

4628

4629

4630

4631

4632

4633

4634

4635

4636

4637

4638

4639

4640

4641

4642

4643

4644

4645

4646

4647

4648

4649

4650

4651

4652

4653

4654

4655 coronoid may have done so once or not at all, the intertemporal (in the expanded taxon sample) twice, once or never. Perhaps unexpectedly, the shortest trees that are constrained against the LH (and consequently support the TH: Analysis R2) additionally require the postparietal, the postfrontal and the tabular of Eocaecilia to be interpreted as reversals; expanding the taxon sample (Analysis R5) further extends this list to include the jugal found in Eocaecilia and Albanerpetidae. Conversely, given the expanded taxon sample, the lost palatine and the lost angular each returned twice according to our unconstrained results (R4), and once or twice according to constrained analyses (R5), but issues with our taxon and character samples are evident.

\section{Other recently discussed characters that are included in this matrix}

The character that describes whether the maxilla and the quadratojugal touch at the ventrolateral margin of the skull (ch. 72: state JUG 2-6(0)) or the jugal intervenes between them (states 1 and 2 ) is only applicable when all three of these bones are present, a fact neglected by Sigurdsen \& Green (2011). This character does indeed reverse from state 1 to state 0 in Eocaecilia in the MPTs from Analysis R4 (and R6), while it does not reverse in those from Analysis R5, which support the TH; however, it also reverses seven additional times elsewhere in the MPTs from Analyses R4 and R6, compared to six in the MPTs from Analysis R5, out of a total of 24 steps in each of the three analyses. We see no reason to ascribe great subjective significance to this character, which is also known to have changed states within Amniota several times; indeed, the occurrence of state 0 in *Caseasauria is unambiguously optimized as a reversal in all of our trees. The holotype of * Lydekkerina even has state 0 on the left and state 1 on the right side (Hewison, 2007).

Sigurdsen \& Green (2011) divided the length/width ratio of the vomer into two states. In our matrix this character, 102 (VOM 1-13), has three states (because RC07 rendered it as two characters). The widest vomers (state 0) occur in *Ymeria, Ventastega, Colosteus, *Spathicephalus, the "core amphibamids" (Eoscopus, Platyrhinops, Amphibamus, Doleserpeton, *Gerobatrachus; homologous among them), the seymouriamorph Ariekanerpeton, and all lissamphibians (unknown in Albanerpetidae and Triadobatrachus) except Eocaecilia and *Pangerpeton. Under the LH (Analysis R4; Fig. 30M), the occurrence of the intermediate state (1) in Eocaecilia is plesiomorphic, and that in *Pangerpeton is equally parsimoniously optimized as plesiomorphic or a reversal. Under the TH (Analysis R5; Fig. 30O), state 0 is homologous among amphibamids and lissamphibians, and state 1 in Eocaecilia and * Pangerpeton requires either two reversals or (equally parsimoniously) a shared reversal followed by a return to state 1. Under the PH (Analysis R6; Fig. 30P), state 1 in *Pangerpeton is 
4656 unambiguously a reversal from state 0, while state 1 in Eocaecilia is not. Altogether, this ordered

4657

4658

4659

4660

4661

4662

4663

4664

4665

4666

4667

4668

4669

4670

4671

4672

4673

4674

4675

4676

4677

4678

4679

4680

4681

4682

4683

4684

4685

4686

4687

4688

4689

4690

4691

4692

4693

4694

4695

4696

4697

4698

4699

4700

4701

three-state character requires 21 steps in Analysis R4 and 20 in R5 and R6. This character is, in other words, rather inconclusive.

The length and curvature of the ribs was given special attention by Sigurdsen \& Green (2011; and references therein), in that they connected short, straight ribs with buccal pumping as the mode of lung ventilation seen in extant amphibians, and long, curved ones with aspiration by active expansion of the ribcage as seen, plesiomorphically, in amniotes. We find much to disagree with here. First, long straight ribs exist (e.g. Diplocaulus), as do short curved ribs (e.g. Acherontiscus); consequently, $\mathrm{RC} 07$ treated these features as two characters, which remain in our matrix as ch. 246 (RIB 3) - "Ribs mostly straight (0) or ventrally curved in at least part of the trunk (1)" - and 250 (RIB 7) - "Trunk ribs longer (0) or shorter (1) than three successive articulated vertebrae in adults". Second, short ribs (straight or not) may require buccal pumping, but long curved ribs do not require aspiration breathing and do not even automatically make it possible, as the trivial cases of the long but entirely immobile ribs of **turtles and such **pterosaurs as **Pteranodon demonstrate; as long as the necessary research on the mobility of the ribs and their muscle attachment sites has not been done, we see no reason to think that such animals as anthracosaurs or lysorophians breathed air in a way much different from **extant caecilians, which use buccal pumping and inhale several times for each time they exhale, thus compensating for the fact that their heads are much smaller than their lungs (Carrier \& Wake, 1995). Third, such research may be difficult in taxa without ossified rib heads; that includes even such terrestrial animals as the chroniosuchian **Bystrowiella (Witzmann \& Schoch, 2017). The hypothesis that buccal pumping is not a lissamphibian or temnospondyl autapomorphy, but rather an ancient plesiomorphy (in agreement with Lebedev \& Coates, 1995, and Witzmann, 2015), is further supported by the fact that it forms the last stage of air inhalation in the extant actinopterygian **Polypterus (Graham et al., 2014). - In any case, states RIB 3(0) and RIB 7(1), both found in all lissamphibians in this matrix except *Chelotriton, support the TH and the $\mathrm{PH}$ over the LH by one step each in the trees with the expanded taxon sample (Fig. 30M, O, P); the $\mathrm{PH}$ ranks with the TH here because these states are optimized as synapomorphies of Eocaecilia and Aïstopoda under the PH.

It may prove interesting that the ribs of **Chinlestegophis, briefly mentioned but not illustrated by Pardo, Small \& Huttenlocker (2017), are curved (state RIB 3(1); Fig. 30I-K) and considerably longer than those of any lissamphibians except certain salamandrids like *Chelotriton, though it is not clear whether state RIB 7(0) was reached (J. Pardo, pers. comm.).

\section{Preaxial polarity in limb development}

Although not included in our matrix, this character has featured prominently in several recent publications, so we take the opportunity to review the latest developments here.

In frogs and amniotes today, the digits form in a roughly postaxial-to-preaxial (caudocranial) sequence: IV first, then III and V, then II, then I. In salamanders with free-living larvae, the opposite is observed: first I and II, then III, then IV, then (in the foot) V. Fröbisch, Carroll \& Schoch (2007) documented the latter (as an ossification sequence) in the dissorophoid temnospondyl Apateon and proposed that this could be a synapomorphy of (at least some) temnospondyls and salamanders. Marjanović \& Laurin (2013a) discussed this, finding the optimization ambiguous due to the lack of an outgroup, and pointed out that distal carpal/tarsal I ossified first in the few temnospondyls, but also anthracosaurs and colosteids, of which 
4702 incompletely ossified carpi/tarsi are known; distal carpal/tarsal 4 is known to ossify first only in 4703 amniotes and diadectomorphs. Recently, Glienke (2015: fig. 6O-S) has documented that distal 4704 tarsal $1 \mathrm{and} /$ or 2 ossified first in the "lepospondyl" Batropetes. This bolsters the inference that 4705 the resemblance of Apateon and salamanders is symplesiomorphic, and that the amniote/frog 4706 pattern of development (see Marjanović \& Laurin, 2013a, for further discussion) is an

4707

4708

4709

4710

4711

4712

4713

4714

4715

4716

4717

4718

4719

4720

4721

4722

4723

4724

4725

4726

4727

4728

4729

4730

4731

4732

4733

4734

4735

4736

4737

4738

4739

4740

4741

4742

4743

4744

4745

4746

4747 autapomorphy of Amniota + Diadectomorpha as well as of Salientia or part thereof.

Olori (2015) found a long delay in the ossification of the fifth toe in the "lepospondyls" Microbrachis and Hyloplesion; because this is shared with Sauropsida, Olori (2015) suggested that these "microsaurs" may have shared the frog/amniote pattern (repeated without further discussion by Fröbisch et al., 2015). We do not find this convincing, especially in the light of Batropetes.

At this opportunity we would like to report that distal tarsal 2 appears to be the only ossified tarsal in NHMW 1983/32/11, a large articulated specimen of Sauropleura scalaris (Fig. 31,32 ). At least for the time being, our analyses continue to find Sauropleura as a "lepospondyl". Further, we wonder if a poorly mineralized round object proximal to metacarpal I in * Casineria is distal carpal 1; other carpals are not mineralized (Fig. 6; Paton, Smithson \& Clack, 1999: fig. 2, 3).

Jia \& Gao (2016b) pointed out that the ossified parts of radius and tibia are considerably longer and wider than those of ulna and fibula, respectively, in the smallest known larva of the Early Cretaceous urodele **Nuominerpeton. They considered this fact to be evidence of a preaxial-to-postaxial sequence in development. If this inference holds, preaxial polarity is very clearly plesiomorphic: state RAD 2(0), radius longer than ulna (see ch. 222 in App. S1), is found in our matrix in Eusthenopteron, Panderichthys, Acanthostega, Crassigyrinus (whose endochondral skeleton is generally poorly ossified) and the skeletally immature temnospondyl *Tungussogyrinus.

\section{Other characters that are potentially important for the origin of lissamphibians but not} considered in this matrix

Several matrices for the analysis of the phylogeny of the limbed vertebrates have coded phalangeal formulae as one or more characters. It appears that the first four toes plesiomorphically have 2-3-4-5- in the pentadactyl clade, as retained by most amniotes and most "lepospondyls", while reductions in the number of phalanges (2-2-3-4- or fewer) occur in temnospondyls and modern amphibians. However, intermediate states are found in Albanerpetidae, where Celtedens - the only representative for which feet are known - has lost only one phalanx (2-3-4-4-; McGowan, 2002), and in Batropetes, which has lost two (2-3-4-3-; Glienke, 2015: fig. 6O-S). We have not looked into this further, but so far it seems that phalangeal formulae do not support the TH or the PH over the LH.

Sigurdsen \& Bolt (2009) have pointed out that lissamphibians share a particularly large radial condyle on the humerus with dissorophid and amphibamid temnospondyls, and Sigurdsen \& Green (2011) used this as a character in their supermatrix. Marjanović \& Laurin (2013a) have discussed complications. D. M. has since seen two humeri of Diadectes (AMNH 4380 and AMNH 4709); their radial condyles are proportionally at least as large as those of Doleserpeton or **Dissorophus (Sigurdsen \& Bolt, 2009: fig. 3).

Sigurdsen (2008) drew renewed attention to the fact that the perilymphatic duct in the inner ear runs on different sides of the lagena in lissamphibians and amniotes, and presented 
4748 evidence that it was positioned in Doleserpeton as in frogs in particular. The conclusion that this

4749

4750

4751

4752

4753

4754

4755

4756

4757

4758

4759

4760

4761

4762

4763

4764

4765

4766

4767

4768

4769

4770

4771

4772

4773

4774

4775

4776

4777

4778

4779

4780

4781

4782

4783

4784

4785

4786

4787

4788

4789

4790

4791

4792

4793

resemblance must be synapomorphic rather than symplesiomorphic rests on the assumption that the amphibian and the amniote conditions must have evolved independently from the lack of a perilymphatic duct as seen in ${ }^{* *}$ Latimeria. We are not convinced by this latter inference; it is not clear to us why the duct could not have twisted around the lagena at some point, or conversely if the condition is homologous among all amniote clades.

Marjanović \& Laurin (2013b) discussed the branchial denticles and their occasional confusion with gill rakers (which most recently occurs in Jia \& Gao, 2016a; for an animal that has branchial denticles and mineralized gill rakers, see **Amia - Grande \& Bemis, 1998), but did not comment on an interesting fact: Plesiomorphically, there is one row of denticle-bearing dermal bone plates on each of the four ceratobranchials (per side). In branchiosaurids, the branchial denticles were apparently used for filter-feeding (the way gill rakers often are), so that each of the three gill slits (per side) was framed by two rows of denticle-bearing plates ceratobranchials 1 and 4 bearing one each, ceratobranchials 2 and 3 bearing two each, for a total of six. Of the four lissamphibians (all of them currently considered crown-group salamanders) known to retain these plates, *Beiyanerpeton has four rows (Gao \& Shubin, 2012), and apparently so does **Qinglongtriton (not quite clear from the illustrations or the text of Jia \& Gao, 2016a) - but **Chunerpeton (Gao \& Shubin, 2003) and **Seminobatrachus (Skutschas \& Gubin, 2012) have six. Homoplasy must be assumed under both the TH and the LH (and even under the $\mathrm{PH}$ ).

Alone among modern amphibians, most anurans have a tympanic ear, and apparently so did at least Notobatrachus among stem-salientians (Báez \& Nicoli, 2004). Osteological correlates of this character complex have often been thought to occur in various temnospondyls, including but not limited to Doleserpeton and **Chinlestegophis. We mostly disagree with these identifications, which we will discuss at length in a future work; an incomplete draft is included in the first preprint version of this paper (Marjanović \& Laurin, 2015).

\section{Conclusions}

\section{Matrix accuracy}

Morphological data matrices for phylogenetic analyses routinely contain problematic scores and questionably constructed characters (e.g. Maddison, 1993; Strong \& Lipscomb, 1999; O'Keefe \& Wagner, 2001; Wiens, 2001; Vallin \& Laurin, 2004; Wiens, Bonett \& Chippindale, 2005; Pawley, 2006; Marjanović \& Laurin, 2008, 2009; Brazeau, 2011; Sigurdsen \& Green, 2011; Bardin, Rouget \& Cecca, 2014; Dilkes, 2015a; Watanabe, 2015; Simões et al., 2016a; Mortimer, 2017; Langer et al., 2017; Spindler et al., 2018: online resource 3). The matrix by RC07 is no exception. In nine years of work, we have found it necessary (App. S1) to merge many redundant characters (the main cause for decreasing the total number of parsimony-informative characters from 333 to 277 without loss of information), redefine nonadditive binary characters (of which one state was described while the other state was scored for all other conditions in RC07), order several potentially continuous characters (Slowinski, 1993; Wiens, 2001; Grand et al., 2013; Rineau et al., 2015; Baron, Norman \& Barrett, 2017: supplementary information: 4-9) and make 4200 changes to individual scores (documented and justified in App. S1, shown in App. S2 except for parsimony-uninformative characters, counted in Data S4), which includes splitting each of two composite characters in two and adding states to a few other characters. 
4794

4795

4796

4797

4798

4799

4800

4801

4802

4803

4804

4805

4806

4807

4808

4809

4810

4811

4812

4813

4814

4815

4816

4817

4818

4819

4820

4821

4822

4823

4824

4825

4826

4827

4828

4829

4830

4831

4832

4833

4834

4835

4836

4837

4838

4839

Neither the original matrix nor our changes show evidence of bias (e.g. for or against a hypothesis on lissamphibian origins: Tables $6-12 ; p>0.1$ ). Rather, apart from the changes necessitated by works published since 2007, most of the 2471 scores of RC07 that disagree with the literature or our observations of specimens without our having redefined a state are best explained as typographic or similar accidental, unsystematic errors.

\section{Phylogeny}

The dataset by RC07 is the largest one that has so far been used in an analysis of the phylogeny of early limbed vertebrates, a problem field that contains the controversial question of the origin(s) of the extant amphibian clades. From analyses of our revised version of this dataset (App. S2, Data S3), we conclude:

- After our attempts to improve the quality of coding and scoring, the matrix supports different results from those found by RC07, most notably the "lepospondyl hypothesis" on the origin of Lissamphibia (LH) rather than the "temnospondyl hypothesis" (TH). This does not change when the taxon sample is expanded or when Bayesian inference is used instead of parsimony; the expanded taxon sample includes the lissamphibian-like dissorophoids Gerobatrachus, Micropholis and Tungussogyrinus as well as the extremely peramorphic and therefore temnospondyl-like salamander Chelotriton (Marjanović \& Witzmann, 2015).

- Many parts of the tree are too sparsely sampled in characters to be strongly supported. The bootstrap values along the "trunk" of the tree are low; while the LH and the "polyphyly hypothesis" may entail statistically distinguishable topologies unless taxa are added, the TH is indistinguishable from both (Table 4); and while some Bayesian posterior probabilities are high (notably 1.00 for lissamphibian monophyly and 0.92 for the LH), moderately high values are also found for more debatable groupings (in particular, Aïstopoda, Adelogyrinidae and Acherontiscus are supported as rather close to Lissamphibia by a posterior probability of 0.76). Future analyses with a taxon sample of similar or larger size will need to have much larger character samples.

It follows that the "temnospondyl hypothesis" should not be taken for granted as the default by studies in evolutionary biology.

Similar conclusions hold for less popular questions which nonetheless have sometimes important implications for evolutionary biology:

The chroniosuchians (represented by Chroniosaurus and Bystrowiella in the expanded taxon sample) are neither anthracosaurs (Laurin, 2000; Klembara, Clack \& Čerňanský, 2010) nor lepospondyls (Buchwitz et al., 2012) nor seymouriamorphs (Klembara et al., 2014) in our trees; instead, they lie between the more rootward gephyrostegids, which may be followed rootward by the temnospondyls, and the more crownward Solenodonsaurus, which is followed crownward (not rootward as in RC07) by Seymouriamorpha (or may even be a seymouriamorph, as weakly supported by our Bayesian analysis). Character conflict causes either the aquatic anthracosaurs or the ecologically diverse temnospondyls to lie more rootward depending on the taxon sample, the analysis method, and even constraints on the positions of extant amphibians. Caerorhachis lies in the same region of the tree; it may or may not be a temnospondyl as found by Pawley (2006). Rather than Casineria being close to amniote origins, we cannot so far distinguish it from Caerorhachis (as previously noted by Pawley, 2006); we find it in the gephyrostegid grade, rootward of the chroniosuchians. This is corroborated by three (possibly four) previously overlooked features of Casineria that would be unexpected for a close relative of Amniota. 
4840 Westlothiana retains its position from RC07: it is the sister-group to all other amphibians. 4841 Iberospondylus emerges particularly close to Dissorophoidea; Nigerpeton and Saharastega are 4842 well supported as sister-groups, and recovered around Eryops or around or even in 4843 Stereospondylomorpha in the most parsimonious trees, but with weak bootstrap support for a 4844 position next to Edopoidea and weak Bayesian support for a position in it; temnospondyl phylogeny otherwise remains largely mysterious and very strongly depends on the relative positions of temnospondyls and anthracosaurs, though a sister-group relationship of Eryops (the only sampled eryopid) and Stereospondylomorpha is likely. "Nectridean" monophyly is never found. "Microsaurian" monophyly is sometimes approximated, but the brachystelechids usually cannot even be considered part of a "microsaur" grade; neither can Utaherpeton. Despite the

4850

4851 variety of results, Tuditanomorpha, Microbrachomorpha and Recumbirostra are not found;

4852

4853 Microbrachis and Hyloplesion are sister-groups, however. Perittodus emerges rootward of Ichthyostega; Densignathus lies just crownward or just rootward of Whatcheeriidae, followed crownward by Tulerpeton, all of which are rootward of Crassigyrinus and Colosteidae;

4854

4855 Sigournea, Doragnathus and Diploradus are found as baphetoids close to Spathicephalus, apparently baphetids; the "St. Louis tetrapod" and Aytonerpeton are unambiguous colosteids; the 4856 4857 4858 "Parrsboro jaw" may be close to temnospondyl origins and/or to Caerorhachis.

Within the LH, the closest relatives of lissamphibians including albanerpetids are not the lysorophians alone (represented by Brachydectes), but most parsimoniously a clade composed of the latter and the brachystelechid "microsaurs" (Batropetes, Carrolla and Quasicaecilia); the Bayesian analysis even nests Lissamphibia within Brachystelechidae with rather strong support (posterior probability $=0.88$ ). Within the $\mathrm{TH}$, our constrained analyses find Doleserpeton and Gerobatrachus to be equally good (or bad) candidates for the lissamphibian sister-group.

4863

4864

4865

4866

4867

4868

4869

4870

4871

4872

4873

4874

\section{Phylogenetics}

In spite of generally low support, several poorly known taxa are placed unambiguously in our trees. Isolated lower jaws and the like, not to mention headless skeletons like Casineria or the "microsaur" Trihecaton, should not be excluded from phylogenetic analyses a priori; they are by no means guaranteed to add more noise than signal, let alone noise that conflicts with the signal. Wildcard behavior is hard to predict and does not, in our dataset, correlate with amounts or proportions of missing data.

Not all settings for phylogenetic analyses make it likely that all, or any, of the most parsimonious trees will be found. Maddin, Jenkins \& Anderson (2012) recovered neither any of the MPTs that are consistent with their matrix, nor the vast majority of the trees that have the length of the shortest trees they reported (Marjanović \& Laurin, 2018); similarly, at least one of the analyses performed by Skutschas \& Gubin (2012) and Schoch (2013) failed to find any trees of the shortest possible length (reported but not commented on by Skutschas \& Gubin, 2012, and Schoch, 2013, respectively), and Baron, Norman \& Barrett (2017) found only 93 of 16,632

4880 MPTs (Watanabe, 2017a, b; Langer et al., 2017). Care should be taken to run enough additionsequence replicates to find all optimal islands, and to allow sufficient time for the branchswapping algorithms to explore these islands thoroughly. However, the "parsimony ratchet" procedure of RC07 found all MPTs that were compatible with their matrix - even though it did not find any MPTs, or any trees less than 35 steps longer than the MPTs, when applied to the much smaller matrix of Skutschas \& Gubin (2012, as reported there). 
4885

4886

4887

4888

4889

4890

4891

4892

4893

4894

4895

4896

4897

4898

4899

4900

4901

4902

4903

4904

4905

4906

4907

4908

4909

4910

4911

4912

4913

4914

4915

4916

4917

4918

4919

4920

4921

4922

4923

4924

4925

4926

4927

4928

4929

4930

We encourage testing of the behavior of Bayesian inference with large paleontological matrices (missing data clustered by body part; non-coeval tips), which is currently not well understood. Homoplasy-free characters are uncommon enough in our matrix that a prerequisite for parsimony with implied weights is most likely not fulfilled.

Further progress on the questions of phylogeny discussed above - and on many others will require the description of new fossils and the redescription of known ones, but also the mergers of existing matrices to increase the character sample (and to a lesser degree the taxon sample) together with a special effort to keep data matrices free from typographic errors and similar accidental misscores, from the effects of heterochrony and from redundant characters.

In the meantime, we hope to have laid a solid base for future additions of data by creating a "reasonably clean" dataset that does not contradict current knowledge. We have documented all of our changes to the matrix of RC07 in App. S1 and S2 and invite further scrutiny. A matrix of morphological data is a matrix of hypotheses and must not be taken for granted as a set of measured facts; and it must not be assumed that any cell in a matrix could not influence the resulting trees.

\section{Acknowledgments}

This work began as chapter V of the doctoral thesis of Damien Germain supervised by M. L. (Germain, 2008a). D. Germain did not have time to complete this large task, so additional work came to be done as chapter 5 of the doctoral thesis of D. M., again supervised by M. L. (Marjanović, 2010; parts were presented in Marjanović \& Laurin, 2013a). This, too, remained incomplete for lack of time. Further work was later done by D. M.; this is presented here for the first time. We thank Damien Germain for sharing his data and expertise with us; he did not feel that he had contributed enough to this study to coauthor it.

We warmly thank José Grau (Museum für Naturkunde, Berlin) for coming up with, and implementing, ways to run the bootstrap and Bayesian analyses in parallel on a computer he has access to. Previously, they took a week each, even though we had restricted the bootstrap analyses to very few heuristic replicates per bootstrap replicate and extremely few rearrangements per heuristic replicate, restrictions that were no longer necessary this time.

Valentin Rineau (CR2P, Paris) kindly calculated the ITRI (Table 5); hardware and software issues prevented us from doing that ourselves.

We are indebted to Ronan Allain and Bernard Battail for prolonged access to casts of Triadobatrachus and Karaurus at the MNHN (as well as to the original of Triadobatrachus for shorter periods). D. M. further thanks Jasons Anderson and Pardo, Andrew and Angela Milner, Marcello Ruta, Rainer Schoch, Trond Sigurdsen, Florian Witzmann, David Černý, Arjan Mann, Ralf Werneburg, John Nyakatura and Sabine Glienke for discussions, Andrew Milner in particular for discussion of the postcranium of Isodectes and David Černý for discussion of the current state of Bayesian inference in phylogenetics (in comments to Mortimer, 2017); Florian Witzmann, Oliver Hampe and the librarian Annegret Henkel (now retired) for access to dead-tree literature; Ursula Göhlich, Mathias Harzhauser and Alexander Lukeneder for access to specimens of Microbrachis, Hyloplesion, Sauropleura (Fig. 31, 32), Sparodus (Fig. 3, 4) and others at the NHMW, Ursula Göhlich for permission to publish photos (Fig. 3, 4, 31, 32), Amy Henrici and Matt Lamanna for access to many specimens of Chenoprosopus, Isodectes, Diceratosaurus, Ptyonius, Tseajaia and others at the CM (twice), Michael Brett-Surman and Hans-Dieter Sues for access to specimens of Chenoprosopus, Isodectes, Eryops, Tuditanus, 
4931 Odonterpeton and others at the USNM, J. Chris Sagebiel and Timothy Rowe for access to

4932

4933

4934

4935

4936

4937

4938

4939

4940

4941

4942

4943

4944

4945

4946

4947

4948

4949

4950

4951

4952

4953

4954

4955

4956

4957

4958

4959

4960

4961

4962

4963

4964

4965

4966

4967

4968

4969

4970

4971

4972

4973

4974

4975

4976

specimens of Acanthostega, Ichthyostega, Greererpeton, Gephyrostegus, Eryops, Seymouria, Diadectes, Paleothyris, Carrolla and others at the BEG/TMM, Carl Mehling for access to specimens of Ichthyostega, Colosteus, Trimerorhachis, Eryops, Phonerpeton, Doleserpeton, Platyrhinops, Diadectes, Tuditanus, Diceratosaurus and others at the AMNH, Mark Norell for access to a microscope in his office at the AMNH (that's the office with the amazing view), Jessica Cundiff for access to specimens of Ichthyostega, Edops (including the large, heavy skull on exhibit and its separately kept right stapes), Eryops, Acheloma, Phonerpeton, Eocaecilia, Archeria, Paleothyris, Ptyonius, Lethiscus, Eothyris, Oedaleops and others at the MCZ, Stig Walsh for access to Casineria and a microscope with a digital camera at the NMS, for explaining that the almost $Я$-shaped glyph in the specimen number on the part and counterpart of Casineria is a $G$ and for permission to publish photos (Fig. 5-7), Florian Witzmann, Oliver Hampe, Thomas Schossleitner and Rodrigo Soler-Gijón for access to specimens of Gephyrostegus, Batropetes, Scincosaurus, Diceratosaurus, Iberospondylus, Sclerocephalus and Chelotriton at the Museum für Naturkunde, Berlin, Chris Sidor, Meredith Rivin and Ron Eng for access to the collection of the Burke Museum at the University of Washington (Seattle) where all known material of Nigerpeton and Saharastega is currently kept, Jason Anderson for access and transportation to a specimen of Asaphestera (currently on loan in his lab at the University of Calgary), not to mention others that will feature in future publications, Frederik Spindler, Ralf Werneburg, Ronny Rößler and Jörg Schneider for access to and discussion of the only known skull of Madygenerpeton during a conference-associated excursion in 2014, during which the abovementioned two aïstopod specimens from Chemnitz (Rößler et al., 2012) were also shown, and Antonia Bookbinder and family, Keating Holland, Maryann Haggerty and Erin Machniak for hospitality during collection visits. Adam Bodzioch and Jakub Kowalski kindly enabled D. M. to participate in the excavations in Krasiejów (Poland) of 2015 and to partially prepare a Metoposaurus clavicle discovered there by Weronika Kulikowska. Finally, D. M. would like to thank the security staff at the AMNH for successfully wrestling with the new badge system in 2013.

Andrew Milner, Laura Nicoli, Jasons Anderson and Pardo, Florian Witzmann, Jorge Bar, Guilhem Carbasse, José R. Guzmán Gutiérrez, Graeme Lloyd, Krzysztof Rogoż, Thiago Carlisbino, Eduardo Ascarrunz and Ralf Werneburg sent electronic reprints, some of which would have been impossible to acquire otherwise; Eduardo Ascarrunz and John Nyakatura shared their digital models of Triadobatrachus (Ascarrunz et al., 2016) and Orobates (Nyakatura et al., 2015); Trond Sigurdsen sent a version of the NEXUS file of RC07 to clear up our confusion about different-looking versions. The authors of Ascarrunz et al. (2016) and Pardo \& Anderson (2016) kindly allowed us to use their manuscripts before publication.

We apologize for any omissions from these lists; their order is meant to be chronological.

D. M. thanks David Peters for making a claim about Eocaecilia and Microbrachis that turned out to be incorrect but led D. M. to consulting the literature and correcting two scores for Acherontiscus and Adelospondylus.

The reviewers Alexander Pyron, Michael Buchwitz and Jason Pardo took the time to read the huge manuscript three times (J. Pardo five times), for which we are very grateful; their comments helped clarify our explanations. We also greatly appreciate Erik Seiffert's handling of the manuscript as editor during its most difficult period.

\section{References}


4977

4978

4979

4980

4981

4982

4983

4984

4985

4986

4987

4988

4989

4990

4991

4992

4993

4994

4995

4996

4997

4998

4999

5000

5001

5002

5003

5004

5005

5006

5007

5008

5009

5010

5011

5012

5013

5014

5015

5016

5017

5018

5019

5020

5021

This is not the complete list of our sources for score changes; additional references are cited in App. S1.

Ahlberg PE. 1995. Elginerpeton pancheni and the earliest tetrapod clade. Nature 373:420-425. DOI 10.1038/373420a0

Ahlberg PE. 1998. Postcranial stem tetrapod remains from the Devonian of Scat Craig, Morayshire, Scotland. Zoological Journal of the Linnean Society 122:99-141. DOI 10.1111/j.1096-3642.1998.tb02526.x

Ahlberg PE. 2011. Humeral homology and the origin of the tetrapod elbow: a reinterpretation of the enigmatic specimens ANSP 21350 and GSM 104536. Special Papers in Palaeontology 86:17-29.

Ahlberg PE, Clack JA. 1998. Lower jaws, lower tetrapods - a review based on the Devonian genus Acanthostega. Transactions of the Royal Society of Edinburgh: Earth Sciences 89:11-46. DOI 10.1017/S0263593300002340

Ahlberg PE, Friedman M, Blom H. 2005. New light on the earliest known tetrapod jaw. Journal of Vertebrate Paleontology 25:720-724. DOI 10.1671/02724634(2005)025[0720:NLOTEK]2.0.CO;2

Ahlberg PE, Lukševičs E, Lebedev O. 1994. The first tetrapod finds from the Devonian (Upper Famennian) of Latvia. Philosophical Transactions of the Royal Society B 343:303-328. DOI 10.1098/rstb.1994.0027

Ahlberg PE, Clack JA, Lukševičs E, Blom H, Zupiņš I. 2008. Ventastega curonica and the origin of tetrapod morphology. Nature 453:1199-1204. DOI 10.1038/nature06991

Anderson JS. 2001. The phylogenetic trunk: maximal inclusion of taxa with missing data in an analysis of the Lepospondyli (Vertebrata, Tetrapoda). Systematic Biology 50:170-193. DOI 10.1080/10635150119889

Anderson JS. 2002. Revision of the aïstopod genus Phlegethontia (Tetrapoda-Lepospondyli). Journal of Paleontology 76:1029-1046. DOI 10.1666/00223360(2002)076<1029:ROTAGP $>2.0 . \mathrm{CO} ; 2$

Anderson JS. 2003a. Cranial anatomy of Coloraderpeton brilli, postcranial anatomy of Oestocephalus amphiuminus, and reconsideration of Ophiderpetontidae (Tetrapoda: Lepospondyli: Aistopoda). Journal of Vertebrate Paleontology 23:532-543. DOI $10.1671 / 1752$

Anderson JS. 2003b. A new aïstopod (Tetrapoda: Lepospondyli) from Mazon Creek, Illinois. Journal of Vertebrate Paleontology 23:79-88. DOI 10.1671/02724634(2003)23[79:ANATLF]2.0.CO;2

Anderson JS. 2007a. Direct evidence of the rostral anatomy of the aïstopod Phlegethontia, with a new cranial reconstruction. Journal of Paleontology 81:408-410. DOI 10.1666/00223360(2007)81[408:DEOTRA]2.0.CO;2

Anderson JS. 2007b. Incorporating ontogeny into the matrix: a phylogenetic evaluation of developmental evidence for the origin of modern amphibians. In: Anderson JS, Sues HD, ed. Major transitions in vertebrate evolution. Bloomington: Indiana University Press, 182-227.

Anderson JS, Carroll RL, Rowe TB. 2003. New information on Lethiscus stocki (Tetrapoda: Lepospondyli: Aistopoda) from high-resolution computed tomography and a 
5022

5023

5024

5025

5026

5027

5028

5029

5030

5031

5032

5033

5034

5035

5036

5037

5038

5039

5040

5041

5042

5043

5044

5045

5046

5047

5048

5049

5050

5051

5052

5053

5054

5055

5056

5057

5058

5059

5060

5061

5062

5063

5064

5065

5066

5067 phylogenetic analysis of Aistopoda. Canadian Journal of Earth Sciences $\mid$ Revue canadienne des Sciences de la Terre 40:1071-1083. DOI 10.1139/e03-023

Anderson JS, Pardo JD, Holmes R. 2018. An enigmatic tetrapod from Five Points, Ohio (Upper Carboniferous), further supports aïstopod placement among the tetrapod stem group [abstract]. Vertebrate Anatomy Morphology Palaeontology 5:9 [volume number given as 6 throughout the abstracts]. DOI of all abstracts 10.18435/vamp29338

Anderson JS, Reisz RR, Scott D, Fröbisch NB, Sumida SS. 2008a. A stem batrachian from the Early Permian of Texas and the origin of frogs and salamanders. Nature 453:515518. DOI 10.1038/nature06865

Anderson JS, Henrici AC, Sumida SS, Martens T, Berman DS. 2008b. Georgenthalia clavinasica, a new genus and species of dissorophoid temnospondyl from the Early Permian of Germany, and the relationships of the family Amphibamidae. Journal of Vertebrate Paleontology 28:61-75. DOI 10.1671/02724634(2008)28[61:GCANGA]2.0.CO;2

Anderson JS, Smithson T, Mansky CF, Meyer T, Clack J. 2015. A diverse tetrapod fauna at the base of 'Romer's gap'. PLOS ONE 10:e0125446. DOI 10.1371/journal.pone.0125446

Andrews SM, Carroll RL. 1991. The order Adelospondyli: Carboniferous lepospondyl amphibians. Transactions of the Royal Society of Edinburgh 82:239-275.

Ascarrunz E, Rage J-C, Legreneur P, Laurin M. 2016. Triadobatrachus massinoti, the earliest known lissamphibian (Vertebrata: Tetrapoda) re-examined by $\mu \mathrm{CT}$ scan, and the evolution of trunk length in batrachians. Contributions to Zoology 85:201-234. http://www.contributionstozoology.nl/vol85/nr02/a04

Averianov A, Sues H-D. 2012. Correlation of Late Cretaceous continental vertebrate assemblages in Middle and Central Asia. Journal of Stratigraphy 36:462-485.

Báez AM, Basso NG. 1996. The earliest known frogs of the Jurassic of South America: Review and cladistic appraisal of their relationships. Münchner Geowissenschaftliche Abhandlungen A 30:131-158.

Báez AM, Nicoli L. 2004. A new look at an old frog: the Jurassic Notobatrachus Reig from Patagonia. Ameghiniana 41:257-270.

Báez AM, Nicoli L. 2008. A new species of Notobatrachus (Amphibia, Salientia) from the Middle Jurassic of northwestern Patagonia. Journal of Paleontology 82:372-376.

Baird D. 1962. A rhachitomous amphibian, Spathicephalus, from the Mississippian of Nova Scotia. Breviora of the Museum of Comparative Zoology 157:1-9+ one unnumbered plate.

Barberena MC. 1998. Australerpeton cosgriffi n.g., n.sp., a Late Permian Rhinesuchoid [sic] amphibian from Brazil. Anais da Académia Brasileira das Ciências 70:125-137.

Bardin J, Rouget I, Cecca F. 2014. Cladistics in ammonoids: back to the future. Neues Jahrbuch für Geologie und Paläontologie Abhandlungen 274:239-253. DOI 10.1127/njgpa/2014/0449

Bardin J, Rouget I, Yacobucci MM, Cecca F. 2014. Increasing the number of discrete character states for continuous characters generates well-resolved trees that do not reflect phylogeny. Integrative Zoology 9:531-541. DOI 10.1111/1749-4877.12076

Baron MG, Norman DB, Barrett PM. 2017. A new hypothesis of dinosaur relationships and early dinosaur evolution. Nature 543:501-506. DOI 10.1038/nature21700

Beaumont EH. 1977. Cranial morphology of the Loxommatidae (Amphibia: Labyrinthodontia). Philosophical Transactions of the Royal Society B 280:29-101. 
5068 Beaumont EH, Smithson TR. 1998. The cranial morphology and relationships of the aberrant

5069

5070

5071

5072

5073

5074

5075

5076

5077

5078

5079

5080

5081

5082

5083

5084

5085

5086

5087

5088

5089

5090

5091

5092

5093

5094

5095

5096

5097

5098

5099

5100

5101

5102

5103

5104

5105

5106

5107

5108

5109

5110

5111

5112

5113
Carboniferous amphibian Spathicephalus mirus Watson. Zoological Journal of the

Linnean Society 122:187-209.

Beerbower JR. 1963. Morphology, paleoecology, and phylogeny of the Permo-Pennsylvanian amphibian Diploceraspis. Bulletin of the Museum of Comparative Zoology 130:33-108.

Berman DS. 2013. Diadectomorphs, amniotes or not? In: Lucas SG, DiMichele WA, Barrick JE, Schneider JW, Spielmann JA, eds. The Carboniferous-Permian transition. Bulletin of the New Mexico Museum of Natural History and Science 60:22-35.

Berman DS, Eberth DA, Brinkman DB. 1988. Stegotretus agyrus[,] a new genus and species of microsaur (amphibian) from the Permo-Pennsylvanian of New Mexico. Annals of Carnegie Museum 57:293-323. [Comma missing in the paper, but present in the table of contents.]

Berman DS, Reisz RR, Eberth DA. 1985. Ecolsonia cutlerensis, an Early Permian dissorophid amphibian from the Cutler Formation of north-central New Mexico. Circular of the New Mexico Bureau of Mines \& Mineral Resources 191:1-31.

Berman DS, Reisz RR, Scott D. 2010. Redescription of the skull of Limnoscelis paludis Williston (Diadectomorpha: Limnoscelidae) from the Pennsylvanian of Cañon del Cobre, northern New Mexico. New Mexico Museum of Natural History and Science Bulletin 49:185-210.

Berman DS, Sumida SS. 1990. A new species of Limnoscelis (Amphibia, Diadectomorpha) from the late Pennsylvanian Sangre de Cristo Formation of central Colorado. Annals of Carnegie Museum 59:303-341.

Berman DS, Sumida SS, Lombard RE. 1992. Reinterpretation of the temporal and occipital regions in Diadectes and the relationships of diadectomorphs. Journal of Paleontology 66:481-499.

Berman DS, Sumida SS, Martens T. 1998. Diadectes (Diadectomorpha: Diadectidae) from the Early Permian of central Germany, with description of a new species. Annals of Carnegie Museum 67:53-93.

Berman DS, Henrici AC, Kissel RA, Sumida SS, Martens T. 2004. A new diadectid (Diadectomorpha), Orobates pabsti, from the Early Permian of Central Germany. Bulletin of Carnegie Museum 35:1-36. DOI 10.2992/01459058(2004)35[1:ANDDOP]2.0.CO;2

Bernardi M, Angielczyk KD, Mitchell JS, Ruta M. 2016. Phylogenetic stability, tree shape, and character compatibility: a case study using early tetrapods. Systematic Biology 65:737-758. DOI 10.1093/sysbio/syw049

Bishop PJ. 2014. The humerus of Ossinodus pueri, a stem tetrapod from the Carboniferous of Gondwana, and the early evolution of the tetrapod forelimb. Alcheringa 38:209-238.

Boisvert CA. 2005. The pelvic fin and girdle of Panderichthys and the origin of tetrapod locomotion. Nature 438:1145-1147.

Boisvert CA. 2009. The humerus of Panderichthys in three dimensions and its significance in the context of the fish-tetrapod transition. Acta Zoologica (Stockholm) 90 (suppl. 1): 297-305.

Boisvert CA, Mark-Kurik E, Ahlberg PE. 2008. The pectoral fin of Panderichthys and the origin of digits. Nature 456:636-638.

Bolt JR, Chatterjee S. 2000. A new temnospondyl amphibian from the Late Triassic of Texas. Journal of Paleontology 74:670-683. 
5114 Bolt JR, Lombard RE. 1985. Evolution of the amphibian tympanic ear and the origin of frogs.

5115

5116

5117

5118

5119

5120

5121

5122

5123

5124

5125

5126

5127

5128

5129

5130

5131

5132

5133

5134

5135

5136

5137

5138

5139

5140

5141

5142

5143

5144

5145

5146

5147

5148

5149

5150

5151

5152

5153

5154

5155

5156

5157 Biological Journal of the Linnean Society 24:38-99.

Bolt JR, Lombard RE. 1992. Nature and quality of the fossil evidence for otic evolution in early tetrapods. In: Webster DB, Fay RR, Popper AN, ed. The evolutionary biology of hearing. New York/Berlin: Springer, 377-403.

Bolt JR, Lombard RE. 2000. Palaeobiology of Whatcheeria deltae, a primitive Missisippian tetrapod. In: Heatwole H, Carroll RL, ed. Amphibian Biology. Chipping Norton: Surrey Beatty \& Sons, 1044-1052.

Bolt JR, Lombard RE. 2001. The mandible of the primitive tetrapod Greererpeton, and the early evolution of the tetrapod lower jaw. Journal of Paleontology 75:1016-1042.

Bolt JR, Lombard RE. 2006. Sigournea multidentata, a new stem tetrapod from the upper Mississippian of Iowa, USA. Journal of Paleontology 80:717-725.

Bolt JR, Lombard RE. 2010. Deltaherpeton hiemstrae, a new colosteid tetrapod from the Mississippian of Iowa. Journal of Paleontology 84:1135-1151.

Bolt JR, Rieppel O. 2009. The holotype skull of Llistrofus pricei Carroll and Gaskill, 1978 (Microsauria: Hapsidopareiontidae). Journal of Paleontology 83:471-483.

Bossy KV. 1976. Morphology, paleoecology, and evolutionary relationships of the Pennsylvanian urocordylid nectrideans (subclass Lepospondyli, class Amphibia). Doctoral thesis, Yale University.

Bossy KV, Milner AC. 1998. Order Nectridea. In: Carroll RL, Bossy KV, Milner AC, Andrews SM, Wellstead CF, ed. Lepospondyli - part 4 of Wellnhofer P, ed. Handbuch der Paläoherpetologie | Encyclopedia of Paleoherpetology. Stuttgart: Gustav Fischer, 73131.

Botelho JF, Ossa-Fuentes L, Soto-Acuña S, Smith-Paredes D, Nuñez-León D, SalinasSaavedra M, Ruiz-Flores M, Vargas AO. 2014. New developmental evidence clarifies the evolution of wrist bones in the dinosaur-bird transition. PLOS Biology 12:e1001957. DOI 10.1371/journal.pbio.1001957

Boy JA. 1972. Die Branchiosaurier (Amphibia) des saarpfälzischen Rotliegenden (Perm, SWDeutschland). Abhandlungen des hessischen Landesamts für Bodenforschung 65:1-137.

Boy JA. 1986. Studien über die Branchiosauridae (Amphibia: Temnospondyli). 1. Neue und wenig bekannte Arten aus dem mitteleuropäischen Rotliegenden (?oberstes Karbon bis unteres Perm). Paläontologische Zeitschrift 60:131-166.

Boy JA. 1987. Studien über die Branchiosauridae (Amphibia: Temnospondyli; Ober-KarbonUnter-Perm) 2. Systematische Übersicht. Neues Jahrbuch für Geologie und Paläontologie Abhandlungen 174:75-104.

Boy JA. 1993. Über einige Vertreter der Eryopoidea (Amphibia: Temnospondyli) aus dem europäischen Rotliegend (?höchstes Karbon-Perm). 4. Cheliderpeton latirostre. Paläontologische Zeitschrift 67:123-143.

Boy JA. 1995. Über die Micromelerpetontidae (Amphibia: Temnospondyli). 1. Morphologie und Paläoökologie des Micromelerpeton credneri (Unter-Perm; SW-Deutschland). Paläontologische Zeitschrift 69:429-457.

Boy JA. 1996. Ein neuer Eryopoide (Amphibia: Temnospondyli) aus dem saarpfälzischen Rotliegend (Unter-Perm; Südwest-Deutschland). Mainzer geowissenschaftliche Mitteilungen 25:7-26. 
5158

5159

5160

5161

5162

5163

5164

5165

5166

5167

5168

5169

5170

5171

5172

5173

5174

5175

5176

5177

5178

5179

5180

5181

5182

5183

5184

5185

5186

5187

5188

5189

5190

5191

5192

5193

5194

5195

5196

5197

5198

5199

5200

5201

5202

Boy JA, Bandel K. 1973. Bruktererpeton fiebigi n. gen. n. sp. (Amphibia: Gephyrostegida), der erste Tetrapode aus dem rheinisch-westfälischen Karbon (Namur B; W.-Deutschland). Palaeontographica A 145:39-77+ plates 7-8.

Boyd MJ. 1982. Morphology and relationships of the Upper Carboniferous aïstopod amphibian Ophiderpeton nanum. Palaeontology 25:209-214.

Brazeau MD. 2011. Problematic character coding methods in morphology and their effects. Biological Journal of the Linnean Society 104:489-498.

Brazeau MD, Ahlberg PE. 2006. Tetrapod-like middle ear architecture in a Devonian fish. Nature 439:318-321.

Brazeau MD, Guillerme T, Smith MR. 2017. Morphological phylogenetic analysis with inapplicable data. bioR $\chi$ iv. DOI 10.1101/209775

Brocklehurst N, Romano M, Fröbisch J. 2016. Principal component analysis as an alternative treatment for morphometric characters: phylogeny of caseids as a case study. Palaeontology 59:877-886. DOI 10.1111/pala.12264

Brocklehurst N, Reisz RR, Fernandez V, Fröbisch J. 2016. A re-description of 'Mycterosaurus' smithae, an Early Permian eothyridid, and its impact on the phylogeny of pelycosaurian-grade synapsids. PLOS ONE 11:e0156810. DOI 10.1371/journal.pone. 0156810

Brown JW, Parins-Fukuchi C, Stull GW, Vargas OM, Smith SA. 2017. Bayesian and likelihood phylogenetic reconstructions of morphological traits are not discordant when taking uncertainty into consideration: a comment on Puttick et al. Proceedings of the Royal Society B 284:20170986. DOI 10.1098/rspb.2017.0986

Buchwitz M, Foth C, Kogan I, Voigt S. 2012. On the use of osteoderm features in a phylogenetic approach on the internal relationships of the Chroniosuchia (Tetrapoda: Reptiliomorpha). Palaeontology 55:623-640.

Bulanov VV. 2003. Evolution and systematics of seymouriamorph parareptiles. Paleontological Journal 37 (Suppl. 1): S1-S105.

Bulanov VV. 2014. New finds of Microphon exiguus (Seymouriamorpha, Kotlassidae) in the Severodvinian Beds of the Sukhona River Basin, Russia. Paleontological Journal 48:633-644.

Buscalioni ÁD. 2017. The Gobiosuchidae in the early evolution of Crocodyliformes. Journal of Vertebrate Paleontology 37:e1324459. DOI 10.1080/02724634.2017.1324459

Butler RJ, Upchurch P. 2007. Highly incomplete taxa and the phylogenetic relationships of the theropod dinosaur Juravenator starki. Journal of Vertebrate Paleontology 27:253-256.

Bystrow AP. 1944. Kotlassia prima Amalitzky. Bulletin of the Geological Society of America 55:379-416.

Callier V, Clack JA, Ahlberg PE. 2009. Contrasting developmental trajectories in the earliest known tetrapod forelimbs. Science 324:364-367.

Carrier DR, Wake MH. 1995. Mechanism of lung ventilation in the caecilian Dermophis mexicanus. Journal of Morphology 226:289-295.

Carroll RL. 1964. Early evolution of the dissorophid amphibians. Bulletin of the Museum of Comparative Zoology 131:161-250.

Carroll RL. 1967. Labyrinthodonts from the Joggins Formation. Journal of Paleontology 41:111-142.

Carroll RL. 1969a. A new family of Carboniferous amphibians. Paleontology 12:537-548. 
5203 Carroll RL. 1969b. A Middle Pennsylvanian captorhinomorph, and the interrelationships of primitive reptiles. Journal of Paleontology 43:151-170.

Carroll RL. 1970. The ancestry of reptiles. Philosophical Transactions of the Royal Society B 257:267-308.

Carroll RL. 1988. An articulated gymnarthrid microsaur (Amphibia) from the Upper Carboniferous of Czechoslovakia. [Italics in the original.] Acta Zoologica Cracoviensia 31:441-450 + plate XII.

Carroll RL. 1991. Batropetes from the Lower Permian of Europe - a microsaur, not a reptile. Journal of Vertebrate Paleontology 11:229-242.

Carroll RL. 1998a. Cranial anatomy of ophiderpetontid aïstopods: Palaeozoic limbless amphibians. Zoological Journal of the Linnean Society 122:143-166.

Carroll RL. 1998b. Order Aïstopoda MIALL 1875. In: Carroll RL, Bossy KV, Milner AC, Andrews SM, Wellstead CF, ed. Lepospondyli - part 4 of Wellnhofer P, ed. Handbuch der Paläoherpetologie | Encyclopedia of Paleoherpetology. Stuttgart: Gustav Fischer, 163-182.

Carroll RL. 2007. The Palaeozoic ancestry of salamanders, frogs and caecilians. Zoological Journal of the Linnean Society 150 (Suppl. 1): 1-140.

Carroll RL, Chorn J. 1995. Vertebral development in the oldest microsaur and the problem of "lepospondyl" relationships. Journal of Vertebrate Paleontology 15:37-56.

Carroll RL, Bybee P, Tidwell WD. 1991. The oldest microsaur (Amphibia). Journal of Paleontology 65:314-322.

Carroll RL, Gaskill P. 1978. The order Microsauria. Philadelphia: American Philosophical Society.

Carroll RL, Holmes R. 1980. The skull and jaw musculature as guides to the ancestry of salamanders. Zoological Journal of the Linnean Society 68:1-40.

Case EC. 1910. New or little known reptiles and amphibians from the Permian (?) of Texas. Bulletin of the American Museum of Natural History 28:163-181.

Case EC. 1911. A revision of the Cotylosauria of North America. Carnegie Institute of Washington publication 145:1-122.

Case EC, Williston SW. 1912. A description of the skulls of Diadectes lentus and Animasaurus carinatus. American Journal of Science 33:339-348.

Cau A. 2018a. The assembly of the avian body plan: A 160-million-year long process. Bollettino della Società Paleontologica Italiana 57:1-25. DOI 10.4435/bspi.2018.01

Cau A. 2018b. [comment to Mortimer, 2018]. http://theropoddatabase.blogspot.com/2018/05/testing-alternative-stembird.html?showComment=1526498961231\#c5174312511096268432

Cau A, Beyrand V, Voeten DFAE, Fernandez V, Tafforeau P, Stein K, Barsbold R, Tsogtbaatar Kh, Currie PJ, Godefroit P. 2017. Synchrotron scanning reveals amphibious ecomorphology in a new clade of bird-like dinosaurs. Nature 552:395-399. DOI 10.1038/nature24679

Chase JN. 1965. Neldasaurus wrightae, a new rhachitomous labyrinthodont from the Texas Lower Permian. Bulletin of the Museum of Comparative Zoology 133:153-225.

Chen J, Bever GS, Yi H-Y, Norell MA. 2016. A burrowing frog from the late Paleocene of Mongolia uncovers a deep history of spadefoot toads (Pelobatoidea) in East Asia. Scientific Reports 6:19209. DOI 10.1038/srep19209 
5249

5250

5251

5252

5253

5254

5255

5256

5257

5258

5259

5260

5261

5262

5263

5264

5265

5266

5267

5268

5269

5270

5271

5272

5273

5274

5275

5276

5277

5278

5279

5280

5281

5282

5283

5284

5285

5286

5287

5288

5289

5290

5291

5292

5293

Cisneros JC, Marsicano C, Angielczyk KD, Smith RMH, Richter M, Fröbisch J, Kammerer CF, Sadleir RW. 2015. New Permian fauna from tropical Gondwana. Nature Communications 6:8676. DOI 10.1038/ncomms9676

Clack JA. 1987a. Two new specimens of Anthracosaurus (Amphibia: Anthracosauria) from the Northumberland Coal Measures. Palaeontology 30:15-26.

Clack JA. 1987b. Pholiderpeton scutigerum Huxley, an amphibian from the Yorkshire coal measures. Philosophical Transactions of the Royal Society B 318:1-107.

Clack JA. 1998. The Scottish Carboniferous tetrapod Crassigyrinus scoticus (Lydekker)_ cranial anatomy and relationships. Transactions of the Royal Society of Edinburgh: Earth Sciences 88:127-142.

Clack JA. 2001. Eucritta melanolimnetes from the Early Carboniferous of Scotland, a stem tetrapod showing a mosaic of characteristics. Transactions of the Royal Society of Edinburgh: Earth Sciences 92:75-95.

Clack JA. 2003. A new baphetid (stem tetrapod) from the Upper Carboniferous of Tyne and Wear, U.K., and the evolution of the tetrapod occiput. Canadian Journal of Earth Sciences $\mid$ Revue canadienne des Sciences de la Terre 40:483-498.

Clack JA. 2011a. A new microsaur from the Early Carboniferous (Viséan) of East Kirkton, Scotland, showing soft-tissue evidence. Special Papers in Palaeontology 86:45-55.

Clack JA. 2011b. A Carboniferous embolomere tail with supraneural radials. Journal of Vertebrate Paleontology 31:1150-1153.

Clack JA, Finney SM. 2005. Pederpes finneyae, an articulated tetrapod from the Tournaisian of Western Scotland. Journal of Systematic Paleontology 2:311-346.

Clack JA, Klembara J. 2009. An articulated specimen of Chroniosaurus dongusensis and the morphology and relationships of the chroniosuchids. Special Papers in Palaeontology $81: 15-42$.

Clack JA, Milner AR. 2010. Morphology and systematics of the Pennsylvanian amphibian Platyrhinops lyelli (Amphibia: Temnospondyli). Earth and Environmental Science Transactions of the Royal Society of Edinburgh 100:275-295.

Clack JA, Milner AR. 2015. Basal Tetrapoda. Part 3A1 of Sues H-D, ed. Handbook of Paleoherpetology. Munich: Dr. Friedrich Pfeil.

Clack JA, Ahlberg PE, Blom H, Finney SM. 2012a. A new genus of Devonian tetrapod from north-east Greenland, with new information on the lower jaw of Ichthyostega. Palaeontology 55:73-86.

Clack JA, Witzmann F, Müller J, Snyder D. 2012b. A colosteid-like early tetrapod from the St. Louis Limestone (Early Carboniferous, Meramecian), St. Louis, Missouri, USA. Fieldiana: Life and Earth Sciences 5:17-39.

Clack JA, Bennett CE, Carpenter DK, Davies SJ, Fraser NC, Kearsey TI, Marshall JEA, Millward D, Otoo BKA, Reeves EJ, Ross AJ, Ruta M, Smithson KZ, Smithson TR, Walsh SA. 2016. Phylogenetic and environmental context of a Tournaisian tetrapod fauna. Nature Ecology \& Evolution 1:0002. DOI 10.1038/s41559-016-0002

Cloutier R, Béchard I. 2013. A new piece of the Devonian fish-to-tetrapod puzzle: the discovery of a complete specimen of Elpistostege [abstract]. Journal of Vertebrate Paleontology, Program and Abstracts 2013, 107. PDF of all abstracts: http://vertpaleo.org/Annual-Meeting/Future-Past-Meetings/MeetingPdfs/SVP-2013merged-book-10-15-2013.aspx 
5294

5295

5296

5297

5298

5299

5300

5301

5302

5303

5304

5305

5306

5307

5308

5309

5310

5311

5312

5313

5314

5315

5316

5317

5318

5319

5320

5321

5322

5323

5324

5325

5326

5327

5328

5329

5330

5331

5332

5333

5334

5335

5336

5337

5338

5339

Cloutier R, Long JA, Béchard I, Clement A. 2016. Rates of phenotypic evolution during the fish to tetrapod transition: the elpistostegalian fast-lane [abstract]. Journal of Vertebrate Paleontology, Program and Abstracts 2016, 118. PDF of all abstracts: http://vertpaleo.org/PDFS/2016/SVP-2016-Program-Book-v10-with-covers.aspx

Coates MI. 1996. The Devonian tetrapod Acanthostega gunnari Jarvik: postcranial anatomy, basal tetrapod interrelationships and patterns of skeletal evolution. Transactions of the Royal Society of Edinburgh 87:363-421.

Coates MI, Clack JA. 1995. ROMER's gap: tetrapod origins and terrestriality. Bulletin du Muséum national d'Histoire naturelle, Paris, $4^{e}$ série 17:373-388.

Coates MI, Ruta M, Friedman M. 2008. Ever since Owen: changing perspectives on the early evolution of tetrapods. Annual Review of Ecology, Evolution, and Systematics 39:571592. DOI 10.1146/annurev.ecolsys.38.091206.095546

Conrad JL, Norell MA. 2015. Anguimorpha (Squamata) and the importance of fossils [abstract]. Journal of Vertebrate Paleontology, Program and Abstracts 2015, 109. PDF of all abstracts: http://vertpaleo.org/PDFS/SVP-2015-Program-and-Abstract-Book-9-222015.aspx

Daeschler EB. 2000. Early tetrapod jaws from the Late Devonian of Pennsylvania, USA. Journal of Paleontology 74:301-308.

Daeschler EB, Clack JA, Shubin NH. 2009. Late Devonian tetrapod remains from Red Hill, Pennsylvania, USA: how much diversity? Acta Zoologica (Stockholm) 90:306-317.

Daly E. 1994. The Amphibamidae (Amphibia: Temnospondyli), with a description of a new genus from the Upper Pennsylvanian of Kansas. University of Kansas Museum of Natural History Special Publication 85:1-59.

Damiani R, Sidor CA, Steyer JS, Smith RMH, Larsson HCE, Maga A, Ide Ou. 2006. The vertebrate fauna of the Upper Permian of Niger. V. The primitive temnospondyl Saharastega moradiensis. Journal of Vertebrate Paleontology 26:559-572.

Danto M, Witzmann F, Müller J. 2012. Redescription and phylogenetic relationships of Solenodonsaurus janenschi Broili, 1924, from the Late Carboniferous of Nýřany, Czech Republic. Fossil Record 15:45-59.

Dawson JW. 1863. Air-breathers of the coal period: a descriptive account of the remains of land animals found in the coal formation of Nova Scotia, with remarks on their bearing on theories of the formation of coal and of the origin of species. Montreal: Dawson Brothers.

Dias EV, Schultz CL. 2003. The first Paleozoic temnospondyl postcranial skeleton from South America. Revista Brasileira de Paleontologia 6:29-42.

Diaz RE, Trainor PA. 2015. Hand/foot splitting and the 're-evolution' of mesopodial skeletal elements during the evolution and radiation of chameleons. BMC Evolutionary Biology 15:184.

Dilkes DW. 1990. A new trematopsid amphibian (Temnospondyli: Dissorophoidea) from the Lower Permian of Texas. Journal of Vertebrate Paleontology 10:222-243.

Dilkes D. 2015a. Carpus and tarsus of Temnospondyli. Vertebrate Anatomy Morphology Palaeontology 1:51-87. DOI 10.18435/B5MW2Q

Dilkes D. 2015b. 'Dissorophus' angustus (Temnospondyli, Dissorophoidea) and increasing variability of dissorophid osteoderms [abstract]. Journal of Vertebrate Paleontology, Program and Abstracts 2015, 117. PDF of all abstracts: http://vertpaleo.org/PDFS/SVP2015-Program-and-Abstract-Book-9-22-2015.aspx

PeerJ reviewing PDF | (2015:12:8254:6:0:NEW 20 Jul 2018) 
5340 Dong L, Roček Z, Wang Y, Jones MEH. 2013. Anurans from the Lower Cretaceous Jehol

5341

5342

5343

5344

5345

5346

5347

5348

5349

5350

5351

5352

5353

5354

5355

5356

5357

5358

5359

5360

5361

5362

5363

5364

5365

5366

5367

5368

5369

5370

5371

5372

5373

5374

5375

5376

5377

5378

5379

5380

5381

5382

5383
Group of western Liaoning, China. PLOS ONE 8:e69723. DOI

10.1371/journal.pone.0069723

Douthitt H. 1917. The structure and relationships of Diplocaulus. Contributions from Walker Museum II:3-41. [Lack of italics in the original.]

Efremov JA. 1932. Über die Labyrinthodonten der U. d. S. S. R. - II. Permische Labyrinthodonten des früheren Gouvernements Wjatka. Труды палеозоологического института Академии наук СССР [Works of the Paleozoological Institute of the Academy of Sciences of the USSR] II:117-173. [In German with Russian summary.]

Eltink E, Langer MC. 2014. A new specimen of the temnospondyl Australerpeton cosgriffi from the Late Permian of Brazil (Rio do Rasto Formation, Paraná Basin): comparative anatomy and phylogenetic relationships. Journal of Vertebrate Paleontology 34:524-538.

Eltink E, Dias EV, Dias-da-Silva S, Schultz CL, Langer MC. 2016. The cranial morphology of the temnospondyl Australerpeton cosgriffi (Tetrapoda: Stereospondyli) from the Middle-Late Permian of Paraná Basin and the phylogenetic relationships of Rhinesuchidae. Zoological Journal of the Linnean Society 176:835-860. DOI 10.1111/zoj.12339

Estes R, Hoffstetter R. 1976. Les Urodèles du Miocène de La Grive-Saint-Alban (Isère, France). Bulletin du Muséum national d'Histoire naturelle, $3^{e}$ série 398 (Sciences de la Terre 57): 297-343.

Estes R, Reig OA. 1973. The early fossil record of frogs: A review of the evidence. In: Vial JL, ed. Evolutionary Biology of the Anurans. Columbia: University of Missouri Press, 11-63.

Evans SE, Borsuk-Białynicka M. 2009. The Early Triassic stem-frog Czatkobatrachus from Poland. Palaeontologia Polonica 65:79-105.

Evans SE, Milner AR. 1996. A metamorphosed salamander from the early Cretaceous of Las Hoyas, Spain. Philosophical Transactions of the Royal Society B 351:627-646.

Ezcurra MD. 2016. The phylogenetic relationships of basal archosauromorphs, with an emphasis on the systematics of proterosuchian archosauriforms. PeerJ 4:e1778. DOI 10.7717 peerj. 1778

Fox RC, Naylor BG. 1982. A reconsideration of the relationships of the fossil amphibian Albanerpeton. Canadian Journal of Earth Sciences | Revue canadienne des Sciences de la Terre 19:118-128.

Fracasso MA. 1983. Cranial osteology, functional morphology, systematics and paleoenvironment of Limnoscelis paludis Williston. Doctoral thesis, Yale University.

Fröbisch NB, Bickelmann C, Witzmann F. 2014. Early evolution of limb regeneration in tetrapods: evidence from a 300-million-year-old amphibian. Proceedings of the Royal Society B 281:20141550. DOI 10.1098/rspb.2014.1550

Fröbisch NB, Carroll RL, Schoch RR. 2007. Limb ossification in the Paleozoic branchiosaurid Apateon (Temnospondyli) and the early evolution of preaxial dominance in tetrapod limb development. Evolution \& Development 9:69-75. DOI 10.1111/j.1525142X.2006.00138.X

Fröbisch NB, Reisz RR. 2012. A new species of dissorophid (Cacops woehri) from the Lower Permian Dolese Quarry, near Richards Spur, Oklahoma. Journal of Vertebrate Paleontology 32:35-44. DOI 10.1080/02724634.2012.633586 
5384 Fröbisch NB, Schoch RR. 2009a. Testing the impact of miniaturization on phylogeny:

5385

5386

5387

5388

5389

5390

5391

5392

5393

5394

5395

5396

5397

5398

5399

5400

5401

5402

5403

5404

5405

5406

5407

5408

5409

5410

5411

5412

5413

5414

5415

5416

5417

5418

5419

5420

5421

5422

5423

5424

5425

5426

5427

5428

5429
Paleozoic dissorophoid amphibians. Systematic Biology 58:312-327. DOI

10.1093/sysbio/syp029

Fröbisch NB, Schoch RR. 2009b. The largest specimen of Apateon and the life history pathway of neoteny in the Paleozoic temnospondyl family Branchiosauridae. Fossil Record 12:83-90. DOI 10.1002/mmng.200800012

Fröbisch NB, Bickelmann C, Olori JC, Witzmann F. 2015. Deep-time evolution of regeneration and preaxial polarity in tetrapod limb development. Nature 527:231-234. DOI 10.1038/nature15397

Gao K, Chen J. 2017. A new crown-group frog (Amphibia: Anura) from the Early Cretaceous of northeastern Inner Mongolia, China. American Museum Novitates 3876:1-39. DOI $10.1206 / 3876.1$

Gao K, Shubin NH. 2003. Earliest known crown-group salamanders. Nature 422:424-428. DOI 10.1038/nature01491

Gao K, Shubin NH. 2012. Late Jurassic salamandroid from western Liaoning, China. Proceedings of the National Academy of Sciences of the United States of America 109:5767-5772. DOI 10.1073/pnas.1009828109

Gardner JD. 2001. Monophyly and affinities of albanerpetontid amphibians (Temnospondyli; Lissamphibia). Zoological Journal of the Linnean Society 131:309-352. DOI 10.1111/j.1096-3642.2001.tb02240.x

Gardner JD, Evans SE, Sigogneau-Russell D. 2003. New albanerpetontid amphibians from the Early Cretaceous of Morocco and Middle Jurassic of England. Acta Palaeontologica Polonica 48:301-319.

Germain D. 2008a. Anatomie des Lépospondyles et origine des Lissamphibiens. Doctoral thesis, Muséum national d'Histoire naturelle (Paris). http://www.theses.fr/2008MNHN0028

Germain D. 2008b. A new phlegethontiid specimen (Lepospondyli, Aistopoda) from the Late Carboniferous of Montceau-les-Mines (Saône-et-Loire, France). Geodiversitas 30:669680.

Germain D. 2010. The Moroccan diplocaulid: the last lepospondyl, the single one on Gondwana. Historical Biology 22:4-39. DOI 10.1080/08912961003779678

Glienke S. 2013. A taxonomic revision of Batropetes (Amphibia, Microsauria) from the Rotliegend (basal Permian) of Germany. Neues Jahrbuch für Geologie und Paläontologie Abhandlungen 269:73-96. DOI 10.1127/0077-7749/2013/0336

Glienke S. 2015. Two new species of the genus Batropetes (Tetrapoda, Lepospondyli) from the Central European Rotliegend (basal Permian) in Germany. Journal of Vertebrate Paleontology 35:e918041. DOI 10.1080/02724634.2014.918041

Godefroit P, Cau A, Hu D-Y, Escuillié F, Wu W, Dyke G. 2013. A Jurassic avialan dinosaur from China resolves the early phylogenetic history of birds. Nature 498:359-362. DOI 10.1038 /nature 12168

Godfrey SJ. 1989. The postcranial skeletal anatomy of the Carboniferous tetrapod Greererpeton burkemorani Romer, 1969. Philosophical Transactions of the Royal Society B 323:75133.

Godfrey SJ. 2003. A diminutive temnospondyl amphibian from the Pennsylvanian of Illinois. Canadian Journal of Earth Sciences | Revue canadienne des Sciences de la Terre 40:507-514. DOI 10.1139/E02-064 
5430 Godfrey SJ, Fiorillo AR, Carroll RL. 1987. A newly discovered skull of the temnospondyl

5431

5432

5433

5434

5435

5436

5437

5438

5439

5440

5441

5442

5443

5444

5445

5446

5447

5448

5449

5450

5451

5452

5453

5454

5455

5456

5457

5458

5459

5460

5461

5462

5463

5464

5465

5466

5467

5468

5469

5470

5471

5472

5473

5474

5475 amphibian Dendrerpeton acadianum Owen. Canadian Journal of Earth Sciences $\mid$ Revue canadienne des Sciences de la Terre 24:796-805.

Godfrey SJ, Holmes RB. 1989. A tetrapod lower jaw from the Pennsylvanian (Westphalian A) of Nova Scotia. Canadian Journal of Earth Sciences | Revue canadienne des Sciences de la Terre 26:1036-1040.

Godfrey SJ, Reisz RR. 1991. The vertebral morphology of Gephyrostegus bohemicus Jaekel 1902, with comments on the atlas-axis complex in primitive tetrapods. Historical Biology $5: 27-36$.

Goldman N, Anderson JP, Rodrigo AG. 2000. Likelihood-based tests of topologies in phylogenetics. Systematic Biology 49:652-670. DOI 10.1080/106351500750049752

Goloboff PA, Torres A, Arias JS. 2017. Weighted parsimony outperforms other methods of phylogenetic inference under models appropriate for morphology. Cladistics online early (31 pp.). DOI 10.1111/cla.12205

Goodrich EW. 1916. On the classification of the Reptilia.. Proceedings of the Royal Society of London B 89:261-276.

Graham JB, Wegner NC, Miller LA, Jew CJ, Lai NC, Berquist RM, Frank LR, Long JA. 2014. Spiracular air breathing in polypterid fishes and its implications for aerial respiration in stem tetrapods. Nature Communications 5:3022. DOI $10.1038 /$ ncomms 4022

Grand A, Corvez A, Duque Velez LM, Laurin M. 2013. Phylogenetic inference using discrete characters: performance of ordered and unordered parsimony and of three-item statements. Biological Journal of the Linnean Society 110:914-930.

Grande L, Bemis WE. 1998. A comprehensive phylogenetic study of amiid fishes (Amiidae) based on comparative skeletal anatomy. An empirical search for interconnected patterns of natural history. Society of Vertebrate Paleontology Memoir 4 (suppl. to issue 1 of Journal of Vertebrate Paleontology 18). Society of Vertebrate Paleontology. DOI 10.1080/02724634.1998.10011114

[Gubin YuM] Губин ЮМ. 1991. Пермские архегозавроидные амфибии СССР [Реттіап archegosauroid amphibians of the USSR]. [Equals volume 249 of Tpydbl палеонтологического института Академии наук СССP-Works of the Paleontological Institute of the Academy of Sciences of the USSR.] Moscow: Наука [Nauka].

Harjunmaa E, Seidel K, Häkkinen T, Renvoisé E, Corfe IJ, Kallonen A, Zhang Z-Q, Evans AR, Mikkola ML, Salazar-Ciudad I, Klein OD, Jernvall J. 2014. Replaying evolutionary transitions from the dental fossil record. Nature 512:44-48. DOI 10.1038 /nature13613

Hewison RH. 2007. The skull and mandible of the stereospondyl Lydekkerina huxleyi, [sic] (Tetrapoda: Temnospondyli) from the Lower Triassic of South Africa, and a reappraisal of the family Lydekkerinidae, its origin, taxonomic relationships and phylogenetic importance. Self-published ("Journal of Temnospondyl Palaeontology") 1:1-80. http://www.lydekkerina.com/images/Vol\%201\%20Number\%201.pdf

Hewison RH. 2008. The sacral region, pelvis and hind limb of the stereospondyl Lydekkerina huxleyi (Tetrapoda: Temnospondyli) from the Lower Triassic of South Africa. Selfpublished ("Journal of Temnospondyl Palaeontology") 2:1-26. http://www.lydekkerina.com/images/Vol\%202\%20Number\%201-1.pdf 
5476

5477

5478

5479

5480

5481

5482

5483

5484

5485

5486

5487

5488

5489

5490

5491

5492

5493

5494

5495

5496

5497

5498

5499

5500

5501

5502

5503

5504

5505

5506

5507

5508

5509

5510

5511

5512

5513

5514

5515

5516

5517

5518

5519

5520

5521

Holmes R. 1980. Proterogyrinus scheelei and the early evolution of the labyrinthodont pectoral limb. In: Panchen AL, ed. The Terrestrial Environment and the Origin of Land Vertebrates. London and New York: Academic Press, 351-376.

Holmes R. 1984. The Carboniferous amphibian Proterogyrinus scheelei Romer, and the early evolution of tetrapods. Philosophical Transactions of the Royal Society B 306:431-527.

Holmes R. 1989. The skull and axial skeleton of the Lower Permian anthracosauroid amphibian Archeria crassidisca Cope. Palaeontographica A 207:161-206.

Holmes R, Berman DS, Anderson JS. 2013. A new dissorophid (Temnospondyli, Dissorophoidea) from the Early Permian of New Mexico (United States). Comptes Rendus Palevol 12:419-435. DOI 10.1016/j.crpv.2013.07.002

Holmes R, Carroll R. 1977. A temnospondyl amphibian from the Mississippian of Scotland. Bulletin of the Museum of Comparative Zoology 147:489-511. http://biostor.org/reference/656

Holmes RB, Carroll RL. 2010. An articulated embolomere skeleton (Amphibia: Anthracosauria) from the Lower Pennsylvanian (Bashkirian) of Nova Scotia. Canadian Journal of Earth Sciences | Revue canadienne des Sciences de la Terre 47:209-219.

Holmes RB, Carroll RL, Reisz RR. 1998. The first articulated skeleton of Dendrerpeton acadianum (Temnospondyli, Dendrerpetontidae) from the Lower Pennsylvanian locality of Joggins, Nova Scotia, and a review of its relationships. Journal of Vertebrate Paleontology 18:64-79.

Holmes R, Godfrey S, Baird D. 1995. Tetrapod remains from the late Mississippian Pomquet Formation near Grand Étang, Nova Scotia. Canadian Journal of Earth Sciences $\mid$ Revue canadienne des Sciences de la Terre 32:913-921.

Hook RW. 1983. Colosteus scutellatus (Newberry), a primitive temnospondyl amphibian from the Middle Pennsylvanian of Linton, Ohio. American Museum Novitates 2770:1-41.

Hook RW. 1993. Chenoprosopus lewisi, a new cochleosaurid amphibian (Amphibia: Temnospondyli) from the Permo-Carboniferous of North-Central Texas. Annals of Carnegie Museum 62:273-291.

Hook RW, Baird D. 1984. Ichthycanthus platypus Cope, 1877, reidentified as the dissorophoid amphibian Amphibamus lyelli. Journal of Paleontology 58:697-702.

Huelsenbeck J, Larget B, van der Mark P, Ronquist F, Simon D, Teslenko M. 2015. MrBayes: Bayesian Inference of Phylogeny. Version 3.2.6. http://mrbayes.sourceforge.net/

Huttenlocker AK, Small BJ, Pardo JD, Anderson JS. 2013. Cranial morphology of recumbirostrans (Lepospondyli) from the Permian of Kansas and Nebraska, and early morphological evolution inferred by micro-computed tomography. Journal of Vertebrate Paleontology 33:540-552. DOI 10.1080/02724634.2013.728998

Irisarri I, Baurain D, Brinkmann H, Delsuc F, Sire J-Y, Kupfer A, Petersen J, Jarek M, Meyer A, Vences M, Philippe H. 2017. Phylotranscriptomic consolidation of the jawed vertebrate timetree. Nature Ecology \& Evolution 1:1370-1378. DOI 10.1038/s41559017-0240-5

International Commission on Zoological Nomenclature. 1999. International Code of Zoological Nomenclature. $4^{\text {th }}$ edition. London: International Trust for Zoological Nomenclature. http://www.nhm.ac.uk/hosted-sites/iczn/code/

Ivachnenko MF. 1978 (English translation 1979). Urodelans from the Triassic and Jurassic of Soviet central Asia. Paleontological Journal 1978:362-368. 
5522 [Ivachnenko MF, Tverdochlebova GI] Ивахненко МФ, Твердохлебова ГИ. 1980.

5523

5524

5525

5526

5527

5528

5529

5530

5531

5532

5533

5534

5535

5536

5537

5538

5539

5540

5541

5542

5543

5544

5545

5546

5547

5548

5549

5550

5551

5552

5553

5554

5555

5556

5557

5558

5559

5560

5561

5562

5563

5564

5565

5566

5567
Систематика, морфология и стратиграфическое значение верхнепермских хрониозухов востока европейской части ССCP [Systematics, morphology and stratigraphic significance of the Upper Permian chroniosuchians of the east of the European part of the USSR]. Saratov: Издательство саратовского университета [Izdatel'stvo saratovskogo universiteta].

Jaekel O. 1903. Ueber Ceraterpeton [sic], Diceratosaurus und Diplocaulus. [Lack of italics in the original.] Neues Jahrbuch für Mineralogie, Geologie und Palaeontologie 1903:109$134+$ plates II-V.

Jarvik E. 1996. The Devonian tetrapod Ichthyostega. Fossils \& Strata 40:1-206.

Jeannot AM, Damiani R, Rubidge BS. 2006. Cranial anatomy of the Early Triassic stereospondyl Lydekkerina huxleyi (Tetrapoda: Temnospondyli) and the taxonomy of South African lydekkerinids. Journal of Vertebrate Paleontology 26:822-838.

Jenkins FA Jr, Walsh DM, Carroll RL. 2007. Anatomy of Eocaecilia micropodia, a limbed caecilian of the Early Jurassic. Bulletin of the Museum of Comparative Zoology 158:285365. DOI 10.3099/0027-4100(2007)158[285:AOEMAL]2.0.CO;2

Jia J, Gao K-Q. 2016a. A new basal salamandroid (Amphibia, Urodela) from the Late Jurassic of Qinglong, Hebei Province, China. PLOS ONE 11:e0153834. DOI 10.1371/journal.pone.0153834

Jia J, Gao K-Q. 2016b. A new hynobiid-like salamander (Amphibia, Urodela) from Inner Mongolia, China, provides a rare case study of developmental features in an Early Cretaceous fossil urodele. PeerJ 4:e2499. DOI 10.7717/peerj.2499

Johanson Z, Joss J, Boisvert CA, Ericsson R, Sutija M, Ahlberg PE. 2007. Fish fingers: digit homologues in sarcopterygian fish fins. Journal of Experimental Zoology B 308:757768 .

Kangas AT, Evans AR, Thesleff I, Jernvall J. 2004. Nonindependence of mammalian dental characters. Nature 432:211-214. DOI 10.1038/nature02927

Kennedy NK. 2010. Redescription of the postcranial skeleton of Limnoscelis paludis Williston (Diadectomorpha: Limnoscelidae) from the upper Pennsylvanian of El Cobre Canyon, northern New Mexico. New Mexico Museum of Natural History and Science Bulletin 49:211-220.

Kissel R. 2010. Morphology, Phylogeny and Evolution of Diadectidae (Cotylosauria: Diadectomorpha). Doctoral thesis, University of Toronto. http://hdl.handle.net/1807/24357

Klembara J. 1993. The subdivisions and fusions of the exoskeletal skull bones of Discosauriscus austriacus (Makowsky 1876) and their possible homologues in rhipidistians. Paläontologische Zeitschrift 67:145-168.

Klembara J. 1997. The cranial anatomy of Discosauriscus Kuhn, a seymouriamorph tetrapod from the Lower Permian of the Boskovice Furrow (Czech Republic). Philosophical Transactions of the Royal Society B 352:257-302.

Klembara J. 2009. New cranial and dental features of Discosauriscus austriacus (Seymouriamorpha, Discosauriscidae) and the ontogenetic conditions of Discosauriscus. Special Papers in Palaeontology 81:61-69.

Klembara J. 2011. The cranial anatomy, ontogeny, and relationships of Karpinskiosaurus secundus (Amalitzky) (Seymouriamorpha, Karpinskiosauridae) from the Upper Permian of European Russia. Zoological Journal of the Linnean Society 161:184-212. 
5568

5569

5570

5571

5572

5573

5574

5575

5576

5577

5578

5579

5580

5581

5582

5583

5584

5585

5586

5587

5588

5589

5590

5591

5592

5593

5594

5595

5596

5597

5598

5599

5600

5601

5602

5603

5604

5605

5606

5607

5608

5609

5610

5611

5612

5613

Klembara J, Bartík I. 2000. The postcranial skeleton of Discosauriscus Kuhn, a seymouriamorph tetrapod from the Lower Permian of the Boskovice Furrow (Czech Republic). Transactions of the Royal Society of Edinburgh 90:287-316.

Klembara J, Clack JA, Čerňanský A. 2010. The anatomy of [the] palate of Chroniosaurus dongusensis (Chroniosuchia, Chroniosuchidae) from the Upper Permian of Russia. Palaeontology 53:1147-1153.

Klembara J, Ruta M. 2004a. The seymouriamorph tetrapod Utegenia shpinari from the ?Upper Carboniferous - Lower Permian of Kazakhstan. Part I: cranial anatomy and ontogeny. Transactions of the Royal Society of Edinburgh 94:45-74.

Klembara J, Ruta M. 2004b. The seymouriamorph tetrapod Utegenia shpinari from the ?Upper Carboniferous - Lower Permian of Kazakhstan. Part II: Postcranial anatomy and relationships. Transactions of the Royal Society of Edinburgh 94:75-93.

Klembara J, Ruta M. 2005a. The seymouriamorph tetrapod Ariekanerpeton sigalovi from the Lower Permian of Tadzhikistan. Part I: Cranial anatomy and ontogeny. Transactions of the Royal Society of Edinburgh 96:43-70.

Klembara J, Ruta M. 2005b. The seymouriamorph tetrapod Ariekanerpeton sigalovi from the Lower Permian of Tadzhikistan. Part II: Postcranial anatomy and relationships. Transactions of the Royal Society of Edinburgh 96:71-93.

Klembara J, Steyer JS. 2012. A new species of Sclerocephalus (Temnospondyli: Stereospondylomorpha) from the Early Permian of the Boskovice Basin (Czech Republic). Journal of Paleontology 86:302-310.

Klembara J, Tomášik A, Kathe W. 2002. Subdivisions, fusions and extended sutural areas of dermal skull bones in Discosauriscus KUHN (Seymouriamorpha). Neues Jahrbuch für Geologie und Paläontologie Abhandlungen 223:317-349.

Klembara J, Berman DS, Henrici AC, Čerňanský A. 2005. New structures and reconstructions of the skull of the seymouriamorph Seymouria sanjuanensis Vaughn. Annals of Carnegie Museum 74:217-224.

Klembara J, Berman DS, Henrici AC, Čerňanský A, Werneburg R. 2006. Comparison of cranial anatomy and proportions of similarly sized Seymouria sanjuanensis and Discosauriscus austriacus. Annals of Carnegie Museum 75:37-49.

Klembara J, Berman DS, Henrici AC, Čerňanský A, Werneburg R, Martens T. 2007. First description of skull of Lower Permian Seymouria sanjuanensis (Seymouriamorpha: Seymouriidae) at an early juvenile growth stage. Annals of Carnegie Museum 76:53-72.

Klembara J, Clack JA, Milner AR, Ruta M. 2014. Cranial anatomy, ontogeny, and relationships of the Late Carboniferous tetrapod Gephyrostegus bohemicus Jaekel, 1902. Journal of Vertebrate Paleontology 34:774-792. DOI 10.1080/02724634.2014.837055

[Konzhukova YeD] Конжукова ЕД. 1955. Platyops stuckenbergi Trautsch.архегозавроидный лабиринтодонт нижних зон верхней перми Приуралья [Platyops stuckenbergi Trautsch(old). - an archegosauroid labyrinthodont of the lower zones of the Upper Permian of the Ural foreland]. Труды палеонтологического института Академии наук ССCP [Works of the Paleontological Institute of the Academy of Sciences of the USSR] XLIX:89-127.

Krätschmer K. 2006. Oestocephalus guettleri n. sp. - Erster artikulierter Aistopode aus dem Rotliegend des südwestdeutschen Saar-Nahe-Becken[s]. Geowissenschaftliche Beiträge zum [s]aarpfälzischen Rotliegenden 4:47-76. [In German with short English abstract.] http://permfossil.de/images/stories/fossil/Band_4_2_Oestocephalus.pdf 
5614 Langer MC, Ezcurra MD, Rauhut OWM, Benton MJ, Knoll F, McPhee BW, Novas FE,

5615

5616

5617

5618

5619

5620

5621

5622

5623

5624

5625

5626

5627

5628

5629

5630

5631

5632

5633

5634

5635

5636

5637

5638

5639

5640

5641

5642

5643

5644

5645

5646

5647

5648

5649

5650

5651

5652

5653

5654

5655

5656

5657

5658

5659
Pol D, Brusatte SL. 2017. Untangling the dinosaur family tree. Nature 551:E1-E3. DOI 10.1038/nature24011

Langston W Jr. 1953. Permian amphibians from New Mexico. University of California Publications - Bulletin of the Department of Geology 29:349-416.

Langston W Jr. 1965. Oedaleops campi (Reptilia: Pelycosauria) new genus and species from the Lower Permian of New Mexico, and the family Eothyrididae. Bulletin of the Texas Memorial Museum 9:3-47.

Laurin M. 1996a. A redescription of the cranial anatomy of Seymouria baylorensis, the best known seymouriamorph (Vertebrata: Seymouriamorpha). PaleoBios 17:1-16.

Laurin M. 1996b. A reevaluation of Ariekanerpeton, a Lower Permian seymouriamorph (Tetrapoda: Seymouriamorpha) from Tadzhikistan. Journal of Vertebrate Paleontology 16:653-665.

Laurin M. 1996c. A reappraisal of Utegenia, a Permo-Carboniferous seymouriamorph (Tetrapoda: Batrachosauria) from Kazakhstan. Journal of Vertebrate Paleontology 16:374-383.

Laurin M. 1998a. The importance of global parsimony and historical bias in understanding tetrapod evolution. Part I. Systematics, middle ear evolution, and jaw suspension. Annales des Sciences Naturelles, Zoologie, 13e Série 19:1-42.

Laurin M. 1998b. A reevaluation of the origin of pentadactyly. Evolution 52:1476-1482.

Laurin M. 2000. Seymouriamorphs. In: Heatwole H, Carroll RL, ed. Amphibian Biology. Chipping Norton: Surrey Beatty \& Sons, 1064-1080.

Laurin M. 2002. Tetrapod phylogeny, amphibian origins, and the definition of the name Tetrapoda. Systematic Biology 51:364-369.

Laurin M, Reisz RR. 1999. A new study of Solenodonsaurus janenschi, and a reconsideration of amniote origins and stegocephalian evolution. Canadian Journal of Earth Sciences | Revue canadienne des Sciences de la Terre 36:1239-1255.

Laurin M, Soler-Gijón R. 2001. The oldest stegocephalian from the Iberian Peninsula: evidence that temnospondyls were euryhaline. Comptes Rendus de l'Académie des Sciences de Paris, Sciences de la vie/Life Sciences 324:495-501.

Laurin M, Soler-Gijón R. 2006. The oldest known stegocephalian (Sarcopterygii: Temnospondyli) from Spain. Journal of Vertebrate Paleontology 26:284-299.

Lebedev OA, Coates MI. 1995. The postcranial skeleton of the Devonian tetrapod Tulerpeton curtum Lebedev. Zoological Journal of the Linnean Society 114:307-348.

Lee MSY, Cau A, Naish D, Dyke GJ. 2014. Sustained miniaturization and anatomical innovation in the dinosaurian ancestors of birds. Science 345:562-566. DOI $10.1126 /$ science. 1252243

Lillich R, Schoch R. 2007. Finally grown up - the significance of adult Micromelerpeton [abstract]. Journal of Vertebrate Paleontology 27 (suppl. to issue 3): 106A. PDF of all abstracts: http://vertpaleo.org/Annual-Meeting/Future-Past-Meetings/SVP-67th-AnnualMeeting-Summary/JVPabstracts2007.aspx

Lombard RE, Bolt JR. 1979. Evolution of the tetrapod ear: an analysis and reinterretation. Biological Journal of the Linnean Society 11:19-76.

Lombard RE, Bolt JR. 1988. Evolution of the stapes in Paleozoic tetrapods - conservative and radical hypotheses. In: Fritzsch B, Ryan MJ, Wilczynski W, Walkoviak W, ed. The evolution of the amphibian auditory system. New York: Wiley, 37-67. 
5660 Lombard RE, Bolt JR. 1995. A new primitive tetrapod, Whatcheeria deltae, from the Lower

5661

5662

5663

5664

5665

5666

5667

5668

5669

5670

5671

5672

5673

5674

5675

5676

5677

5678

5679

5680

5681

5682

5683

5684

5685

5686

5687

5688

5689

5690

5691

5692

5693

5694

5695

5696

5697

5698

5699

5700

5701

5702

5703 Carboniferous of Iowa. Palaeontology 38:471-494.

Lombard RE, Bolt JR. 1999. A microsaur from the Mississippian of Illinois and a standard format for morphological characters. Journal of Paleontology 73:908-923.

Lombard RE, Bolt JR. 2006. The mandible of Whatcheeria deltae, an early tetrapod from the late Mississippian of Iowa. In: Carrano MT, Gaudin TJ, Blob RW, Wible JR, ed. Amniote paleobiology: perspectives on the evolution of mammals, birds, and reptiles. Chicago: The University of Chicago Press, 21-52.

Lukševičs E, Ahlberg PE, Clack JA. 2003. The dermal skull roof and braincase of the early tetrapod Ventastega curonica from the Late Devonian of Latvia. Ichthyolith Issues, Special Publication 7.

MacIver MA, Schmitz L, Mugan U, Murphey TD, Mobley CD. 2017. Massive increase in visual range preceded the origin of terrestrial vertebrates. Proceedings of the National Academy of Sciences of the United States of America 114:E2375-E2384. DOI 10.1073/pnas.1615563114

Maddin HC, Anderson JS. 2012. Evolution of the amphibian ear with implications for lissamphibian phylogeny: insight gained from the caecilian inner ear. Fieldiana Life and Earth Sciences 5:59-76. DOI 10.3158/2158-5520-5.1.59

Maddin HC, Jenkins FA Jr, Anderson JS. 2012. The braincase of Eocaecilia micropodia (Lissamphibia, Gymnophiona) and the origin of caecilians. PLOS ONE 7:e50743. DOI 10.1371/journal.pone.0050743

Maddin HC, Olori JC, Anderson JS. 2011. A redescription of Carrolla craddocki (Lepospondyli: Brachystelechidae) based on high-resolution CT, and the impacts of miniaturization and fossoriality on morphology. Journal of Morphology 272:722-743. DOI 10.1002/jmor.10946

Maddin HC, Reisz RR, Anderson JS. 2010. Evolutionary development of the neurocranium in Dissorophoidea (Tetrapoda: Temnospondyli), an integrative approach. Evolution \& Development 12:393-403. DOI 10.1111/j.1525-142X.2010.00426.x

Maddin HC, Venczel M, Gardner JD, Rage J-C. 2013a. Micro-computed tomography study of a three-dimensionsally preserved neurocranium of Albanerpeton (Lissamphibia, Albanerpetontidae) from the Pliocene of Hungary. Journal of Vertebrate Paleontology 33:568-587. DOI 10.1080/02724634.2013.722899

Maddin HC, Fröbisch NB, Evans DC, Milner AR. 2013b. Reappraisal of the Early Permian amphibamid Tersomius texensis and some referred material. Comptes Rendus Palevol 12:447-461. DOI 10.1016/j.crpv.2013.06.007

Maddison WP. 1993. Missing data versus missing characters in phylogenetic analysis. Systematic Biology 42:567-581.

Maddison WP, Maddison DR. 2017. Mesquite: a modular system for evolutionary analysis. Version 3.31. http://mesquiteproject.wikispaces.com/

Marjanović D. 2010. Phylogeny of the limbed vertebrates with special consideration of the origin of the modern amphibians. Doctoral thesis, Université Pierre et Marie Curie (Paris) and Universität Wien (Vienna, Austria). http://othes.univie.ac.at/12920

Marjanović D, Laurin M. 2007. Fossils, molecules, divergence times, and the origin of lissamphibians. Systematic Biology 56:369-388. DOI 10.1080/10635150701397635 
5704 Marjanović D, Laurin M. 2008. A reevaluation of the evidence supporting an unorthodox

5705

5706

5707

5708

5709

5710

5711

5712

5713

5714

5715

5716

5717

5718

5719

5720

5721

5722

5723

5724

5725

5726

5727

5728

5729

5730

5731

5732

5733

5734

5735

5736

5737

5738

5739

5740

5741

5742

5743

5744

5745

5746

5747

5748 hypothesis on the origin of extant amphibians. Contributions to Zoology 77:149-199. http://www.contributionstozoology.nl/vol77/nr03/a02

Marjanović D, Laurin M. 2009. The origin(s) of modern amphibians: a commentary. Evolutionary Biology 36:336-338. DOI 10.1007/s11692-009-9065-8

Marjanović D, Laurin M. 2013a. The origin(s) of extant amphibians: a review with emphasis on the "lepospondyl hypothesis". Geodiversitas 35:207-272. DOI 10.5252/g2013n1a8

Marjanović D, Laurin M. 2013b (printed 2014). An updated paleontological timetree of lissamphibians, with comments on the anatomy of Jurassic crown-group salamanders (Urodela). Historical Biology 26:535-550. DOI 10.1080/08912963.2013.797972

Marjanović D, Laurin M. 2015. Reevaluation of the largest published morphological data matrix for phylogenetic analysis of Paleozoic limbed vertebrates. PeerJ PrePrints 3:e1596v1. DOI 10.7287/peerj.preprints.1596v1 [first preprint version of the present work]

Marjanović D, Laurin M. 2016. Reevaluation of the largest published morphological data matrix for phylogenetic analysis of Paleozoic limbed vertebrates. PeerJ PrePrints 4:e1596v2. DOI 10.7287/peerj.preprints.1596v2 [second preprint version of the present work]

Marjanović D, Laurin M. 2018. Reproducibility in phylogenetics: reevaluation of the largest published morphological data matrix for phylogenetic analysis of Paleozoic limbed vertebrates. PeerJ Preprints 6:e1596v3. DOI 10.7287/peerj.preprints.1596v3 [third preprint version of the present work]

Marjanović D, Witzmann F. 2015. An extremely peramorphic newt (Urodela: Salamandridae: Pleurodelini) from the latest Oligocene of Germany, and a new phylogenetic analysis of extant and extinct salamandrids. PLOS ONE 10:e137068. DOI 10.1371/journal.pone.0137068

Martín C, Alonzo-Zarazaga MA, Sanchiz B. 2012. Nomenclatural notes on living and fossil amphibians. Graellsia 68:159-180. DOI 10.3989/graellsia.2012.v68.056

Matsumoto R, Evans SE. 2018. The first record of albanerpetontid amphibians (Amphibia: Albanerpetontidae) from East Asia. PLOS ONE 13:e0189767. DOI 10.1971/journal.pone.0189767

Matsumoto R, Buffetaut É, Escuillié F, Hervet S, Evans SE. 2013. New material of the choristodere Lazarussuchus (Diapsida, Choristodera) from the Paleocene of France. Journal of Vertebrate Paleontology 33:319-339. DOI 10.1080/02724634.2012.716274

McGowan GJ. 2002. Albanerpetontid amphibians from the Lower Cretaceous of Spain and Italy: a description and reconsideration of their systematics. Zoological Journal of the Linnean Society 135:1-32.

McHugh JB. 2012. Temnospondyl ontogeny and phylogeny, a window into terrestrial ecosystems during the Permian-Triassic mass extinction. Doctoral thesis, The University of Iowa. http://ir.uiowa.edu/etd/2942

Meckert D. 1993. Der Schultergürtel des Sclerocephalus haeuseri GoLDFUSS, $1847 \mathrm{im}$ Vergleich mit Eryops COPE, 1877 (Eryopoida [sic], Amphibia, Perm). Palaeontographica A 229:113-140.

Milner AR. 1980. The temnospondyl amphibian Dendrerpeton from the Upper Carboniferous of Ireland. Palaeontology 23:125-141. 
5749

5750

5751

5752

5753

5754

5755

5756

5757

5758

5759

5760

5761

5762

5763

5764

5765

5766

5767

5768

5769

5770

5771

5772

5773

5774

5775

5776

5777

5778

5779

5780

5781

5782

5783

5784

5785

5786

5787

5788

5789

5790

5791

5792

5793

5794

Milner AR. 1996. A revision of the temnospondyl amphibians from the Upper Carboniferous of Joggins, Nova Scotia. Special Papers in Palaeontology 52:81-103.

Milner A[R]. 2007. Mordex laticeps and the base of the Trematopidae [abstract]. Journal of Vertebrate Paleontology 27 (suppl. to issue 3): 118A. PDF of all abstracts: http://vertpaleo.org/Annual-Meeting/Future-Past-Meetings/SVP-67th-Annual-MeetingSummary/JVPabstracts2007.aspx

Milner AC, Lindsay W. 1998. Postcranial remains of Baphetes and their bearing on the relationships of the Baphetidae (=Loxommatidae). Zoological Journal of the Linnean Society 122:211-235.

Milner AC, Milner AR, Walsh SA. 2009. A new specimen of Baphetes from Nýřany, Czech Republic, and the intrinsic relationships of the Baphetidae. Acta Zoologica (Stockholm) 90 (Suppl. 1): 318-334.

Milner AC, Ruta M. 2009. A revision of Scincosaurus (Tetrapoda, Nectridea) from the Moscovian of Nýrany, Czech Republic, and the phylogeny and interrelationships of nectrideans. Special Papers in Palaeontology 81:71-89.

Milner AR, Schoch RR. 2013. Trimerorhachis (Amphibia: Temnospondyli) from the Lower Permian of Texas and New Mexico: cranial osteology, taxonomy and biostratigraphy. Neues Jahrbuch für Geologie und Paläontologie Abhandlungen 270:91-128.

Milner AR, Sequeira SEK. 1994. The temnospondyl amphibians from the Viséan of East Kirkton, West Lothian, Scotland. Transactions of the Royal Society of Edinburgh: Earth Sciences 84:331-361.

Milner AR, Sequeira SEK. 2003. Revision of the amphibian genus Limnerpeton (Temnospondyli) from the Upper Carboniferous of the Czech Republic. Acta Palaeontologica Polonica 48:123-141.

Milner AR, Sequeira SEK. 2011. The amphibian Erpetosaurus radiatus (Temnospondyli, Dvinosauria) from the middle Pennsylvanian of Linton, Ohio: morphology and systematic position. Special Papers in Palaeontology 86:57-73.

Mondéjar-Fernández J, Clément G, Sanchez S. 2014. New insights into the scales of the Devonian tetrapod Tulerpeton curtum Lebedev, 1984. Journal of Vertebrate Paleontology 34:1454-1459. DOI 10.1080/02724634.2014.877474

Montanari SA. 2012. Paleobiology, paleoecology, and morphology of vertebrates: new approaches to old questions. Doctoral thesis, American Museum of Natural History. http://hdl.handle.net/2246/6471

Mortimer M. 2006. [Re: Juravenator: or How not to perform a phylogenetic analysis] [mailinglist post]. In: Rowe M, Kirkaldy M, moderators. Dinosaur Mailing List. http://dml.cmnh.org/2006Mar/msg00244.html

Mortimer M. 2017. Ornithoscelida Tested- [sic] Adding taxa and checking characters [blog post]. In: Mortimer M. The Theropod Database Blog. http://theropoddatabase.blogspot.com/2017/03/ornithoscelida-tested-adding-taxaand.html

Mortimer M. 2018. Testing alternative stem bird topologies in Cau, 2018 [blog post]. In: Mortimer M. The Theropod Database Blog. http://theropoddatabase.blogspot.com/2018/05/testing-alternative-stem-bird.html

Moss JL. 1972. The morphology and phylogenetic relationships of the lower Permian tetrapod Tseajaia campi Vaughn (Amphibia: Seymouriamorpha). University of California Publications: Bulletin of the Department of Geology 98:1-71. 
5795

5796

5797

5798

5799

5800

5801

5802

5803

5804

5805

5806

5807

5808

5809

5810

5811

5812

5813

5814

5815

5816

5817

5818

5819

5820

5821

5822

5823

5824

5825

5826

5827

5828

5829

5830

5831

5832

5833

5834

5835

5836

5837

5838

5839

Motulsky H. 2018. QuickCalcs. La Jolla: GraphPad Software. https://www.graphpad.com/quickcalcs/binomial1/

Müller J, Reisz RR. 2006. The phylogeny of early eureptiles: comparing parsimony and Bayesian approaches in the investigation of a basal fossil clade [sic - basal fossil members of an extant clade]. Systematic Biology 55:503-511.

Nyakatura JA, Allen VR, Lauströer J, Andikfar A, Danczak M, Ullrich H-J, Hufenbach W, Martens T, Fischer MS. 2015. A three-dimensional skeletal reconstruction of the stemamniote Orobates pabsti (Diadectidae): analyses of body mass, centre of mass position, and joint mobility. PLOS ONE 10:e0137284. DOI 10.1371/journal.pone.0137284

O'Keefe FR, Wagner PJ. 2001. Inferring and testing hypotheses of cladistic character dependence by using character compatibility. Systematic Biology 50:657-675.

Olori JC. 2015. Skeletal morphogenesis of Microbrachis and Hyloplesion (Tetrapoda: Lepospondyli), and implications for the developmental patterns of extinct, early tetrapods. PLOS ONE 10:e0128333. DOI 10.1371/journal.pone.0128333

Olson EC. 1941. The family Trematopsidae. Journal of Geology 49:149-176.

Olson EC. 1947. The family Diadectidae and its bearing on the classification of reptiles. Fieldiana: Geology 11:1-53.

O'Reilly JE, Puttick MN, Parry L, Tanner AR, Tarver JE, Fleming J, Pisani D, Donoghue PCJ. 2016. Bayesian methods outperform parsimony but at the expense of precision in the estimation of phylogeny from discrete morphological data. Biology Letters 12:20160081. DOI 10.1098/rsbl.2016.0081

O’Reilly JE, Puttick MN, Pisani D, Donoghue PCJ. 2017 (printed 2018). Probabilistic methods surpass parsimony when assessing clade support in phylogenetic analyses of discrete morphological data. Palaeontology 61:105-118. DOI 10.1111/pala.12330

Ossa-Fuentes L, Mpodozis J, Vargas AO. 2015. Bird embryos uncover homology and evolution of the dinosaur ankle. Nature Communications 6:8902. DOI $10.1038 /$ ncomms 9902

Panchen AL. 1964. The cranial anatomy of two Coal Measure anthracosaurs. Philosophical Transactions of the Royal Society of London B 247:593-636.

Panchen AL. 1972. The skull and skeleton of Eogyrinus attheyi Watson (Amphibia: Labyrinthodontia). Philosophical Transactions of the Royal Society of London B 263:279-326.

Panchen AL. 1975. A new genus and species of anthracosaur amphibian from the Lower Carboniferous of Scotland and the status of Pholidogaster pisciformis Huxley. Philosophical Transactions of the Royal Society of London B 269:581-637.

Panchen AL. 1977. On Anthracosaurus russelli Huxley (Amphibia: Labyrinthodontia) and the family Anthracosauridae. Philosophical Transactions of the Royal Society of London B 279:447-512.

Panchen AL. 1985. On the amphibian Crassigyrinus scoticus Watson from the Carboniferous of Scotland. Philosophical Transactions of the Royal Society of London B 309:505-568.

Panchen AL, Smithson TR. 1990. The pelvic girdle and hind limb of Crassigyrinus scoticus (Lydekker) from the Scottish Carboniferous and the origin of the tetrapod pelvic skeleton. Transactions of the Royal Society of Edinburgh 81:31-44.

Pardo J. 2011. The morphology and relationships of the Carboniferous-Permian nectridean Diploceraspis burkei [abstract]. Journal of Vertebrate Paleontology, Program and 
5840

5841

5842

5843

5844

5845

5846

5847

5848

5849

5850

5851

5852

5853

5854

5855

5856

5857

5858

5859

5860

5861

5862

5863

5864

5865

5866

5867

5868

5869

5870

5871

5872

5873

5874

5875

5876

5877

5878

5879

5880

5881

5882

5883

5884

5885

Abstracts 2011, 170-171. PDF of all abstracts: http://vertpaleo.org/AnnualMeeting/Future-Past-Meetings/MeetingPdfs/SVP11Abstracts_Full.aspx

Pardo JD. 2014. Morphology, ontogeny, and phylogenetic relationships of the PermoCarboniferous tetrapod Brachydectes newberryi from the Council Grove Group, Nebraska, USA. MSc thesis, University of Calgary. http://hdl.handle.net/11023/1914

Pardo JD, Anderson JS. 2016. Cranial morphology of the Carboniferous-Permian tetrapod Brachydectes newberryi (Lepospondyli, Lysorophia): new data from $\mu \mathrm{CT}$. PLOS ONE 11:e0161823. DOI 10.1371/journal.pone.0161823

Pardo JD, Small BJ, Huttenlocker AK. 2017. Stem caecilian from the Triassic of Colorado sheds light on the origins of Lissamphibia. Proceedings of the National Academy of Sciences of the United States of America 114:E5389-E5395. DOI 10.1073/pnas. 1706752114

Pardo JD, Szostakiwskyj M, Anderson JS. 2015. Cranial morphology of the brachystelechid 'microsaur' Quasicaecilia texana Carroll provides new insights into the diversity and evolution of braincase morphology in recumbirostran 'microsaurs'. PLOS ONE 10:e0130359. DOI 10.1371/journal.pone.0130359

Pardo JD, Szostakiwskyj M, Ahlberg PE, Anderson JS. 2017. Hidden morphological diversity among early tetrapods. Nature 546:642-645. DOI 10.1038/nature22996

Pardo JD, Carter A, Lennie K, Sallan LC, Anderson JS. 2018. New $\mu$ CT data on nectrideans reveals unappreciated complexities in early tetrapod evolution [abstract]. Vertebrate Anatomy Morphology Palaeontology 5:41 [volume number given as 6 throughout the abstracts]. DOI of all abstracts 10.18435/vamp29338

Paton RL, Smithson TR, Clack JA. 1999. An amniote-like skeleton from the Early Carboniferous of Scotland. Nature 398:508-513. DOI 10.1038/19071

Pawley K. 2006. The postcranial skeleton of temnospondyls (Tetrapoda: Temnospondyli). Doctoral dissertation, La Trobe University. http://hdl.handle.net/1959.9/405644

Pawley K. 2007. The postcranial skeleton of Trimerorhachis insignis Cope, 1878 (Temnospondyli: Trimerorhachidae): a plesiomorphic temnospondyl from the Lower Permian of North America. Journal of Paleontology 81:873-894.

Pawley K, Warren A. 2005. A terrestrial stereospondyl from the Lower Triassic of South Africa: the postcranial skeleton of Lydekkerina huxleyi (Amphibia: Temnospondyli). Palaeontology 48:281-298.

Pawley K, Warren A. 2006. The appendicular skeleton of Eryops megacephalus (Temnospondyli: Eryopoidea) from the Lower Permian of North America. Journal of Paleontology 80:561-580.

Pereira Pacheco C, Eltink E, Müller RT, Dias-da-Silva S. 2016 (printed 2017). A new Permian temnospondyl with Russian affinities from South America, the new family Konzhukoviidae, and the phylogenetic status of Archegosauroidea. Journal of Systematic Palaeontology 15:241-256. DOI 10.1080/14772019.2016.1164763

Perseus Digital Library (Crane GR, ed.; no year given). Tufts University. http://www.perseus.tufts.edu

Pierce SE, Clack JA, Hutchinson JR. 2012. Three-dimensional limb joint mobility in the early tetrapod Ichthyostega. Nature 486:523-526. DOI 10.1038/nature11124

Pierce SE, Ahlberg PE, Hutchinson JR, Molnar JL, Sanchez S, Tafforeau P, Clack JA. 2013. Vertebral architecture in the earliest stem tetrapods. Nature 494:226-229. DOI 10.1038 /nature1 1825 
5886 Planet PJ. 2006. Tree disagreement: Measuring and testing incongruence in phylogenies.

5887

5888

5889

5890

5891

5892

5893

5894

5895

5896

5897

5898

5899

5900

5901

5902

5903

5904

5905

5906

5907

5908

5909

5910

5911

5912

5913

5914

5915

5916

5917

5918

5919

5920

5921

5922

5923

5924

5925

5926

5927

5928

5929
Journal of Biomedical Informatics 39: 86-102. DOI 10.1016/j.jbi.2005.08.008

Polley BP, Reisz RR. 2011. A new Lower Permian trematopid (Temnospondyli:

Dissorophoidea) from Richards Spur, Oklahoma. Zoological Journal of the Linnean

Society 161:789-815. DOI 10.1111/j.1096-3642.2010.00668.x

Porro LB, Clack JA, Rayfield EJ. 2015. Anatomy and three-dimensional reconstruction of the skull of the stem tetrapod Crassigyrinus scoticus [abstract]. Journal of Vertebrate Paleontology, Program and Abstracts 2015, 197. PDF of all abstracts: http://vertpaleo.org/PDFS/SVP-2015-Program-and-Abstract-Book-9-22-2015.aspx

Porro LB, Rayfield EJ, Clack JA. 2015. Descriptive anatomy and three-dimensional reconstruction of the skull of the early tetrapod Acanthostega gunnari Jarvik, 1952. PLOS ONE 10:e0118882. DOI 10.1371/journal.pone.0118882

Puttick MN, O'Reilly JE, Tanner AR, Fleming JF, Clark J, Holloway L, LozanoFernandez J, Parry LA, Tarver JE, Pisani D, Donoghue PCJ. 2017. Uncertain-tree: discriminating among competing approaches to the phylogenetic analysis of phenotype data. Proceedings of the Royal Society B 284:20162290. DOI 10.1098/rspb.2016.2290

Pyron RA. 2014. Biogeographic analysis reveals ancient continental vicariance and recent oceanic dispersal in amphibians. Systematic Biology 63:779-797. DOI 10.1093/sysbio/syu042

Rage J-C, Roček Z. 1989. Redescription of Triadobatrachus massinoti (Piveteau, 1936)[,] an anuran amphibian from the Early Triassic. Palaeontographica A 206:1-16.

Rasmussen C, Huttenlocker AK, Irmis R. 2016. A new species of Eryops from the Lower Permian Cedar Mesa Sandstone (Cutler Group) of southeastern Utah and its implications for the phylogeny and biogeography of eryopids [abstract]. Journal of Vertebrate Paleontology, Program and Abstracts 2016, 211. PDF of all abstracts: http://vertpaleo.org/PDFS/2016/SVP-2016-Program-Book-v10-with-covers.aspx

Raven TJ, Maidment SCR. 2018. The systematic position of the enigmatic thyreophoran dinosaur Paranthodon africanus, and the use of basal exemplifiers in phylogenetic analysis. PeerJ 6:e4529. DOI 10.7717/peerj.4529

Reisz RR. 1981. A diapsid reptile from the Pennsylvanian of Kansas. University of Kansas Museum of Natural History Special Publication 7:1-74.

Reisz RR. 2007. The cranial anatomy of basal diadectomorphs and the origin of amniotes. In: Anderson JS, Sues H-D, ed. Major Transitions in Vertebrate Evolution. Bloomington: Indiana University Press, 228-252 + plates 25-27.

Reisz RR, Berman DS, Henrici AC. 2005. A new skull of the cochleosaurid amphibian Chenoprosopus (Amphibia: Temnospondyli) from the Early Permian of New Mexico. New Mexico Museum of Natural History and Science Bulletin 30 (Lucas SG, Zeigler KE, ed. The Nonmarine Permian): 253-255.

Reisz RR, Dilkes DW. 2003. Archaeovenator hamiltonensis, a new varanopid (Synapsida: Eupelycosauria) from the Upper Carboniferous of Kansas. Canadian Journal of Earth Sciences | Revue canadienne des Sciences de la Terre 40:667-678.

Reisz RR, Fröbisch J. 2014. The oldest caseid synapsid from the late Pennsylvanian of Kansas, and the evolution of herbivory in terrestrial vertebrates. PLOS ONE 9:e94518. DOI 10.1371/journal.pone.0094518 
5930 Reisz RR, Godfrey SJ, Scott D. 2009. Eothyris and Oedaleops: do these Early Permian

5931

5932

5933

5934

5935

5936

5937

5938

5939

5940

5941

5942

5943

5944

5945

5946

5947

5948

5949

5950

5951

5952

5953

5954

5955

5956

5957

5958

5959

5960

5961

5962

5963

5964

5965

5966

5967

5968

5969

5970

5971

5972

5973 synapsids from Texas and New Mexico form a clade? Journal of Vertebrate

Paleontology 29:39-47. DOI 10.1671/039.029.0112

Rineau V, Grand A, Zaragüeta R, Laurin M. 2015. Experimental systematics: sensitivity of cladistic methods to polarization and character ordering schemes. Contributions to Zoology 84:129-148. http://www.ctoz.nl/vol84/nr02/a03

Robinson J, Ahlberg PE, Koentges G. 2005. The braincase and middle ear region of Dendrerpeton acadianum (Tetrapoda: Temnospondyli). Zoological Journal of the Linnean Society 143:577-597. DOI 10.1111/j.1096-3642.2005.00156.x

Roček Z, Rage J-C. 2000. Proanuran stages (Triadobatrachus, Czatkobatrachus). In: Heatwole H, Carroll RL, ed. Amphibian Biology. Chipping Norton: Surrey Beatty \& Sons, 12831294.

Roček Z, Wuttke M. 2010. Amphibia of Enspel (Late Oligocene, Germany). Palaeobiodiversity and Palaeoenvironments 90:321-340. DOI 10.1007/s12549-010-0042-0

Romer AS. 1930. The Pennsylvanian tetrapods of Linton, Ohio. Bulletin of the American Museum of Natural History LIX:77-147.

Romer AS. 1957. The appendicular skeleton of the Permian embolomerous amphibian Archeria. Contributions from the Museum of Paleontology, University of Michigan XIII:103-159.

Romer AS. 1963. The larger embolomerous amphibians of the American Carboniferous. Bulletin of the Museum of Comparative Zoology 128:415-454.

Romer AS. 1964. The skeleton of the Lower Carboniferous labyrinthodont Pholidogaster pisciformis. Bulletin of the Museum of Comparative Zoology 131:129-159.

Romer AS, Witter RV. 1942. Edops, a primitive rhachitomous amphibian from the Texas red beds. Journal of Geology (Chicago) 50:925-960.

Rößler R, Zierold T, Feng Z, Kretzschmar R, Merbitz M, Annacker V, Schneider JW. 2012. A snapshot of an Early Permian ecosystem preserved by explosive volcanism: new results from the Chemnitz Petrified Forest, Germany. Palaios 27:814-834. DOI 10.2110/palo.2011.p11-p112r

Ruta M. 2009. Patterns of morphological evolution in major groups of Palaeozoic Temnospondyli (Amphibia: Tetrapoda). Special Papers in Palaeontology 81:91-120.

Ruta M. 2011. Phylogenetic signal and character compatibility in the appendicular skeleton of early tetrapods. Special Papers in Palaeontology 86:31-43. DOI 10.1111/j.14754983.2011.01071.x

Ruta M, Bolt JR. 2006. A reassessment of the temnospondyl amphibian Perryella olsoni from the Lower Permian of Oklahoma. Transactions of the Royal Society of Edinburgh: Earth Sciences 97:113-165. DOI 10.1017/S0263593300001437

Ruta M, Clack JA. 2006. A review of Silvanerpeton miripedes, a stem amniote from the Lower Carboniferous of East Kirkton, West Lothian, Scotland. Transactions of the Royal Society of Edinburgh: Earth Sciences 97:31-63. DOI 10.1017/S0263593300001395

Ruta M, Coates MI. 2007. Dates, nodes and character conflict: addressing the lissamphibian origin problem. Journal of Systematic Palaeontology 5:69-122. DOI $10.1017 /$ S 1477201906002008

Ruta M, Coates MI, Quicke DLJ. 2003. Early tetrapod relationships revisited. Biological Reviews 78:251-345. DOI 10.1017/S1464793102006103 
5974 Ruta M, Milner AR, Coates MI. 2002. The tetrapod Caerorhachis bairdi Holmes and Caroll

5975

5976

5977

5978

5979

5980

5981

5982

5983

5984

5985

5986

5987

5988

5989

5990

5991

5992

5993

5994

5995

5996

5997

5998

5999

6000

6001

6002

6003

6004

6005

6006

6007

6008

6009

6010

6011

6012

6013

6014

6015

6016

6017

6018 from the Lower Carboniferous of Scotland. Transactions of the Royal Society of

Edinburgh: Earth Sciences 92:229-261. DOI 10.1017/S0263593300000249

Salisbury BA, Kim J. 2001. Ancestral state estimation and taxon sampling density. Systematic Biology 50:557-564. DOI 10.1080/10635150119819

Sawin HJ. 1941. The cranial anatomy of Eryops megacephalus. Bulletin of the Museum of Comparative Zoology 88:407-463.

Schoch RR. 2002a. The stapes and middle ear of the Permo-Carboniferous tetrapod Sclerocephalus. Neues Jahrbuch für Geologie und Paläontologie Monatshefte 2002:671680.

Schoch RR. 2006. A complete trematosaurid amphibian from the Middle Triassic of Germany. Journal of Vertebrate Paleontology 26:29-43.

Schoch RR. 2009. Evolution of life cycles in early amphibians. Annual Reviews of Earth and Planetary Sciences 37:135-162.

Schoch RR. 2012. Character distribution and phylogeny of the dissorophid temnospondyls. Fossil Record 15:121-137.

Schoch RR. 2013. The evolution of major temnospondyl clades: an inclusive phylogenetic analysis. Journal of Systematic Palaeontology 11:673-705. DOI 10.1080/14772019.2012.699006

Schoch RR, Fröbisch NB. 2006. Metamorphosis and neoteny: alternative pathways in an extinct amphibian clade. Evolution 60:1467-1475.

Schoch RR, Milner AR. 2000. Stereospondyli. Part 3B of Wellnhofer P, ed. Handbuch der Paläoherpetologie | Encyclopedia of Paleoherpetology. Munich: Dr. Friedrich Pfeil.

Schoch RR, Milner AR. 2008. The intrarelationships and evolutionary history of the temnospondyl family Branchiosauridae. Journal of Systematic Palaeontology 6:409-431. DOI 10.1017/S1477201908002460

Schoch RR, Milner AR. 2014. Temnospondyli I. Part 3A2 of Sues H-D, ed. Handbook of Paleoherpetology. Munich: Dr. Friedrich Pfeil.

Schoch RR, Poschmann M, Kupfer A. 2015. The salamandrid Chelotriton paradoxus from Enspel and Randeck Maars (Oligocene-Miocene, Germany). Palaeobiodiversity and Palaeoenvironments 95:77-86. DOI 10.1007/s12549-014-0182-8

Schoch RR, Rubidge BS. 2005. The amphibamid Micropholis from the Lystrosaurus Assemblage Zone of South Africa. Journal of Vertebrate Paleontology 25:502-522. DOI 10.1671/0272-4634(2005)025[0502:TAMFTL]2.0.CO;2

Schoch RR, Voigt S, Buchwitz M. 2010. A chroniosuchid from the Triassic of Kyrgyzstan and analysis of chroniosuchian relationships. Zoological Journal of the Linnean Society 160:515-530. DOI 10.1111/j.1096-3642.2009.00613.x

Schoch RR, Witzmann F. 2009a. Osteology and relationships of the temnospondyl genus Sclerocephalus. Zoological Journal of the Linnean Society 157:135-168. DOI 10.1111/j.1096-3642.2009.00535.x

Schoch RR, Witzmann F. 2009b. The temnospondyl Glanochthon from the Lower Permian Meisenheim Formation of Germany. Special Papers in Palaeontology 81:121-136.

Schoch RR, Witzmann F. 2010 (printed 2011). Bystrow's Paradox - gills, fossils, and the fishto-tetrapod transition. Acta Zoologica (Stockholm) 92:251-265. DOI 10.1111/j.14636395.2010.00456.x 
6019 Sequeira SEK. 1998. The cranial morphology and taxonomy of the saurerpetontid Isodectes

6020

6021

6022

6023

6024

6025

6026

6027

6028

6029

6030

6031

6032

6033

6034

6035

6036

6037

6038

6039

6040

6041

6042

6043

6044

6045

6046

6047

6048

6049

6050

6051

6052

6053

6054

6055

6056

6057

6058

6059

6060

6061

6062

6063

6064 obtusus comb. nov. (Amphibia: Temnospondyli) from the Lower Permian of Texas.

Zoological Journal of the Linnean Society 122:237-259.

Sequeira SEK. 2004. The skull of Cochleosaurus bohemicus Frič, a temnospondyl from the Czech Republic (Upper Carboniferous) and cochleosaurid interrelationships.

Transactions of the Royal Society of Edinburgh: Earth Sciences 94:21-43.

Sequeira SEK. 2009. The postcranium of Cochleosaurus bohemicus Frič, a primitive Upper Carboniferous temnospondyl from the Czech Republic. Special Papers in Palaeontology 81:137-153.

Sequeira SEK, Milner AR. 1993. The temnospondyl amphibian Capetus from the Upper Carboniferous of the Czech Republic. Palaeontology 36:657-680.

Shelley SL, Williamson TE, Brusatte S. 2016. The anatomy of Periptychus carinidens with comments on functional morphology and the phylogeny of 'archaic' Paleocene mammals [abstract]. Journal of Vertebrate Paleontology, Program and Abstracts 2016, 223. PDF of all abstracts: http://vertpaleo.org/PDFS/2016/SVP-2016-Program-Book-v10-withcovers.aspx

Shishkin MA, Rubidge BS, Kitching JW. 1996. A new lydekkerinid (Amphibia, Temnospondyli) from the lower [sic] Triassic of South Africa: implications for evolution of the early capitosauroid cranial pattern. Philosophical Transactions of the Royal Society of London B 351:1635-1659.

Sidor CA. 2013. The vertebrate fauna of the Upper Permian of Niger - VIII. Nigerpeton ricqlesi (Temnospondyli: Cochleosauridae) and tetrapod biogeographic provinces. Comptes Rendus Palevol 12:463-472. DOI 10.1016/j.crpv.2013.05.005

Sigurdsen T. 2008. The otic region of Doleserpeton (Temnospondyli) and its implications for the evolutionary origin of frogs. Zoological Journal of the Linnean Society 154:738-751. DOI 10.1111/j.1096-3642.2008.00459.x

Sigurdsen T, Bolt JR. 2009. The lissamphibian humerus and elbow joint, and the origins of modern amphibians. Journal of Morphology 270:1443-1453. DOI 10.1002/jmor.10769

Sigurdsen T, Bolt JR. 2010. The Lower Permian amphibamid Doleserpeton (Temnospondyli: Dissorophoidea), the interrelationships of amphibamids, and the origin of modern amphibians. Journal of Vertebrate Paleontology 30:1360-1377. DOI $10.1080 / 02724634.2010 .501445$

Sigurdsen T, Green DM. 2011. The origin of modern amphibians: a re-evaluation. Zoological Journal of the Linnean Society 162:457-469. DOI 10.1111/j.1096-3642.2010.00683.x

Sigurdsen T, Green DM, Bishop PJ. 2012. Did Triadobatrachus jump? Morphology and evolution of the anuran forelimb in relation to locomotion in early salientians. Fieldiana Life and Earth Sciences 5:77-89. DOI 10.3158/2158-5520-5.1.77

Simmons MP. 2011a (printed 2012). Misleading results of likelihood-based phylogenetic analyses in the presence of missing data. Cladistics 28:208-222. DOI 10.1111/j.10960031.2011.00375.x

Simmons MP. 2011b (printed 2012). Radical instability and spurious branch support by likelihood when applied to matrices with non-random distributions of missing data. Molecular Phylogenetics and Evolution 62:472-484. DOI 10.1016/j.ympev.2011.10.017

Simões TR, Caldwell MW, Palci A, Nydam RL. 2016a (printed 2017). Giant taxon-character matrices: quality of character constructions remains critical regardless of size. Cladistics 33:198-219. DOI 10.1111/cla.12163 
6065 Simões TR, Caldwell MW, Nydam RL, Jimenez Huidobro P. 2016b. Osteology, phylogeny

6066

6067

6068

6069

6070

6071

6072

6073

6074

6075

6076

6077

6078

6079

6080

6081

6082

6083

6084

6085

6086

6087

6088

6089

6090

6091

6092

6093

6094

6095

6096

6097

6098

6099

6100

6101

6102

6103

6104

6105

6106

6107

6108

6109

6110

and functional morphology of two Jurassic lizard species indicate the early evolution of scansoriality in geckoes [abstract]. Journal of Vertebrate Paleontology, Program and Abstracts 2016, 225. PDF of all abstracts: http://vertpaleo.org/PDFS/2016/SVP-2016Program-Book-v10-with-covers.aspx

Skutschas PP, Gubin YuM. 2012. A new salamander from the late Paleocene-early Eocene of Ukraine. Acta Palaeontologica Polonica 57:135-148. DOI 10.4202/app.2010.0101

Slowinski JB. 1993. "Unordered” versus “ordered” characters. Systematic Biology 42:155-165. DOI 10.1093/sysbio/42.2.155

Smithson TR. 1980. A new labyrinthodont amphibian from the Carboniferous of Scotland. Palaeontology 23:915-923.

Smithson TR. 1982. The cranial morphology of Greererpeton burkemorani Romer (Amphibia: Temnospondyli). Zoological Journal of the Linnean Society 76:29-90.

Smithson TR. 1985. The morphology and relationships of the Carboniferous amphibian Eoherpeton watsoni Panchen. Zoological Journal of the Linnean Society 85:317-410.

Smithson TR. 1994. Eldeceeon rolfei, a new reptiliomorph from the Viséan of East Kirkton, West Lothian, Scotland. Transactions of the Royal Society of Edinburgh: Earth Sciences 84:377-382.

Smithson TR, Clack JA. 2013. Tetrapod appendicular skeletal elements from the Early Carboniferous of Scotland. Comptes Rendus Palevol 12:405-417. DOI 10.1016/j.crpv.2013.06.006

Smithson TR, Carroll RL, Panchen AL, Andrews SM. 1994. Westlothiana lizziae from the Viséan of East Kirkton, West Lothian, Scotland, and the amniote stem. Transactions of the Royal Society of Edinburgh: Earth Sciences 84:383-412.

Smithson TR, Browne MAE, Davies SJ, Marshall JEA, Millward D, Walsh SA, Clack JA. 2017. A new Mississippian tetrapod from Fife, Scotland, and its environmental context. Papers in Palaeontology 3:547-557. DOI 10.1002/spp2.1086

Sookias RB, Böhmer C, Clack JA. 2014. Redescription and phylogenetic analysis of the mandible of an enigmatic Pennsylvanian (Late Carboniferous) tetrapod from Nova Scotia, and the lability of Meckelian jaw ossification. PLOS ONE 9:e109717. DOI 10.1371/journal.pone.0109717

Spindler F, Falconnet J, Fröbisch J. 2015 (printed 2016). Callibrachion and Datheosaurus, two historical and previously mistaken basal caseasaurian synapsids from Europe. Acta Palaeontologica Polonica 61:597-616. DOI 10.4202/app.00221.2015

Spindler F, Werneburg R, Schneider JW, Luthardt L, Annacker V, Rößler R. 2018. First arboreal 'pelycosaurs' (Synapsida: Varanopidae) from the early [sic] Permian Chemnitz Fossil Lagerstätte, SE Germany, with a review of varanopid phylogeny. Paläontologische Zeitschrift 92:315-364. DOI 10.1007/s12542-018-0405-9

Steyer JS, Damiani R, Sidor CA, O'Keefe FR, Larsson HCE, Maga A, Ide Ou. 2006. The vertebrate fauna of the Upper Permian of Niger. IV. Nigerpeton ricqlesi (Temnospondyli: Cochleosauridae), and the edopoid colonization of Gondwana. Journal of Vertebrate Paleontology 26:18-28. DOI 10.1671/0272-4634(2006)26[18:TVFOTU]2.0.CO;2

Strong EE, Lipscomb D. 1999. Character coding and inapplicable data. Cladistics 15:363-371. DOI 10.1111/j.1096-0031.1999.tb00272.x

Sumida SS, Pelletier V, Berman DS. 2014. New information on the basal pelycosaurian-grade synapsid Oedaleops. In: Kammerer CF, Angielczyk KD, Fröbisch J, eds. Early 
6111

6112

6113

6114

6115

6116

6117

6118

6119

6120

6121

6122

6123

6124

6125

6126

6127

6128

6129

6130

6131

6132

6133

6134

6135

6136

6137

6138

6139

6140

6141

6142

6143

6144

6145

6146

6147

6148

6149

6150

6151

6152

6153

6154

6155

6156

evolutionary history of the Synapsida. Dordrecht, Heidelberg, New York, London: Springer, 7-23.

Swofford DL. 2003. PAUP*: Phylogenetic Analysis Using Parsimony (*and other methods). Version 4.0b10. Sunderland, Massachusetts: Sinauer Associates.

Swofford DL. 2017. PAUP*: Phylogenetic Analysis Using Parsimony (*and other methods). Version 4.0a158. http://phylosolutions.com/paup-test/

Szostakiwskyj M, Pardo JD, Anderson JS. 2015. Micro-CT study of Rhynchonkos stovalli (Lepospondyli, Recumbirostra), with description of two new genera. PLOS ONE 10:e0127307. DOI 10.1371/journal.pone.0127307

Tschopp E, Mateus O, Benson RBJ. 2015. A specimen-level phylogenetic analysis and taxonomic revision of Diplodocidae (Dinosauria, Sauropoda). PeerJ 3:e857. DOI $10.7717 /$ peerj. 857

Tykoski RL. 2005. Anatomy, ontogeny, and phylogeny of coelophysoid theropods. Doctoral dissertation, The University of Texas at Austin. http://hdl.handle.net/2152/3992

Vallin G, Laurin M. 2004. Cranial morphology and affinities of Microbrachis, and a reappraisal of the phylogeny and lifestyle of the first amphibians. Journal of Vertebrate Paleontology 24:56-72. DOI 10.1671/5.1

Venczel M, Gardner JD. 2005. The geologically youngest albanerpetontid amphibian, from the Lower Pliocene of Hungary. Palaeontology 48:1273-1300. DOI 10.1111/j.14754983.2005.00512.x

Vorobyeva E, Schultze H-P. 1991. Description and systematics of panderichthyid fishes with comments on their relationship to tetrapods. In: Schultze H-P, Trueb L, ed. Origins of the Higher Groups of Tetrapods-Controversy and Consensus. Ithaca: Cornell University Press, 68-109.

Wake DB. 2009. What salamanders have taught us about evolution. Annual Review of Ecology, Evolution, and Systematics 40:333-352. DOI 10.1146/annurev.ecolsys.39.110707.173552

Wang Y, Evans SE. 2006. A new short-bodied salamander from the Upper Jurassic/Lower Cretaceous of China. Acta Palaeontologica Polonica 51:127-130. http://app.pan.pl/article/item/app51-127.html

Warren A. 1999. Karoo tupilakosaurid: a relict from Gondwana. Transactions of the Royal Society of Edinburgh: Earth Sciences 89:145-160. DOI 10.1017/S0263593300007094

Warren A. 2007. New data on Ossinodus pueri, a stem tetrapod from the Early Carboniferous of Australia. Journal of Vertebrate Paleontology 27:850-862. DOI 10.1671/02724634(2007)27[850:NDOOPA]2.0.CO;2

Warren A, Rozefelds AC, Bull S. 2011. Tupilakosaur-like vertebrae in Bothriceps australis, an Australian brachyopid stereospondyl. Journal of Vertebrate Paleontology 31:738-753. DOI 10.1080/02724634.2011.590563

Warren A, Turner S. 2004. The first stem tetrapod from the Lower Carboniferous of Gondwana. Palaeontology 47:151-184. DOI 10.1111/j.0031-0239.2004.00353.x

Watanabe A. 2015 (printed 2016). The impact of poor sampling of polymorphism on cladistic analysis. Cladistics 32:317-334. DOI 10.1111/cla.12130

Watanabe A. 2017a. [tweet]. https://twitter.com/akiopteryx/status/844954395431391232

Watanabe A. 2017b. [tweet]. https://twitter.com/akiopteryx/status/845284614319063040

Wellstead CF. 1982. A Lower Carboniferous aïstopod amphibian from Scotland. Palaeontology 25:193-208. 
6157 Wellstead CF. 1991. Taxonomic revision of the Lysorophia, Permo-Carboniferous lepospondyl

6158

6159 amphibians. Bulletin of the American Museum of Natural History 209:1-90.

6160

6161

6162

6163

6164

6165

6166

6167

6168

6169

6170

6171

6172

6173

6174

6175

6176

6177

6178

6179

6180

6181

6182

6183

6184

6185

6186

6187

6188

6189

6190

6191

6192

6193

6194

6195

6196

6197

6198

6199

6200

6201

Werneburg R. 1991. Die Branchiosaurier aus dem Unterrotliegend des Döhlener Beckens bei Dresden. Veröffentlichungen des Naturhistorischen Museums Schleusingen 6:75-99.

Werneburg R. 2007a. Timeless design: colored pattern of skin in Early Permian branchiosaurids (Temnospondyli: Dissorophoidea). Journal of Vertebrate Paleontology 27:1047-1050. DOI 10.1671/0272-4364(2007)27[1047:TDCPOS]2.0.CO;2

Werneburg R. 2007b. Der „Manebacher Saurier“ - ein neuer großer Eryopide (Onchiodon) aus dem Rotliegend (Unter-Perm) des Thüringer Waldes. Veröffentlichungen des Naturhistorischen Museums Schleusingen 22:3-40.

Werneburg R. 2009. The Permotriassic branchiosaurid Tungussogyrinus Efremov, 1939 (Temnospondyli, Dissorophoidea) from Siberia restudied. Fossil Record 12:105-120. DOI 10.1002/mmng.200900001

Werneburg R. 2012a. Dissorophoide Amphibien aus dem Westphalian [sic] D (Ober-Karbon) von Nýřany in Böhmen (Tschechische Republik) - der Schlüssel zum Verständnis der frühen ,Branchiosaurier'. Semana - Naturwissenschaftliche Veröffentlichungen des Naturhistorischen Museums Schleusingen 27:3-50.

Werneburg R. 2012b. Eryopid temnospondyls from different ecosystems of Pennsylvanian up to Upper Permian Laurasia [abstract]. Terra Nostra 2012(3):196.

Werneburg R, Berman DS. 2012. Revision of the aquatic eryopid temnospondyl Glaukerpeton avinoffi Romer, 1952, from the Upper Pennsylvanian of North America. Annals of Carnegie Museum 81:33-60. DOI 10.2992/007.081.0103

Werneburg R, Steyer JS. 2002. Revision of Cheliderpeton vranyi FRITSCH, 1877 (Amphibia, Temnospondyli) from the Lower Permian of Bohemia (Czech Republic). Paläontologische Zeitschrift 76:149-162. DOI 10.1007/BF02988193

Werneburg R, Steyer JS, Sommer G, Gand G, Schneider JW, Vianey-Liaud M. 2007. The earliest tupilakosaurid amphibian with diplospondylous vertebrae from the Late Permian of southern France. Journal of Vertebrate Paleontology 27:26-30. DOI 10.1671/02724634(2007)27[26:TETAWD]2.0.CO;2

White TE. 1939. Osteology of Seymouria baylorensis Broili. [Lack of italics in the original.] Bulletin of the Museum of Comparative Zoölogy 85:325-409.

Wiens JJ. 2001. Character analysis in morphological phylogenetics: problems and solutions. Systematic Biology 50:689-699. DOI 10.1080/106351501753328811

Wiens JJ. 2011. Re-evolution of lost mandibular teeth in frogs after more than 200 million years, and re-evaluating Dollo's law. Evolution 65:1283-1296. DOI 10.1111/j.15585646.2011.01221.x

Wiens JJ, Bonett RM, Chippindale PT. 2005. Ontogeny discombobulates phylogeny: paedomorphosis and higher-level salamander relationships. Systematic Biology 54:91110. DOI 10.1080/10635150590906037

Williston SW. 1909. The skull and extremities of Diplocaulus. Transactions of the Kansas Academy of Science 22:122-131.

Witzmann F. 2006. Cranial morphology and ontogeny of the Permo-Carboniferous temnospondyl Archegosaurus decheni Goldfuss, 1847 from the Saar-Nahe Basin, Germany. Transactions of the Royal Society of Edinburgh: Earth Sciences 96:131-162. DOI 10.1017/S0263593300001279 
6202 Witzmann F. 2007. The evolution of the scalation pattern in temnospondyl amphibians.

6203

6204

6205

6206

6207

6208

6209

6210

6211

6212

6213

6214

6215

6216

6217

6218

6219

6220

6221

6222

6223

6224

6225

6226

6227

6228

6229

6230

6231

6232

6233

6234

6235

6236

6237

6238

6239

6240

Zoological Journal of the Linnean Society 150:815-834. DOI 10.1111/j.10963642.2007.00309.x

Witzmann F. 2010 (printed 2011). Morphological and histological changes of dermal scales during the fish-to-tetrapod transition. Acta Zoologica (Stockholm) 92:281-302. DOI 10.1111/j.1463-6395.2010.00460.x

Witzmann F. 2013. Phylogenetic patterns of character evolution in the hyobranchial apparatus of early tetrapods. Earth and Environmental Science Transactions of the Royal Society of Edinburgh 104:145-167. DOI 10.1017/S1755691013000480

Witzmann F. 2015 (printed 2016). $\mathrm{CO}_{2}$-metabolism in early tetrapods revisited: inferences from osteological correlates of gills, skin and lung ventilation in the fossil record. Lethaia 49:492-506. DOI 10.1111/let.12161

Witzmann F, Schoch RR. 2006a. Skeletal development of the temnospondyl Acanthostomatops vorax from the Lower Permian Döhlen Basin of Saxony. Transactions of the Royal Society of Edinburgh: Earth Sciences 96:365-385. DOI 10.1017/S0263593300001358

Witzmann F, Schoch RR. 2006b. The postcranium of Archegosaurus decheni, and a phylogenetic analysis of temnospondyl postcrania. Palaeontology 49:1211-1235. DOI 10.1111/j.1475-4983.2006.00593.x

Witzmann F, Schoch RR. 2017. Skull and postcranium of the bystrowianid Bystrowiella schumanni from the Middle Triassic of Germany, and the position of chroniosuchians within Tetrapoda. Journal of Systematic Palaeontology 16:711-739. DOI 10.1080/14772019.2017.1336579

Witzmann F, Schoch RR, Maisch MW. 2008. A relict basal tetrapod from Germany: first evidence of a Triassic chroniosuchian outside Russia. Naturwissenschaften 95:67-72. DOI 10.1007/s00114-007-0291-6

Wright AM, Hillis DM. 2014. Bayesian analysis using a simple likelihood model outperforms parsimony for estimation of phylogeny from discrete morphological data. PLOS ONE 9:e109210. DOI 10.1371/journal.pone.0109210

Yates A (as "Adam” only, but known to be Yates). 2007. [Comment to Naish D. 2007. Temnospondyls the early years (part II)]. In: Naish D. Tetrapod Zoology. http://scienceblogs.com/tetrapodzoology/2007/07/09/temnospondyls-the-early-years1/\#comment-1935

Yates A, Warren AA. 2000. The phylogeny of the 'higher' temnospondyls (Vertebrata: Choanata) and its implications for the monophyly and origins of the Stereospondyli. Zoological Journal of the Linnean Society 128: 77-121. DOI 10.1006/zjls1998.0184

Zaragüeta-Bagils R, Ung V, Grand A, Vignes-Lebbe R, Cao N, Ducasse J. 2012. LisBeth: New cladistics for phylogenetics and biogeography. Comptes Rendus Palevol 11:563566. DOI 10.1016/j.crpv.2012.07.002 - version 01/2013 is available at https://lisupmc.snv.jussieu.fr/?q=en/resources/software/lisbeth/download 


\section{Figure legends}

6243

6244

6245

6246

6247

6248

6249

6250

6251

6252

6253

6254

6255

6256

6257

6258

6259

6260

6261

6262

6263

6264

6265

6266

6267

6268

6269

6270

6271

6272

6273

6274

6275

6276

6277

6278

6279

6280

6281

6282

6283

6284

6285

6286

6287
Figure 1: Strict consensus of the MPTs (length: 1621 steps including polymorphisms) found by RC07 and by our unconstrained reanalysis of their unchanged data matrix (Analysis O1; see Table 1). The topology is identical to RC07: fig. 5, 6. Names of extant taxa are in boldface in this and all following tree figures. In this figure and all following ones, Albanerpetidae and Dendrerpetidae are each a single OTU (called "Albanerpetontidae" and "Dendrerpeton" by RC07). The name Edopoidea (and, in some other figures, Eutemnospondyli) is placed under the assumption that Mastodonsaurus (not included in any of our analyses) would be closest to Eryops as found by Schoch (2013); "Dissorophoidea (S13)" is placed according to Schoch's (2013) definition, "Dissorophoidea (YW00)" is placed according to the definition by Yates \& Warren (2000), and "Dissorophoidea (content)", only shown where different from Dissorophoidea (YW00), is the smallest clade that has the traditional contents of that taxon (trematopids, Broiliellus, amphibamids/branchiosaurids, Micromelerpeton). The origin of digits cannot be narrowed down to a single internode in this and several other figures. For easier orientation, Ichthyostega, Baphetes and Eryops are written in purple. The color scheme of the background boxes is consistent across all figures. Occasional abbreviations: Gymnophio., Gymnophionomorpha; Liss., Lissamphibia; micr. or microbr., microbrachomorphs; Ph., Pholiderpeton. All "microsaurs" are underlain in the same shade of gray, but some (in some figures) are not labeled as such due to lack of space.

Figure 2: Hypotheses on the origin(s) of the extant amphibians, and their compatibility with molecular and morphological data. The names Amphibia and Lissamphibia do not apply in E. A: Consensus of all recent molecular studies (e.g. Pyron, 2014: supplementary file amph_shl.tre; Irisarri et al., 2017). B: Part of the consensus of all recent morphological studies (e.g. our results, RC07, and references in both); modern amphibians not shown. C: Extant amphibians added to B according to the "temnospondyl hypothesis"; note compatibility with A. D: "Lepospondyl hypothesis" mapped to $\mathbf{B}$; note compatibility with $\mathbf{A}$. E: "Polyphyly hypothesis" mapped to $\mathbf{B}$; note lack of compatibility with A. In earlier versions of the "polyphyly hypothesis", Caudata often lies next to Gymnophionomorpha instead of Salientia; in that case, the name Batrachia does not apply (or becomes a synonym of the tetrapod crown-group).

Figure 3: NHMW 1899/0003/0006 (formerly 1899-III-6), a specimen referred to Sparodus validus. For an interpretative drawing, see Carroll (1988: pl. XII), which, however, makes the vertebrae appear much flatter than they are and omits the unusually well preserved scales. Some of the scales are only visible as striations in the matrix (see Fig. 4), which is usual in other "microsaurs" and in micromelerpetid temnospondyls; but most retain thick bone. Photo taken by D. M.

Figure 4: Ventral view of the palate and lingual view of the left lower jaw of the Sparodus specimen shown in Fig. 3. Carroll (1988: fig. 1A) provided an interpretative drawing of a latex cast. The photograph was taken by holding a digital camera to an ocular of a binocular microscope. Abbreviations: 1., left; r., right; .c, largest caniniform tooth (on the maxilla); .t, tusk (one of two per bone); cor, unidentified coronoid; d, dentary; hu, humerus; j, jugal; mx, maxilla; orb, orbit; pal, palatine; pmx, premaxilla; po, postorbital; pof, postfrontal; sc, scales preserved as striations. Photo taken by D. M. 
Figure 5: Caudal trunk vertebrae of NMS G 1993.54.1cp, the holotype of Casineria kiddi

6290

6291

6292

6293 (counterpart plate), in partly right lateral, partly interior view. The neural arches end dorsally in an ossification front marked by black material (an iron sulfide?) just dorsal to the level of the postzygapophyses, indicating that the individual was immature; the pleuro- and intercentra are reminiscent of those of the anthracosaur Proterogyrinus and the temnospondyl Neldasaurus

6294

6295 (Chase, 1965), although their precise shapes are difficult to determine because the vertebrae are split lengthwise and probably recrystallized. Abbreviations: ic, intercentrum; nsp, neural spine; pc, pleurocentrum; poz, postzygapophysis; prz, prezygapophysis. The scale bar is approximate. Photo taken by D. M.

6298

6299

Figure 6: Left forelimb of NMS G $1993.54 .1 \mathrm{cp}$, the holotype of Casineria kiddi (counterpart

6300

6301

6302 plate), in plantar view. The carpus is unossified except for what may be an incipiently mineralized distal carpal 1 . The inset shows phalanx IV-5 at a higher magnification (length

6303 approximately $1 \mathrm{~mm}$ ); note that the tip, although curved plantarly, is blunt, unlike in amniotes

6304 and contrary to Paton, Smithson \& Clack (1999). Abbreviation: mc, metacarpal. The scale bar is 6305

6306

6307 approximate. Photos taken by D. M.

6308

6309

6310

6311

Figure 7: Right cleithrum of NMS G $1993.54 .1 \mathrm{cp}$, the holotype of Casineria kiddi (counterpart plate), in caudal view, showing postbranchial lamina (compare fig. 70-2.4 of Pawley, 2006). Total length about $11.5 \mathrm{~mm}$. Photo taken by D. M.

6312

6313

Figure 8: Topological constraints used in Analyses O2, O3, R2, R3, R5 and R6 (see Table 1). These are backbone constraints: the OTUs not mentioned in the constraints were free to be positioned anywhere within the constraint tree (including at its root), and the polytomies were allowed to be resolved in all mathematically possible ways. A: Constraint against the LH,

6314

6315

6316 allowing both TH and PH; used in Analyses R2 and R5 (both of which found the TH). Whether Lepospondyli is extinct depends on the unconstrained positions of the caudates. With the

6317 positions of Brachydectes and Doleserpeton exchanged, this constraint instead enforces the LH and was used in Analysis O2. B: Constraint for the PH, used in Analyses O3, R3 and R6.

6318

6319

6320

Figure 9: Strict consensus of the MPTs (length: 1622 steps including polymorphisms) found by

6321 our reanalysis of the unchanged matrix of RC07 constrained for the LH (Analysis O2). The constraint forced the lysorophian "lepospondyl" Brachydectes to be closer to the three salientians

6322

6323

6324

6325

6326

6327 (Triadobatrachus, Notobatrachus, Vieraella) than the dissorophoid temnospondyl Doleserpeton (Fig. 8A with the positions of Brachydectes and Doleserpeton exchanged).

6328

6329

Figure 10: Representation of some of the MPTs (length: 2182 steps) from Analysis R1, performed on our revised matrix - specifically the ones where Anthracosauria is rootward of Temnospondyli. The remaining MPTs from R1, where Anthracosauria is crownward of Temnospondyli, are shown in the next figure. The taxon sample was unchanged from RC07; no constraint was enforced.

This tree, like those in the following figures, aims to represent all of the information contained in the MPTs, more than any consensus tree can. The uninterrupted part of the tree

6332 (black lines), polytomies excepted, is present in all MPTs represented in this figure as a

6333 backbone on which the branches connected only by colored underlays have varying positions; all 
6334 nodes underlain in color are absent from the strict consensus. The cyan underlay in this figure

6335

6336

6337

6338

6339

6340

6341

6342

6343

6344

6345

6346

6347

6348

6349

6350

6351

6352

6353

6354

6355

6356

6357

6358

6359

6360

6361

6362

6363

6364

6365

6366

6367

6368

6369

6370

6371

6372

6373

6374

6375

6376

6377

6378

6379 connects the two positions of Ichthyostega that occur in different MPTs: one node more rootward, or one node more crownward, than Ventastega and Acanthostega; the green underlay connects the two positions of Capetus in the upper inset, the blue one the two of the (Neldasaurus + Isodectes) clade, the yellow one the two of Doleserpeton.

In this and the following figures, branches with only two neighboring positions in different MPTs are indicated with blue lines: for example, the clade of all seymouriamorphs except Kotlassia is the sister-group of Kotlassia or of the tetrapod crown-group. Trichotomies that are resolved in all three possible ways in different MPTs (none in this figure) are shown in the usual way (without a blue line).

Equally parsimonious alternatives that would be confusing if shown on the same tree are shown in the insets to the left, concerning in this case Temnospondyli and Caerorhachis, which may or may not (with equal parsimony) be a temnospondyl.

Figure 11: Representation of those MPTs from Analysis R1 where Anthracosauria lies crownward of Temnospondyli. See legend of Fig. 10 for more information. Note the additional position of Ichthyostega (immediately crownward of Ventastega and rootward of Acanthostega) and the fixed position of Caerorhachis (outside of Temnospondyli, though close to it). Dark blue underlay: Platyrhinops (3 positions); yellow: Kotlassia (3 positions); red: (Leptoropha + Microphon) (3 positions).

Figure 12: Representation of all MPTs (length: 2191 steps) from Analysis R2, performed on our revised matrix. The taxon sample was unchanged from RC07. A constraint forced the three salientians (Triadobatrachus, Notobatrachus, Vieraella) to be closer to the dissorophoid temnospondyl Doleserpeton than to the lysorophian "lepospondyl" Brachydectes; this allowed both the $\mathrm{TH}$ and the $\mathrm{PH}$.

Cyan underlay as in Fig. 10 and 11.

Figure 13: Representation of all MPTs (length: 2194 steps) from Analysis R3, performed on our revised matrix. Only the parts different from the results of R1 (Fig. 10,11) are shown. Outside the shown clades, the entire range of resolutions of R1 also occurs in R3. Procera lies in the place where Lissamphibia is found in R1. The taxon sample was unchanged from RC07. A constraint forced the dissorophoid temnospondyl Doleserpeton to be closer to the three salientians (Triadobatrachus, Notobatrachus, Vieraella) than the lysorophian "lepospondyl" Brachydectes, and additionally forced the gymnophionomorph Eocaecilia to be closer to Brachydectes than to Doleserpeton; this allowed only the PH.

Figure 14: Representation of all MPTs (length: 3011 steps) from Analysis R4 (revised matrix, expanded taxon sample, no constraint). The insets at the left show the two remaining parsimonious alternatives to the three aïstopod topologies shown in the main tree.

In this and the following figures, the names Stereospondylomorpha and Limnarchia are placed according to prevailing usage, not according to the definitions by Yates \& Warren (2000) or Schoch (2013). According to the former, Stereospondylomorpha would in this analysis probably exclude *Sclerocephalus and *Cheliderpeton, possibly also *Glanochthon; all three were included in the different topology of Yates \& Warren (2000: fig. 1). Limnarchia would be a synonym of Stereospondylomorpha as used here, but would exclude the originally included 
6380 dvinosaurs. Stereospondylomorpha as redefined by Schoch (2013) would have the contents

6381 shown here, but this does not apply to some of the following figures. Similarly, Dvinosauria 6382 would under its original definition (Yates \& Warren, 2000) be a synonym of Dissorophoidea as 6383 defined in the same work; as redefined by Schoch (2013), it would have the contents shown here, 6384 but not quite in Fig. 16.

6385 The cyan underlay connects the four positions of Ossinodus (a whatcheeriid sister to the 6386 rest, one node more crownward than Whatcheeriidae, sister to *Densignathus, or one node more 6387 crownward than it). The two positions of the "Parrsboro jaw" (see text) are connected by the red 6388 underlay. The green underlay connects the two positions of (*Nigerpeton $+*$ Saharastega), the dark blue one the four of *Casineria, the brown one the two of *Trihecaton.

6390

6391

6392

6393

6394

6395

6396

6397

6398

6399

6400

6401

6402

6403

6404

6405

6406

6407

6408

6409

6410

6411

6412

6413

Figure 15: Representation of some MPTs (length: 3021 steps) resulting from Analysis R5 (revised matrix, expanded taxon sample, constraint against the LH as in Analysis R2). The insets at the left show two equally parsimonious alternative to parts of the temnospondyl topology shown in the main tree. The remaining MPTs from R5 are represented in Fig. 16; the differences are again limited to eutemnospondyl phylogeny (Eutemnospondyli being the sister-group of Edops, as defined by Schoch, 2013).

Cyan, red, green and dark blue underlays as in Fig. 14, though note a fourth position for $(*$ Nigerpeton $+*$ Saharastega $)$ in the lower inset. Further underlay colors: magenta:

*Palatinerpeton (3 positions); violet: *Tungussogyrinus (3); light brown: *Cheliderpeton (2); dark brown: (Eocaecilia + Albanerpetidae) (2).

Figure 16: Eutemnospondyl phylogeny from those MPTs from Analysis R5 that are not represented in Fig. 15; see the legend of Fig. 15 for more information.

Figure 17: Representation of all MPTs (length: 3026 steps) from Analysis R6 (revised matrix, expanded taxon sample, constraint for the PH as in Analysis R3).

Underlays as in Fig. 15.

Figure 18: Bootstrap tree from Analysis B1 (revised matrix, original taxon sample, no constraint; compare Fig. 10). Clades with a bootstrap percentage below 50 are included if they are compatible with those above 50; percentages of 50 and above are in boldface.

6414

6415

6416

6417

6418

Figure 19: Bootstrap tree from Analysis B2 (revised matrix, expanded taxon sample, no constraint; compare Fig. 14). See legend of Fig. 18 for more information.

6419

6420

6421

6422

6423

6424

Figure 20: Topology and posterior probabilities from Analysis EB (revised matrix, expanded taxon sample, no constraint; compare Fig. 14, 19). The numbers are posterior probabilities (in $\%$ ), in boldface if 75 or higher. For branch lengths see Fig. 21. Abbreviations: br., branchiosaurids; microbr., microbrachomorphs.

Figure 21: Branch lengths from Analysis EB. For nomenclature and branch support see Fig. 20.

Figure 22: Homoplasy distribution in our matrix. The number of extra steps (x-axis) is the number of observed steps (in Mesquite, on the tree in Data S1 for each analysis) minus the 6425 minimum possible number of steps, which is the number of states minus one (for ordered and 
6426 unordered characters as well as for both of our stepmatrix characters). The number of characters

6427

6428

6429

6430

6431

6432

6433

6434

6435

6436

6437

6438

6439

6440

6441

6442

6443

6444

6445

6446

6447

6448

6449

6450

6451

6452

6453

6454

6455

6456

6457

6458

6459

6460

6461

6462

6463

6464

6465

6466

6467

6468

6469

6470

6471

that have each number of extra steps is plotted on the y-axis. The line between the data points is meaningless, but makes it easier to compare the distributions to an exponential curve. Compare Goloboff, Torres \& Arias (2017: fig. 1(a)). A: original taxon sample (Analysis R1); the highest number of extra steps is 47, but we plot to 69 for comparison to B. B: expanded taxon sample (Analysis R4); the highest number of extra steps is 69.

Figure 23: Hypotheses on the relationships of Devonian limbed and possibly limbed vertebrates. (Whatcheeriidae and *Perittodus, which have limbs, are only known from the Carboniferous with certainty.) Equally parsimonious positions of the same OTU are highlighted in color in this and the following figures. A: RC07 and references therein. B: our results from the same taxon sample as RC07 (Analyses R1-R3). Numbers below internodes are BPO, in boldface if 50 or higher. C: our results from the expanded taxon sample (R4-R6). Numbers below internodes are BPE $\backslash P$, BPE in boldface if 50 or higher, PP in boldface if 75 or higher. Analysis B2 places *Ymeria next to Ventastega (BPE: 19), Acanthostega one node crownward of them (BPE: 11) and *Densignathus one node rootward of Whatcheeriidae (BPE: 13); EB places *Ymeria next to *Metaxygnathus (PP: 58), Acanthostega one node crownward of Ventastega (PP: 76) and *Perittodus one node crownward of Ichthyostega (PP: 58).

Figure 24: Hypotheses on the relationships of post-Devonian limbed vertebrates, and distribution of several character states. A: RC07; the entire clade shown here equals the "post-Devonian limbed vertebrates" of Fig. 23A. Anthr., Anthracosauria. B: our results from the original taxon sample (R1-R3). The entire clade shown here equals the "other post-Devonian limbed vertebrates" of Fig. 23B. Numbers are BPO, in boldface if 50 or higher. Analysis B1 finds Caerorhachis next to the other temnospondyls, but places Temnospondyli in the more rootward of the two indicated positions and also finds Silvanerpeton next to the other anthracosaurs (BPO: 10). C: our results from the expanded taxon sample (R4-R6). The entire clade shown here equals the "other post-Devonian limbed vertebrates" of Fig. 23C. In B and C, the extant amphibians are shown indirectly as the three positions of the tetrapod crown-group; although not rendered in boldface, "(rest of) Temnospondyli" is extant in R2, R3, R5 and R6, and "Lepospondyli" is extant in R1, R3, R4 and R6. Parentheses show which of these positions are found in which analyses. The numbers of these analyses are written in black if these analyses find a single position; for any position found as the single position by any analyses, the parentheses themselves are also black. Numbers are BPE\PP, BPE in boldface if 50 or higher, PP in boldface if 75 or higher.

The rectangles indicate state 0 (filled) or 1 (empty) of characters 31 (PAR 1 - cyan), 147 (PSYM 1 - teal), 192 (CLE 2 - blue), 212 (HUM 10 - purple), 214 (HUM 12-15 - red), 260 (TRU VER 8 - orange) and 277 (CAU FIN 1 - green); absence of a rectangle means missing data. Where known, Devonian limbed vertebrates have state 0 of each of these characters.

Character 31: supratemporal/postparietal suture (0) or tabular/parietal suture (1); 147: presence (0) or absence (1) of parasymphysial; 192: presence (0) or absence (1) of postbranchial lamina on cleithrum; 212: humerus not (0) waisted (1); 214: humerus L-shaped (0) or not (1); 260: absence ( 0 ) or presence (1) of fusion between left and right pleurocentra in ventral midline; 277: presence (0) or absence (1) of tail fin skeleton (supraneural radials, lepidotrichia). See App. S1 for more precise definitions and discussion. Note that, of all adelospondyls, character 260 is only known in Acherontiscus; the adelogyrinids share state 1 if their single-piece centra are

PeerJ reviewing PDF | (2015:12:8254:6:0:NEW 20 Jul 2018) 
6472 pleurocentra, but they have state 0 if their centra are intercentra, a question we cannot presently 6473 decide. Although all anthracosaurs in this matrix are unknown for character 277, we here show 6474 state 0 because of **CM 34638 (Clack, 2011b).

6475

Further derivations occur within some of the composite clades shown here: the highly

6476

6477 nested temnospondyl Micromelerpeton is polymorphic for 31, and the diadectomorph Tseajaia

6478

6479 has state 31(0); Pederpes (nested within Whatcheeriidae) has state 192(1); Urocordylidae and the diplocaulid Keraterpeton ("lepospondyls") have state 212(0); Eusthenopteron, Panderichthys

6480 and the embolomeres Archeria and Pholiderpeton scutigerum have state 212(1), while Limnoscelis and Orobates (Diadectomorpha) as well as the diplocaulids Keraterpeton and

6482 Diceratosaurus have state 212(0); and the highly nested temnospondyls Doleserpeton and

6483 *Gerobatrachus have state 260(1). State 2 of the ordered character 214 is not shown (Eucritta,

6484 which has state 0 or 1 , is therefore shown as unknown); all the OTUs that have this state (an

6485

6486

6487 extra-long humerus) are nested well within clades for which 214(1) is plesiomorphic.

6488

Figure 25: Hypotheses on temnospondyl phylogeny since 2006. Taxa absent from our expanded taxon sample are omitted, those we added to the sample of RC07 are marked with an asterisk.

6489 Dvinosauria and Dissorophoidea are collapsed where possible. The colored rectangles (labeled in D) and the terms "Dissorophoidea (content)" and "Dissorophoidea (S13)" are consistent with Figures 1 and 10-20. The matrices of $\mathbf{A}-\mathbf{C}$ are ultimately based on that of Ruta, Coates \& Quicke (2003), so they - and ours (Fig. 26) - should not be considered fully independent of each other. Numbers below internodes are bootstrap percentages; those below 50 were, except in $\mathbf{G}$, not published. A: strict consensus of RC07; non-temnospondyls omitted. Dissorophoidea is marked in boldface for being extant because it contains Lissamphibia; this is the only analysis shown here that included any lissamphibians. RC07 (app. 4) performed a bootstrap analysis but did not publish the results for the part of the tree shown here. B: Pawley (2006: chapter 5). "*Cheliderpeton + *Glanochthon" was a single OTU called "Cheliderpeton spp."; the name Glanochthon had not yet been coined. *Acanthostomatops was included as part of a Zatracheidae OTU. C: Ruta (2009), strict consensus of analysis without reweighting; non-temnospondyls omitted. *Glanochthon latirostris was called by its previous name, "Cheliderpeton latirostre". The "rest-dendrerpetid OTU" was called "Dendrerpeton acadianum", but may have contained information from other dendrerpetids as well; see Material and methods: Treatment of OTUs: Dendrerpetidae. D: McHugh (2012); non-temnospondyl omitted. Dissorophidae (as two clades, one of which contains Broiliellus brevis) and Trematopidae (also as two clades) are nested in three different places within Amphibamidae. All bootstrap percentages of the nodes shown here are below 50 and were not published. E, F: all equally parsimonious topologies (8 MPTs) from the analysis of 48 taxa in Dilkes (2015a); non-temnospondyls omitted. Note (in F) that Dendrerpetidae and Balanerpeton are sister-groups in all MPTs. To see the individual MPTs, we repeated the analysis (calculation time: 00:04:36.5), finding MPTs of the same number, length and indices as published by Dilkes (2015a). The bootstrap percentages are from Dilkes (2015a: fig. 11B), which shows an analysis where Capetus and *Iberospondylus were omitted; the topology is otherwise identical to E. G: Pereira Pacheco et al. (2016), focused on stereospondylomorphs. Two species of *Platyoposaurus and three of *Konzhukovia were included as separate OTUs. The tree by Eltink et al. (2016) is fully compatible. H: Pardo, Small \& Huttenlocker (2017); non-temnospondyls and lissamphibians omitted (the MPTs differ only in the positions of the lissamphibians and ${ }^{* *}$ Chinlestegophis). Numbers are bootstrap percentages followed by Bayesian posterior probabilities in $\%$ (boldface if $\geq 75$, not published $<50$ ). 
6518

6519

6520

6521

6522

6523

6524

6525

6526

6527

6528

6529

6530

6531

6532

6533

6534

6535

6536

6537

6538

6539

6540

6541

6542

6543

6544

6545

6546

6547

6548

6549

6550

6551

6552

6553

6554

6555

6556

6557

6558

6559

6560

6561

6562

6563
Figure 26: Temnospondyl phylogeny in our analyses, distribution of the intertemporal bone, and the application of the name Temnospondyli. Dissorophoidea and Stereospondylomorpha are collapsed; modern amphibians and various "lepospondyls" (see Fig. 12) are omitted. Filled rectangles indicate presence of the intertemporal (state 0 of ch. 32 - PAR 2/POSFRO 3/INTEMP 1/SUTEMP 1), empty rectangles its absence (state 1, or state 2 in the case of *Erpetosaurus; see App. S1); the absence of a rectangle for *Palatinerpeton and the *Parrsboro jaw represents missing data. T., Temnospondyli as defined by Schoch (2013); the entire depicted clade is Temnospondyli as defined by Yates \& Warren (2000). A: topology found in Analyses R1-R3 (original taxon sample) in MPTs where Temnospondyli is closer to Amniota than Anthracosauria. Only one position of Caerorhachis is shown; the other is far outside Temnospondyli. Numbers are BPO. B: additional topologies found in R1 and R3 (original taxon sample) in MPTs where Temnospondyli is closer to Amniota than Anthracosauria. Numbers are BPO of nodes absent from A. C: topologies found in R1 and R3 (original taxon sample) and R4R6 (expanded taxon sample) in MPTs where Anthracosauria is closer to Amniota than Temnospondyli. Only one position of the *Parrsboro jaw is shown, the other being well outside Temnospondyli. Numbers are BPO/BPE $\backslash P P$, or BPE $\backslash$ PP where BPO is inapplicable. D: additional topologies found in R5 (Fig. 16). Numbers are BPE $\backslash$ PP.

Figure 27: Amniote relationships and distribution of the intertemporal bone. Taxa not included in our analyses are omitted; those included in the expanded but not the original taxon sample are marked with an asterisk. Several named clades are collapsed. The matrices of $\mathbf{B}, \mathbf{C}, \mathbf{F}$ and $\mathbf{G}$ are ultimately based on that of Ruta, Coates \& Quicke (2003), so they should not be considered fully independent of each other. Numbers are bootstrap percentages. Filled rectangles indicate presence of the intertemporal (state 0 of ch. 32 - PAR 2/POSFRO 3/INTEMP 1/SUTEMP 1; see App. S1), empty rectangles its absence (any other state); the absence of a rectangle indicates missing data. A., Amniota; D., Diadectomorpha; Saur., Sauropsida. A: Vallin \& Laurin (2004). B: Pawley (2006: chapter 6). C: RC07; "rest of Lepospondyli" excludes the adelospondyls (see Fig. 1, 24A), bootstrap values were not published. D: Clack et al. (2012b; lacking *Bystrowiella) and Witzmann \& Schoch (2017); all bootstrap percentages in this part of the tree are below 50 in both versions and were not published. E: Klembara et al. (2014). Bootstrap percentages below 50 were not published, but note that a sister-group relationship of Chroniosaurus and Gephyrostegidae (G.) is supported by a bootstrap value of $\mathbf{5 3} \%$. F: topologies from our Analysis R2 and some MPTs from R1 and R3, where Temnospondyli is closer to Amniota than Anthracosauria. Numbers are BPO/BPE\PP. G: topologies found in Analyses R4-R6 and the remaining MPTs from R1 and R3. Numbers below nodes are BPO/BPE\PP; the space for BPO is omitted where inapplicable. "D." indicates Diadectomorpha for the original but not the extended taxon sample.

Figure 28: Hypotheses on "lepospondyl" relationships since 2004. Various named clades are collapsed. Taxa not included in our analyses are omitted; those included in the expanded but not the original taxon sample are marked with an asterisk. A., Amphibia; HPSA13, Huttenlocker et al. (2013); L., Lepospondyli; MJA12, Maddin, Jenkins \& Anderson (2012); ML09, Marjanović \& Laurin (2009). A: Vallin \& Laurin (2004). The other position of Westlothiana lies outside Amphibia and is not shown (see Fig. 27A). Numbers are bootstrap percentages. B: Pawley (2006: fig. 88). Numbers are bootstrap percentages from fig. 63 (those below 50 were not 
6564 reported), which shows an analysis that omitted the taxa written in dark green but found a 6565 congruent topology. C: Pawley (2006: fig. 91): cranial characters and data as in B, but the 6566 postcranial ones unchanged from Ruta, Coates \& Quicke (2003). D: RC07. E: Milner \& Ruta 6567 (2009); entire analysis except the aïstopod **Ophiderpeton. Adelospondyls and lysorophians 6568 were not included. H., Holospondyli; N., Nectridea. Numbers are bootstrap percentages. F: 6569 matrices derived from that of Anderson et al. (2008a), namely: 1) ML09; 2) MJA12 as

6570 reanalyzed by Marjanović \& Laurin (2018), lacking the corrections proposed by ML09 and those 6571 by Sigurdsen \& Green (2011) but containing those by Maddin \& Anderson (2012) and new ones 6572 by MJA12; 3) HPSA13, lacking all of the proposed corrections mentioned here but containing 6573 new ones. Note that ML09 found the LH, MJA12 the TH and HPSA13 the PH, and that ML09 6574 and HPSA13 did not find Eocaecilia in the same position. Numbers are bootstrap percentages 6575 from ML09, MJA12 and HPSA13 in this order (the latter two did not report percentages below 6576 50). - Pardo, Small \& Huttenlocker (2017: fig. S6) analyzed an unpublished matrix based on 6577 MJA12 with some updates based on HPSA13; the MPTs and the Bayesian tree are almost fully 6578 congruent with this tree, especially with MJA12, and neither bootstrap values nor posterior 6579 probabilities were published.

6580

6581

6582

Figure 29: The relationships of "lepospondyls" in our analyses, and the distribution of the supratemporal bone. Various named clades are collapsed. Filled rectangles indicate presence of

6584 the supratemporal (state 2 or lower of ch. 32 - PAR 2/POSFRO 3/INTEMP 1/SUTEMP 1; see

6585 App. S1), empty rectangles its absence (state 3 or 4 ); the absence of a rectangle indicates missing data. A., Amphibia; B., Batrachia; H., Holospondyli; L., Lepospondyli. A: topology found in Analyses R1 and R3. Numbers below internodes are BPO; compare unsupported nodes to Fig. 18. B: topology found in R2. C: topology found in R4-R6. Numbers are BPE $\backslash P P$; compare unsupported nodes to Fig. 19, 20. Numbers in red or brown depend on *Trihecaton or Eocaecilia, respectively, not being members of the clades in question; the opposite holds for clade names in color.

Figure 30: The phylogeny of modern amphibians and their closest relatives in recently published $(\mathbf{A}-\mathbf{K})$ and our $(\mathbf{L}-\mathbf{P})$ analyses, and the distribution of several character states. Albanerpetidae is a composite OTU throughout. Numbers in $\mathbf{A}, \mathbf{D}, \mathbf{E}, \mathbf{G}, \mathbf{H}$ are bootstrap percentages. B., Batrachia; G., Gymnophionomorpha (not shown when Eocaecilia is the only included member); L., Lissamphibia; m., modern amphibians (when not synonymous with L.); P., Procera. A: Vallin \& Laurin (2004); the two unnumbered "caudate" nodes have bootstrap values below $30 \%$. B:

6599 Pawley (2006: fig. 88; see Fig. 28B). C: Pawley (2006: fig. 91; see Fig. 28C). D: RC07. We write " $<50$ " where RC07 (app. 4) wrote "no bootstrap support in a $50 \%$ majority-rule

6601 consensus" and "-" where they wrote "no boots[t]rap support compatible with a $50 \%$ majority-

6602 rule consensus". Caudate monophyly is found in some MPTs and, as shown here, $61 \%$ of the

6603 bootstrap replicates. E: Marjanović \& Laurin (2009). F: Sigurdsen \& Green (2011: fig. 2B, 4).

6604 Numbers are bootstrap percentages from unweighted parsimony (left; not published if below 50)

6605

6606

6607

6608

6609 and Bayesian posterior probabilities (right; in \%). G: Maddin, Jenkins \& Anderson (2012). Bootstrap percentages below 50 were not published. H: Huttenlocker et al. (2013). Bootstrap percentages below 50 were not published. I-K: MPTs from Pardo, Small \& Huttenlocker (2017); see Marjanović \& Laurin (2018). Numbers in $\mathbf{J}$ are bootstrap percentages (left; not published if below 50) and Bayesian posterior probabilities (right; in \%). The ribs of **Rileymillerus are scored under the assumption that the tentatively referred postcrania really 
6610 belong to a juvenile of the same taxon as the holotype, which is a skull (Bolt \& Chatterjee, 6611 2000); this is at least not contradicted by the postcrania of **Chinlestegophis (Pardo, Small \& 6612 Huttenlocker, 2017). L: R1 (unconstrained), R3 (constrained for the PH; both original taxon 6613 sample). Numbers are BPO given that Salientia has the position it has in R1. M: R4

6614 (unconstrained, expanded taxon sample). Numbers are BPE $\backslash$ PP. N: R2 (constrained against the 6615 LH, original taxon sample). O: R5 (constrained against the LH, expanded taxon sample);

6616 Micropholis also has two positions outside the clade shown here. P: R6 (constrained for the PH, 6617 expanded taxon sample).

6618 Except where mentioned otherwise below, the rectangles show state 0 (filled) or 1 6619 (empty) of characters 18 (LAC 1 - cyan), 39 (POSPAR 1-2 - teal), 45 (POSFRO 1 - blue), 53

6620 (TAB 1/SQU 4 - violet), 60 (POSORB 1 - reddish purple), 71 (JUG 1 - magenta), 72 (JUG 2-6 6621 - red), 77 (QUAJUG 1 - orange), 102 (VOM 1-13 - bright green and greenish yellow, see 6622 below), 160 (ANG 1 - dark green), 246 (RIB 3 - brown) and 250 (RIB 7 - black). Absence of a

6623

6624

6625

6626

6627

6628

6629 rectangle means missing data, inapplicability or ambiguous optimization at the root of a collapsed clade, except that *Lydekkerina is polymorphic for character 72 .

State 1 of the unordered character 39 does not occur in this figure, so empty rectangles show state 2. State 2 of the ordered character 72 is limited to Pantylidae in this figure and therefore shown as state 1 . The ordered character 102 has three states: vomer about as wide as long or wider ( 0 , filled greenish yellow rectangles); intermediate (1, empty bright green rectangles); at least $2^{1 / 2}$ times as long as wide (2, filled bright green rectangles). Rectangles with

6630

6631

6632 a bright green rim filled in with greenish yellow indicate ambiguous optimization of state 1 or 2 . Character 18: presence (0) or absence (1) of lacrimal; 39: presence (0) or absence (2) of

6633 postparietals; 45: presence (0) or absence (1) of postfrontal; 53: presence (0) or absence (1) of

6634

6635 tabular; 60: presence (0) or absence (1) of postorbital; 71: presence (0) or absence (1) of jugal; 72: contact between maxilla and quadratojugal at ventrolateral edge of skull (0), jugal separates maxilla and quadratojugal and forms part of ventrolateral edge of skull (1 and 2); 77: presence

6636

6637

6638 (0) or absence (1) of quadratojugal; 102: see above; 160: presence (0) or absence (1) of angular; 246: ribs mostly straight ( 0 , empty rectangles) or ventrally curved in at least part of the trunk ( 1 , filled rectangles); 250: trunk ribs longer (0) or shorter (1) than three successive articulated

6639 vertebrae in adults. Note that we do not distinguish absence due to loss of an ossification center

6640 from absence as a separate bone due to ontogenetic fusion; in more or less all cases the ontogeny

6641 is insufficiently well known to decide.

6642 For further derivations within Caudata and Salientia, see the text. Adelospondylus has state 60(1), Notobatrachus is polymorphic for 77. The optimizations for character 72 differ for

6644 Aïstopoda between $\mathbf{P}$ and the rest due to the larger taxon sample in $\mathbf{P}$; likewise, the optimizations 6645 6646

6647 Figure 31: NHMW 1983/32/11, a natural mold of an articulated skeleton referred to Sauropleura 6648 6649 scalaris. Abbreviations: 1.cl, left clavicle; r.cl, right clavicle. Photo taken by D. M.

6651

6652

Figure 32: Area around the left hindlimb of a latex cast of the Sauropleura specimen shown in Fig. 31. Abbreviation: mt, metatarsal. Photo taken by D. M. 


\section{Supplementary Information}

6654

6655

6656

6657

6658

6659

6660

6661

6662

6663

6664

6665

6666

6667

6668

6669

6670

6671

6672

6673

6674

6675

6676

6677

6678

6679

6680

6681

6682

6683

6684

6685

6686

6687

6688

6689

6690

6691

6692

6693

6694

6695

6696

6697

6698

Appendix S1: Complete annotated list of characters and modifications to the data matrix of RC07, with reports of new observations of specimens.

Appendix S2: Human-readable version of the revised data matrix for parsimony analyses (the computer-readable version is contained in Data S3).

Data S3: Computer-readable version of the revised data matrix for parsimony analyses. NEXUS file (plain text) containing our revised data matrix, one MPT from each of Analyses R1-R6 and the settings used for these analyses. Executing the file in PAUP* performs the statistical comparisons (Kishino-Hasegawa test, Templeton test, winning-sites test) of these trees and then repeats Analyses R1-R6 in this order. In addition, the bootstrap trees from Analyses B1 and B2, and the tree from Analysis EB are included. So are eight trees illustrating the necessary numbers of extra steps for various rearrangements, all made by hand in Mesquite, followed by subtree pruning and regrafting of various branches in Mesquite but not tested by a constrained analysis in PAUP*: "R1-adelo-colo" (adelospondyls moved to colosteids on the tree from R1); "R1weirdo-colo" (adelospondyls, aïstopods and urocordylids moved to colosteids); "R1-adelo-coloaisto-uro-whatcheer" (adelospondyls moved to colosteids, aïstopods + urocordylids moved into the whatcheeriid grade); "R1-weirdo-whatcheer" (adelospondyls, aïstopods and urocordylids moved into the whatcheeriid grade); "R2-normal" (lepospondyl monophyly restored and all modern amphibians moved next to Doleserpeton on the tree from R2); "R4-adelo-colo", "R4weirdo-colo" and "R4-weirdo-whatcheer" (analogous to their R1-based counterparts).

Data S4: Excel file for counting the red, green and blue scores of App. S2 per OTU and per character, including the deleted characters not shown in App. S2. On each sheet, the matrix is reproduced in cells B1-JR103, the presence of a change of the kind in question marked by " 1 " while all other cells remain empty; the matrix is surrounded by sums and ranked lists with a few comments. On the sheet "green", the changes caused by the removal of all postcranial scores from Rhynchonkos are not counted. See the introduction of App. S2 for more information.

\section{Data S5: Excel file containing our measurements and their ratios relevant to characters} PREMAX 7 and SKU TAB 1.

On the sheet "Data", the OTUs are listed such that the line numbers are the same as the numbers the OTUs have in the matrix; OTUs that cannot be measured for any of the parameters are represented by blank lines. Calculations are underlain in yellow or blue. The raw measurements, in $\mathrm{cm}$, will mostly be difficult to reproduce: they were taken from illustrations (we preferred reconstruction drawings to avoid the effects of diagenetic distortion) on paper or on a screen, in the latter cases usually but not always at a magnification such as $150 \%, 200 \%$ or $300 \%$. The ratios, however, should be fairly well reproducible. Column B is the distance (at a right angle to the sagittal plane) between the lateral extremities of the premaxillae, measured in ventral view when the premaxillae are insufficiently exposed in dorsal view. Column $\mathrm{C}$ is the maximum width of the dermatocranium in dorsal view. Column $\mathrm{D}$ is the maximum width of the skull table; when sharp edges between the table and the temporal regions are absent or unknown, this can be measured across the "tabular horns", across the supratemporals across the rostral ends of the temporal notches, or across the intertemporals, whichever is widest. When possible, we 
6699 have consulted lateral views to determine where the dorsal and the lateral surfaces of the skull 6700 roof meet. Column E cites our sources (all of them are also cited in App. S1 and therefore listed 6701 in its References section). Column $\mathrm{G}$ is the ratio of premaxillary width to skull roof width (B 6702 divided by $\mathrm{C}$ ), which we decided to use as the raw data for PREMAX 7 (App.-Table 1 under ch. 67033 in App. S1). Column $\mathrm{H}$ is the ratio of premaxillary width to skull table width (B/D). Column I 6704 is the postorbital skull table length, in other words the rostrocaudal distance between the caudal 6705 margins of the orbits or orbitotemporal fenestrae (averaged if necessary) and the caudal end of 6706 the skull table in the midline. In salientians, the rostral margin of the otic capsule was assumed to 6707 lie at the caudal margin of the orbitotemporal fenestra, not at that of the lateral process of the 6708 parietal which covers only the caudal or caudomedial part of the otic capsule. Column $\mathrm{J}$ is the 6709 postorbital skull roof length, in other words the rostrocaudal distance between the caudal margins 6710 of the orbits (averaged if necessary) and the caudalmost extent of the dermatocranium, which 6711 may be the caudal end of the skull table in the midline, the tips of "tabular horns" (averaged if 6712 necessary), or the caudal ends of the suspensoria excluding the quadrates (averaged if necessary). Column $\mathrm{K}$ is the ratio of skull roof width to postorbital skull roof length $(\mathrm{C} / \mathrm{J})$. Column $\mathrm{L}$ is the ratio of skull table width to postorbital skull table length (D/I), which we decided to use as the raw data for SKU TAB 1 (App.-Table 3 under ch. 95 in App. S1). Column M is the ratio of skull roof width to postorbital skull table length $(\mathrm{C} / \mathrm{I})$, and column $\mathrm{N}$ is the ratio of skull table width to postorbital skull roof length $(\mathrm{D} / \mathrm{J})$. OTUs are represented by their most complete and skeletally

6719 most mature known members, except that Sauropleura is represented by $S$. scalaris rather than

6720 the morphological outlier S. pectinata (which is measured in line 153); Dendrerpetidae is

6721 represented by Dendrysekos, Albanerpetidae by Celtedens, *Caseasauria by Eothyris.

On all other sheets, OTUs scored 0 by RC07 are underlain in blue (for PREMAX 7 on the

6722

6723

6724

6725

6726

6727

6728

6729

6730

6731

6732

6733

6734 sheets "pmx-roof" and "pmx-table", for SKU TAB 1 on the others), OTUs scored 1 by RC07 are underlain in yellow, and OTUs scored as unknown by RC07 as well as those that we have added retain a white background. On each sheet, the values from one of the calculated columns on "Data" are ordered by size in column B (from highest to lowest for PREMAX 7, from lowest to highest for SKU TAB 1, in agreement with the original state definitions) and plotted; the line between the data points is of course meaningless, but we included it in order to see more easily where morphological gaps would lie. Column C on the sheets "pmx-roof" (for PREMAX 7) and "po table" (for SKU TAB 1) shows the state we have assigned to each OTU. Sheet "pmx-roof" is column G of the sheet "Data", "pmx-table" is H, "po roof" is K, "po table" is L, "roof width, table length" is M, "table width, roof length" is N.

Data S6: Revised data matrix for Bayesian analysis. NEXUS file (plain text) containing our revised data matrix, a tree from Analysis EB in two formats (one with the posterior probabilities

6735

6736

6737 as node labels), and the settings used for this analysis. The stepmatrix characters (32 and 134 in both Appendices and Data S3) are split into two or three characters each, as explained in the text and App. S1. Executing the file in MrBayes repeats Analysis EB.

6738

6739

Data S7: Original data matrix of RC07. NEXUS file (plain text) containing the original data 6740 matrix of RC07, one MPT from each of Analyses O1, O2 and O3, as well as the settings for these analyses. Executing the file in PAUP* performs the statistical comparisons (KishinoHasegawa test, Templeton test, winning-sites test) of these trees and then repeats Analyses O16744 $\mathrm{O} 3$ in this order. In addition, a topologically very different MPT from Analysis O3 is included. 
6745 Data S8: Homoplasy per character in our revised matrix given an MPT from Analysis R1 6746 (unconstrained, original taxon sample) and one from R4 (unconstrained, expanded taxon 6747 sample). The line numbers are the numbers of the characters (1-277). Column A shows the 6748 minimum of necessary steps per character (its number of states minus one); column B shows the 6749 steps actually found in R1 or R4 on the trees included in Data S3; column C is the difference 6750 between A and B, sorted from lowest to highest in column E, counted in columns G and $\mathrm{H}$ and 6751 plotted in the file as well as in Fig. 22. The line between the data points in the plots is 6752 meaningless, but makes it easier to compare the distributions to an exponential curve. Compare 6753 Goloboff, Torres \& Arias (2017: fig. 1(a)). 


\section{Figure 1 (on next page)}

Strict consensus of the MPTs (length: 1621 steps including polymorphisms) found by RC07 and by our unconstrained reanalysis of their unchanged data matrix (Analysis 01; see Table 1).

The topology is identical to RC07: fig. 5, 6. Names of extant taxa are in boldface in this and all following tree figures. In this figure and all following ones, Albanerpetidae and Dendrerpetidae are each a single OTU (called "Albanerpetontidae" and "Dendrerpeton" by RC07). The name Edopoidea (and, in some other figures, Eutemnospondyli) is placed under the assumption that Mastodonsaurus (not included in any of our analyses) would be closest to Eryops as found by Schoch (2013); "Dissorophoidea (S13)" is placed according to Schoch's (2013) definition, "Dissorophoidea (YW00)" is placed according to the definition by Yates \& Warren (2000), and "Dissorophoidea (content)", only shown where different from Dissorophoidea (YW00), is the smallest clade that has the traditional contents of that taxon (trematopids, Broiliellus, amphibamids/branchiosaurids, Micromelerpeton). The origin of digits cannot be narrowed down to a single internode in this and several other figures. For easier orientation, Ichthyostega, Baphetes and Eryops are written in purple. The color scheme of the background boxes is consistent across all figures. Occasional abbreviations: Gymnophio., Gymnophionomorpha; Liss., Lissamphibia; micr. or microbr., microbrachomorphs; Ph., Pholiderpeton. All "microsaurs" are underlain in the same shade of gray, but some (in some figures) are not labeled as such due to lack of space. 
Eusthenopteron

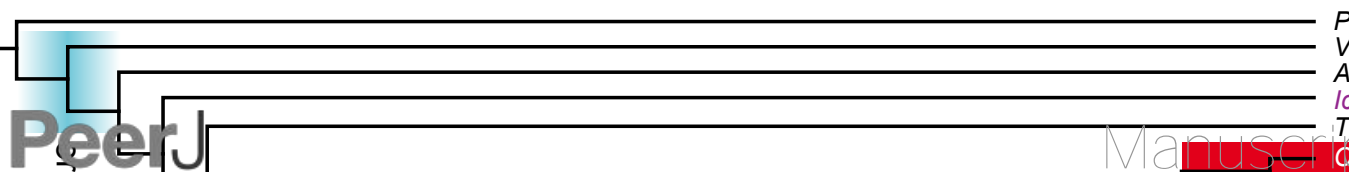

derichthys

Ventastega

Acanthostega

chthyostega

क्ञ

을. Tulerpeton bre cervileyvive Greererpeton

Acherontiscus

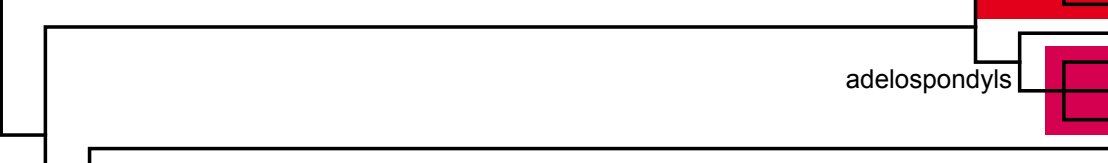
Adelogyrinus Dolichopareias

Crassigyrinus

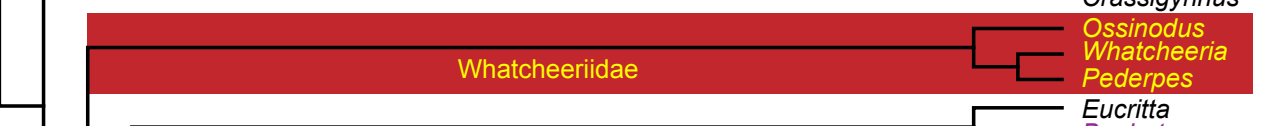

Eucritta

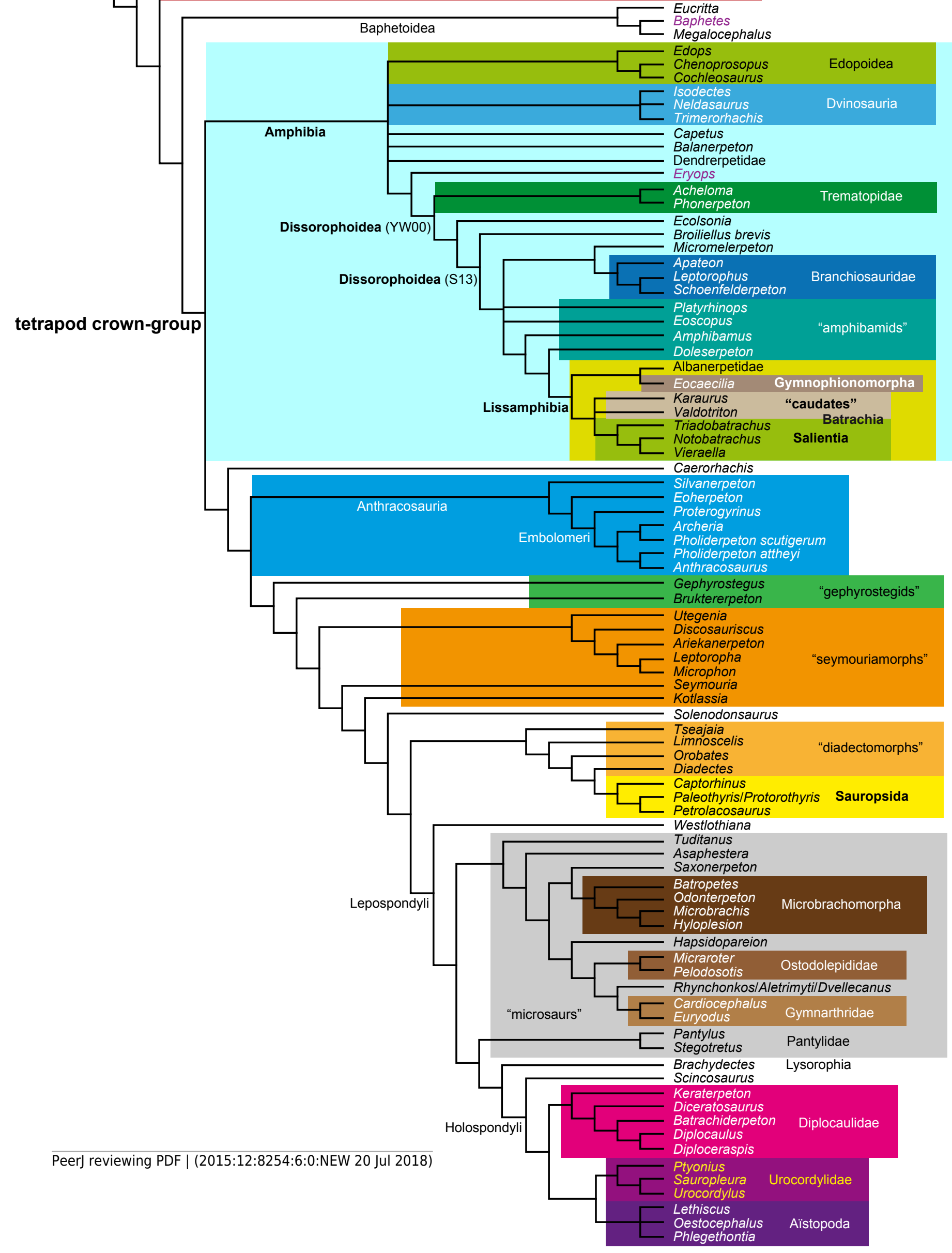




\section{Figure 2 (on next page)}

Hypotheses on the origin(s) of the extant amphibians, and their compatibility with molecular and morphological data.

The names Amphibia and Lissamphibia do not apply in E. A: Consensus of all recent molecular studies (e.g. Pyron, 2014: supplementary file amph_shl.tre; Irisarri et al., 2017). B: Part of the consensus of all recent morphological studies (e.g. our results, RC07, and references in both); modern amphibians not shown. C: Extant amphibians added to $\mathbf{B}$ according to the "temnospondyl hypothesis"; note compatibility with A. D: "Lepospondyl hypothesis" mapped to B; note compatibility with A. E: "Polyphyly hypothesis" mapped to B; note lack of compatibility with A. In earlier versions of the "polyphyly hypothesis", Caudata often lies next to Gymnophionomorpha instead of Salientia; in that case, the name Batrachia does not apply (or becomes a synonym of the tetrapod crown-group). 



\section{Figure 3}

NHMW 1899-0003-0006 (formerly 1899-III-6), a specimen referred to Sparodus validus.

For an interpretative drawing, see Carroll (1988: pl. XII), which, however, makes the vertebrae appear much flatter than they are and omits the unusually well preserved scales. Some of the scales are only visible as striations in the matrix (see Fig. 4), which is usual in other "microsaurs" and in micromelerpetid temnospondyls; but most retain thick bone. Photo taken by $\mathrm{D}$. M. 


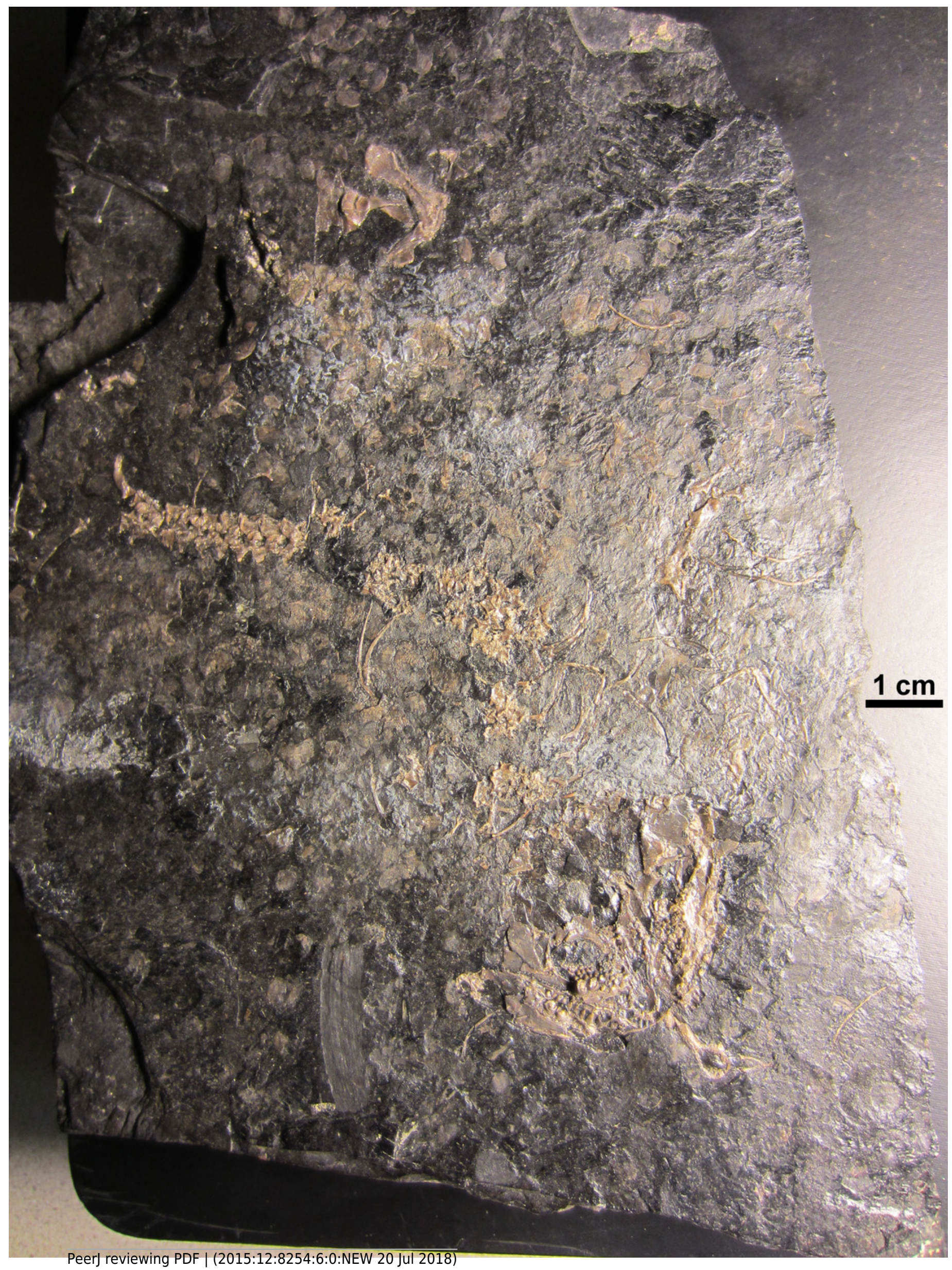




\section{Figure 4}

Ventral view of the palate and lingual view of the left lower jaw of the Sparodus specimen shown in Fig. 3.

Carroll (1988: fig. 1A) provided an interpretative drawing of a latex cast. The photograph was taken by holding a digital camera to an ocular of a binocular microscope. Abbreviations: I., left; r., right; .c, largest caniniform tooth (on the maxilla); .t, tusk (one of two per bone); cor, unidentified coronoid; d, dentary; hu, humerus; j, jugal; mx, maxilla; orb, orbit; pal, palatine; pmx, premaxilla; po, postorbital; pof, postfrontal; sc, scales preserved as striations. Photo taken by $\mathrm{D}$. M. 


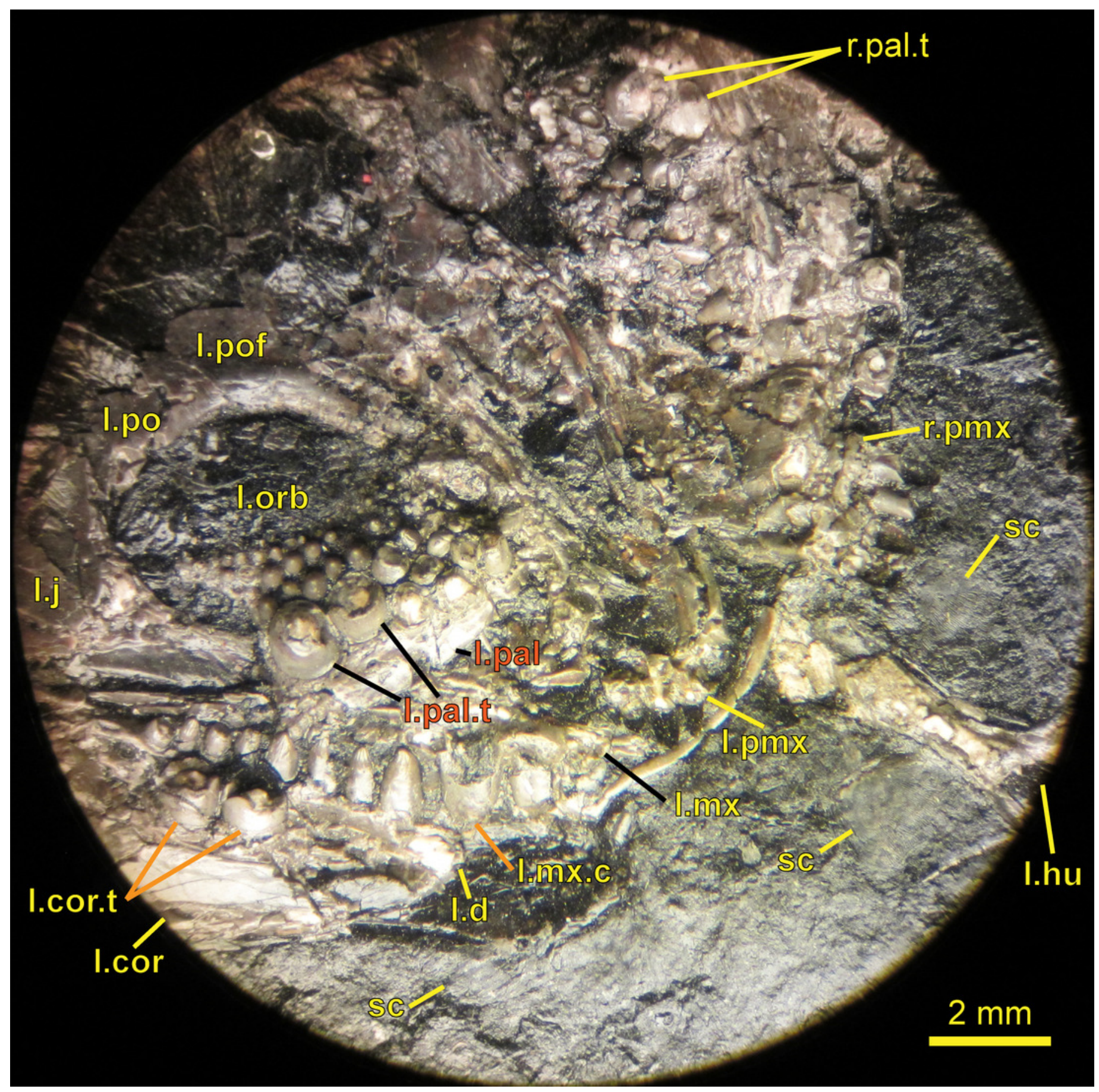




\section{Figure 5}

Caudal trunk vertebrae of NMS G 1993.54.1cp, the holotype of Casineria kiddi (counterpart plate), in partly right lateral, partly interior view.

The neural arches end dorsally in an ossification front marked by black material (an iron sulfide?) just dorsal to the level of the postzygapophyses, indicating that the individual was immature; the pleuro- and intercentra are reminiscent of those of the anthracosaur Proterogyrinus and the temnospondyl Neldasaurus (Chase, 1965), although their precise shapes are difficult to determine because the vertebrae are split lengthwise and probably recrystallized. Abbreviations: ic, intercentrum; nsp, neural spine; pc, pleurocentrum; poz, postzygapophysis; prz, prezygapophysis. The scale bar is approximate. Photo taken by D. M. 


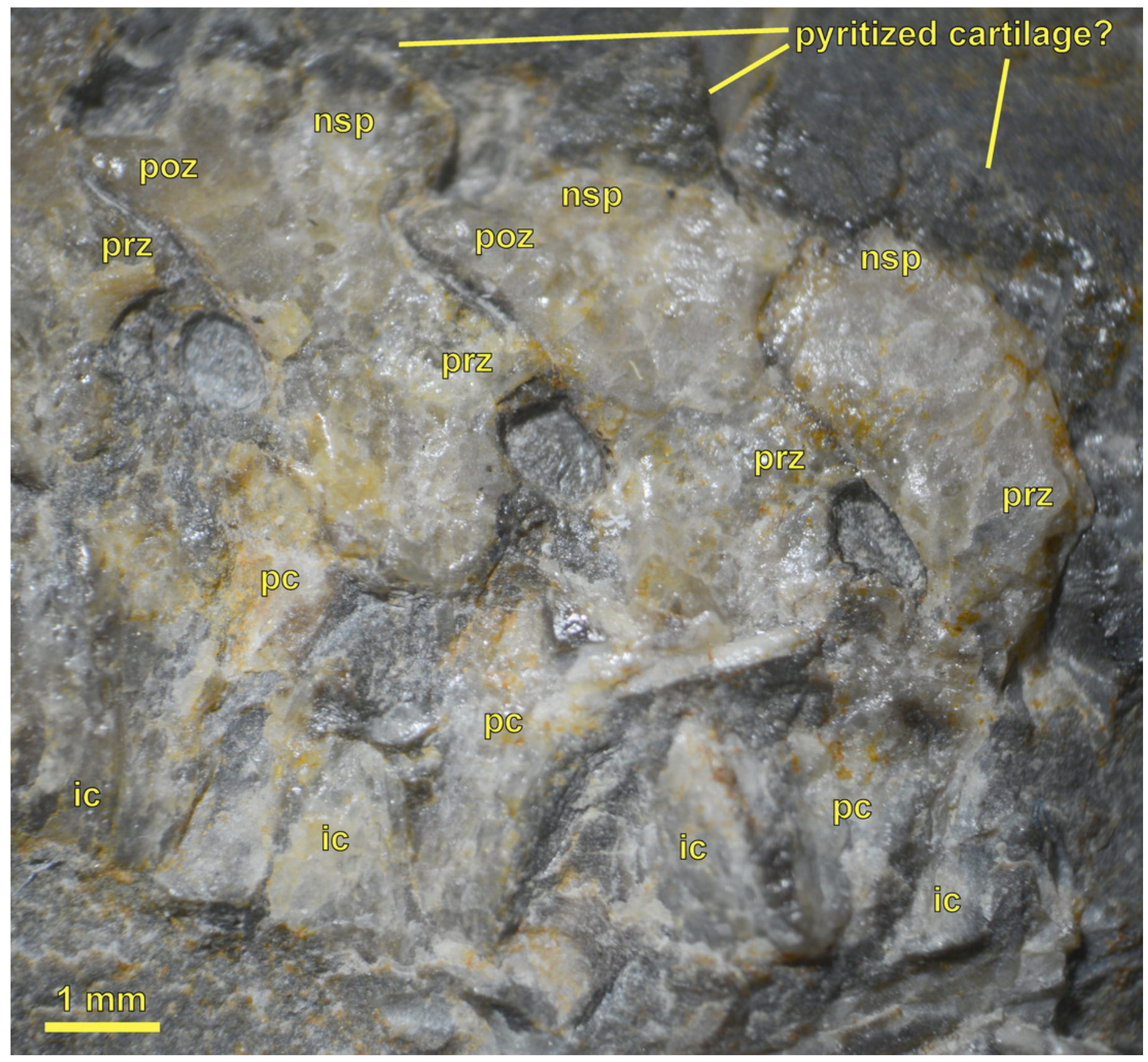




\section{Figure 6}

Left forelimb of NMS G 1993.54.1, the holotype of Casineria kiddi, in plantar view.

The carpus is unossified except for what may be an incipiently mineralized distal carpal 1 . The inset shows phalanx IV-5 at a higher magnification (length approximately $1 \mathrm{~mm}$ ); note that the tip, although curved plantarly, is blunt, unlike in amniotes and contrary to Paton, Smithson \& Clack (1999). Abbreviation: mc, metacarpal. The scale bar is approximate. Photos taken by $\mathrm{D}$. M.

*Note: Auto Gamma Correction was used for the image. This only affects the reviewing manuscript. See original source image if needed for review. 


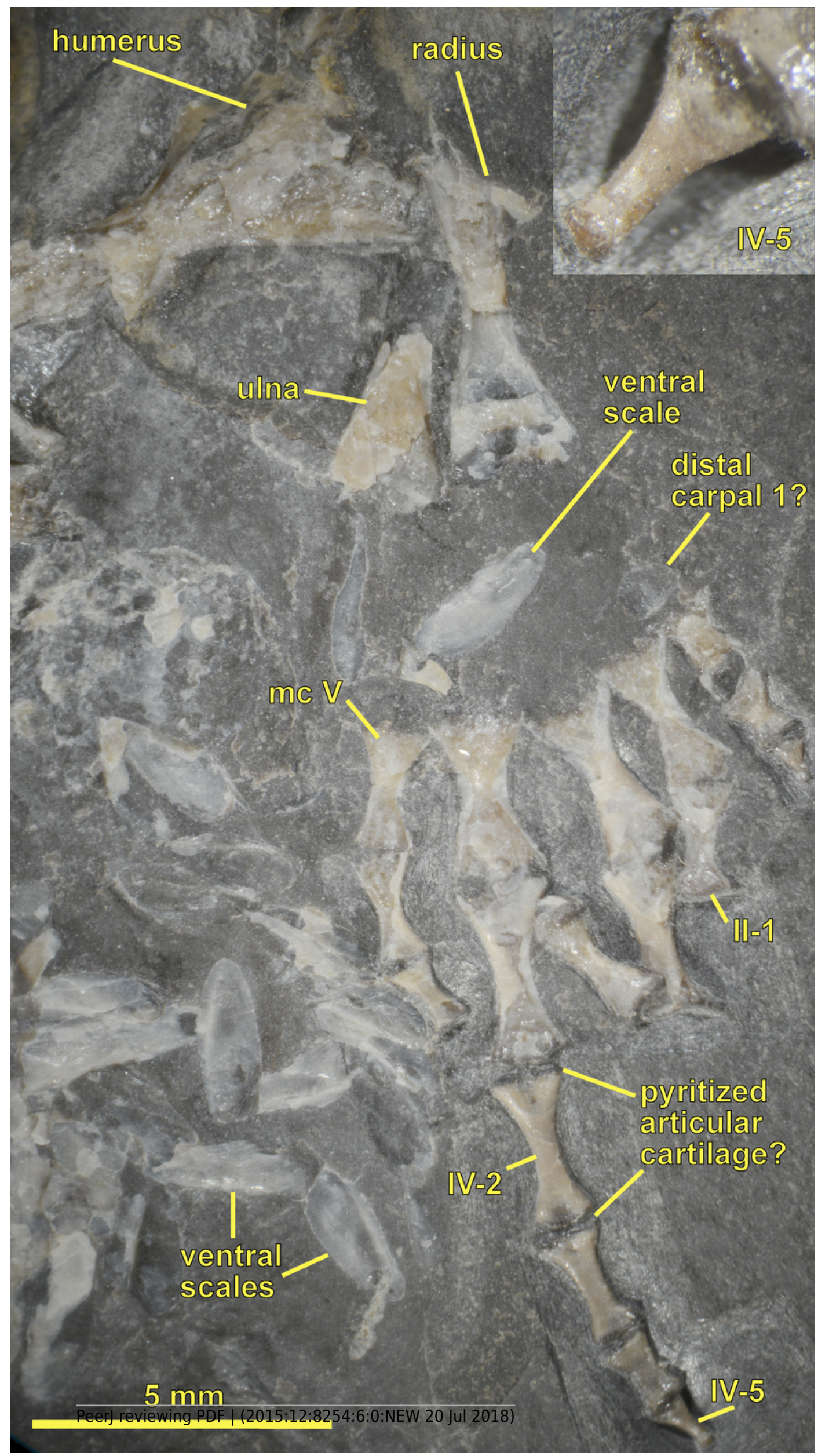




\section{Figure 7}

Right cleithrum of NMS G 1993.54.1, the holotype of Casineria kiddi, in caudal view, showing postbranchial lamina (compare fig. 70-2.4 of Pawley, 2006).

Total length about $11.5 \mathrm{~mm}$. Photo taken by D. M.

*Note: Auto Gamma Correction was used for the image. This only affects the reviewing manuscript. See original source image if needed for review.

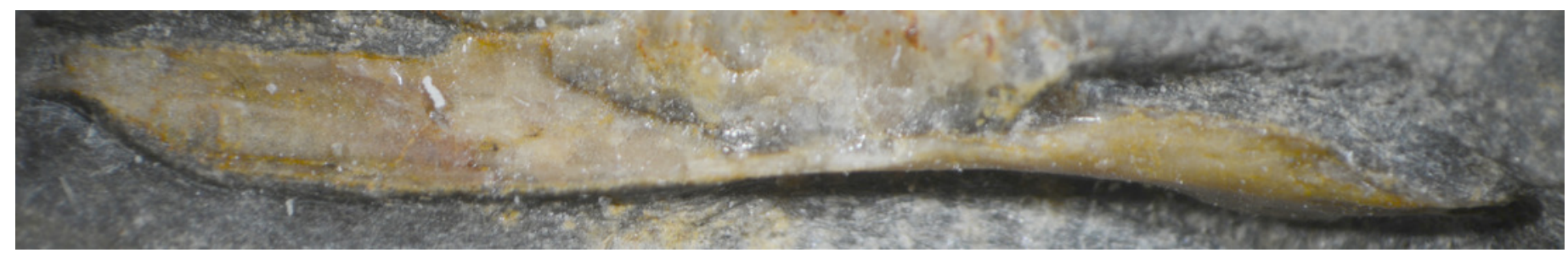




\section{Figure 8 (on next page)}

Topological constraints used in Analyses 02, 03, R2, R3, R5 and R6 (see Table 1).

These are backbone constraints: the OTUs not mentioned in the constraints were free to be positioned anywhere within the constraint tree (including at its root), and the polytomies were allowed to be resolved in all mathematically possible ways. A: Constraint against the $\mathrm{LH}$, allowing both TH and PH; used in Analyses R2 and R5 (both of which found the TH). Whether Lepospondyli is extinct depends on the unconstrained positions of the caudates. With the positions of Brachydectes and Doleserpeton exchanged, this constraint instead enforces the LH and was used in Analysis 02. B: Constraint for the PH, used in Analyses 03, R3 and R6. 


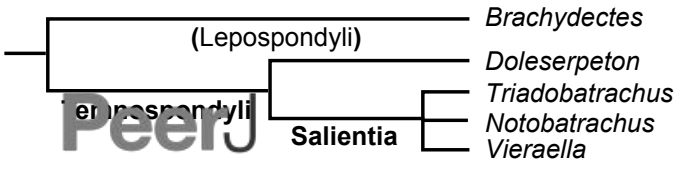

A

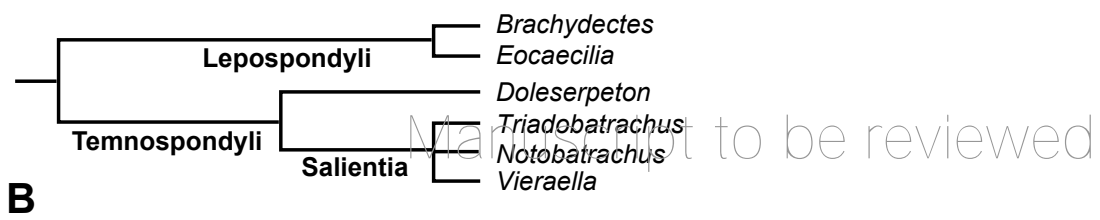


Figure 9 (on next page)

Strict consensus of the MPTs (length: 1622 steps including polymorphisms) found by our reanalysis of the unchanged matrix of RC07 constrained for the LH (Analysis 02).

The constraint forced the lysorophian "lepospondyl" Brachydectes to be closer to the three salientians (Triadobatrachus, Notobatrachus, Vieraella) than the dissorophoid temnospondyl Doleserpeton (Fig. 8A with the positions of Brachydectes and Doleserpeton exchanged). 


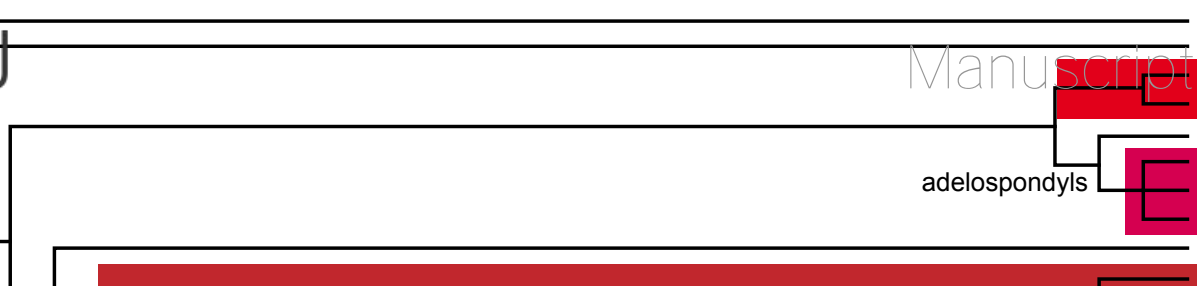

Acanthostega

lchthyostega

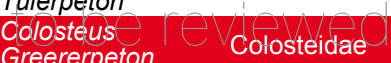

Acherontiscus

Adelospondylus

Adelogyrinus

Dolichopareias

Adelogyrinidae

Crassigyrinus
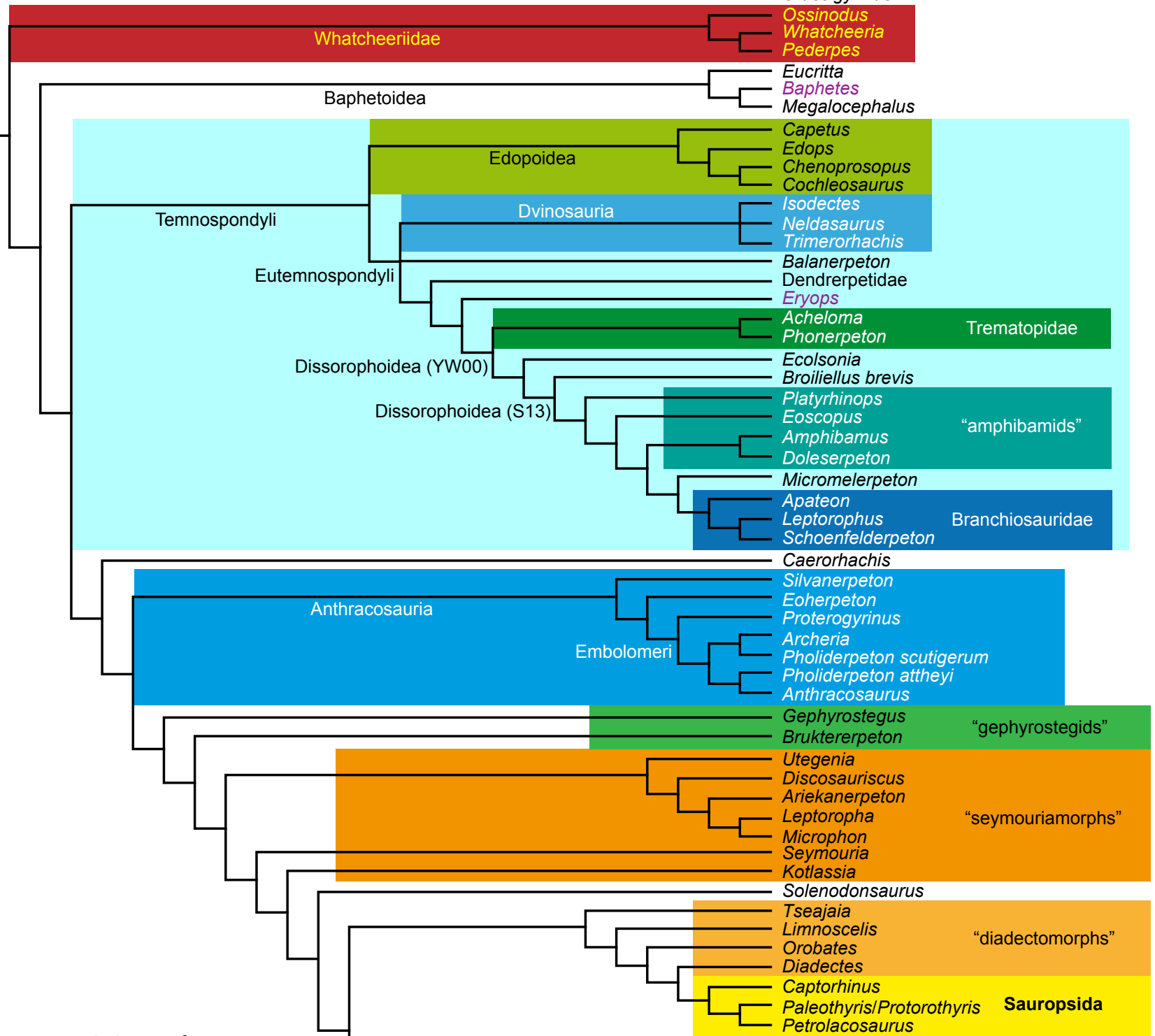

tetrapod crown-group

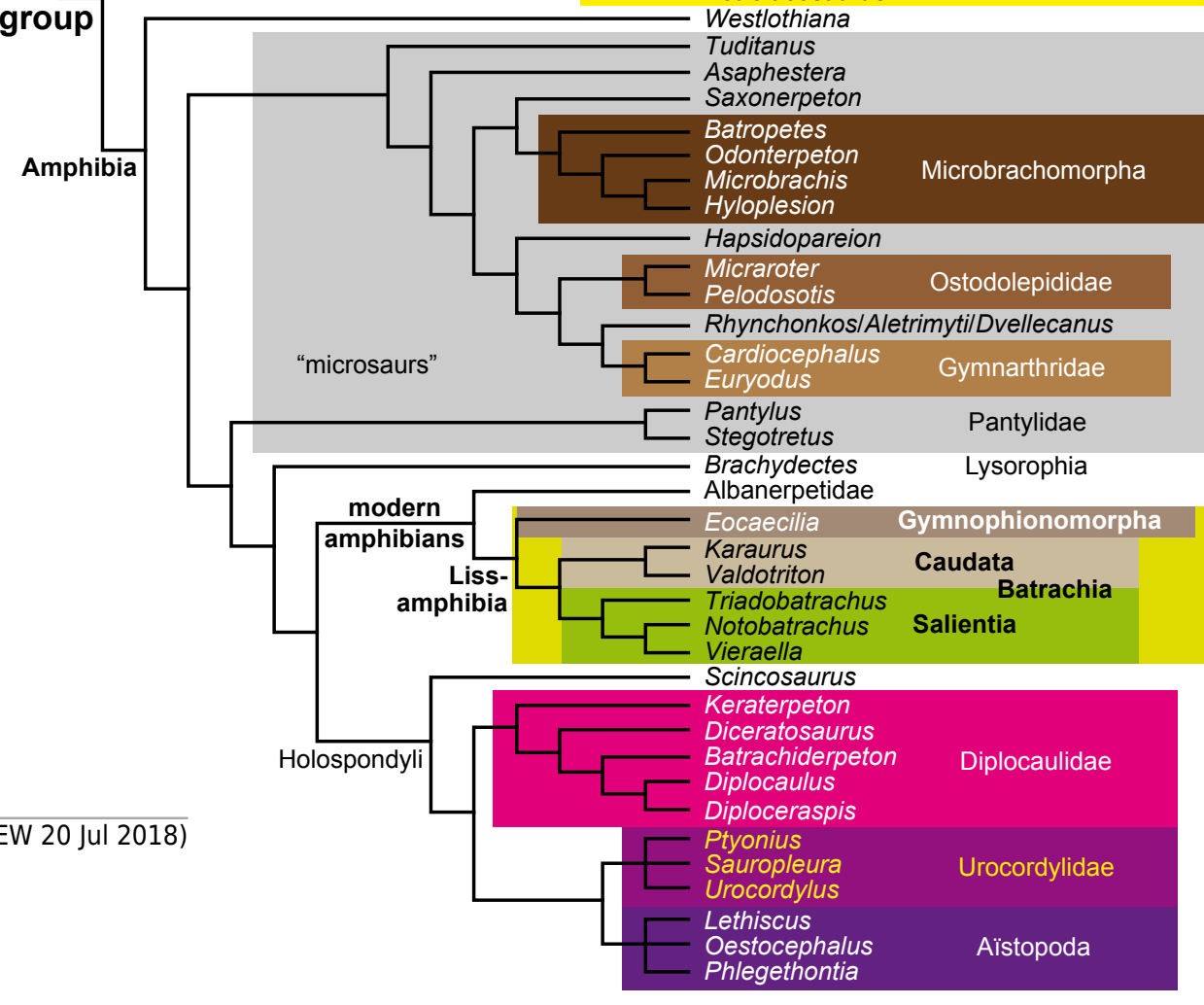




\section{Figure 10 (on next page)}

\section{Representation of some of the MPTs (length: 2182 steps) from Analysis R1, performed} on our revised matrix - specifically the ones where Anthracosauria is rootward of Temnospondyli.

The remaining MPTs from R1, where Anthracosauria is crownward of Temnospondyli, are shown in the next figure. The taxon sample was unchanged from RC07; no constraint was enforced.

This tree, like those in the following figures, aims to represent all of the information contained in the MPTs, more than any consensus tree can. The uninterrupted part of the tree (black lines), polytomies excepted, is present in all MPTs represented in this figure as a backbone on which the branches connected only by colored underlays have varying positions; all nodes underlain in color are absent from the strict consensus. The cyan underlay in this figure connects the two positions of Ichthyostega that occur in different MPTs: one node more rootward, or one node more crownward, than Ventastega and Acanthostega; the green underlay connects the two positions of Capetus in the upper inset, the blue one the two of the (Neldasaurus + Isodectes) clade, the yellow one the two of Doleserpeton.

In this and the following figures, branches with only two neighboring positions in different MPTs are indicated with blue lines: for example, the clade of all seymouriamorphs except Kotlassia is the sister-group of Kotlassia or of the tetrapod crown-group. Trichotomies that are resolved in all three possible ways in different MPTs (none in this figure) are shown in the usual way (without a blue line).

Equally parsimonious alternatives that would be confusing if shown on the same tree are shown in the insets to the left, concerning in this case Temnospondyli and Caerorhachis, which may or may not (with equal parsimony) be a temnospondyl. 


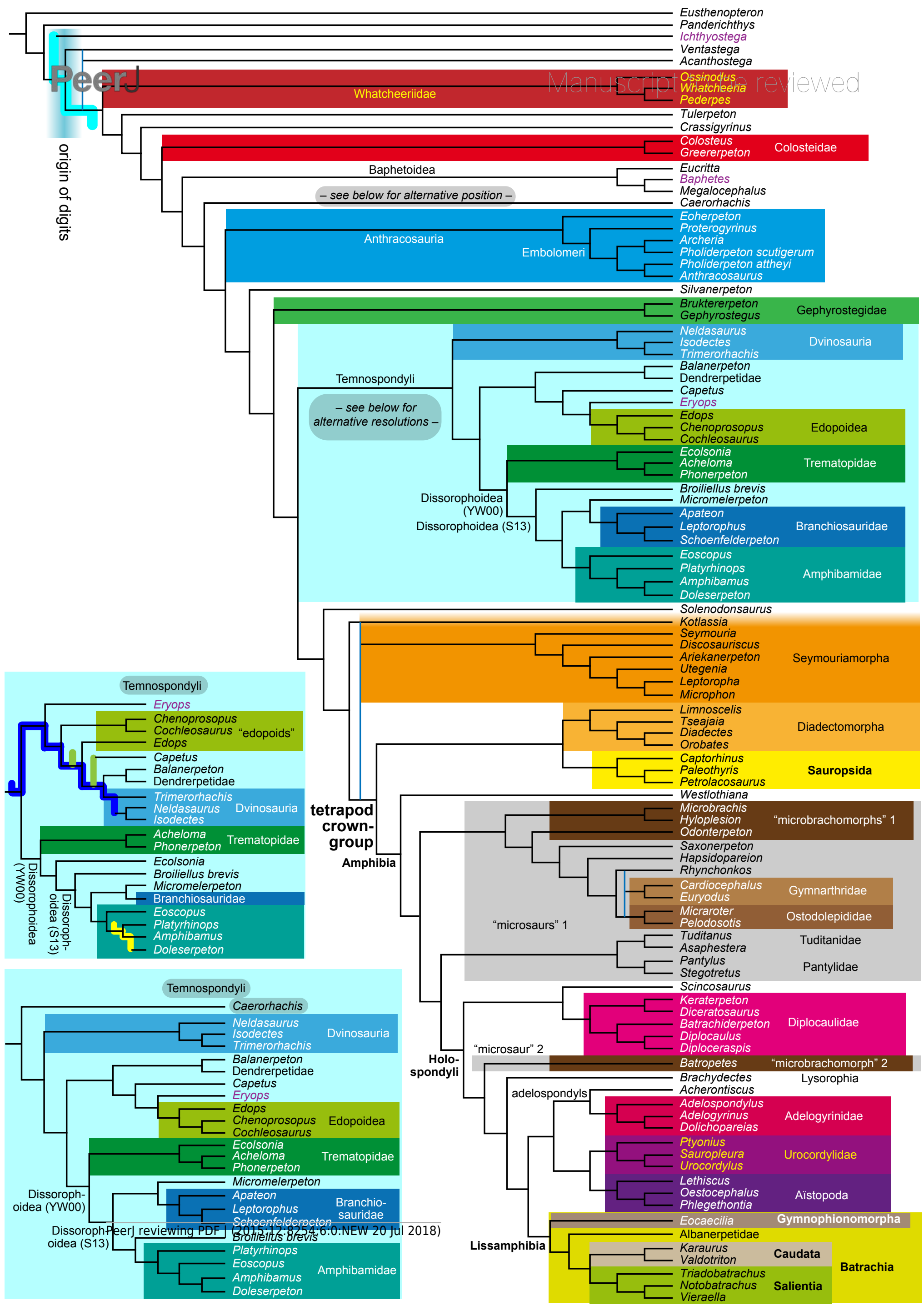


Figure 11(on next page)

Representation of those MPTs from Analysis R1 where Anthracosauria lies crownward of Temnospondyli.

See legend of Fig. 10 for more information. Note the additional position of Ichthyostega (immediately crownward of Ventastega and rootward of Acanthostega) and the fixed position of Caerorhachis (outside of Temnospondyli, though close to it). Dark blue underlay: Platyrhinops (3 positions); yellow: Kotlassia (3 positions); red: (Leptoropha + Microphon) (3 positions). 
Eusthenopteron

음.
$\frac{0}{5}$
$\frac{0}{7}$
흐.
क. Panderichthys

Ichthyostega

Ventastega

Acanthostega

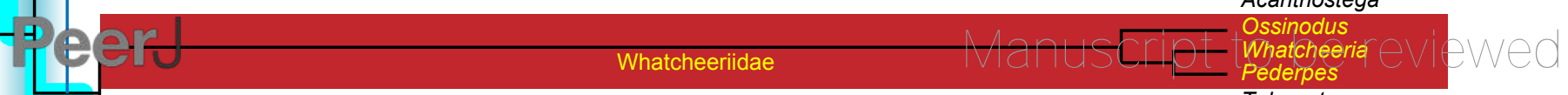

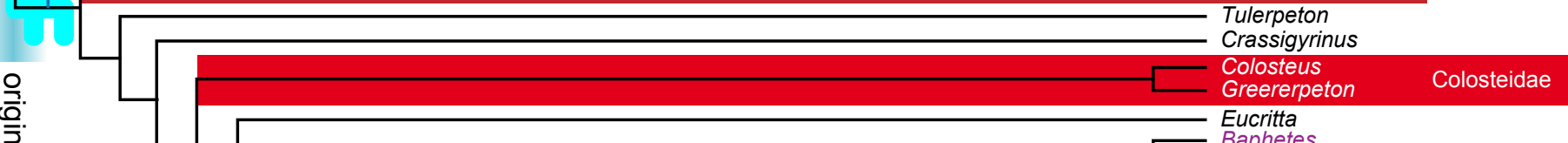

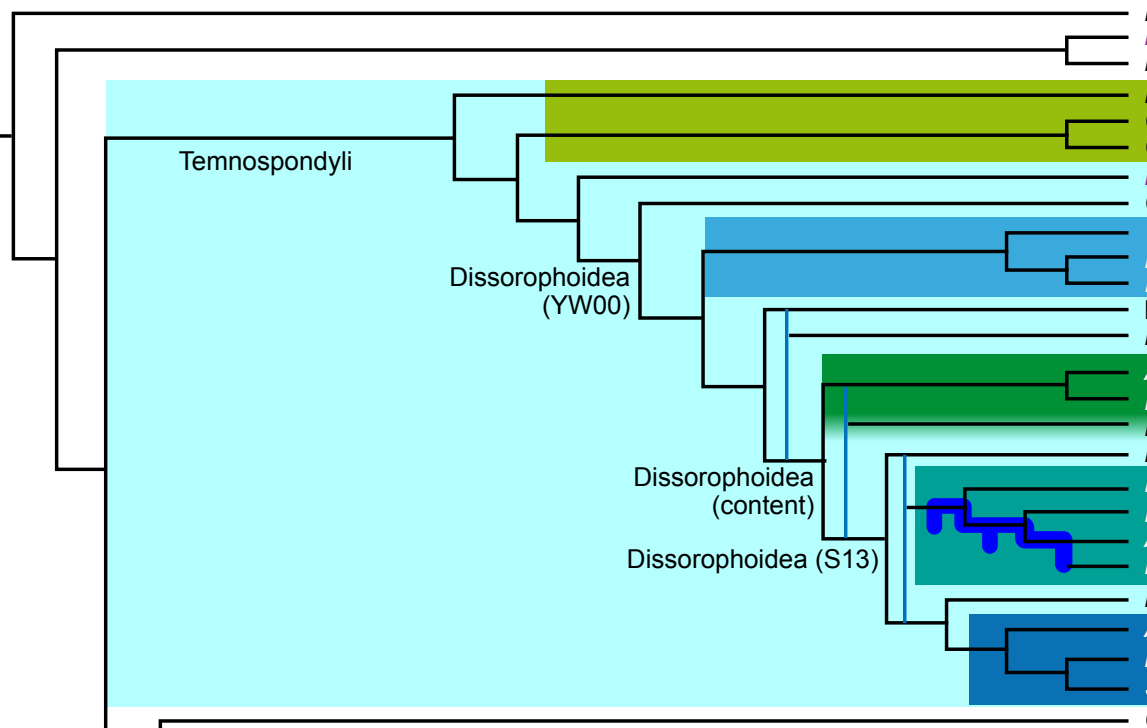

Megalocephalus

Edops

Chenoprosopus "edopoids"

Cochleosaurus

Eryops

Capetus

Trimerorhachis

sodectes

Dendrerpetidae

Balanerpeton

Acheloma

Phonerpeton

Broiliellus brevis

Eoscopus

Doleserpeton

Amphibamus

Platyrhinops

Micromelerpeton

Apateon

Leptorophus

Schoenfelderpeton

Caerorhachis

Eoherpeton

Eoherpeton
Archeria

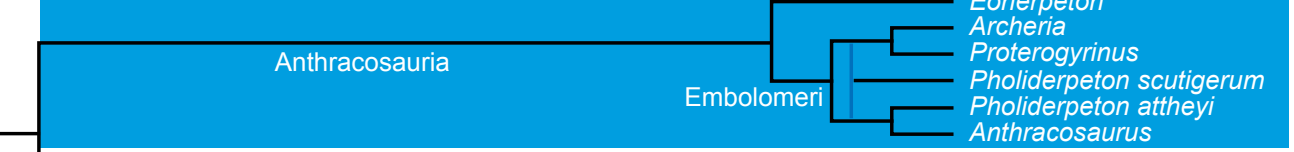

Silvanerpeton

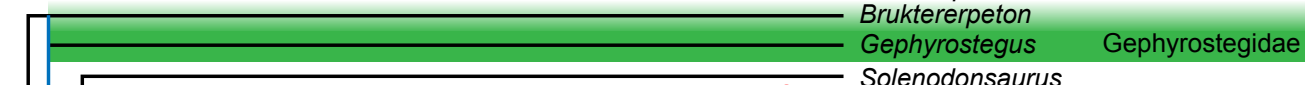

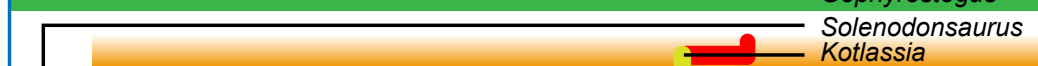

Seymouria

Discosauriscus

Ariekanerpeton

Utegenia

Leptoropha

Microphon

Limnoscelis

Tseajaia

Diadectes

Orobates

Captorhinus

Paleothyris

Petrolacosaurus

Seymouriamorpha

Amphibamidae

Branchiosauridae

tetrapod crown-group Westlothiana

Hyloplesion "microbrachomorphs" 1 Odonterpeton Saxonerpeton

Saxonerpeton
Hapsidopareion

Rhynchonkos

Amphibia

Holospondyli

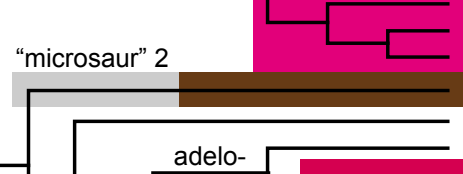

Cardiocephalus

Euryodus

Micraroter

Pelodosotis

Tuditanus

Asaphestera

Pantylus

Stegotretus

Diadectomorpha

Sauropsida

Scincosaurus

Keraterpeton

Diceratosaurus

Batrachiderpeton Diplocaulidae

Diplocaulus

Diploceraspis

Batropetes

Brachydectes

Acherontiscus

Adelospondylus

Adelogyrinus

Dolichopareias

Ptyonius

Sauropleura

Urocordylus

Lethiscus

Oestocephalus

Pestocephalus

Gymnarthridae

Ostodolepididae

Tuditanidae

Pantylidae

"microbrachomorph"

Lysorophia

Adelogyrinidae

Urocordylidae

Aïstopoda

Gymnophionomorpha

Elbanerpetidae

Albanerpetida

Karaurus

Valdotriton Batrachia

Notobatrachus Salientia 
Figure 12 (on next page)

Representation of all MPTs (length: 2191 steps) from Analysis R2, performed on our revised matrix.

The taxon sample was unchanged from RC07. A constraint forced the three salientians (Triadobatrachus, Notobatrachus, Vieraella) to be closer to the dissorophoid temnospondyl Doleserpeton than to the lysorophian "lepospondyl" Brachydectes; this allowed both the TH and the PH.

Cyan underlay as in Fig. 10 and 11. 
Eusthenopteron

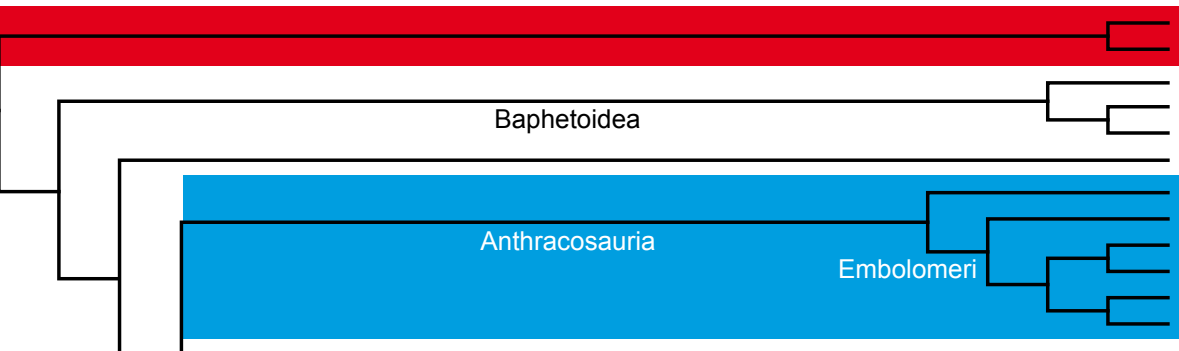

Tulerpeton

Crassigyrinus

Eucritta

Megalocephalus

Caerorhachis

Eoherpeton

Proterogyrinus

Archeria

Pholiderpeton scutigerum

Pholiderpeton attheyi

Anthracosaurus

Silvanerpeton

Bruktererpeton Gephyrostegidae

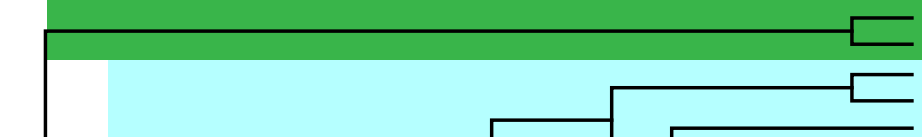

Balanerpeton

Dendrerpetidae

- Capetus

- Eryops

Chenoprosopus Edopoidea
Cochleosaurus

- Acheloma

Phonerpeton Trematopidae

Broiliellus brevis

Eoscopus

Amphibamus Amphibamidae

(YW00)

Amphibia Dissorophoidea (S13)

Platyrhinops

Micromelerpeton

Apateon

Leptorophus Branchiosauridae

Schoenfelderpeton

Neldasaurus

Isodectes

Trimerorhachis

Acherontiscus

Adelospondylus

Adelogyrinus

Dolichopareias

Ptyonius

Sauropleura

Urocordylus

tetrapod crown-group 
Figure 13(on next page)

Representation of all MPTs (length: 2194 steps) from Analysis R3, performed on our revised matrix.

Only the parts different from the results of R1 (Fig. 10,11) are shown. Outside the shown clades, the entire range of resolutions of R1 also occurs in R3. Procera lies in the place where Lissamphibia is found in R1. The taxon sample was unchanged from RC07. A constraint forced the dissorophoid temnospondyl Doleserpeton to be closer to the three salientians (Triadobatrachus, Notobatrachus, Vieraella) than the lysorophian "lepospondyl" Brachydectes, and additionally forced the gymnophionomorph Eocaecilia to be closer to Brachydectes than to Doleserpeton; this allowed only the $\mathrm{PH}$. 


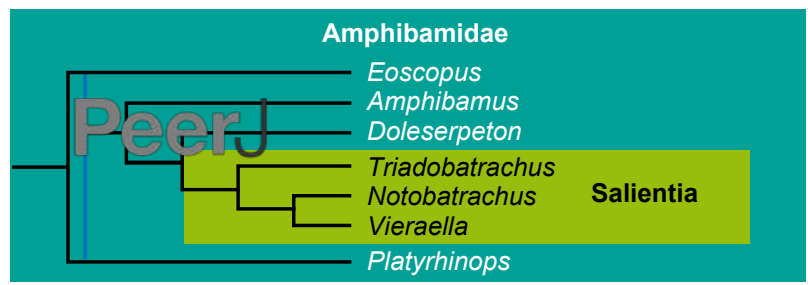

Procera

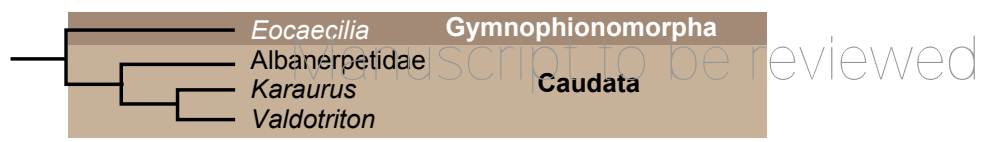




\title{
Figure 14(on next page)
}

\author{
Representation of all MPTs (length: 3011 steps) from Analysis R4 (revised matrix, \\ expanded taxon sample, no constraint).
}

The insets at the left show the two remaining parsimonious alternatives to the three aïstopod topologies shown in the main tree.

In this and the following figures, the names Stereospondylomorpha and Limnarchia are placed according to prevailing usage, not according to the definitions by Yates \& Warren (2000) or Schoch (2013). According to the former, Stereospondylomorpha would in this analysis probably exclude *Sclerocephalus and *Cheliderpeton, possibly also *Glanochthon; all three were included in the different topology of Yates \& Warren (2000: fig. 1). Limnarchia would be a synonym of Stereospondylomorpha as used here, but would exclude the originally included dvinosaurs. Stereospondylomorpha as redefined by Schoch (2013) would have the contents shown here, but this does not apply to some of the following figures. Similarly, Dvinosauria would under its original definition (Yates \& Warren, 2000) be a synonym of Dissorophoidea as defined in the same work; as redefined by Schoch (2013), it would have the contents shown here, but not quite in Fig. 16.

The cyan underlay connects the four positions of Ossinodus (a whatcheeriid sister to the rest, one node more crownward than Whatcheeriidae, sister to *Densignathus, or one node more crownward than it). The two positions of the "Parrsboro jaw" (see text) are connected by the red underlay. The green underlay connects the two positions of (*Nigerpeton $+*$ Saharastega), the dark blue one the four of *Casineria, the brown one the two of *Trihecaton. 

Figure 15(on next page)

Representation of some MPTs (length: 3021 steps) resulting from Analysis R5 (revised matrix, expanded taxon sample, constraint against the LH as in Analysis R2).

The insets at the left show two equally parsimonious alternative to parts of the temnospondyl topology shown in the main tree. The remaining MPTs from R5 are represented in Fig. 16; the differences are again limited to eutemnospondyl phylogeny (Eutemnospondyli being the sister-group of Edops, as defined by Schoch, 2013). Cyan, red, green and dark blue underlays as in Fig. 14, though note a fourth position for (*Nigerpeton $+*$ Saharastega) in the lower inset. Further underlay colors: magenta: *Palatinerpeton (3 positions); violet:

*Tungussogyrinus (3); light brown: *Cheliderpeton (2); dark brown: (Eocaecilia + Albanerpetidae) (2). 

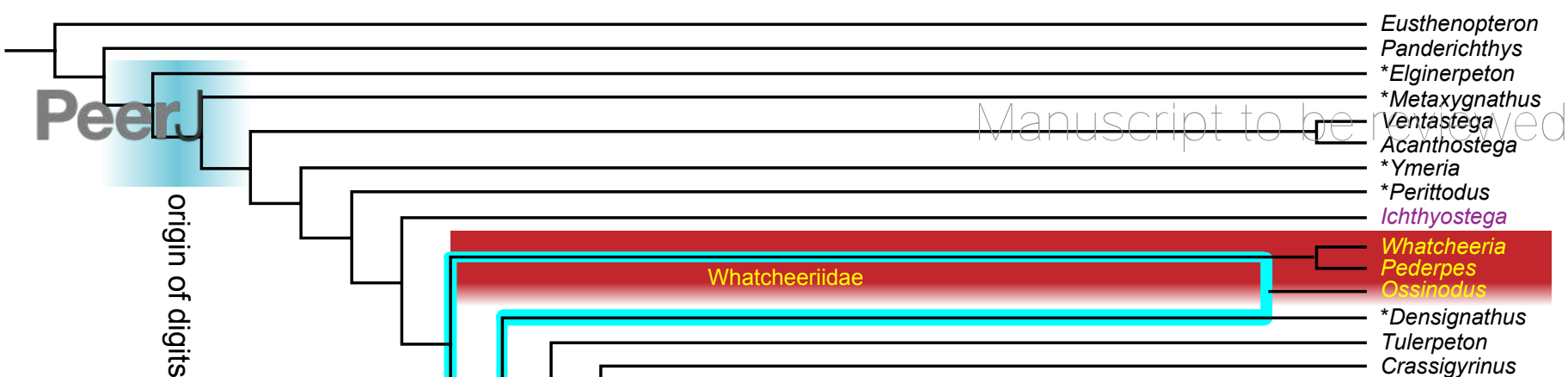

产.

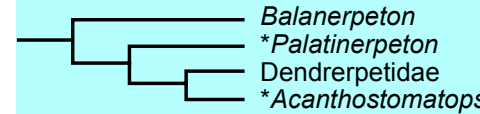

\section{tetrapod crown-group}
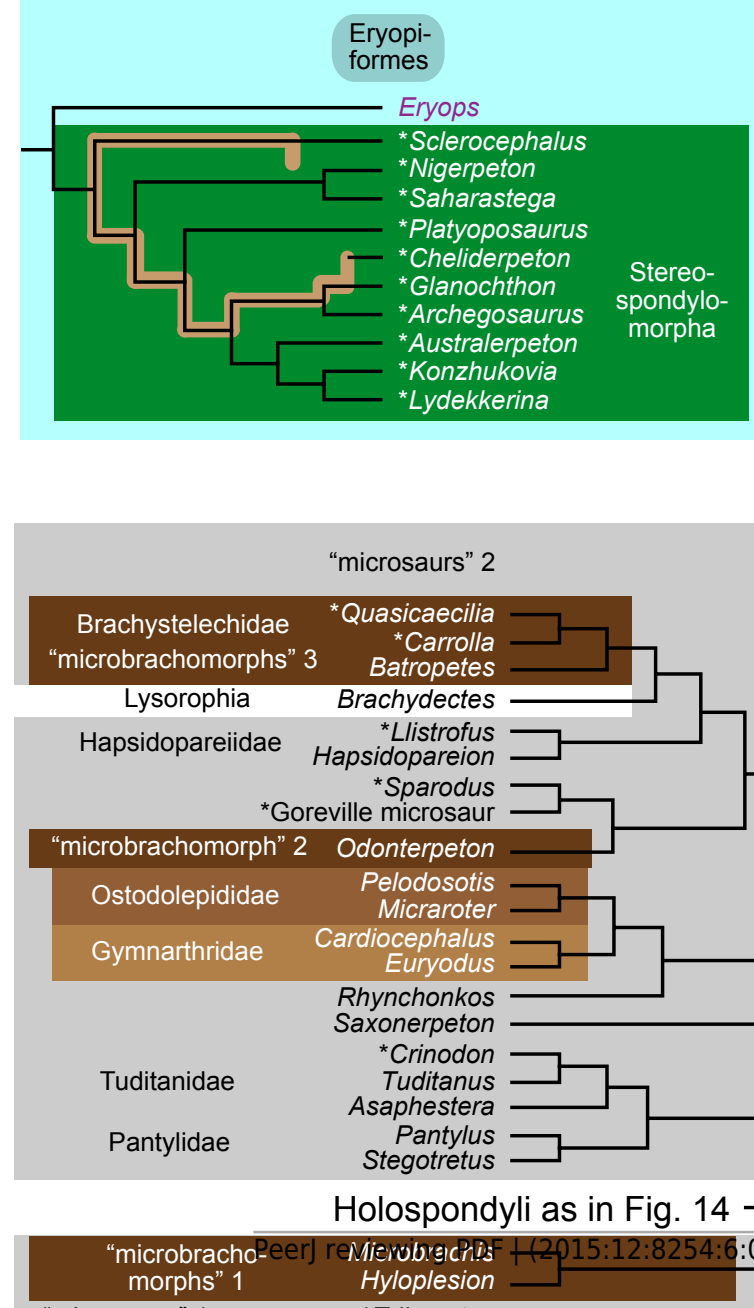

\section{"microsaurs" 2}

Holospondyli as in Fig. 14

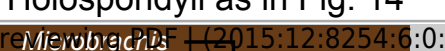
Mierubrechis
Hyloplesion *Trihecaton

Westlothiana
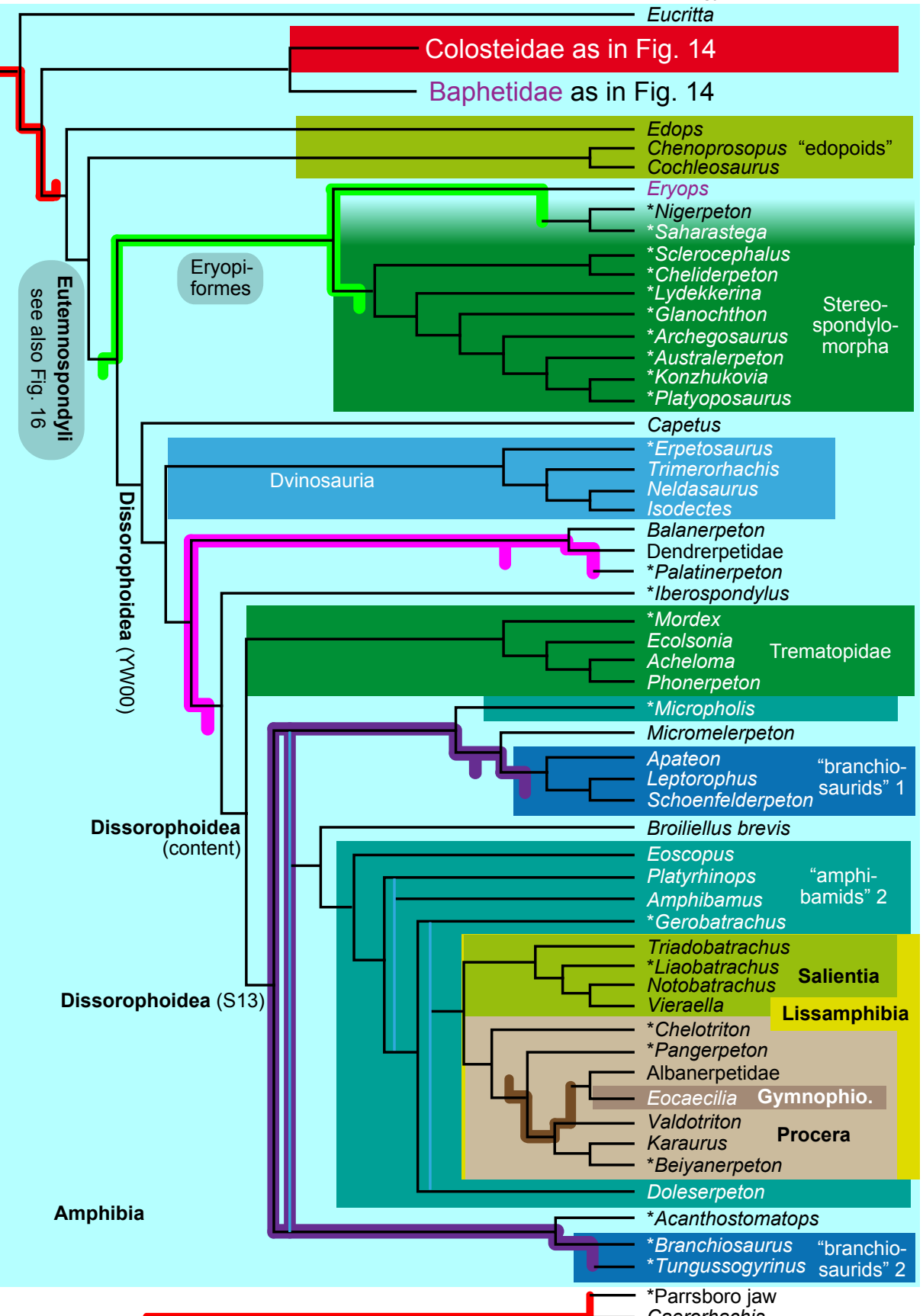
Figure 16(on next page)

Eutemnospondyl phylogeny from those MPTs from Analysis R5 that are not represented in Fig. 15; see the legend of Fig. 15 for more information. 


\section{PeerJ}
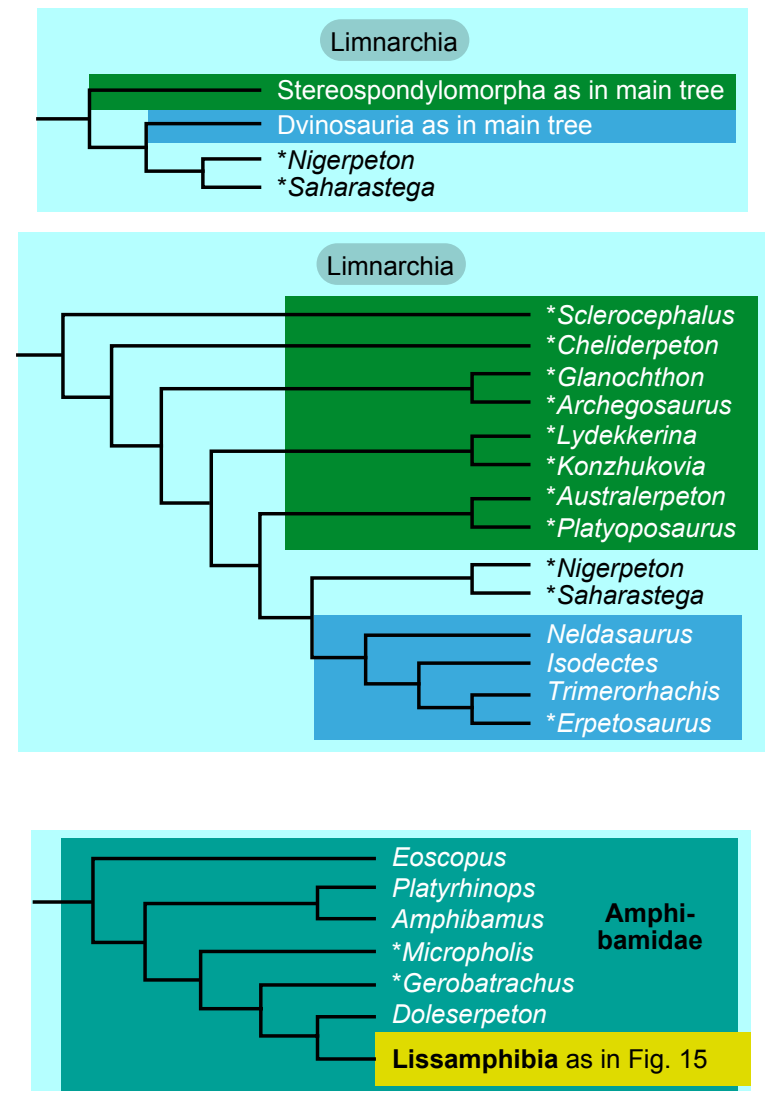

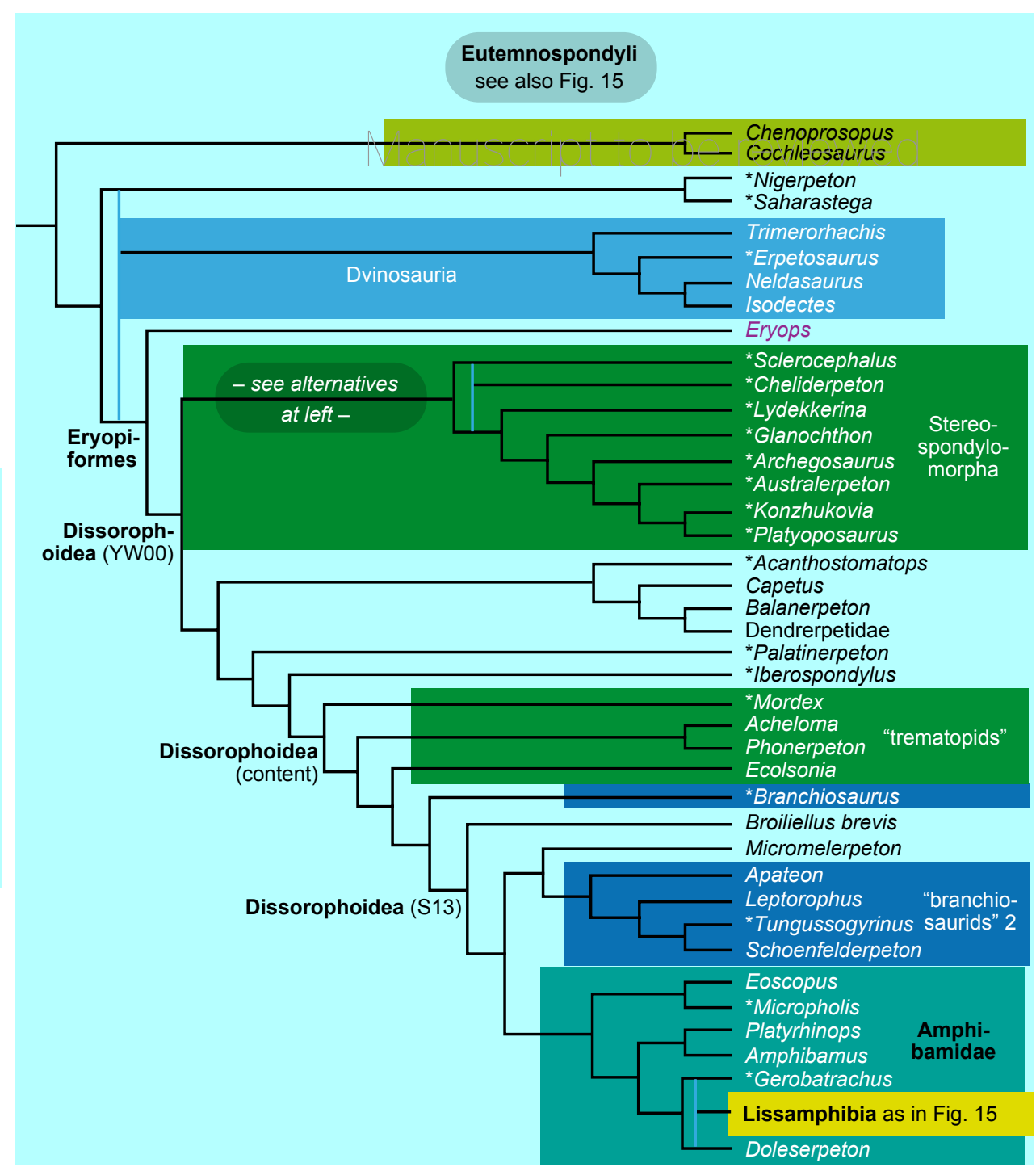


Figure 17(on next page)

Representation of all MPTs (length: 3026 steps) from Analysis R6 (revised matrix, expanded taxon sample, constraint for the PH as in Analysis R3).

Underlays as in Fig. 15. 


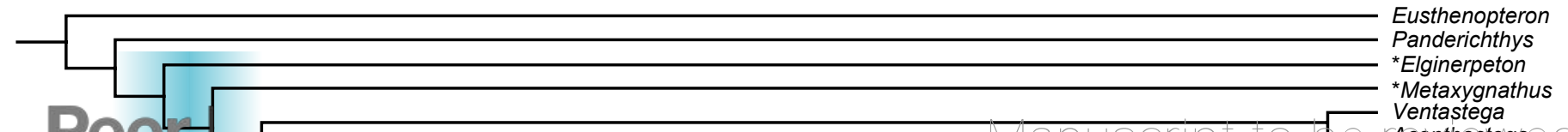
Panderichthys *Elginerpeton Ventastega

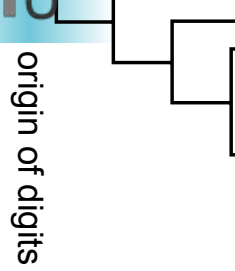

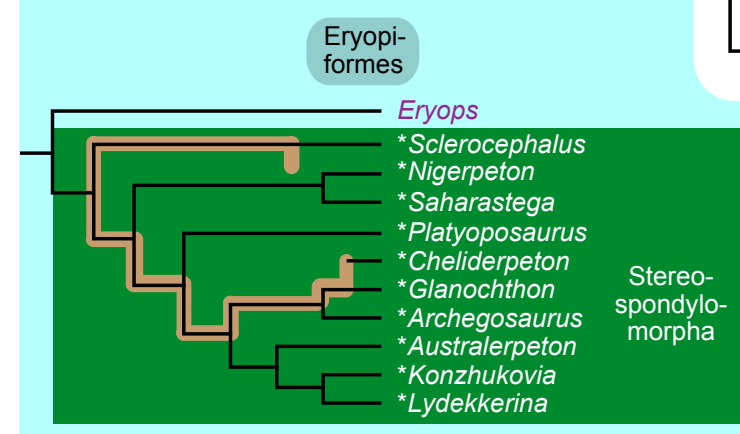

tetrapod crown-group

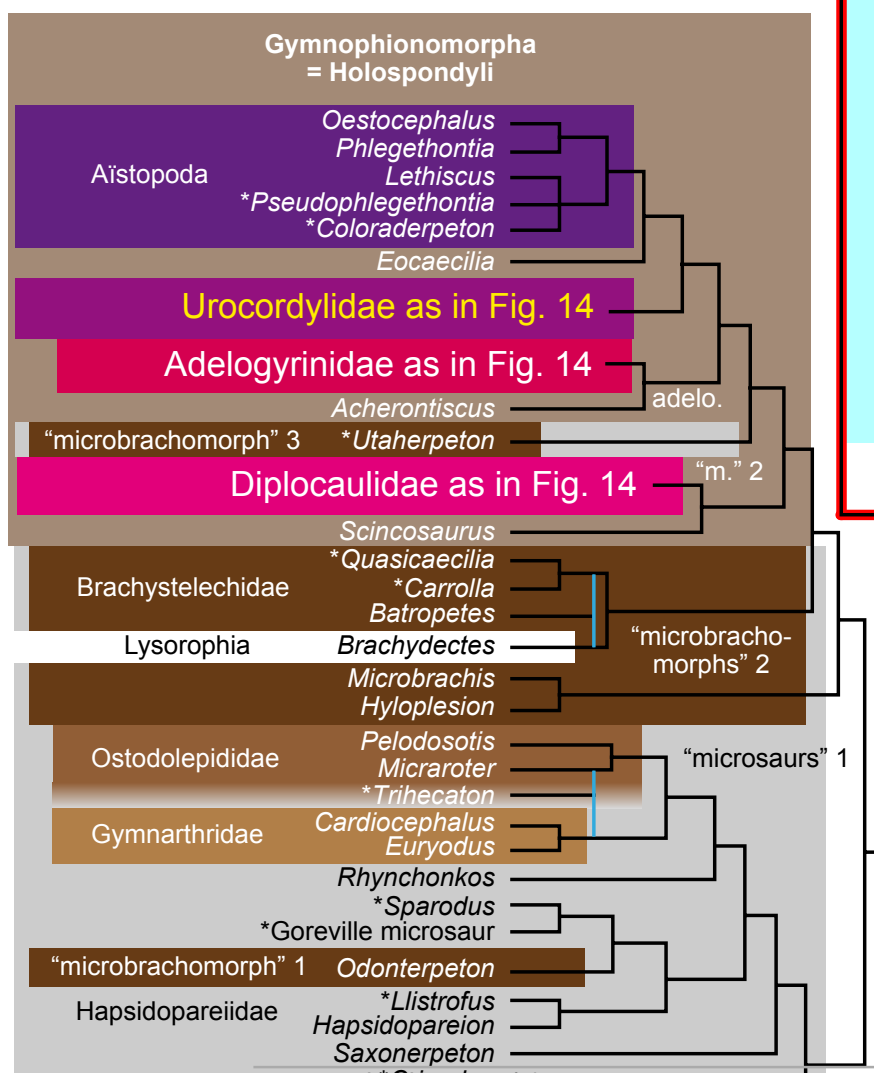

Saxonerpeton

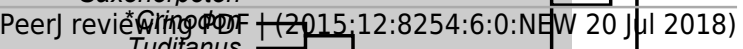

Asaphestera

Pantylidae

Colosteidae as in Fig. 14
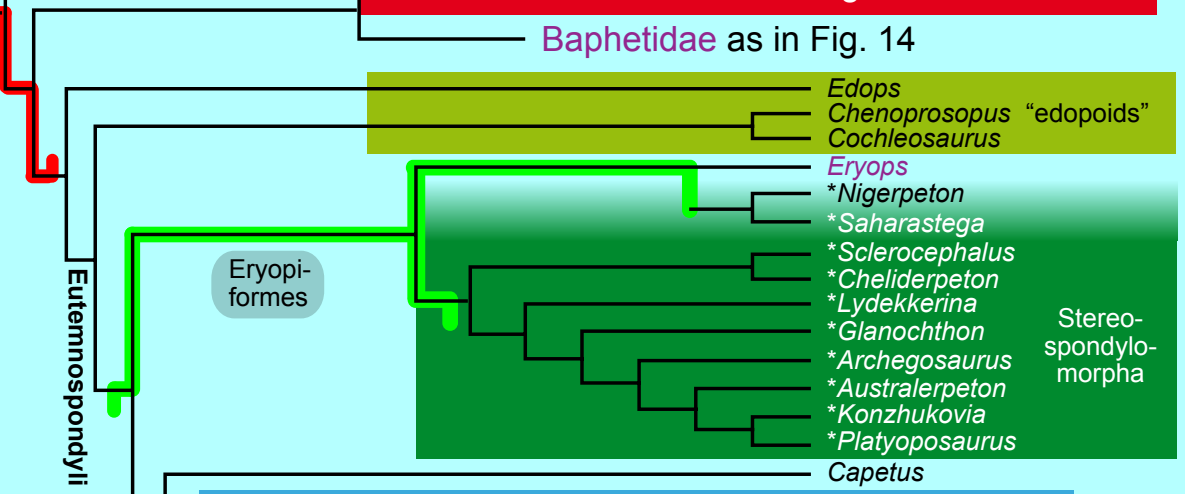

Temnospondyli

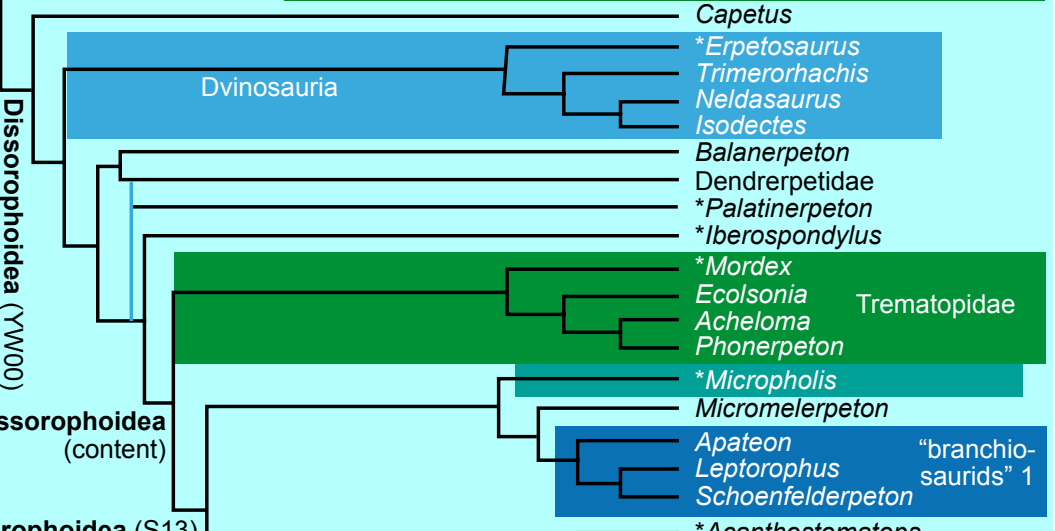

Dissorophoidea (S13)

*Acanthostomatops

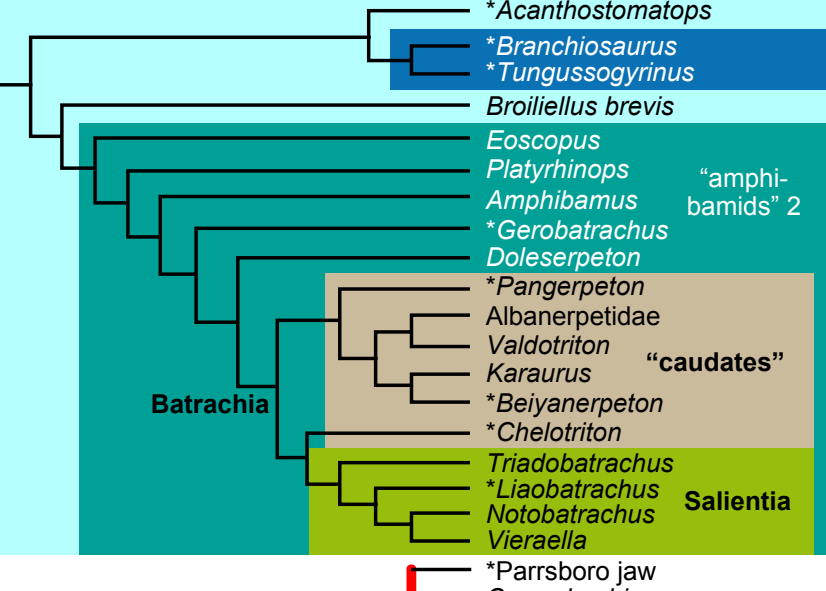

_ Caerorhachis

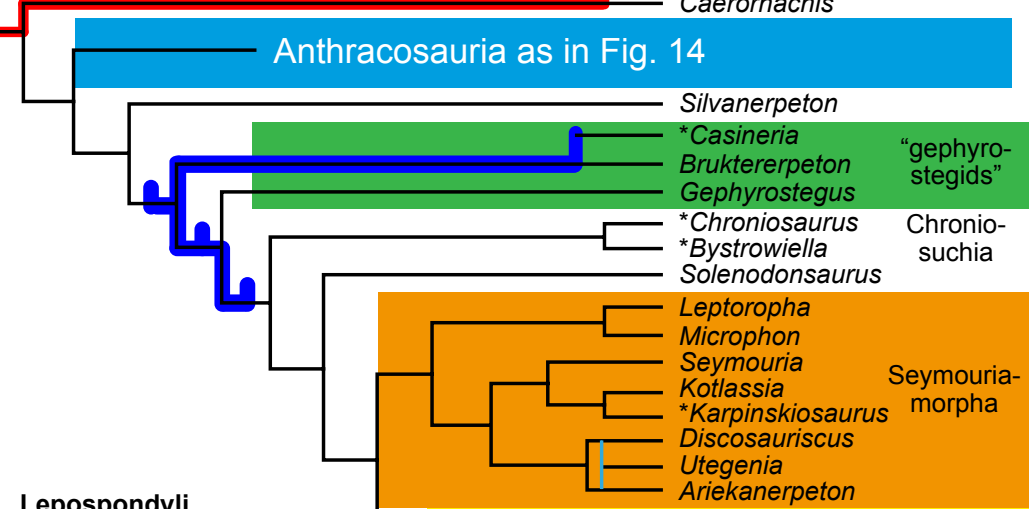

Lepospondyli

Amniota incl. Diadectomorpha as in Fig. 14 
Figure 18 (on next page)

Bootstrap tree from Analysis B1 (revised matrix, original taxon sample, no constraint; compare Fig. 10).

Clades with a bootstrap percentage below 50 are included if they are compatible with those above 50; percentages of 50 and above are in boldface. 
Figure 19(on next page)

Bootstrap tree from Analysis B2 (revised matrix, expanded taxon sample, no constraint; compare Fig. 14).

See legend of Fig. 18 for more information. 
Figure 20 (on next page)

Topology and posterior probabilities from Analysis EB (revised matrix, expanded taxon sample, no constraint; compare Fig. 14, 19).

The numbers are posterior probabilities (in \%), in boldface if 75 or higher. For branch lengths see Fig. 21. Abbreviations: br., branchiosaurids; microbr., microbrachomorphs. 
Eusthenopteron

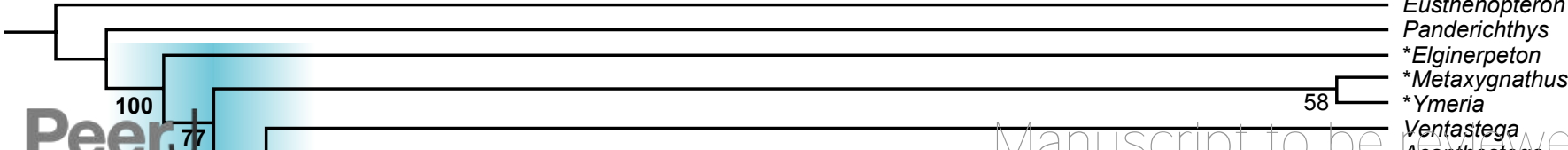

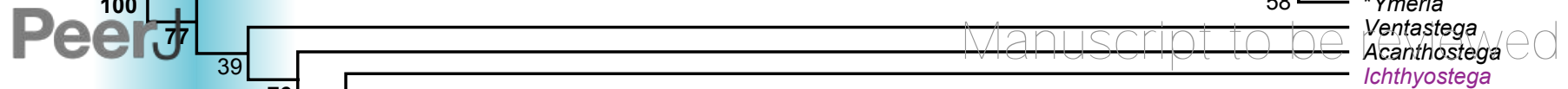

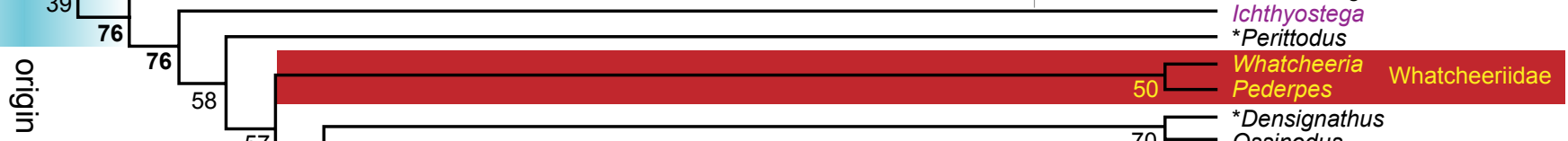

至

을.

57

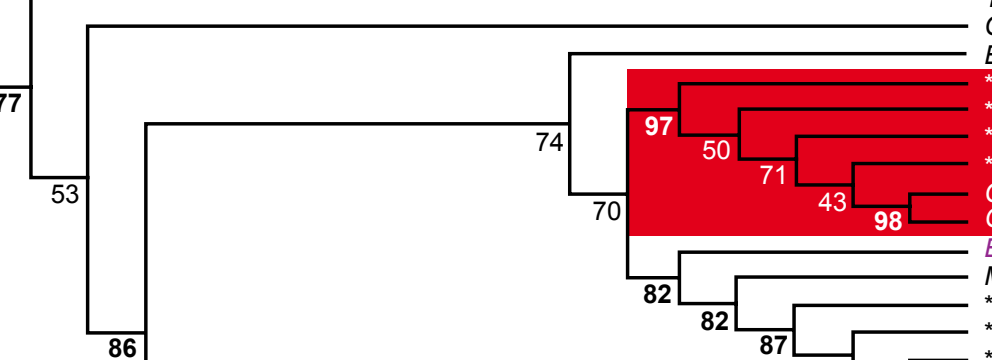

Tulerpeton

Crassigyrinus

Eucritta

77

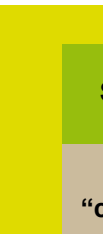

Lissamphibia

Salientia * Notobatrachus $\quad$ Vieraella $\longrightarrow 100$

Liaobatrachus \begin{tabular}{l} 
*Chelotriton \\
Karaurus \\
\hline 467
\end{tabular}

"caudates" * Karaurus

*Pangerpeton Valdotriton

Albanerpetidae

Gymnophionomorpha Eocaecilia "microbr." 3

${ }^{*}$ Carrolla
Batropetes

Lysorophia

Brachydectes Diploceraspis

Diplocaulidae Batrachiderpeton Keraterpeton Diceratosaurus Scincosaurus

*Pseudophlegethontia

\section{Aïstopoda} Lethiscus
Phlegethontia Phlegethontia "Coloraderpeton
Oestocephalus Sauropleura Sauropleura
Urocordylus Ptyonius Adelogyrinus

"urocordylids"

Adelogyrinidae Adelospondylus Dolichopareias Acherontiscus *Utaherpeton Pantylus Stegotretus *Sparodus Microbrachis Hyloplesion Odonterpeton * Llistrofus Hapsidopareion Saxonerpeton

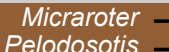
Pelodosotis
Rhynchonkos ardiocephalus Euryodus Asaphestera

*Goreville microsaur ${ }^{*}$ Crinodon Tuditanus Westlothiana Orobates

Diadecto- Limnoscelis

morpha Diadectes

(2015:12:82\$446:0:NEW 20 Jul 2018)

*Caseasauria

Archaeovenator $\square 75$

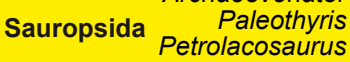
75 Amniota

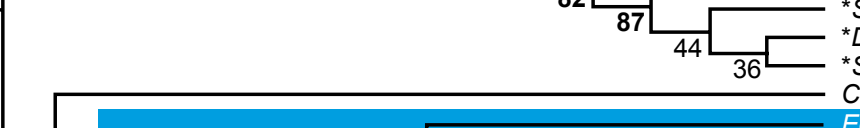
Lotrapod

\section{Ay}

Dytonerpeton

Deltaherpeton

Greererpeto

Baphetes

Megalocephalus

*Diploradus

*Sigournea

*Doragnathus

Caerorhachis

Eoherpeton

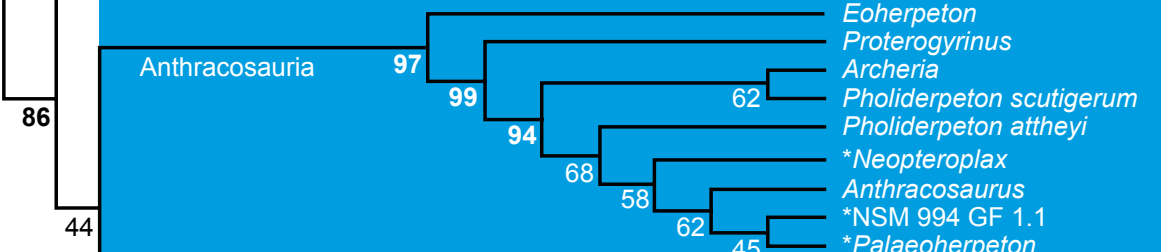

$\frac{4}{48}$

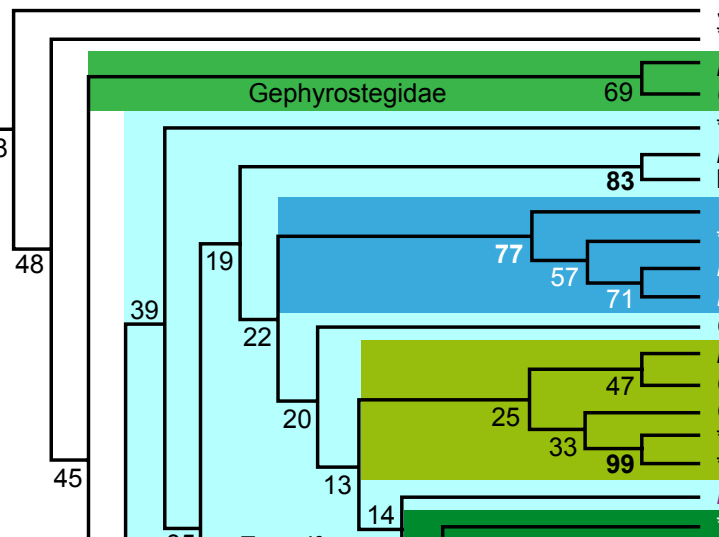
*Palaeoherpeton Silvanerpeton

Parrsboro jaw

Bruktererpeton

Gephyrostegus

Palatinerpeton

Balanerpeton

Trimerorhachis

Baphetidae

Eryopiformes Erpetosaurus sodectes Capetus

Edops

Cochleosaurus

Chenoprosopus Edopoidea Nigerpeton

Saharastega

Dvinosauria
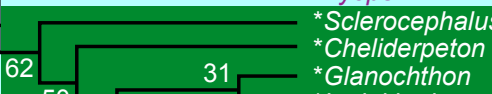

Cheliderpeton

* Glanochthon

Stereo-

57 *Archegosaurus $\begin{array}{r}\text { spondylo- } \\ \text { morpha }\end{array}$

*Platyoposaurus

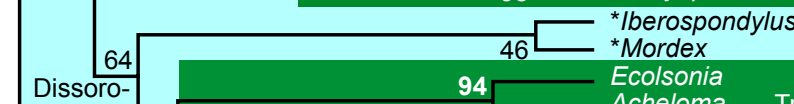

18 phoidea

(YW00) 38

Dissoro-

phoidea
(content)

$40 \quad$ Dissorophoidea (S13)

Ecolsonia

Trematopidae

*Acanthostomatops

*Branchiosaurus

Broiliellus brevis

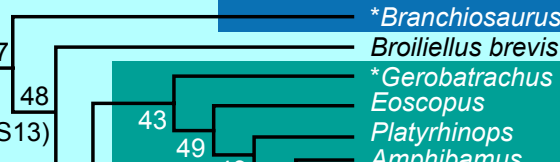

60

30

Platyrhinops

$49 \square+$ Doleserpeton

"amphi-

bamids"

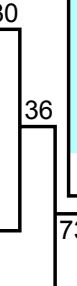

Temno-

spondyli

${ }^{*}$ Micropholis

*Tungussogyrinus

"br." 2

Leptorophus

Schoenfelderpeto

"br." 3

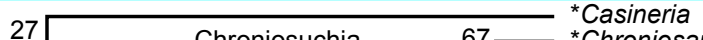

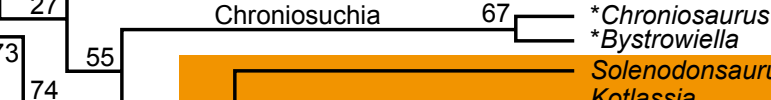

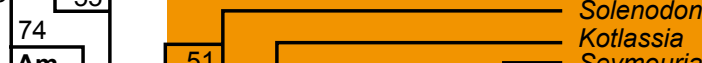

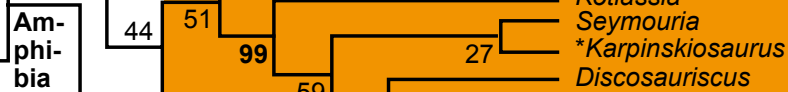

tetrapod 46

crown-

Discosauriscus

6

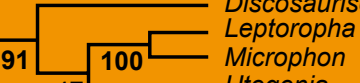

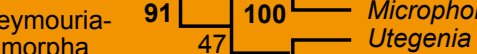

Ariekanerpeton 
Figure 21 (on next page)

Branch lengths from Analysis EB.

For nomenclature and branch support see Fig. 20. 
Figure 22 (on next page)

Homoplasy distribution in our matrix.

The number of extra steps ( $x$-axis) is the number of observed steps (in Mesquite, on the tree in Data S1 for each analysis) minus the minimum possible number of steps, which is the number of states minus one (for ordered and unordered characters as well as for both of our stepmatrix characters). The number of characters that have each number of extra steps is plotted on the $y$-axis. The line between the data points is meaningless, but makes it easier to compare the distributions to an exponential curve. Compare Goloboff, Torres \& Arias (2017: fig. 1(a)). A: original taxon sample (Analysis R1); the highest number of extra steps is 47 , but we plot to 69 for comparison to B. B: expanded taxon sample (Analysis R4); the highest number of extra steps is 69 . 

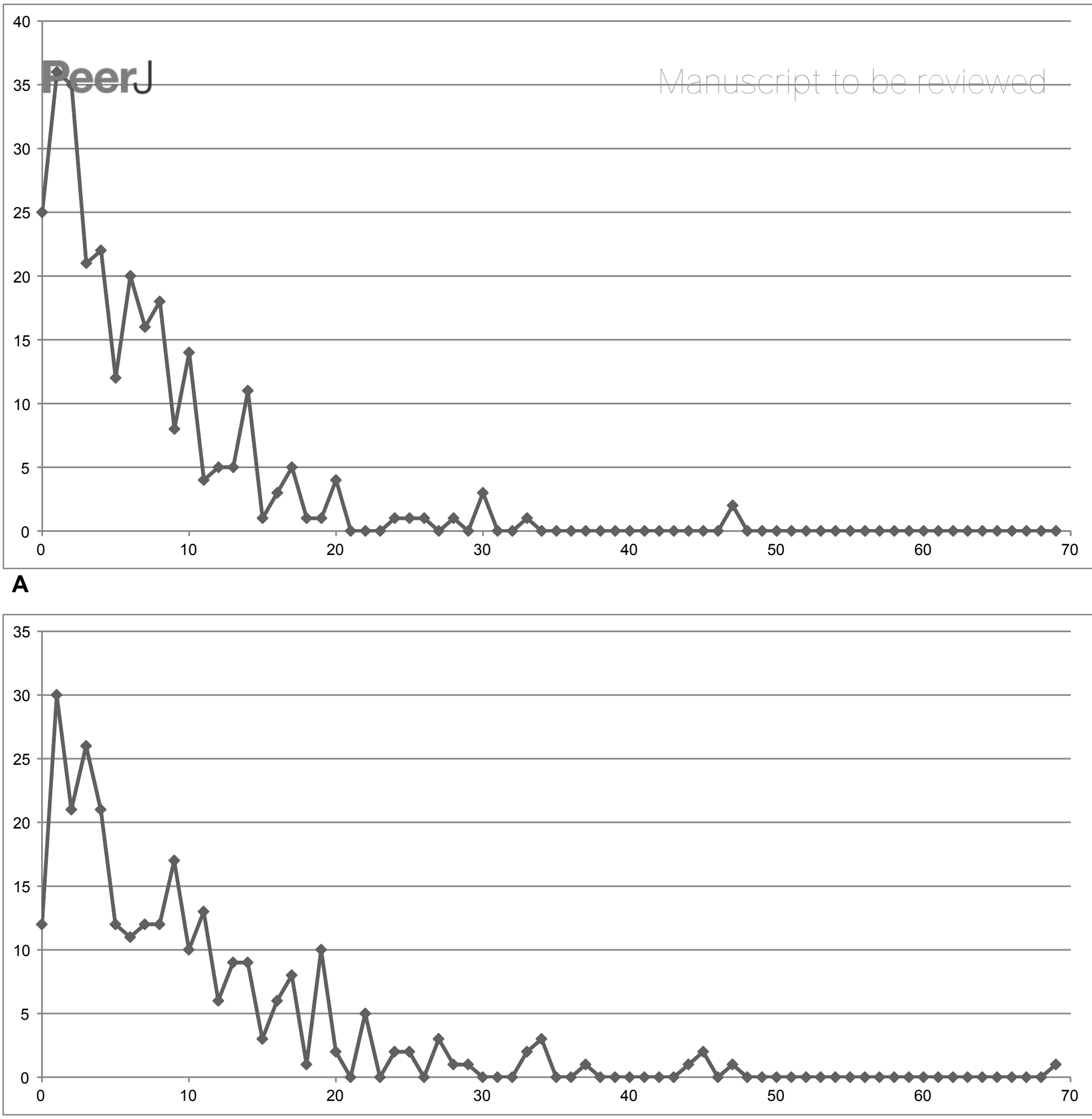

B 


\section{Figure 23(on next page)}

Hypotheses on the relationships of Devonian limbed and possibly limbed vertebrates.

(Whatcheeriidae and $*$ Perittodus, which have limbs, are only known from the Carboniferous with certainty.) Equally parsimonious positions of the same OTU are highlighted in color in this and the following figures. A: RC07 and references therein. B: our results from the same taxon sample as RC07 (Analyses R1-R3). Numbers below internodes are BPO, in boldface if 50 or higher. C: our results from the expanded taxon sample (R4-R6). Numbers below internodes are BPE PP, BPE in boldface if 50 or higher, PP in boldface if 75 or higher. Analysis B2 places *Ymeria next to Ventastega (BPE: 19), Acanthostega one node crownward of them (BPE: 11) and *Densignathus one node rootward of Whatcheeriidae (BPE: 13); EB places *Ymeria next to *Metaxygnathus (PP: 58), Acanthostega one node crownward of Ventastega (PP: 76) and *Perittodus one node crownward of Ichthyostega (PP: 58). 


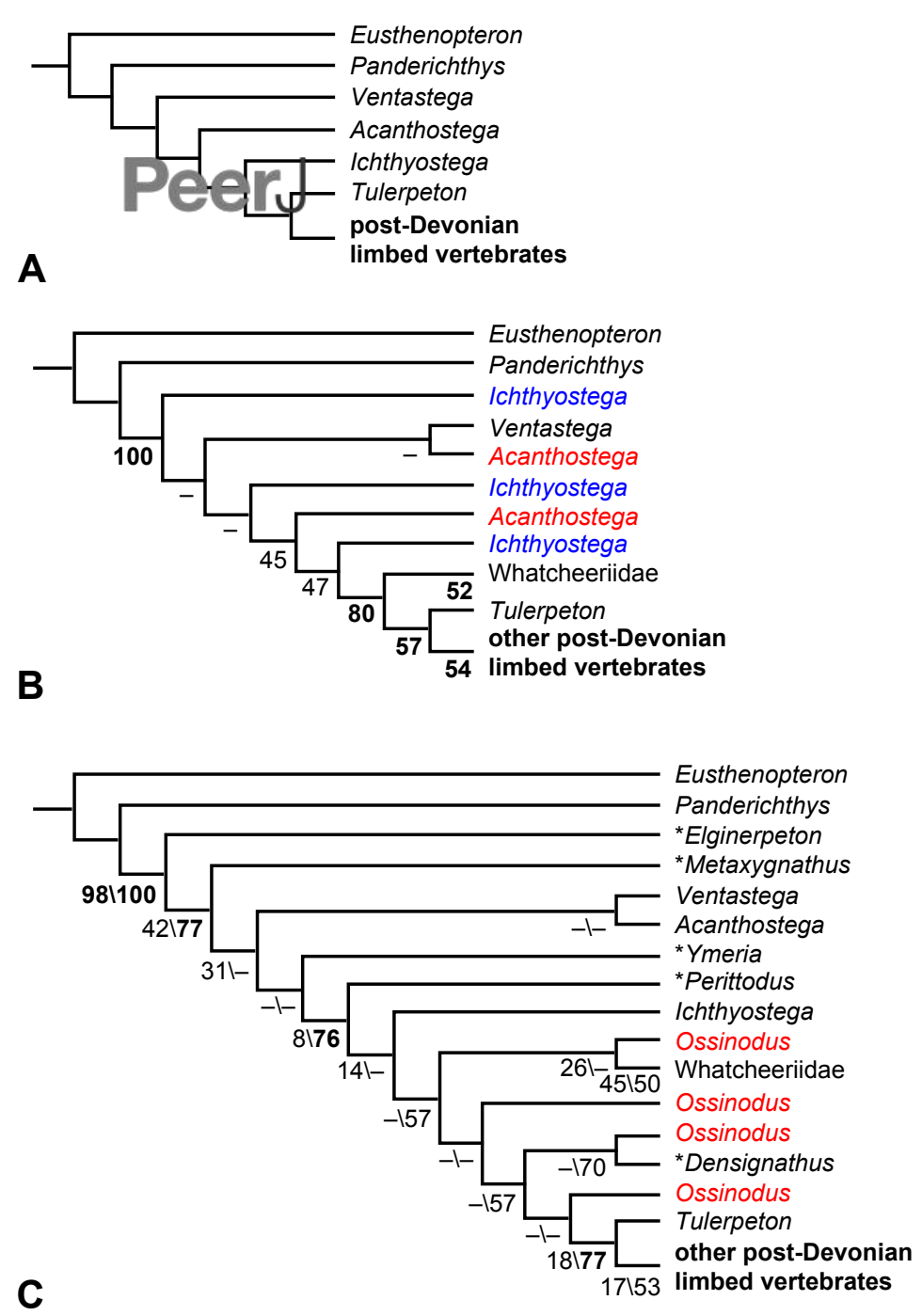




\title{
Figure 24(on next page)
}

\section{Hypotheses on the relationships of post-Devonian limbed vertebrates, and distribution of several character states.}

\begin{abstract}
A: RC07; the entire clade shown here equals the "post-Devonian limbed vertebrates" of Fig. 23A. Anthr., Anthracosauria. B: our results from the original taxon sample (R1-R3). The entire clade shown here equals the "other post-Devonian limbed vertebrates" of Fig. 23B. Numbers are BPO, in boldface if 50 or higher. Analysis B1 finds Caerorhachis next to the other temnospondyls, but places Temnospondyli in the more rootward of the two indicated positions and also finds Silvanerpeton next to the other anthracosaurs (BPO: 10). C: our results from the expanded taxon sample (R4-R6). The entire clade shown here equals the "other post-Devonian limbed vertebrates" of Fig. 23C. In B and C, the extant amphibians are shown indirectly as the three positions of the tetrapod crown-group; although not rendered in boldface, "(rest of) Temnospondyli" is extant in R2, R3, R5 and R6, and "Lepospondyli" is extant in R1, R3, R4 and R6. Parentheses show which of these positions are found in which analyses. The numbers of these analyses are written in black if these analyses find a single position; for any position found as the single position by any analyses, the parentheses themselves are also black. Numbers are BPEIPP, BPE in boldface if 50 or higher, PP in boldface if 75 or higher.
\end{abstract}

The rectangles indicate state 0 (filled) or 1 (empty) of characters 31 (PAR 1 - cyan), 147 (PSYM 1 - teal), 192 (CLE 2 - blue), 212 (HUM 10 - purple), 214 (HUM 12-15 - red), 260 (TRU VER 8 - orange) and 277 (CAU FIN 1 - green); absence of a rectangle means missing data. Where known, Devonian limbed vertebrates have state 0 of each of these characters.

Character 31: supratemporal/postparietal suture (0) or tabular/parietal suture (1); 147: presence (0) or absence (1) of parasymphysial; 192: presence (0) or absence (1) of postbranchial lamina on cleithrum; 212 : humerus not (0) waisted (1); 214: humerus L-shaped (0) or not (1); 260: absence (0) or presence (1) of fusion between left and right pleurocentra in ventral midline; 277: presence (0) or absence (1) of tail fin skeleton (supraneural radials, lepidotrichia). See App. S1 for more precise definitions and discussion. Note that, of all adelospondyls, character 260 is only known in Acherontiscus; the adelogyrinids share state 1 if their single-piece centra are pleurocentra, but they have state 0 if their centra are intercentra, a question we cannot presently decide. Although all anthracosaurs in this matrix are unknown for character 277 , we here show state 0 because of ${ }^{* *}$ CM 34638 (Clack, 2011b).

Further derivations occur within some of the composite clades shown here: the highly nested temnospondyl Micromelerpeton is polymorphic for 31, and the diadectomorph Tseajaia has state 31(0); Pederpes (nested within Whatcheeriidae) has state 192(1); Urocordylidae and the diplocaulid Keraterpeton ("Iepospondyls") have state 212(0); Eusthenopteron, Panderichthys and the embolomeres Archeria and Pholiderpeton scutigerum have state 212(1), while Limnoscelis and Orobates (Diadectomorpha) as well as the diplocaulids Keraterpeton and Diceratosaurus have state 212(0); and the highly nested temnospondyls Doleserpeton and *Gerobatrachus have state $260(1)$. State 2 of the ordered character 214 is not shown (Eucritta, which has state 0 or 1 , is therefore shown as unknown); all the OTUs that have this state (an extra-long humerus) are nested well within clades for which $214(1)$ is plesiomorphic. 


\section{A}

A

B

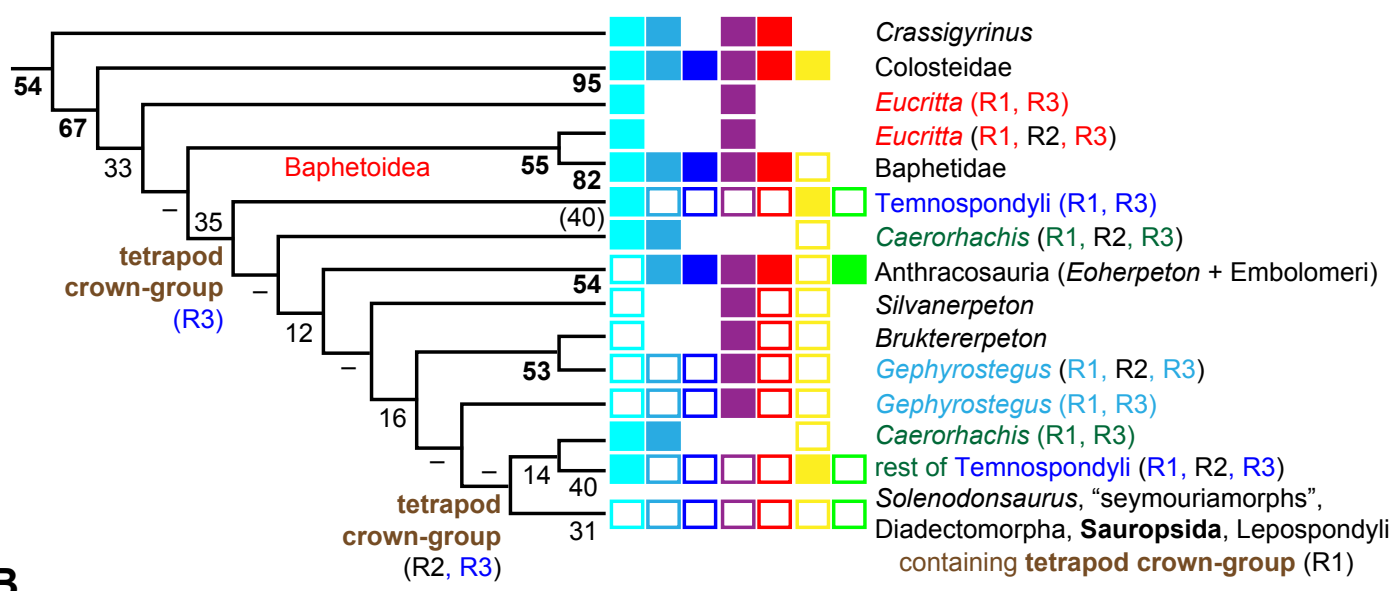




\section{Figure 25 (on next page)}

Hypotheses on temnospondyl phylogeny since 2006.

Taxa absent from our expanded taxon sample are omitted, those we added to the sample of RC07 are marked with an asterisk. Dvinosauria and Dissorophoidea are collapsed where possible. The colored rectangles (labeled in $\mathbf{D}$ ) and the terms "Dissorophoidea (content)" and "Dissorophoidea (S13)" are consistent with Figures 1 and 10-20. The matrices of A-C are ultimately based on that of Ruta, Coates \& Quicke (2003), so they - and ours (Fig. 26) should not be considered fully independent of each other. Numbers below internodes are bootstrap percentages; those below 50 were, except in $\mathbf{G}$, not published. A: strict consensus of RC07; non-temnospondyls omitted. Dissorophoidea is marked in boldface for being extant because it contains Lissamphibia; this is the only analysis shown here that included any lissamphibians. RC07 (app. 4) performed a bootstrap analysis but did not publish the results for the part of the tree shown here. B: Pawley (2006: chapter 5). "*Cheliderpeton + *Glanochthon" was a single OTU called "Cheliderpeton spp."; the name Glanochthon had not yet been coined. *Acanthostomatops was included as part of a Zatracheidae OTU. C: Ruta (2009), strict consensus of analysis without reweighting; non-temnospondyls omitted. *Glanochthon latirostris was called by its previous name, "Cheliderpeton latirostre". The "rest-dendrerpetid OTU" was called "Dendrerpeton acadianum", but may have contained information from other dendrerpetids as well; see Material and methods: Treatment of OTUs: Dendrerpetidae . D: McHugh (2012); non-temnospondyl omitted. Dissorophidae (as two clades, one of which contains Broiliellus brevis) and Trematopidae (also as two clades) are nested in three different places within Amphibamidae. All bootstrap percentages of the nodes shown here are below 50 and were not published. E, F: all equally parsimonious topologies (8 MPTs) from the analysis of 48 taxa in Dilkes (2015a); non-temnospondyls omitted. Note (in F) that Dendrerpetidae and Balanerpeton are sister-groups in all MPTs. To see the individual MPTs, we repeated the analysis (calculation time: 00:04:36.5), finding MPTs of the same

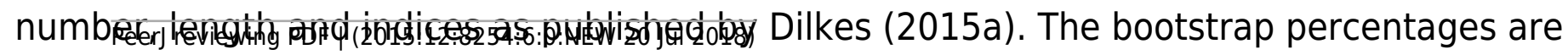


from Dilkes (2015a: fig. 11B), which shows an analysis where Capetus and *Iberospondylus were omitted; the topology is otherwise identical to E. G: Pereira Pacheco et al. (2016), focused on stereospondylomorphs. Two species of *Platyoposaurus and three of *Konzhukovia were included as separate OTUs. The tree by Eltink et al. (2016) is fully compatible. H: Pardo, Small \& Huttenlocker (2017); non-temnospondyls and lissamphibians omitted (the MPTs differ only in the positions of the lissamphibians and ${ }^{* *}$ Chinlestegophis). Numbers are bootstrap percentages followed by Bayesian posterior probabilities in \% (boldface if $\geq 75$, not published $<50$ ). 
PeerJ

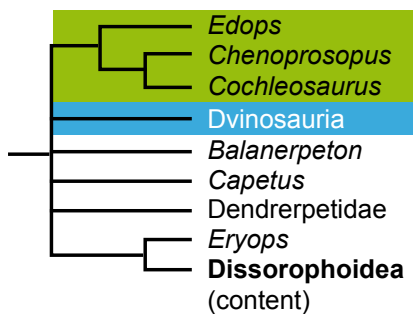

A

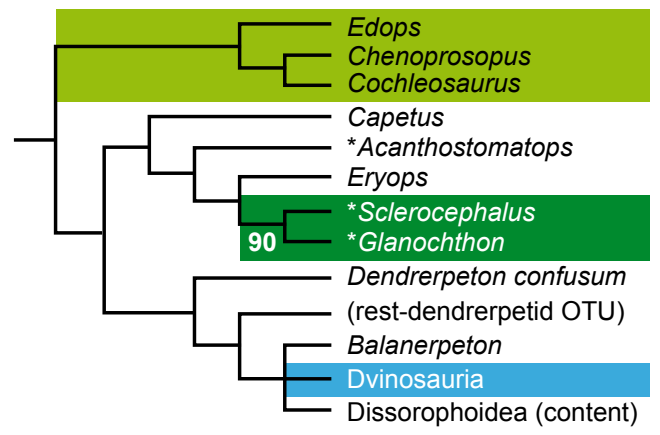

C
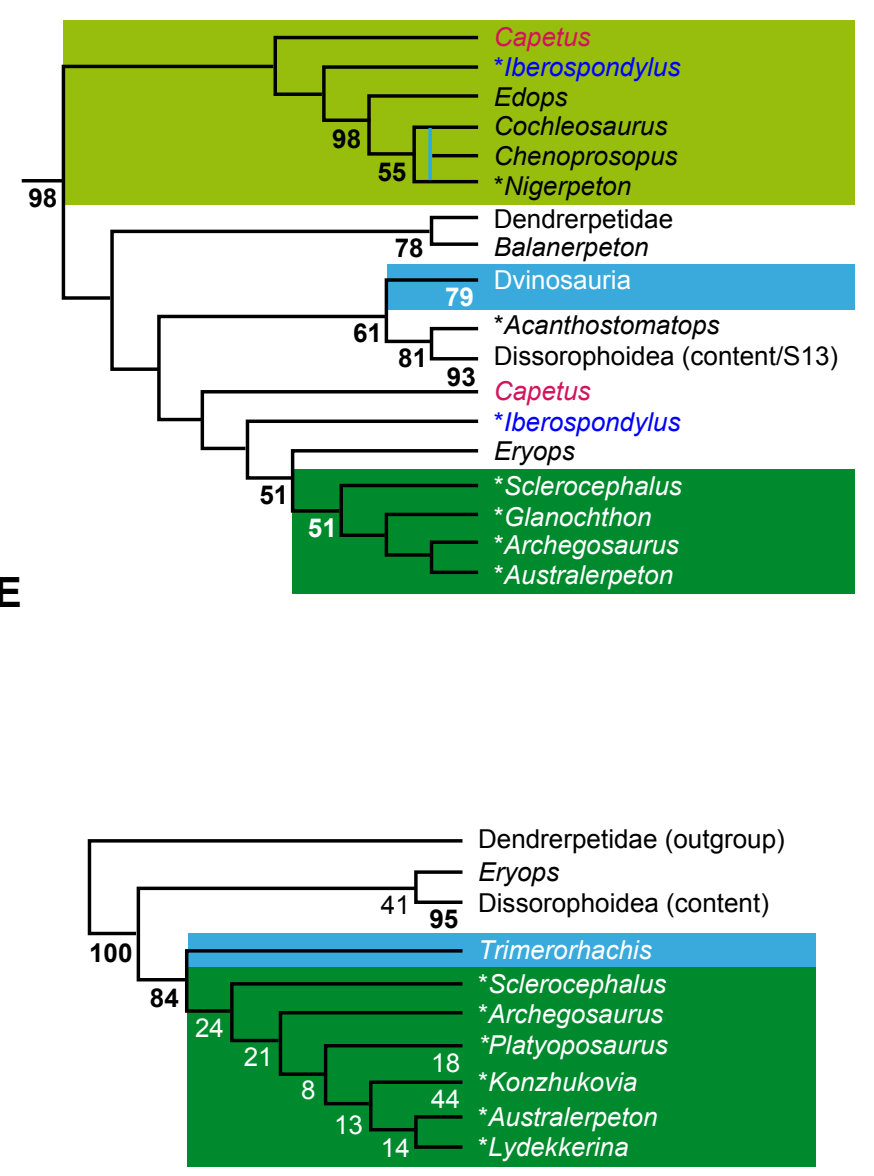

PeerJ reviewing PDF | (2015:12:8254:6:0:NEW 20 Jul 2018)
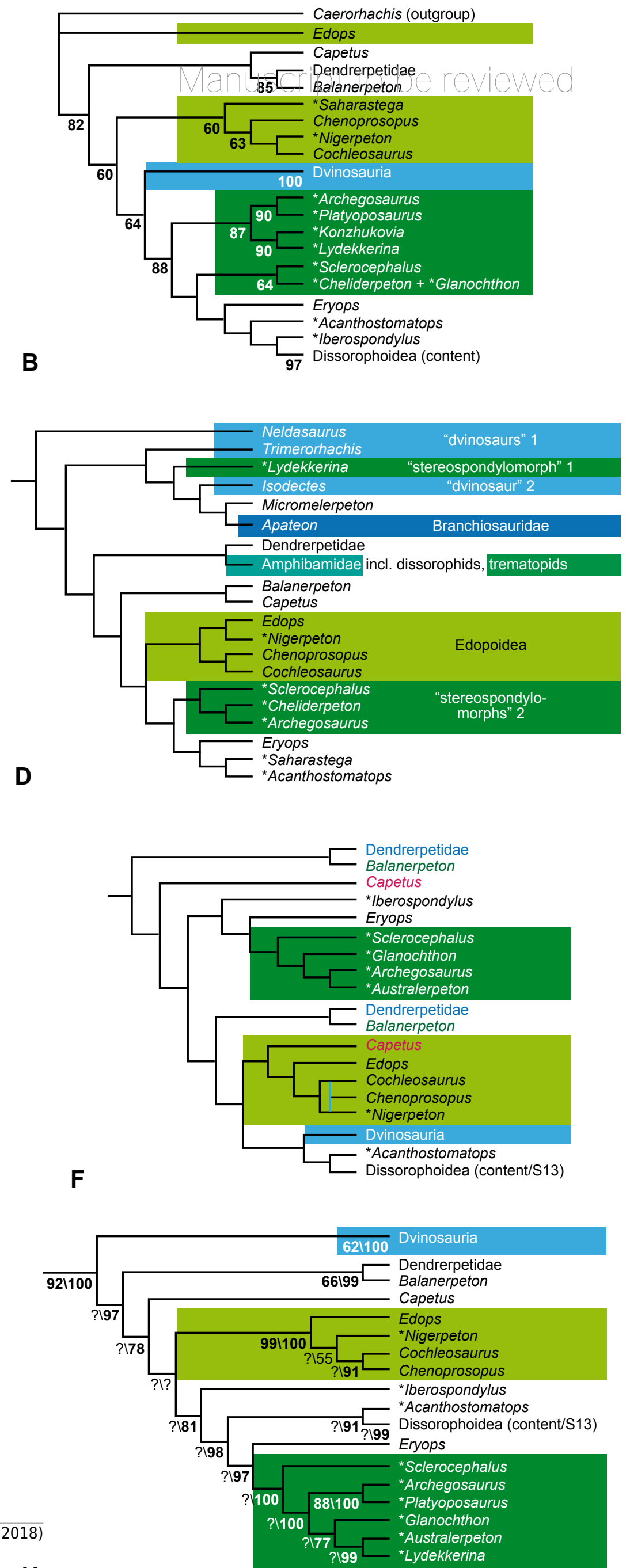


\section{Figure 26(on next page)}

Temnospondyl phylogeny in our analyses, distribution of the intertemporal bone, and the application of the name Temnospondyli.

Dissorophoidea and Stereospondylomorpha are collapsed; modern amphibians and various "lepospondyls" (see Fig. 12) are omitted. Filled rectangles indicate presence of the intertemporal (state 0 of ch. 32 - PAR 2/POSFRO 3/INTEMP 1/SUTEMP 1), empty rectangles its absence (state 1, or state 2 in the case of *Erpetosaurus; see App. S1); the absence of a rectangle for *Palatinerpeton and the *Parrsboro jaw represents missing data. T., Temnospondyli as defined by Schoch (2013); the entire depicted clade is Temnospondyli as defined by Yates \& Warren (2000). A: topology found in Analyses R1-R3 (original taxon sample) in MPTs where Temnospondyli is closer to Amniota than Anthracosauria. Only one position of Caerorhachis is shown; the other is far outside Temnospondyli. Numbers are BPO. B: additional topologies found in R1 and R3 (original taxon sample) in MPTs where Temnospondyli is closer to Amniota than Anthracosauria. Numbers are BPO of nodes absent from A. C: topologies found in R1 and R3 (original taxon sample) and R4-R6 (expanded taxon sample) in MPTs where Anthracosauria is closer to Amniota than Temnospondyli. Only one position of the *Parrsboro jaw is shown, the other being well outside Temnospondyli. Numbers are BPO/BPEIPP, or BPEIPP where BPO is inapplicable. D: additional topologies found in R5 (Fig. 16). Numbers are BPEIPP. 


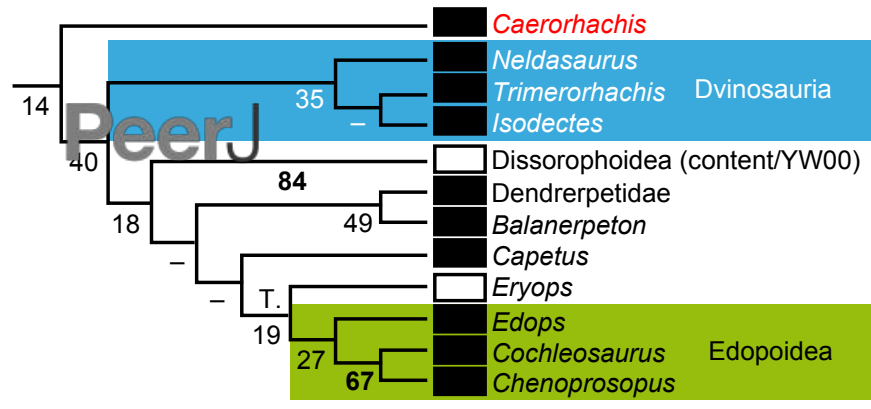

A

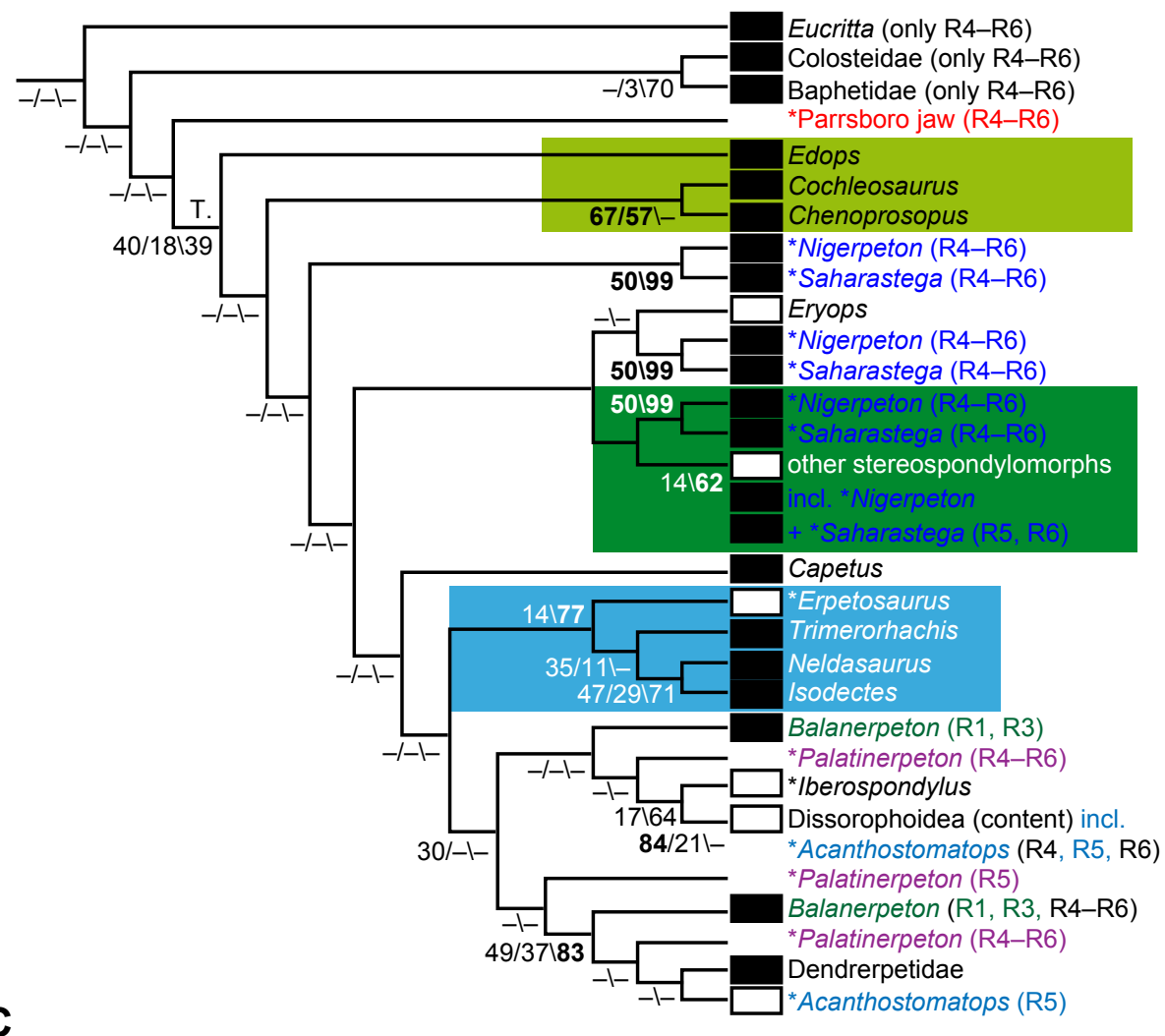

C

B
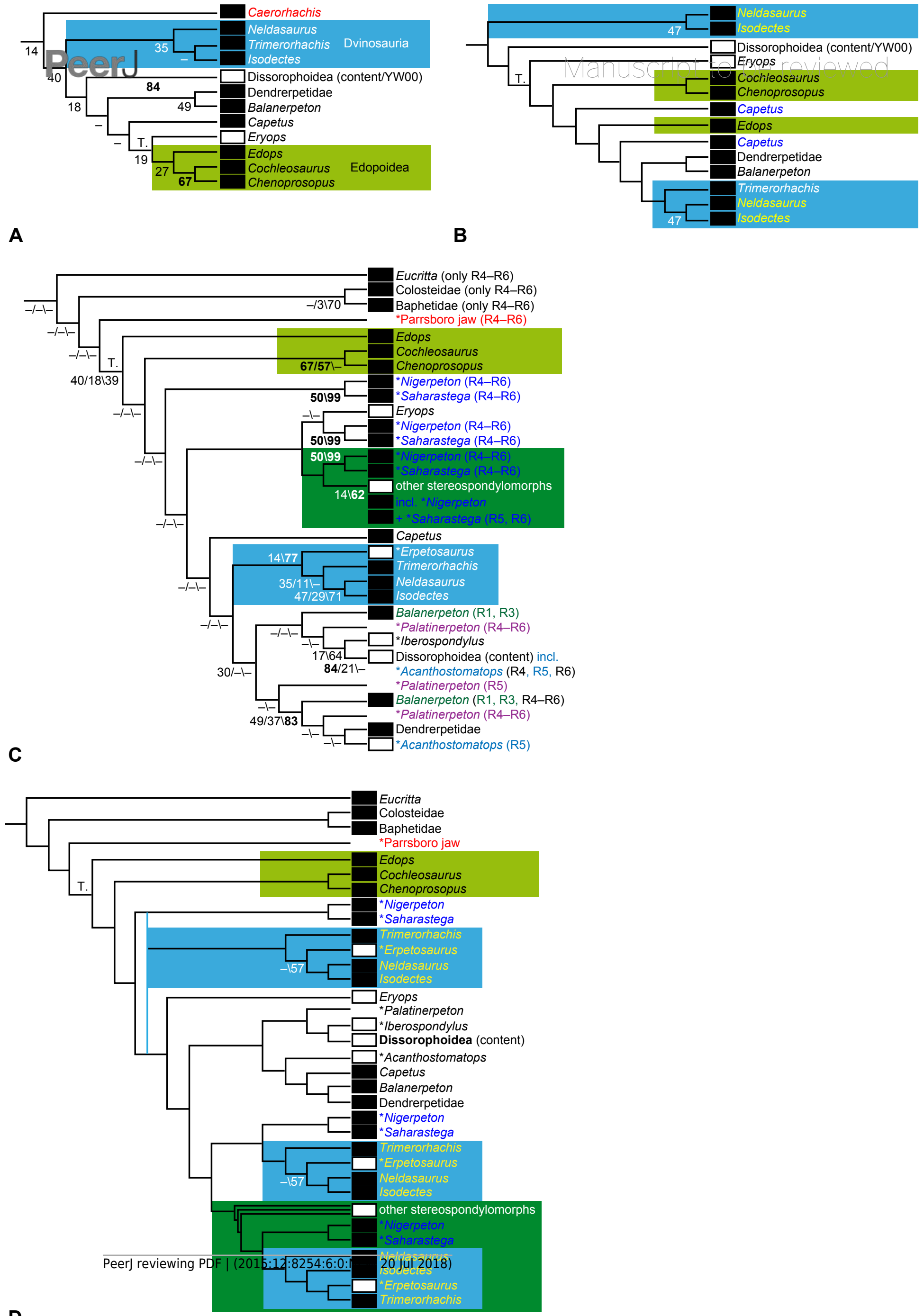


\section{Figure 27 (on next page)}

Amniote relationships and distribution of the intertemporal bone.

Taxa not included in our analyses are omitted; those included in the expanded but not the original taxon sample are marked with an asterisk. Several named clades are collapsed. The matrices of B, C, F and $\mathbf{G}$ are ultimately based on that of Ruta, Coates \& Quicke (2003), so they should not be considered fully independent of each other. Numbers are bootstrap percentages. Filled rectangles indicate presence of the intertemporal (state 0 of ch. 32 - PAR 2/POSFRO 3/INTEMP 1/SUTEMP 1; see App. S1), empty rectangles its absence (any other state); the absence of a rectangle indicates missing data. A., Amniota; D., Diadectomorpha; Saur., Sauropsida. A: Vallin \& Laurin (2004). B: Pawley (2006: chapter 6). C: RC07; "rest of Lepospondyli" excludes the adelospondyls (see Fig. 1, 24A), bootstrap values were not published. D: Clack et al. (2012b; lacking *Bystrowiella) and Witzmann \& Schoch (2017); all bootstrap percentages in this part of the tree are below 50 in both versions and were not published. E: Klembara et al. (2014). Bootstrap percentages below 50 were not published, but note that a sister-group relationship of Chroniosaurus and Gephyrostegidae (G.) is supported by a bootstrap value of $\mathbf{5 3} \%$. F: topologies from our Analysis R2 and some MPTs from R1 and R3, where Temnospondyli is closer to Amniota than Anthracosauria. Numbers are BPO/BPEIPP. G: topologies found in Analyses R4-R6 and the remaining MPTs from R1 and R3. Numbers below nodes are BPO/BPE\PP; the space for BPO is omitted where inapplicable. “D." indicates Diadectomorpha for the original but not the extended taxon sample. 
A
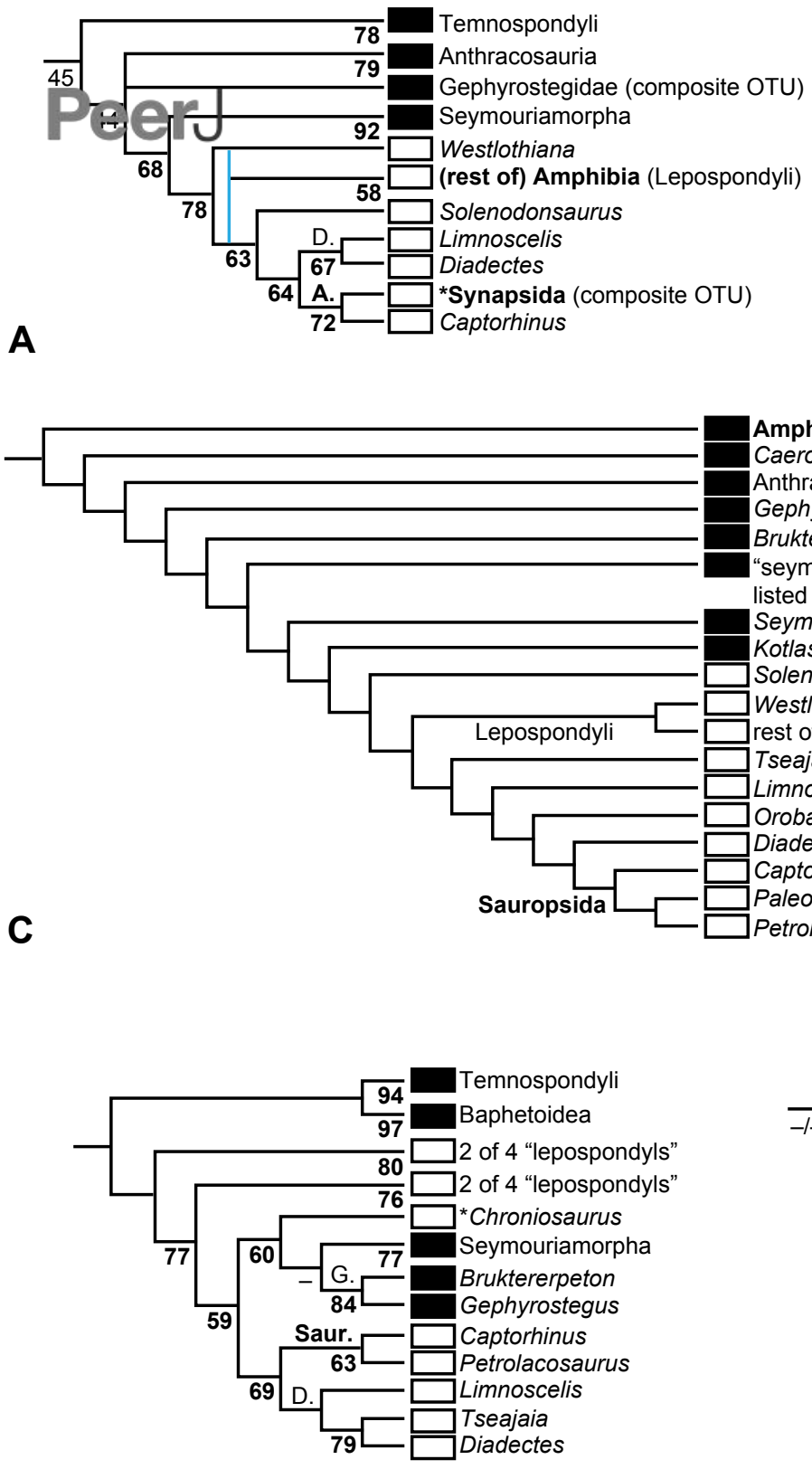

E

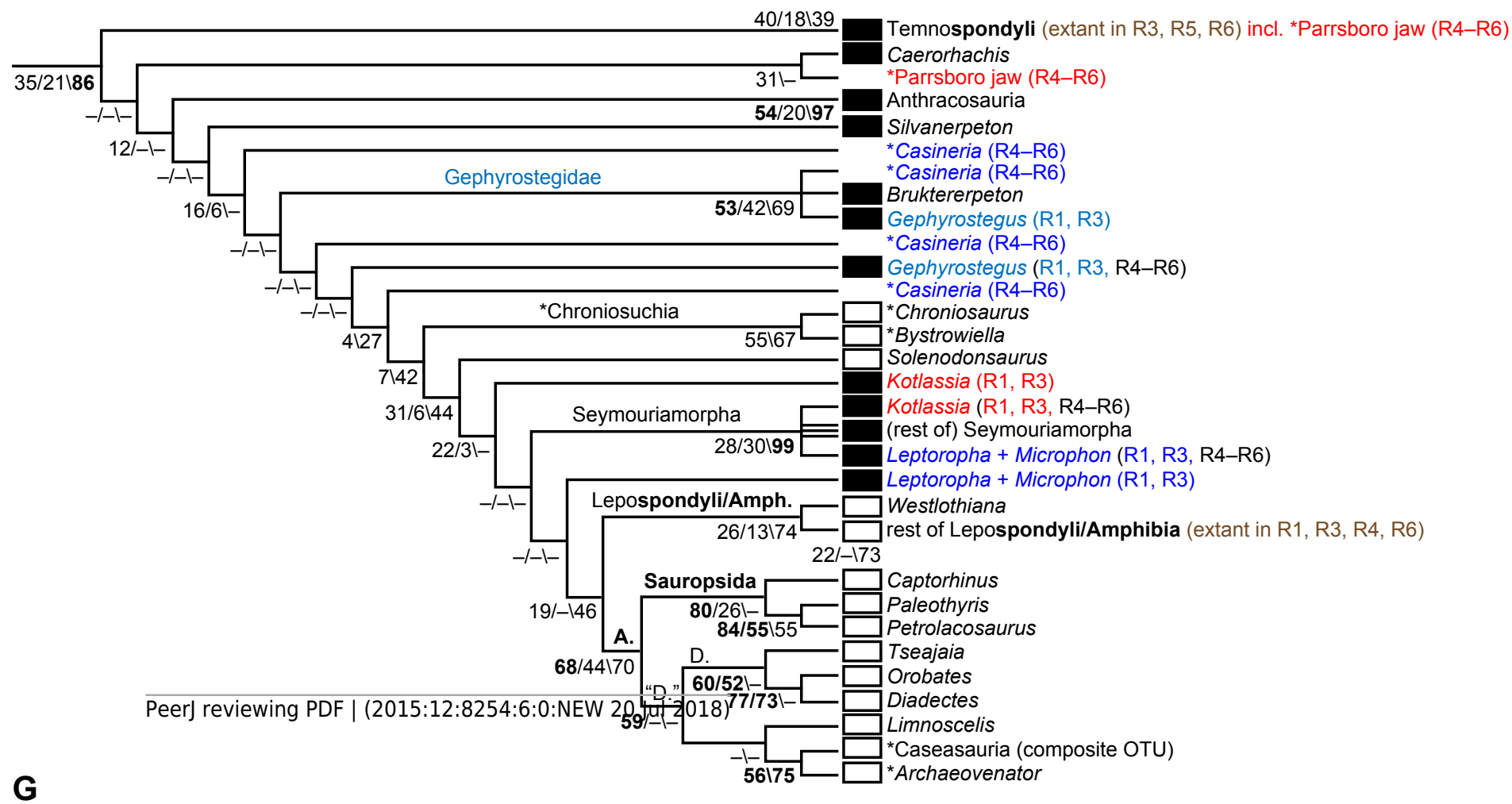

Amphibia (Temnospondyli)

Caerorhachis

Anthracosauria

Bruktererpeton

"seymouriamorphs" not

Seymouria

Kotlassia

Solenodonsaurus

Westlothiana

Tseajaia

Limnoscelis

Orobates

Diadectes

Captorhinus

B

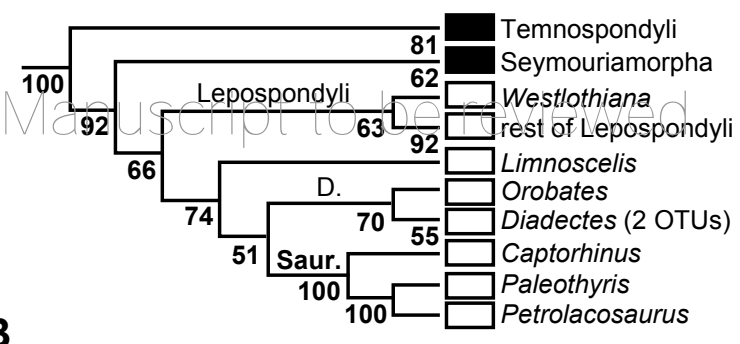

Petrolacosaurus

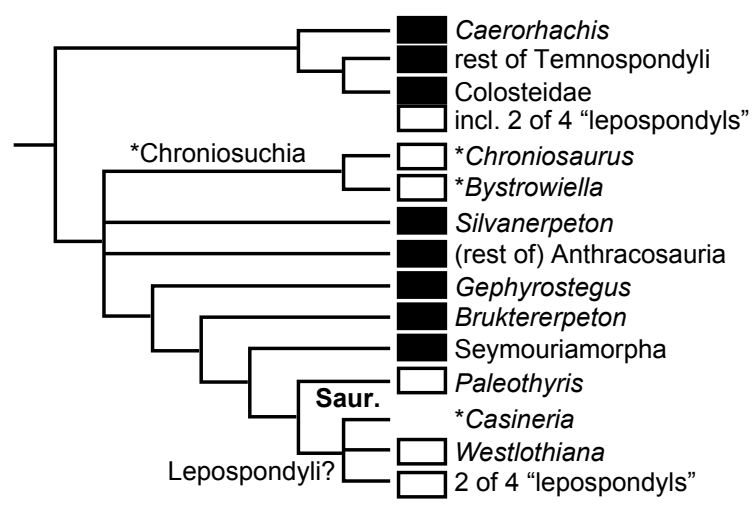

D

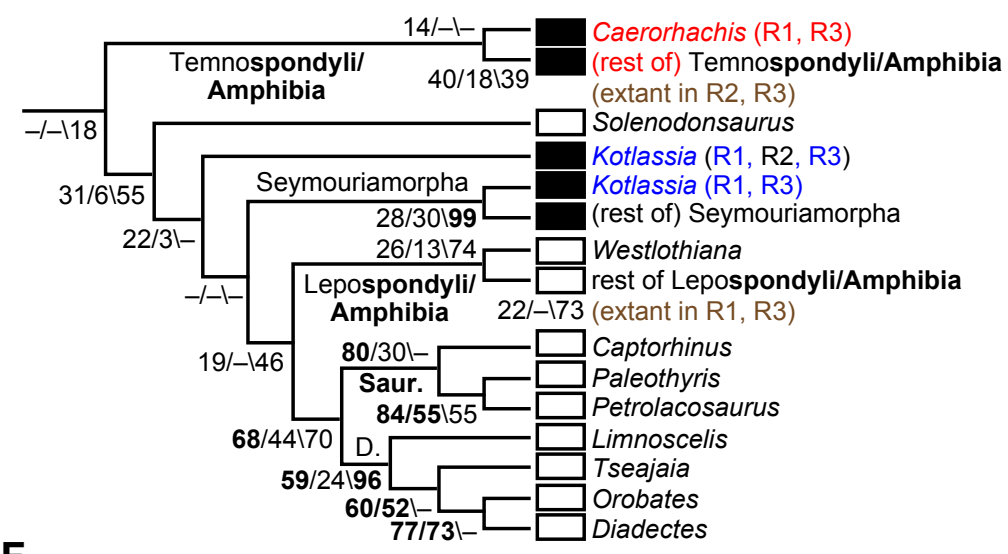

$\mathbf{F}$
Gephyrostegus

listed below

rest of Lepospondyli 


\section{Figure 28(on next page)}

Hypotheses on "lepospondyl" relationships since 2004.

Various named clades are collapsed. Taxa not included in our analyses are omitted; those included in the expanded but not the original taxon sample are marked with an asterisk. A., Amphibia; HPSA13, Huttenlocker et al. (2013); L., Lepospondyli; MJA12, Maddin, Jenkins \& Anderson (2012); ML09, Marjanović \& Laurin (2009). A: Vallin \& Laurin (2004). The other position of Westlothiana lies outside Amphibia and is not shown (see Fig. 27A). Numbers are bootstrap percentages. B: Pawley (2006: fig. 88). Numbers are bootstrap percentages from fig. 63 (those below 50 were not reported), which shows an analysis that omitted the taxa written in dark green but found a congruent topology. C: Pawley (2006: fig. 91): cranial characters and data as in B, but the postcranial ones unchanged from Ruta, Coates \& Quicke (2003). D: RC07. E: Milner \& Ruta (2009); entire analysis except the aïstopod **Ophiderpeton. Adelospondyls and lysorophians were not included. H., Holospondyli; N., Nectridea. Numbers are bootstrap percentages. F: matrices derived from that of Anderson et al. (2008a), namely: 1) ML09; 2) MJA12 as reanalyzed by Marjanović \& Laurin (2018), lacking the corrections proposed by ML09 and those by Sigurdsen \& Green (2011) but containing those by Maddin \& Anderson (2012) and new ones by MJA12; 3) HPSA13, lacking all of the proposed corrections mentioned here but containing new ones. Note that MLO9 found the $L H$, MJA12 the TH and HPSA13 the PH, and that ML09 and HPSA13 did not find Eocaecilia in the same position. Numbers are bootstrap percentages from ML09, MJA12 and HPSA13 in this order (the latter two did not report percentages below 50). - Pardo, Small \& Huttenlocker (2017: fig. S6) analyzed an unpublished matrix based on MJA12 with some updates based on HPSA13; the MPTs and the Bayesian tree are almost fully congruent with this tree, especially with MJA12, and neither bootstrap values nor posterior probabilities were published. 

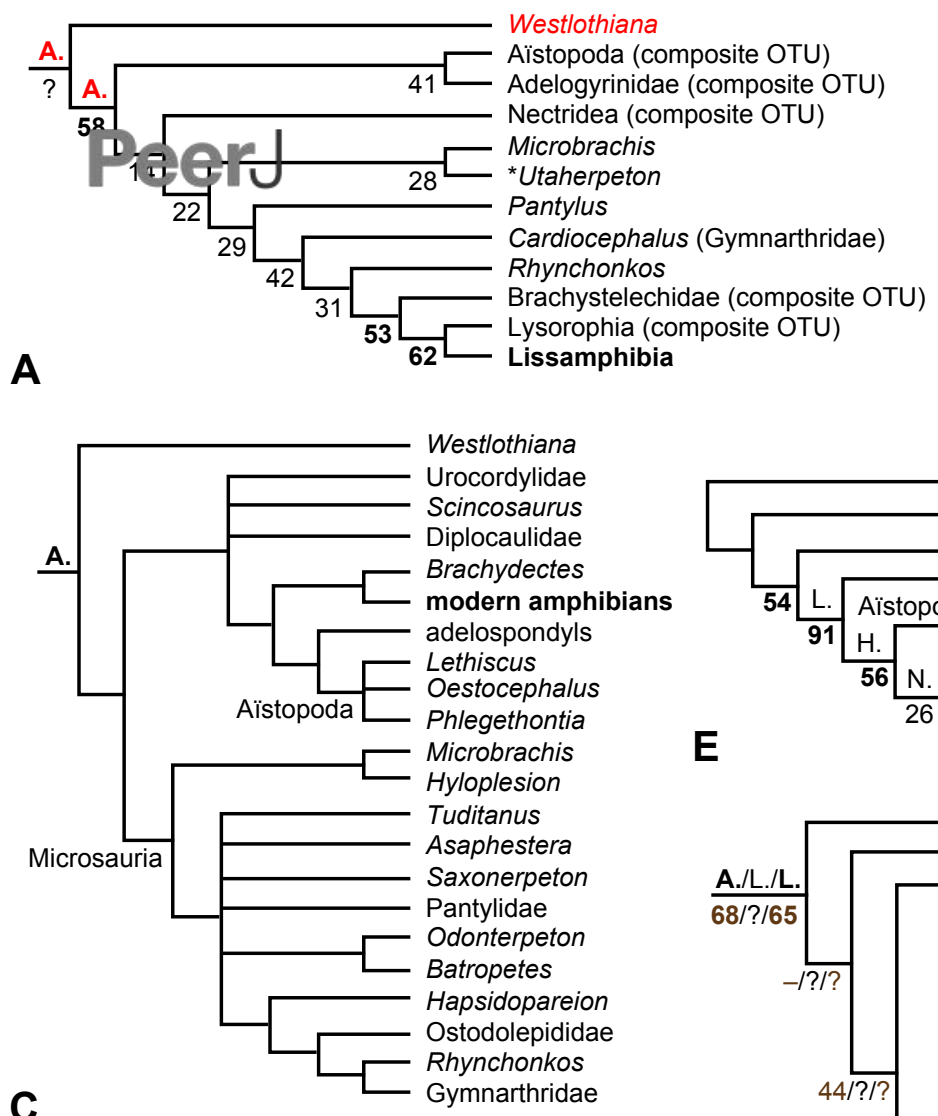

C

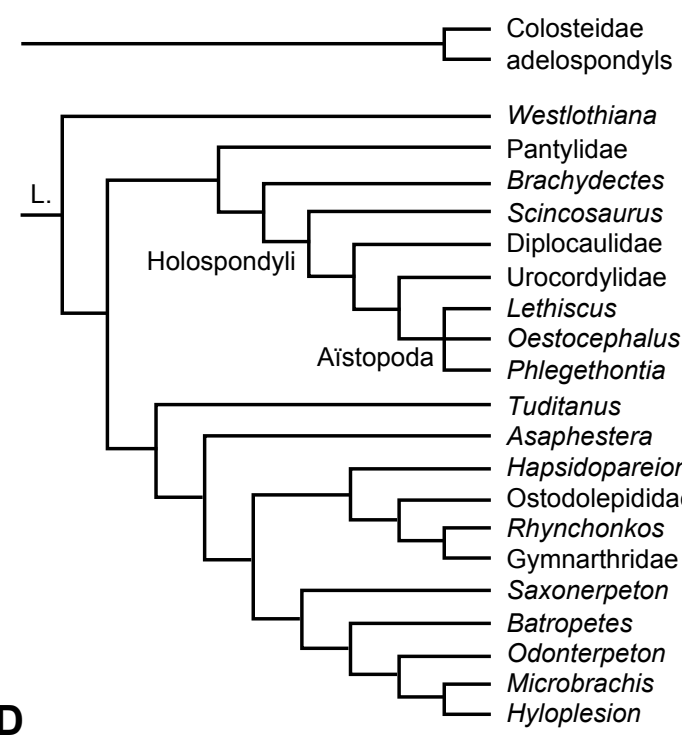

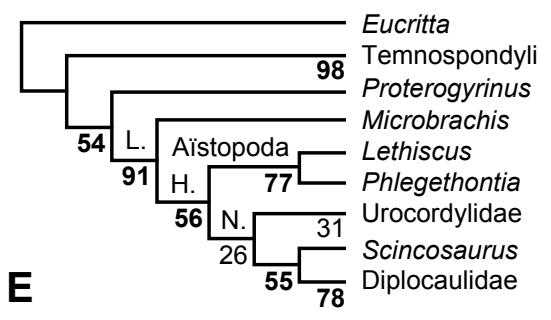
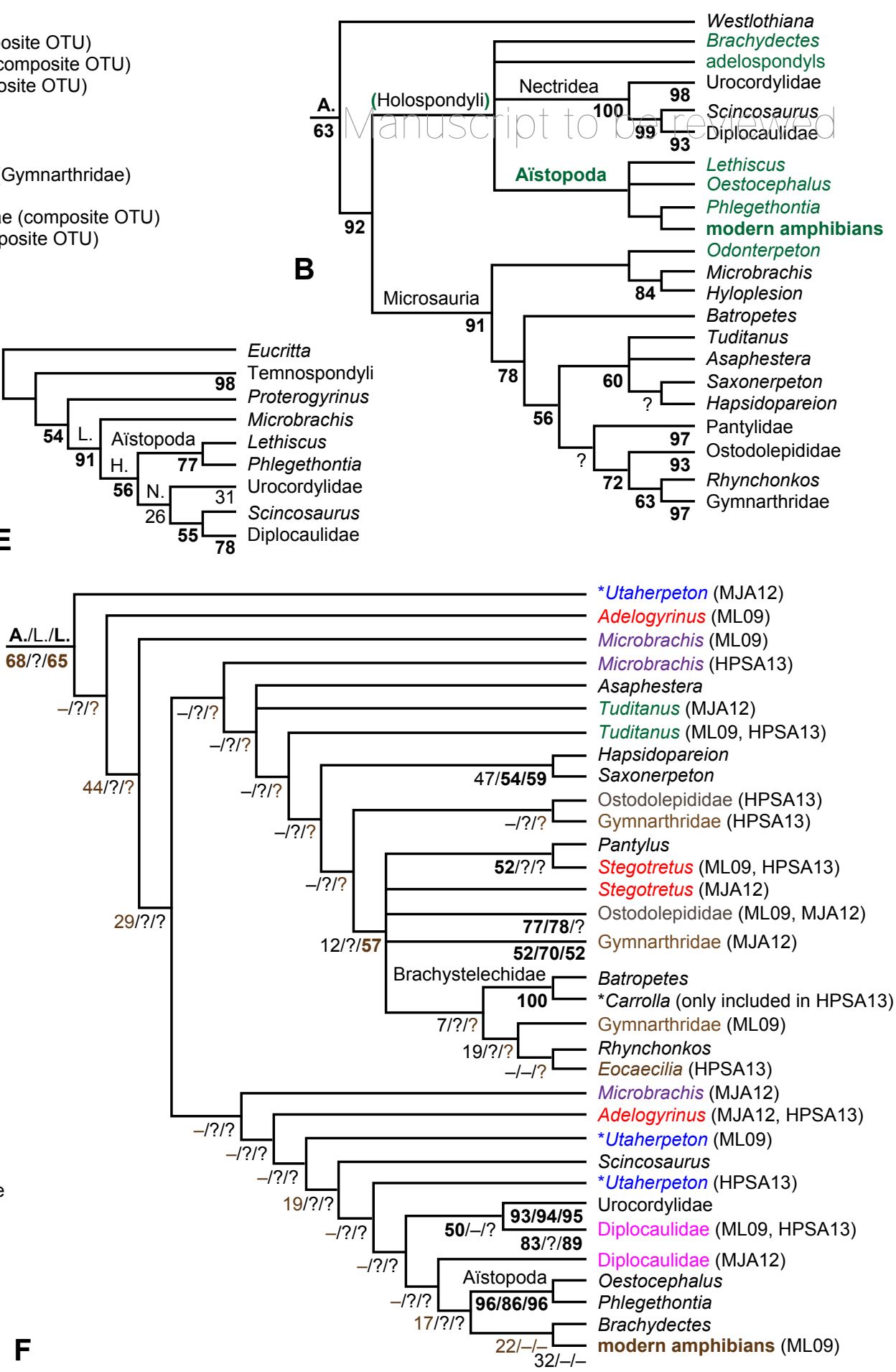
Figure 29 (on next page)

The relationships of "lepospondyls" in our analyses, and the distribution of the supratemporal bone.

Various named clades are collapsed. Filled rectangles indicate presence of the supratemporal (state 2 or lower of ch. 32 - PAR 2/POSFRO 3/INTEMP 1/SUTEMP 1; see App. S1), empty rectangles its absence (state 3 or 4); the absence of a rectangle indicates missing data. A., Amphibia; B., Batrachia; H., Holospondyli; L., Lepospondyli. A: topology found in Analyses R1 and R3. Numbers below internodes are BPO; compare unsupported nodes to Fig. 18. B: topology found in R2. C: topology found in R4-R6. Numbers are BPEIPP; compare unsupported nodes to Fig. 19, 20. Numbers in red or brown depend on *Trihecaton or Eocaecilia, respectively, not being members of the clades in question; the opposite holds for clade names in color. 


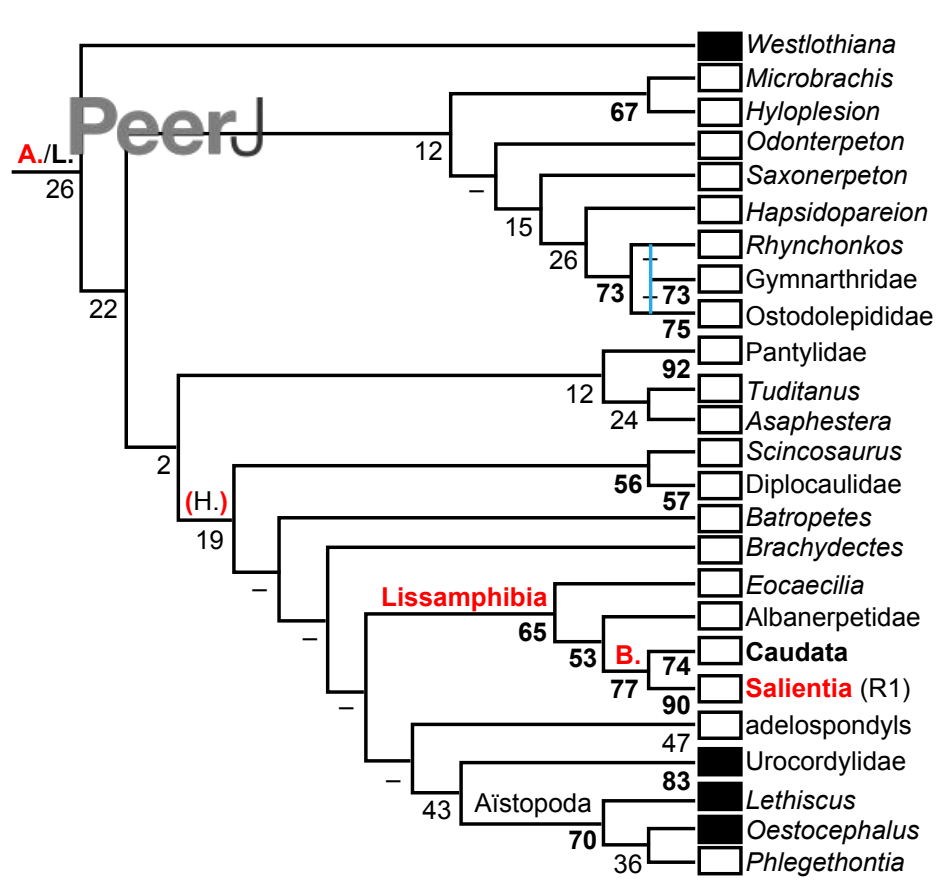

A

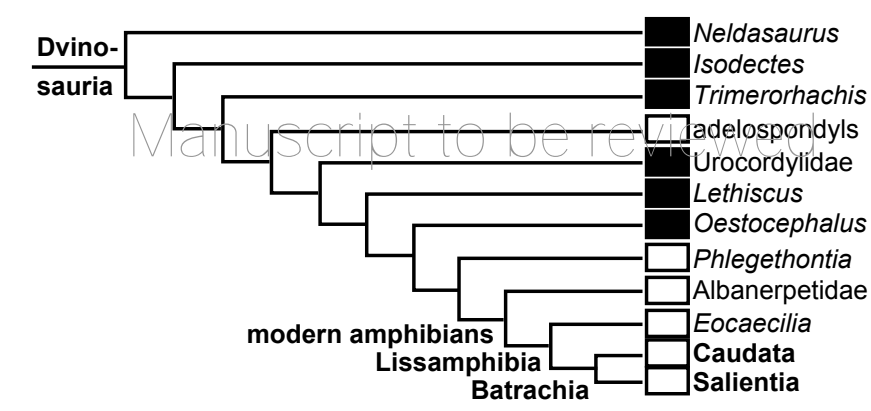

Batrachia

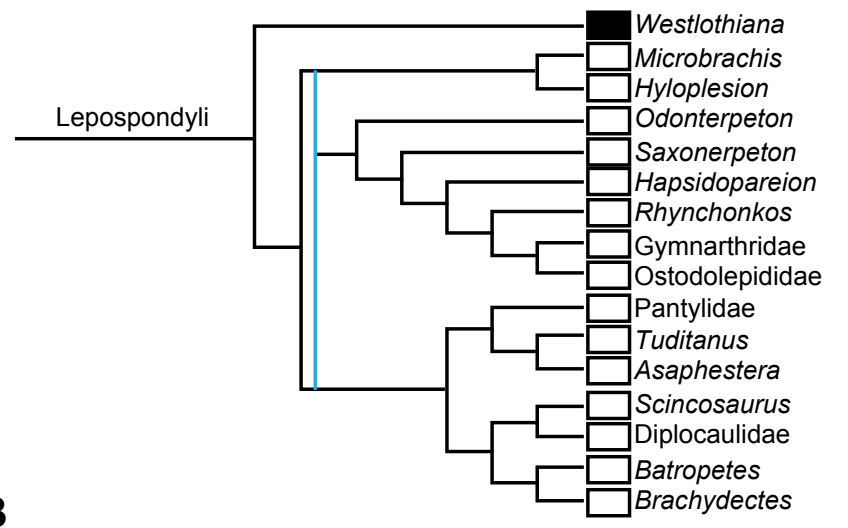

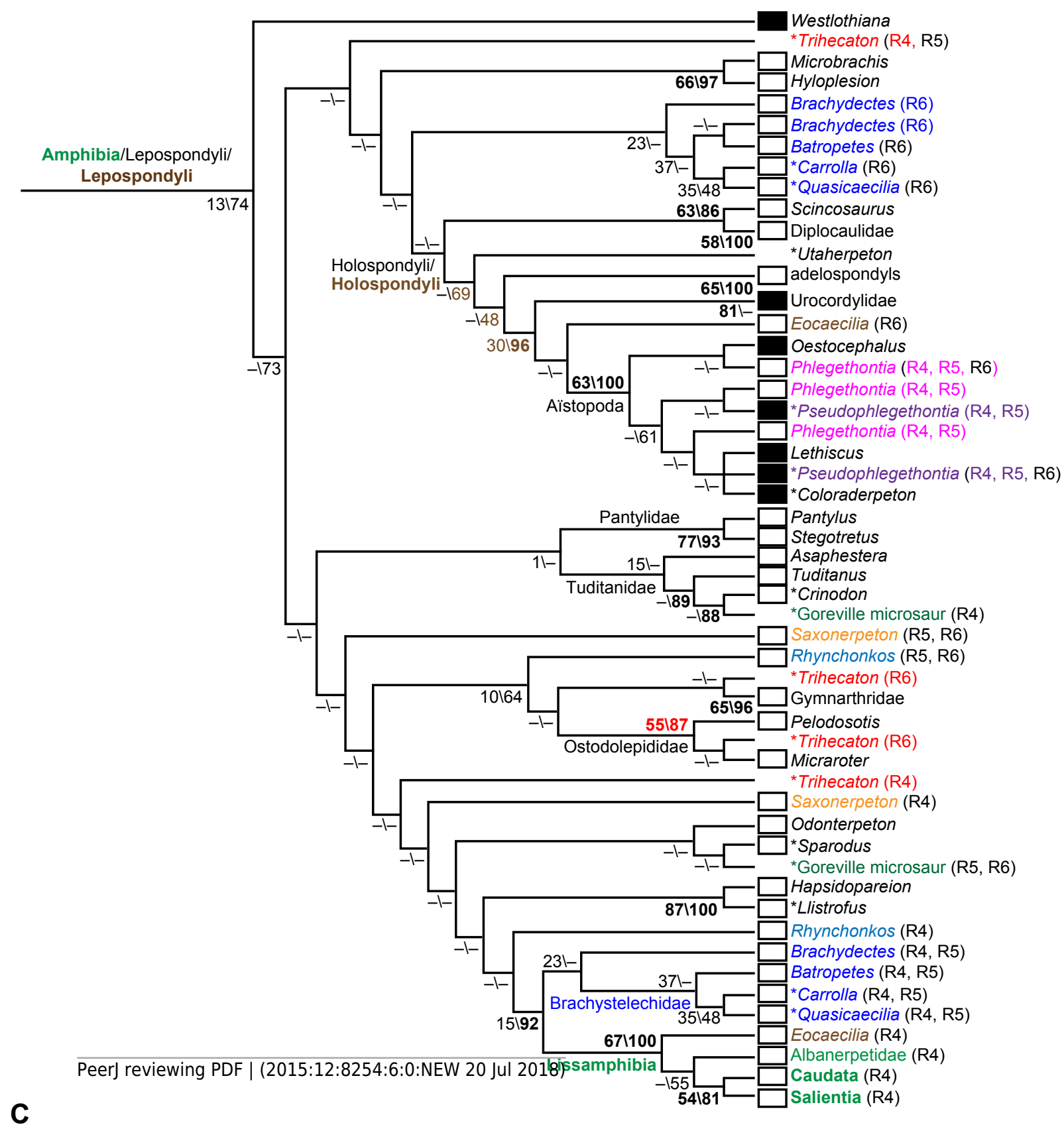




\section{Figure 30(on next page)}

\section{The phylogeny of modern amphibians and their closest relatives in recently published $(\mathrm{A}-\mathrm{K})$ and our (L-P) analyses, and the distribution of several character states.}

Albanerpetidae is a composite OTU throughout. Numbers in A, D, E, G, H are bootstrap percentages. B., Batrachia; G., Gymnophionomorpha (not shown when Eocaecilia is the only included member); L., Lissamphibia; m., modern amphibians (when not synonymous with L.); P., Procera. A: Vallin \& Laurin (2004); the two unnumbered "caudate" nodes have bootstrap values below 30\%. B: Pawley (2006: fig. 88; see Fig. 28B). C: Pawley (2006: fig. 91; see Fig. 28C). D: RC07. We write "< 50" where RC07 (app. 4) wrote "no bootstrap support in a $50 \%$ majority-rule consensus" and "_" where they wrote "no boots[t]rap support compatible with a 50 \% majority-rule consensus". Caudate monophyly is found in some MPTs and, as shown here, $\mathbf{6 1} \%$ of the bootstrap replicates. E: Marjanović \& Laurin (2009). F: Sigurdsen \& Green (2011: fig. 2B, 4). Numbers are bootstrap percentages from unweighted parsimony (left; not published if below 50) and Bayesian posterior probabilities (right; in \%). G: Maddin, Jenkins \& Anderson (2012). Bootstrap percentages below 50 were not published. H: Huttenlocker et al. (2013). Bootstrap percentages below 50 were not published. I-K: MPTs from Pardo, Small \& Huttenlocker (2017); see Marjanović \& Laurin (2018). Numbers in J are bootstrap percentages (left; not published if below 50) and Bayesian posterior probabilities (right; in $\%)$. The ribs of $* *$ Rileymillerus are scored under the assumption that the tentatively referred postcrania really belong to a juvenile of the same taxon as the holotype, which is a skull (Bolt \& Chatterjee, 2000); this is at least not contradicted by the postcrania of **Chinlestegophis (Pardo, Small \& Huttenlocker, 2017). L: R1 (unconstrained), R3 (constrained for the PH; both original taxon sample). Numbers are BPO given that Salientia has the position it has in R1. M: R4 (unconstrained, expanded taxon sample). Numbers are BPE\PP. N: R2 (constrained against the LH, original taxon sample). O: R5 (constrained against the LH, expanded taxon sample); Micropholis also has two positions outside the clade shown here. P: R6 (constrained for the $\mathrm{PH}$, expanded taxon sample).

Except where mentioned otherwise below, the rectangles show state 0 (filled) or 1 (empty) of characters 18 (LAC 1 - cyan), 39 (POSPAR 1-2 - teal), 45 (POSFRO 1 - blue), 53 (TAB 1/SQU 4 - violet), 60 (POSORB 1 reddish purple), 71 (JUG 1 - magenta), 72 (JUG 2-6 - red), 77 (QUAJUG 1 - orange), 102 (VOM 1-13 - bright green and greenish yellow, see below), 160 (ANG 1 - dark green), 246 (RIB 3 - brown) and 250 (RIB 7 black). Absence of a rectangle means missing data, inapplicability or ambiguous optimization at the root of a collapsed clade, except that *Lydekkerina is polymorphic for character 72 .

State 1 of the unordered character 39 does not occur in this figure, so empty rectangles show state 2 . State 2 of the ordered character 72 is limited to Pantylidae in this figure and therefore shown as state 1 . The ordered character 102 has three states: vomer about as wide as long or wider (0, filled greenish yellow rectangles); intermediate ( 1 , empty bright green rectangles); at least $2 \frac{1}{2} 2$ times as long as wide (2, filled bright green rectangles). Rectangles with a bright green rim filled in with greenish yellow indicate ambiguous optimization of state 1 or 2 .

Character 18: presence (0) or absence (1) of lacrimal; 39: presence (0) or absence (2) of postparietals; 45 : presence (0) or absence (1) of postfrontal; 53: presence (0) or absence (1) of tabular; 60: presence (0) or absence (1) of postorbital; 71: presence (0) or absence (1) of jugal; 72: contact between maxilla and quadratojugal at ventrolateral edge of skull (0), jugal separates maxilla and quadratojugal and forms part of ventrolateral edge of skul! ( 1 and 2$)$. 77 : presence (0) or absence (1) of quadratojugal; 102: see above; 160: presence (0) or absence (1) of angular; 246 ! ribs mostly straight $(0$, empty rectangles) or ventrally curved in 
at least part of the trunk (1, filled rectangles); 250: trunk ribs longer (0) or shorter (1) than three successive articulated vertebrae in adults. Note that we do not distinguish absence due to loss of an ossification center from absence as a separate bone due to ontogenetic fusion; in more or less all cases the ontogeny is insufficiently well known to decide.

For further derivations within Caudata and Salientia, see the text. Adelospondylus has state 60(1), Notobatrachus is polymorphic for 77 . The optimizations for character 72 differ for Aïstopoda between $\mathbf{P}$ and the rest due to the larger taxon sample in $\mathbf{P}$; likewise, the optimizations for character 160 vary for Caudata/Urodela depending on taxon samples. 

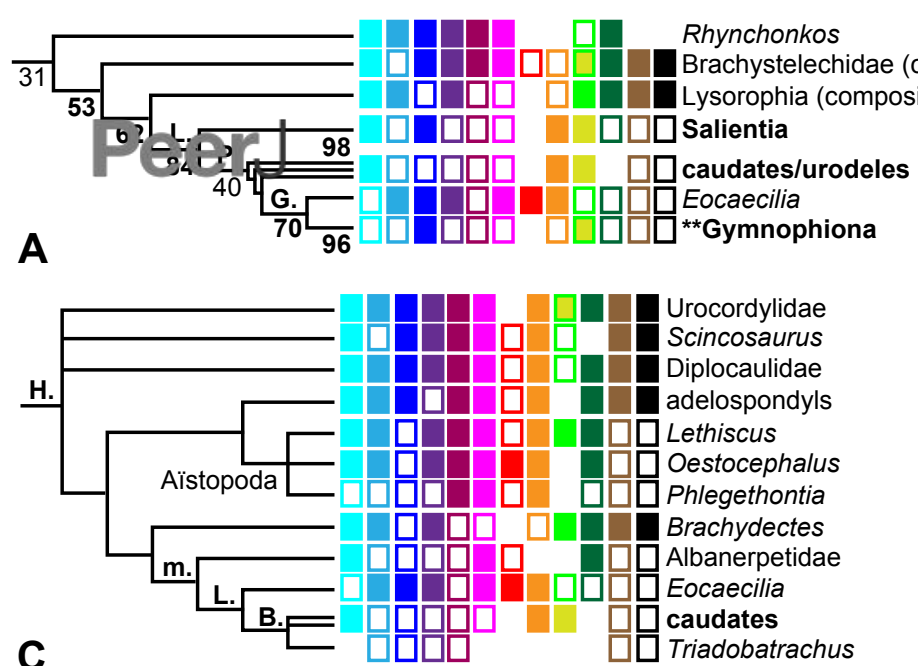

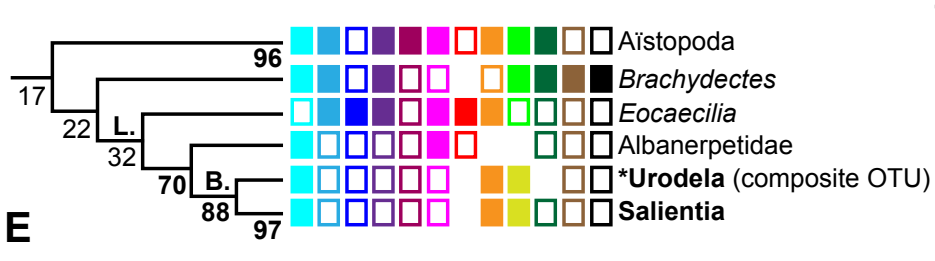
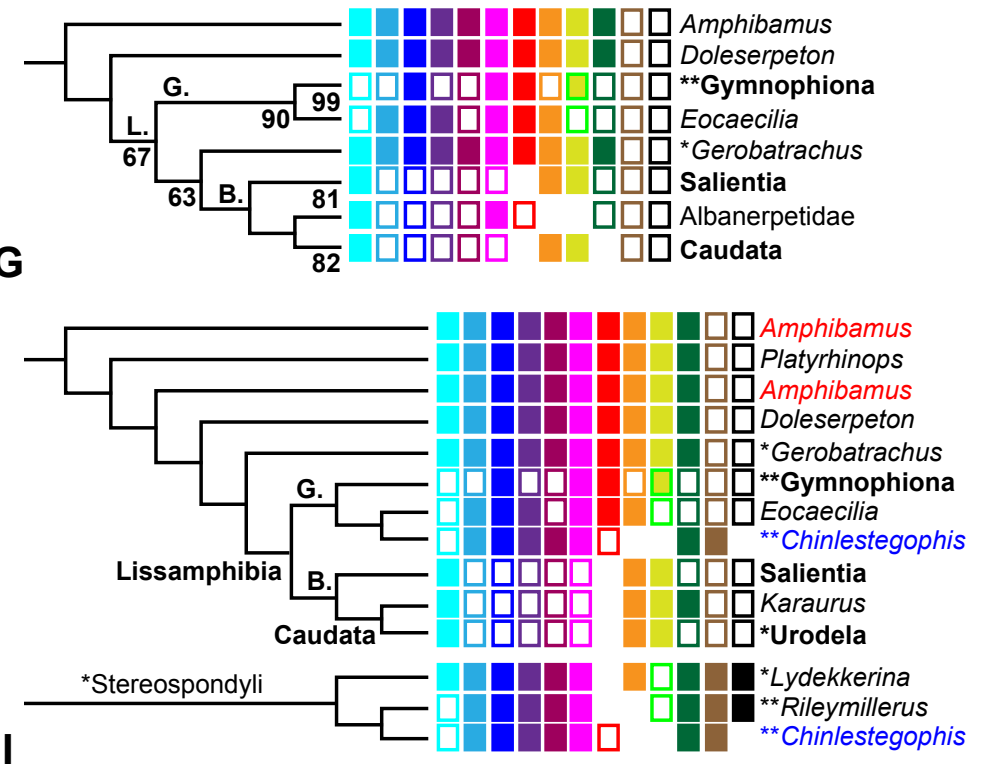

K
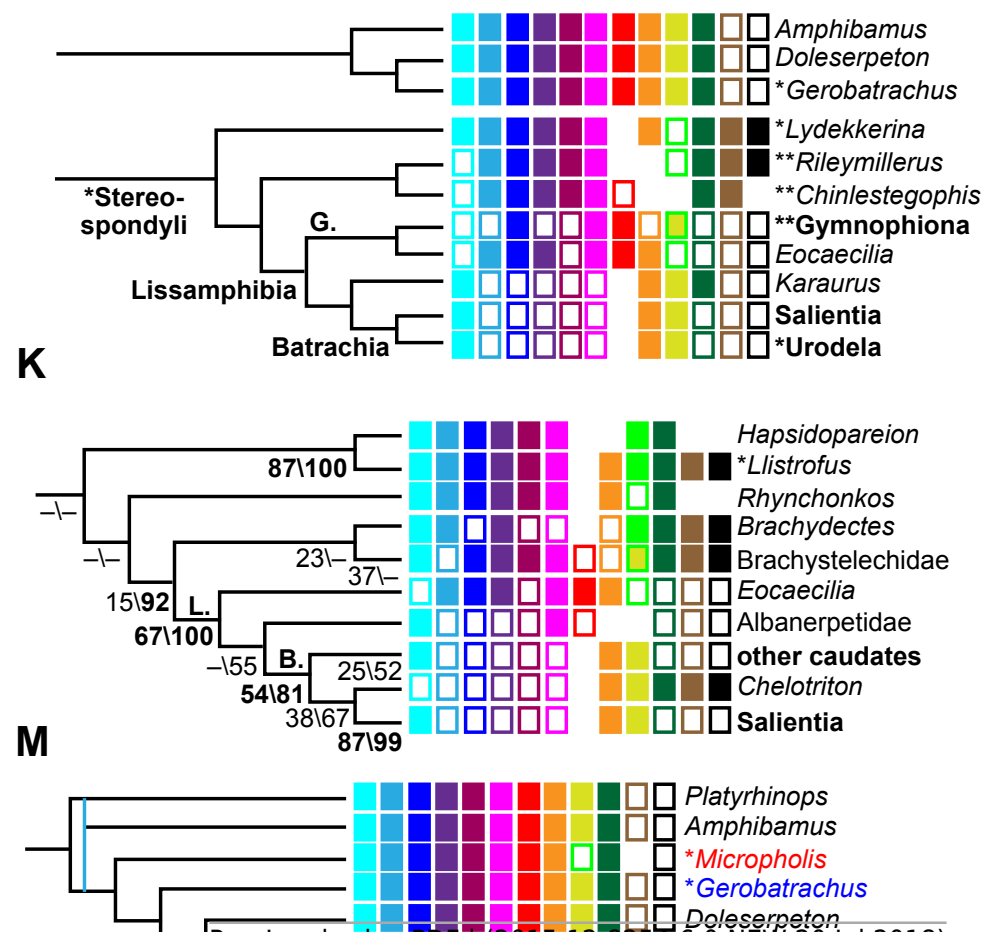

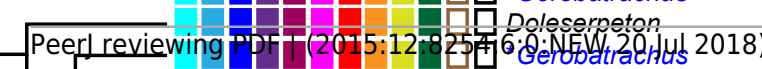

- C Caudata $=$ Procera incl.

0

पD प्रिए :

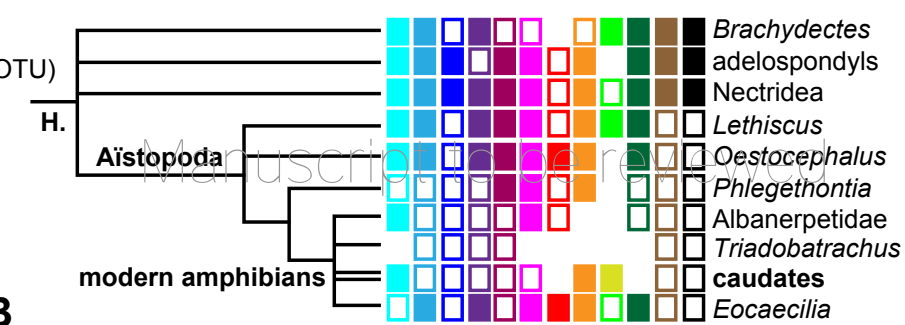

B

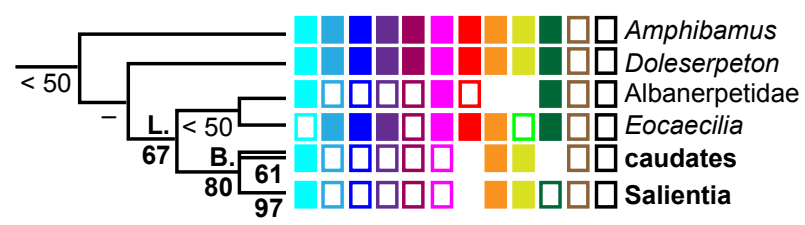

D
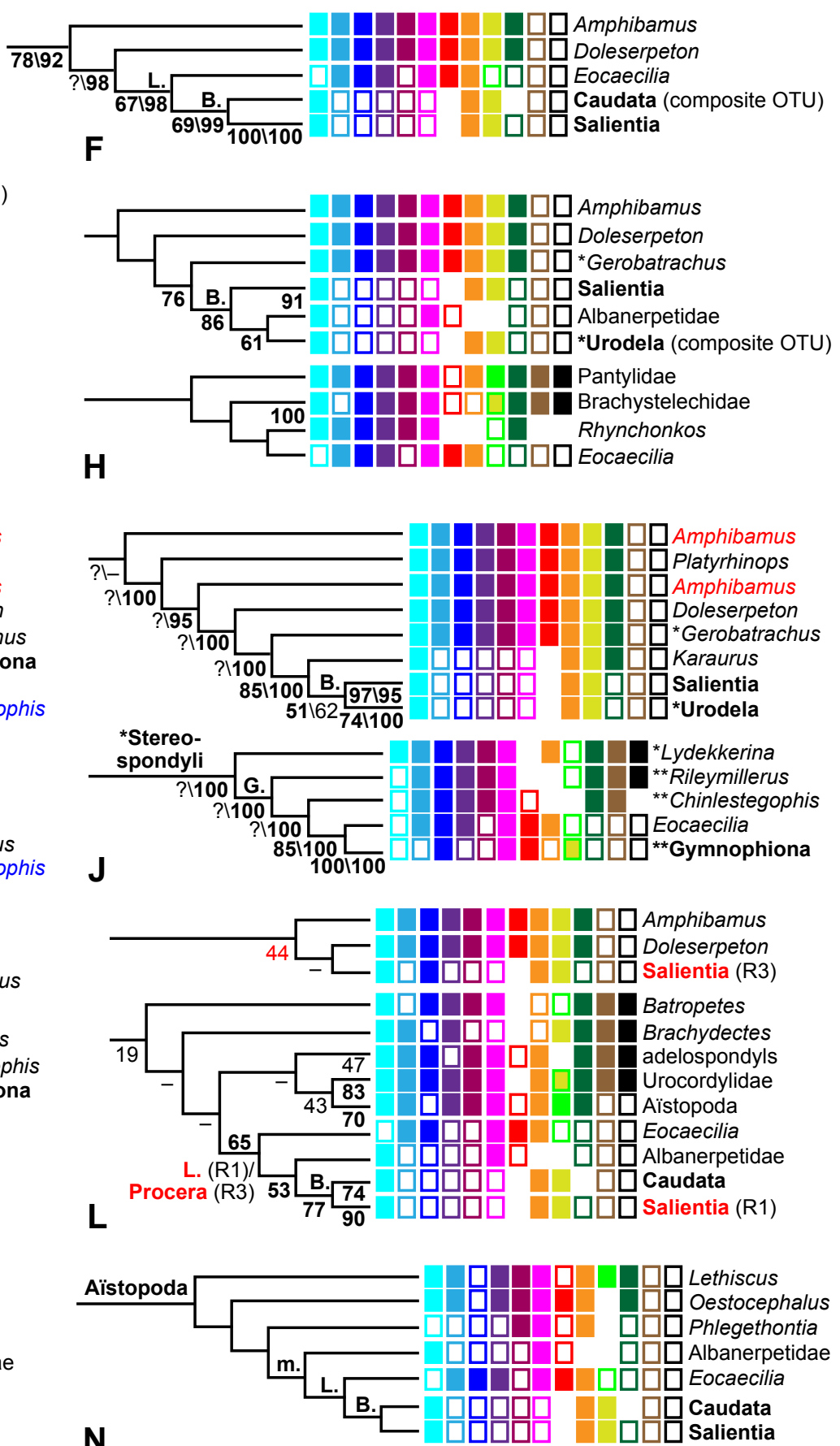

$\mathbf{N}$

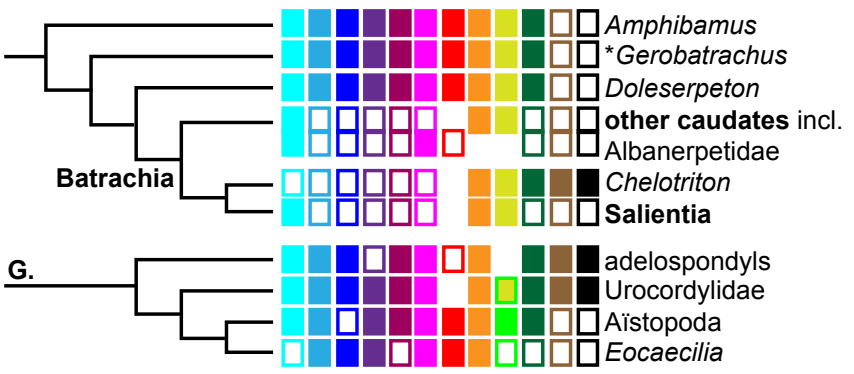




\section{Figure 31}

NHMW 1983/32/11, a natural mold of an articulated skeleton referred to Sauropleura scalaris.

Abbreviations: I.cl, left clavicle; r.cl, right clavicle. Photo taken by D. M.

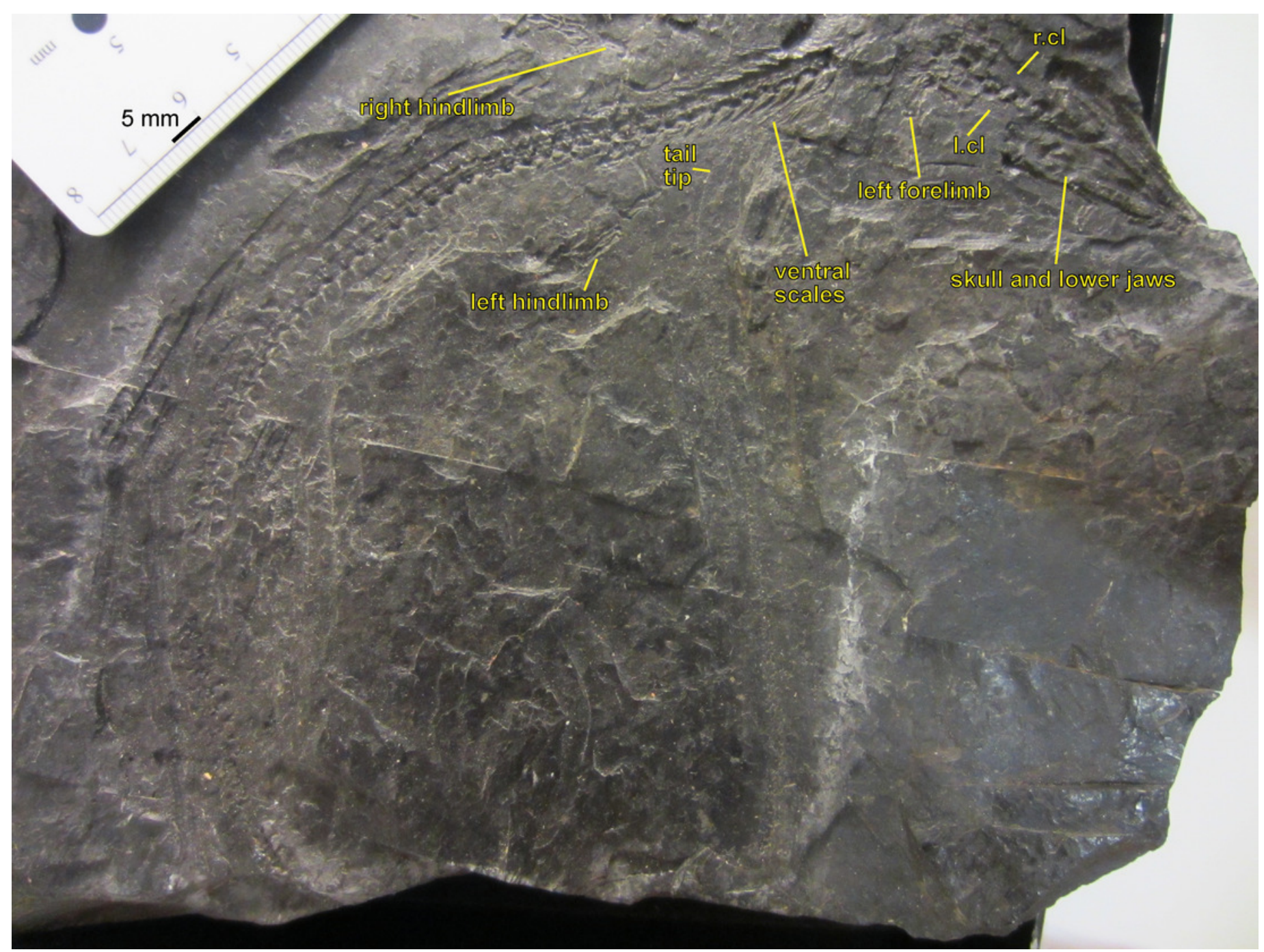




\section{Figure 32}

Area around the left hindlimb of a latex cast of the Sauropleura specimen shown in Fig. 31.

Abbreviation: $\mathrm{mt}$, metatarsal. Photo taken by D. M.

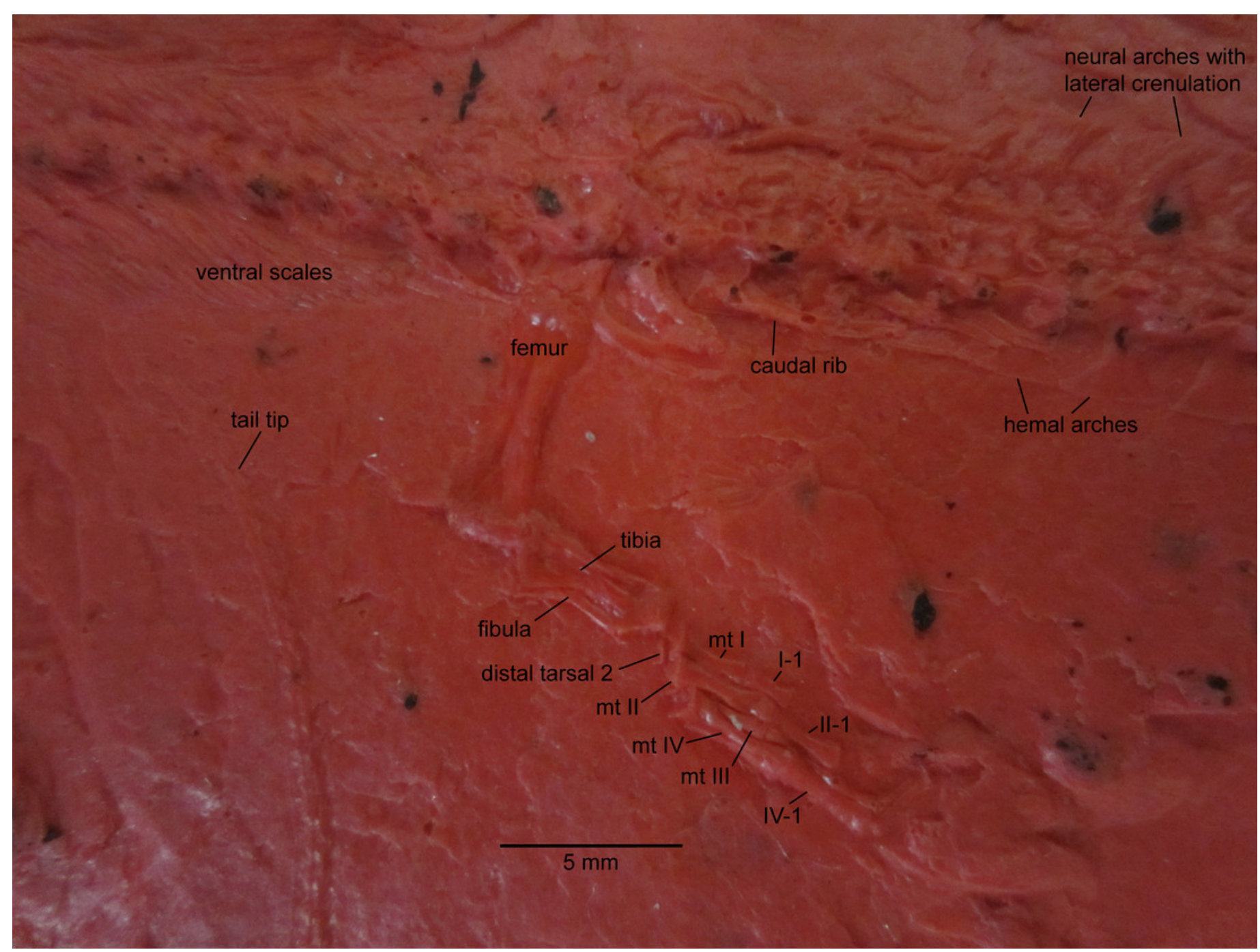




\section{Table $\mathbf{1}$ (on next page)}

Overview of analysis settings and results concerning lissamphibian origins.

See Table 3 for results concerning other questions. Steps were counted in PAUP* (Swofford, 2003), partial uncertainty was distinguished from polymorphism. "Parsimony" refers to equally weighted maximum parsimony, "bootstrap" to equally weighted maximum parsimony bootstrap, "Bayesian" to Bayesian inference. 
1 Overview of analysis settings and results concerning lissamphibian origins. See Table 3 for 2 results concerning other questions. Steps were counted in PAUP* (Swofford, 2003), partial 3 uncertainty was distinguished from polymorphism. "Parsimony" refers to equally weighted 4 maximum parsimony, "bootstrap" to equally weighted maximum parsimony bootstrap, 5 "Bayesian" to Bayesian inference.

6

\begin{tabular}{|c|c|c|c|c|c|c|c|}
\hline Matrix & $\begin{array}{l}\text { Taxon } \\
\text { sample }\end{array}$ & Analysis & Method & Constraint & Steps & Result & Figures \\
\hline \multirow[t]{3}{*}{$\mathrm{RC} 07$} & \multirow{7}{*}{$\begin{array}{l}\text { RC07 } \\
(102 \\
\text { OTUs) }\end{array}$} & 01 & \multirow[t]{6}{*}{ parsimony } & none & 1621 & $\mathrm{TH}$ & 2 \\
\hline & & 02 & & against $\mathrm{TH}^{1}$ & 1622 & LH & 9 \\
\hline & & 03 & & for $\mathrm{PH}$ & 1633 & $\mathrm{PH}$ & - \\
\hline \multirow{9}{*}{ revised } & & R1 & & none & 2182 & LH & 10,11 \\
\hline & & $\mathbf{R 2}$ & & against $\mathrm{LH}^{2}$ & 2191 & $\mathrm{TH}^{3}$ & 12 \\
\hline & & R3 & & for $\mathrm{PH}$ & 2194 & $\mathrm{PH}$ & 13 \\
\hline & & B1 & bootstrap & none & 2210 & LH & 18 \\
\hline & \multirow{5}{*}{$\begin{array}{l}\text { expanded } \\
\text { (158 } \\
\text { OTUs) }\end{array}$} & R4 & \multirow[t]{3}{*}{ parsimony } & none & 3011 & LH & 14 \\
\hline & & R5 & & against $\mathrm{LH}^{2}$ & 3021 & $\mathrm{TH}$ & 15,16 \\
\hline & & R6 & & for $\mathrm{PH}$ & 3026 & $\mathrm{PH}$ & 17 \\
\hline & & B2 & bootstrap & none & 3089 & LH & 19 \\
\hline & & EB & Bayesian & none & $(3075)^{4}$ & LH & 20,21 \\
\hline
\end{tabular}

7

$8{ }^{1}$ This constraint is aimed at enforcing the LH, but it is also compatible with the (never proposed)

9 "inverse polyphyly hypothesis", where the salientians would be lepospondyls and the

10 gymnophionomorphs (represented by Eocaecilia) would be temnospondyls.

$11{ }^{2}$ This constraint is aimed at enforcing the $\mathrm{TH}$, but it is also compatible with the $\mathrm{PH}$.

$12{ }^{3}$ A highly unusual version of the TH, see Results, Discussion and Table 3.

$13{ }^{4}$ This is the parsimony treelength calculated for this topology by PAUP*. The more appropriate

14 Bayesian treelength is not comparable to the other lengths presented here. 


\section{Table 2 (on next page)}

List of specimens used by D. M. to change scores in the matrix (as cited in App. 1) or to score added taxa.

The OTUs are in the same order as in the matrix, which is unchanged from the nonalphabetical version of the matrix file of RC07 for the shared taxa (Acanthostega through Tseajaia). Specimens that do not contradict any scores of RC07 (including "unknown") or the literature on the added taxa (Nigerpeton through Casineria) are not listed except in cases of polymorphism; a few such specimens are mentioned in the text. *, type specimen of the type species; $^{* *}$, type and only known specimen of the only known species. 
1 List of specimens used by D. M. to change scores in the matrix (as cited in App. 1) or to score 2 added taxa. The OTUs are in the same order as in the matrix, which is unchanged from the non3 alphabetical version of the matrix file of RC07 for the shared taxa (Acanthostega through 4 Tseajaia). Specimens that do not contradict any scores of RC07 (including "unknown") or the 5 literature on the added taxa (Nigerpeton through Casineria) are not listed except in cases of 6 polymorphism; a few such specimens are mentioned in the text. *, type specimen of the type 7 species; **, type and only known specimen of the only known species.

\begin{tabular}{|c|c|}
\hline OTU & Specimens \\
\hline Acanthostega & TMM 41766-1 (cast of MGUH VP 6033*, formerly “A. 33”) \\
\hline Ichthyostega & $\begin{array}{l}\text { AMNH 23100; MCZ 3361; TMM 41224-2 (all of them casts of MGUH VP } \\
6055 \text {, formerly "A. 55") }\end{array}$ \\
\hline Greererpeton & TMM 41574-1 \\
\hline Edops & $\begin{array}{l}\text { MCZ 1378, 1769, 1781, 1782, 6489, 6493, 7126, 7128, 7136, 7143, 7158, } \\
7162,7197,7258,7259,7264,7274 ; \text { USNM 23309 }\end{array}$ \\
\hline Chenoprosopus & CM 34909; USNM 437646 \\
\hline Isodectes & $\begin{array}{l}\text { CM 81430, 81512; MCZ } 6044 \text { (cast of USNM 4481); unnumbered MCZ } \\
\text { cast of AMNH } 6935 \text { before etching; USNM 4471, 4474, } 4555\end{array}$ \\
\hline Trimerorhachis & AMNH 4565*, 4572; TMM 40031-80, 40031-81, 40998-39 \\
\hline Eryops & $\begin{array}{l}\text { AMNH 4180, 4183, 4186, 4189*, 4673, 23529; MCZ 1126, 1129, 1536, } \\
\text { 2588, 2638, 2682, 2766; TMM 31225-33, 31226-12, 31227-11, 31227-14, } \\
\text { 40349-20; USNM uncatalogued "Texas '84 \#40", "Texas '86 \#77" }\end{array}$ \\
\hline Phonerpeton & $\begin{array}{l}\text { AMNH 7150; MCZ 1414, 1419*, 1485, 1548, 1771, 2313, 2474; USNM } \\
437796\end{array}$ \\
\hline Ecolsonia & CM 38017,38024 \\
\hline Broiliellus brevis & MCZ 3272 \\
\hline Doleserpeton & AMNH 24969, 29466, 29470; BEG 40882-25 \\
\hline Platyrhinops & AMNH 2002 \\
\hline Eocaecilia & MNA V8066* (Museum of Northern Arizona; formerly MCZ 9010) \\
\hline Karaurus & unnumbered MNHN cast $^{1}$ of PIN 2585/2** \\
\hline Triadobatrachus & MNHN.F.MAE126** (natural mold and cast) ${ }^{1}$ \\
\hline Archeria & MCZ 2049, other MCZ specimens² \\
\hline Gephyrostegus & MB.Am.641; TMM 41733-1 (cast) \\
\hline Seymouria & BEG 30966-176 \\
\hline Diadectes & AMNH 4352, 4839; BEG 31222-56 \\
\hline Paleothyris & TMM 45955-2 (cast of MCZ 3482) \\
\hline Batropetes & B. palatinus: MB.Am.1232 (including casts) \\
\hline Tuditanus & CM 29592 \\
\hline Stegotretus & CM 34901 (all others were on loan) \\
\hline Asaphestera & $\begin{array}{l}\text { NMC } 10041 \text { (National Museum of Canada; currently on loan to J. Anderson, } \\
\text { University of Calgary) }\end{array}$ \\
\hline Microbrachis & MB.Am.840 \\
\hline Hyloplesion & NHMW 1983/82/54, other NHMW specimens \\
\hline Odonterpeton & USNM $4465+4467 * * 3$ \\
\hline Scincosaurus & MB.Am.29 \\
\hline
\end{tabular}




\begin{tabular}{|l|l|}
\hline Diceratosaurus & $\begin{array}{l}\text { AMNH 6933*; CM 25468, 26231, 29593, 29876, 34617, 34656, 34668, } \\
\text { 34696, 34670, 67157, 67169, 72608, 81504, 81507, 81508; MB.Am.776, } \\
\text { MB.Am.778 }\end{array}$ \\
\hline Ptyonius & MCZ 3721 (cast of “AMNH 6871 (85466)") \\
\hline Sauropleura & CM 25312 \\
\hline Lethiscus & MCZ 2185** \\
\hline Phlegethontia & USNM 17097 \\
\hline Tseajaia & CM 38033 \\
\hline Nigerpeton & $\begin{array}{l}\text { MNN MOR } 69^{*} \text { (including unpublished intercentrum and unprepared skull } \\
\text { fragments), 70, 82, 83, 108 }\end{array}$ \\
\hline Saharastega & MNN MOR 73** (including two unpublished mandible pieces) \\
\hline Iberospondylus & PU-ANF 2, 14*, 154 \\
\hline Caseasauria & $\begin{array}{l}\text { Eothyris: MCZ 1161**; Oedaleops: unnumbered MCZ cast of UCMP } \\
\text { 35758* (University of California Museum of Paleontology) }\end{array}$ \\
\hline Sparodus & NHMW 1899/0003/0006 (Fig. 3, 4) \\
\hline Carrolla & TMM 40031-54** \\
\hline Sclerocephalus & MB.Am.1346 \\
\hline Chelotriton & MB.Am.45 (natural mold and cast) \\
\hline Trihecaton & CM 47681*, 47682 (probably part of the same individual) \\
\hline St. Louis tetrapod & MB.Am.1441** (natural mold and cast) \\
\hline Casineria & NMS G $1993.54 .1 * *$ (part and counterpart; Fig. 5-7) \\
\hline
\end{tabular}

$10{ }^{1}$ Observed together with M. L.

$11 \quad{ }^{2}$ All were used to score the same characters.

$12{ }^{3}$ The apparently unpublished specimens CM 81525 and CM 81526 are also labeled

13 Odonterpeton, and are kept together with a note by Donald Baird (dated 1991) which says that

14 further small skeletons attributed to Brachydectes may belong to Odonterpeton as well. More

15 likely, CM 81525 - a limbless skeleton without a trace of limbs or girdles, whose skull is only

16 preserved as an indistinct impression - is instead a juvenile aïstopod with ribs that are not yet k-

17 shaped, and the disarticulated CM 81526 is a juvenile Brachydectes after all (D. M., pers. obs.; J.

18 Pardo, pers. comm. after seeing photos taken by D. M.); a more detailed study is in preparation.

$19{ }^{4}$ All three were observed together with Rodrigo Soler-Gijón; additionally, they had been studied

20 earlier by M. L. (Laurin \& Soler-Gijón, 2001, 2006). 


\section{Table 3(on next page)}

Summary and comparison of analysis results.

See Table 1 for the settings of these analyses and for the numbers of the figures that represent the trees. The "modern amphibians" column gives the closest one or two relatives of the clade of modern amphibians (TH, LH) or of the two clades of modern amphibians (PH); the Batrachia column indicates whether Caudata and Salientia form a clade to the exclusion of Eocaecilia; the Kotlassia column states whether Kotlassia is crownward of, rootward of or a member of Seymouriamorpha; the Limnoscelis column states whether Limnoscelis is crownward of, rootward of or a member of Diadectomorpha; the last column states whether Ichthyostega is rootward or crownward of Acanthostega and Ventastega. Note that whenever Amniota is closer to Temnospondyli than to CAS, Caerorhachis is found as a temnospondyl. Brachystelechidae contains Batropetes, *Carrolla and *Quasicaecilia. OTUs unique to the expanded taxon sample are marked with an asterisk. Abbreviations: \#, symbol of the analysis (as used in the text and in Tables 1, 4-12); AS, Anthracosauria and Silvanerpeton (Caerorhachis is found as a temnospondyl); CAS, Caerorhachis, Anthracosauria and Silvanerpeton; Diad., Diadectomorpha; Seym., Seymouriamorpha; T, Temnospondyli; (U, A), a clade formed exclusively by Urocordylidae and Aïstopoda. Numbers in curly braces are bootstrap percentages, shown in boldface if 50 or higher, except for Analysis EB, where they are posterior probabilities (in \%), shown in boldface if 75 or higher. 
Summary and comparison of analysis results. See Table 1 for the settings of these analyses and for the numbers of the figures that represent the trees. The "modern amphibians" column gives the closest one or two relatives of the clade of modern amphibians (TH, $\mathrm{LH})$ or of the two clades of modern amphibians (PH); the Batrachia column indicates whether Caudata and Salientia form a clade to the exclusion of Eocaecilia; the Kotlassia column states whether Kotlassia is crownward of, rootward of or a member of

Seymouriamorpha; the Limnoscelis column states whether Limnoscelis is crownward of, rootward of or a member of Diadectomorpha; the last column states whether Ichthyostega is rootward or crownward of Acanthostega and Ventastega. Note that whenever Amniota is closer to Temnospondyli than to CAS, Caerorhachis is found as a temnospondyl. Brachystelechidae contains Batropetes, *Carrolla and * Quasicaecilia. OTUs unique to the expanded taxon sample are marked with an asterisk. Abbreviations: \#, symbol of the analysis (as used in the text and in Tables 1, 4-12); AS, Anthracosauria and Silvanerpeton (Caerorhachis is found as a temnospondyl); CAS, Caerorhachis, Anthracosauria and Silvanerpeton; Diad., Diadectomorpha; Seym., Seymouriamorpha; T, Temnospondyli; (U, A), a clade formed exclusively by Urocordylidae and Aïstopoda. Numbers in curly braces are bootstrap percentages, shown in boldface if 50 or higher, except for Analysis EB, where they are posterior probabilities (in \%), shown in boldface if 75 or higher.

\begin{tabular}{|c|c|c|c|c|c|c|c|c|}
\hline$\#$ & $\begin{array}{l}\text { Modern } \\
\text { amphibians }\end{array}$ & $\begin{array}{l}\text { Batra } \\
\text { chia }\end{array}$ & $\begin{array}{l}\text { Albanerpetidae } \\
\text { closest to: }\end{array}$ & $\begin{array}{l}\text { Amniota } \\
\text { closer to: }\end{array}$ & Kotlassia & Limnoscelis & $\begin{array}{l}\text { adelospondyls } \\
\text { closest to: }\end{array}$ & Ichthyostega \\
\hline 01 & TH: Doleserpeton & yes & Eocaecilia & CAS & crownward & rootward & Colosteidae & crownward \\
\hline $\mathbf{O 2}$ & LH: Holospondyli & yes & Lissamphibia & CAS & crownward & rootward & Colosteidae & crownward \\
\hline $\mathbf{O 3}$ & $\begin{array}{l}\text { PH: Doleserpeton; } \\
\text { Brachydectes, Holo- } \\
\text { spondyli or both }\end{array}$ & yes & Eocaecilia & CAS & crownward & rootward & Colosteidae & crownward \\
\hline R1 & $\begin{array}{l}\text { LH: (adelospondyls } \\
(\mathrm{U}, \mathrm{A}))\end{array}$ & yes & Batrachia & $\begin{array}{l}\text { 1) CAS; } \\
\text { 2) } \mathrm{T}\end{array}$ & $\begin{array}{l}\text { 1) } \\
\text { rootward; } \\
\text { 2) Seym. }\end{array}$ & Diad. & $(\mathrm{U}, \mathrm{A})$ & $\begin{array}{l}\text { 1) crownward; 2) } \\
\text { rootward }\end{array}$ \\
\hline $\mathbf{R 2}$ & TH: Phlegethontia ${ }^{1}$ & yes & Lissamphibia & $\mathrm{T}$ & rootward & Diad. & 1 & $\begin{array}{l}\text { 1) crownward; 2) } \\
\text { rootward }\end{array}$ \\
\hline $\mathbf{R 3}$ & $\begin{array}{l}\mathrm{PH}: \text { Doleserpeton; } \\
\text { (adelospondyls (U, } \\
\mathrm{A}))^{2}\end{array}$ & $\mathrm{no}^{2}$ & Caudata & $\begin{array}{l}\text { 1) } \mathrm{CAS} \text {; } \\
\text { 2) } \mathrm{T}\end{array}$ & $\begin{array}{l}\text { 1) } \\
\text { rootward; } \\
\text { 2) Seym. }\end{array}$ & Diad. & $(\mathrm{U}, \mathrm{A})$ & $\begin{array}{l}\text { 1) crownward; 2) } \\
\text { rootward }\end{array}$ \\
\hline B1 & $\begin{array}{l}\mathrm{LH}\{\mathbf{8 4}\}^{3}: \\
\text { (Brachydectes }+ \\
\text { Batropetes) }\{20\}^{4}\end{array}$ & $\begin{array}{l}\text { yes } \\
\{77\}\end{array}$ & Batrachia $\{\mathbf{5 3}\}$ & AS $\{12\}$ & Seym. $\{28\}$ & Diad. $\{\mathbf{5 9}\}$ & $\begin{array}{l}\text { Holospondyli } \\
+ \text { crownward } \\
\{35 ; 25\}^{5} \\
\end{array}$ & crownward $\{47\}$ \\
\hline R4 & $\begin{array}{l}\text { LH: (Brachydectes } \\
+\end{array}$ & yes & Batrachia & CAS & Seym. & crownward & $(\mathrm{U}, \mathrm{A})$ & crownward \\
\hline
\end{tabular}




\begin{tabular}{|c|c|c|c|c|c|c|c|c|}
\hline & Brachystelechidae) & & & & & & & \\
\hline $\mathbf{R 5}$ & $\begin{array}{l}\text { TH: Doleserpeton } \\
\text { or * Gerobatrachus }\end{array}$ & no & Eocaecilia & CAS & Seym. & crownward & $(\mathrm{U}, \mathrm{A})$ & crownward \\
\hline R6 & $\begin{array}{l}\text { PH: Doleserpeton; } \\
\text { Aïstopoda }^{6}\end{array}$ & yes $^{6}$ & Valdotriton & CAS & Seym. & crownward & $(\mathrm{U}, \mathrm{A})$ & crownward \\
\hline B2 & $\begin{array}{l}\mathrm{LH}\left\{21^{3}, 25^{7}\right\}: \\
\text { (Brachydectes }+ \\
\text { Brachystelechidae) } \\
\{15\}^{4}\end{array}$ & $\begin{array}{l}\text { yes } \\
\{\mathbf{5 4}\}\end{array}$ & $\begin{array}{l}\text { Lissamphibia } \\
\{67\}\end{array}$ & $\mathrm{T}\{6\}$ & Seym. $\{30\}$ & Diad. $\{24\}$ & $\begin{array}{l}\text { all other } \\
\text { amphibians } \\
\{20 ; 10\}^{5}\end{array}$ & $\begin{array}{l}\text { crownward of } \\
\text { Ventastega }\{11\} \\
\text { and Acanthostega } \\
\{10\}\end{array}$ \\
\hline EB & $\begin{array}{l}\text { LH }\{\mathbf{9 2}\}: \\
(* \text { Carrolla }+ \\
* \text { Quasicaecilia }) \\
\{\mathbf{8 8}\}^{4}\end{array}$ & $\begin{array}{l}\text { yes } \\
\{\mathbf{8 1}\}\end{array}$ & Batrachia $\{55\}$ & $\mathrm{T}\{48\}$ & Seym. $\{99\}$ & Diad. $\{96\}$ & $\begin{array}{l}\text { (“U”, A) }\{48 ; \\
69 ; \mathbf{8 6}\}^{8}\end{array}$ & $\begin{array}{l}\text { crownward of } \\
\text { Ventastega } \text { and } \\
\text { Acanthostega } \\
\text { \{both } \mathbf{7 6} \text { \} }\end{array}$ \\
\hline
\end{tabular}

${ }^{1}$ An entirely novel arrangement where Lissamphibia and the strangest "lepospondyls" are nested among the dvinosaurian temnospondyls, closest to Trimerorhachis; see text and Fig. 12.

${ }^{2}$ Salientia (as constrained) is nested within Temnospondyli (next to Doleserpeton), while Eocaecilia (as constrained), Caudata and Albanerpetidae are nested within Lepospondyli.

${ }^{3}$ Dissorophoidea.

${ }^{4} 20$ (B1) and 15 (B2) are the bootstrap percentages of the smallest clade that contains Batropetes, Brachydectes and Lissamphibia, which has a PP of 92; (Batropetes + Brachydectes) respectively (Brachystelechidae + Brachydectes) have 37 (B1) or 23 (B2), while (Lissamphibia (*Carrolla, ${ }^{*}$ Quasicaecilia)) has a PP of $\mathbf{8 8}$.

${ }^{5}$ The highest bootstrap percentage that keeps the adelospondyls away from Colosteidae is 35 (B1) or 20 (B2), the highest that keeps them away from (U, A) is $25(\mathrm{~B} 1)$ or $10(\mathrm{~B} 2)$.

${ }^{6}$ Salientia (as constrained) and Caudata including Albanerpetidae are nested within Temnospondyli (next to Doleserpeton), while Eocaecilia (as constrained) is nested within Lepospondyli.

${ }^{7}$ Amphibamidae (not including *Micropholis).

${ }^{8}$ The urocordylids come out as paraphyletic $(\mathrm{PP}=65)$. The PP of the adelospondyls being the sister-group of (Ptyonius (Urocordylus (Sauropleura, Aïstopoda))) is 48 , the PP of that clade $+*$ Utaherpeton is 69 , the highest PP that keeps them away from Colosteidae is 86. 


\section{Table 4 (on next page)}

Summary of parsimony support for hypotheses on lissamphibian origins.

See Table 1 for analysis settings. The second column subtracts the MPT length found by the second analysis in each line from that found by the first. The rightmost column summarizes the $p$ values from the one-tailed Kishino-Hasegawa, Templeton and winning-sites tests (listed in the text; our use of the tests is discussed in Materials and methods: Robustness analyses). Bootstrap values of 0.5 or higher are given in boldface, as are $p$ values that do not certainly fail to reject the null hypothesis at the (arbitrary) 0.05 level; the null hypothesis is that the topology differences cannot be distinguished from random variations. Note that a cutoff value of 0.1 instead of 0.05 would have accepted the comparisons of 03 to 01 and 02 , but otherwise given the same results, except that the Templeton and the winning-sites test distinguish R4 and R6 at a minimum of $p=0.0565$ and $p=0.0966$. Conversely, a cutoff value of 0.03 would have rejected the comparison of R1 and R3 according to the KishinoHasegawa ( $p=0.0352)$ and Templeton $(p=0.0362)$ tests as well as the comparison of 02 and 03; a cutoff value of 0.01 would have rejected all. 
1 Summary of parsimony support for hypotheses on lissamphibian origins. See Table 1 for analysis 2 settings. The second column subtracts the MPT length found by the second analysis in each line 3 from that found by the first. The rightmost column summarizes the $\mathrm{p}$ values from the one-tailed 4 Kishino-Hasegawa, Templeton and winning-sites tests (listed in the text; our use of the tests is 5 discussed in Materials and methods: Robustness analyses). Bootstrap values of 0.5 or higher are 6 given in boldface, as are $\mathrm{p}$ values that do not certainly fail to reject the null hypothesis at the 7 (arbitrary) 0.05 level; the null hypothesis is that the topology differences cannot be distinguished 8 from random variations. Note that a cutoff value of 0.1 instead of 0.05 would have accepted the 9 comparisons of $\mathrm{O} 3$ to $\mathrm{O} 1$ and $\mathrm{O} 2$, but otherwise given the same results, except that the

10 Templeton and the winning-sites test distinguish R4 and R6 at a minimum of $\mathrm{p}=0.0565$ and $\mathrm{p}=$ 11 0.0966. Conversely, a cutoff value of 0.03 would have rejected the comparison of R1 and R3 12 according to the Kishino-Hasegawa $(p=0.0352)$ and Templeton $(p=0.0362)$ tests as well as the 13 comparison of $\mathrm{O} 2$ and $\mathrm{O} 3$; a cutoff value of 0.01 would have rejected all.

\begin{tabular}{|c|c|c|c|}
\hline Analyses & $\begin{array}{l}\text { Difference in } \\
\text { number of } \\
\text { steps }\end{array}$ & $\begin{array}{l}\text { Highest bootstrap } \\
\text { support for difference in } \\
\text { topology }\end{array}$ & $\begin{array}{l}\text { Difference in topology } \\
\text { indistinguishable from } \\
\text { random at } p=0.05 ?\end{array}$ \\
\hline $\mathbf{O 1}(\mathrm{TH}), \mathbf{O 2}(\mathrm{LH})$ & 1 & $<0.5$ (RC07: app. 4) & certainly $(\mathrm{p}>0.44)$ \\
\hline $\mathbf{O 1}(\mathrm{TH}), \mathbf{O 3}(\mathrm{PH})$ & 12 & $\begin{array}{l}\text { 0.67 (modern amphibians } \\
\text { - RC07: app. 4) }\end{array}$ & certainly $(0.06>p>0.054)$ \\
\hline $\mathbf{O 2}(\mathrm{LH}), \mathbf{O 3}(\mathrm{PH})$ & 11 & $\begin{array}{l}\text { 0.67 (modern amphibians } \\
\text { - RC07: app. 4) }\end{array}$ & $\begin{array}{l}\text { one test: certainly, two } \\
\text { others: not certainly }(0.065 \\
>p>\mathbf{0 . 0 4 6 )}\end{array}$ \\
\hline R1 (LH), R2 (TH) & 9 & $\begin{array}{l}0.47 \text { (Isodectes }+ \\
\text { Neldasaurus) }\end{array}$ & certainly $(0.225>p>0.133)$ \\
\hline R1 (LH), R3 (PH) & 12 & $\mathbf{0 . 8 4}$ (Dissorophoidea) & $\begin{array}{l}\text { not certainly }(0.035>p> \\
0.017)\end{array}$ \\
\hline R2 (TH), R3 (PH) & 3 & $\mathbf{0 . 8 4}$ (Dissorophoidea) & certainly $(p>0.4)$ \\
\hline R4 (LH), R5 (TH) & 10 & $\begin{array}{l}0.40 \text { (Doleserpeton }+ \\
* \text { Gerobatrachus) }\end{array}$ & certainly $(0.227>p>0.183)$ \\
\hline R4 (LH), R6 (PH) & 15 & $\mathbf{0 . 6 7}$ (modern amphibians) & certainly $(0.133>p>0.056)$ \\
\hline R5 (TH), R6 (PH) & 5 & 0.67 (modern amphibians) & certainly $(\mathfrak{p}>0.31)$ \\
\hline
\end{tabular}




\section{Table 5 (on next page)}

Maximum similarities of trees from our analyses expressed as ITRIs (in \%).

The trees used here are the ones used for the statistical tests for lack of distinguishability (text and Table 4), meaning they are chosen for being as similar as possible and are included in Data S3. The ITRI is asymmetric; the columns show the reference trees, the lines represent the trees compared to each reference tree. Comparisons represented by empty cells were deemed uninteresting and not made. See Tables 1 and 3 or the text for more information about the analyses. 
1 Maximum similarities of trees from our analyses expressed as ITRIs (in \%). The trees used here 2 are the ones used for the statistical tests for lack of distinguishability (text and Table 4), meaning 3 they are chosen for being as similar as possible and are included in Data S3. The ITRI is 4 asymmetric; the columns show the reference trees, the lines represent the trees compared to each 5 reference tree. Comparisons represented by empty cells were deemed uninteresting and not 6 made. See Tables 1 and 3 or the text for more information about the analyses.

7

\begin{tabular}{|l|l|l|l|l|l|l|l|l|}
\hline$\downarrow$ compared to $\rightarrow$ & $\begin{array}{l}\text { O1 } \\
(\mathrm{TH})\end{array}$ & $\begin{array}{l}\text { O2 } \\
(\mathrm{LH})\end{array}$ & $\begin{array}{l}\text { O3 } \\
(\mathrm{PH})\end{array}$ & $\begin{array}{l}\text { R1 } \\
(\mathrm{LH})\end{array}$ & $\begin{array}{l}\text { R2 } \\
(\mathrm{TH})\end{array}$ & $\begin{array}{l}\text { R3 } \\
(\mathrm{PH})\end{array}$ & $\begin{array}{l}\text { R4 } \\
(\mathrm{LH})\end{array}$ & $\begin{array}{l}\text { R5 } \\
(\mathrm{TH})\end{array}$ \\
\hline O2 $(\mathrm{LH})$ & 86.7 & & & & & & & \\
\hline O3 $(\mathrm{PH})$ & 95.5 & 92.8 & & & & & & \\
\hline R1 (LH) & 72.0 & 92.8 & & & & & & \\
\hline R2 $(\mathrm{TH})$ & 76.7 & 82.0 & & 86.5 & & & & \\
\hline R3 $(\mathrm{PH})$ & & & 78.9 & 95.7 & 85.3 & & & \\
\hline R4 (LH) & 75.6 & 88.2 & & 92.7 & & & & \\
\hline R5 (TH) & 90.2 & 80.1 & & & 79.8 & & 87.8 & \\
\hline R6 (PH) & & & 88.0 & & & 88.6 & 87.5 & 97.5 \\
\hline EB $(\mathrm{LH})$ & 71.2 & 81.3 & & 96.7 & & & 84.2 & \\
\hline
\end{tabular}

8 


\section{Table 6(on next page)}

OTUs with the highest numbers (up to a rank of 20) of score changes that are marked red in App. S2, as counted in Data S4.

In these cases the score in RC07 disagrees with the definition or description of the respective character in RC07, without further interpretation of the character. References are provided in the text (Material and methods: Modifications to individual cells) and in App. S1. 
1 OTUs with the highest numbers (up to a rank of 20) of score changes that are marked red in App.

2 S2, as counted in Data S4. In these cases the score in RC07 disagrees with the definition or

3 description of the respective character in $\mathrm{RC} 07$, without further interpretation of the character.

4 References are provided in the text (Material and methods: Modifications to individual cells) and

5 in App. S1.

6

\begin{tabular}{|c|c|c|c|}
\hline Rank & OTU & $\begin{array}{l}\text { Number } \\
\text { of red } \\
\text { scores }\end{array}$ & $\begin{array}{l}\text { Relevance to lissamphibian origins if any, main } \\
\text { sources of changes }\end{array}$ \\
\hline 1 & Ventastega & 104 & $\begin{array}{l}\text { New material described 2008; description } 1994 \text { had only } \\
\text { partially been used. }\end{array}$ \\
\hline 2 & Lethiscus & 96 & $\begin{array}{l}\text { Redescribed 2017; redescription } 2003 \text { had not been used } \\
\text { either. }\end{array}$ \\
\hline 3 & Cochleosaurus & 68 & $\begin{array}{l}\text { Postcranium described 2009; skull redescription } 2004 \text { had } \\
\text { not been used either. }\end{array}$ \\
\hline 4 & Batropetes & 67 & Close to Lissamphibia in LH. Redescribed 2013, 2015. \\
\hline 5 & Kotlassia & 58 & Redescription 2003 had not been used. \\
\hline 6 & Trimerorhachis & 57 & Redescribed 2007, 2013; pers. obs. \\
\hline 7 & Ossinodus & 54 & New material described 2007, 2014. \\
\hline 8 & Doleserpeton & 52 & Close to Lissamphibia in TH. Redescribed 2008, 2010. \\
\hline 9 & Albanerpetidae & 50 & $\begin{array}{l}\text { Next to or in Lissamphibia. New material described } \\
\text { 2013; descriptions 2002, } 2003 \text { and } 2005 \text { had not been } \\
\text { used. }\end{array}$ \\
\hline 10 & Isodectes & 49 & Pers. obs. of nearly complete undescribed postcrania. \\
\hline 11 & Orobates & 45 & Digital model 2015 showing previously unpublished data. \\
\hline 12 & Diplocaulus & 43 & Descriptions 1909, 1917 had not been used. \\
\hline 13 & Platyrhinops & 42 & Close to Lissamphibia in TH. Redescribed 2010, 2012. \\
\hline 13 & Cardiocephalus & 42 & Effect of scoring both species instead of just one. \\
\hline 15 & Edops & 41 & $\begin{array}{l}\text { Pers. obs. especially of neglected postcrania briefly } \\
\text { described in } 1942 \text {. }\end{array}$ \\
\hline 16 & Eocaecilia & 40 & Lissamphibian. Redescribed 2007; pers. obs. \\
\hline 17 & Acheloma & 38 & Second species described 2010,2011 \\
\hline 17 & Triadobatrachus & 38 & $\begin{array}{l}\text { Lissamphibian. Redescribed 2012, } 2016 \text { (with digital } \\
\text { model); pers. obs. }\end{array}$ \\
\hline 19 & Baphetes & 35 & $\begin{array}{l}\text { Redescribed 2009; new material published } 1998 \text { had not } \\
\text { been fully taken into account either. }\end{array}$ \\
\hline 20 & Limnoscelis & 34 & $\begin{array}{l}\text { Redescribed 2007, 2010; redescription } 1983 \text { (thesis) had } \\
\text { only partially been used. }\end{array}$ \\
\hline
\end{tabular}




\section{Table 7 (on next page)}

Characters with the highest numbers of score changes (up to a rank of 20) that are marked red in App. S2, as counted in Data S4.

In these cases the score in RC07 disagrees with the definition or description of the respective character in RC07, but we did not redefine any states, and ontogeny had no impact on our score changes. Columns $\mathrm{O}$ and $\mathrm{R}$ show the relative support for the hypotheses on lissamphibian origins in the original ( 0 ; in case of mergers we present the net total support from all component characters) and our revised matrix ( $R$ ) found by optimizing each character on the first three trees in Data S7 (from Analyses 01-03) and Data S3 (from Analyses R1-R3). Abbreviations: LP 1: the character has one fewer step on the trees supporting the LH and the PH in Data S7 (column O) or Data S3 (column R) than on the respective tree supporting the $\mathrm{TH} ; \mathrm{T} 4, \mathrm{P} 1$ : the character has one fewer step on the tree supporting the $\mathrm{PH}$ than on the one supporting the $\mathrm{LH}$, and four fewer on the one supporting the TH than on the one supporting the $\mathrm{LH}$; and so on; -: no difference between the three trees (equal to LTP 0). 
1 Characters with the highest numbers of score changes (up to a rank of 20) that are marked red in

2 App. S2, as counted in Data S4. In these cases the score in RC07 disagrees with the definition or 3 description of the respective character in $\mathrm{RC} 07$, but we did not redefine any states, and ontogeny

4 had no impact on our score changes. Columns $\mathrm{O}$ and $\mathrm{R}$ show the relative support for the

5 hypotheses on lissamphibian origins in the original $(\mathrm{O}$; in case of mergers we present the net

6 total support from all component characters) and our revised matrix (R) found by optimizing

7 each character on the first three trees in Data S7 (from Analyses O1-O3) and Data S3 (from

8 Analyses R1-R3). Abbreviations: LP 1: the character has one fewer step on the trees supporting

9 the LH and the PH in Data S7 (column O) or Data S3 (column R) than on the respective tree supporting the TH; T 4, P 1: the character has one fewer step on the tree supporting the PH than on the one supporting the $\mathrm{LH}$, and four fewer on the one supporting the $\mathrm{TH}$ than on the one supporting the LH; and so on; -: no difference between the three trees (equal to LTP 0).

\begin{tabular}{|c|c|c|c|c|c|}
\hline Rank & Character & $\begin{array}{l}\text { Number } \\
\text { of red } \\
\text { scores }\end{array}$ & $\mathbf{O}$ & $\mathbf{R}$ & Main sources of changes \\
\hline 1 & $\begin{array}{l}\text { 257: TRU VER } \\
4\end{array}$ & 50 & - & - & $\begin{array}{l}\text { Description says "centra"; had been scored } \\
\text { only for pleurocentra, possibly under } \\
\text { untenable assumption that hemal arches } \\
\text { are homologous to intercentra or parts } \\
\text { thereof. Discussed in App. S1. }\end{array}$ \\
\hline 2 & 215: HUM 13 & 37 & - & - & $20^{\text {th }}$-century literature. \\
\hline 3 & 101: SC 2 & 34 & - & - & $\begin{array}{l}\text { Literature of all ages; misinterpretation by } \\
\text { Ruta, Coates \& Quicke (2003) of wording } \\
\text { in CG78, discussed in App. S1. }\end{array}$ \\
\hline 3 & 225: ILI 3 & 34 & - & - & $\begin{array}{l}\text { Homology across the taxon sample left } \\
\text { unclear by RC07, discussed in App. S1. }\end{array}$ \\
\hline 5 & $\begin{array}{l}\text { 40: POSPAR 3- } \\
6\end{array}$ & 32 & LP 1 & - & Literature mostly from 2003 and later. \\
\hline 6 & $\begin{array}{l}\text { 276: DIG 1-2-3- } \\
4\end{array}$ & 30 & - & - & Mostly $20^{\text {th }}$-century literature; pers. obs. \\
\hline 7 & 100: SC 1 & 29 & - & - & Literature of all ages. \\
\hline 7 & 102: VOM 1-13 & 29 & T 2 & - & Literature mostly from 2003 and later. \\
\hline 7 & 119: PTE 3-9 & 29 & LT 1 & LP 1 & Literature of all ages and pers. obs. \\
\hline 10 & $\begin{array}{l}\text { 134: EXOCC 2- } \\
3-4-5 / \text { BASOCC } \\
1-5\end{array}$ & 26 & T 2 & LT 1 & Literature of all ages and pers. obs. \\
\hline 11 & $\begin{array}{l}\text { 146: JAW ART } \\
\text { 1/SQU 2/DEN } 8 \\
\end{array}$ & 25 & L 1 & $\begin{array}{l}\text { T } 4, \\
\text { P } 1\end{array}$ & $20^{\text {th }}$-century literature. \\
\hline 12 & 17: PREFRO 10 & 24 & T 1 & - & $20^{\text {th }}$-century literature. \\
\hline 12 & 66: POSORB 7 & 24 & - & T 1 & Literature of all ages. \\
\hline 12 & $\begin{array}{l}\text { 219: HUM } \\
\text { 18/DIG } 1\end{array}$ & 24 & T 1 & LP 3 & $20^{\text {th }}$-century literature. \\
\hline 15 & 34: PAR 5 & 23 & - & LP 1 & Literature of all ages. \\
\hline 16 & 27: FRO 2 & 22 & $\begin{array}{l}\text { L 2, } \\
\text { P } 1\end{array}$ & LP 1 & Literature of all ages. \\
\hline
\end{tabular}




\begin{tabular}{|l|l|l|l|l|l|}
\hline 16 & 43: POSPAR 9 & 22 & - & - & Literature of all ages. \\
\hline 16 & 130: CHO 1 & 22 & - & - & Literature of all ages. \\
\hline 16 & $\begin{array}{l}\text { 272: TRU VER } \\
27\end{array}$ & 22 & - & - & $\begin{array}{l}\text { Artificial missing data filled in from } \\
\text { literature of all ages and pers. obs. }\end{array}$ \\
\hline 20 & $\begin{array}{l}\text { 105: VOM 5- } \\
10 / \text { PTE 10-12- } \\
18 / \text { INT VAC 1 }\end{array}$ & 21 & T 2 & T 2 & Literature mostly from 2006 and earlier. \\
\hline
\end{tabular}

14 


\section{Table 8 (on next page)}

OTUs with the highest numbers of score changes (up to a rank of 20) that are marked green in App. S2, as counted in Data S4.

These changes involve, or potentially involve, redefinitions of character states. "Complete enough to be affected by many redefinitions" applies to all OTUs in this table and is only mentioned in the absence of other major causes. 
1 OTUs with the highest numbers of score changes (up to a rank of 20) that are marked green in

2 App. S2, as counted in Data S4. These changes involve, or potentially involve, redefinitions of

3 character states. "Complete enough to be affected by many redefinitions" applies to all OTUs in

4 this table and is only mentioned in the absence of other major causes.

5

\begin{tabular}{|c|c|c|c|}
\hline Rank & OTU & $\begin{array}{l}\text { Number } \\
\text { of green } \\
\text { scores }\end{array}$ & $\begin{array}{l}\text { Relevance to lissamphibian origins if any, main causes } \\
\text { of changes }\end{array}$ \\
\hline 1 & Brachydectes & 33 & $\begin{array}{l}\text { Close to Lissamphibia in LH. Very many scores } \\
\text { inapplicable. Redescribed } 2016 .\end{array}$ \\
\hline 2 & Batropetes & 29 & $\begin{array}{l}\text { Close to Lissamphibia in LH. Many scores inapplicable. } \\
\text { Redescribed } 2013,2015 .\end{array}$ \\
\hline 3 & Lethiscus & 27 & $\begin{array}{l}\text { Unusual anatomy, some scores inapplicable. Redescribed } \\
\text { 2017; redescription } 2003 \text { had not been used either. }\end{array}$ \\
\hline 4 & Trimerorhachis & 23 & Some unusual anatomy. Redescribed 2007, 2013. \\
\hline 5 & Baphetes & 22 & $\begin{array}{l}\text { Unusual anatomy, a few scores inapplicable. Redescribed } \\
\text { 2009; new material published } 1998 \text { had not been fully } \\
\text { taken into account either. }\end{array}$ \\
\hline 5 & Valdotriton & 22 & Lissamphibian. Very many scores inapplicable. \\
\hline 5 & Gephyrostegus & 22 & $\begin{array}{l}\text { Skull redescribed 2014; redescription of axial skeleton } \\
1991 \text { had not been used either. }\end{array}$ \\
\hline 8 & Eryops & 21 & Appendicular skeleton redescribed 2006. \\
\hline 8 & Archeria & 21 & Complete enough to be affected by many redefinitions. \\
\hline 10 & Megalocephalus & 20 & Unusual anatomy, a few scores inapplicable. \\
\hline 10 & Cochleosaurus & 20 & $\begin{array}{l}\text { Postcranium described 2009; skull redescription } 2004 \text { had } \\
\text { not been used either. }\end{array}$ \\
\hline 10 & Acheloma & 20 & Redescribed 2010, 2011 \\
\hline 10 & Albanerpetidae & 20 & $\begin{array}{l}\text { Next to or in Lissamphibia. Very many scores } \\
\text { inapplicable. New material described 2013; descriptions } \\
2002,2003 \text { and } 2005 \text { had not been used. }\end{array}$ \\
\hline 10 & Triadobatrachus & 20 & $\begin{array}{l}\text { Lissamphibian. Very many scores inapplicable. } \\
\text { Redescribed 2012, } 2016 \text { (with digital model); pers. obs. }\end{array}$ \\
\hline 10 & Eoherpeton & 20 & Complete enough to be affected by many redefinitions. \\
\hline 10 & Kotlassia & 20 & Redescription 2003 had not been used. \\
\hline 10 & Limnoscelis & 20 & $\begin{array}{l}\text { Redescribed 2007, 2010; redescription } 1983 \text { (thesis) had } \\
\text { only partially been used. }\end{array}$ \\
\hline 10 & Phlegethontia & 20 & Unusual anatomy, very many scores inapplicable. \\
\hline 10 & Orobates & 20 & Digital model 2015. \\
\hline 20 & Ventastega & 19 & $\begin{array}{l}\text { New material described 2008; description } 1994 \text { had only } \\
\text { partially been used. }\end{array}$ \\
\hline 20 & Acanthostega & 19 & Skull redescribed 2015 \\
\hline 20 & Ichthyostega & 19 & $\begin{array}{l}\text { Partial redescriptions 2009, 2011, } 2012 \text { twice, 2013, } \\
\text { 2015, pers. obs. }\end{array}$ \\
\hline 20 & Dendrerpetidae & 19 & $\begin{array}{l}\text { Effect of making sure all species are scored; occiput } \\
\text { redescribed } 2005 .\end{array}$ \\
\hline 20 & Platyrhinops & 19 & Close to Lissamphibia in TH. Redescribed 2010, 2012. \\
\hline
\end{tabular}




\begin{tabular}{|l|l|l|l|}
\hline 20 & Karaurus & 19 & $\begin{array}{l}\text { Lissamphibian. Very many scores inapplicable; pers. } \\
\text { obs. }\end{array}$ \\
\hline 20 & Oestocephalus & 19 & Unusual anatomy, some scores inapplicable. \\
\hline 20 & Notobatrachus & 19 & $\begin{array}{l}\text { Lissamphibian. Very many scores inapplicable; } \\
\text { redescribed 2004, 2008. }\end{array}$ \\
\hline
\end{tabular}

6 
Table 9 (on next page)

Characters with the highest numbers of score changes (up to a rank of 20) that are marked green in App. S2, as counted in Data S4.

These characters have redefined or possibly redefined states. Columns 0 and $R$ as in Table 7. 
1 Characters with the highest numbers of score changes (up to a rank of 20) that are marked green

2 in App. S2, as counted in Data S4. These characters have redefined or possibly redefined states.

3 Columns $\mathrm{O}$ and $\mathrm{R}$ as in Table 7.

4

\begin{tabular}{|c|c|c|c|c|c|}
\hline Rank & Character & $\begin{array}{l}\text { Number } \\
\text { of green } \\
\text { scores }\end{array}$ & $\mathbf{O}$ & $\mathbf{R}$ & Main cause of changes \\
\hline 1 & $\begin{array}{l}\text { 85: MED } \\
\text { ROS } 1\end{array}$ & 102 & & - & Split off from INT FEN 1 (ch. 84, O: L1, R: -). \\
\hline 2 & $\begin{array}{l}\text { 190: TEETH } \\
10\end{array}$ & 99 & & - & $\begin{array}{l}\text { Split off from TEETH } 3 \text { (ch. 183, O: T1, R: TP } \\
\text { 2). }\end{array}$ \\
\hline 3 & $\begin{array}{l}\text { 95: SKU TAB } \\
1\end{array}$ & 98 & - & $\begin{array}{l}\text { LP } \\
2\end{array}$ & States redefined, completely rescored; see text. \\
\hline 4 & 25: MAX 8 & 97 & T 1 & - & $\begin{array}{l}\text { States redefined to make scores reproducible, } \\
\text { completely rescored. }\end{array}$ \\
\hline 5 & $\begin{array}{l}\text { 3: PREMAX } \\
7\end{array}$ & 94 & - & - & States redefined, completely rescored; see text. \\
\hline 6 & $\begin{array}{l}\text { 1: PREMAX } \\
1-2-3\end{array}$ & 87 & $\begin{array}{l}\text { LP } \\
1\end{array}$ & $\begin{array}{l}\text { LP } \\
1\end{array}$ & $\begin{array}{l}\text { States redefined to account for entire taxon } \\
\text { sample, completely rescored. }\end{array}$ \\
\hline 7 & $\begin{array}{l}\text { 145: PASPHE } \\
14\end{array}$ & 86 & T 1 & P 1 & $\begin{array}{l}\text { States redefined to account for entire taxon } \\
\text { sample, completely rescored. }\end{array}$ \\
\hline 8 & 10: NAS 5 & 68 & $\begin{array}{l}\text { TP } \\
1\end{array}$ & T 1 & $\begin{array}{l}\text { Redefined to make scores reproducible and } \\
\text { avoid massive redundancy, completely } \\
\text { rescored. }\end{array}$ \\
\hline 9 & $\begin{array}{l}\text { 64: POSORB } \\
5\end{array}$ & 62 & - & - & $\begin{array}{l}\text { Interpreted as reductive to avoid massive } \\
\text { redundancy, see text. }\end{array}$ \\
\hline 10 & 122: PTE 13 & 48 & P 1 & - & Interpreted as reductive to avoid redundancy. \\
\hline 11 & 124: PTE 16 & 44 & - & - & Interpreted as reductive to avoid redundancy. \\
\hline 12 & 83: NOS 4 & 39 & P 1 & - & $\begin{array}{l}\text { Redefined to increase applicability and avoid } \\
\text { correlation with snout length. }\end{array}$ \\
\hline 12 & 131: CHO 2 & 39 & $\begin{array}{l}\mathrm{TP} \\
1\end{array}$ & $\begin{array}{l}\text { LP } \\
1\end{array}$ & States defined to make scores reproducible. \\
\hline 14 & 56: TAB 6 & 37 & - & - & Redefined to make more useful. \\
\hline 14 & 69: SQU 3 & 37 & $\begin{array}{l}\text { LP } \\
1\end{array}$ & P 1 & $\begin{array}{l}\text { States defined to make scores reproducible and } \\
\text { avoid nonadditive binary coding. }\end{array}$ \\
\hline 14 & $\begin{array}{l}\text { 231: FEM 1- } \\
2-6\end{array}$ & 37 & L 1 & $\begin{array}{l}\text { LP } \\
1\end{array}$ & $\begin{array}{l}\text { States redefined to be primarily homologous } \\
\text { across taxon sample and to avoid redundancy. }\end{array}$ \\
\hline 17 & $\begin{array}{l}\text { 253: CER } \\
\text { VER } 4\end{array}$ & 31 & $\begin{array}{l}\text { LP } \\
1\end{array}$ & $\mathrm{~L} 1$ & Interpreted as reductive to avoid redundancy. \\
\hline 18 & 123: PTE 14 & 29 & $\begin{array}{l}\text { LT } \\
1\end{array}$ & - & States defined to make scores reproducible. \\
\hline 19 & $\begin{array}{l}\text { 265: TRU } \\
\text { VER 13-14 }\end{array}$ & 26 & $\mathrm{~L} 1$ & - & $\begin{array}{l}\text { Single-piece centra no longer assumed to be } \\
\text { necessarily pleurocentra. }\end{array}$ \\
\hline 20 & 31: PAR 1 & 22 & T 1 & $\begin{array}{l}\text { LP } \\
1\end{array}$ & Redefined to avoid redundancy. \\
\hline
\end{tabular}




\section{Table $\mathbf{1 0}$ (on next page)}

Complete list of OTUs with score changes that are marked blue in App. S2, as counted in Data S4.

These changes involve interpretations of ontogeny or heterochrony. 
1 Complete list of OTUs with score changes that are marked blue in App. S2, as counted in Data

2 S4: these changes involve interpretations of ontogeny or heterochrony.

3

\begin{tabular}{|c|c|c|c|}
\hline Rank & OTU & $\begin{array}{l}\text { Number } \\
\text { of blue } \\
\text { scores }\end{array}$ & $\begin{array}{l}\text { Relevance to lissamphibian origins if any, main } \\
\text { causes of changes }\end{array}$ \\
\hline 1 & Schoenfelderpeton & 8 & $\begin{array}{l}\text { Close to Lissamphibia in TH. Skeletally immature, } \\
\text { possibly neotenic. }\end{array}$ \\
\hline 1 & Micromelerpeton & 8 & $\begin{array}{l}\text { Somewhat close to Lissamphibia in TH. Known } \\
\text { mostly from immature individuals. }\end{array}$ \\
\hline 3 & Apateon & 7 & $\begin{array}{l}\text { Close to Lissamphibia in TH. Known mostly from } \\
\text { immature individuals, one species known to be } \\
\text { neotenic (Fröbisch \& Schoch, 2009b). }\end{array}$ \\
\hline 3 & Utegenia & 7 & Skeletally immature, possibly neotenic. \\
\hline 5 & Discosauriscus & 6 & Known mostly from immature individuals. \\
\hline 6 & Ariekanerpeton & 5 & Skeletally immature, possibly neotenic. \\
\hline 6 & Microphon & 5 & Skeletally immature, possibly neotenic. \\
\hline 8 & Amphibamus & 4 & $\begin{array}{l}\text { Close to Lissamphibia in TH. Known mostly from } \\
\text { immature individuals. }\end{array}$ \\
\hline 8 & Leptorophus & 4 & $\begin{array}{l}\text { Close to Lissamphibia in TH. Skeletally immature, } \\
\text { possibly neotenic. }\end{array}$ \\
\hline 8 & Leptoropha & 4 & Skeletally immature, possibly neotenic. \\
\hline 11 & Eucritta & 3 & $\begin{array}{l}\text { Skeletally immature, known from incomplete growth } \\
\text { series. }\end{array}$ \\
\hline 11 & Trimerorhachis & 3 & $\begin{array}{l}\text { Slow-growing; postcranium redescribed } 2007 \text { with } \\
\text { reports of more mature individuals than known before. }\end{array}$ \\
\hline 13 & Doleserpeton & 2 & $\begin{array}{l}\text { Close to Lissamphibia in TH. New growth stage of } \\
\text { ch. } 238 \text { described in } 2010 ; \text { ch. } 252 \text { known in subadult } \\
\text { but not adult specimens. }\end{array}$ \\
\hline 13 & Pederpes & 2 & $\begin{array}{l}\text { Known humerus (ch. 205) and ulna (ch. 224) } \\
\text { incompletely ossified. }\end{array}$ \\
\hline 15 & Crassigyrinus & 1 & Known ulna incompletely ossified (ch. 224). \\
\hline 15 & Chenoprosopus & 1 & Ch. 69 changes state in ontogeny. \\
\hline 15 & Broiliellus & 1 & Known humerus incompletely ossified (ch. 205). \\
\hline 15 & Archeria & 1 & Ch. 205 changes state in ontogeny. \\
\hline 15 & Kotlassia & 1 & $\begin{array}{l}\text { State of ch. } 105 \text { probably not mature in known } \\
\text { specimen. }\end{array}$ \\
\hline 15 & Batropetes & 1 & $\begin{array}{l}\text { Close to Lissamphibia in LH. Ch. } 263 \text { changes state } \\
\text { in ontogeny. }\end{array}$ \\
\hline 15 & Hapsidopareion & 1 & $\begin{array}{l}\text { Somewhat close to Lissamphibia in LH. State of ch. } \\
95 \text { probably not mature in known specimens. }\end{array}$ \\
\hline 15 & Microbrachis & 1 & Ch. 263 changes state in ontogeny. \\
\hline 15 & Hyloplesion & 1 & Ch. 122 changes state in ontogeny. \\
\hline 15 & Odonterpeton & 1 & Known ulna incompletely ossified (ch. 224). \\
\hline 15 & Silvanerpeton & 1 & Known ulna incompletely ossified (ch. 224). \\
\hline
\end{tabular}




\section{Table $\mathbf{1 1}$ (on next page)}

Complete list of characters with score changes that are marked blue in App. S2, as counted in Data S4.

These changes involve interpretations of ontogeny or heterochrony. Columns $\mathrm{O}$ and $\mathrm{R}$ as in Table 7. 
1 Complete list of characters with score changes that are marked blue in App. S2, as counted in 2 Data S4. These changes involve interpretations of ontogeny or heterochrony. Columns $\mathrm{O}$ and $\mathrm{R}$ 3 as in Table 7.

4

\begin{tabular}{|c|c|c|c|c|c|}
\hline Rank & Character & $\begin{array}{l}\text { Number } \\
\text { of blue } \\
\text { scores }\end{array}$ & $\mathbf{O}$ & $\mathbf{R}$ & Causes of changes \\
\hline 1 & 205: HUM 2 & 9 & - & LP 1 & $\begin{array}{l}\text { Distal end of humerus generally ossifies } \\
\text { late, if ever. }\end{array}$ \\
\hline 1 & 224: ULNA 1 & 9 & L 1 & - & $\begin{array}{l}\text { Proximal end of ulna generally ossifies } \\
\text { late. }\end{array}$ \\
\hline 3 & 100: SC 1 & 6 & - & - & $\begin{array}{l}\text { Lateral-line canals often disappear in } \\
\text { ontogeny. }\end{array}$ \\
\hline 3 & $\begin{array}{l}\text { 105: VOM 5- } \\
\text { 10/PTE 10-12- } \\
\text { 18/INT VAC } 1\end{array}$ & 6 & T 2 & T 2 & $\begin{array}{l}\text { Interpterygoid vacuities closed in } \\
\text { seymouriamorph and possibly baphetoid } \\
\text { ontogeny. }\end{array}$ \\
\hline 5 & 101: SC 2 & 5 & - & - & $\begin{array}{l}\text { Lateral-line canals often disappear in } \\
\text { ontogeny. }\end{array}$ \\
\hline 5 & $\begin{array}{l}\text { 146: JAW ART } \\
\text { 1/SQU 2/DEN } 8\end{array}$ & 5 & L 1 & $\begin{array}{l}\text { T } 4, \\
\text { P } 1\end{array}$ & $\begin{array}{l}\text { Jaw joint very often moves caudally in } \\
\text { ontogeny. }\end{array}$ \\
\hline 5 & $\begin{array}{l}\text { 263: TRU VER } \\
11\end{array}$ & 5 & - & - & $\begin{array}{l}\text { Neural arches fused to centra during } \\
\text { ontogeny in amniotes, at least some } \\
\text { "microsaurs" and probably } \\
\text { seymouriamorphs. }\end{array}$ \\
\hline 8 & $\begin{array}{l}\text { 22: MAX 5/PAL } \\
5\end{array}$ & 2 & T 1 & LT 1 & $\begin{array}{l}\text { Apateon changed to state } 2 \text { in ontogeny, } \\
\text { Schoenfelderpeton only known from } \\
\text { morphologically immature individuals } \\
\text { showing the immature state } 1 \text {. }\end{array}$ \\
\hline 8 & 27: FRO 2 & 2 & $\begin{array}{l}\text { L } 2, \\
\text { P } 1\end{array}$ & LP 1 & $\begin{array}{l}\text { Apateon changed to state } 2 \text { (already } \\
\text { scored in RC07) in ontogeny; } \\
\text { Leptorophus and Schoenfelderpeton only } \\
\text { known from less mature individuals. }\end{array}$ \\
\hline 8 & 43: POSPAR 9 & 2 & - & - & $\begin{array}{l}\text { Microphon changed from state } 0 \text { to } 1 \text { in } \\
\text { ontogeny; Leptoropha only known from } \\
\text { individuals that have state } 0 \text { and are } \\
\text { ontogenetically comparable to those of } \\
\text { Microphon with state } 0 \text {. }\end{array}$ \\
\hline 8 & 69: SQU 3 & 2 & LP 1 & P 1 & $\begin{array}{l}\text { Temporal embayment often became } \\
\text { narrower notch in ontogeny, affecting } \\
\text { Chenoprosopus and Micromelerpeton. }\end{array}$ \\
\hline 8 & 189: ТЕETH 9 & 2 & - & T 1 & $\begin{array}{l}\text { Number of maxillary teeth increased in } \\
\text { ontogeny in Apateon and Leptorophus. }\end{array}$ \\
\hline 8 & 261: TRU VER 9 & 2 & LP 1 & LP 1 & $\begin{array}{l}\text { Trunk pleurocentra fused middorsally in } \\
\text { seymouriamorph ontogeny, including } \\
\text { Utegenia and probably Ariekanerpeton. }\end{array}$ \\
\hline 14 & 14: PREFRO 3 & 1 & P 1 & - & Micromelerpeton changed from 1 to 0 in \\
\hline
\end{tabular}




\begin{tabular}{|c|c|c|c|c|c|}
\hline & & & & & ontogeny. \\
\hline 14 & 23: MAX 6 & 1 & - & - & $\begin{array}{l}\text { Schoenfelderpeton scored as unknown } \\
\text { following closure of maxillary arcade in } \\
\text { ontogeny of Apateon. }\end{array}$ \\
\hline 14 & 37: PAR 8 & 1 & T 1 & LP 2 & $\begin{array}{l}\text { Suture became more interdigitated in } \\
\text { ontogeny of Apateon. }\end{array}$ \\
\hline 14 & 54: TAB 2-3-9 & 1 & - & - & $\begin{array}{l}\text { Tabular "horns" became longer and more } \\
\text { pointed in ontogeny of Apateon. }\end{array}$ \\
\hline 14 & 66: POSORB 7 & 1 & - & T 1 & $\begin{array}{l}\text { Postorbital became wider in ontogeny of } \\
\text { Apateon. }\end{array}$ \\
\hline 14 & 72: JUG 2-6 & 1 & LP 1 & - & $\begin{array}{l}\text { Schoenfelderpeton skeletally too } \\
\text { immature to be scored. }\end{array}$ \\
\hline 14 & 74: JUG 4 & 1 & - & T 1 & Eucritta has juvenile eye size. \\
\hline 14 & 87: ORB 2 & 1 & P 1 & $\begin{array}{l}\text { P 2, } \\
\text { T } 1\end{array}$ & Eucritta has juvenile eye size. \\
\hline 14 & 88: ORB 3/LAC 5 & 1 & - & - & Eucritta has juvenile eye size. \\
\hline 14 & 95: SKU TAB 1 & 1 & - & LP 2 & $\begin{array}{l}\text { Postorbital part of Hapsidopareion skull } \\
\text { became longer in ontogeny. }\end{array}$ \\
\hline 14 & 103: VOM 3 & 1 & - & LP 1 & $\begin{array}{l}\text { Amphibamus grew vomerine tusks in } \\
\text { ontogeny. }\end{array}$ \\
\hline 14 & 110: PAL 1 & 1 & - & LP 1 & $\begin{array}{l}\text { Amphibamus grew palatine tusks in } \\
\text { ontogeny. }\end{array}$ \\
\hline 14 & 113: PAL 7 & 1 & $\begin{array}{l}\text { P } 2, \\
\text { L } 1\end{array}$ & P 1 & $\begin{array}{l}\text { Shape of palatine, unknown in the most } \\
\text { mature Amphibamus specimens, may } \\
\text { depend on tusks. }\end{array}$ \\
\hline 14 & 122: PTE 13 & 1 & P 1 & - & $\begin{array}{l}\text { Interpterygoid vacuities of Hyloplesion } \\
\text { become wider in ontogeny. }\end{array}$ \\
\hline 14 & $\begin{array}{l}\text { 137: PASPHE 2- } \\
12\end{array}$ & 1 & - & LT 1 & $\begin{array}{l}\text { Amphibamus changed from state } 2 \text { to } 1 \text { in } \\
\text { ontogeny. }\end{array}$ \\
\hline 14 & 196: INTCLA 3 & 1 & - & T 1 & $\begin{array}{l}\text { Schoenfelderpeton skeletally too } \\
\text { immature to be scored. }\end{array}$ \\
\hline 14 & 204: HUM 1 & 1 & - & - & $\begin{array}{l}\text { Trimerorhachis acquired state } 1 \text { rather } \\
\text { early in ontogeny. }\end{array}$ \\
\hline 14 & 215: HUM 13 & 1 & - & - & $\begin{array}{l}\text { Entepicondyle kept ossifying in large } \\
\text { Trimerorhachis. }\end{array}$ \\
\hline 14 & 238: FIB 3 & 1 & - & - & Doleserpeton grew a ridge in ontogeny. \\
\hline 14 & 250: RIB 7 & 1 & T 1 & T 1 & Ribs of Micromelerpeton grew longer. \\
\hline 14 & 252: CER VER 3 & 1 & LT 1 & L 1 & Ontogeny of Doleserpeton unclear. \\
\hline 14 & $\begin{array}{l}\text { 262: TRU VER } \\
10\end{array}$ & 1 & - & - & $\begin{array}{l}\text { Utegenia scored as unknown following } \\
\text { swelling of neural arches in ontogeny of } \\
\text { Discosauriscus. }\end{array}$ \\
\hline
\end{tabular}




\section{Table 12 (on next page)}

Tests of the hypotheses that our revisions of scores have favored the $\mathrm{LH}$ or the $\mathrm{TH}$.

These tests rest on a binomial distribution of the probability of finding patterns at least as asymmetrical as the ones observed in Tables 7, 9 and 11 separately and together (last test). $\mathrm{H}_{0}$ is that the changes are random, $\mathrm{H}_{1}$ is that they are biased in favor of the $\mathrm{LH}$ or the $\mathrm{TH}$; all four tests fail to reject $\mathrm{H}_{0}\left(0.10<\mathrm{p}<0.69\right.$; two-tailed because $\mathrm{H}_{1}$ consists of two diametrically opposed hypotheses corresponding with the two tails of the distribution). We have not tested bias in favor of the PH because there is no reason to expect it either in RC07 or in our revision (Tables 1,4). "Changes" are the changes between columns 0 and $\mathrm{R}$ of the respective Tables. See the text for more information. 
1 Tests of the hypotheses that our revisions of scores have favored the LH or the TH. These tests

2 rest on a binomial distribution of the probability of finding patterns at least as asymmetrical as

3 the ones observed in Tables 7, 9 and 11 separately and together (last test). $\mathrm{H}_{0}$ is that the changes

4 are random, $\mathrm{H}_{1}$ is that they are biased in favor of the $\mathrm{LH}$ or the $\mathrm{TH}$; all four tests fail to reject $\mathrm{H}_{0}$

$5 \quad\left(0.10<\mathrm{p}<0.69\right.$; two-tailed because $\mathrm{H}_{1}$ consists of two diametrically opposed hypotheses

6 corresponding with the two tails of the distribution). We have not tested bias in favor of the $\mathrm{PH}$

7 because there is no reason to expect it either in RC07 or in our revision (Tables 1,4). "Changes"

8 are the changes between columns $\mathrm{O}$ and $\mathrm{R}$ of the respective Tables. See the text for more

9 information.

10

\begin{tabular}{|c|c|c|c|}
\hline \multicolumn{4}{|c|}{ Table 7} \\
\hline & Character & LH & TH \\
\hline 5 & 40 & & 1 \\
\hline 7 & 102 & 2 & \\
\hline 7 & 119 & 1 & \\
\hline 10 & 134 & 2 & \\
\hline 11 & 146 & & 5 \\
\hline 12 & 17 & 1 & \\
\hline 12 & 66 & & 1 \\
\hline 12 & 219 & 4 & \\
\hline 15 & 34 & 1 & \\
\hline 16 & 27 & & 1 \\
\hline \multicolumn{2}{|l|}{ Total } & 11 & 8 \\
\hline \multicolumn{4}{|c|}{$p=0.6476$} \\
\hline \multicolumn{4}{|c|}{ Table 9} \\
\hline \multirow[t]{2}{*}{ Rank } & \multirow{2}{*}{ Character } & \multicolumn{2}{|l|}{ Changes favoring } \\
\hline & & LH & TH \\
\hline 3 & 95 & 2 & \\
\hline 4 & 25 & 1 & \\
\hline 7 & 145 & 1 & \\
\hline 12 & 131 & 2 & \\
\hline 14 & 69 & & 1 \\
\hline 19 & 265 & & 1 \\
\hline 20 & 31 & 2 & \\
\hline \multicolumn{2}{|c|}{ Total } & 8 & 2 \\
\hline \multicolumn{4}{|c|}{$p=0.1094$} \\
\hline \multicolumn{4}{|c|}{ Table 11} \\
\hline \multirow[t]{2}{*}{ Rank } & \multirow[t]{2}{*}{ Character } & \multicolumn{2}{|l|}{ Changes favoring } \\
\hline & & LH & TH \\
\hline 1 & 205 & 1 & \\
\hline 1 & 224 & & 1 \\
\hline 5 & 146 & & 5 \\
\hline 8 & 22 & 1 & \\
\hline 8 & 27 & & 1 \\
\hline
\end{tabular}




\begin{tabular}{|c|c|c|c|}
\hline 8 & 69 & & 1 \\
\hline 8 & 189 & & 1 \\
\hline 14 & 37 & 3 & \\
\hline 14 & 66 & & 1 \\
\hline 14 & 72 & & 1 \\
\hline 14 & 74 & & 1 \\
\hline 14 & 87 & & 1 \\
\hline 14 & 95 & 2 & \\
\hline 14 & 103 & 1 & \\
\hline 14 & 110 & 1 & \\
\hline 14 & 113 & & 1 \\
\hline 14 & 196 & & 1 \\
\hline 14 & 252 & 1 & \\
\hline \multicolumn{2}{|c|}{ Total } & 10 & 15 \\
\hline \multicolumn{4}{|c|}{$p=0.4244$} \\
\hline \multicolumn{4}{|c|}{ Global test } \\
\hline & & \multicolumn{2}{|l|}{ Changes favoring } \\
\hline & & LH & TH \\
\hline Table 7 & & 11 & 8 \\
\hline Table 9 & & 8 & 2 \\
\hline Table 11 & & 10 & 15 \\
\hline Total & & 29 & 25 \\
\hline \multicolumn{4}{|c|}{$p=0.6835$} \\
\hline
\end{tabular}

